\title{
28th Annual Conference of the International Society for Quality of Life Research
}

\section{Cutting Edge Research Plenary Session}

(1) Developing a multimedia patient-reported outcomes measure for low literacy patients

Chao Long, MD MPH, The Johns Hopkins Hospital and The Curtis National Hand Center, Baltimore, Maryland, USA; Laura Beres, PhD, MPH, Johns Hopkins Bloomberg School of Public Health, Baltimore, Maryland, USA; Albert Wu, MD, MPH, Johns Hopkins Bloomberg School of Public Health, Baltimore, Maryland, USA; Aviram Giladi, MD, MS, The Curtis National Hand Center, MedStar Union Memorial Hospital, Baltimore, Maryland, USA

Aims: Almost all patient-reported outcomes measures (PROMs) are text based. This poses a barrier to accurate completion in low literacy populations. We developed the Multimedia Adaptation Protocol (MAP) for adapting validated, text-based PROMs to multimedia versions that can be self-administered in mixed literacy populations. We aimed to execute the first stage of the MAP, forward adaptation, to adapt the Patient-Reported Outcomes Measurement Information System Upper Extremity Short Form (PROMIS-UE) to a multimedia version (mPROMIS-UE) for a mixed literacy hand surgery patient population in Baltimore, Maryland (USA). Methods: Taking a community-engaged and human-centered design approach, forward adaptation included six phases undertaken in a serial, iterative fashion (Table 1). Direct observations were documented in memos which underwent rapid thematic analysis. Interviews were audio recorded and documented using analytic memos; framework analysis was used to identify inductive and deductive themes. Themes were further analyzed to identify personas and distilled into design challenges to guide ideation and prototyping that involved multidisciplinary research team members. To ensure the credibility of our findings, we consulted our community advisory board (CAB) and collected additional interviews including member checking of initial findings. Results: We conducted $12 \mathrm{~h}$ of observations. We interviewed 17 adult English-speaking participants (12 patients, 3 caregivers, 2 staff) of mixed literacy (age 20-81 years). We identified two distinct user personas and three distinct literacy personas; we developed the mPROMIS-UE with these personas in mind. Themes from interviews were distilled into four broad design challenges: surrounding literacy, customizability, convenience, and shame. We identified features (audio, animations, icons, avatars, progress indicator) that addressed the design challenges. These features were synthesized into a prototype that underwent four iterations of refinement. Conclusion: We successfully adapted the PROMIS-UE to an mPROMIS-UE that addresses the challenges identified in a mixed literacy hand surgery population. We demonstrated the feasibility of adapting PROMs to multimedia versions. The mPROMIS-UE is ready to undergo the remaining stages of the MAP (back adaptation, qualitative evaluation, validation). A validated mPROMIS-UE will expand clinicians' and investigators' ability to capture PROs in mixed literacy populations.

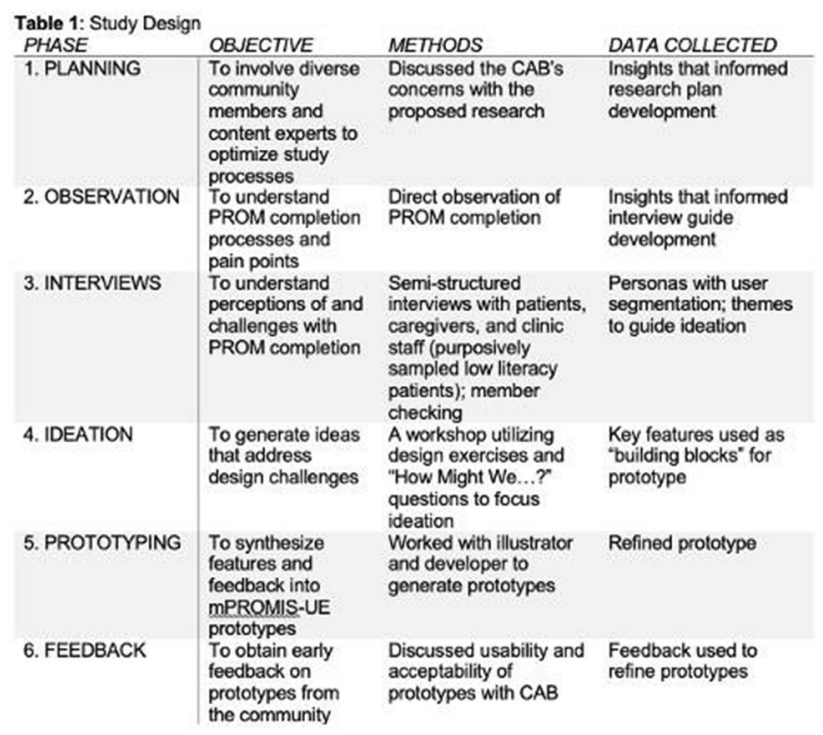

(2) Fortitude in the face of a pandemic: mediation modeling to characterize resilience during COVID-19

Katrina Borowiec, MA, Boston College, Chestnut Hill, Massachusetts, USA; Roland Stark, M.Ed., DeltaQuest Foundation, Concord, Massachusetts, USA; Bruce Rapkin, Ph.D., Department of Epidemiology and Population Health, Albert Einstein College of Medicine, Bronx, New York, USA; Carolyn Schwartz, Sc.D., DeltaQuest Foundation, Concord, Massachusetts, USA

Aims: We are living in extraordinary times. The novel coronavirus disease 2019 (COVID) has led to a world-wide health crisis that has required enormous changes in our lives. By dint of its different repercussions associated with sociodemographic and other personal characteristics, COVID presents a unique opportunity to study resilience. This study evaluated the differential impact of stressors and 
psychosocial resources on quality-of-life (QOL) outcomes, and investigated whether attitudes, perspectives, and behaviors relevant to wellness protect one from the negative aspects of the pandemic. Methods: This cross-sectional study done Spring/Summer 2020 recruited patients and caregivers of people with chronic medical conditions and a nationally representative comparison sample of USA adults. Linear regression investigated the associations between COVID-specific variables and QOL outcomes, after covariate adjustment. Structural Equation Modeling investigated whether the links between Resilience and COVID-specific variables were mediated by attitudes, perspectives, and behaviors relevant to wellness, as measured by the DeltaQuest Wellness Measure. Results: The sample included 3,085 chronically ill patients, 685 caregivers, 191 patient/caregivers, and 855 in the comparison group. The sample had a mean age of $51.6(\mathrm{SD}=14.2) ; 82 \%$ were female; $89 \%$ white, $6 \%$ black, $4 \%$ other, and 5\% were Hispanic. Participants seemed knowledgeable of and adherent to the practices endorsed by publichealth experts. COVID-specific Hardship, Interpersonal Conflict, and Worry were associated with worse QOL outcomes, and Growth, Social Support, and Coping were associated with better. Wellness was the most salient predictor of Resilience, functioning both as a main effect and mediator for COVID-specific predictors. People with lower levels of Worry and/or higher levels of Social Support tended to have better-than-expected daily performance in the face of the pandemic. These two predictors acted in large part through the attitudes, perspectives, and behaviors relevant to Wellness. Conclusion: Our findings support the idea that cultivating Wellness by dint of one's perspective, attitudes, and behaviors can be an important buffer to challenging times during a pandemic. Wellness seems to support resilience in its own right in addition to being a mechanism through which other factors can do so. Enhancing this type of Wellness may dovetail with the goals of mindfulness interventions.

\section{(3) Impact of Racial and Ethnic Disparities on Patient-Reported Outcomes Following Cervical Spine Surgery Using the Data from National Spine Registry}

Jacquelyn S. Pennings, Ph.D., Vanderbilt University Medical Center, Nashville, Tennessee, USA; Hiral Master, PT, PhD, Vanderbilt University Medical Center, Nashville, Tennessee, USA; Claudia Davidson, MPH, Vanderbilt University Medical Center, Nashville, Tennessee, USA; Rogelio Coronado, PT, PhD, Vanderbilt University Medical Center, Nashville, Tennessee, USA; Clinton Devin, MD, Steamboat Orthopaedic Spine Institute, Steamboat Springs, Colorado, USA; Kristin Archer, PhD, DPT, Vanderbilt University Medical Center, Nashville, Tennessee, USA

Aims: The aim of the study is to investigate the association of race and ethnicity with patient-reported health outcomes after cervical spine surgery. Methods: Data from 14,136 QOD participants (age = $56.8 \pm 11.5 ; \mathrm{BMI}=30.2 \pm 6.4 ; 49 \%$ females) across 28 states in the Unites States who underwent elective surgery for degenerative cervical spine disease and completed 1-year follow-up were retrospectively analyzed. Participants self-identified as Hispanic
( $n=343)$, non-Hispanic Black $(n=1191)$, Asian $(n=112)$, or White $(n=12,490)$. PROMs were used for assessing disability (Neck Disability Index: NDI), neck, and arm pain (11-point Numeric Rating Scale), and health-related quality of life (EuroQol EQ-5D) at the preoperative and 1-year time point after surgery. Minimal symptom state at 1 year was defined as achieving a score of $\leq 20$ on NDI, and neck and arm pain scores $\leq 2$. Satisfaction was assessed using a single-item question at 1 year after surgery (NASS scale). Multivariable linear and logistic regression analyses were conducted to compute effect estimates and 95\% confidence interval (CI) to determine the association of racial/ethnic groups with PROs. Covariates included sociodemographic items (e.g. age, sex, BMI, insurance, education, employment status), U.S. census regions, surgical variables (e.g. surgical approach, pathology), opioid use, and preop PROs. Multiple imputation was used for missing data $(<5 \%$ missing). Results: Of the 14,136 participants, $48 \%$ achieved minimal symptom state at 1 year. Trajectories of continuous outcomes by group are shown in Fig. 1. Black non-Hispanic had 57\% (odds ratio[95\% CI]: $1.57[1.37,1.81])$ and Hispanics had $31 \%(1.31[1.02,1.69])$ higher odds of not achieving minimal symptom state at 1-year follow-up compared to White non-Hispanic (Fig. 2). Similar findings for Black or Hispanics vs. White groups were observed for other PROs and satisfaction. There were no differences in any outcome measures between Asian and White. Conclusion: Compared to White, Asian did not have increased likelihood, but Hispanics and Black groups did have increased likelihood of reporting poor outcomes following cervical spine surgery, even after adjusting for potential confounders. The findings of this study suggest the need to account for inherent racial and ethnic disparities to better understand the recovery trajectories following cervical spine surgery. Also, strategies to minimize racial disparities are needed to ensure equitable access to postoperative care in this surgical population.

Figure 1. Means of continuous PROMs over time by group
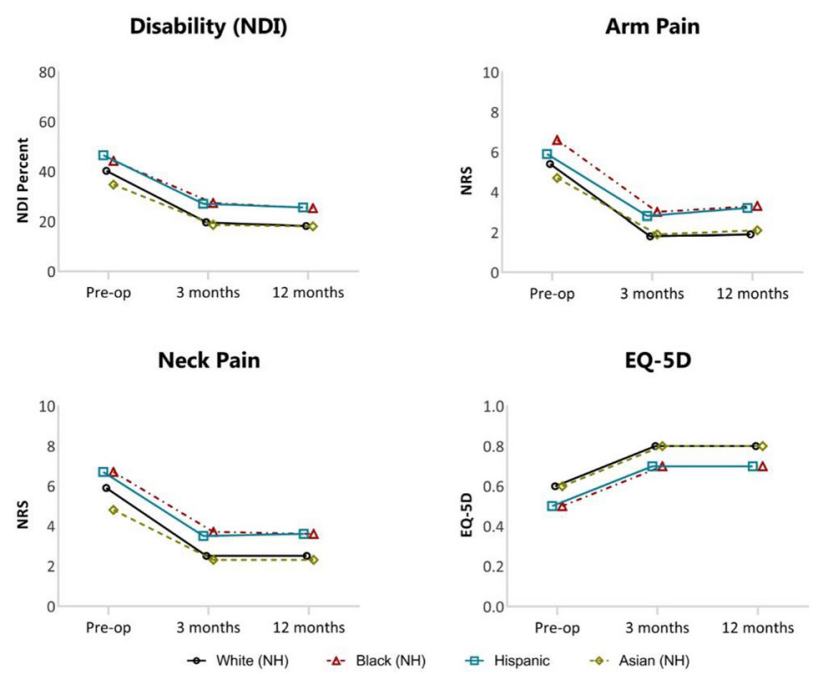
Figure 2. Adjusted odds ratios $(95 \% \mathrm{Cl})$ of race/ethnic groups on PROMs

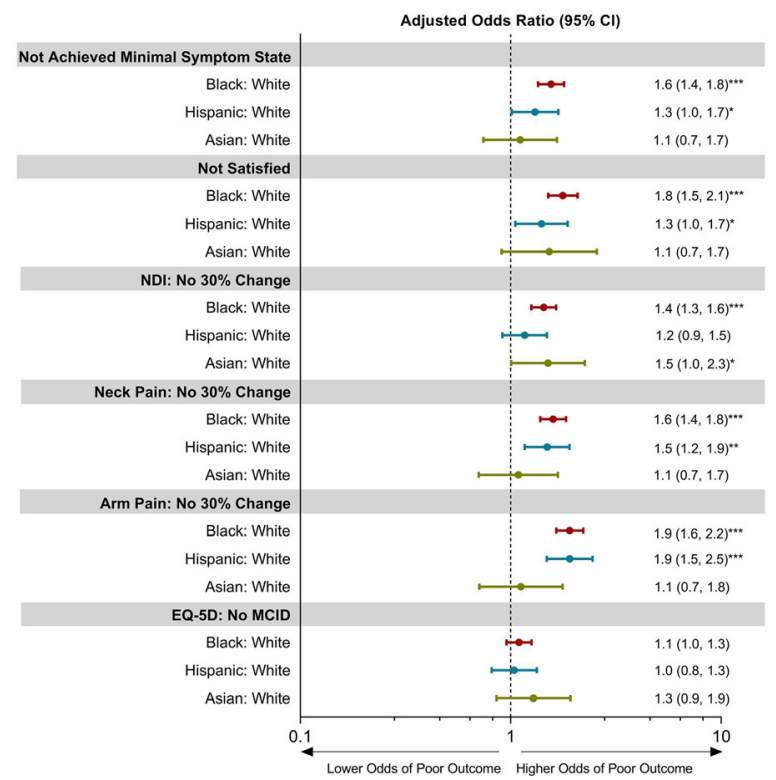

(4) Personalized Symptom Management Strategies by Bayesian Nonparametric Model (BAM)

Yuelin Li, PhD, Memorial Sloan Kettering Cancer Center, New York, New York, USA; Kevin Liou, MD, Memorial Sloan Kettering Cancer Center, New York, New York, USA; Thomas M. Atkinson, PhD, Memorial Sloan Kettering Cancer Center, New York, New York, USA; Jun Mao, MD, Memorial Sloan Kettering Cancer Center, New York, New York, USA

Aims: Personalized treatments based on an individual's PRO characteristics may be more effective than a one-size-fits-all approach, especially among cancer survivors who frequently experience multiple "clustering" of co-morbid symptoms. We describe an application of Bayesian nonparametric method, which leverages novel Machine Learning algorithms, to identify symptom clusters and to evaluate whether treatment responses differ by symptom clusters. Methods: This study is a secondary analysis of a PCORI-funded comparative effectiveness trial that evaluated acupuncture versus cognitive behavioral therapy for insomnia (CBT-I) (CER-1403-14292-IC). The trial evenly randomized 160 cancer survivors with a DSM-IV insomnia diagnosis to acupuncture or CBT-I. Interventions were delivered over 8 weeks. Outcomes included insomnia (primary outcome), fatigue, anxiety, and pain, assessed at baseline and posttreatment (weeks 8 and 20). We used a Bayesian nonparametric technique with a Dirichlet Process prior to identify symptom clusters and to evaluate whether treatment responses to acupuncture or CBT-I differed by symptom clusters. Results: Mean age was 62.5 years $(\mathrm{SD}=11.7), 91(57 \%)$ were women, $113(71 \%)$ were white. BAM identified three symptom clusters at baseline: (1) "insomnia-predominant" patients $(N=84)$, characterized by high insomnia severity and low severity of other symptoms; (2) "high symptom burden" patients $(N=54)$, characterized by high severity across all symptoms; and (3) "insomnia-pain" patients $(N=21)$, characterized by high severity of insomnia and pain. CBT-I was more effective than acupuncture at reducing insomnia among "insomnia-predominant" patients (Fig. 1(a); posterior mean $=-3.4,85 \%$ Bayesian Highest Density Interval [HDI]: $-6.8,-0.3)$ and among "insomnia-pain" patients (Fig. 1(a); - 3.8, 80\% HDI: - 7.3, - 0.2). However, acupuncture was more effective than CBT-I at reducing pain among "insomnia-pain" patients (Fig. 1(d); -1.46 , 95\% HDI: -2.85 ,
- 0.27). CBT-I and acupuncture were equally effective for the "high symptom burden" cluster. Conclusion: Our BAM method revealed distinct symptom clusters that responded differently to insomnia treatments. Among cancer survivors with insomnia alone or cooccurring with pain, CBT-I was more effective at reducing insomnia, but acupuncture was more effective at reducing cooccurring pain. Bayesian nonparametric approaches can inform personalized management strategies tailored to individuals and their unique symptom clusters, which can be applied in numerous other areas of PRO research in acute and chronic illnesses.

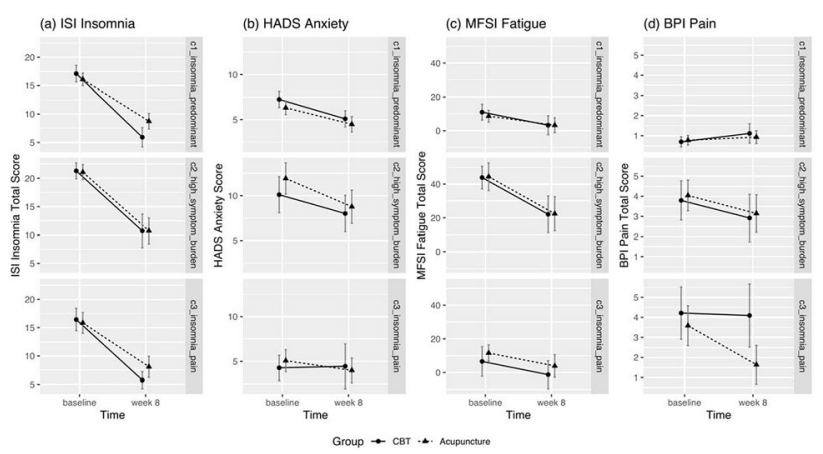

\section{Symposium Sessions}

\section{Symposium 01: Young people in the time of COVID}

Moderator: John Eric Chaplin, $\mathrm{PhD}$, Sahlgrenska Academy at University of Gothenburg, Gothenburg, Sweden

Overview: It was inevitable that COVID-19 would have an effect on the fate of young people. In this symposium, we provide evidence and lessons learned from young people's reported experiences of living through the time of COVID. The studies presented are from both Europe and North America, employing different research designs, methods and instruments, including PROMIS ${ }^{8}$, Kidscreen, CAQ, SDQ, SCARED, CES-DC and ACHWM. These studies will illuminate present concerns about the mental health and quality of life of young people by examining their reported experiences and the lessons learned leading to interventions.

Data will be presented from Sweden that highlights the importance of maintaining school and family routines and how communities need to focus on supporting adolescents to reconnect with education. A presentation from Germany will compare results gathered during the pandemic to those of a large survey conducted prior to the pandemic. Conclusions will be drawn for health promotion in children and adolescents-especially for those at risk - which take into consideration the reported needs of parents to support their children during the pandemic. A presentation from the The Netherlands will consider the longitudinal aspects of the pandemic and the importance of understanding mental and social health to guide interventions. A presentation from Canada will explore how indigenous communities have learnt lessons which have resulted in responding to their young people's unique needs by tailoring and scaffolding culturally appropriate support services. The final presentation will be by young participants in a youth research advisory panel in British Columbia. The panel engages young people in achieving appropriate health research and health promotion through prioritizing the needs and ideas of young people themselves.

The impact of living through the time of COVID is multifaceted. Young people have had to cope with close friendships being weakened, disruption to broader peer group membership, loss of education and even feeling of responsibility for virus transmission. This 
symposium will present important insights into how young people experience living through a pandemic and how the development of behavioural interventions in different healthcare contexts is being used to mitigate the mental health impact of COVID on young people.

\section{Individual Presentations}

\section{The impact of anxiety in relation to COVID-19 on the life- situation of young people in Sweden}

Malin Berghammer, Asso. professor Queen Silvias Childrens Hospital, Gothenburg, Sweden; Margaretha Jenholt Nolbris, Asso. professor Institute of Health and Care Sciences, Sahlgrenska Academy, University of Gothenburg, Gothenburg, Sweden; Patrica Olaya Contreras, PhD, Institute of Health and Care Sciences, Sahlgrenska Academy, University of Gothenburg, Gothenburg, Sweden; Maria Forsner, Ass professor Department of Nursing, Umeå University, Umeå, Sweden; Anna-Clara Rullander, MD, Department of Nursing, Umeå University, Umeå, Sweden; Susanne Ragnarsson, MD, Department of Nursing, Umeå University, Umeå, Sweden; Janet Mattsson, Ass professor The Swedish Red Cross University College, Department of Health Sciences, Stockholm, Sweden; Lise-Lott Rydström, MD, Department of Neurobiology, Care Sciences and Society, Karolinska Institutet, Stockholm, Sweden; Anna-Lena Brorson, MD, Department of Neurobiology, Care Sciences and Society, Karolinska Institutet, Stockholm, Sweden; Inger Kull, professor Department of Clinical Science and Education, Karolinska Institutet, Södersjukhuset, Stockholm, Sweden; Anna LindholmOlinder, Ass professor Department of Clinical Science and Education, Karolinska Institutet, Södersjukhuset, Stockholm, Sweden; Marla Andreia Garcia de Avila, Ass professor São Paulo State, Botucatu Medical School, Department of Nursing, Sao Paulo, Brazil; Stefan Nilsson, Ass professor Institute of Health and Care Sciences, Sahlgrenska Academy, University of Gothenburg, Gothenburg, Sweden

Aims: In general, COVID-19 symptoms are milder in children than in adults, but the experience of the pandemic could increase anxiety and significantly affect the life situation of children and adolescents. It could also lead to a long-term negative effect on their health. To study how the corona pandemic affected the life situation of children and adolescents in Sweden. Methods: A self-reported online survey was performed July-November 2020. Cross-sectional data were collected using non-probability and convenience sampling methods. The sample consisted of children 6-14 years and their guardians, and adolescents 15-19 years. The questionnaire covered items regarding the life situation including demographics, school situation, social isolation, and an open-ended question to provide a subjective expression of the living situation. A standardized measurement of anxiety was collected using the Children's Anxiety Questionnaire (CAQ) (scores range 4-12) and the Numeric Rating Scale (NRS) (scores range 0-10). Data were analyzed by descriptive statistics and with qualitative manifest content analysis. Results: In total, 1487 participants, 768 children with guardians and 719 adolescents participated. Most of the participants, 754 children (97.2\%) and 634 adolescents $(89.2 \%)$ attended school where a mixture of physical attendance and distance learning was reported by 79 children $(10.2 \%)$ and 261 adolescents $(36.7 \%)$. Two children $(0.3 \%)$ and 298 adolescents $(41.9 \%)$ reported only having distance learning. A larger proportion of children $(n=339,43.9 \%)$ and adolescents $(n=420$, $59.2 \%$ ) reported abstaining from leisure activities, while a minority of children $(n=103,13 \%)$ and adolescent $(n=135,19 \%)$ reported experiencing a feeling of 'social isolation'. These experiences of changes in daily routine were prominent in the qualitative result. Social restrictions and loss of contact with older relatives led to fear and anxiety. However, for young children, their lives continued to revolve around the everyday things in life rather than the corona pandemic, for the adolescents; however, their life situation was negatively affected by isolation from peer groups and the loss of school routine. Conclusion: The experiences by children due to COVID-19 in Sweden highlight the importance that children continue living their lives as unchanged as possible and that particularly adolescents need receiving greater support with the maintenance of an educational routine.

\section{A longitudinal study on mental and social health of children and adolescents: a Dutch general population}

Lotte Haverman, PhD, Emma Children's Hospital, Amsterdam UMC, Amsterdam, The Netherlands; Hedy van Oers, PhD, Emma Children's Hospital, Amsterdam UMC, Amsterdam, The Netherlands; Michiel Luijten, MSc, Emma Children's Hospital Amsterdam UMC, Amsterdam, The Netherlands; Maud van Muilekom, MSc, Emma Children's Hospital, Amsterdam UMC, Amsterdam, The Netherlands; Lorynn Teela, MSc, Emma Children's Hospital, Amsterdam UMC, Amsterdam, The Netherlands; Josjan Zijlmans, PhD, Amsterdam UMC, Vrije Universiteit, Amsterdam, The Netherlands; Tinca Polderman, PhD, Amsterdam UMC, Vrije Universiteit, Amsterdam, The Netherlands; Kim Oostrom, PhD, Emma Children's Hospital, Amsterdam UMC, Amsterdam, The Netherlands; Arne Popma, MD $\mathrm{PhD}$, Emma Children's Hospital, Amsterdam UMC, Amsterdam, The Netherlands

Aims: As shown in previous studies, the COVID-19 outbreak profoundly impacts the mental and social health of children and adolescents. However, the longitudinal effect is yet unknown. Therefore, this study aimed to compare mental and social health of children and adolescents in the Dutch general population during the first COVID-19 lockdown (April 2020) to a follow-up measurement (November 2020). Methods: In April and November 2020, children and adolescents aged 8-18y, representative of the Dutch population, were asked to complete six PROMIS ${ }^{\circledR}$ domains (Global Health, Peer Relationships, Anxiety, Depressive Symptoms, Anger, Sleep-Related Impairment) and an open question on the impact of COVID-19 regulations on daily life. Parents were asked to complete sociodemographic questions about themselves (age, ethnicity, educational level) and their child (age, gender) and to answer COVID-19 specific questions such as consequences for employment and if a relative/friend was infected with COVID-19. PROMIS T-scores between the two measurement occasions will be compared, associated factors will be identified, and the impact of COVID-19 regulations on daily life will be investigated. Results: In total, 844 (April 2020) and 505 (November 2020) children and adolescents completed the questionnaires. Data management and analyses are currently being carried out. Results will be shown at the conference. Conclusion: A better understanding of how the governmental restrictions during the COVID-19 pandemic affect children's and adolescents' mental and social health longitudinally can help guide future interventions and inform policy makers.

\section{Responding to challenges faced by indigenous children \& youth during COVID}

Dr. Nancy Young, PhD, Laurentian University, Sudbury, Ontario, Canada; Lisa Puchalski Ritchie, MD PhD, Li Ka Shing Knowledge Institute, Toronto, Ontario, Canada; Skye Barbic, PhD, University of British Columbia, Vancouver, British Columbia, Canada; Samantha Fowler, MSCom, ECHO Research Centre, Laurentian University, Sudbury, Ontario, Canada; Mia Bourque, MA candidate, 
ECHO Research Centre, Laurentian University, Sudbury, Ontario, Canada; Kiana Seidel, MBA candidate, ECHO Research Centre, Laurentian University, Sudbury, Ontario, Canada; Submitted on behalf of the VIEW Study Team

Aims: One of the most sustained consequences of the COVID-19 pandemic will be its impact on mental wellness. The impact is magnified among Indigenous children and youth in rural and remote regions of Canada due to geographic isolation, limited internet, and lack of access to comprehensive health supports. Methods: The Aaniish Naa Gegii: the Children's Health and Well-being Measure (ACHWM) is a patient-reported outcome (PRO) for school-aged Indigenous children in Canada. Our project sought to leverage our PRO research and website (www.ACHWM.ca) to improve local mental health services for Indigenous children and youth. (a) We conducted a scoping review to identify mental wellness group programs relevant to this population. We applied critical analysis to determine each program's feasibility in rural and remote communities and documented each program's effectiveness. (b) We co-created new resources to support this population that reflect their cultures. (c) We updated our website to share the ACHWM (a PRO for Indigenous children 8 to 18 years of age) with more Indigenous health leaders, and to create a special portal for Indigenous children and youth. Results: This project produced a compendium of 52 mental wellness group programs that are grounded in culture and evidence, and tailored for use in rural and remote Indigenous communities. We developed tips sheets about each program's goals, level of need, resources required, and level of evidence. Our website also includes a Resources Hub where we share new culturally relevant colouring pages, workbooks, calming activity pages, and tips sheets that integrate Indigenous cultures. This Hub also includes positive messaging materials to reduce the stigma associated with mental health. We automated the www.ACHWM.ca site to ensure Indigenous leaders have better access to our PRO app and full control of their ACHWM data. Through a special portal, Indigenous children and youth now have direct access, to resources that respect, reflect their cultures, and promote wellness. Conclusion: This project responded to the challenges faced by Indigenous children and youth during COVID and produced culturally relevant information that will inform stepped care models. We are helping Indigenous leaders help their next generation to paddle upstream towards wellness.

\section{The key to creating a COVID research agenda in 2021} and beyond: safe, intentional, and meaningful engagement

Alisha Daya, The University of British Columbia, Burnaby, British Columbia, Canada; Jenna Pillainayagam, The University of British Columbia, Vancouver, British Columbia, Canada; Dan Nixon, BA, The University of British Columbia, Vancouver, British Columbia, Canada; Skye Barbic, PhD, The University of British Columbia, Vancouver, British Columbia, Canada

Aims: There is imminent risk during the COVID-19 pandemic that health models, services, and policies are being designed and implemented without considering the perspectives of children and young people they are targeted towards. In response, a Youth Research Advisory Panel (YRAP) was created to ensure that youth are meaningfully positioned to inform and guide Canadian COVID-19 research. The aims of the YRAP are to (1) ensure the highest patient engagement standards are achieved and (2) establish a research culture that prioritizes the needs and ideas of all young people during and after the pandemic. This presentation will highlight early lessons from the YRAP and our immediate impact. Methods: We recruited a youth lead and ten diverse youth with lived or living experience of mental health and substance use, including LBGTQ2IA youth, non-binary youth, and racialized youth. After collating panel members, we developed a terms of reference, workflow, and communications strategy to share the opportunity for researchers to engage with the panel. After connecting with the panel, researchers work alongside youth panel members to guide grant proposal creation to ensure that meaningful engagement practices and youth voice are included in the entire research process. Evaluation for this project is ongoing. Results: To date, one youth lead and 10 youth panel members (ages 13-24 years) have been hired. Lessons learned include (1) clear description of roles and responsibilities for panel members, (2) need youth-engagement education for researchers, and (3) need for reciprocal education to panel members on research methods, cultural safety, and implementation science. The project has evolved through the leadership of the youth, to move beyond just consultation and towards supporting the co-creation of research agendas and policies that inform, shape, and create research that has direct impacts on youth outcomes and experiences. Conclusion: The YRAP is challenging the status quo of engagement within global research beyond just transactional relationships. The potential that the YRAP has to create safe, meaningful, and impactful research programs is remarkable. In the aftermath of COVID-19, the YRAP continues to capture and share robust engagement practices, while ensuring research that truly serves young people and prioritizes their unique needs and perspectives.

Symposium 02: Who cares? Moving beyond concordance studies in caregiver and proxy research

Moderator: Jessica Roydhouse, PhD, University of Tasmania, Hobart, Australia

Overview: Caregivers provide critical support to patients across disease contexts. The caregiver experience is wide ranging and includes the impact of providing care on caregiver health and quality of life outcomes and providing proxy reports of patient health and quality of life for patients who cannot self-report. A shift toward dyadic illness management and an effort toward greater understanding of both members of patient-caregiver dyads, as well as the dynamic nature of the dyadic relationship itself, have highlighted areas where further research and methods are needed. This includes unaddressed measurement and methodological challenges. The measurement challenges include identifying and selecting appropriate instruments for measuring the caregiver experience, determining when to transition from patient to proxy report for patients, and ascertaining the impact of question phrasing and perspective taking on proxy reporting. The methodological challenges include reducing bias when proxies are used. This symposium will examine key issues in outcomes research with caregivers and proxies, and their possible solutions. The symposium will also feature a discussion of developments and challenges in the field, and what is needed to move the field forward, particularly for working with caregivers who may need to report on their own outcomes and patient outcomes.

\section{Individual Presentations}

Identifying appropriate measures for caregivers in a lung cancer longitudinal study

Bellinda King-Kallimanis, PhD, LUNGevity Foundation, Bethesda, Maryland, USA; Anne-Marie Baird, PhD, School of Medicine, Translational Medicine Institute, Trinity College Dublin, Dublin, Ireland; Martha Belesis, Patient advocate, New York, USA; Upal Basu-Roy, PhD, LUNGevity Foundation, New York, USA 
Aims: Caregivers to people impacted by cancer play an important role in the patient's care trajectory. Studies focused on the caregiver experience have mostly concentrated on 3 key areas: caregiver-focused outcomes (e.g., health/well-being, and coping), patient-focused outcomes as a proxy reporter (e.g., symptoms and healthcare utilization), or patient-caregiver relationship-focused outcomes (e.g., patient-family-healthcare clinician concordance). LUNGevity Foundation has developed a longitudinal study aiming to include caregivers' experience at all stages of the care trajectory. We investigated the concepts, reliability, and validity of instruments for the Palliative Care Research Cooperative Groups caregiver measures inventory in relation to lung cancer. Methods: We first developed a lung cancer caregiver map in context to a patient's care trajectory and defined three types of caregivers: active treatment caregiver-defined as someone providing care to a patient undergoing active treatment, non-active treatment caregiver-defined as someone providing care to a patient with stable disease or who was no evidence of disease (NED), and caregiver to a deceased patient/caregiver in bereavement. We analyzed 16 measures in the inventory that were deemed reliable and valid and determined how applicable these were to the three caregiver groups to learn about their quality of life (QoL). Results: We identified reliable and valid instruments for two of our three groups. There were measures available to assess QoL (i.e., support, emotional distress, activity restriction, finances) of caregivers in the active treatment phase. For caregivers in bereavement, there were measures focused on the benefits, hope, and meaning of the experience. However, there were no measures included in our review that assessed the experience of a non-active treatment caregiver. Conclusion: We did not find an off-the-shelf instrument that fits all types of caregivers that captured their QoL. The issue was that concerns are different for caregivers who have moved into the non-active phase, for example, the return of disease versus side effects or management of treatment, which are relevant for those receiving active treatment. Ideally, a core set of items that can be tracked along the care trajectory are needed with supplementation of additional items dependent on the caregiving phase.

\section{Among study participants with dementia, at what level of cognitive impairment should family caregiver proxy report be used instead of patient self-report for the assessment symptoms, function, and quality of life?}

\section{Antonia Bennett, PhD, University of North Carolina, Chapel Hill,} USA

Aims: Mild cognitive impairment (MCI) and dementia are common among older adults. The objective of this research is to identify at what level of cognitive impairment study participants can no longer provide valid and reliable responses on patient-reported outcome (PRO) assessments. This information will guide the use of family caregiver proxy reporting of patient outcomes in clinical trials. Methods: A narrative review of multiple studies will be presented that evaluate the validity and reliability of PROs for individuals within three groups: MCI mild dementia or moderate dementia, and compared to individuals with normal cognitive function. The comprehension of items from PROMIS ${ }^{\circledR}$, the Geriatric Depression Scale (GDS), and the PHQ-9 was evaluated through cognitive interviews of 40 patients receiving care at a geriatric specialty clinic. Factor structure and differential item functioning (DIF) of the GDS were evaluated by cognitive group in a matched sample of 24,674 adults that included patients with severe dementia. The psychometric properties of the FACT-General and FACT-Brain module from two radiotherapy trials of brain metastases $(n=407)$ will be compared across cognitive function groups. Results: In cognitive interviews, participants rarely misunderstood simple concepts (e.g., "feel tired")(2\%) and simple recall periods (e.g., "now") (2\%), or misused simple response options (e.g., "yes/no")(8\%); however, patients with dementia were more likely than patients with normal cognitive impairment or MCI to misunderstand complex concepts ( $25 \%$ vs $2 \%$, $p<0.001)$ and complex recall periods $(60 \%$ vs $20 \%, p<0.007)$, and to misuse complex response options ( $34 \%$ vs $24 \%, p<0.096$ ). A onefactor CFA/IRT model of the GDS fit well to all groups except those with severe cognitive impairment, and the item about memory problems showed significant moderate to large DIF effects. Conclusion: This formative work evaluated the validity and reliability of self-reported PRO data in adults with MCI or dementia. Simple items are more accessible than complex items. The factor structure of scales may vary across level of cognitive impairment and DIF can occur when the PRO assesses symptoms also present in dementia. A brief cognitive function screening tool should be developed to identify individuals unable to provide valid and reliable self-report at each study assessment time point.

Optimal methods for reducing proxy-introduced bias on patientreported outcome measurements for group-level analyses

Brittany Lapin, PhD MPH, Cleveland Clinic, Cleveland, Ohio, USA; Nicolas Thompson, MS, Cleveland Clinic, Cleveland, Ohio, USA; Andrew Schuster, BA, Cleveland Clinic, Cleveland, Ohio, USA; Irene Katzan, MD, Cleveland Clinic, Cleveland, Ohio, USA

Aims: Patient-reported outcomes (PROs) are utilized to assess outcomes following stroke; however, a caregiver, or proxy, often completes PROs on the patient's behalf. Research has demonstrated proxies rate patient outcomes worse than patients rate their own outcomes. To improve interpretability of PROs in group-level analyses, our study aimed to identify optimal approaches for reducing this bias in the analysis of PROs. Methods: Data were simulated using a cross-sectional survey study of 200 stroke patients and their proxies who both completed 9 PROMIS domains. Bias was evaluated through simulating 1000 datasets according to the observed model (patientonly responses) and estimating regression coefficients from the simulated data based on the linear regression model: Patient-Reported Domain $\quad$ Score $=\beta 0+\beta 1$ age $+\beta 2 \mathrm{sex}+\beta 3 \mathrm{mRS}+\varepsilon \quad$ (where $\mathrm{mRS}=$ modified Rankin scale, a measure of stroke disability). Six approaches for handling proxy-completions were investigated: (1) excluding proxy response; (2) proxy substitution; (3) Method 2 plus proxy adjustment; (4) Method 3 including inverse-probability of treatment weighting (IPTW); (5) multiple imputation; and (6) linear equating. The sample size was varied as 50, 100, 200, and 500, and the proportion of patients with proxy-respondents was varied as $10 \%$, $20 \%$, and $50 \%$. The methods were evaluated by comparing mean domain $\mathrm{T}$-scores and percent bias for each regression coefficient as $100 *(\hat{\beta} x-\beta x) / \beta x$. Results: Overall mean T-score differences ranged from 0.0002 to 2.36 . The range of mean differences varied by the six methods with methods 2 and 3 having the lowest difference (0-1.46 T-score points), and method 4 having the largest difference (0.15-2.36 T-score points). In regression models, IPTW and multiple imputation resulted in less accurate estimations while complete case analysis and proxy substitution provided less proxy-introduced bias. Bias remained low across domain and varying sample sizes but increased with larger percentages of proxy respondents. Conclusion: Our study found modest proxy-introduced bias when estimating PRO scores or regression estimates across 9 domains of health. This bias remained low, even when sample size was 50 and there were large proportions of proxy completions. While many methods can be chosen for including proxies in stroke PRO research, proxy 
substitution with adjustment resulted in low bias and performed best with larger proportions of proxy respondents.

\section{A matter of perspective? Assessing the role of phrasing on proxy- reported health experiences}

Jessica Roydhouse, PhD, University of Tasmania, Hobart, Australia

Aims: Patient-reported experience measures (PREMs) are increasingly used in assessments of health care quality. Surveys with PREMs often use proxies when patients cannot self-report. Assessment of proxy-reported health outcome data has identified the perspective taken by the proxy may affect how they report. This study aimed to evaluate the impact of phrasing and perspective taking for proxyreported experiences. Methods: Data from a prospective cohort study of patients with advanced cancer were used. Respondents completed questionnaires at two time points: (1) shortly after diagnosis or progression to an advanced stage of disease, and (2) after the completion of at least one treatment cycle. Kendall's tau was used for correlation between items. Completion and descriptive statistics across differently phrased items were used for comparison. Results: Ninety-nine proxies completed questionnaires at both time points. Proxies were mostly the patient's spouse $(n=65,66 \%)$ and most reported always attending oncologist visits with the patient $(n=60,61 \%)$. For care coordination outcomes at time $1,71 \%$ of proxies endorsed the top/ best score for a neutrally phrased question, compared to $82 \%$ and $80 \%$ for negatively phrased questions. Missingness was lower for the negatively phrased questions $(4-5 \%)$ compared to the neutrally phrased question (9\%). Results for these questions were similar at time 2. Ratings of oncologist communication with the patient (mean 8.9/10) were similar to ratings of oncologist communication with the proxy (mean 8.6/10), and the items were highly correlated (0.68). Completion for ratings of provider provision of emotional support varied by how the question was phrased. Missingness was higher for phrasing referring to the proxy $(n=14)$ compared to phrasing referring to the patient $(n=8)$, although the ratings were highly correlated (0.81). Results for these questions were similar at time 2 . Conclusion: Results for proxy-reported care experience were similar when neutral or negative phrasing for questions was used, although completion was slightly better for negatively phrased questions. However, completion was better for questions referring to the patient rather than the proxy. These findings suggest that caution should be taken when using experience questions referring to the proxy rather than the patient.

\section{Symposium 03: PROMs in telehealth}

\section{Moderator: Elizabeth Unni, PhD, Touro College of Pharmacy, New} York, USA

Overview: The COVID-19 pandemic has accelerated the adoption of telehealth initiatives in the health care system. It has also emphasized the need and relevance of patient-reported outcome measures (PROMs) in routine clinical practice. PROMs in telehealth could be central to the COVID-19 response by facilitating the remote monitoring of symptoms and long-term effects. Although remote patient management has been used for decades in telemedicine solutions in the healthcare system, the use of PROMs in telehealth is a relatively new initiative in clinical practice. PROMs via telehealth allow new opportunities such as real-time monitoring of symptoms, flexible scheduling of hospital appointments, early detection of problems, prompt clinical intervention if needed, and provision of automated tailored advice to support self-management and enhance self-efficacy.
In addition, PROMs in telehealth could contribute to the reorganization of the healthcare system for follow-up activities in patients with long-term conditions by prioritizing or optimizing the use of healthcare resources and promoting patient-centered care.

This symposium aims to provide insight into and discuss the use of PROMs in telehealth. Members of the ISOQOL QOL in Clinical Practice Special Interest Group will share experiences with PROMs in telehealth initiatives and discuss challenges and opportunities. The symposium will contribute with insight into

- Applications of PROMs in telehealth

- State of the art: what is the impact of using PROMs in telehealth?

- Experiences from a collection of PROMs in telehealth initiatives across oncology, kidney disease, and cystic fibrosis will be drawn upon

The intended target audience for this symposium are clinicians and researchers. The presenters will focus on the effect of using PROMs in telehealth, development of PROMs in telehealth interventions, feasibility in clinical practice, and considerations regarding effectiveness research, e.g., selection of outcome measures. Moreover, challenges and considerations of using PROMs in telehealth will be discussed. The symposium will provide an opportunity for attendees to discuss and consider the next steps forward.

\section{Individual Presentations}

Current evidence supporting the implementation of electronic patient-reported outcome measures (ePROMs) in the management of chronic diseases: a structured review

Olalekan Lee Aiyegbusi, MBChB, PhD, University of Birmingham, Birmingham, UK; Devika Nair, MD, Vanderbilt University Medical Center, Division of Nephrology and Hypertension, Nashville, TN, USA; John Devin Peipert, PhD, Department of Medical Social Sciences, Northwestern University Feinberg School of Medicine, Chicago, IL, USA; Kara Schick-Makaroff, RN, PhD, University of Alberta, Faculty of Nursing, Edmonton, Alberta, Canada; Istvan Mucsi, MD, PhD, Multiorgan Transplant Program, University Health Network and Division of Nephrology, Department of Medicine, University of Toronto, Ontario, Canada

Aims: Numerous studies have demonstrated that the use of ePROMs in routine clinical practice is both acceptable and feasible with patients increasingly expressing a preference for an electronic mode of administration. However, despite these advancements, doubts about the quantifiable benefits of implementing PROMs/ePROMs for use in routine clinical practice persist. The aim of this review is to summarise recent evidence of the impact of ePROMs on clinical parameters and outcomes relevant to chronic diseases. To explore recently published literature regarding issues that may influence the robust implementation of ePROMs for routine clinical practice. Methods: Forward citation searches of three key articles (Boyce and Brown 2013, Basch et al. 2016, 2017) were conducted in September 2020 on Web of Science database (All Databases version) and Google Scholar. Randomised controlled trials (RCTs) and observational studies of any chronic medical condition were included if they reported the impact of ePROMs on clinical parameters and/or patient outcomes and/or healthcare resources. Study findings were extracted and narrative synthesis conducted. Results: Fifteen articles were included most of which were oncology based. A few focused on diseases such as rheumatoid arthritis, suspected/confirmed severe acute respiratory syndrome coronavirus-2 (SARS-CoV-2) infection, epilepsy, and sleep apnoea. All except one study were conducted in outpatient settings and most were RCTs. There was evidence to 
support use of ePROMs in routine clinical practice. Potential benefits included improvement in patient survival, treatment adherence and its monitoring, symptom management and individualised care, efficient utilisation of healthcare resources, and reduction of risk of disease transmission during outbreaks and epidemics. Conclusion: There is increasing evidence that the use of electronic patient-reported outcome (ePROMs) could have significant impacts on outcomes valued by patients, healthcare providers, and researchers. However, there are a number of challenges that need to be adequately addressed to ensure seamless implementation and integration of ePROMs into a health system. Further research is required to demonstrate the long-term cost-effectiveness of using ePROMs in routine clinical practice.

\section{Remote monitoring of symptoms from both disease and treatment in men with advanced prostate cancer: a single-arm clinical trial}

Natasha Roberts, PhD, University of Queensland, Herston, Australia

Aims: Consistent with international literature, research data in our institution identified that patients diagnosed with advanced prostate cancer had persistent unmet needs and a high symptom burden. When telehealth was rapidly introduced to all oncology patients through our oncology outpatients' departments in response to the COVID-19 pandemic in March 2020, telephone or virtual meeting platforms replaced face-to-face visits for most of these men. In response, we used the increased accessibility of routine telehealth, to investigate the use of a structured PROMs and telehealth model of care to directly address the unmet needs and symptom management in this group of men. Methods: All men diagnosed with advanced prostate cancer attending our outpatients' departments were eligible. Participation included a screening PROM on a monthly basis with a specialist nurse consultation by telehealth. Health-related quality of life study outcomes are measured, including a Supportive Care Needs Survey (SCNS-SF, SCNS-SFC) at baseline, 1 months, 3 months and 6 months. Feasibility was measured by the number of assessments completed. Results: 42 men were recruited to participate. Unmet needs were improved at 3 months $(p=0.01)$ and symptom burden decreased at 6 months $(p=0.05)$. 85\% of men completed all assessments. Marital status was the only demographic to influence preferences for telehealth, with single or divorced men preferring telehealth at baseline. This model is feasible and can inform a multisite randomised controlled trial to measure effectiveness. Conclusion: Health service changes to accommodate the COVID-19 pandemic can be harnessed to investigate alternative care models. Telehealth supported by PROMs can be used to address unmet needs for men with prostate cancer. Qualitative interviews will be conducted with approximately 10 men and their carers.

\section{Electronic patient-reported outcome measure use during the management of patients with advanced chronic kidney disease: a pilot/feasibility trial}

Derek Kyte, PhD, University of Worcester, Worcester, UK; Nicola Anderson, University Hospitals Birmingham NHS Foundation Trust, Birmingham, UK; Jon Bishop, Birmingham Clinical Trials Unit (BCTU), Institute of Applied Health Research, University of Birmingham, Birmingham, UK; Andrew Bissell, Patient Advisory Group, Centre for Patient-Reported Outcomes Research, Institute of Applied Health Research, University of Birmingham, Birmingham, UK; Elizabeth Brettell, Birmingham Clinical Trials Unit (BCTU), Institute of Applied Health Research, University of Birmingham, Birmingham, UK; Melanie Calvert, Centre for Patient-Reported Outcomes Research, Institute of Applied Health Research, University of Birmingham, Birmingham, UK; Marie Chadburn, Birmingham
Clinical Trials Unit (BCTU), Institute of Applied Health Research, University of Birmingham, Birmingham, UK; Paul Cockwell, University Hospitals Birmingham NHS Foundation Trust, Birmingham, UK; Mary Dutton, University Hospitals Birmingham NHS Foundation Trust, Birmingham, UK; Helen Eddington, University Hospitals Birmingham NHS Foundation Trust, Birmingham, UK; Elliot Forster, University Hospitals Birmingham NHS Foundation Trust, Birmingham, UK; Gabby Hadley, University Hospitals Birmingham NHS Foundation Trust, Birmingham, UK; Natalie Rowland, Birmingham Clinical Trials Unit (BCTU), Institute of Applied Health Research, University of Birmingham, Birmingham, UK; Louise Jackson, Health Economics Unit, Institute of Applied Health Research, University of Birmingham, Birmingham, UK; Sonia O'Brien, Patient Advisory Group, Centre for Patient-Reported Outcomes Research, Institute of Applied Health Research, University of Birmingham, Birmingham, UK; Gary Price, Patient Advisory Group, Centre for Patient-Reported Outcomes Research, Institute of Applied Health Research, University of Birmingham, Birmingham, UK; Keeley Sharpe, Patient Advisory Group, Centre for PatientReported Outcomes Research, Institute of Applied Health Research, University of Birmingham, Birmingham, UK; Stephanie Stringer, University Hospitals Birmingham NHS Foundation Trust, Birmingham, UK 4 Birmingham Clinical Trials Unit (BCTU), Institute of Applied Health Research, University of Birmingham, Birmingham, UK; Rav Verdi, Patient Advisory Group, Centre for Patient-Reported Outcomes Research, Institute of Applied Health Research, University of Birmingham, Birmingham, UK; Judi Waters, Patient Advisory Group, Centre for Patient-Reported Outcomes Research, Institute of Applied Health Research, University of Birmingham, Birmingham, UK; Adrian Wilcockson, Birmingham Clinical Trials Unit (BCTU), Institute of Applied Health Research, University of Birmingham, Birmingham, UK

Aims: Use of routine remote follow-up for patients with chronic kidney disease (CKD) has increased in recent years and has further accelerated in response to COVID-19. It has been suggested that electronic patient-reported outcomes (ePROs) could be used in parallel, to facilitate real-time symptom monitoring aimed at improving care. We tested the feasibility of this approach in a pilot trial of ePRO symptom monitoring versus usual care in patients with advanced CKD (pre-dialysis). Methods: We conducted a pilot randomized controlled trial and qualitative sub-study. Participants included adult patients with advanced CKD (eGFR $\geq 6$ and $\leq 15 \mathrm{~mL} / \mathrm{min} / 1.73 \mathrm{~m}^{2}$, or a projected risk of progression to end-stage renal failure (ESRF) within 2 years $\geq 20 \%$ ). The intervention involved monthly online ePRO symptom reporting, including automated feedback of tailored self-management advice and triggered clinical notifications in the advent of severe symptoms. Real-time ePRO data were made available to the clinical team via the electronic medical record. Trial outcomes included: feasibility and intervention adherence/acceptance; health-related quality of life; healthcare utilization; routine renal lab results, and clinical event data. The qualitative sub-study explored patient and health care practitioner (HCP) thoughts/experiences regarding the trial processes and intervention; all data were analyzed by the lead author using conventional content analysis. Results: 52 patients were randomized (31\% of approached). Case report form returns were high $(99.5 \%)$. No patients were excluded from the intention to treat analysis. Overall, $>70 \%$ of expected ePRO questionnaires were received. Intervention adherence was high beyond 90 days $(74 \%)$ and 180 days $(65 \%)$, but dropped beyond 270 days (46\%). Patients reported 579 symptoms, the most prevalent of which included fatigue, shortness of breath, itchy/dry skin, and pain. There were 19 triggered notifications for severe symptoms, which were predominantly managed by staff via telephone consultations supporting patient self-management. $n=25$ qualitative interviews supported proof of concept and intervention acceptability 
but highlighted necessary changes aimed at enhancing overall functionality/scalability. Conclusion: Pilot trial findings suggest that it is possible to randomize patients to ePRO reporting with real-time feedback or usual care, with high levels of data completion up to 12 months, and that a RCT is feasible. Patient/HCP feedback has provided important information to guide intervention improvements.

\section{Remote follow-up using patient-reported outcome measures in patients with chronic kidney disease: The PROKID study: an ongoing non-inferiority pragmatic randomised controlled trial}

Birgith Grove, Phd student, AmbuFlex/Center for Patient-Reported Outcome, Herning, Denmark; Niels Henrik Hjøllund, professor AmbuFlex/WestChronic, Occupational Medicine, University Research Clinic, Aarhus University, Herning, Denmark and Department of Clinical Epidemiology, Aarhus University Hospital, Herning, Denmark; Liv Marit Schougaard, RN, MHSc, $\mathrm{PhD}$, Center for Patient-Reported Outcome, Herning, Denmark; Per Ivarsen, $\mathrm{MD}, \mathrm{PhD}$, associate professor Department of Nephrology, Aarhus University Hospital, Aarhus, Denmark; Derek Kyte, PhD, University of Worcester, Worcester, UK; Annette De Thurah, MPH, $\mathrm{PhD}$, associate professor Department of Rheumatology, Aarhus University Hospital, Department of Clinical Medicine, Aarhus University, Aarhus, Denmark

Aims: Outpatient care is steadily changing from hospital consultations to other platforms. It is prudent to maintain quality of care with such initiatives. Currently, patients with chronic kidney disease (CKD) have frequent scheduled visits, but it may be possible to optimise the frequency of hospital consultations using information from patient-reported outcome (PRO) questionnaires filled in at home (PRO-based follow-up). We aimed to evaluate the effectiveness of the quality of care, use of resources and patient outcomes associated with PRO-based follow-up in patients with CKD. Methods: A protocol has been published and the study commenced January 2019. The study is a pragmatic, non-inferiority, randomised controlled trial in outpatients with CKD. Totally, 150 newly referred patients with an estimated glomerular filtration rate (eGFR) of $\leq 40 \mathrm{ml} / \mathrm{min} 1.73 \mathrm{~m} 2$ will be randomised to 1 . PRO-based remote follow-up; 2 . PRO-based telephone consultation or 3. usual outpatient follow-up (control group). A disease-specific questionnaire completed by the patient at home substitutes for usual outpatient follow-up visits. The questionnaire is partly used as a screening tool to identify patients in need of outpatient contact and to identify symptom burden. Responses from the questionnaire are processed according to a disease-specific algorithm and assigned green, yellow, or red status according to patients' needs. The primary outcome is loss of renal function evaluated by eGFR. Secondary outcomes are (i) clinical outcomes, including initiation of acute dialyses and mortality, (ii) utilisation of healthcare resources and (iii) PRO measures, primarily quality of life (Euroqol EQ-5D). Results: This study is ongoing and up until now 110 patients are randomised (70\% of approached). Mean age 69 years, (range 31-92) and $36 \%$ female. Totally, 17 patients exited trial during follow-up: End-Stage Renal Disease $(n=2)$, death $(n=3)$, comorbidity $(n=4)$, discharge from outpatient follow-up $(n=5)$, patient or clinician wish $(n=3) .100 \%$ of the PRO questionnaire were returned and assessed by the clinicians. However, the use of reminders has been required. The study is expected to be completed December 2022. Conclusion: If PRO-based follow-up proves non-inferior to usual outpatient followup, then a reorganisation of routine clinical practice in nephrology outpatient clinics may occur.
Remote monitoring of cystic fibrosis (CF) due to COVID-19: use of telephone consultations combined with patient-reported outcome measures (PROMs) - a feasibility study

Liv Marit Valen Schougaard, AmbuFlex, Regional Hospital West Jutland, Herning, Denmark; Majbritt Jeppesen, Infectious Diseases, Aarhus University Hospital, Aarhus, Denmark; Lotte Oerneborg Rodkjaer, Infectious Diseases, Aarhus University Hospital, Aarhus, Denmark

Aims: Patients with cystic fibrosis (CF) are considered a Covid-19 high-risk patient group. In March 2020, it was, therefore, decided to develop a fast-track PROM solution for patients with CF to ensure that they only visited the hospital if they had an acute or subacute need. The overall aim was to investigate the feasibility of an urgent outpatient reorganization of in-person appointments to remote monitoring using telephone consultations combined with PROMs among patients with $\mathrm{CF}$. We investigated patients and healthcare professionals' acceptance, as well as recruitment rate, response rate, missing data, and attrition. Methods: We included adult patients diagnosed with CF from the Department of Infectious Diseases at Aarhus University Hospital from April to June 2020. Patients filled in a disease-specific questionnaire including clinical aspects relevant to patients with CF 2-3 days before a scheduled telephone consultation. Twelve participants who had completed the questionnaire and have had a telephone consultation were interviewed, and three doctors and three nurses from the $\mathrm{CF}$ clinical team participated in a focus group interview. Results: A total of 80 patients were recruited to remote monitoring, and 41 patients filled in at least one questionnaire during the period. Overall, both patients and health care professionals found remote monitoring and use of PROMs acceptable and useful. Patients experienced greater flexibility, they found the questionnaire relevant and understandable, but pointed out the need for items regarding mental health status and more adequate information about change in follow-up and work flow. Conclusion: Urgent reorganization of outpatient follow-up among patients with CF due to COVID-19 was feasible in routine clinical practice. However, patient involvement should be a future point of attention to ensure a sustainable telehealth PROM solution.

Symposium 04: One size does not always fit all: Are we measuring what matters in patient experiences?

Moderator: Maria J. Santana, PhD, Cumming School of Medicine, University of Calgary, Calgary, Alberta, Canada

Overview: Improving the patient experience is a key objective of the Institute for Healthcare Improvement's (IHI) Triple Aim Framework. To realize this goal, many healthcare organizations routinely collect patient experience survey data. Unfortunately, despite the vast amounts of data collected, knowledge of these surveys and how the data may be translated into improvement actions remains low. Furthermore, surveys often rely on a random sample, and may not capture the experiences of certain groups (e.g., non-English speakers, persons living with cognitive impairments).

In this symposium, we will examine and discuss (a) the various methods which are used to capture the experiences of patients and family members, and (b) how novel methods beyond surveys can be used to gain a better understanding of what matters to patients and family members during their healthcare journey.

To achieve these learning objectives, four individual case-study presentations will take place:

(1) A patient-partner discussion of his experiences and what healthcare systems can learn from patients; 
(2) The systematic collection of hospital experience data in Alberta, Canada;

(3) Analysis of patient concerns data using machine learning and sentiment analysis in Alberta, Canada;

(4) The theory and development of smart patient experience tools to maximize actionable insights in patient experience assessment when deployed at scale.

Capturing the experiences of patients and families is crucial in providing high-quality, value-based, patient-centered care. Through this symposium, attendees can expect to learn more about how systematic measurement and analysis of patient experiences can inform positive changes in healthcare systems. Recognizing that surveys may not answer all questions and may not capture the experiences of all equally, other methods for measuring patient experiences and applied examples of qualitative work to supplement quantitative survey activities will be presented. A moderated question and answer period at the conclusion of the session will facilitate a fruitful discussion of learnings, challenges, generation of new ideas, and proposed next steps in the measurement of healthcare experiences.

\section{Individual Presentations}

\section{A patients/family perspective}

D'Arcy Duquette, Health Quality Council of Alberta, Calgary, Alberta, Canada

Aims: The patient is the central player in their own healthcare. As such, they are the only person who is present at all steps in their journey and who experiences all care processes. Capturing patientreported experiences helps to determine what is working well and what are the gaps, providing opportunities to improve care. These experiential patient-reported data are invaluable for learning health systems and the effective delivery of patient-centered care. This session highlights the importance of capturing and acting upon what matters most to patients. The patient reporting his experiences receiving care will set the stage for the panel discussions which follow. Methods: Our patient partner has had experiences with both colon and lung cancer. He brings these personal experiences and his knowledge to his various roles as a champion and advocate for patient safety and quality healthcare. This includes work as a Patient Advisor with Alberta Health Services, the Health Quality Council of Alberta, the Alberta Surgical Safety Action Team, and as a member the Patient and Community Engagement Research (PaCER) program at the University of Calgary. Results: The presentation will focus upon multiple elements of the care experience, and the presenter's own reflections upon these and how his experiences have informed change in the healthcare system. Topics such as interactions with healthcare staff, coordination of care, information needs, shared decision-making, integration of family members, and ongoing care will be highlighted. Conclusion: These patient reports will be compared and contrasted with other ways to capture patient-reported experiences, presented by the other panelists.

\section{Measuring experiences of hospitalized patients in Alberta, Canada using validated surveys}

Kyle Kemp, PhD, University of Calgary, Calgary, Alberta, Canada
Aims: A successful person-centered, learning health system is evidence based, people informed, and responsive to issues that matter to its users. Surveys are widely used to capture what matters to patients and to obtain feedback about healthcare services. Information derived from the survey data is extremely important in the delivery of personcentered care. The aims of this session are to (a) present a comprehensive survey program to capture the experiences of those who are hospitalized in Alberta, Canada, and (b) share key findings, insights, and gaps which have resulted from our team's research partnership with our local health authority. Methods: Since 2006, Alberta Health Services have surveyed adults and parents/guardians of children who have been hospitalized. This is done via telephone, using modified versions of the Canadian Patient Experiences Survey of Inpatient Care (CPES-IC) and Child Hospital Consumer Assessment of Healthcare Providers and Systems (Child HCAHPS) instruments within 6 weeks of discharge from hospital. Each year, approximately 25,000 adult and 2500 child surveys are collected from 93 hospitals across the province. Survey data are collected and analyzed to inform quality improvement efforts across the organization. Additionally, research projects by our group have examined the data in isolation, and in combination with other administrative data sources (e.g., inpatient records, emergency department visits) to examine specific topics and clinical populations. Results: Over the past 6 years, our collaborative research program has produced over 15 published manuscripts and 50 conference abstracts. In this session, the main clinical and demographic drivers of overall experience, specific processes of care associated with positive experiences, areas identified for improvement, and the association of elements of patient experiences with other health system outcomes (e.g., patient safety indicators, unplanned hospital readmissions) will be presented. Future directions for our research, limitations of the survey methodology, and perceived gaps will also be highlighted. Conclusion: This program of research has answered many questions and has expanded our understanding of the experiences of patients and families across our province. It has also spurred novel ideas for examining patient experiences in other ways. These will be presented and discussed in the presentations which follow as part of this symposium.

\section{Categorizing patient concerns using natural language processing techniques}

Paul Fairie, PhD, University of Calgary, Calgary, Alberta, Canada; Zilong Zhang, M.Eng., Centre for Health Informatics, University of Calgary, Calgary, Alberta, Canada; Adam G. D'Souza, PhD, Centre for Health Informatics, University of Calgary, Calgary, Alberta, Canada; Tara Walsh, BA, Alberta Health Services, Edmonton, Alberta, Canada; Hude Quan, $\mathrm{PhD}$, Centre for Health Informatics, University of Calgary, Calgary, Alberta, Canada; Maria J. Santana, PhD, University of Calgary, Calgary, Alberta, Canada

Aims: Patient feedback is critical to identify and resolve patient safety and experience issues in healthcare systems. However, unstructured text data can pose problems for manual analysis. In this presentation, we will discuss the results of using a topic-modeling approach to analyze a large text corpus of patient feedback. Methods: Patient concerns were received by Alberta Health Services (AHS) between 2011 and $2018(N=76,163)$, regarding 806 care facilities in 163 municipalities, including hospitals, clinics, community care centers, and retirement homes, in a province of 4.4 million. Their existing 
framework requires manual labeling of pre-defined categories. We compared the usefulness of this approach to an automated Latent Dirichlet Allocation algorithm with 40 underlying topics. Results: Following interpretation by researchers, the model produced 28 coherent topics. The most frequent topics identified were communication issues causing delays (frequency: $10.58 \%$ ), community care for elderly patients $(8.82 \%)$, interactions with nurses $(8.80 \%)$, and emergency department care $(7.52 \%)$. Many patient concerns were categorized into multiple topics. Some were more specific versions of categories from the existing framework (e.g., communication issues causing delays), while others were novel (e.g., smoking in inappropriate settings). Conclusion: LDA-generated topics were more nuanced than the manual categories. For example, LDA found that concerns with community care were related to concerns about nursing for seniors, providing opportunities for insight and action. Our findings outline the range of concerns patients share in a large health system and demonstrate the usefulness of using LDA to identify categories of patient concerns. As part of this symposium, this presentation will contribute to our understandings of new approaches to understanding the patient experience, and in this case, an approach that combines expert opinion with automated natural language processing techniques.

\section{Innovative System for Patient-Reported Experience in Surgery (INSPiRES): semi-structured emotional reporting system for surgical patients and trainees}

Christopher Gibbons, The University of Texas MD Anderson Cancer Center, Houston, USA; Atkinson Rachel, MD, Harvard Medical School, Boston, USA; Andrea Pusic, MD, Harvard Medical School, Boston, USA

Aims: Typical patient-reported experience measures are designed for benchmarking and not provide granular insights into the drivers of patient and trainee experience. Attempts to utilize open-text experience reports have been met with challenges in both human processing and computational interpretation due to the high volume of brief context-free comments. We aimed to develop a system which could provide broad yet interpretable assessment of experience. Methods: We developed a system named INSPiRES to collect experience in a structed sequential manner assessing (a) a patient or trainees' emotional state (e.g., happy, frustrated, confused), (b) their explanation of that emotion, and finally (c) what event had led to their emotional response. For example, a patient may report that they felt confused because of a lack of information provided by their surgeon and a surgical trainee may report that they were happy to have been patiently mentored when learning to suture. The system is designed to collect data which will both facilitate comparisons between providers and provide detailed information into drivers of performance. Results: We collected data from 2452 patients and trainees at an academic medical center in Boston Massachusetts who reported an emotional response to 5302 specific events. We were able to visualize emotional responses to different events alongside a succinct explanation derived from open text. Our data demonstrate that INSPiRES is suitable both for comparing the performance of different providers and deriving actionable insights. Conclusion: Structured assessment of emotional responses to events surrounding surgical care and training is feasible and may enhance the ability of machine learning algorithms to automatically evaluate open-text reports of experience from both patients and trainees.
Symposium 05: Advancing the science of patient-centered measurement methods

Moderator: Danielle Lavallee, PharmD, PhD, British Columbia Academic Health Sciences Network, Vancouver, British Columbia, Canada.

Overview: Patient-reported experience and patient-reported outcomes are increasingly recognized as important measures of care quality. Research to advance the science of person-centered measurement is needed to ensure that effective strategies are identified for both collecting and leveraging such data to focus improvement on what matters to patients and their families/caregivers. In 2018, workshops were conducted across every region of British Columbia to identify priorities for research to advance the science of patient-centered measurement methods; separate workshops were conducted with patients and with stakeholders (comprising healthcare leaders and researchers). A call for patient-oriented research proposals aligned with 11 priority areas identified from analysis of the findings from the workshops included integration of measurement and data in clinical care, equity in measurement, cultural safety of measurement, and inclusion of care partner voices. This symposium provides an overview of the community informed process for generating research priorities for patient-centered measurement and highlights selected projects funded to address the priority topics identified. As patientoriented research initiatives, each project will be co-presented by researcher and patient team members. Moderated discussion will follow presentations to examine experiences with patient-partnered research, advancements to patient-centered measurement research, and opportunities for future work.

\section{Individual Presentations}

\section{Priorities for advancing methods for measuring what matters:} patient and researcher perspectives

Lena Cuthbertson, BHSc (OT), MEd, OT(C), PMP, Office of PatientCentred Measurement, British Columbia Ministry of Health,

Vancouver, British Columbia, Canada; Richard Sawatzky, PhD, RN, Trinity Western University, Langley, British Columbia, Canada

Aims: This presentation reports on the outcomes of a large-scale engagement effort to explore patient-oriented methodological priorities for advancing the science of patient-centred measurement, including the use of patient-reported experience and outcome measures, from diverse perspectives of patients and other research community stakeholders. Findings informed a call for proposals for research projects to address priority themes for enhancing patientcentred measurement methods in the province of British Columbia (BC), Canada. Methods: Workshops were conducted with two types of stakeholder groups in every region of BC:7 workshops with patients and family caregivers $(N=74)$, and 7 workshops with users of patient-reported data $(N=96)$, including researchers from academic or healthcare organizations, patient experience, or quality improvement leaders, and decision makers in health services administration. The workshops were recorded, transcribed verbatim, and subsequently analyzed using a qualitative descriptive approach. Results: Diverse perspectives of both groups of stakeholders were categorized into 11 priority themes. Four themes focus explicitly on the involvement of patients, family caregivers and communities: Measurement that is patient driven. Giving "voice" to patient, family caregiver, and community stories. Ensuring patients feel safe to 
provide feedback. Ensuring the data collected accurately reflects what is important to patient. Four themes address the need for methodological advancements to enhance equity, inclusivity, and representativeness in patient-centred measurement: individualized or tailored measurement in diverse populations; representation of marginalized, vulnerable, or hard-to-reach populations; measuring across the journey of care; and indigenous methodologies for patientcentred measurement. Three additional priorities for methodological advancements focus on improvements in the analysis, reporting, and use of patient-centred measurement data: improving use of patientcentred measurement data for research, clinical, evaluation, and administrative purposes; integration of patient-reported data with clinical and administrative data; and innovative technology for patient-reported data collection, analysis, reporting, and use. Conclusion: This consultation effort revealed the value of engaging diverse stakeholders, including patients, in identifying research priorities for advancing the science of patient-centred measurement methods. The call for proposals yielded 10 research projects and 4 research fellowships. Project descriptions and further information are available

https://bcsupportunit.ca/about/methods-clusters/patient-centredmeasurement.

\section{Considerations for the assessment and sharing of caregiver- reported outcomes: a qualitative interpretive description study}

Fuchsia Howard, PhD, RN, University of British Columbia, Vancouver, British Columbia, Canada

Aims: Primary caregivers, key family members or friends, can experience high levels of caregiver burden across the cancer trajectory. Beginning at the time of diagnosis and then at different points in the cancer journey, it is unknown how and when screening for caregiver-reported outcomes (CROs) would best be done. CROs refer to a caregiver's assessment of their own health status as a result of supporting a patient with cancer. Our purpose was to describe how CROs could be integrated into cancer care in a manner that meets caregivers' needs. Caregivers of patients with colorectal cancer (CRC) were our focus because CRC is one of the most commonly diagnosed cancers and caregiving can be particularly demanding in this context. Methods: As guided by a qualitative Interpretive Description approach, our team of researchers, clinicians, and patient and caregiver partners conducted and analyzed semi-structured interviews with 25 caregivers, 37 patients with CRC, and 16 healthcare providers (HCPs) using inductive coding and constant comparative techniques. Results: Among both the caregiver and patient participants, contrasting perspectives about the assessment and sharing of CROs with patients were expressed. Transparency in the sharing of CROs was considered by some caregivers and patients to be essential for understanding and appreciating caregiver challenges. In contrast, others preferred that the assessment and discussion of CROs remain private, rather than shared with the patient. For caregivers, this preference for privacy arose out of concern about burdening the patient and guilt if the patient was aware of caregiver struggles. Patients who preferred not to know the results of CROs expressed feeling unable to hear, appreciate, or attend to caregiver needs. HCPs were aware of variation in caregiver and patient preferences, and they described strategies for informally assessing CROs that were sensitive to these preferences. Conclusion: The integration of CROs into cancer care would benefit from careful consideration of caregiver and patient preferences regarding the transparency or privacy of CRO assessment and sharing of results to prevent additional caregiver burden.

\section{Making visible the needs of vulnerable and marginalized populations}

Kelli Stajduhar, $\mathrm{PhD}$, University of Victoria, Victoria, British Columbia, Canada; Kara Whitlock, BSW, University of Victoria, Victoria, British Columbia, Canada

Aims: This integrated knowledge translation project aimed to advance methods in the implementation of patient-reported outcome measures (PROMS) and patient-reported experience measures (PREMS) (i.e., recruitment, data collection, determining measures, and engagement of the target population) for people with chronic illness who have lived experience of homelessness. This presentation focuses specifically on the patient-oriented co-development process for testing PROMs and PREMs. Methods: Over the course of 14 two-hour meetings, an advisory committee of persons with lived experience met virtually with two research team members and a co-chair with lived experience. Meetings were recorded, transcribed, and thematically analyzed. NVivo 12 was used to organize and code data. Transcripts were re-read to generate an initial codebook, which was refined in an iterative fashion throughout the analysis. Results: Participants began by discussing concepts related to PROMs and PREMs and identifying the most important concepts for persons with lived experience of homelessness who also experience chronic illness. As concepts were explored and refined, the group transitioned to discussing examples of PROMs and PREMs. These discussions generated a series of themes, as well as specific recommendations for the design and delivery of PROMs and PREMs. Participants elaborated on the meaning of key PROM and PREM concepts, including quality of life and quality of care, in the context of homelessness, poverty, and intersecting vulnerabilities. Several existing PROM and PREM tools were discussed for relevance; and strengths, weaknesses, and opportunities for adaptation were proposed. Experience measures were identified as a top priority by advisory committee members, as were relational PROM and PREM measures including being listened to, being treated with dignity, having a trusting relationship with health care providers, and having overlapping health issues addressed at one site or in one visit. Conclusion: People who experience structural vulnerability face severe disadvantage as their health declines, lack trust in the health care system and its providers, and feel judged related to vulnerabilities such as mental health issues or substance use. PROMs and PREMs offer a potential avenue for making the needs and concerns of structurally vulnerable persons visible within the health care system.

\section{Centering lived expertise and equity in health metrics}

Saraswathi Vedam, RM, PhD, FACNM, Birth Place Lab, University of British Columbia, Vancouver, British Columbia, Canada; Rachel Olson, PhD, DirectOR Firelight Research Group; Co-PI, Decolonizing Birth Research, Vancouver, British Columbia, Canada; Aldabaran Tatum, BA, Colourwave Consulting and Birth Place Lab, Vancouver, British Columbia, Canada; Kathrin Stoll, PhD, Birth Place Lab at UBC, Vancouver, British Columbia, Canada; Submitted on behalf of the Steering Councils, RESPCCT Study and Decolonizing Birth Research

Aims: Poor access to respectful care has emerged as a critical factor associated with adverse health outcomes in childbearing communities. Research highlights that disenfranchised populations are more 
likely to experience barriers and mistreatment when accessing or receiving care. People with lived experiences of care are increasingly acknowledged as the "experts" who are best positioned to inform patient-oriented, community-responsive research, knowledge translation, and quality improvement. We aim to study healthcare outcomes through an equity lens. Methods: Our research cluster specializes in health services participatory action research. Projects include a provincial study of experiences of maternity care; a national study where service users with a broad range of identities, circumstances and backgrounds, report on their experiences of childbearing care; and an international study examining experiences of respect, discrimination, and mistreatment during childbearing care among communities of color. Transdisciplinary study teams developed novel person-centered measures across domains that have been understudied. These measures are now being adapted to study respect and mistreatment in gender and sexual minority populations in the Birth includes US study, and the Decolonizing Birth Research project, coled by Indigenous researchers that explores the applicability of these methods and measures in BC Indigenous communities. Results: Our team has developed a systematic process for engagement, training, and support for community researchers to participate in study design, recruitment, analysis and interpretation of data, and reporting, including community-led design of images and messaging via social media and arts-based KT tools. We will report findings on best practices in equity-based participatory research along 4 themes: trust building; centering the lived experience throughout the research process; community-responsive instrument development; and codifying equity approaches within the research unit. Conclusion: Intentional, relationship-based, and continuous inclusion of community partners, knowledge keepers, and decision makers, throughout the research process highlighted the importance of attention to cultural safety, and reproductive justice training and support for community partners and staff. By applying these principles of participatory action research, we can elevate the concerns and perspectives of the most marginalized, increase the integrity and availability of data in understudied domains, and enhance the responsiveness and quality of health care for all.

\section{Oral Sessions}

\section{1: Application and Methods of Mental Health}

(101.1) Impact of Item Non-Response on the Detection of Differential Item Functioning in Multi-Item Patient-Reported Outcome Measures

Olawale F. Ayilara, University of Manitoba, Winnipeg, Manitoba, Canada; Mohammad Jafari Jozani, PhD, University of Manitoba, Winnipeg, Manitoba, Canada; Tolulope T. Sajobi, PhD, University of Calgary, Calgary, Alberta, Canada; Ruth Barclay, PhD, University of Manitoba, Winnipeg, Manitoba, Canada; Lisa M. Lix, PhD, University of Manitoba, Winnipeg, Manitoba, Canada

Aims: Differential item functioning (DIF) arises when the probability of item response in a multi-item measure varies amongst groups after controlling for a latent variable effect. Item non-response (i.e., missing data) can mask DIF detection. This study addressed incomplete ordinal item-level patient-reported outcome measures using a machine-learning method and compared its performance for DIF detection with commonly used missing data methods. Methods: Monte Carlo simulation was used to assess the accuracy of a graded response model (GRM) for DIF detection using four missing data methods. Simulation parameters included the magnitude of DIF, sample size, and percentage of missing observations (0-50\%). Item non-response was addressed using (a) list-wise deletion (LD), (b) half-mean imputation (HMI), (c) full information maximum likelihood (FIML), and (d) non-negative matrix factorization (NNMF); the latter is an unsupervised machine-learning method that uses optimization techniques to detect a low-dimensional structure from the data. Robustness of Type I error rates (i.e., probability of erroneously rejecting a true null hypothesis) were evaluated using a liberal criterion [0.025-0.075] for $\alpha=0.05$. We compared the power of the GRM with and without adoption of a missing data method; differences $\leq 10 \%$ were considered negligible while those $>10 \%$ were considered non-negligible. Results: Type I error rates for DIF tests using LD, FIML, and NNMF missing data methods were within the bounds of the liberal criterion for more than $90 \%$ of investigated simulation conditions. The HMI method resulted in inflated error rates with $50 \%$ missing data. Statistical power to detect DIF increased as the magnitude of DIF and sample size increased. Power differences to detect moderate DIF effects for the LD, FIML, and NNMF methods were non-negligible with $50 \%$ missing data and negligible for smaller amounts of missing data. Conclusion: The NNMF method performed as well as commonly used methods to address ordinal item nonresponse when testing for DIF. This method has the advantage of being computationally efficient. However, given that no single method was optimal for all simulation conditions, we recommend that researchers compare two or more missing data methods for ordinal item non-response in sensitivity analyses.

(101.2) Optimizing the efficiency of computerized adaptive tests using real data: A machine learning approach

Michiel Luijten, MSc., Amsterdam UMC, Amsterdam, The Netherlands; Benjamin Schalet, PhD, Northwestern University, Chicago, Illinois, USA; Leo Roorda, PhD, Reade Amsterdam, Amsterdam, The Netherlands; Martha Grootenhuis, Prof., Princess Máxima Center for Pediatric Oncology, Amsterdam, The Netherlands; Lotte Haverman, $\mathrm{PhD}$, Emma Children's Hospital, Amsterdam UMC, Amsterdam, The Netherlands; Caroline Terwee, PhD., Amsterdam UMC, Amsterdam, The Netherlands;

Aims: To reduce administrative burden and increase efficiency of Computerized Adaptive Tests (CAT) our aim is to develop an additional stopping rule for CATs based on change in SE of a person's estimated score $(\theta)$ after each administered item. Methods: In April 2020 and November 2020 Patient-Reported Outcomes Measurement Information System (PROMIS) CATs for several domains were administered to assess the mental and social health of children in the The Netherlands during the COVID-19 pandemic ( $n$-range: 3212-3429). The stopping rules consisted of a standard error of measurement $(\operatorname{SE}(\theta))<0.32$ (which equals $90 \%$ reliability) or a maximum of 12 items administered. Data on item selection, item responses, $\theta$ and $\operatorname{SE}(\theta)$ of each step within the CAT were extracted. Per domain, the dataset was randomly split into a training and test set. Using a machine-learning procedure the efficiency $((1-\operatorname{SE}(\theta) 2) /$ nitems) was maximized against the change in $\operatorname{SE}(\theta)$ in the training set to determine the optimal change in $\operatorname{SE}(\theta)$ to be used as a stopping rule. This stopping rule was subsequently applied to the test set (in addition to standard stopping rules) and the amount of participants reliably estimated $(\operatorname{SE}(\theta)<0.32)$, average test length and relative efficiency were compared to using only the original stopping parameters. We applied this procedure to one domain with relatively low discrimination parameters (Anxiety) and one domain with high discrimination parameters (Depressive Symptoms) as it is likely that the optimal stopping rule of change in $\operatorname{SE}(\theta)$ is influenced by the discrimination parameters within the item response theory (IRT) model. Results: Preliminary results show that on Depressive 
Symptoms/Anxiety, respectively $1193(35.9 \%) / 1369(39.7 \%)$ of participants had 12 items administered of which $33.9 \% / 18.9 \%$ were due to floor effects. For these floor effects, a change in SE of 0.01 would respectively reduce the amount of items administered from $12(\operatorname{SE}(\theta)=0.588 /(\operatorname{SE}(\theta)=0.566))$ to $4(\operatorname{SE}(\theta)=0.619) / 5(\operatorname{SE}(\theta)=$ $0.592)$, which results in a difference of $T$-score estimates of 3.3(32.0 vs 35.3)/3.4(31.9 vs 34.3). Conclusion: Optimizing CAT efficiency by adding an additional stopping rule based on the change in $\operatorname{SE}(\theta)$, may reduce the burden of PROMIS administration, while retaining precise, reliable measurements. Further optimizing the stopping rule will likely result in better trade-offs with fewer negative consequences to $\mathrm{T}$-score estimates.

(101.3) Measuring what matters to young people: development of a measurement curriculum for service providers working within integrated youth health services

Skye Barbic, PhD, The University of British Columbia, Vancouver, British Columbia, Canada; Eran Tal, PhD, McGill University, Montreal, Quebec, Canada; Steve Mathias, MD, Foundry, Vancvouer, British Columbia, Canada; Sebastian Rodriguez Duque, MA, McGill University, Montreal, Quebec, Canada; Kyle Dewsnap, The University of British Columbia, Vancouver, British Columbia, Canada; Sophie Osiecki, McGill University, Montreal, Quebec, Canada; Darius Valevicius, McGill University, Montreal, Quebec, Canada; Danielle Celone, McGill University, Montreal, Quebec, Canada

Aims: Canada's enthusiasm for measuring what matters to youth accessing integrated youth health services (IYHS) has never been higher. One barrier to measuring youth outcomes is a lack of training on the behalf of service providers for how to select, interpret, and use measures in practice. The aim of this presentation is to summarize a unique collaboration between young people, service providers, philosophers, and metrologists to design a curriculum to teach service providers about measurement. Specific objectives are to: (1) Describe why a measurement curriculum designed for Canadian IYHS service providers is needed; (2) Understand how the curriculum and training strategy was co-designed, and (3) Describe the implementation of the training within a large IYHS network. Methods: After hiring five youth trainees, two service providers, and two measurement experts, we developed an outline for the training and preliminary content, with embedded human design methods to guide integrated knowledge translation activities. In our first expert workshop, we invited experts from diverse backgrounds (psychiatry, primary care, psychology, rehabilitation, anthropology, ethics, law, philosophy, and psychometrics. Experts were asked to review content and comment in both presentation and written format. After this workshop, we used humancentred participatory research methods, specifically weekly co-design meetings to engage young people, clinicians, and decision-maker from across Canada in the co-design of knowledge products for the training for immediate at one IYHS organization called Foundry. Forty service providers from across Foundry were invited to the pilot training and the information from the pilot informed a training plan across the Foundry network (19 communities and a virtual service). Results: A training curriculum, includes a guidebook, training videos, youth-centred infographics and knowledge products, and a virtual training workshop resulted. Conclusion: Our study provides preliminary evidence for a training curriculum to support clinicians to learn about the application of measurement in practice. Our study provides a foundation to begin to understand the knowledge needs for clinical. As well, the methodology presented in our study may have wide scale applicability for co-designing training curriculums that are clinically relevant and can inform clinical care for end-user stakeholders in mental health and beyond.
(101.4) Parents of children with medical complexity: a qualitative exploration of impact on health and well-being from caregiving and care coordination

Oksana Hlyva, MSc, PhD, Department of Pediatrics, McMaster University, Hamilton, Ontario, Canada; Charlene Rae, MSc, Department of Pediatrics, McMaster University, Hamilton, Ontario, Canada; Shelby Deibert, MHK, Department of Pediatrics, McMaster University, Hamilton, Ontario, Canada; Haniah Shaikh Shaikh, Bachelor of Health Science, Michael G. DeGroote School of Medicine, McMaster University, Hamilton, Ontario, Canada; Rakhshan Kamran, BSc (Hons), Michael G. DeGroote School of Medicine, McMaster University, Hamilton, Ontario, Canada; Anne Klassen, BA (Hons), DPhil, Department of Pediatrics, McMaster University, Hamilton, Ontario, Canada; Audrey Lim, MD, Masters of Community Health Sciences, Department of Pediatrics, McMaster University, Hamilton, Ontario, Canada

Aims: To explore parent perspectives on how both providing and coordinating care for their child with medical complexity (CMC) affects parents' health and well-being. Methods: The study was guided by interpretive description, a qualitative research methodology designed to respond to experience-based questions of interest to a practice-based discipline. A purposive sampling strategy was employed. In-depth 90-min interviews were conducted using a semistructured interview guide to learn from parents about their experience of caring for CMC, the impact of this experience on their lives and health status, and family experiences of care and care coordination. The interviews took place at parents' homes, at the clinic, or by phone. The interviews were audio-recorded, transcribed verbatim, and coded line-by-line using NVivo 10. The coded data were analyzed inductively and iteratively, generating study themes. Results: Seventeen parents completed the interviews. Participants resided in rural and urban areas in Southern Ontario. Participants represented a variety of families, including foster and single parents. The majority were white females 26 to 43 years of age. Most were college or university educated. The age of the children ranged from 1 to 10 years. The children had a broad range of genetic, neuromuscular, respiratory, and craniofacial conditions. Eleven children were dependent on tracheostomy and four children required home mechanical ventilation.Several themes and subthemes were identified, highlighting multiple ways caring and coordinating care for CMC impact their lives. The themes include the following: (i) day-to-day caregiving and parent roles (as a "paramedic", care coordinatOR and advocate); (ii) additional burden of care (home nursing paucity; financial stress); (iii) parent functioning and health (physical, mental, social and emotional health); (iv) potential ways to alleviate the burden. Conclusion: CMC family caregivers report experiencing a detrimental impact on their health and well-being from poorly supported caregiving at home as well as from their very complex role coordinating multifaceted fragmented healthcare for their children in both clinical and community settings. The study participants also underscored the vital importance of family-centered coordinated complex care clinics and tailored caregiver health-related supports at home and in the community as potential ways to alleviate the burden.

(101.5) Unsupervised learning methods for assessing differential item Functioning in Hospital Anxiety and Depression Scale

Tolu Sajobi, PhD, University of Calgary, Calgary, Alberta, Canada; Ridwan A Sanusi, PhD, University of Manitoba, Winnipeg, Manitoba, Canada; Nancy Mayo, PhD, McGill University, Montreal, Quebec, Canada; Richard Sawatzky, PhD, Trinity Western University, Langley, British Columbia, Canada; Lene Kongsgaard Nielsen, MD, 
Quality of Life Research Center, Odense University Hospital, Odense, Denmark; Veronique Sebille, PhD, EA 4275 SPHERE, methods in Patient-centered outcomes and HEalth ResEarch, Nantes University, Nantes, France; Juxin Liu, PhD, Department of Mathematics \& Statistics, University of Saskatchewan, Saskatoon, Saskatchewan, Canada; Eric Bohm, MD, MSc, Rady Faculty of Health Sciences, University of Manitoba, Winnipeg, Manitoba, Canada; Stephen Wilton, MD, MSc, Department of Cardiac Sciences, University of Calgary, Calgary, Alberta, Canada; Matthew. T James, $\mathrm{MD}, \mathrm{PhD}$, Department of Community Health Sciences, University of Calgary, Calgary, Alberta, Canada; Colleen. M Norris, RN, PhD, Faculty of Nursing, University of Alberta, Edmonton, Alberta, Canada; Lisa Lix, PhD, Department of Community Health Sciences, University of Manitoba, Winnipeg, Manitoba, Canada

Aims: Unsupervised learning methods, including latent class analysis and machine learning models, have recently been proposed for examining differential item functioning (DIF) in patient-reported outcome (PRO) data when the predictors associated with DIF are not known a priori. These procedures are expected to differ in their sensitivity to identify homogeneous subgroups because of differences in their underlying algorithms. This study compared the latent class Rasch and tree-based Rasch models for identifying demographic and clinical variables that may predict DIF in PRO measures Methods: Data were from 4487 individuals with coronary artery disease (CAD) in the Alberta Provincial Project on Outcome Assessment in Coronary Heart Disease registry who completed the Hospital Anxiety and Depression Scale (HADS) following a cardiac angiogram procedure. Latent class Rasch model and a Rasch tree model based on recursive partitioning were used to identify (1) subgroups of patients with DIF on the 7 items of the HADS depression subscale and (2) demographic and clinical variables associated with DIF. For latent class model, the best fitting model fit was determined using lowest Bayesian Information Criterion and the Vuong-Lo-Mendell-Rubin likelihood ratio test that compares models with $\mathrm{k}$ and $\mathrm{k}-1$ classes. Bonferroni-corrected structural change tests were used to prevent overfitting in the tree-based model. Weighted and unweighted analysis of variance and chi square tests were used to test the variables associated with subgroup differences in latent class and Rasch tree models, respectively. Results: The median (IQR) age was $64.5(15.7)$ years, 3522 $(78.5 \%)$ patients were male, and 3102(69.1\%) had unstable angina. A 4-class Rasch model had better fit than a one-class Rasch model. The Rasch tree model identified nine subgroups (nodes) via recursive partitioning using age, sex, type of treatment received, and smoking as splitting variables. For both methods, between-subgroup differences were explained by age, type of treatment received, disease indication, smoking status, and comorbid diabetes, congestive heart failure, and chronic obstructive pulmonary disease. Conclusion: Latent class Rasch and tree-based Rasch models were similar in identifying multiple demographic and clinical variables that may explain DIF. Future research will use computer simulations to assess the accuracy of these models for DIF detection for various data features.

\section{(101.6) Application of an HRQoL measure to inform connections to services for First Nations children in Canada}

Nancy Young, CHEO Research Institute, Ottawa, Ontario, Canada; Gerrilynn Manitowabi, BHSc, MPH, NAANDWECHIGE-GAMIG Wikwemikong Health Centre, Wiikwemkoong Unceded Territory, Ontario, Canada; Diane Jacko, BA, NAANDWECHIGE-GAMIG Wikwemikong Health Centre, Wiikwemkoong Unceded Territory, Ontario, Canada; Samantha Fowler, BSc, MScCom, ECHO Research Centre, Laurentian University, Sudbury, Ontario, Canada; Marnie Anderson, BPHE, Laurentian University, Sudbury, Ontario, Canada;
Mary Jo Wabano, MHK, Naandwechige-Gamig Wikwemikong Health Centre, Wiikwemkoong Unceeded Territory, Ontario, Canada; Trisha Trudeau, MA, Naandwechige-Gamig Wikwemikong Health Centre, Wiikwemkoong Unceeded Territory, Ontario, Canada; Ranjeeta Mallick, PhD, The Ottawa Hospital Research Institute, Ottawa, Ontario, Canada; Peter Szatmari, MD, Hospital for Sick Children, Toronto, Ontario, Canada; Koyo Usuba, MHK, Laurentian University, Sudbury, Ontario, Canada; Lorrilee McGregOR PhD, Northern Ontario School of Medicine, Sudbury, Ontario, Canada; Brenda Restoule, PhD, First People's Wellness Circle, Nipissing First Nation, Ontario, Canada; Annie Roy-Charland, $\mathrm{PhD}$, Université d'Moncton, Moncton, New Brunswick, Canada; Skye Barbic, PhD, University of British Columbia, Vancouver, British Columbia, Canada; Kednapa Thavorn, PhD, The Ottawa Hospital Research Institute, Ottawa, Ontario, Canada; Christopher Mushquash, PhD, Lakehead University, Thunder Bay, Ontario, Canada; Stephen Ritchie, PhD, Laurentian University, Sudbury, Ontario, Canada; submitted on behalf of "The ACHWM IMPACT study team"

Aims: First Nations health leaders are working to promote wellness among their children and youth. An HRQoL measure was developed for Indigenous children to support this goal; the Aaniish Naa Gegii: the Children's Health and Well-being Measure (ACHWM). We sought to determine whether the ACHWM identified children's needs for support earlier in their wellness journey. Methods: Children 8 to 18 years of age were recruited from the community (schools, youth centre and community events in Wiikwemkoong) and through the local mental health clinic. All children completed the ACHWM then met with a local mental health worker who determined whether their health was at-risk. ACHWM Emotional Quadrant Scores (EQS) were compared to determine if children identified in the community as at-risk had higher (healthier) scores than children who were already accessing local mental health services. We also compared both groups to their healthy peers. Results: Three groups of children were formed: a healthy peers (HP) group who were not at-risk $(n=134)$; a newly identified needs (NIN) group who were at-risk and not previously identified $(n=35)$; and a typical treatment group who were already receiving support $(n=58)$. The mean EQS were: 80.1 (SD 11.25) in the HP group; 67.2 $(\mathrm{SE}=13.27)$ in the NIN group; and 66.2, $(\mathrm{SD}=16.30)$ in the TT group. The HP group had significantly higher (healthier) EQS than NIN and TT groups $(p<0.0001)$. The NIN and TT groups EQS did not differ from each other $(p=0.76)$. Conclusion: The ACHWM was able to distinguish between healthy children and those in need of support. This study identified needs for support among 35 children. These children were then connected to local services earlier than would have occurred through the standard referral process. The similarity of EQS in the NIN and TT groups highlights the value of community screening to optimize access to services. Future research will examine the impact of this process over the subsequent year in these groups. This measure provides an effective option for connecting First Nations children to local supports.

(101.7) Employment and family life course typologies in the 25 years following the German reunification: Associations with subjective health, wellbeing, and social context

Laura Altweck, Greifswald University, Greifswald, Germany; Stefanie Hahm, Dipl.-Psych., Greifswald University, Greifswald, Germany; Holger Mühlan, Dr., Greifswald University, Greifswald, Germany; Silke Schmidt, Prof. Dr., Greifswald University, Greifswald, Germany

Aims: The German reunification 1989/90 was followed by extensive socio-structural transformations like mass unemployment and dissolution of social networks, which especially affected the East Germans 
joining the West German system. Because health and wellbeing are a product of life course trajectories, we examined sociodemographic variables, subjective health, and wellbeing in relation to employment and family life course typologies in the 25 years following the reunification. Methods: To determine life course clusters, sequence and cluster analysis was performed on the annual states of employment, marital, and children in household (waves 1990-2014) of the German Socio-Economic Panel data $(N=12.298 ;$ age $=49.37,52.87 \%$ female). Using multinomial logistic regression analyses, associations between clusters and sociodemographic variables, social context (East vs. West German), subjective mental and physical health, and wellbeing (general and domain-specific life satisfaction, affective wellbeing) (wave 2014) were examined. Results: Seven clusters were identified: mixed employment $\&$ separated $(4.26 \%)$, mixed employment $\&$ single (20.90\%), mixed employment \& married (7.82\%), non-employment \& married $(25.04 \%)$, full-time employment \& married (reference, $17.84 \%$ ), full-time employment \& mixed family (18.51\%), and parttime employment \& married, no children (5.64\%). Compared to fulltime employment \& married, the East versus West German sample was less likely to belong to all other life course clusters (OR [0.20-0.51]); especially mixed employment \& married (OR 0.20) and part-time employment \& married, no children (OR 0.27). Distinct differences in subjective physical health and wellbeing emerged for cluster membership. For instance, while both mixed employment \& separated and non-employment \& married were associated with lower subjective physical health and satisfaction with life, health, and sleep, the former was also related to lower affective wellbeing and satisfaction with family, while the latter to greater satisfaction with family. Subjective mental health only showed few associations. Conclusion: Distinct types of life courses and differential associations with sociodemographic data, subjective health, and wellbeing emerged. While subjective physical health, general, and domain-specific life satisfaction were significantly related to life course typologies, less implications were seen for subjective mental health. The analyses reveal a burden on individuals who experienced partnership separation and non-employment and, thus, present important target groups for prevention, e.g., for physical health problems.

\section{2: Application of Clinical Research in Children's Health}

\section{(102.1) Characterizing free-text aspirations of Duchenne} Muscular Dystrophy patients, their siblings, and the general population: How do humans and Natural Language Processing compare?

Yuelin Li, PhD, Memorial Sloan Kettering Cancer Center, New York, New York, USA; Roland B. Stark, M.Ed., DeltaQuest Foundation, Boston, Massachusetts, USA; Elijah Biletch, DeltaQuest Foundation, Boston, Massachusetts, USA; Richard B. B. Stuart, DeltaQuest Foundation, Boston, Massachusetts, USA; Carolyn E. Schwartz, Sc.D., DeltaQuest Foundation, Boston, Massachusetts, USA

Aims: Qualitative methods can enhance our understanding of constructs that have not been well portrayed and enable nuanced depiction of experience from study participants who have not been broadly studied. However, qualitative data require time and effort to train raters to achieve validity and reliability. This study compares recent advances in Natural Language Processing (NLP) models with human coding. Methods: This web-based study $(N=1,638 ; 6942$ free-text entries) included people with Duchenne Muscular Dystrophy (DMD), their siblings, and a representative comparison group. Human raters $(n=6)$ were trained over multiple sessions in content analysis as per a comprehensive codebook. Four prompts addressed distinct aspects of participants' aspirations. Unsupervised NLP was implemented using Latent Dirichlet Allocation (LDA), which extracts latent topics across all the free-text entries. Supervised NLP was done using Bidirectional Encoder Representations from Transformers (BERT) model, which requires training the algorithm to recognize relevant human-coded themes across all the free-text entries. Preliminary analysis using a portion of the dataset was randomly divided into training $(n=620)$ and validation ( $n=157)$ sets for BERT. We compared the human-, LDA-, and BERT-coded themes. Results: Study sample contained 286 people with DMD, 355 DMD siblings, and 997 comparison participants, age 8-69. Human coders generated 95 codes across the four prompts and had an average inter-rater reliability (Fleiss's kappa) of 0.77 , with minimal rater-effect $(\mathrm{R} 2=2 \%)$. LDA extracted seven latent topics related to Material, Quality of Life, Brother's Lot, World Problems, Normative Self-Aspirations, Normative Others-Aspiration, and Work/ Purpose. In preliminary trials, BERT accurately learned $93 \%$ of the training set of human-annotated free-text statements but correctly predicted only $31 \%$ of the validation set. LDA and BERT took approximately $1.15 \mathrm{~min}$ per open-text entry, compared to $1.18 \mathrm{~min}$ for human raters Conclusion: LDA and BERT provide potentially viable approaches to analyzing large-scale qualitative data, but both have limitations. When text entries are short, LDA yields latent topics that are hard to interpret. Even though BERT was able to achieve high accuracy during learning, it accurately identified only about one-third of neverencountered new statements. Humans provided reliable and cost-effective coding in the web-based context. Future research will examine NLP's predictive accuracy given different quantities of training data.

(102.2) Psychometric Evaluation of the Pediatric RSV Severity and Outcomes Rating System Version 7 (PRESORSv7.1, Observer-Reported and PRESORSv7 Clinician- Reported Forms) Scores in Young Children Hospitalized With Respiratory Syncytial Virus Infection

Shanshan Qin, PhD, RTI Health Solutions, Research Triangle Park, North Carolina, USA; Lauren Nelson, PhD, RTI Health Solutions, Research Triangle Park, North Carolina, USA; Lori McLeod, PhD, RTI Health Solutions, Research Triangle Park, North Carolina, USA; Jia Ma, PhD, RTI Health Solutions, Research Triangle Park, North Carolina, USA; Beatriz López Sánchez, Janssen Research \& Development, Leiden, The Netherlands; Mohamed Gamil, Janssen Research \& Development, Beerse, Belgium; Marita Stevens, Janssen Research \& Development, Beerse, Belgium; Jane Scott, Janssen Global Services, High Wycombe, Buckinghamshire, UK

Aims: RSV-related lower respiratory tract infection is a major cause of hospital admissions and death in young children worldwide. To meet the need for a comprehensive assessment of respiratory and behavioral signs for infants or young children with RSV, two clinical outcome assessment measures, the PRESORS ObsRO and ClinRO, have been developed according to guidelines set forth by the Food and Drug Administration. The objective was to assess the psychometric properties of a classification system for symptom resolution (Resolved or Not Resolved scores) for the two PRESORS measures. Methods: The analyses were conducted on the Resolved/Not Resolved scores of each PRESORS measure for individual symptoms and summaries of Key RSV Symptoms, Respiratory Symptoms, General Illness Behaviors, and Overall RSV Symptoms, using baseline to Day 14 blinded data of 106 hospitalized children aged $\geq 28$ days to 36 months from a phase 2 clinical trial for rilematovir, an RSV fusion inhibitor. Descriptive statistics were examined for distribution abnormality. Test-retest reliability was analyzed in stable patients defined by several criteria. Correlations and cross-tabulation for construct validity and for the ability to detect change were computed with supporting measures (e.g., global ratings, supplemental oxygen/feeding/hydration, and discharge readiness). 
Results: Ceiling effects (best outcomes) were minimal at baseline for all PRESORS ObsRO and ClinRO summaries ( $\leq 12.3 \%$ resolved). Summaries generally demonstrated moderate to substantial test-retest reliability (79-95.2\% absolute agreement; simple kappa, 0.38-0.68) in patients with no change in global RSV severity or health status. Expected frequency patterns and moderate to strong correlations ( $|r|=0.39-1.00)$ were observed between all PRESORS summaries and the global ratings and discharge readiness at Day 5. A higher proportion of unresolved cases was observed among inpatients and particularly in patients with supplemental oxygen across time points, supporting discriminating ability. Compared with changes in global RSV severity or health status from baseline to Day 8/end of treatment, responsiveness correlations were mostly moderate to strong $(|r|=0.39-0.63)$ with anticipated change patterns for all PRESORS summaries. Conclusion: The results support the PRESORS ObsRO and ClinRO as appropriate measures to monitor RSV signs/symptoms resolution for hospitalized pediatric patients in clinical research

(102.3) Asthma control and health-related quality of life in pediatric patients and its relationship with the mother's educational level: ARCA Study (Asthma Research in Children and Adolescents)

Catalina Lizano-Barrantes, PharmD, IMIM- Hospital del Mar Medical Research Institute, Barcelona, Spain; Karina Mayoral, IMIMHospital del Mar Medical Research Institute, Barcelona, Spain; Laura Valdesoiro, Hospital Parc Taulí, Barcelona, Spain; Inés de Mir, Hospital Vall d'Hebron, Barcelona, Spain; María Araceli Caballero, Hospital del Mar, Barcelona, Spain; José Antonio Castillo, Hospital Miguel Servet, Zaragoza, Spain; Pilar Ortiz, Centro de Salud Dos de Mayo, Madrid, Spain; Àngels Pont, IMIM- Hospital del Mar Medical Research Institute, Barcelona, Spain; Olatz Garin, IMIM- Hospital del Mar Medical Research Institute, Barcelona, Spain; Montse Ferrer, IMIM- Hospital del Mar Medical Research Institute, Barcelona, Spain; ARCA Group, IMIM- Hospital del Mar Medical Research Institute, Barcelona, Spain

Aims: The objective of the study is to evaluate inequalities in the health of children/adolescents with asthma, according to the educational level of the mother. Methods: Participants from ARCA, a multicenter, observational, longitudinal, prospective study of children/adolescents (6-14 years old) with persistent asthma were included. In the telephone interview at baseline, we collected the highest level of education of the parents and the Asthma Control Questionnaire (ACQ), which assesses the frequency of asthma symptoms with an overall score from 0 to 6. Through the ARCA App, designed to monitor the participants, we administered the specific questionnaire on quality of life for pediatric asthma of the PatientReported Outcomes Measurement Information System (PROMIS), with a total raw score of 8 to 40 . We evaluated differences between groups of children/adolescents defined according to the mother's educational level (A- primary or less, B- secondary, and C-university) with ANOVA. Results: Of the 179 participants included, 25 were located in group A, 69 in group B, and 85 in group C. The ACQ presented statistically significant differences between groups in the mean global score (A: 1,2, B: 0,9, y C: 0,6, $p=0.004$ ). The pediatric asthma PROMIS was completed by 119 participants, with no statistically significant differences between group mean scores (A: 12.7, B: 13.0, and $\mathrm{C}: 12.5, p=0.897)$. Conclusion: These results show that the mother's higher educational level is positively associated with asthma control in children/adolescents, but does not affect health-related quality of life.Funding: Instituto de Salud Carlos III FEDER: Fondo Europeo de Desarrollo Regional (PI15/00449 and FI16/00071), Universidad de Costa Rica (OAICE-85-2019).
(102.4) Understanding outcomes and experiences of care important to transgender and gender diverse youth

Shelby Deibert, MHK, McMaster University, Hamilton, Ontario, Canada; Manraj Kaur, PT, PhD, Brigham and Women's Hospital, Boston, Massachusetts, USA; Oksana Hlyva, PhD, McMaster University, Hamilton, Ontario, Canada; Leah Algu, BHSc, University of Toronto, Toronto, Ontario, Canada; Natasha Johnson, MD, FAAP, FRCPC, McMaster University, Hamilton, Ontario, Canada; Shane Morrison, MD, MS, University of Michigan Medical Center, Ann ArbOR Michigan, USA; Andrea Pusic, MD, MHS, FACS, FRCSC, Brigham and Women's Hospital, Boston, USA; Anne Klassen, BA, DPhil, McMaster University, Hamilton, Ontario, Canada

Aims: Gender-affirming hormones and surgery are provided to transgender and gender diverse teenagers and adults in many countries. Such treatments can reduce gender dysphoria and allow people to live more comfortably with their body by changing their voice and how their face and body look and function. Given the lack of available PROMs to evaluate treatment outcomes, our team is developing the GENDER-Q, which will provide a means to evaluate outcomes and healthcare experiences in gender-affirming treatment. To develop the GENDER-Q, interviews were conducted with 84 transgender and gender diverse people from 4 countries. In the sample, 10 participants were teenagers aged 16-18 years old. This abstract describes the concepts identified as important to youth through the teenager interviews. The findings from these interviews were used to inform a new study to develop a PROM for younger transgender and gender diverse youth that is currently underway. Methods: An interpretive description approach was used. Interviews were audio-recorded, transcribed verbatim, line-by-line coded using constant comparison, and analyzed concurrently with data collection. The coded data were analyzed and used to develop a conceptual framework covering concepts of interest important to this sample of participants. Results: Of the 10 participants, the majority identified as trans male $(n=7)$ and were from Canada $(n=7)$. Most participants were receiving hormone blockers or gender-affirming hormones $(n=8)$ and almost all participants planned to have gender-affirming surgery in the future $(n=9)$. Concepts elicited during the interviews covered a range of healthrelated quality of life concerns, including dissatisfaction with appearance (e.g., hair, face, body) and voice, gender dysphoria, binding (positive and negative impacts), and physical, psychological, social, and sexual function. In addition to outcomes, concepts related to the healthcare experience were hugely important (e.g., access to treatment, teen-centred care). Conclusion: Given the significant challenges faced by transgender and gender diverse youth, a comprehensive PROM that covers a full range of concerns is needed to provide a means to evaluate treatment outcomes. The concepts identified from the interviews with teenagers in the GENDER-Q study were used to inform an interview guide for a new study with transgender and gender diverse youth aged 8-18 years old.

\section{3: Applied Clinical Care for Different Health Conditions}

(103.1) An innovative approach to develop, deploy and evaluate personalised, patient-centred, outcome measurement: an international, multicentered study with the CLEFT-Q Computerised Adaptive Test

Conrad Harrison, University of Oxford, Oxford, UK; Inge Apon, Department of Oral and Maxillofacial Surgery, the Dutch Craniofacial Center, Erasmus University Medical Center, Rotterdam, The Netherlands; Kenny Ardouin, Cleft Lip and Palate Association, London, UK; Chris Sidey-Gibbons, MD Anderson Center for INSPiRED Cancer Care, the University of Texas, Houston, Texas, 
USA; Anne Klassen, Department of Pediatrics, McMaster University, Hamilton, Ontario, Canada; Stefan Cano, Modus Outcomes, Letchworth Garden City, UK; Karen Wong Riff, Department of Plastic and Reconstructive Surgery, Hospital for Sick Children, Toronto, Ontario, Canada; Andrea Pusic, Patient-Reported Outcomes, Values \& Experience Center, Department of Surgery, Brigham and Women's Hospital, Harvard Medical School, Boston, USA; Sarah Versnel, Department of Plastic and Reconstructive Surgery, the Dutch Craniofacial Center, Erasmus University Medical Center, Rotterdam, The Netherlands; Maarten Koudstaal, Department of Oral and Maxillofacial Surgery, the Dutch Craniofacial Center, Erasmus University Medical Center, Rotterdam, The Netherlands; Alexander Allori, Division of Plastic, Maxillofacial \& Oral Surgery, Duke University Hospital \& Children's Health Center, Durham, USA; Carolyn RogersVizena, Department of Plastic and Oral Surgery, Boston Children's Hospital, Boston, USA; Marc Swan, The Spires Cleft Centre, John Radcliffe Hospital, Oxford University Hospitals, Oxford, UK; Dominic Furniss, Nuffield Department of Orthopaedics, Rheumatology and Musculoskeletal Sciences, University of Oxford, Oxford, UK; Jeremy Rodrigues, Warwick Clinical Trials Unit, Warwick Medical School, University of Warwick, Coventry, UK

Aims: The CLEFT-Q is a patient-reported outcome measure (PROM) for use in cleft lip and/or palate. Its sub-scales constitute nine of the eleven PROMs in the International Consortium for Health Outcomes Measurement core outcome set for CL/P. Response burden has been identified as a major barrier to CLEFT-Q implementation internationally. In the UK and Ireland, only 4 of 18 cleft centers routinely use the CLEFT-Q. We aimed to develop, validate and deploy a computerized adaptive test (CAT) version of the CLEFT-Q, to reduce response burden and facilitate greater uptake. Methods: CAT algorithms were developed from CLEFT-Q field test data comprising 2434 individuals and refined in Monte Carlo simulations with simulated datasets. They were externally validated in an independent sample of 551 CLEFT-Q response sets collected from three sites across the USA and the The Netherlands. CAT stopping rules were based on consensus achieved at a bespoke multistakeholder workshop, with patient and public involvement as well as surgeons, therapists, psychologists and researchers. A user interface was built for the CAT using the open-source Concerto platform, and Shiny apps were developed to compare a respondent's score to the score distributions of populations with similar clinical and demographic characteristics. The platform was deployed in Oxford (UK) and Rotterdam (the The Netherlands). Semi-structured interviews were conducted with patients and healthcare providers who had used the CAT and analyzed thematically to evaluate end-user experience. Results: Multistakeholder consensus-based CAT stopping rules reduced the total length of the CLEFT-Q from 110 to 86 items across 12 scales. At this limit, CATs for each scale reproduced linear assessment scores with close agreement (root mean squared error between CAT and linear assessment scores was 2-5 out of 100 CLEFT-Q points). Patients and providers felt that the platform improved the PROM's usability and would facilitate its uptake in routine clinical practice. Conclusion: We have developed, validated and deployed a CAT, exclusively with open-source software, which reliably reduces response burden and increases patient and provider engagement with the CLEFT-Q. This process could be quickly and cost-efficiently replicated for other PROMs that meet the structural assumptions of modern test theory.
(103.2) A toolkit to support the implementation of patientreported outcome measures in routine HIV care

Duncan Short, PhD, ViiV Healthcare, London, UK; Heidi Crane, MD, MPH, University of Washington, Seattle, Washington, USA; William Lober, MD, University of Washington, Seattle, Washington, USA; Justin McReynolds, MS, University of Washington, Seattle, Washington, USA; Sierramatice Karras, BS, University of Washington, Seattle, Washington, USA; Emma Fitzsimmons, BA, University of Washington, Seattle, Washington, USA; Rob Fredericksen, PhD, MPH, University of Washington, Seattle, USA

Aims: Implementing patient-reported outcomes and measures within routine HIV care can generate clinically important insights for healthcare providers that they may not otherwise be aware of. By improving the information exchange between patients and providers, PROs have been shown to positively impact patient care in areas such as depression, suicidal ideation, inadequate antiretroviral (ART) adherence, and substance use. Successfully implementing PROs within clinical practice, however, presents significant knowledge and practical barriers to adoption. To address this gap, the PROgress Study Team created an implementation toolkit as a pragmatic resource to support the introduction of clinical PRO assessments into routine HIV care. Methods: The PROgress Implementation Toolkit was developed on an iterative basis during the course of a multifaceted collaborative PRO workstream between 2018 and 2020. An initial targeted literature review covering publications between 2006 and 2017 focused upon the use and utility of PROs implemented within routine care settings across multiple disease areas. This, and prior experience of the study team in implementing PROs within clinical trials, research studies, and large academic centers such as CFAR CNICS sites then informed the development of a prospective study of the value and feasibility of implementing PROs within routine care at 2 North American HIV outpatient clinics. As part of the implementation, the experiences, decision points and challenges were captured and these formed the basis of a toolkit that was reviewed by the study steering group which included patients living with HIV, HIV clinic leaders, care providers and healthcare researchers. Results: The final toolkit is available at https://progresshivcare.org and provides resources, tips, and effective practices to help clinics implement PROs. The toolkit describes iterative stages of the implementation process, including: (1) The assessment of clinic readiness; (2) Engaging and communicating with stakeholders (3) Building a technical infrastructure; (4) Creating a PRO assessment; (5) Clinic workflow integration; (6) Training; (7) Monitoring and evaluation. The Toolkit weighs the pros and cons surrounding decisions related to implementation, with respect to the varying needs and challenges faced by individual HIV clinics. Conclusion: The PROgress Implementation Toolkit provides a practical resource to support the integration of PROs within routine HIV care.

\section{(103.3) PROs to predict hospital admissions and ED visits}

Irene Katzan, Cleveland Clinic, Cleveland, Ohio, USA; Nicolas Thompson, MS, Cleveland Clinic, Cleveland, Ohio, USA; Andrew Schuster, BS, Cleveland Clinic, Cleveland, Ohio, USA; Belinda Udeh, PhD, Cleveland Clinic, Cleveland, Ohio, USA; Elizabeth Pfoh, 
$\mathrm{PhD}$, Cleveland Clinic, Cleveland, Ohio, USA; Brittany Lapin, $\mathrm{PhD}$, Cleveland Clinic, Cleveland, Ohio, USA

Aims: Identification of patients at increased risk of emergency department (ED) visits or hospital admissions allows implementation of strategies to reduce this risk. Health care systems have relied primarily on administrative and clinical data to identify these high-risk patients. Self-reports of health provide information not available in these data-sources, which may improve risk prediction. The objective of our study was to evaluate the utility of PROMIS Global Health $(\mathrm{GH})$ to predict important utilization outcomes within 1-year when added to other readily available clinical variables. Methods: A retrospective cohort study was performed of adult patients within the Cleveland Clinic Health System who were seen at least once between January 2016 and December 2018 and who completed the PROMIS GH scale as part of routine care. Separate multivariable logistic regression models were developed for following outcomes: avoidable ED visits (based on NYU ED Algorithm), unplanned admissions, and ambulatory-care-sensitive admissions for different time-frames: 30 days, 90 days, 180 days, and 1 year. Additional predictor variables in all models included age, sex, race, marital status, insurance, median income by ZIP Code, smoking status, BMI, any ED visit in prior year, and any inpatient admission in prior year. C-statistics were corrected for optimism using bootstrap internal validation, using 40 bootstrap resamples. Results: The study cohort consisted of 478,631 patients with mean age 54.2 years (SD 16.7), and mean PROMIS GH physical health and mental health summary T-scores 45.7 (SD 9.4) and 48.6 (SD 9.8), respectively. In the year following PROMIS GH completion, there were 134,032 ED visits (77, 528 patients, $16.1 \%$ ) and 77,422 hospital admissions (50, 689 patients, 10.5\%). Ambulatory-care-sensitive admission models had highest optimism-corrected c-statistics (range 0.872-0.886); while avoidable ED visits had lowest (0.751-0.762). Performance of models was generally better at shorter time periods (see Table). Calibration plots revealed good calibration for all models. Conclusion: A simple prediction model that includes PROMIS GH and several readily available clinical variables can predict future potentially avoidable ED visits and hospital admissions. Next step is to identify effective interventions to reduce future ED and hospital admissions in patients identified as high-risk.

Multivariable logistic regression models of future ED visit or hospital admission, optimism-corrected C-statistics

\begin{tabular}{|l|c|c|c|c|}
\hline Outcome & $\mathbf{3 0 ~ d}$ & $\mathbf{9 0 ~ d}$ & $\mathbf{1 8 0} \mathbf{d}$ & $\mathbf{3 6 5} \mathbf{d}$ \\
\hline Avoidable ED Visit & 0.761 & 0.762 & 0.759 & 0.751 \\
\hline $\begin{array}{l}\text { Unplanned hospital } \\
\text { admission }\end{array}$ & 0.803 & 0.799 & 0.796 & 0.791 \\
\hline $\begin{array}{l}\text { Ambulatory Care Sensitive } \\
\text { Hospital Admission }\end{array}$ & 0.874 & 0.886 & 0.883 & 0.872 \\
\hline
\end{tabular}

*Based on NYU ED Algorithm

(103.4) Identifying the factors contributing to diagnostic delays: a qualitative study of patients with inflammatory bowel disease

Rebecca Purc-Stephenson, PhD, University of Alberta, Camrose, Alberta, Canada

Aims: Inflammatory bowel disease (IBD) is a chronic disease affecting the gastrointestinal tract. IBD is characterized by unpredictable flare-ups with common symptoms of diarrhea, fatigue, abdominal pain, and rectal bleeding. Although Canada has one of the highest prevalence rates of IBD in the world, diagnostic delays (DD) are common. Research shows a diagnosis can range from three months to 11 years. A timely diagnosis can reduce the risk of hospitalization, surgery, and improve quality of life. In this study, we aimed to explored the symptom-appraisal behaviOR medical help- seeking, and patient-practitioner communication among individuals with IBD to understand their diagnostic experience and to identify the factors that contributed to DD. Methods: We conducted a qualitative study using a grounded theory approach. We recruited a purposive sample of 48 adults diagnosed with IBD in Canada. Our analytic process followed Lincoln and Guba's (1985) recommended steps for ensuring rigor and trustworthiness of the data. Results: We identified five themes that underscored the diagnostic experience: symptom ambiguity, fear and denial, patient-provider communication breakdown, self-advocacy, and diagnostic journey. We reviewed previous research examining the diagnostic experiences of patients with other chronic diseases (e.g., fibromyalgia, lupus, etc.), and found similarities to what our participants reported. In other words, the diagnostic experiences of IBD patients were not necessarily unique from the diagnostic experiences of patients with other chronic diseases. Next, we used our data to generate a new model to characterize the journey that patients with IBD — or other chronic diseases - experience when seeking a diagnosis. We called this model the Diagnostic Pathways Model, and it illustrates the interacting roles of patient, provider, and service factors involved in receiving a diagnosis, and highlights the places in the process where DD commonly occur. Conclusion: Compared to the standard diagnostic model in primary-care, whereby a clinical encounter is depicted as six successive phases (i.e., opening, problem presentation, history and exams, diagnosis, treatment, and closing), our model depicts the diagnosis process for chronic diseases as nonlinear and iterative, and incorporates the behaviors that patients tend to do in the time between their medical consultations. Implications for physician-patient communication and public information are discussed.

(103.5) Barriers and facilitators to using patient-reported data for quality improvement: informing the implementation of person-centred quality indicators in Canada

Kimberly Manalili, PhD Candidate, University of Calgary, Calgary, Alberta, Canada; Maeve O'Beirne, University of Calgary, Calgary, Alberta, Canada; Catherine Scott, University of Calgary, Calgary, Alberta, Canada; Brenda Hemmelgarn, University of Alberta, Edmonton, Alberta, Canada; Maria J. Santana, University of Calgary, Calgary, Alberta, Canada

Aims: Person-Centred Quality Indicators (PC-QIs) use data obtained from Patient-Reported Experience Measures (PREMs) to identify gaps in person-centred care and facilitate improvements. However, implementation and use of these measures is impacted by barriers and facilitators to implementation. These factors may differ based on the context of implementation. Thus, it is important to understand potential barriers and facilitators to implementing PC-QIs to optimize the uptake and use of patient-reported data to improve quality of care. Methods: The Consolidated Framework for Implementation Research (CFIR) was used as the guiding framework for this study, as it comprises constructs related to understanding the barriers and facilitators to implementation through an exploration of context. Semistructured interviews regarding barriers and facilitators to implementing PC-QIs were conducted with representatives from regional healthcare authorities (providing a system-level perspective) across Canada and a representative sample of primary care clinics and support organizations across the province of Alberta, Canada, to provide a clinical perspective. Framework analysis was conducted using the CIFR to identify key factors for consideration in implementation. Results: A total of ten regional healthcare authorities across Canada and eleven primary care clinics/organizations in Alberta participated in the interviews. From both a system-level (regional) and primary care clinical perspective, key barriers to 
implementation include: resources to support the administration and collection of PREM data and for reporting this data using the PC-QIs, and measurement burden, considering the relative priority among other measures of quality required by health authorities. Across all participants, leadership engagement at the clinical and regional levels was seen as critical for obtaining buy-in. For regional healthcare authorities, alignment with provincial/regional policy would be important to facilitate implementation. Among primary care participants, lack of time and capacity for quality improvement was identified as a major barrier for primary care participants. However, in general, most regional and primary care organizations perceived person-centred care to be a high priority. Conclusion: The implementation of patient-reported measures require consideration of the resources and capacity required, the importance of leadership engagement, and the alignment with existing policy. By understanding these barriers and facilitators, implementation can be optimized to effectively use patient-reported data for improving person-centred care.

\section{4: COVID-19 Application in Clinical Research}

(104.1) The impact of COVID-19 on health-related quality of life: a pre-post cohort study with a propensity score matched control group

Brittany Lapin, PhD MPH, Cleveland Clinic, Cleveland, Ohio, USA; Irene Katzan, MD, Cleveland Clinic, Cleveland, USA

Aims: Research suggests a large proportion of people experience long-term health effects following COVID-19 infection; however, studies have been hindered by a lack of pre-infection data. The impact of COVID-19 on health-related quality of life (HRQOL), including who may experience ongoing symptoms, is unknown. Our study aimed to identify change in HRQOL following COVID-19 compared to pre-infection HRQOL, and identify predictors of patients who worsen. Methods: Patients diagnosed with COVID-19 at a large healthcare system were included in our pre-post cohort study if they completed PROMIS Global Health following infection with COVID19 as well as in the year prior to their infection. Predictors of COVID19 patients who meaningfully worsened $(5+T$-score points) on global mental (GMH) and physical health (GPH) summary scores were identified through multivariable logistic regression models. Propensity score match was utilized to identify a 1:1 control cohort of patients who completed PROMIS GH at two time points but did not have COVID-19. HRQOL following COVID-19 was compared with matched controls using generalized estimating equations. Results: There were 3926 patients (mean age $53 \pm 15$; 65\% female) diagnosed with COVID-19 between March and December 2020 who completed PROMIS GH in the year prior (median 11.1 months) as well as after diagnosis (median 1.5 months). Compared to before infection, COVID-19 patients had a significant reduction in GMH and GPH ( -1.16 vs $-0.23 \mathrm{~T}$-score points, respectively) with a clinically meaningful reduction experienced by $28 \%$ and $23 \%$ of patients, respectively. Predictors of worsening on GMH included being female, having depression, low back pain, and better pre-COVID GMH. Predictors of worsening on GPH included older age, being female, having more comorbidities including obesity, being hospitalized for COVID-19, and having better pre-COVID GPH. Compared to the control group, there was no difference in change in GMH but a significantly larger decline in GPH following COVID-19 ( -0.12 and $-0.98 \mathrm{~T}$-score points, respectively). Conclusion: A quarter of patients with COVID-19 experienced meaningful reductions in global health. Changes in GMH were similar to a control group; however, GPH was significantly worse following COVID-19. Additionally, we identified predictors of patients who worsen, which may assist clinicians treating patients at risk of worse HRQOL following COVID-19.

(104.2) Using the AQOL-MHS to measure long-term effects of multiple stressful events in a clinical sample of adolescents

Ligia Chavez, Ph.D., University of Puerto Rico, San Juan, Puerto Rico; Keilyn Vale, M.S., University of Puerto Rico, San Juan, Puerto Rico; Pedro Garcia, M.A., University of Puerto Rico, San Juan, Puerto Rico

Aims: Disasters disrupt life. This study provides a rare opportunity to examine post disaster HRQOL longitudinally in a sample of adolescents receiving mental health services. This vulnerable Puerto Rican population experienced a devastating hurricane in September 2017, a swarm of destructive earthquakes starting in December 2019, and more recently, the COVID-19 Pandemic. The emergence and persistence of psychological reactions in response to disasters have been associated with increased symptomatology, decreased quality of life, and the experience of post-traumatic stress. We examine the implications for this population. Methods: Adolescents (12-18 years, $N=228$ ) were evaluated with the AQOL-MHS in three domains: Emotional Regulation (ER), Self-Concept (SC), and Social Context (SoC). Data collection spans over a two-year period. Data across time were included in the analysis and linear time trends were examined using three models. Model A includes main effects of time, sex, age, and diagnostic; Model B adds Time by DX (TD), and Model C, Time by PTSD (TP). Results: In our sample, $13.6 \%$ of the children reported PTSD and $49.3 \%$ were scored as having a psychiatric disorder. Girls were disproportionately affected in all models for SC and SoC domains. Both having a diagnosis, or PTSD, are strong indicators of worse ER and SC QOL. For the TP interaction models, results for both ER $(p \leq 0.05)$ and SC $(p \leq 0.01)$ indicate that those with PTSD are improving over time at a greater rate than those without PTSD There is also a time trend that those with a diagnosis (TD) improved their ER across time. Conclusion: Children are known to adapt to their environmental circumstances and better cope with their limitations. The lack of improvement in general in our sample is of concern. The most stagnant domain is Social Context. Although only $7.3 \%$ of our sample was reporting QOL during pandemic circumstances, they were still burdened by prolonged recovery efforts. Results also pinpoint a clear gender disparity in detriment of girls. One lesson learned is that disasters and pandemics have far reaching implications. Knowing those who are at greater risk can allow for focused allotment of resources and the creation of targeted population-based mitigation and preparedness strategies.

(104.3) The COVID-19 pandemic does not affect the quality of life reportings of Danish patients with multiple myeloma

Louise Redder, Odense University Hospital Department of Hematology, Odense, Denmark; Sören Möller, OPEN, Odense Patient data Explorative Network, Odense University Hospital and Department of Clinical Research, University of Southern Denmark, Odense, Denmark; Henrik Rode Eshoj, Quality of Life Research Center, Department of Haematology, Odense University Hospital, Odense, Denmark; Mary Jarden, Department of Haematology, Copenhagen University Hospital, Copenhagen, Denmark; Christen Lykkegaard Andersen, Department of Haematology, Zealand University Hospital, Roskilde, Denmark; Henrik Frederiksen, Quality of Life Research Center, Department of Haematology, Odense University Hospital, Odense, Denmark; Henrik Gregersen, Department of Haematology, Aalborg University Hospital, Aalborg, Denmark; Anja Klostergaard, 
Department of Haematology, Aarhus University Hospital, Aarhus, Denmark; Morten Saaby Steffensen, Department of Haematology, Regional Hospital West Jutland, Holstebro, Denmark; Per Trøllund Pedersen, Department of Haematology, South West Jutland Hospital, Esbjerg, Denmark; Maja Hinge, Department of Haematology, Vejle Hospital, Vejle, Denmark; Mikael Frederiksen, Department of Haematology, Hospital of Southern Jutland, Aabenraa, Denmark; Bo Amdi Jensen, Department of Haematology, Zealand University Hospital, Roskilde, Denmark; Carsten Helleberg, Department of Haematology, Herlev Hospital, Herlev, Denmark; Anne Kaergaard Mylin, Department of Haematology, Copenhagen University Hospital, Copenhangen, Denmark; Niels Abildgaard, Quality of Life Research Center, Department of Haematology, Odense University Hospital, Odense, Denmark; Lene Kongsgaard Nielsen, Quality of Life Research Center, Department of Haematology, Odense University Hospital and Research Unit for Multimorbidity, Department of Internal Medicine and Cardiology, Viborg Regional Hospital, Odense, Denmark

Aims: Patients with multiple myeloma (MM) have increased risk of dying if infected with COVID-19, and the pandemic has, to some extent, changed standard of care towards extended use of oral regimens and limiting hospital visits. We aimed to analyze patientreported quality of life (QoL) in Danish patients with MM preCOVID and during-COVID. Methods: The study was a natural experiment using a cross-sectional study design, where the QoL of Danish patients with MM was compared pre-COVID and duringCOVID. The data originated from the ongoing survey study, "Quality of life in Danish patients with Multiple Myeloma" (QoL-MM), initiated in February 2017. QoL-MM data is collected at enrolment, when new treatment is initiated, and subsequently at 12 follow-up time points over a two-year period. Twenty-five QoL domains are included, assessed by the European Organisation for Research and Treatment of Cancer Quality of life QLQ-C30, the Multiple Myeloma module QLQ-MY20, the Chemotherapy-Induced Peripheral Neuropathy module and the Short-form health survey version 2.The QoL data was analyzed using mixed effects linear regression, with a yearperiod-interaction. Pre-COVID versus during-COVID QoL mean score difference was considered evident, if the difference was both statistically significant $(p$-value $<0.05$ ) and clinically relevant using minimal important difference in accordance with Cohens MID criteria (0.3 standard deviation of the mean score of each domain for the entire group). Results: A total of 1,685 questionnaires were analyzed corresponding to a completion rate of $95 \%$. Mean age was 68 years, $40 \%$ were females, and $75 \%$ were married or cohabiting. No statistically significant and clinically relevant differences were found, when comparing the mean scores from April-June 2019 (pre-COVID) to the mean scores from April-June 2020 (COVID 1st wave) in any of the investigated domains. Likewise, for the second wave, see Table 1. Conclusion: Based on our data, the pandemic has not affected the reported QoL in patients with MM. Part of the reason may be that the questionnaires used are not developed to capture the impact of the pandemic on QoL. Importantly, our results suggest that QoL collected in clinical trials during the pandemic allow interpretation without adjusting for the impacts of the pandemic.

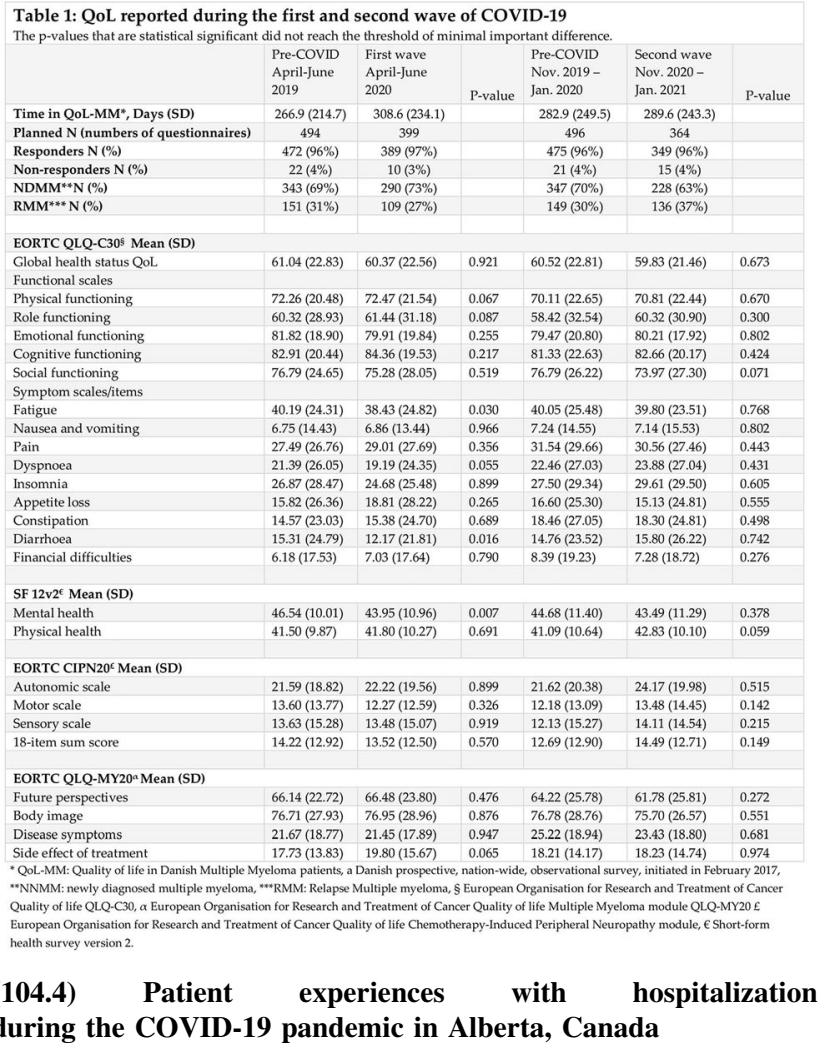

Kyle Kemp, PhD, University of Calgary \& Health Quality Council of Alberta, Calgary, Alberta, Canada; Paul Fairie, University of Calgary, Calgary, Alberta, Canada; Brian Steele, University of Calgary, Calgary, Alberta, Canada; Jeff Bakal, Alberta Health Services, Edmonton, Alberta, Canada; Erik Youngson, Alberta Health Services, Edmonton, Alberta, Canada; Hude Quan, University of Calgary, Calgary, Alberta, Canada; Maria Santana, University of Calgary, Calgary, Alberta, Canada

Aims: Despite assurances that healthcare services remained safe and effective, many avoided accessing care during the outset of the COVID-19 pandemic. Our aims were to a) report on the experiences of patients who were hospitalized in the early months of the COVID19 , and b) compare with historical results, in a Canadian province of 4.4 million residents. Methods: A random sample of patients were surveyed within six weeks of discharge from 93 hospitals, using a modified version of the Canadian Inpatient Experiences SurveyInpatient Care instrument. Surveys were linked with administrative records. Discharges from April to September 2020 comprised the "during COVID-19" patient cohort, while those from April to September 2019 formed the historical one. We examined 39 survey questions and reported results as percent in "top box", as represented by the most positive answer choice. Odds of reporting a "top box" response (COVID-19 vs. historical) were calculated, while controlling for demographic and clinical features. Adjusted odds ratios and $95 \%$ confidence intervals were generated using logistic regression. Results: In total, 23,412 surveys (11,344 during COVID-19, 12,068 historical) were obtained. For the overall rating of care, $65.3 \%$ of "during COVID-19" and $62.5 \%$ of historical responses were classified as "top box". Those hospitalized during COVID-19 had higher odds of "top box" ratings on 15 questions, which included overall ratings of nurses and doctors, pain control, and cleanliness and quietness of the hospital. Of the remaining questions, 22 showed no difference between the two cohorts, and the "during COVID-19 group" had lower odds 
of reporting a "top box" rating on two questions (information about the admission process, inclusion of family/friends in care decisions). Complete results for all questions are provided in the accompanying table. Conclusion: Our results indicate that patients who were hospitalized during the early months of the COVID-19 pandemic had experiences that were largely better or comparable to those hospitalized prior to the pandemic. This highlights the dedication and resilience of the staff who provided care during a very challenging period. The findings may assist in mitigating fears among those who may be hesitant to seek medical care during future pandemics or public health emergencies.

\begin{tabular}{|c|c|c|c|c|}
\hline Item Description & Top Box Response & During COVID- 19 & Historical & $a O R *(95 \% C D$ \\
\hline \multicolumn{5}{|l|}{ Global ratings of care } \\
\hline $\begin{array}{l}\text { Overall rating } \\
\text { On care }\end{array}$ & 9 or 10 & 65.3 & 62.5 & $1.16(1.10-1.23)$ \\
\hline Recommendation of hospital & Definitely yes & 74.4 & 71.8 & $1.17(1.10-1.24)$ \\
\hline Helped by hospital stay & 9 or 10 & 74.1 & 72.9 & $1.10(1.04-1.17)$ \\
\hline Overall hospital experience & 9 or 10 & 67.5 & 65.7 & $1.12(1.05-1.18)$ \\
\hline \multicolumn{5}{|l|}{ Care from nurses } \\
\hline Nurse courtesy \& respect & Always & 85.4 & 84.8 & $1.08(1.00-1.16)$ \\
\hline Nurse listening & Always & 74.8 & 74.3 & $1.05(0.99-1.12)$ \\
\hline Nurse explanations & Always & 75.4 & 76.1 & $0.98(0.92-1.04)$ \\
\hline Call button assistance & Always & 59.9 & 60.3 & $1.02(0.96-1.09)$ \\
\hline Timely bathroom assistance & Always & 67.1 & 67.1 & $1.05(0.97-1.14)$ \\
\hline Overall rating of nursing care & 9 or 10 & 69.4 & 68.0 & $1.10(1.03-1.16)$ \\
\hline \multicolumn{5}{|l|}{ Care from doctors } \\
\hline Doctor courtesy \& respect & Always & 84.1 & 83.6 & $1.07(1.00-1.15)$ \\
\hline Doctor listening & Always & 76.7 & 76.7 & $1.04(0.97-1.11)$ \\
\hline Doctor explanations & Always & 74.5 & 75.4 & $0.99(0.93-1.05)$ \\
\hline Overall rating of doctor care & 9 or 10 & 75.9 & 75.2 & $1.08(1.01-1.15)$ \\
\hline \multicolumn{5}{|l|}{ Hospital environment } \\
\hline Room cleanliness & Always & 63.7 & 62.2 & $1.07(1.01-1.13)$ \\
\hline Room quietness & Always & 50.7 & 48.3 & $1.11(1.05-1.17)$ \\
\hline \multicolumn{5}{|l|}{ Pain } \\
\hline Pain well control & Always & 61.7 & 61.2 & $1.07(1.01-1.14)$ \\
\hline Staff helped with pain & Always & 76.8 & 76.8 & $1.05(0.98-1.13)$ \\
\hline Purpose of new medications & Always & 76.8 & 76.3 & $1.04(0.96-1.13)$ \\
\hline Side effects of new medications & Always & 50.3 & 50.0 & $1.03(0.95-1.10)$ \\
\hline
\end{tabular}

\begin{tabular}{|c|c|c|c|c|}
\hline Admission to hospital & & & & \\
\hline $\begin{array}{l}\text { Information about admit process } \\
\text { Admission was organized }\end{array}$ & Completely & $\begin{array}{l}58.3 \\
76.8\end{array}$ & $\begin{array}{l}65.7 \\
79.1\end{array}$ & $\begin{array}{l}0.77(0.70-0.83) \\
0.92(0.84-1.02)\end{array}$ \\
\hline Care in emergency department & & & & \\
\hline Information about condition & Completely & 49.2 & 51.4 & $0.93(0.86-1.00)$ \\
\hline Information about admit process & Completely & 50.8 & 52.6 & $0.96(0.89-1.03)$ \\
\hline Wait for admission to hospital & & 80.3 & 75.2 & $1.35(1.23-1.48)$ \\
\hline Transfer to bed was organized & Completely & 75.3 & 72.0 & $1.21(1.11-1.31)$ \\
\hline Processes of care & & & & \\
\hline Communication between staff & Always & 63.2 & 61.9 & $1.10(1.04-1.17)$ \\
\hline Staff informed/up to date & Always & 60.6 & 59.6 & $1.08(1.03-1.14)$ \\
\hline Tests/procedures done on time & Always & 71.8 & 70.3 & $1.11(1.04-1.19)$ \\
\hline Info about condition/treatment & Always & 69.7 & 69.9 & $1.04(0.98-1.10)$ \\
\hline Support for anxieties/worries/fears & Always & 63.2 & 62.6 & $1.07(1.00-1.15)$ \\
\hline Patient involvement in care & Always & 67.4 & 67.2 & $1.06(1.00-1.12)$ \\
\hline Family/friend involvement in care & Always & 60.0 & 73.7 & $0.54(0.50-0.58)$ \\
\hline Discharge from hospital & & & & \\
\hline Help needed after discharge & Yes & 83.8 & 84.4 & $0.98(0.91-1.06)$ \\
\hline Received written discharge info & Yes & 78.7 & 80.2 & $0.94(0.88-1.01)$ \\
\hline Understanding of medications & Completely & 77.5 & 77.9 & $1.01(0.94-1.08)$ \\
\hline Info about post-discharge worries & Completely & 70.1 & 71.5 & $0.98(0.92-1.04)$ \\
\hline Better understanding of condition & Completely & 64.1 & 65.0 & $1.00(0.94-1.06)$ \\
\hline Patient concerns & & & & \\
\hline Had a concern about care & No & 80.7 & 79.1 & $1.12(1.05-1.19)$ \\
\hline
\end{tabular}

(104.5) Communication with emergency department nurses and doctors before and during the COVID-19 pandemic: ensuring measurement equivalence

Jeanette Jackson, Health Quality Council of Alberta, Calgary, Alberta, Canada; Carmelle Bolo, Health Quality Council of Alberta, Calgary, Alberta, Canada; Ben Higgins, Health Quality Council of Alberta, Calgary, Alberta, Canada; Jack Ma, Health Quality Council of Alberta, Calgary, Alberta, Canada; Kara Schick-Makaroff, Faculty of Nursing, University of Alberta, Edmonton, Alberta, Canada; Lisa Corscadden, Office of Patient-Centred Measurement, British Columbia Ministry of Health, Vancouver, British Columbia, Canada;
Rick Sawatzky, School of Nursing, Trinity Western University, Vancouver, British Columbia, Canada

Aims: The Health Quality Council of Alberta (HQCA) has the mandate to promote and improve patient safety, person-centred care, and health service quality. The HQCA surveys Albertans about their experiences in emergency departments (EDs) to inform ongoing improvements. This control chart suggests an improvement in overall patient experience during COVID-19 compared to a period of one year prior to the pandemic:Motivated by this observation, we explored whether observed improvements in ED experience could be a result of suboptimal measurement invariance. Specifically, our objective was to evaluate measurement invariance by comparing the measurement of patient experience in EDs in four quarterly periods during and prior to the COVID-19 pandemic. Methods: The HQCA ED experience survey was administered to patients one year before and one year during the pandemic $(N=12,149$ and 7722 , respectively). 2187(7.8\%) were younger than $24,7605(27 \%)$ older than 65 , and $14,553(52 \%)$ female. The analysis focused on 8 items measuring quality of communication with nurses and doctors, as these constructs have been shown to be strong drivers of overall patient experience in the ED. We tested measurement invariance using a 2-factor multigroup confirmatory factor analysis (CFA) model to compare following seasonal subgroups: Spring 2019 vs. $2020(n=3318 ; n=2329)$, Summer 2019 vs. $2020(n=3499 ; n=2214)$, Fall 2019 vs. 2020 $(n=2598 ; n=3013)$, and Winter 2020 vs. $2021 \quad(n=3085$; $n=3223$ ). Comparative fit indices were used to sequentially evaluate measurement invariance constraints between the subgroups. Results: Multi-group CFA showed no evidence against measurement equivalence when comparing one year before and one year during the pandemic. However, when testing season subgroups, scalar invariance was violated when comparing Spring 2020 with 2019. The invariance test comparing Summer 2019 vs. 2020, Fall 2019 vs. 2020, or Winter 2020 vs. 2021 were not statistically significant. The results indicate no statistically significant means differences in standardized factor scores for quality of communication with nurses and doctors for Spring and Summer of 2019 vs. 2020, and small but statistically significant improvements comparing Fall 2019 vs. 2020 (nurses =0.15; doctors $=0.21$ ) and Winter 2020 vs. $2021 \quad$ (nurses $=0.24$; doctors $=0.22$ ). Conclusion: The findings suggest that Albertans changed the way they responded to questions about their experience with communication in the ED during the first 3 months of the pandemic, a time where healthcare professionals were celebrated as heroes.

(104.6) Social inequalities in mental and physical health: measured with EQ-5D-5L-derived from the COVID-19 pandemic in Spain beyond infectious disease

Isabel Moreira, MD, Parc de Salut Mar PSMAR, Barcelona, Spain; Gemma Vilagut, Health Services Research Unit, IMIM-Institut Hospital del Mar d'Investigacions Mèdiques, Barcelona, Spain; Philippe Mortier, Health Services Research Unit, IMIM-Institut Hospital del Mar d'Investigacions Mèdiques, Barcelona, Spain; Josep-Maria Haro, Parc Sanitari Sant Joan de Déu, Barcelona, Spain; Jordi Alonso, Health Services Research Unit, IMIM-Institut Hospital del Mar d'Investigacions Mèdiques, Barcelona, Spain; Montse Ferrer, Health Services Research Unit, IMIM-Institut Hospital del Mar d'Investigacions Mèdiques, Barcelona, Spain; submitted on behalf of "MINDCOVID Working group"

Aims: Understanding the impact of the COVID-19 crisis on general population's health involves conducting longitudinal studies to evaluate the evolution of inequalities that may have been exacerbated by the pandemic. The objective of this study is to examine differences by 
education level in mental and physical health derived from the COVID-19 pandemic in Spanish general population, beyond infectious disease. Methods: Study of the Spanish adult general population $(18+y s)$ within the MINDCOVID project. A specialized company carried out the computer-assisted telephone interviews (CATI) after the first wave in June 2020 (baseline) and 8-9 months later in February-March 2021. The EQ-5D-5L measures five dimensions of health (mobility, self-care, usual activities, pain/discomfort, and anxiety/depression) with five levels of severity. We calculated absolute and relative differences between extreme groups of education level (Secondary education or lower vs University degree) in the prevalence of reported problems for each EQ-5D-5L dimension: prevalence difference for absolute inequalities and prevalence ratio for relative inequalities and their 95\% Confidence Intervals (95\% CI). Analyses were stratified by gender. Results: 3.500 persons completed the CATI at baseline, and $2.000(57 \%)$ at follow-up. Among women, prevalence difference in June 2020 between extreme groups of education level ranged from $23.57 \%$ in Pain/Discomfort to $6.08 \%$ in Selfcare; in relative terms, prevalence ratio was the highest for Self-care (4.67) and the lowest for Anxiety/Depression (1.40). Prevalence differences had diminished in February-March 2021, especially in Pain/ Discomfort, from $23.57 \%$ (95\% CI 18.31-28.83) to $13.93 \%$ (95\% CI 6.49-21.38). Changes on prevalence ratios were in the same direction, but smaller. Among men, prevalence difference in June 2020 ranged from $15.43 \%$ in Pain/Discomfort to $4.41 \%$ in Anxiety/Depression; in relative terms, prevalence ratio was the highest for Self-care (5.52) and the lowest for Anxiety/Depression (1.33). In February-March 2021, prevalence differences were very similar in all dimensions except in Pain/Discomfort that decreased from $15.43 \%$ (95\% CI 9.11-21.76) to $7.60 \%$ (95\% CI - 1.07 to 16.26). Conclusion: These results suggest that the greatest inequalities in health took place during the lockdown and are decreasing over time. More analysis is needed to elucidate how much of this change in inequalities is explained by the selection bias introduced by the drop out in the follow-up.

\section{5: Cancer Clinical Care Applications}

\section{(105.1) Using Patient-Reported Outcomes (PROs) in Early-Phase Dose-finding Oncology Trials (EDOT): a global survey}

Julia Lai-Kwon, MBBS, Drug Development Unit, The Institute of Cancer Research and Royal Marsden Hospital, London, UK; Alyssa Vanderbeek, Clinical Trials and Statistics Unit, The Institute of Cancer Research, London, UK; Anna Minchom, Drug Development Unit, The Institute of Cancer Research and Royal Marsden Hospital, London, UK; Christina Yap, Clinical Trials and Statistics Unit, The Institute of Cancer Research, London, UK

Aims: Patient-reported adverse events may be a useful adjunct for assessing a drug's tolerability in EDOT. Collecting PROs in EDOT is feasible and acceptable to patients. However, overall use remains limited. Little is known about the reasons for limited PRO use. We conducted a global online survey of key stakeholders to understand attitudes towards PROs in EDOT. Methods: A 35-question survey of clinicians, trial managers, statisticians, funders and regulators involved in EDOT in hospitals, academia or industry was distributed via professional bodies. Questions examined prior experience of designing, conducting and reporting EDOT with PROs, attitudes towards benefits and barriers to PRO use and their potential role in defining tolerable doses. Adaptive questioning was used. No identifiable data were collected. Results: 112 responses from 15 September to 30 November 2020; 103 trialists [48 clinicians (42.9\%), 38 statisticians $(34.0 \%), 17$ trial managers $(15.2 \%)], 7$ regulators $(6.3 \%)$, and 2 funders (1.8\%). Most had worked in EDOT for 6-10 years (35,
$31.3 \%)$ in the UK $(65,58.0 \%)$ or USA $(22,19.6 \%)$. Most trialists reported no prior experience designing $(73,70.9 \%)$, conducting $(52$, $50.5 \%$ ), or reporting $(88,85.4 \%)$ PROs in EDOT. Respondents 'strongly agreed' or 'agreed' that PROs could identify new toxicities $(75,67.0 \%)$, provide data regarding frequency $(86,76.8 \%)$ and duration $(81,72.3 \%)$ of toxicities, especially in agents with moderate, chronic or delayed toxicities $(77,68.8 \%)$. Respondents agreed that PROs should be reviewed when making dose-escalation decisions $(61,54.5 \%)$, when determining the Maximum Tolerated Dose (63, $56.3 \%)$ and Recommended Phase 2 Dose $(76,67.9 \%)$. The top 5 barriers were lack of guidance regarding PRO selection (73/103, $70.9 \%)$, missing PRO data $(79 / 112,70.5 \%)$, overburdening staff $(68 /$ $103,66.0 \%)$ or patients $(57 / 103,55.3 \%)$, and analysis and publication (58/112, 51.8\%). Conclusion: Key stakeholders reported minimal experience designing, conducting or analysing PROs in EDOT. Despite this, broad support was expressed for using PROs to inform selection of tolerable doses, particularly the Recommended Phase 2 Dose. Future research should explore patient attitudes towards PROs in EDOT and identify the optimal settings in which to use PROs. Guidelines are needed to standardise selection, analysis and reporting and increase efficiency of PRO collection.

(105.2) Using the theoretical domains framework to understand assessment and management of long-term and late effects in breast cancer survivorship care

Eden Brauer, UCLA, Los Angeles, California, USA; Laura Petersen, MS, UCLA Cancer Prevention and Control, Los Angeles, California, USA; Patricia A. Ganz, MD, UCLA, Los Angeles, California, USA

Aims: Assessment and management of physical and psychosocial long-term and late effects are an essential component of cancer survivorship care. Breast cancer-specific survivorship guidelines provide recommendations in this area, but adherence in clinical practice remains low. The Theoretical Domains Framework (TDF) was developed for implementation research to identify influences on clinical adoption of evidence-based recommendations. In this study, we conducted a TDF-based survey of medical oncologists to understand key influences on the assessment and management of long term and late effects in breast cancer survivorship care. Methods: Medical oncologists who treat breast cancer patients were recruited to participate in a web-based survey. Survey development was informed by preliminary TDF-based qualitative work and utilized items from existing generic TDF questionnaires. Average scores for each theoretical domain were calculated for respondents. Outcomes included self-reported frequencies of assessing and managing (1) physical and (2) psychosocial long term and late effects in practice. Descriptive summaries, including means, standard deviations, and frequencies, of demographic characteristics, theoretical domains, and outcome variables were performed. Linear regression models were used to examine associations between theoretical domains and outcomes. Results: 217 medical oncologists completed the survey. Of these, 52\% believed breast cancer patients are well informed about long-term and late effects. The 'skills' $(b=0.293, p=0.007)$, 'social/professional role and identity' ( $b=0.273, p=0.012)$, and 'environmental context and resources' ( $b=0.288, p=0.012)$ domains were significantly associated with increased assessment and management of physical long-term and late effects. For psychosocial long-term and late effects, the domains of 'skills' $(b=0.493, p<0.001)$ and 'social/ professional role and identity' $(b=0.356, p=0.004)$ were significantly associated with increased assessment and management. In the 'environmental' domain, the majority $(67 \%)$ of oncologists reported low use and high perceived value regarding potential resources, such as standardized symptom checklists and patient-reported outcomes, to 
improve care of long-term and late effects. Conclusion: Findings support using the TDF to understand oncologists' behaviors and perceived barriers in addressing long-term and late effects in practice. Targeting relevant domains in future implementation efforts may increase the uptake of guideline-concordant survivorship care for breast cancer patients.

(105.3) An honest look in the mirror: patient-reported outcome measurements are rarely used in pediatric cancer clinical trialsa systematic review of clinical trial registries

Maria Rothmund, Medical University Innsbruck, Innsbruck, Austria; David Riedl, Medical University Innsbruck, Innsbruck, Austria; Anne-Sophie Darlington, School of Health Sciences, University of Southampton, Southampton, UK; Samantha C. Sodergren, School of Health Sciences, University of Southampton, Southampton, UK; Roman Crazzolara, Medical University Innsbruck, Innsbruck, Austria; Teresa de Rojas, Children's University Hospital Niño Jesús, Madrid, Spain; submitted on behalf of "the EORTC Quality of Life Group"

Aims: Patient-reported outcomes (PROs) are the gold standard to assess patients' subjective health status. While both the Food and Drug Administration (FDA) and the European Medicines Agency (EMA) recommend the use of PROs as endpoints in pediatric clinical trials to support claims for medical product labeling, it is not known how often PROs are actually used. The aim of this study was to assess the use of PRO instruments in childhood cancer clinical trials investigating anti-cancer treatments. Methods: In June 2020, ClinicalTrials and EudraCT were systematically searched for all trials registered since 2007 investigating children and adolescents with cancer ( $\leq 21$ years). To investigate which trial characteristics are associated with the use of PROs, Chi-Square or Fisher exact tests and a multivariate binary logistic regression were calculated. Results: Of 4789 identified trials, 711 were included. The most frequent reason for exclusion was age limitation (upper age limit $>21$ years). Of all included trials, only $8.2 \%$ used PROs as endpoints and only $0.6 \%$ as the primary endpoint. The most commonly used questionnaire was the PedsQLTM (32.8\%), followed by PROMIS scales (12.1\%). Another $24.1 \%$ reported measuring quality of life without specifying the tool to be used. In $19.0 \%$ of the trials using PROs, only pain or acceptability was assessed. No association was observed between the use of PROs and trial region, number of centers, trial phase, time period, or intervention type (all $\mathrm{p}>0.05$ ). The use of PROs did not substantially increase over time. Only $20.3 \%$ of the closed studies had published their results. Conclusion: Despite recommendations of regulatory agencies, PRO assessment is extremely rare in pediatric oncology clinical trials. The results shed light on the delay of progress in pediatric oncology due to its dependency from adult oncology. As research in adult cancer patients has already shown that PROMs are easily applicable and useful tools both for research and clinical practice, more efforts should be undertaken to facilitate implementation of PRO in pediatric trials to guarantee patient-centered research and treatments.

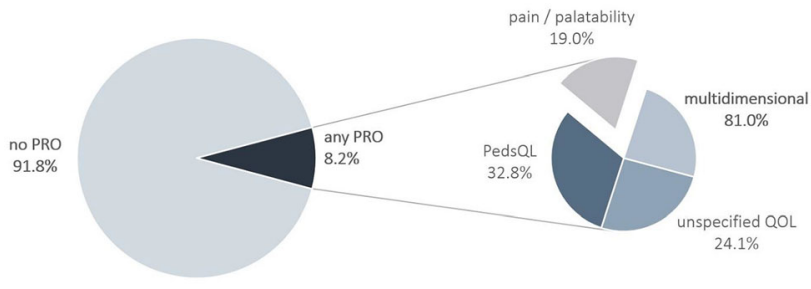

\section{(105.4) The relationship between age and ePRO use}

Ian Kudel, PhD, Varian, Palo Alto, New York, USA; Toni Perry, Varian Medical Systems, Palo Alto, New York, USA

Aims: To determine whether age is meaningfully associated with different aspects of noona, an ePRO is used to communicate treatment-related cancer symptoms Methods: Noona is an ePRO that has been installed in over 65 clinics worldwide. It is currently available in 8 languages and has over 23,000 active users. Clinical staff at each site onboards patients and assists with creating a profile. Participants $(n=3349)$ are experienced noona users, which are defined as having an active account for more than 30 days, using one of the primary features, and reporting satisfaction with the application. Additionally, patients were undergoing active cancer treatment (systemic or radiotherapy) in the USA. Data were derived from logic within noona that passively collects user activity in the system and administers a satisfaction question using a single minimally invasive question, "How likely are you to recommend noona to another patient" using an 11-point visual analog scale (VAS). Data were collected between January 1st (the first day objective data and patient satisfaction/loyalty were both collected) and March 17th, when the data were downloaded and analyzed. A linear regression tested whether 5 variables: (1) use of a CTCAE-based noona symptom questionnaires (NSQ) that incorporates branching logic, (2) device used at the last login, (3) days since app activation (days), (4) time on app (minutes), and (5) patient satisfaction with the app (0-10 visual analog scale) predicted respondent age. Results: Participants were generally senior citizens $(M=64.87, \quad S D=12.05)$, with active accounts for 322.58 days $(\mathrm{SD}=156.52)$ and used the app for $39.37 \mathrm{~min}(\mathrm{SD}=$ 77.28). Mean use of the NSQ was 0.41 (SD $=1.61)$. Participants were more likely to use smartphones/tablets $(n=786 ; 72.7 \%)$ than computers the last time they logged in. The regression found that device use had a weak, but significant relationship regarding device use. The rest of the standardized betas indicates no meaningful relationships and that significance was a function of sample size (Table). Conclusion: In a large sample of cancer patients, age is associated with using a smartphone/tablet and not meaningfully related to noona use (days since login or use in minutes), app satisfaction, and symptom treatment reporting.

\begin{tabular}{|c|c|c|c|c|c|c|c|}
\hline & \multicolumn{7}{|c|}{$\begin{array}{l}95.0 \% \text { Confidence } \\
\text { Interval for B }\end{array}$} \\
\hline & B & Std. Error & Beta & $\mathrm{t}$ & Sig. & $\begin{array}{l}\text { Lower } \\
\text { Bound }\end{array}$ & $\begin{array}{l}\text { Upper } \\
\text { Bound }\end{array}$ \\
\hline Device & 4.60 & 0.82 & 0.17 & 5.59 & 0.00 & 2.98 & 6.21 \\
\hline Days since activation & 0.01 & 0.00 & 0.11 & 3.81 & 0.00 & 0.00 & 0.01 \\
\hline App time & 0.00 & 0.01 & 0.01 & 0.40 & 0.69 & -0.01 & 0.01 \\
\hline Satisfaction/loyalty & 0.42 & 0.15 & 0.09 & 2.89 & 0.00 & 0.14 & 0.71 \\
\hline NSQ use & -0.40 & 0.13 & -0.09 & -3.15 & 0.00 & -0.64 & -0.15 \\
\hline
\end{tabular}

\section{6: Cancer Methods in General Populations}

\section{(106.1) Confirming fit of the EORTC CAT Core models in independent data}

Morten Petersen, Palliative Care Research Unit, Department of Geriatrics and Palliative Medicine GP, Bispebjerg og Frederiksberg Hospital, Copenhagen NV, Denmark; Neil K. Aaronson, Division of Psychosocial Research \& Epidemiology, The The Netherlands Cancer Institute, Amsterdam, The Netherlands; Thierry Conroy, Université de Lorraine, APEMAC, EA4360 and Medical Oncology Department, Institut de cancérologie de Lorraine, Vandoeuvre-lès-Nancy, France; Anna Costantini, Psychoncology Unit, Sant'Andrea Hospital, Faculty of Medicine and Psychology Sapienza University, Rome, Italy; Johannes M. Giesinger, Department of Psychiatry, Psychotherapy and Psychosomatics, University Hospital of Psychiatry II, Innsbruck, 
Austria; Eva Hammerlid, Department of Otolaryngology Head and Neck Surgery, Sahlgrenska University Hospital, Göteborg University, Göteborg, Sweden; Bernhard Holzner, Department of Psychiatry, Psychotherapy and Psychosomatics, University Hospital of Psychiatry II, Innsbruck, Austria; Colin D. Johnson, Surgical Unit, Faculty of Medicine, University of Southampton, Southampton, UK; Jacobien M. Kieffer, Division of Psychosocial Research \& Epidemiology, The The Netherlands Cancer Institute, Amsterdam, The Netherlands; Marieke van Leeuwen, Division of Psychosocial Research \& Epidemiology, The The Netherlands Cancer Institute, Amsterdam, The Netherlands; Sandra Nolte, Medical Department, Division of Psychosomatic Medicine, Charité-Universitätsmedizin Berlin, Corporate Member of Freie Universität Berlin, Humboldt-Universität zu Berlin, and Berlin Institute of Health, Berlin, Germany; John K. Ramage, Department of Gastroenterology, Hampshire Hospitals NHS Trust, Kings College Hospital ENETS centre of Excellence, Basingstoke, UK; Krzysztof A. Tomaszewski, Faculty of Medicine and Health Sciences, Andrzej Frycz Modrzewski Krakow University, Kraków, Poland; Annika Waldmann, Hamburg Cancer Registry, Hamburg, Germany; Teresa Young, East \& North Hertfordshire NHS Trust incorporating Mount Vernon Cancer Centre, Middlesex, UK; Paola Zotti, ientific Directorate, Centro di Riferimento Oncologico di Aviano (CRO) IRCCS, Aviano, Italy; Mogens Groenvold, The Research Unit, Department of Palliative Medicine, Bispebjerg \& Frederiksberg Hospital, University of Copenhagen, Copenhagen, Denmark; submitted on behalf of "the EORTC Quality of Life Group"

Aims: The EORTC CAT Core measures the same domains as the static EORTC QLQ-C30 questionnaire and includes 14 item banks (a total of 260 items) calibrated for computer adaptive test (CAT) and short-form measurement. The basis for the EORTC CAT is unidimensional item response theory (IRT)-generalized partial credit models, calibrated and evaluated in large, heterogeneous, international samples of cancer patients. The general measurement properties were confirmed in an international field study. However, the general applicability of the IRT model parameters has not yet been examined and confirmed in independent data. The aim of this study was to conduct the first cross-validation of the EORTC CAT Core IRT models using independent data. Methods: Model validation was based on a heterogeneous sample of 699 cancer patients recruited across seven European countries for the field study evaluating the measurement properties (focusing on known-groups validity) of the EORTC CAT Core. Using CAT, participants responded to 5-13 items dynamically selected from each item bank. Items with responses from $\geq 400$ participants were examined. Observed and expected item responses were compared overall and across the score continuums for indications of systematic bias. The impact for domain score estimation of potentially problematic items was assessed. Results: Of 77 items with $\geq 400$ responses, two items had mean differences between observed and expected responses $>10 \%$ of the item score range and seven had differences $>5 \%$. Comparisons across the score continuums revealed deviations for some scores for several items but few indications of systematic bias. Based on these comparisons, four items (two physical functioning, one dyspnoea, and one financial difficulties item) were flagged as having potentially biased parameters. Comparisons of domain score estimates with and without these items did not indicate systematically biased score estimates when including the items (mean score differences $<0.1 \mathrm{SD}$ ). Conclusion: Few indications of systematically biased item parameters were observed in this crossvalidation, and there was no evidence that these would result in biased score estimates. The results indicate that the IRT models used in the EORTC CAT Core are generally applicable and produce valid score estimates.
(106.2) Evaluating sub-banks of the EORTC emotional functioning item bank: a supplement to the EORTC CAT Core?

Mogens Groenvold, Palliative Care Research Unit, Department of Geriatrics and Palliative Medicine GP, Bispebjerg og Frederiksberg Hospital, Copenhagen NV, Denmark; Eva-Maria Gamper, Department of Psychiatry, Psychotherapy and Psychosomatics, University Hospital of Psychiatry II, Innsbruck, Austria; Anna Costantini, Psychoncology Unit, Sant'Andrea Hospital, Faculty of Medicine and Psychology Sapienza University, Rome, Italy; Johannes M. Giesinger, Department of Psychiatry, Psychotherapy and Psychosomatics, University Hospital of Psychiatry II, Innsbruck, Austria; Bernhard Holzner, Department of Psychiatry, Psychotherapy and Psychosomatics, University Hospital of Psychiatry II, Innsbruck, Austria; Colin D. Johnson, Surgical Unit, Faculty of Medicine, University of Southampton, Southampton, UK; Monika Sztankay, Department of Psychiatry, Psychotherapy and Psychosomatics, University Hospital of Psychiatry II, Innsbruck, Austria; Hugo Vachon, Quality of Life Department, European Organisation for Research and Treatment of Cancer, Brussels, Belgium; Teresa Young, East \& North Hertfordshire NHS Trust incorporating Mount Vernon Cancer Centre, Middlesex, UK; Morten Aa. Petersen, Palliative Care Research Unit, Department of Geriatrics and Palliative Medicine GP, Bispebjerg og Frederiksberg Hospital, Copenhagen NV, Denmark; submitted on behalf of "the EORTC Quality of Life Group"

Aims: The EORTC CAT Core includes 14 item banks for computer adaptive test (CAT) and short-form measurement. During development, it was a prerequisite that the item banks were conceptually compatible with the domains of the EORTC QLQ-C30 questionnaire. This not only ensured measurement within a well-established conceptual framework but also limited the range of items that could be included. The emotional functioning (EF) bank includes 24 items covering anxiety, depression, and general distress. The aim in this study was to evaluate whether unidimensional item banks for these three subdomains could be formed and potentially include items discarded in the development of the existing EF item bank. Methods: We followed the EORTC approach for psychometric validation of CAT item banks. Based on a large international sample of cancer patients, we evaluated dimensionality of the hypothesized subdomains, item response theory (IRT) (generalized partial credit) model fit, differential item functioning (DIF), measurement precision, and known groups validity. Results: A total of 935 cancer patients from Austria, Denmark, Italy, and the UK responded to $33 \mathrm{EF}$ items. Factor analyses indicated acceptable fit of three unidimensional models: three factors explained $72 \%$ of the variation, fit indices CFI/TLI ranged 0.97-0.99, and RMSEA ranged 0.06-0.10. Inspection of observed and expected item responses and infit and outfit indices generally indicated acceptable fit of the IRT models with a few indications of minor redundancy/misfit. Of about 300 tests for DIF, 19 indicated significant DIF. However, evaluations indicated the possible impact of DIF to be trivial, i.e., items seemed to work similarly across groups. Compared to the QLQ-C30 item(s) of each subdomain, the item banks may reduce sample size requirements with up to $40 \%$ (anxiety), 50\% (depression), and 25\% (general distress) without reducing power. Conclusion: The study confirmed that three unidimensional item banks (33 items in total) covering anxiety, depression, and general distress can be administered as CATs or as short forms with markedly increased power compared to the QLQ-C30 items. The final composition of these EF sub-banks may be adjusted based on input from conceptual experts. 
(106.3) Choosing appropriate estimators for estimands in PRO endpoints

Konstantina Skaltsa, PhD, IQVIA, Barcelona, Spain; Cristina Ivanescu, IQVIA, Amsterdam, The Netherlands

Aims: Patient-reported outcome (PRO) objectives sometimes are primary or key secondary endpoints in clinical trials, in which case they should be described using the estimand framework, following the adoption of the ICH E9(R1) addendum by regulators. The addendum emphasizes the need to account for intercurrent events (ICEs), i.e. post-randomization events potentially related to treatment and outcome. The authors have provided thoughts on how to apply the estimand framework in PRO endpoints (Lawarence 2020; Fiero 2020). The aim of this work is to propose some estimators that align with the strategies described in the addendum. An example case study on an oncology trial will be shown; potential scientific questions of interest will be set, strategies for handling ICEs will be discussed, and appropriate methodology will be proposed to estimate a reliable treatment effect for each scientific question. Methods: The addendum specifies 5 strategies for dealing with ICEs: treatment policy, hypothetical, composite, principal stratification, and while on treatment. A review of methods proposed in the literature (Mallinckrodt 2019) will be discussed for PRO endpoints, including common analyses, such as mixed models etc. Results: Treatment policy ignores the occurrence of ICEs and requires post-ICE data to be collected. Some data will still be missing, in which case appropriate methods reflecting the ICE occurrence when modelling missing outcomes are needed, e.g. reference-based imputation. Hypothetical strategies do not require postICE data; these are modelled via reference-based imputation, likelihood-based methods and multiple imputation. The remaining strategies do not face missing data issues. Composite approaches include non-responder imputation, composite time-to-event endpoints, or assigning poor values to post-ICE outcomes, e.g. reversion to baseline. Rank-based approaches constitute another alternative. While-on-treatment approaches focus on outcomes up to the ICE occurrence, for which summary measures (e.g. Area Under the Curve) may be used, or ANCOVA at the last visit, if only last value is of interest. Conclusion: As the estimand framework is adopted for PRO endpoints, scientific questions should be thoughtfully decided upfront, handling of ICEs considered carefully and appropriate estimators that align with the selected strategies should be employed. This work provides an overview of potential methods that could help researchers answer the scientific question of interest.

(106.4) Latent class analysis of symptom patterns in the adult Ontario population

Sara Wallström, PhD, University of Gothenburg, Gothenburg, Sweden; Jason Sutherland, University of British Columbia, Vancouver, British Columbia, Canada; Aslam Anis, University of British Columbia, Vancouver, British Columbia, Canada; Jacek Kopec, University of British Columbia, Vancouver, British Columbia, Canada; Rick Sawatzky, Trinity Western University, Langley, British Columbia, Canada

Aims: Much research on cancer-related symptoms has focused on prevalence, severity, and patterns of symptoms assuming a homogenous population. Less is known about socio-demographic factors associated with different symptom patterns. The aims of the study were (1) to distinguish subgroups of patients in the Cancer Care Ontario population who have different symptom patterns based on their first response to the Edmonton Symptom Assessment Scalerevised (ESAS-r) and (2) identify socio-demographic factors associated with the subgroups. Methods: ESAS-r data and socio- demographic variables were obtained via the Ontario Cancer Registry and linked health data files. Latent class analysis was used to identify the subgroups of patients. All differences are statistically significant $(p<0.0001)$. Results: In total, 216000 patients were included in the study. The cohort consisted of $45.9 \%$ men and $54.1 \%$ women with a mean age of 64.5. The analyses identified a 6-class model. The symptom patterns are shown in Fig. 1 . There are several socio-demographic differences between the classes. Class 1 includes the youngest patients (mean age 62) and Class 4 the oldest (mean age 69). Gender varies between 48.6 to $57.6 \%$ women in all classes except Class 3, which includes $70.6 \%$ women. Patients in Class 3 are also most likely to live in a city of more than 500,000 inhabitants. Patients in Class 4 are most likely to live in a smaller city or rural area. Patients in Class 1 are most likely to live in a neighborhood within the lowest income quintile, while patients in Class 5 are the most likely to live in the highest income neighborhood. Class 1 has the highest proportion of patients who live in a neighborhood that falls within the highest tercile of proportion foreign born, while the patients in Class 4 are most likely to live in a neighborhood that falls within the lowest tercile of foreign born. Conclusion: This study shows that latent class analysis can be used to identify subgroups of people with different symptom profiles that would not be possible to identify by just looking at the symptom assessments. Symptom patterns are associated with various socio-demographic differences, including age, gender, neighborhood income, and rurality of residence.

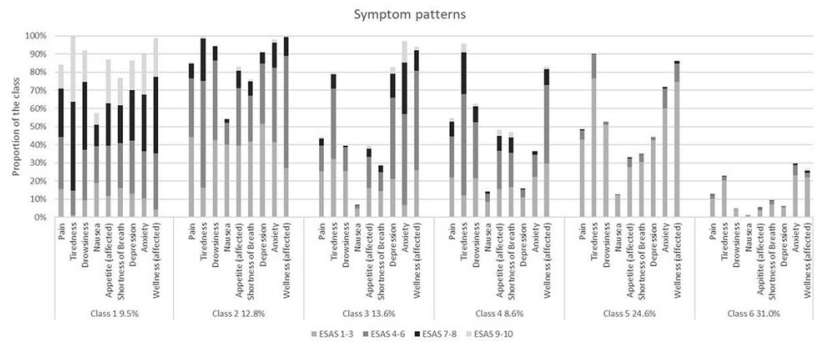

(106.5) An estimand perspective on the Mixed-Model Repeated Measures (MMRM) for the analysis of longitudinal PRO data in clinical trials

Konstantina Skaltsa, PhD, IQVIA, Barcelona, Spain; Libby Floden, Clinical Outcomes Solutions, Tucson, Arizona, USA; Rachael Lawrance, Adelphi Values, Bollington, UK

Aims: In clinical trials, Patient-Reported Outcome (PRO) data are typically collected at several timepoints. The use of Mixed-Model Repeated Measures (MMRM) for estimating the treatment effect has been endorsed by many, in part because of the way the model accounts for missing PRO data. At the same time, recommendations for measuring the patient experience not only while on treatment but also after discontinuation or clinical events such as disease progression have been increasingly implemented. Questions arise as to when and how to include such data. The ICH E9(R1) addendum on estimands, which defines intercurrent events (ICEs) as postrandomization events that may affect the interpretation of a variable, can help. The aim of this work is to illustrate the scientific questions that the MMRM can answer and how it can handle ICEs, considering implications to interpretation when including assessments that occur after certain ICEs. Methods: An MMRM is applied under two scenarios: (1) PRO data are not available after the ICE (e.g. death), (2) PRO data are available after the ICE (e.g. after discontinuation). The MMRM model uses information from all available data to calculate estimates by assuming missing outcomes are a weighted combination of the patients' previous observed values and values from similar patients in the same treatment arm. Results: When PRO data are not 
available (e.g. death) or not used after the ICE in the MMRM model, the hypothetical strategy is implied, estimating treatment effects "if the patient had not experienced the ICE". When PRO data are collected after the ICE (e.g. treatment discontinuation), and used in the MMRM, strategy will target treatment policy, estimating treatment effects "regardless of treatment discontinuation". Conclusion: When using the commonly applied MMRM model to assess PRO treatment differences, it is important to understand what treatment effects are being estimated and how the inclusion or exclusion of data after ICEs affects interpretation. If data are available post-ICE, the MMRM estimates the treatment effect as if the ICE did not occur when omitting these data, or ignoring the ICE (treatment policy) when included. If other scientific questions are of interest, then alternative statistical methods should be sought.

\section{7: Methodological Studies}

(107.1) Evaluating assumptions of growth mixture models for process-outcome data in a rehabilitation trial

Jan Boehnke, School of Health Sciences, University of Dundee, Dundee, UK; Christine Comer, Leeds Community Healthcare NHS Trust Musculoskeletal Service; Faculty of Medicine and Health, University of Leeds, Leeds, UK; Esther Williamson, Botnar Research Centre, Nuffield Department of Orthopaedics Rheumatology \& Musculoskeletal Sciences, University of Oxford, Oxford, UK; Sallie Lamb, College of Medicine and Health, University of Exeter, Exeter, UK

Aims: Neurogenic Claudication is a term used to describe leg pains when walking caused by spinal stenosis - a common degenerative condition in the lumbar spine. A recent trial evaluated a combined physical and psychological intervention for older adults living with the condition and secondary analyses of this trial investigate whether there are distinct trajectories of change in intermediate rehabilitation outcomes (self-management, walking disability). In this presentation, we discuss how different model specifications were evaluated to inform the main analysis strategy. Methods: $N=435$ entered the trial and data of $N=292$ participants in the intervention condition were analyzed. The intervention consisted of twelve 90 min sessions. Selfreport outcomes were assessed at baseline and sessions 3-, 6-, 9-, and $12(n=198 ; 67.8 \%$ contributed at least three out of four session assessments). We estimated a standard growth mixture model (GMM; linear and loglinear trends) and varied key parameter choices: (i) autocorrelation between assessments and (ii) class-specific random effects. For each combination of parameters, we estimated an increasing number of classes and used BIC evidence weights to evaluate the relative fit of models. Results: Across the set of models, a standard GMM with two classes was the best-fitting model for the self-management outcome, and a three-class GMM with class-specific random effects for walking disability. Within parametrizations, between one and two classes were identified for self-management and between two and four classes for walking capacity. Comparing the classification of participants across different parametrizations (e.g., Fig. 1) showed in some cases considerable differences in inferences about the trajectories. Conclusion: The presented work follows recent methodological reviews and simulations (Herle et al. 2020, European Journal of Epidemiology, 35, 205-222; Sijbrandij et al. 2020, BMC Medical Research Methodology, 20, 276), which highlighted the impact of parameter choices in these models and stressed that the results of these models should not be treated as confirmed phenotypes. Implications for GMM-based research will be discussed as well as extensions of this work evaluating congruence with other longitudinal latent class models, the role of drop-out, and the predictive power with view to the six-month trial outcomes.
Figure 1. Spaghetti plots of the observed data $(\mathrm{N}=292)$ and overlayed overall optimal (first column) and next-best (with different parametrization; second column) growth mixture model estimates. Selfmanagement trajectories (top row) show two classes for linear and loglinear trends with strong consistency across models ( $\mathrm{n}=3 ; 1.0 \%$, not classified to a similarly shaped trajectory). Walking disability trajectories (bottom row) dapict (bottom row) depict classes with class-specific and constant random-effect estimates with low consistency
across models (at least $\mathrm{n}=47 ; 16.1 \%$, not classified to a similarly shaped trajectory).
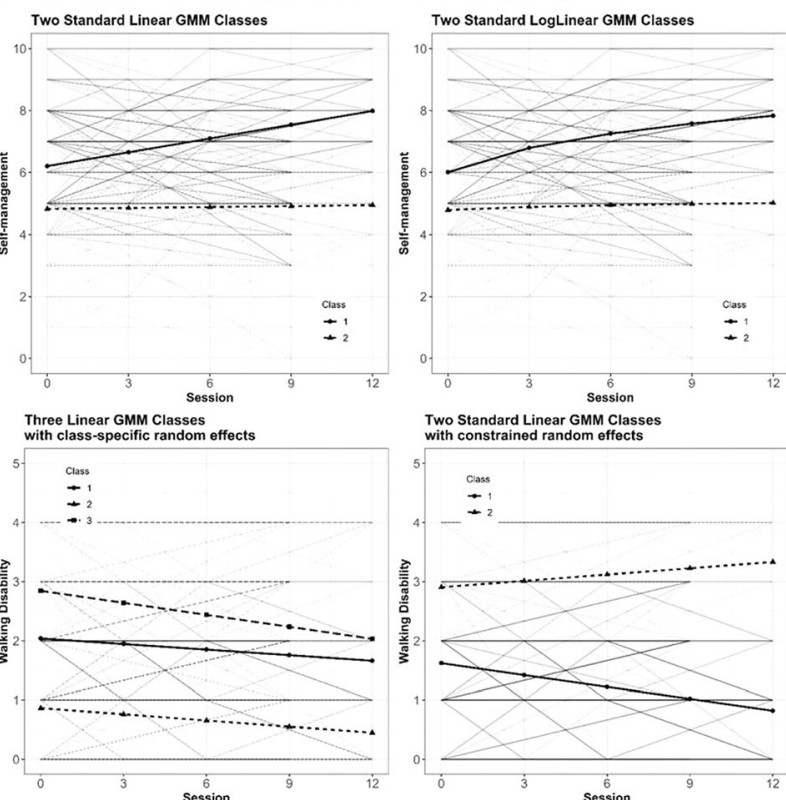

(107.2) Are my patients really getting better? Using plausible values to assess true change of patient-reported outcomes

Emily Ho, PhD, Northwestern University, Chicago, Illinois, USA; Jay Verkuilen, PhD, CUNY Graduate Center, New York, New York, USA; Felix Fischer, PhD, Charite - Universitätsmedizin Berlin, Berlin, Germany

Aims: One core advantage of IRT-based/PROMIS measures is that each estimate of the latent trait is associated with a standard errOR reflecting uncertainty in the measurement. Such uncertainty needs to be acknowledged and quantified when assessing individual patients. In this study, we use plausible values to analyze the probability of true within-individual change over time. Methods: We used a freely available dataset of $N=188$ COPD patients (Yount et al. 2019). We compute individual's physical function and fatigue PROMIS T-scores with established PROMIS item parameters (see Fig. 1 for longitudinal probability of true change for a sample patient). At each measurement, we impute 1000 plausible values from a normal approximation to the scores' posterior distribution. Plausible values were then used to calculate probability of true change, for individuals and groups at various levels of confidence/uncertainty and across the $T$-Score metric. Results: Uncertainty quantification across three measures of physical function and fatigue suggests that CAT has the most favorable properties, with a constant theta change of approximately 5 points reflecting a $95 \%$ probability of improvement. For short forms, theta change associated with $95 \%$ certainty of true change can vary considerably depending on the $T$-score. A comparison of reliability precision across two PROMIS domains, Physical Function and Fatigue, suggests differing thresholds for meaningful change across the same $T$-Score for different health domains. Conclusion: Plausible values offer a flexible way to include measurement error in analysis of individuals and on a group level and offer a useful complement to existing distribution-based approaches by providing an assessment of probability of true change. This method facilitates ease of interpretation and allows for a finer-grained comparison of improvement or decline than previously possible. 


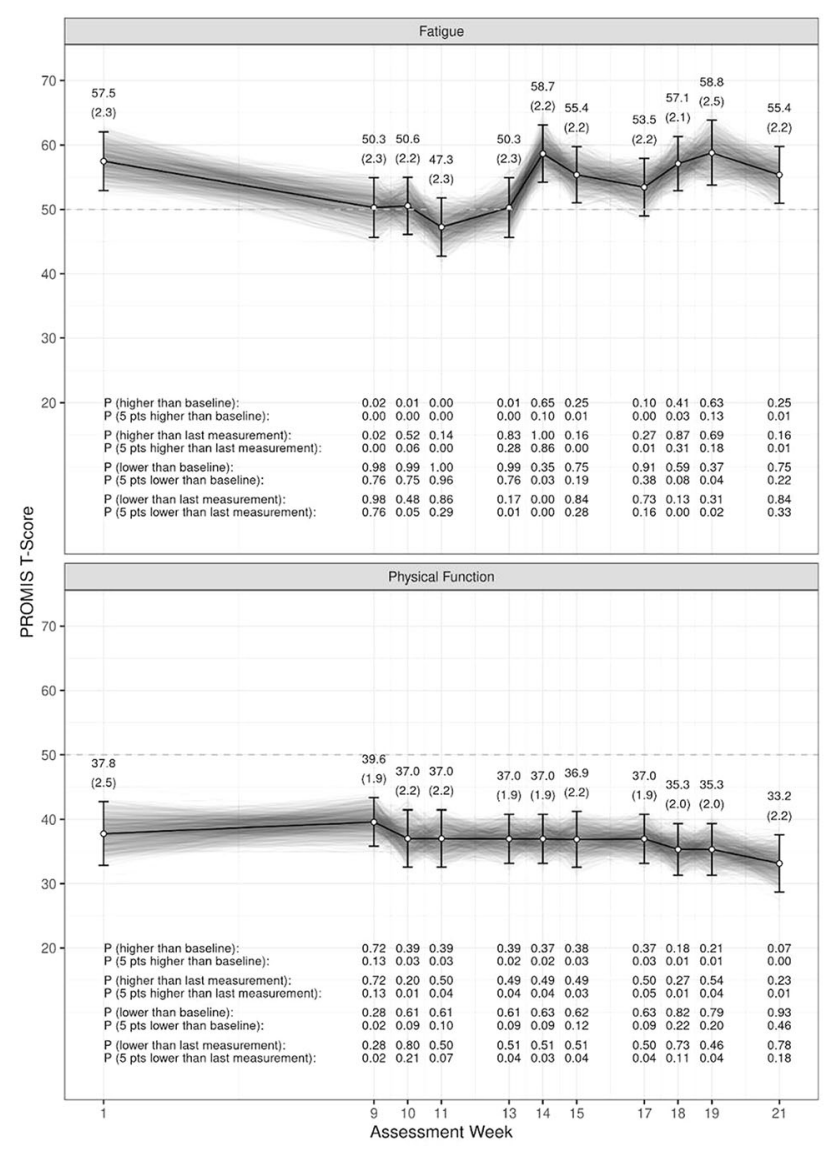

(107.3) Computerised Adaptive Testing for the UK National PROMs programme oxford hip and knee scores dramatically reduces the number of items needed without compromising precision

Jonathan Evans, MBChB, MSc, MD, FRCS, University of Exeter Medical School, Exeter, UK; Chris Gibbons, PhD, MD Anderson Center for INSPiRED Cancer Care (Integrated Systems for PatientReported Data), Houston, Texas, USA; Jose Valderas, MD, MPH, $\mathrm{PhD}$, Health Policy and Research Group, University of Exeter Medical School, Exeter, UK

Aims: The NHS England Patient-Reported Outcome Measures (PROMs) programme is the world's largest repository of hip and knee replacement outcome scores with over 160,000 participants per year. We used a modern psychometric approach to develop Computerised Adaptive Tests for joint replacement outcomes with the aim of reducing the patient and infrastructure burden associated with large PROMs datasets. Methods: The 2018/19 National PROMs programme 12-item Oxford Hip and Knee Scores (OHS and OKS), recorded before a primary joint replacement, were evaluated for dimensionality, local dependency, item fit, and marginal reliability. We used the Graded Response Model to develop item-specific difficulty and discrimination parameters. We used patient data from 2017/2018 to simulate a CAT. We compared the item responses, precision, scale scores, and length of both CAT and full-length versions of the OHS and the OKS. Results: We conducted IRT analysis using 40,432 OHS and 44,714 OKS observations. Primary OHS and OKS were both unidimensional (Root-Mean-Square Error of Approximation (RMSEA) of 0.08 and 0.07 , respectively). Marginal reliability (MR) was 0.914 and 0.903 , respectively. The CAT, with a precision of standard error of measurement 0.32 and 0.45 , required a median of 3 items (range 1-12) $(r=0.96)$ and median 2 items $(1-7)$ $(r=0.90)$, respectively for the OHS, and median of 4 items $(1-12)$ $(r=0.96)$ and 2 items $(1-12)(r=0.91)$ for the OKS. This represents a potential $82 \%$ reduction in PROM length, which could equate to over $30,000 \mathrm{~h}$ of reduced data collection time per year. Conclusion: We have established that the application of IRT to the OHS and OKS produces an efficient and substantially reduced CAT. By reducing the number of items required and applying a standardised measurement scale, we have demonstrated a path to reduce burden and potentially increase compliance for these ubiquitous outcome measures.

(107.4) Automatically analyzing online patient experience data with Natural Language Processing: an instrument to investigate health status and help-seeking factors in patients with obesity

Matthias Hartung, Dr., Semalytix GmbH, Bielefeld, Germany; Nathalie Schwering, Dr., Semalytix GmbH, Bielefeld, Germany; Yannick Loonus, Dr., Semalytix GmbH, Bielefeld, Germany; Philipp Cimiano, Prof. Dr., Semalytix GmbH; Bielefeld University, Bielefeld, Germany; Anna A. Jäger, Dr., Boehringer Ingelheim International $\mathrm{GmbH}$, Ingelheim, Germany; Ben Collins, Ph.D., Boehringer Ingelheim International $\mathrm{GmbH}$, Ingelheim, Germany

Aims: Self-reported patient narratives from social media provide a rich source of information towards understanding the real-world experience of patients from an unsolicited first-hand perspective. Due to volume and heterogeneity, automated methods are required for transforming patient-generated content into real-world data to support patient-focused drug development. Using obesity as an example, this study explores the benefit of such data sources for gathering insights into the real-world experience of a disease as reported by large online patient populations. Methods: Purposive sampling yielded $1.8 \mathrm{M}$ unique posts from obesity-related online conversations in six social media sources between January 2018 and February 2020. Using stateof-the-art technology from natural language processing, this corpus was reduced to approx. 22,000 narratives by patients with obesity, a subset of which $(n=6124)$ contains explicit references to symptoms and co-morbidities. Within this sub-corpus, 1424 documents were automatically mapped to speakers from four BMI classes (25-29.9; $30-34.9 ; \quad 35-39.9 ;>40) ; 3400$ documents were automatically assigned to thematic clusters focusing on doctor-patient relationships $(n=2863)$ and moments of motivation for trying to lose weight $(n=830)$. Results: Quantitative analysis reveals marked differences between the distributions of symptoms and co-morbidities across BMI classes. Cardio-metabolic conditions (diabetes mellitus, hypertension) are consistently overexpressed in BMI classes 35-39.9 (12.8\% vs. $8.8 \%, 9.0 \%$ vs. $3.0 \%$, respectively) and $>40(13.8 \%$ vs. $8.8 \%, 9.8 \%$ vs. $3.0 \%$, respectively) compared to the overall dataset. Depression is strongly overexpressed in higher BMI classes (10.3\% in BMI 35-39.9 vs. $7.2 \%$ all; $17.0 \%$ in BMI $>40$ vs. $7.2 \%$ all), thus, being the most frequently reported burden by morbidly obese patients. Qualitative interpretation of thematic clusters shows that the majority of motives for help seeking are of intrinsic and positive orientation. Supportive behaviors vs. blaming are found as main drivers of a positively or negatively perceived doctor-patient relationship. Conclusion: This study corroborates the potential of automatically analyzing patientgenerated online data to complement and extend traditional instruments in market and outcomes research. In the obesity case, the approach yields confirmatory results about the bi-directional relationship of obesity and depression, while unlocking an additional data source for qualitative research into motivational factors and drivers of effective patient-doctor relationships. 
(107.5) The use of collaborative patient engagement in the development of a PROM to assess the impact of hypoglycaemia on quality of life as part of the HypoRESOLVE project: Patient and researcher perspectives

Jill Carlton, University of Sheffield, Sheffield, UK; Philip Powell, $\mathrm{PhD}$, University of Sheffield, Sheffield, UK; submitted on behalf of "Patient Advisory Committee (PAC)"

Aims: The Hypoglycaemia REdefining SOLutions for better liVEs (Hypo-RESOLVE) project is developing a new patient-reported outcome measure (PROM) suitable for assessing the impact of hypoglycaemia on quality of life (QoL) in people with diabetes. Significant patient engagement has been incorporated within many aspects of the development of the instrument itself. We aim to discuss here both from researcher and patient perspectives the benefits, challenges, and mechanics of achieving successful patient engagement in PROM development. Our discussion will focus on lessons learned as part of the work that can be applied to other instrument development projects. Methods: This will be a practical co-presented discussion of the researcher's and patient's lived experience in working together to try and achieve optimal and reciprocally beneficial patient engagement. We will use illustrative examples to support our claims. Particular attention will be drawn to aspects of the engagement process where things could have been done differently or improved. Results: Patient engagement on the Hypo-RESOLVE project to date has been a notable strength, with an established Patient Advisory Group and patient representatives on other governing bodies, including the Scientific Group and overall Advisory Group. The patients and researchers have further collaborated on methods of effective dissemination, including a recurrent podcast and social media presence. Conclusion: Patient engagement is essential in producing valid and robust PROMs. Existing evidence can portray patient involvement as being a wholly positive experience. However, the reality of patient involvement and engagement can be incredibly challenging for all parties. We hope our frank discussion on patient engagement in the Hypo-RESOLVE project from both the patient and researcher perspective and lessons learned will provide useful and reflective insights for QoL and PROM researchers.

(107.6) The responsiveness and meaningful interpretation of the PROMISnq SF v2.1: Physical Function (MS) 15a in relapsing and progressive Multiple Sclerosis populations

Paul Kamudoni, PhD MSc, Global Evidence \& Value Development, Merck Healthcare KgaA, Darmstadt, Germany; Jeffrey Johns, PhD, Institute of Medicines Development, Cardiff, UK; Karon Cook, PhD, Feral Scholars, Broaddus, Texas, USA; Rana Salem, MA, Department of Rehabilitation Medicine, University of Washington, Seattle, Washington, USA; Sam Salek, PhD, School of Life and Medical Sciences, University of Hertfordshire, Hatfield, UK; Jana Raab, MSc, Global Evidence \& Value Development - R\&D, Merck Healthcare KgaA, Darmstadt, Germany; Rod Middleton, UK MS Register, Swansea Medical School, Swansea, UK; Dagmar Amtmann, PhD, Department of Rehabilitation Medicine, University of Washington, Seattle, Washington, USA; Christian Henke, PhD, Global Evidence \& Value Development - R\&D, Merck Healthcare KgaA, Darmstadt, Germany

Aims: The aim of the study is to evaluate the responsiveness of the PROMISnq SF v2.1-Physical Function (MS) 15a [PROMIS PF(MS)15a] and to establish minimal important difference estimates (MID) and interpretation tools, in relapsing and progressive Multiple Sclerosis (MS) populations Methods: A 96-week longitudinal study was performed in the UK MS Register Cohort as part of an instrument development program, from September 2018 to October 2020. The final version of the PROMIS PF(MS)15a short form was administered from week 52 onwards. Responsiveness and MID were analyzed based on score change from week 52 to week 96. An anchor-based approach was used to establish MIDs and an interpretation guide for the PROMIS PF(MS)15a scores. A T-score map, based on item response theory, showing the most likely limitation for each $T$-score, was developed. Results: At baseline, study participants had a mean age of 49.9 ( $\mathrm{SD}=9.74)$ years, and median patient-reported EDSS score of 5. Six anchors met criteria and were used in the analyses. The PROMIS PF(MS)15a scores were sensitive to worsening ( $\mathrm{SRM}=$ 0.38 to 0.62$)$ as well as improvement (SRM $=-0.28$ to -0.49$)$ in physical function over 52 weeks of follow-up. Mean $T$-score change estimates for minimal worsening, based on two anchors (i.e., GHS everyday physical activities, GHS GPH summary $T$-score), met all criteria for MID estimation. A score change of 2.1 to 2.6 points is proposed as MID criteria for minimal worsening in physical function; this is above the standard error of measurement [SD * $\sqrt{ }(1-$ reliability)] at week 52 (i.e., 1.8). A heatmap, i.e., the T-score map, allowing interpretation of $\mathrm{T}$-scores based on responses on the individual items was developed. For example, a $T$-score of 30 represents physical function characterized by (little difficultly) holding a plate of food, (unable) to exercise hard for half an hour, and (a lot of difficulty) walking on uneven surfaces. Conclusion: This research extends the evidence underpinning the applicability of the PROMIS $\mathrm{PF}(\mathrm{MS}) 15 \mathrm{a}$ in routine clinical practice and clinical research. The score interpretation guide may aid the integration of PROMIS scores into clinical decision making as well as facilitate clinician-patient communication. MID estimates will be useful in evaluating physical function over time.

\section{8: Cancer Clinical Research in Caregivers and Vulnerable Populations}

(108.1) Quality of life in siblings of childhood leukemia survivors, long-term after diagnosis: a French Childhood Cancer Survivor Study For Leukemia (LEA Cohort)

Cindy Faust, Research Unit EA 3279 and Department of Public Health, Aix-Marseille University and Timone Hospital, Marseille, France., Marseille, France; Pascal Auquier, Research Unit EA 3279 and Department of Public Health, Aix-Marseille University and Timone Hospital, Marseille, France; Gérard Michel, Department of Pediatric Hematology and Oncology, Timone Enfants Hospital and Aix-Marseille University, Marseille, France; Yves Bertrand, Department of Pediatric Hematology and Oncology, University Hospital of Lyon, Lyon, France; Marie-Dominique Tabone, Department of Pediatric Hematology and Oncology, A.Trousseau University Hospital, APHP, Paris, France; Sophie Ansoborlo, Department of Pediatric Hematology and Oncology, University Hospital of Bordeaux, Bordeaux, France; André Baruchel, Department of Pediatric Hematology and Oncology, St Louis Hospital, APHP, Paris, France; Virginie Gandemer, Department of Pediatric Hematology and Oncology, University Hospital of Rennes, Rennes, France; JeanHugues Dalle, Pediatric Hematology Department, Robert Debré Hospital, Paris, France; Pascal Chastagner, Department of Pediatric Hematology and Oncology, Children's Hospital of Brabois, Vandoeuvre Les Nancy, France, Nancy, France; Justyna Kanold, Department of Pediatric Hematology and Oncology, CIC Inserm 501, University Hospital of Clermont-Ferrand, Clermont-Ferrand, France; Maryline Poiree, Department of Pediatric Hematology and Oncology, University Hospital L'Archet, Nice, France; Nicolas Sirvent, Department of Pediatric Hematology and Oncology, University Hospital of Montpellier, Montpellier, France; Geneviève Plat, 
Department of Pediatric Hematology and Oncology, University Hospital of Toulouse, Toulouse, France; Isabelle Pellier, Department of Pediatric Hematology-Oncology, University Hospital of Angers, Angers, France; Zeinab Hamidou, Research Unit EA 3279 and Department of Public Health, Aix-Marseille University and Timone Hospital Marseille, France, Marseille, France; Julie Berbis, Research Unit EA 3279 and Department of Public Health, Aix-Marseille University and Timone Hospital Marseille, France, Marseille, France

Aims: The experience of pediatric cancer is a life-altering event for all the family, including siblings. Psychological problems, such as symptoms of post-traumatic stress, have been investigated, and some studies showed a persistence over time. Literature concerning siblings' long-term quality of life (QoL) is still infrequent and methodological limitations are substantial. We aimed to assess QoL of siblings of childhood leukemia survivors long time after the diagnosis compared with reference population and survivors, and then to define clusters of siblings' QoL levels to identify profiles of siblings and to explore determinants of their long-term QoL. Methods: Siblings over 8 years old of survivors from the French multicenter LEA Cohort were asked to complete QoL questionnaires (VSP-A and SF-36, respectively). Results were compared with age- and sexmatched values from (1) reference population and (2) those obtained for survivors. Using the 8-dimension scores of each questionnaire and a new method of interpretable clustering based on unsupervised binary trees (CUBT), siblings were classified in "high," "moderate," and "low" level of QoL. Multivariate analyses explored sibling's and survivor's sociodemographic and health-related and cancer-related characteristics likely to be associated with siblings' QoL levels. Results: We included 689 siblings (mean follow-up time from diagnosis $13.2 \pm 6.6$ years). Compared with reference population, 313 minors showed a good overall QoL $(69.6 \pm 12.8$ vs. $66.9 \pm 5.0$, $p<0.001)$ but reported significantly lower scores for relationships with family, when compared to survivors $(62.7 \pm 22.7$ vs. $67.8 \pm 21.0, p$-value $<0.001)$. Compared with reference population, 376 adults expressed a significantly lower perception regarding psychological domains (MCS $45.2 \pm 9.8$ vs. $47.9 \pm 2.1, p<0.001$ ). Also, their QoL was significantly higher in general health perception and in physical domains than survivors scores. Being a woman seemed to be linked with the lowest QoL level. QoL did not differ according to the treatment received. Conclusion: According to their age category, siblings reported a different perception of their QoL at long term. To avoid late consequences in domains impacted during the transition between childhood and adulthood, the long-term followup already implemented for survivors should also benefit to siblings, who are frequently considered as the "forgotten children".

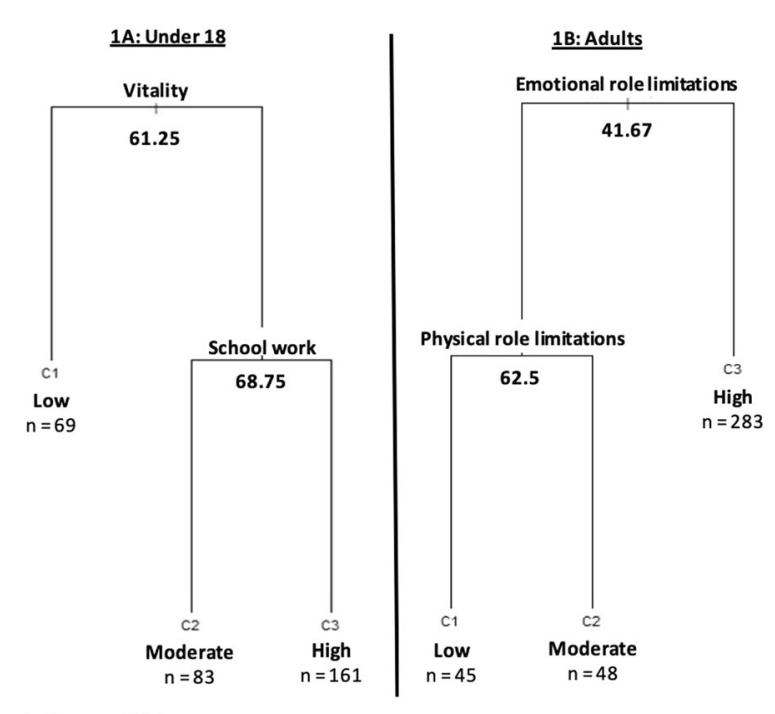

Figure 1. Clusters of QoL

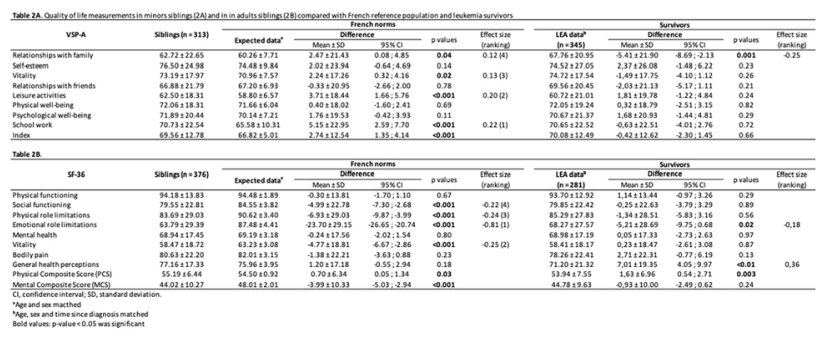

\begin{tabular}{|c|c|c|c|}
\hline VSP-A & $\begin{array}{l}\text { Cluster } 1 \\
\text { Low QoL } \\
(n=69)\end{array}$ & $\begin{array}{c}\text { Cluster 2 } \\
\text { Moderate QoL } \\
(n=83)\end{array}$ & $\begin{array}{l}\text { Cluster } 3 \\
\text { High QoL } \\
(\mathbf{n}=161)\end{array}$ \\
\hline Relationships with family & $45,36 \pm 22,81$ & $63,39 \pm 18,09$ & $69,82 \pm 20,76$ \\
\hline Self-esteem & $53,25 \pm 30,29$ & $80,16 \pm 19,32$ & $84,59 \pm 18,15$ \\
\hline Vitality & $47,23 \pm 12,71$ & $75,93 \pm 9,62$ & $82,90 \pm 11,14$ \\
\hline Relationships with friends & $52,97 \pm 22,93$ & $69,80 \pm 21,39$ & $71,33 \pm 18,98$ \\
\hline Leisure activities & $53,71 \pm 19,21$ & $63,43 \pm 17,84$ & $65,79 \pm 17,01$ \\
\hline Physical well-being & $59,78 \pm 17,63$ & $73,75 \pm 17,52$ & $76,45 \pm 16,68$ \\
\hline Psychological well-being & $52,71 \pm 22,05$ & $75,81 \pm 13,71$ & $78,09 \pm 17,50$ \\
\hline School work & $64,13 \pm 22,68$ & $47,14 \pm 14,65$ & $85,71 \pm 11,34$ \\
\hline Index & $53,64 \pm 10,33$ & $68,68 \pm 7,72$ & $76,84 \pm 8,91$ \\
\hline \multicolumn{4}{|c|}{ Table 3B. SF-36 dimension scores by cluster of QoL } \\
\hline & Cluster 1 & Cluster 2 & Cluster 3 \\
\hline SF-36 & $\begin{array}{l}\text { Low QoL } \\
(n=45)\end{array}$ & $\begin{array}{l}\text { Moderate QoL } \\
\quad(n=48)\end{array}$ & $\begin{array}{l}\text { High QoL } \\
(\mathbf{n}=\mathbf{2 8 3})\end{array}$ \\
\hline Physical functioning & $87,65 \pm 15,50$ & $94,06 \pm 13,93$ & $95,23 \pm 13,29$ \\
\hline Social functioning & $48,06 \pm 19,94$ & $64,06 \pm 19,74$ & $87,19 \pm 17,53$ \\
\hline Physical role limitations & $23,89 \pm 21,29$ & $94,27 \pm 10,62$ & $91,40 \pm 19,54$ \\
\hline Emotional role limitations & $17,78 \pm 16,82$ & $23,61 \pm 15,31$ & $77,92 \pm 15,88$ \\
\hline Mental health & $48,89 \pm 16,56$ & $56,56 \pm 13,87$ & $74,23 \pm 14,55$ \\
\hline Vitality & $38,69 \pm 16,43$ & $48,73 \pm 17,78$ & $63,26 \pm 16,43$ \\
\hline Bodily pain & $59,96 \pm 24,19$ & $81,40 \pm 20,89$ & $83,79 \pm 20,33$ \\
\hline General health perceptions & $61,93 \pm 20,24$ & $73,44 \pm 16,62$ & $80,21 \pm 15,51$ \\
\hline Physical Composite Score (PCS) & $49,02 \pm 6,96$ & $60,15 \pm 5,96$ & $55,33 \pm 5,60$ \\
\hline Mental Composite Score (MCS) & $30,36 \pm 7,08$ & $32,06 \pm 8,32$ & $48,22 \pm 7,00$ \\
\hline
\end{tabular}

(108.2) Patterns in patient-reported outcomes preceding disease progression in lung cancer: preliminary analysis of an ongoing randomized controlled trial (ProWide)

Rasmus Blechingberg Friis, $\mathrm{MD}, \mathrm{PhD}$ student, Department of Oncology, Hospital Unit West Jutland, Herning, Denmark; Helle Pappot, professor MD, Department of Oncology, University Hospital, 
Copenhagen, Copenhagen, Denmark; Niels Henrik Hjøllund, professor MD, AmbuFlex/WestChronic, Occupational Medicine, Herning and Department of Epidemiology, Aarhus University Hospital, Denmark, Herning, Denmark; Skuladottir Halla, D.M.Sc., MD, Department of Oncology, Hospital Unit West Jutland, Herning, Denmark

Aims: In an ongoing national randomised controlled trial (ProWide), we are studying the effects of remote symptom monitoring using electronic patient-reported outcome (PRO) measures in patients with advanced lung cancer. In the intervention arm, patients weekly complete a 13-item electronic questionnaire. A pre-defined severity threshold is used for each item. The software algorithm automatically notifies a nurse if a symptom exceeds the threshold. If symptoms indicate disease progression, a CT scan is performed as soon as possible. The intervention may result in improved disease surveillance by timely detection of disease progression. In an exploratory analysis, we aimed to evaluate if the pre-defined severity-threshold algorithm had the properties to indicate disease progression. Methods: All items were dichotomized into categories with responses exceeding the predefined symptom-severity thresholds and responses not exceeding the thresholds. The prognostic value of all 13 items for finding disease progression was evaluated with a multistage model using a multivariate cox regression analysis with threshold exceedance as time-dependent covariate. In doing so, we assessed the ability of the predefined severity thresholds to predict disease progression. Results: At the time of data extraction, 203 patients in the intervention arm had completed 4,975 weekly symptom reports. Overall weekly compliance was $80 \%$ in the intention-to-monitor population. Progressive disease had been confirmed in 117 patients. The thresholds for five of 13 symptoms were found to have a significant prognostic value indicating disease progression. Pain (HR $2.2, p=0.02$ ), overall health (HR 6.1, $p<0.00$ ), cough (HR 2.6, $p=0.00$ ), hemoptysis (HR 9.5, $p=0.00$ ) and sense of a growing tumour (HR 9.5, $p=0.00$ ). Conclusion: Exceedances of the predefined severity thresholds were found to indicate disease progression for five of 13 symptoms. This may help to reduce the number of necessary items when aiming to detect disease progression in patients with advanced lung cancer.

\section{(108.3) AIRPoRT: Assessment of Information Required by Patients or relatives who have undergone radical lung Radiotherapy Treatment: Patient and carer interviews}

Patricia (Trish) Holch, PhD, Leeds Beckett University, Centre for Psychological Research, Leeds, UK; Lisa Copeland, BSc (Hons) Nursing Practice Sp Prac, Leeds Teaching Hospitals NHS Trust, Leeds, UK; Charlotte Hunting, BSc (Hons) Therapeutic Radiography, Leeds Teaching Hospitals NHS Trust, Leeds, UK; Carole Burnett, PhD Medicine, University of Leeds: Leeds Insitute of Medical Research, Leeds, UK; Helen Melling, BSc (Hons) Therapy Radiography, Leeds Teaching Hospitals NHS Trust, Leeds, UK

Aims: Prior to discharge from radiotherapy (RT), all UK patients are given information about potential side effects and discuss issues with a therapeutic radiographer in an end of treatment discussion (EOTD). However, it is unclear whether the information given is appropriate or timely and supports patient quality of life. Here, we aimed to explore the information and support needs of patients and carers via semistructured interviews to optimise the content and delivery of the EoTD. Methods: Participants $(N=16)$ were recruited as a volunteer sample from a Leeds Cancer Centre RT department consisting of $n=12$ patients undergoing radical lung cancer treatment (mean age 72.33, s.d. 10.17: $61.5 \%$ female and $38.5 \%$ male) and carers $(n=4)$, mean age 65.67 , s.d. $8.32: 100 \%$ female. Semi-structured interviews explored the knowledge and experience of EoTD, post-RT symptoms, emotional and financial issues, and experiences on relative attendance. Interviews were audio recorded and transcribed verbatim and subject to both quantitative content and qualitative thematic analysis. Results: $67 \%$ of patients and $75 \%$ of carers were reassured by the EoTD. The majority felt informed about potential skin changes and fatigue; however, only $33 \%$ were aware of potential post-RT breathing difficulties. Similarly, $42 \%$ of patients felt their concerns were not fully explored: "I think there's always questions you want answering". Emotional issues were evident "I was ready to commit suicide" and "I always fear the worst". 58\% of patients had their carer present and found the "moral support" and ability to distil complex information helpful. Fifty percent of patients experienced financial difficulties and think they should be discussed in the EoTD. Findings also showed a correlation between location of radiotherapy target volume (central/peripheral) and side effects experienced; highlighting a need to tailor EoTD information to individuals' treatment plan. Conclusion: The findings have enabled a deeper understanding of patient and carer need in relation to the EoTD and have informed topic areas for a survey currently underway. The findings from the interviews and survey will enable us to restructure the EoTD to meet patient need and ultimately develop national guidelines for EoTD in RT practice.

\section{(108.4) Patient-reported symptoms during lung cancer chemotherapy: a nested cohort within the PRO-TECT pragmatic trial (AFT-39)}

Gita Mody, MPH MD Assistant professor Director of Thoracic Surgical Oncology, UNC-Chapel Hill, North Carolina, USA; Angela Stover, University of North Carolina, Chapel Hill, North Carolina, USA; Mian Wang, University of North Carolina, Chapel Hill, North Carolina, USA; Jennifer Jansen, University of North Carolina, Chapel Hill, North Carolina, USA; Sydney Henson, University of North Carolina, Chapel Hill, North Carolina, USA; Arlene Chung, University of North Carolina, Chapel Hill, North Carolina, USA; Mattias Jonsson, University of North Carolina, Chapel Hill, North Carolina, USA; Antonia Bennett, University North Carolina, Chapel Hill, North Carolina, USA; Angela Smith, University of North Carolina, Chapel Hill, North Carolina, USA; William Wood, University of North Carolina, Chapel Hill, North Carolina, USA; Allison Deal, University of North Carolina, Chapel Hill, North Carolina, USA; Amylou Dueck, Mayo Clinic, Scottsdale, Arizona, USA; Brenda Ginos, Mayo Clinic, Scottsdale, Arizona, USA; Deborah Schrag, Harvard Medical School, Boston, Massachusetts, USA; Ethan Basch, University of North Carolina, Chapel Hill, North Carolina, USA

Aims: Patients with metastatic lung cancer often have a high symptom burden and diminished quality of life. Electronic patient-reported outcomes (ePROs) afford an opportunity to longitudinally monitor patients remotely and alert clinicians about severe or worsening symptoms to inform management. Methods: Adults receiving chemotherapy for metastatic lung cancer at 26 community oncology sites were invited to participate in a prospective trial of weekly ePRO symptom reporting. Surveys assessing patients' satisfaction with the ePRO system was administered at 3 months. Descriptive statistics were generated for demographics, survey completion rates, symptom occurrence, and provider management approaches. Pairwise relationships between symptom items were evaluated using intraindividual repeated-measures correlation coefficients. Results: Among 118 participating patients, $90 \%$ of weekly ePRO surveys were completed. Nearly all $(97 \%)$ patients reported one or more concerning (severe or worsening) symptoms during participation, with $33 \%$ of surveys including concerning symptoms. Pain was the most 
frequently reported and longest lasting concerning symptom and was associated with reduced activity level. More than half of all alerts to nurses or providers for concerning symptoms led to intervention including discussion with the patient and/or caregiver. The majority of patients $(87 \%)$ would recommend using the remote ePRO system to other lung cancer patients. Patient feedback on the ePRO system results are displayed in Fig. 1. Conclusion: Remote weekly symptom monitoring using ePROs for patients with metastatic lung cancer was feasible and acceptable in a multicenter pragmatic study. A high symptom burden specific to lung cancer was detected, and clinician outreach in response to alerts was common, suggesting that ePROs may be a beneficial strategy for identifying actionable symptoms and allow opportunities to optimize well-being in this population.

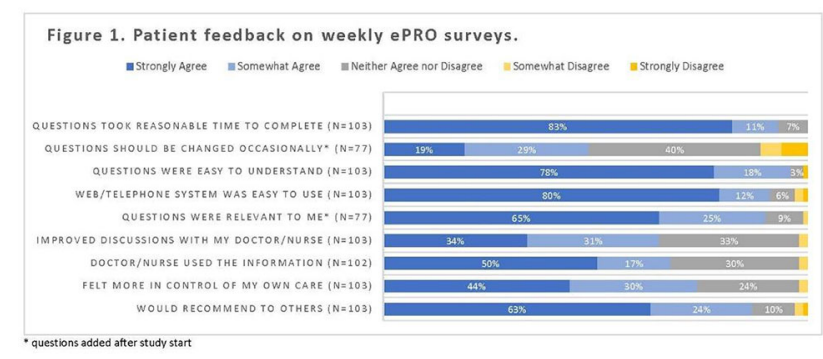

(108.5) Timing of advance care planning for patients with advanced cancer

Ida Korfage, Erasmus MC University Medical Center Rotterdam, Rotterdam, The Netherlands; Agnes van der Heide, Erasmus MC University Medical Center Rotterdam, Rotterdam, The Netherlands; Judith Rietjens, Erasmus MC University Medical Center Rotterdam, Rotterdam, The Netherlands

Aims: Timing of advance care planning (ACP) in patients with advanced cancer is challenging. If the process is initiated too early, patients may not be ready and their quality of life affected. Yet, delaying ACP may leave insufficient time to prepare for the future. Uncertainty about timing of ACP leads to conspiracies of silence among patients, relatives, and health care providers. We aimed to evaluate the experience of timing of ACP conversations among patients with advanced cancer. Methods: We used data from an ACP cluster-RCT across six countries (Belgium, Denmark, Italy, The Netherlands, Slovenia, and UK) including patients with advanced lung or colorectal cancer with a life expectancy of approximately one year. The ACP intervention included scripted ACP conversations between patients, relatives, and certified facilitators. Participants' experience of timing of ACP was measured at 12 and 20 weeks by "How did you feel about the timing of your ACP conversations during the study in relation to where you find yourself in your life at the moment?" Answering options were too early, just about right, and too late. Results: Of 442 intervention patients, 396 (90\%) engaged in ACP of whom $295(75 \%)$ answered the question about timing. Of these, $16 \%$ considered timing to be too early, $75 \%$ just right, and $8 \%$ too late. Experiences of timing were associated with gender (women more often found it too early, $p=0.02$ ) and country (more often too early in Denmark and too late in Slovenia, $p=0.02$ ). Timing was more often considered too late with worse performance status ( $p=0.009)$ and if remaining time until death was shorter $(p=0.007)$. When timing was considered right, conversations were perceived as more helpful $(p<0.001)$ and less stressful $(p=0.01)$, but also when timing was considered too early $64 \%$ of patients considered ACP helpful and $74 \%$ found it not stressful. When timing was considered as too early, advance directives were less often included in medical files. Conclusion: Adequate timing of the initiation of ACP conversations improved acceptance and efficacy of ACP. Evaluation of timing was not only associated with clinical characteristics such as performance status, but also with personal and cultural factors.

\section{9: Application of Clinical Research in Cancer}

(109.1) Predicting 180-day mortality for women with ovarian cancer using machine learning and patient-reported outcome data

Christopher Sidey-Gibbons, PhD, The University of Texas MD Anderson Cancer Center, Houston, Texas, USA; Charlotte Sun, DrPH, University of Texas MD Anderson Cancer Center, Houston, Texas, USA; Sheng-Chieh Lu, PhD., University of Texas MD Anderson Cancer Center, Houston, Texas, USA; Cai Xu, PhD., University of Texas MD Anderson Cancer Center, Houston, Texas, USA; Subbiah Ishwaria, PhD., University of Texas MD Anderson Cancer Center, Houston, Texas, USA; Alexi Wright, MD MPH, Harvard Medical School, Boston, Massachusetts, USA; Larissa Meyer, MD MPH, University of Texas MD Anderson Cancer Center, Houston, Texas, USA

Aims: Contra to national guidelines, women with ovarian cancer often receive aggressive treatment up until death. For clinicians, having to rely solely on clinical acumen for clinical predictions of death is likely a contributing factor. We sought to train machine learning algorithms to predict mortality within 180 days for women with ovarian cancer to facilitate appropriate provision of end-of-life care. Methods: Data were collected from a single academic cancer institution in the USA. Women completed biopsychosocial patientreported outcome measures (PROMs) every 90 days. We randomly partitioned our dataset into training and testing samples with a 2:1 ratio. We used synthetic minority oversampling to reduce class imbalance in the training dataset. We fitted training data to six machine-learning algorithms and combined their classifications on the testing dataset into a voting ensemble. We assessed the accuracy, sensitivity, specificity, and area under the receiver operating characteristic curve (AUROC) for each algorithm using testing data. Results: We recruited 245 patients who completed 1319 assessments. The final voting ensemble performed well across all performance metrics (Accuracy $=0.79$, Sensitivity $=0.71, \quad$ Specificity $=0.80$, AUROC $=0.76$ ). The algorithm correctly identified 25 of the 35 women in the testing dataset who died within 180 days of assessment. Conclusion: Machine learning algorithms trained using PROM data offer state-of-the-art performance in predicting whether a woman with ovarian cancer will reach the end of life within 180 days. We highlight the importance of patient-centered data in ML models of mortality. Our model exhibits substantial improvements in prediction sensitivity compared to other similar models trained using electronic health record data alone. This model could inform clinical decision making and improve the uptake of appropriate end-of-life care. Further research is warranted to expand on these findings in a larger, more diverse sample.

(109.2) Trajectories of symptom and quality-of-life (QOL) recovery after autologous stem cell transplantation (ASCT) in multiple myeloma (MM): secondary analysis of BMT CTN 0702 trial

Anita D'Souza, MD, MS, Medical College of Wisconsin, Milwaukee, Wisconsin, USA; Ruta Brazauskas, Medical College of Wisconsin, Milwaukee, Wisconsin, USA; Edward Stadtmauer, Unniversity of Pennsylvania, Philadelphia, Pennsylvania, USA; Marcelo Pasquini, Medical College of Wisconsin, Milwaukee, Wisconsin, USA; Parameswaran Hari, Medical College of Wisconsin, Milwaukee, 
Wisconsin, USA; Asad Bashey, Northside Hospital, Atlanta, Georgia, USA; Steven Devine, National Marrow Donor Program, Minneapolis, Minnesota, USA; Yvonne Efeberra, Ohio State University, Columbus, Ohio, USA; Siddharta Ganguly, Kansas University Medical Center, Kansas City, Missouri, USA; Cristina Gasparetto, Duke University, Raleigh, North Carolina, USA; Nancy Geller, National Heart Lung and Blood Institute, Bethesda, Maryland, USA; Mary Horowitz, Medical College of Wisconsin, Milwaukee, Wisconsin, USA; John Koreth, Dana Farber Cancer Institute, Boston, Massachusetts, USA; Heather Landau, Memorial Sloan Kettering Cancer Center, New York, New York, USA; Claudio Brunstein, University of Minnesota, Minneapolis, Minnesota, USA; Philip McCarthy, Roswell Park Cancer Center, Rochester, New York, USA; Muzaffar Qazilbash, MD Anderson Cancer Center, Houston, Texas, USA; Sergio Giralt, Memorial Sloan Kettering Cancer Center, New York, New York, USA; Amrita Krishnan, City of Hope Comprehensive Cancer Center, Duarte, California, USA; Kathryn Flynn, Medical College of Wisconsin, Milwaukee, Wisconsin, USA

Aims: Multiple myeloma (MM) is a chronic incurable malignancy of older adults. The use of early autologous stem cell transplantation (ASCT) and continuous post-transplant maintenance therapy is standard but can increase toxicity burden. We sought to describe symptom burden and trajectories in MM patients after ASCT. Methods: The BMT CTN 0702 trial compared outcomes of 3 treatment interventions after a single ASCT in $758 \mathrm{MM}$ patients. The study showed no difference in clinical outcomes including progression-free or overall survival and FACT-BMT scores between the 3 arms [J Clin Oncol. 2019;37(7):589-597]. In this ancillary study, we analyzed the FACTBMT at enrollment and annually for 4 years post-ASCT. In addition to standard FACT-BMT scores, individual symptoms from the FACTBMT survey were considered; scores $0-2$ were categorized as 'good' and scores 3-4 'poor'. Among 17 individual symptoms, patients with no 'poor' symptom ratings were considered to have 'good' health at 1 year. Results: Mean age at enrollment was 55.5 years (SD 8.4) with $17.2 \%$ African Americans. Median follow-up was 6 years (range 0.4-8.5). Baseline FACT-BMT score was associated with race, functional status, and comorbidities (Table 1). All scores, except Social/Family Well-Being showed improvement between enrollment and 1-year remaining stable thereafter (Table 2). At baseline, $27 \%$ patients had good and $73 \%$ patients had poor scores, at 1 year $38 \%$ had good and $62 \%$ poOR, and at 3 years $32 \%$ had good and $68 \%$ poor scores. Predictors of being in good health at 1 year included having good health at baseline: OR $2.71(1.8-4.1), p<0.0001$, older age: OR 2.1 (1.3-3.22), $p=0.0007$, household income $\geq \$ 55,000$ : OR 1.7 (1.2-2.5), $p=0.02$, and was inversely related to 'not employed' status: OR $0.47(0.31-0.72), p=0.0016)$. Conclusion: Overall QOL scores recover to population norms by 1 -year post-ASCT and remain stable thereafter, though many patients continue to report poor health at 1-year post-ASCT and beyond. For clinical utility, tracking specific symptoms can complement FACT-BMT domain scores. These data provide preliminary work to develop a $\mathrm{PRO}$-based intervention using symptom burden in MM patients after ASCT.

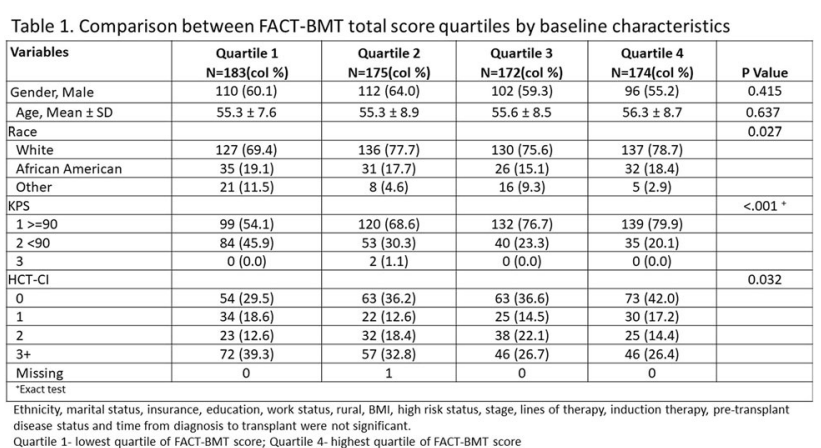

\begin{tabular}{|c|c|c|c|c|c|}
\hline & Baseline ( $N=712)$ & 1 year $(\mathrm{N}=549)$ & 2 year $(\mathrm{N}=465)$ & 3 year $(\mathrm{N}=371)$ & 4 year $(\mathrm{N}=268)$ \\
\hline $\begin{array}{l}\begin{array}{l}\text { Physical Well-being } \\
\text { (norm=21.6) }\end{array} \\
\end{array}$ & $19.3 \pm 5.9$ & $21.6 \pm 5.3$ & $22.0 \pm 4.9$ & $21.6 \pm 5.3$ & $22.0 \pm 4.8$ \\
\hline $\begin{array}{l}\begin{array}{l}\text { Social/family well-being } \\
\text { (norm=24) }\end{array} \\
\end{array}$ & $23.7 \pm 4.3$ & $22.8 \pm 4.8$ & $22.5 \pm 5.3$ & $22.6 \pm 5.1$ & $22.9 \pm 5.5$ \\
\hline \begin{tabular}{|l|}
$\begin{array}{l}\text { Emotional well-being } \\
\text { (norm=18.24) }\end{array}$ \\
\end{tabular} & $18.6 \pm 3.9$ & $19.4 \pm 3.9$ & $19.6 \pm 3.7$ & $19.4 \pm 3.9$ & $19.8 \pm 3.8$ \\
\hline \begin{tabular}{|l|}
$\begin{array}{l}\text { Functional well-being } \\
\text { (norm=17.7) }\end{array}$ \\
\end{tabular} & $16.4 \pm 6.3$ & $19.4 \pm 6.2$ & $19.9 \pm 6.0$ & $19.9 \pm 6.1$ & $20.4 \pm 6.0$ \\
\hline \begin{tabular}{|l|} 
FACT-G total \\
(norm $=81.54$ ) \\
\end{tabular} & $78.1 \pm 15.5$ & $83.2 \pm 16.4$ & $84.0 \pm 16.0$ & $83.6 \pm 16.6$ & $85.2 \pm 16.5$ \\
\hline $\begin{array}{l}\text { FACT-BMT concerns } \\
\text { (norm=27.9) }\end{array}$ & $27.8 \pm 5.6$ & $29.7 \pm 5.6$ & $30.5 \pm 5.3$ & $30.3 \pm 5.3$ & $30.7 \pm 5.6$ \\
\hline \begin{tabular}{|l} 
FACT-BMT total \\
(norm=109.44)
\end{tabular} & $106.0 \pm 20.0$ & $112.9 \pm 21.2$ & $114.5 \pm 20.3$ & $114.0 \pm 21.0$ & $115.9 \pm 21.2$ \\
\hline $\begin{array}{l}\text { FACT-BMT TOI } \\
\text { (norm=67.2) }\end{array}$ & $63.5 \pm 15.5$ & $70.7 \pm 15.4$ & $72.4 \pm 14.4$ & $71.9 \pm 14.9$ & $73.2 \pm 14.9$ \\
\hline
\end{tabular}

(109.3) Application of the Patient-Reported Outcomes Version of the Common Terminology Criteria for Adverse Events (PROCTCAE) in adult survivors of childhood cancer: a content elicitation study

Tegan Reeves, PhD, St. Jude Children's Research Hospital, Memphis, Tennessee, USA; Taylor Mathis, MPH, St. Jude Children's Research Hospital, Memphis, Tennessee, USA; Hailey Bauer, BS, St. Jude Children's Research Hospital, Memphis, Tennessee, USA; Kumar Strivasata, PhD, St. Jude Children's Research Hospital, Memphis, Tennessee, USA; Melissa Hudson, MD, St. Jude Children's Research Hospital, Memphis, Tennessee, USA; Leslie Robison, PhD, St. Jude Children's Research Hospital, Memphis, Tennessee, USA; Justin Baker, MD, St. Jude Children's Research Hospital, Memphis, Tennessee, USA; I-Chan Huang, PhD, St. Jude Children's Research Hospital, Memphis, Tennessee, USA

Aims: The content validity of the current PRO-CTCAE for long-term survivors of childhood cancer who often have high symptom burden related to late medical effects is unknown. This study compared symptom experiences of adult survivors of childhood cancer through in-depth qualitative interviews to the symptom items included in the PRO-CTCAE. Methods: 120 adult survivors of childhood cancer enrolled in the St. Jude Lifetime Cohort Study who endorsed mild to moderate symptom burden in at least three organ-based domains completed semi-structured interviews in 2020-2021. Interviews were transcribed verbatim and analyzed in three steps of grounded coding. These steps included (1) sensitizing structure with a priori fields to organize transcript sections into organ-specific domains, (2) iterative inductive coding using lumping and splitting to catalog persistent meaning-units relevant to symptom experiences, and (3) performing abductive and axial coding using constant comparative matrix-mapping to index unique symptom issues derived by descriptions. The comparative matrix included symptom items from the BioPortal and ICD-10 codes to identify missing contents from the current PRO- 
CTCAE. Results: Survivors reported a total of 219 concepts that captured symptom issues over six organ-based domains (19 in cardiovascular, 47 in respiratory, 48 in gastrointestinal, 39 in head/neck, 28 in neurological, and 38 in musculoskeletal). Among these concepts, 27 were not precisely represented by the PRO-CTCAE but represented by the BioPortal and/or ICD-10, including 1 in cardiovascular (e.g., chest pain/pressure), 5 in respiratory (e.g., dyspnea), 8 in gastrointestinal (e.g., thirst), 1 in head/neck (e.g., facial swelling), 8 in neurological (e.g., tremor), and 4 in musculoskeletal (e.g., muscle weakness) domains. In addition, 12 symptom concepts were not precisely represented by the PRO-CTCAE, BioPortal and/or ICD-10, including 3 in cardiovascular (e.g., swelling in extremities), 4 in respiratory (e.g., rapid respiration), 1 in gastrointestinal (e.g., belching), 6 in head/neck (e.g. dry eyes), 4 in neurological (e.g., neuropathic pain), and 2 in musculoskeletal (e.g., joint instability) domains. Conclusion: Several meaningful symptom concepts elicited from childhood cancer survivors were unavailable in the current PROCTCAE. Our findings facilitate the creation of new items for the PRO-CTCAE toward cognitive debriefing and psychometric/clinical validation in childhood cancer survivors.

\section{(109.4) Cost effectiveness of eRAPID eHealth intervention for symptom management during chemotherapy}

Bryony Dawkins, University of Leeds, Leeds, UK; Claire Hulme, University of Exeter, Exeter, UK; Kate Absolom, University of Leeds, Leeds, UK; Lorraine Warrington, University of Leeds, Leeds, UK; Jenny Hewison, University of Leeds, Leeds, UK; Eleanor Hudson, University of Leeds, Leeds, UK; Andrea Gibson, University of Leeds, Leeds, UK; Marie Holmes, University of Leeds, Leeds, UK; Zoe Rogers, University of Leeds, Leeds, UK; Robert Carter, University of Leeds, Leeds, UK; Julia Brown, University of Leeds, Leeds, UK; Galina Velikova, University of Leeds, Leeds, UK

Aims: A randomized controlled trial (RCT) in UK of eRAPID (electronic patient self-Reporting of Adverse-events: Patient Information and aDvice) online system showed better physical well-being during chemotherapy in patients with colorectal, breast or gynaecological cancers, when compared to usual care (UC). This study evaluated the cost effectiveness of eRAPID being added to UC. Methods: An embedded economic evaluation was conducted alongside the RCT. Costs and quality-adjusted life years (QALYs) were compared over the 18 week trial. Incremental cost-effectiveness ratios (ICERs) were estimated and compared to the National Institute for Health and Care Excellence cost-effectiveness threshold. Uncertainty was explored using non-parametric bootstrapping and sensitivity analyses. Primary analysis was conducted from healthcare provider perspective, with additional analyses conducted from societal perspective to include patient out of pocket care costs. Exploratory analysis over 12 months was conducted for a subset of participants with longer follow-up. Results: Over the 18 week trial of 508 patients, patients with eRAPID had the highest QALY gain and the lowest costs. These differences were small and not statistically significant. eRAPID had a 55-58\% probability of being more cost effective than UC. Patient costs were lower in the eRAPID group, indicating that eRAPID may help patients access support needed within NHS services. Exploratory subgroup analysis by disease status indicated eRAPID may be more cost effective in patients with early cancers (treated with curative intent) than metastatic cancers. Sensitivity analyses did not change the interpretation of the results. Exploratory 12 month analysis showed higher QALY gains with eRAPID but also higher costs. Conclusion: Despite small differences in QALYs and costs, the analyses were indicative of the potential cost effectiveness of eRAPID for this population. Patient and healthcare provider acceptability are also likely to be important in decisions about wider implementation. Trial registration: ISRCTN88520246 Funding source: Supported by the National Institute for Health Research (NIHR), Research Program Grant for Applied Research (RP-PG-0611-20008). The views expressed are those of the author(s) and not necessarily those of the NIHR or the Department of Health and Social Care.

\begin{tabular}{|c|c|c|c|c|c|c|}
\hline $\begin{array}{l}\text { Analysis } \\
\text { (time frame: } \\
\text { perspective) }\end{array}$ & \begin{tabular}{|l}
$\begin{array}{l}\text { Treatment } \\
\text { group }\end{array}$ \\
\end{tabular} & \begin{tabular}{|l|} 
Cost: \\
Mean (SE) (1)
\end{tabular} & \begin{tabular}{|l} 
Incremental \\
cost (2)
\end{tabular} & \begin{tabular}{|l|} 
QALY: \\
Mean (SE) (1)
\end{tabular} & \begin{tabular}{|l} 
Incremental \\
QALY (2)
\end{tabular} & $\begin{array}{l}\text { ICER } \\
\text { (E/QALY) }\end{array}$ \\
\hline \multirow{2}{*}{$\begin{array}{l}\text { 18-weeks: } \\
\text { healthcare } \\
\text { provider }\end{array}$} & UC & \begin{tabular}{|l}
$£ 8330.36(435.23)$ \\
\end{tabular} & & $0.255(0.004)$ & & \\
\hline & eRapid & f8305.08 (450.5) & $\cdot f 25.28$ & $0.259(0.004)$ & 0.003 & \begin{tabular}{|l|} 
eRAPID \\
Dominates
\end{tabular} \\
\hline \multirow{2}{*}{$\begin{array}{l}\text { 18-weeks: } \\
\text { Societal }\end{array}$} & uc & \begin{tabular}{|l|l} 
f9811.67 (453.53) \\
\end{tabular} & & $0.255(0.004)$ & & \\
\hline & eRapid & f9662.24 (463.3) & $-f 149.42$ & $0.259(0.004)$ & 0.003 & \begin{tabular}{|l|} 
eRAPID \\
Dominates
\end{tabular} \\
\hline \multirow{2}{*}{$\begin{array}{l}\text { 12-month: } \\
\text { healthcare } \\
\text { provider }\end{array}$} & UC & £10023.78 (641.66) & & $0.663(0.021)$ & & \\
\hline & eRapid & $f 10635.43$ (699.15) & £611.65 & $0.673(0.022)$ & 0.009 & $664,455.74$ \\
\hline \multirow{2}{*}{$\begin{array}{l}\text { 12-month: } \\
\text { Societal }\end{array}$} & UC & \begin{tabular}{|l|}
$11467.41(669.27)$ \\
\end{tabular} & & $0.663(0.021)$ & & \\
\hline & eRapid & f11843.72 (707.83) & E376.31 & $0.673(0.022)$ & 0.009 & $£ 39,662.81$ \\
\hline
\end{tabular}

(109.5) Higher EQ-5D-5L utility scores at diagnosis are associated with improved overall survival in Australian patients with multiple myeloma: results from the Australian and New Zealand Myeloma and Related Diseases Registry

Elizabeth Moore, PhD, Monash University, Melbourne, Australia; Cameron Wellard, PhD, Monash University, Melbourne, Australia; Adam Irving, $\mathrm{PhD}$, Monash University, Melbourne, Australia; Erica Wood, MBBS, Monash University, Melbourne, Australia; Zoe McQuilten, PhD, Monash University, Melbourne, Australia; Andrew Spencer, MD, Alfred Hospital - Monash University, Melbourne, Australia; submitted on behalf of "the MRDR investigators"

Aims: Multiple myeloma (MM) is an incurable blood cancer with a high disease burden. Early assessment of health-related quality of life (HRQOL) can help to guide therapy planning. EQ-5D-5L (EQ5D) scores are collected in the Australian and New Zealand Myeloma and Related Diseases Registry (MRDR) at diagnosis and follow-up as a non-mandatory item. We describe the use of EQ5D utility scores to assess patients' HRQOL at diagnosis. Methods: Utility scores are a measure of HRQOL, from ' 0 ' (equal to death) to ' 1 ' (full health). Scores were calculated for Australian patients with EQ5D data at diagnosis on the MRDR (February 2013-March 2021) using a scoring algorithm developed for Australia (Norman R, et al. Appl Health Econ Health Policy. 2013). Kaplan-Meier methods were used for survival analysis. Results: Of 2239 Australian patients with MM on the MRDR, 483 had baseline EQ5D within $6 \mathrm{~m}$ of diagnosis with data for all 5 dimensions. Median age was 68y (59-76), 63\% were male, and median overall survival was $63.0 \mathrm{~m}$ (54.1-NR). Figure 1 summarises EQ5D results at baseline. Patient characteristics were compared between EQ5D utility score groups (Q1: $<0.25[n=71]$, Q2: $0.25-0.49$ [ $n=87]$, Q3: $0.50-0.74[n=153]$, Q4: $0.75-1.0$ $[n=172])$ with no difference in median age, gender or disease stage (ISS) between the 4 groups ( $p \geq 0.19$ ). As expected, with increasing utility score (better health), median EQ VAS score (patient-identified health status, 0 to $100,100=$ best health, $0=$ worst $)$ increased, and proportion of patients with ECOG performance status 2-4 (unable to work) decreased $(p<0.001)$. The presence of cardiac disease decreased with higher utility score $(p=0.048)$. Notably, higher utility scores at diagnosis were found to be associated with improved overall survival (OS) (Fig. 2, $p=0.001)$. Conclusion: EQ5D utility scores in Australian MRDR patients with MM were independent of age, gender, and disease stage (ISS $=3$ ), but high scores were associated with higher EQ VAS score, improved ECOG, less cardiac disease, and better overall survival. HRQOL of MM patients at diagnosis has 
prognostic potential with better EQ5D utility scores associated with longer overall survival.
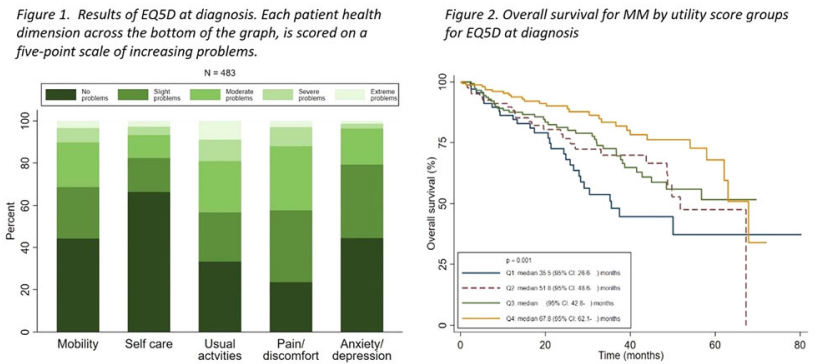

(109.6) Patient-reported outcome-based symptom recovery from surgery for patients with esophageal squamous cell carcinoma

Xi Luo, Sichuan Cancer Hospital, Chengdu, China; Qin Xie, Department of Thoracic Surgery, Sichuan Cancer Hospital and Research Institution, School of Medicine, University of Electronic Science and Technology of China, Chengdu, China; Qiulin Shi, Department of Thoracic Surgery, Sichuan Cancer Hospital and Research Institution, School of Medicine, University of Electronic Science and Technology of China, Chengdu, China; Yan Miao, Department of Thoracic Surgery, Sichuan Cancer Hospital and Institution, Sichuan Cancer Center, School of Medicine, University of Electronic Science and Technology of China, Chengdu, China; Hong Zhou, Department of Thoracic Surgery, Sichuan Cancer Hospital and Institution, Sichuan Cancer Center, School of Medicine, University of Electronic Science and Technology of China, Chengdu, China

Aims: Esophageal squamous cell carcinoma (ESCC) patients have severe symptom burden after esophagectomy; however, longitudinal studies of symptom recovery after surgery are scarce. This study used longitudinal patient-reported outcome (PRO)-based symptoms to identify severe symptoms and profile symptom recovery from esophagectomy. Methods: Data on patient symptoms were extracted from the Sichuan Cancer Hospital's Case Management Registration Database. Assessment time points were pre-surgery and 1, 3, 5, 7, 14, 21, 30, and 90 days post-surgery using the MD Anderson Symptom Inventory. Core symptoms were the top five percent with scores $\geq 4$, which were profiled using mixed-effect models and Kaplan-Meier analysis. Results: The core symptoms after esophagectomy were pain, fatigue, dry mouth, disturbed sleep, and distress. The symptoms that remained more severe at 90 days after surgery than at pre-operation were pain (mean: 1.05 [SD 1.24] vs 0.44 [SD 0.78], $p<0.0001$ ) and fatigue (mean: 1.77 [SD 1.47] vs 0.65 [SD 1.05], $p<0.0001$ ). The recovery probability of pain was lower within 7 days post-surgery. Disturbed sleep and distress persisted from pre-surgery to 90 days after surgery; average sleep recovery time was up to 20 days. Female patients were more likely to report more severe symptoms in pain, fatigue, and disturbed sleep (all $p<0.05$ ). Patients underwent IvorLewis or open Mckeown experienced more severe symptoms pain, fatigue, disturbed sleep, and distress (all $p<0.05$ ). Conclusion: Early postoperative pain management after esophagectomy should be considered. Intervention strategies focused on the characteristics of postoperative fatigue, distress, and disturbed sleep in esophageal cancer patients warranty further studies.
110: Clinical Research Applications in General Populations

(110.1) Acceptability of patient-reported outcome measures in women following pelvic floor procedures

Rasa Ruseckaite, PhD, Monash University, Melbourne, Australia; Claire BavOR Monash University, Melbourne, Australia; Lucy Marsh, Monash University, Melbourne, Australia; Joanne Dean, Monash University, Melbourne, Australia; Oliver Daly, Monash University, Melbourne, Australia; Dora Vasiliadis, Australian Pelvic Floor Procedure Registry, Melbourne, Australia; Susannah Ahern, Monash University, Melbourne, Australia

Aims: Pelvic floor disorders, such as stress urinary incontinence (SUI) and pelvic organ prolapse (POP), are common, with prevalence increasing with parity and age. SUI is associated with a significantly poorer health-related quality of life (HRQoL) for which women frequently undergo anti-incontinence procedures including mesh-based mid-urethral slings than their counterparts without SUI. Patient-Reported Outcome Measures (PROMs) are valuable tools in evaluating the outcomes of surgical treatment HRQoL of women with SUI and may be incorporated into related and may be incorporated into related into clinical quality registries. The aim of this study was to assess the feasibility and acceptability of incorporating PROMs into the Australian Pelvic Floor Procedure Registry (APFPR) for women and surgeons. Methods: Semi-structured qualitative interviews were conducted with women with SUI $(N=12)$ and their managing clinicians $(N=11)$ in Victoria, Australia. Interview topics covered content and face validity, appropriateness, and acceptability of three incontinence-specific, two pain, one anxiety and depression, one sexual function and one patient global impression of improvement instruments identified through the literature to determine their suitability and acceptability for the APFPR. We analysed interview data into topics using conventional content analysis. Results: Study participants agreed that PROMs were needed for the APFPR. Both participant groups suggested that some of the instruments were ambiguous; therefore, based on the results of this study, only three of eight instruments (one incontinence-specific, sexual function and patient global impression of improvement) will be included in the APFPR. Both clinicians and women agreed it would be appropriate to answer PROMs at baseline and then at 6 and 12 months post-operatively. Email, phone call and mail out of the instruments were the preferred options for administration. Conclusion: Most women and clinicians supported the feasibility of incorporating PROMs in the APFPR. Participants believed the PROMs would demonstrate useful aggregate HRQoL data, facilitate research and have potential for use in individual care.

(110.2) "I just literally want, like, one day where it's like, I'm not having to worry about anything": The impact of hypoglycaemia on quality of life in people living with diabetes (a Hypo-RESOLVE study)

Philip Powell, PhD, University of Sheffield, Sheffield, UK; Jill Carlton, PhD, University of Sheffield, Sheffield, UK; submitted on behalf of "Hypo-RESOLVE Patient Advisory Group and HypoRESOLVE Scientific Group"

Aims: Hypoglycaemia-Redefining SOLutions for better liVEs (HypoRESOLVE) is a Pan-European project funded by the Innovative 
Medicines Initiative, designed to better understand the predictors, mechanisms, and consequences of hypoglycaemia in people living with diabetes (PwD). One aim of Hypo-RESOLVE is to assess the impact of hypoglycaemia on quality of life (QoL), in order to inform the development of a new patient-reported outcome measure (PROM). Methods: Semi-structured qualitative interviews were conducted with $32 \mathrm{PwD}$, recruited from the NHS, and purposively sampled by type (22 type 1,10 type 2 ), duration ( $0-4$ to $20+$ years), age (18-30 to $66+$ years), and gender ( 15 men, 17 women). The interviews were recorded and transcribed verbatim. The topic guide was based on a framework developed from a systematic review into the measurement of QoL in hypoglycaemia. Transcripts were analysed independently by two researchers against this framework $(50 \%$ dual coded) using Framework Analysis and further adaptations were made inductively. The transcripts were analysed iteratively alongside data collection in batches of four, followed by meetings to discuss the coding. Patient and scientific advisory groups provided feedback on the analysis following the first four transcripts and at the end of the process. Saturation was monitoring using an a priori stopping rule. Results: Data saturation and sufficient breadth in the sampling framework were reached by 32 interviews. The final QoL framework featured 17 themes organised within 3 categories: Physical functioning, psychological function, and social functioning (see Fig. 1). Differential impacts on QoL were revealed during and just after hypoglycaemia, as well as longer-term impacts. Insights were observed into the heterogeneous effects on QoL of hypos that occurred in the day versus the night, as a result of single versus recurrent hypos, and as a consequence of particular triggering events. Conclusion: Hypoglycaemia affects multiple aspects of people living with diabetes' QoL, including physical, psychological, and social functioning. Existing PROMs may be insufficient to fully capture the framework identified in this study. This framework will be used to generate items for a new PROM as part of the Hypo-RESOLVE project.

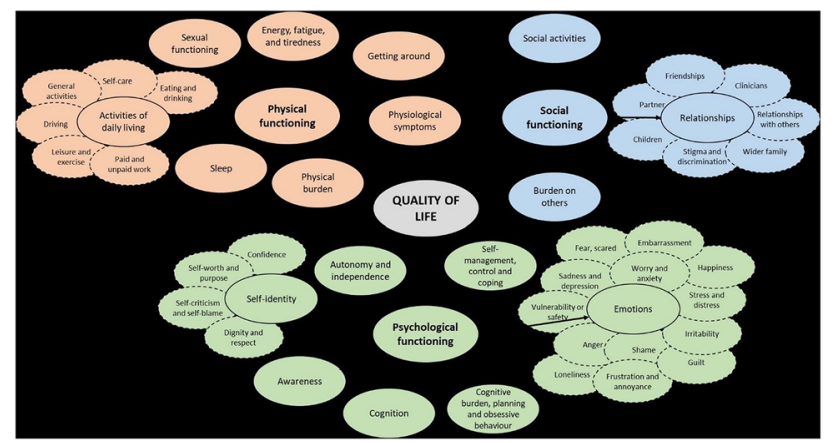

(110.3) Modeling longitudinal trajectories of treatment burden in people with multiple chronic conditions

David Eton, $\mathrm{PhD}$, Mayo Clinic, Rochester, Minnesota, USA; Roger Anderson, PhD, University of Virginia School of Medicine, Charlottesville, Virginia, USA; Jennifer St. Sauver, PhD, Mayo Clinic, Rochester, Minnesota, USA; Elizabeth Rogers, MD, University of Minnesota Medical School, Minneapolis, Minnesota, USA; Minji Lee, PhD, Mayo Clinic, Rochester, Minnesota, USA

Aims: Many people with multiple chronic conditions (MCC) must seamlessly integrate complex treatment and self-care into their lives. The "workload" involved with self-managing chronic conditions (e.g., taking medications, monitoring health, medical appointments) and its "impact" on quality of life (e.g., participating in roles and activities) are key elements of the burden of treatment (BoT). New patient-reported measures are facilitating the study of BoT, yet no study has addressed how BoT fluctuates over an extended period. We conducted a prospective observational study to uncover whether distinct longitudinal patterns of BoT exist in people with MCC, and if so, explore what factors predict them. Methods: Surveys were completed by 439 community-dwelling adults with MCC at baseline and 6,12 , and 24 months later (90\% follow-up response). They included a valid measure of BoT, the Patient Experience with Treatment and Self-management (PETS), and standardized metrics of demographic, psycho-social, and health-related factors. Latent Class Growth Mixture Modeling (LCGMM) was used to determine BoT trajectories for PETS Workload and Impact scores. Multiple binomial logistic regressions were used to identify independent predictors of trajectory class. Results: Three trajectories of PETS Workload were supported by LCGMM: low $(n=294)$, high $(n=70)$, and declining $(n=16)$ burden (Fig. 1). Also, three trajectories of PETS Impact were supported by LCGMM: low $(n=278)$, high $(n=62)$, and increasing $(n=51)$ burden (Fig. 2). Psycho-social and health-related factors were associated with trajectory classification for both PETS Workload and Impact scores. Participants with lower health literacy, lower selfefficacy, and more problems in their relationships with others were more likely to be classified into a trajectory pattern reflecting poorer BoT $($ Ps $<0.05)$. Self-reports of poorer physical health were associated with a pattern of increasing PETS Impact score $(p<0.001)$. Conclusion: There are differences in the way BoT is experienced over time by people with MCC. Psycho-social and health-related factors that predict patterns of BoT hint at ways to intervene. Higher burdened individuals might benefit from efforts to improve health literacy or formal strategies that empower them to successfully selfmanage. Improving the quality of social relationships may hold promise as a means to further mitigate BoT.

Figure 1. Three-trajectory model of PETS Workload ( $\mathrm{N}=380$ )

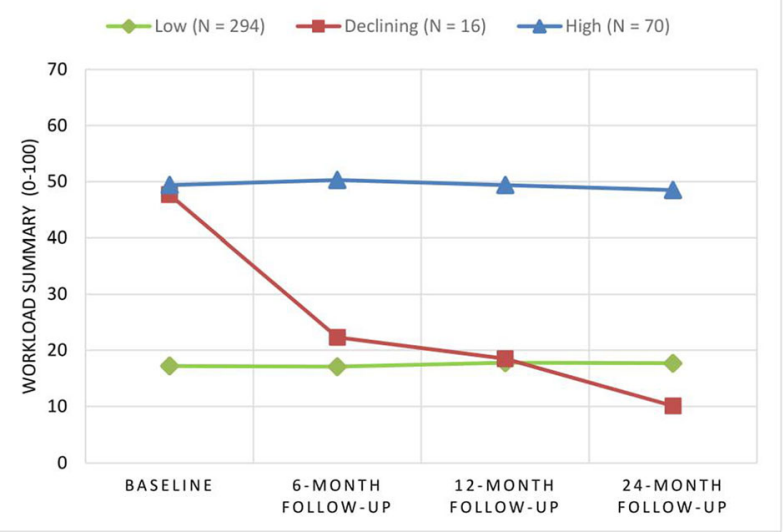


Figure 2. Three-trajectory model of PETS Impact ( $\mathrm{N}=391)$

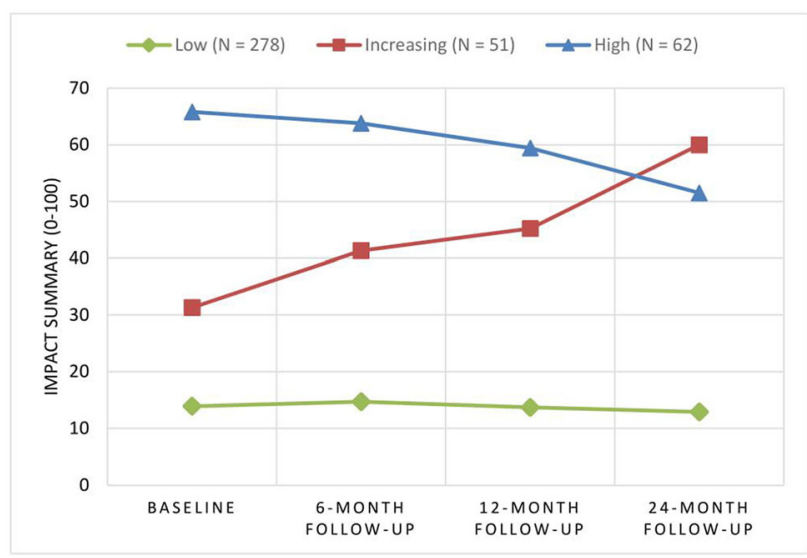

(110.4) The global patient experience of transthyretin amyloidosis (ATTR): symptoms and quality-of-life impacts of ATTR among patients in 11 countries

Lynne Broderick, MPH, QualityMetric, Johnston, Rhode Island, USA; Meaghan O'ConnOR MTS, MPH, QualityMetric, Johnston, Rhode Island, USA; Duncan Brown, PhD, Ionis Pharmaceuticals, Carlsbad, California, USA; Montserrat Vera Llonch, MD, MPH, MSc, Ionis Pharmaceuticals, Carlsbad, California, USA; Kristen Hsu, BS, Amyloidosis Research Consortium, Newton, Massachusetts, USA; Kristen McCausland, PhD, MPH, QualityMetric, Johnston, Rhode Island, USA; Michelle White, $\mathrm{PhD}$, MS, QualityMetric, Johnston, Rhode Island, USA

Aims: Transthyretin amyloidosis (ATTR) is a rare, debilitating condition caused by misfolded protein deposits in different organ systems. There are 2 types of ATTR: hereditary (ATTRv [ $\mathrm{v}$ for variant]) and wild type (ATTRwt). ATTRv genotypes are associated with specific clinical presentations and may be more common in different regions of the world. The objective of this research is to summarize ATTRv and ATTRwt symptoms and impacts described by patients from 11 countries to determine if the patient experience differs geographically and to inform the development of an ATTRspecific patient-reported outcome (PRO) measure. Methods: The research team conducted concept elicitation interviews with 63 patients in 11 countries (Brazil, Canada, France, Germany, Italy, Japan, Portugal, Spain, Sweden, the US, and the UK). Prior to each interview, patients completed and returned a symptoms checklist. During the interviews, patients were asked about the symptoms they experience and impacts of ATTR on their lives. Checklist responses were analyzed by country to identify patterns in geographic differences in symptomology. Interview transcripts were analyzed using thematic analysis to identify the concepts relevant to the patient experience of ATTR and the domains of life impacted by ATTR. Results: The types of symptoms and impacts reported did not differ by country. The average number of ATTR symptoms ranged from 10 to 21 per patient (overall mean $=16$ ). The most frequently reported symptoms included those associated with cardiomyopathy, peripheral neuropathy, and autonomic neuropathy. In all countries, patients reported impacts on physical functioning, social functioning, emotional and mental health, financial well-being, work and productivity, recreational pastimes, and ability to plan for both the short and long term. Conclusion: The results suggest that patient-reported symptoms and impacts of ATTR are similar regardless of where a patient is located. Thus, international clinicians and researchers could rely on a standardized, ATTR-specific PRO to better understand the symptoms and impacts of ATTR and the effectiveness of treatments. Routine use of this disease-specific PRO in clinical practice and research can lead to a more complete understanding of the patient experience, identification of unmet needs, improved patient care, and evidence of treatment effects on patient symptoms and quality of life.

(110.5) Association between knee symptoms, change in knee symptoms over 6-9 years and SF-6D health state utility among middle-aged Australians

Ambrish Singh, Menzies Institute for Medical Research, University of Tasmania, Hobart, Australia; Julie A Campbell, Menzies Institute for Medical Research, University of Tasmania, Hobart, Australia, Hobart, Australia; Alison Venn, Menzies Institute for Medical Research, University of Tasmania, Hobart, Australia, Hobart, Australia; Graeme Jones, Menzies Institute for Medical Research, University of Tasmania, Hobart, Australia, Hobart, Australia; Leigh Blizzard, Menzies Institute for Medical Research, University of Tasmania, Hobart, Australia, Hobart, Australia; Andrew J Palmer, Menzies Institute for Medical Research, University of Tasmania, Hobart, Australia, Hobart, Australia; Terence Dwyer, Menzies Institute for Medical Research, University of Tasmania, Hobart, Australia, Hobart, Australia; Flavia Cicuttini, Department of Epidemiology and Preventive Medicine, Monash University, Melbourne, Australia, Melbourne, Australia; Changhai Ding, Menzies Institute for Medical Research, University of Tasmania, Hobart, Australia, Hobart, Australia; Benny Antony, Menzies Institute for Medical Research, University of Tasmania, Hobart, Australia, Hobart, Australia

Aims: Health state utilities (HSUs) are an input metric for estimating quality-adjusted life years (QALY) in cost-utility analyses. Currently, there is a paucity of data on association of knee-symptoms with HSUs for middle-aged populations. We aimed to describe the association of knee-symptoms and change in knee-symptoms with SF-6D HSUs and described the distribution of HSUs against knee-symptoms' severity. Methods: Participants (36-49-years) were selected from the third follow-up (completed 2019) of Australian Childhood Determinants of Adult Health (CDAH) study. SF-6D HSUs were generated from the participant-reported SF-12. Association between participant-reported WOMAC knee-symptoms' severity, change in knee symptoms over 6-9 years, and HSUs were evaluated using linear regression models. Results: $N=1567$ participants were included in the analysis; mean age 43.5 years, female 54\%, BMI 27.18 (SD: 5.31) $\mathrm{kg} / \mathrm{m}^{2}$. Mean HSUs for normal, moderate, and severe WOMAC scores were 0.82 (0.12), $0.80(0.12)$, and $0.74(0.13)$, respectively. Significant association was observed between worsening knee-symptoms and HSUs in univariable and multivariable analyses after adjustment (age and sex). HSU decrement for normal to severe total-WOMAC and WOMACpain was $-0.080(95 \% \mathrm{CI}-0.10041$ to $-0.05972, \mathrm{p}<0.01)$ and -0.067 ( -0.08497 to $-0.04853, p<0.01)$, exceeding the mean minimal clinically important difference $(0.04)$. Increase in knee pain over 6-9 years was associated with a significant reduction in HSU. Conclusion: In a middle-aged population-based sample, there was an independent negative association between worse knee-symptoms and SF-6D HSUs. Our findings may be used by decision makers to define more realistic and conservative baseline and ongoing HSU values when assessing QALY changes associated with osteoarthritis interventions.

(110.7) Illness perception, health behaviours and health-related quality of life of patients with knee osteoarthritis

Olufunmilola Adaraniwon, MSc, Federal Medical Centre Ebute Metta, Lagos, Nigeria; Adesola Odole, PhD, Department of 
Physiotherapy, College of Medicine, University of Ibadan, Ibadan, Nigeria; Serifu Lamidi, MSc, Department of Epidemiology and Medical Statistics, Faculty of Public Health, College of Medicine University of Ibadan, Ibadan, Nigeria; Oluwagbohunmi Awosoga, $\mathrm{PhD}$, Faculty of health sciences, University of Lethbridge, Lethbridge, Alberta, Canada

Aims: Illness perceptions are mental representations that patients have regarding their illnesses while health behaviours are habits that may affect an individual's physical health. These are important predictors of how patients will behave during their illness experience and are directly associated with several health outcomes. This study was aimed at investigating the associations among illness perception, health behaviours (smoking, alcohol consumption, dietary pattern and physical activity level) and health-related quality of life (HRQoL) in patients with knee osteoarthritis (OA) in Ibadan. Methods: This study was a cross-sectional survey, and purposive sampling method was used to select participants literate in English and Yoruba Language. Dietary pattern and smoking were assessed using the Nigerian Composite Lifestyle CVD Risk Factors Questionnaire while alcohol intake was assessed using the Alcohol Use Identification Test. Physical activity was assessed using the International Physical Activity Questionnaire Short form. Illness perception and health-related quality of life were assessed using Brief Illness Perception Questionnaire and Short-Form 12-Item Health Survey, respectively. Descriptive statistics of mean and standard deviation were used to summarize the data. Chi-square test of association and logistic regression were used for inferential analysis. Results: Majority of the participants $114(91.2 \%)$ were females, $102(81.6 \%)$ were retired $89(71.2 \%)$ married and $86(68.8 \%)$ within the age group $60-79$. Prevalence of alcohol use was $6.4 \%$ while $89(71.2 \%)$ had low physical activity. Participants with high and moderate vegetable consumption were 5 and 6 times more likely to have good mental HRQoL, respectively (OR 5.05, CI 1.29-19.84) (OR 5.91, CI $1.20-29.17)$ compared to those with low vegetable consumption. Also, participants with good illness perception were about 12 and 3 times more likely to have good physical and mental HRQoL, respectively (OR 11.95, CI 4.76-29.98) (OR 3.02, CI 1.42-6.44) compared to those with poor illness perception. Conclusion: Regular consumption of vegetables and good illness perception contribute positively to the HRQoL of patients with knee OA. It is recommended that patient's illness perception should be incorporated in the management of knee OA. Healthy behaviours should also be emphasized and promoted through adequate awareness to improve their HRQoL.

\section{1: Methods for Different Health Conditions}

(111.1) Reflections on the methodological development and utility of the Kidscreen-52 Quality of Life Questionnaire: a crossdisciplinary approach

Filip Kachnic, $\mathrm{PhD}$ Candidate, Institute for Research and Development of Education, Faculty of Education, Charles University, Prague, Czech Republic

Aims: The purpose of this study is to review the methodology for item generation in the KIDSCREEN-52 quality-of-life questionnaire which is currently used in 17 countries (Ravens-Sieberer 2014, 2008; Robitail et al. 2006) and to explore if it might be considered for crosssectional adaptation of questionnaire items with vulnerable populations, especially with immigrant children. The reason for conducting this study is the need for methodological transferrability both in multilingual health-related research (Perneger et al. 1999) and educational research on immigrant children (Liamputtong 2007, 2008). Our reseach questions are as follows: What methodological principles do emerge in evidence-based educational research literature relevant to immigrant children? In what ways are these principles present in the methodology of Kidscreen-52? Methods: Due to the cross-disciplinary uniqueness of our study, we have adopted the intrinsic study design (Creswell 2003).First research question: To answer the first research question, we conducted a rapid review of the evidence-based literature relevant to immigrant children. Second research question: To answer the second research question, we have conducted a methodological examination of the process of item generation in Kidscreen-52 (Bullinger et al. 1998). Results: First research question: The rapid review of educational research has surfaced three methodological principles: providing relational authenticity with research participants (Noddings 2013; Noddings 2015; Valenzuela 1999), ensuring higher level of reflexivity of research activities ( $\mathrm{Li}$ amputtong 2007, 2008), enhancing verbal and non-verbal expression (Punch 2012). Second research question: The methodological examination of the development of Kidscreen-52 yielded the following results: By enabling peer interactions, the Kidscreen-52 focus groups have provided relational authenticity. By covering a greater range of experiences, the Kidscreen-52 focus groups have enhanced reflexivity which is instrumental in capturing cross-cultural experiences of vulnerable populations (Punch 2012). By providing group dynamics, the Kidscreen-52 focus groups have served as a catalyst for verbal and non-verbal expression (Morgan 1988). Conclusion: Researchers often are in need of adapting questionnaire items age appropriately (Bollweg et al. 2020; Nassar-McMillan and Borders 2002). Focus groups utilized for item generation of Kidscreen-52 demonstrated this potential intrinsically and cross-culturally (Detmar et al., 2006).

\section{Bibliography}

Arksey H, O'Malley L. (2005). Scoping studies: towards a methodological framework Int J Soc Res Methodol. 2005;8:19-32. doi:10.1080/1364557032000119616.

Bollweg, T. M., Okan, O., Pinheiro, P., Bröder, J., Bruland, D., Freţian, A. M., \& Bauer, U. (2020). Adapting the European Health Literacy Survey for fourth-grade students in Germany: Questionnaire development and qualitative pretest. HLRP: Health Literacy Research and Practice, 4(2), e119-e128.

Bullinger, M., Alonso, J., Apolone, G., Leplege, A., Sullivan, M., Wood-Dauphinee, S., et al (1998). Translating health status questionnaires and evaluating their quality The IQOLA Project approach.International Quality of Life Assessment. Journal of Clinical Epidemiology, 51(11), 913-923.

Detmar, S. B., Bruil, J., Ravens-Sieberer, U., Gosch, A., \& Bisegger, C (2006). The use of focus groups in the development of the KIDSCREEN HRQL questionnaire. Quality of Life Research, 15(8), 1345-1353.

Dickson-Swift, V., James, E. L., \& Liamputtong, P (2008). Undertaking sensitive research in the health and social sciences: Managing boundaries, emotions and risks.

Gifford, S. M., Bakopanos, C., Kaplan, I., \& Correa-Velez, I (2007). Meaning or measurement? Researching the social contexts of health and settlement among newly-arrived refugee youth in Melbourne, Australia. Journal of refugee studies, $20(3), 414-440$

Harkness, J. A. (2007). Improving the comparability of translations. Measuring attitudes cross-nationally: Lessons from the European Social Survey, 79-95.

Herdman, M., Rajmil, L., Ravens-Sieberer, U., Bullinger, M., Power, M., Alonso, J., \& European Kidscreen and Disabkids groups. (2002). Expert consensus in the development of a European health-related quality of life measure for children and adolescents: a Delphi study. Acta Paediatrica, 91(12), 1385-1390.

Liamputtong, P. (2007). Researching the vulnerable: A guide to sensitive research methods. Sage. 
Liamputtong, P. (2008). Doing research in a cross-cultural context: Methodological and ethical challenges. In Doing cross-cultural research (pp. 3-20). Springer, Dordrecht.

Mbuagbaw, L., Lawson, D. O., Puljak, L., Allison, D. B., \& Thabane, L. (2020). A tutorial on methodological studies: the what, when, how and why. BMC Medical Research Methodology, 20(1), 1-12

Morgan, D. L. (1988). Focus groups as qualitative research. Newbury Park, CA: Sage.

Nassar-McMillan, S. C., \& Borders, L. D. (2002). Use of focus groups in survey item development. The Qualitative Report 7(1). Retrieved from http://www.nova.edu/ssss/QR/QR7-1/nassar.html.

Noddings, N. (2013). Caring: A relational approach to ethics and moral education. Univ of California Press.

Noddings, N. (2015). The challenge to care in schools, 2nd Editon. Teachers College Press.

Perneger, T. V., Leplège, A., \& Etter, J. F. (1999). Cross-cultural adaptation of a psychometric instrument: two methods compared. Journal of clinical epidemiology, 52(11), 1037-1046.

Punch, S. (2012). Studying transnational children: A multi-sited, longitudinal, ethnographic approach. Journal of Ethnic and Migration Studies, 38(6), 1007-1023.

Ravens-Sieberer, U., Herdman, M., Devine, J. et al. (2014). The European KIDSCREEN approach to measure quality of life and well-being in children: development, current application, and future advances. Qual Life Res 23, 791-803. https://doi.org/10.1007/s11136-013-0428-3

Ravens-Sieberer, U., Gosch, A., Rajmil, L., Erhart, M., Bruil, J., Power, M., et al. (2008). The KIDSCREEN-52 quality of life measure for children and adolescents: Psychometric results from a cross-cultural survey in 13 European countries. Value in Health, 11(4), 645-658.

Robitail S Simeoni, M C Erhart M Ravens-Sieberer U Bruil J Auquier P \& Group, E. K. (2006). Validation of the European proxy KIDSCREEN-52 pilot test health-related quality of life questionnaire: first results. Journal of Adolescent Health 39(4), 596-e1.

(111.2) The Health Literacy Questionnaire as a multi-dimensional measure: some implications for valid inference from a bi-factor confirmatory factor analysis

Gerald Elsworth, PhD, Swinburne University of Technology, Melbourne, Australia; Sandra Nolte, $\mathrm{PhD}$, Charité - Universitätsmedizin Berlin, Berlin, Germany; Christina Cheng, Swinburne University of Technology, Melbourne, Australia; Melanie Hawkins, PhD, Swinburne University of Technology, Melbourne, Australia; Richard Osborne, PhD, Swinburne University of Technology, Melbourne, Australia

Aims: Multi-item questionnaires scored by summing items to construct scales frequently encompass several related content domains, but there is ongoing debate about whether to represent multi-dimensional constructs as a single score across all items or as separate domain scores. Health literacy is theorised as both a single and multidimensional construct. The Health Literacy Questionnaire (HLQ) exemplifies both a multi-dimensional health literacy theory and multiscale assessment tool. Evidence of homogeneity and distinctiveness of the nine HLQ scales has typically relied on a nine-factor confirmatory factor analysis (CFA) model. Inter-factor correlations vary across constructs, but some are consistently quite high. This paper aims to contrast validity inferences drawn from the correlated-factors models with those from a bi-factor model (one that includes both a general factor loaded by all items and a specific factor for each construct). Methods: Correlated factor and bi-factor models (allowing correlated specific factors) were fitted to responses to the HLQ provided by 813 clients of community-based healthcare agencies in Victoria, Australia using Bayesian CFA. Results were replicated on an independent sample of 1464 adults in a computer-aided telephone survey conducted within a Regional Primary Health Network in Victoria. Results: All models fitted that data well using established Bayesian fit criteria. The correlated factor models replicated the pattern of previous results, but the bi-factor models provided a sharply different perspective on the factorial composition of items. In the bifactor model of community-agency responses, loadings on the general factor ranged from 0.22 to 0.79 , being generally higher for the items associated with four constructs that use a 'task difficulty' response metric. The domain-specific factors for these items were consequently not as well defined, but each was associated with at least three items with a loading $>0.25$. All five factors for items using an 'agreedisagree' metric were well defined by all hypothesised items. Conclusion: Bi-factor modelling of the HLQ suggests that items represent at least two sources of substantive common factor variance, associated with a general factor related to all items and with a more specific conceptual domain, suggesting that there is unavoidable heterogeneity in self-report questionnaire items. Implications for construct validity studies will be discussed.

(111.3) Response-shift detection using Differential Item Functioning analysis: Comparing QOL of Ravulizumab and Eculizumab in Paroxysmal Nocturnal Hemoglobinuria with the general population

Carolyn Schwartz, ScD, DeltaQuest Foundation and Tufts University School of Medicine, Concord, Massachusetts, USA; Roland Stark, M.Ed., DeltaQuest Foundation, Concord, Massachusetts, USA; Katrina Borowiec, M.A., Boston College Lynch School of Education and Human Development \& DeltaQuest Foundation, Chicopee, Massachusetts, USA; Sandra Nolte, Ph.D., Charité - Universitätsmedizin Berlin, Berlin, Germany; Karl-Johan Myren, M.S., Alexion Pharmaceuticals, Stockholm, Sweden

Aims: Paroxysmal nocturnal hemoglobinuria (PNH) is a rare, lifethreatening intravascular hematologic disorder with significant morbidity and premature mortality. Clinical trials (NCT02946463 and NCT03056040) comparing ravulizumab with eculizumab for PNH have supported the non-inferiority of the former and similar safety and tolerability. This secondary analysis compared PNH trial participants after 26 weeks on either treatment $(n=438)$ to a generalpopulation sample (GenPop) $(n=15,386)$ and investigated responseshift effects. Methods: Multivariate analysis of covariance (MANCOVA) investigated function and symptom scores on the European Organisation for Research and Treatment of Cancer (EORTC) QLQ$\mathrm{C} 30$ of people with $\mathrm{PNH}$ as compared to GenPop, after covariate adjustment. Risk-factor groups were created based on clinical indicators known to be associated with worse PNH outcomes, and separate MANCOVAs were computed for lower- and higher-risk- 
factor groups. Differential item functioning (DIF) analyses examined whether item response varied systematically (1) by treatment, (2) compared to GenPop, and (3) over time, the latter two suggesting and reflecting response-shift effects, respectively. DIF analyses examined 24 items from scales with at least two items. Recalibration response shift was operationalized as uniform DIF over time, reflecting the idea that, for a given group, the difficulty of endorsing an item changes over time, after adjusting for the total subscale score. Reprioritization response shift was operationalized as non-uniform DIF over time, i.e., the relative difficulty of endorsing an item over time changes across the total domain score. Results: Across PNH risk-factor levels, people treated with ravulizumab or eculizumab for 26 weeks reported betterthan-expected functioning and lower symptom burden compared to GenPop. Ravulizumab generally showed larger effect sizes. Results observed were similar for both lower and higher PNH risk factors, with slightly stronger effects in the former. DIF analyses revealed no treatment DIF, but did uncover group DIF ( 9 with uniform DIF, and 11 with non-uniform) and DIF over time (7 with uniform DIF, and 3 with non-uniform). Conclusion: People with $\mathrm{PNH}$ treated with ravulizumab or eculizumab for 26 weeks reported better-than-expected QOL given their overall health. Significant effects of DIF by group and DIF over time support recalibration and reprioritization responseshift effects. These findings suggest that these treatments enabled adaptive changes in QOL.

\section{(111.4) Differences in the conceptual structures perceived by Japanese stakeholders between "patient-reported outcomes" and "quality of life"}

Takako Kaneyasu, Ritsumeikan University, Kusatsu, Shiga, Japan; Shinya Saito, Okayama University Graduate School of Health Sciences, Okayama, Japan; Kikuko Miyazaki, Kyoto University School of Public Health, Department of Health Informatics, Kyoto, Japan; Yoshimi Suzukamo, Tohoku University Graduate School of Medicine, Department of Physical Medicine and Rehabilitation, Sendai, Miyagi, Japan; Mariko Naito, Graduate School of Biomedical and Health Sciences, Hiroshima University, Department of Oral Epidemiology, Hiroshima, Japan; Takashi Kawaguchi, Tokyo University of Pharmacy and Life Sciences, Department of Practical Pharmacy, Hachioji, Tokyo, Japan; Takako E Nakajima, Kyoto University Hospital, Kyoto Innovation Center for Next-Generation Clinical Trials \& iPS Cell Therapy, Kyoto, Japan; Takuhiro Yamaguchi, Tohoku University Graduate School of Medicine, Division of Biostatistics, Sendai, Miyagi, Japan; Kojiro Shimozuma, Ritsumeikan University Department of Biomedical Sciences, College of Life Sciences, Kusatsu, Shiga, Japan

Aims: We launched a task force to develop Japanese patient-reported outcome (PRO) guidelines, funded by the Ministry of Health, Labor and Welfare, and we implemented a two-step survey to investigate the need for guidelines regarding the perception of the concepts of PRO and quality of life (QOL) among Japanese stakeholders. Methods: First, we conducted a pilot survey including 45-min interviews with the following stakeholders: patient advocates, industry representatives, clinical experts, and academic researchers. The interview included questions regarding PRO and QOL perception, interrelation, difficulty of measurement, and multidimensionality. Second, we performed a web-based survey with the same questions used in the interview for 253 medical professionals and 249 pharmaceutical company employees. The pilot survey results were narratively analyzed, and the web-based survey was analyzed descriptively. Results:
The pilot survey revealed an unpredicted dynamic conceptual structure instead of a static structure perceived by patient advocates regarding the intercorrelation of PRO and QOL, i.e., "Occasionally, PRO improves patients' QOL.” The web-based survey supported the results, as the most frequent responses regarding the interrelation between PRO and QOL were "partly different," followed by "QOL includes PRO". The contents that should be included in the Japanese guidelines for medical professionals and pharmaceutical company employees were "the analysis and interpretation of PRO measure results," "the use of PRO measure results in clinical practice," and "the application of PRO measure results in clinical practice guidelines." Conclusion: The results of the two surveys revealed that most Japanese stakeholders perceived that "there are noncongruent areas between PRO and QOL" or that "QOL comprises PRO." These results suggested that Japanese stakeholders possibly have different recognitions from those premised as "PRO comprises QOL" in some international guidelines. In the development of Japanese PRO guidelines, we should consider this unique perception and the needs of Japanese stakeholders as a future direction.

(111.5) Valuation of lost productivity in caregivers: a validation study

Aaron Gelfand, BSc, School of Population and Public Health - UBC, Vancouver, British Columbia, Canada; Julie Sou, MSc, Centre for Health Evaluation \& Outcome Sciences, Vancouver, British Columbia, Canada; Aslam Anis, PhD, School of Population and Public Health - UBC, Vancouver, British Columbia, Canada; Alison Pearce, $\mathrm{PhD}$, University of Sydney, Sydney, Australia; Katrina Prescott, section 2 Productions, Vancouver, British Columbia, Canada; Rick Sawatzky, PhD, Trinity Western University, Vancouver, British Columbia, Canada; Christine Lee, MD, Vancouver Island Health Authority, Vancouver, British Columbia, Canada; Wei Zhang, PhD, School of Population and Public Health - UBC, Vancouver, British Columbia, Canada

Aims: The Valuation of Lost Productivity (VOLP) questionnaire was developed to measure work productivity losses in terms of work time losses in people with health problems. The major losses include absenteeism and presenteeism. Caring for people with health problems affects the work productivity of their caregivers. Existing questionnaires assessing caregiver work productivity losses have limitations. We adapted the VOLP into a caregiver version to capture work productivity losses of caregivers. Methods: After reviewing the VOLP and other existing questionnaires measuring caregiver work productivity losses, the research team drafted an initial questionnaire. This was improved using one-on-one interviews with caregivers and converted to an online format (Qualtrics). The online version was administered to 400 Canadian caregivers (382 valid responses), defined as individuals caring for a family member or friend living with a chronic condition. The Work Productivity and Activity Impairment (WPAI) questionnaire for caregivers was included to test validity.Main outcomes included VOLP and WPAI absenteeism and presenteeism. Absenteeism was measured using work time missed in different recall periods ( 3 months in VOLP vs. 1 week in WPAI). VOLP presenteeism was measured using a direct hour estimating method. WPAI presenteeism was measured using a $0-10$ scale. Similar validation methods for the VOLP patient version were applied. Results: Mean presenteeism estimated by the VOLP was lower than WPAI (VOLP $=8.86 \%$ time loss while working; $\mathrm{WPAI}=37.62 \%$ ). Additional caregiver participant characteristics, 
VOLP and WPAI outcomes are presented (Figs. 1 and 2). We found low Spearman correlations between VOLP and WPAI outcomes (absenteeism $=0.49$; presenteeism $=0.36$ ). Correlations of VOLP and WAPI outcomes, respectively, with time spent on caregiving responsibilities, ranged from negligible to low (VOLP: absenteeism $=0.39 ;$ presenteeism $=0.22 ;$ WPAI: absenteeism $=0.27$; presenteeism $=0.35$ ). Conclusion: The study contributes to more evidence on the feasibility and validity of the VOLP in measuring productivity losses due to caregiving responsibilities, in addition to the previous validation study among patients. Additionally, the VOLP could measure caregiving time, absenteeism and presenteeism for volunteer work, and lost opportunities. Consideration should be given to recall period for caregiving time, potential double-counting issue by including emotional support, and appropriate sample size due to highly skewed data with excess zeros.

\begin{tabular}{|c|c|c|}
\hline \begin{tabular}{|l|} 
Variables $(\mathrm{N}=382)$ \\
\end{tabular} & Mean (SD) & Median (Q1 to Q3) \\
\hline Age & $46.54(10.90)$ & $49.50(39.50-59.50)$ \\
\hline $\begin{array}{l}\text { Total caregiving hours in the past } \\
\text { week }\end{array}$ & $33.64(26.80)$ & $27.0(14.12-43.0)$ \\
\hline \multirow{2}{*}{$\begin{array}{l}\text { Health status of caregivers (1-5 } \\
\text { scale, } 1 \text { being poor, } 5 \text { being } \\
\text { excellent) }\end{array}$} & $3.24(1.04)$ & $3.0(3.0-4.0)$ \\
\hline & $\mathbf{N}$ & $\%$ \\
\hline \begin{tabular}{|l|} 
Female \\
\end{tabular} & 203 & 53.14 \\
\hline Volunteering & 51 & 12.83 \\
\hline \multicolumn{3}{|l|}{$\begin{array}{l}\text { Highest level of education } \\
\text { completed }\end{array}$} \\
\hline Primary or High school & 73 & 19.11 \\
\hline College or technical/trade & 115 & 30.10 \\
\hline University & 132 & 34.55 \\
\hline $\begin{array}{l}\text { Post-graduate or professional } \\
\text { designation }\end{array}$ & 59 & 15.45 \\
\hline \multicolumn{3}{|l|}{\begin{tabular}{|l} 
Ethnicity \\
\end{tabular}} \\
\hline Aboriginal & 15 & 3.93 \\
\hline African & 5 & 1.31 \\
\hline Hispanic, Latino or Spanish & 6 & 1.57 \\
\hline European & 227 & 59.42 \\
\hline East Asian & 55 & 14.40 \\
\hline South Asian & 16 & 4.19 \\
\hline West Asian & 9 & 2.36 \\
\hline Other & 37 & 9.69 \\
\hline $\begin{array}{l}\text { Prefer not to answer/ do not } \\
\text { know }\end{array}$ & 18 & 4.71 \\
\hline \multicolumn{3}{|l|}{\begin{tabular}{|l|} 
Province of Residence \\
\end{tabular}} \\
\hline Alberta & 46 & 12.04 \\
\hline Atlantic Region* & 28 & 7.33 \\
\hline British Columbia & 52 & 13.61 \\
\hline Manitoba & 14 & 3.66 \\
\hline Ontario & 163 & 42.67 \\
\hline Quebec & 72 & 18.85 \\
\hline Saskatchewan & 7 & 1.83 \\
\hline \multicolumn{3}{|l|}{\begin{tabular}{|l|}
$\begin{array}{l}\text { Severity of care recipients' } \\
\text { chronic conditions }\end{array}$ \\
\end{tabular}} \\
\hline Mild & 57 & 14.92 \\
\hline Moderate & 234 & 61.26 \\
\hline Severe & 77 & 20.16 \\
\hline
\end{tabular}

* includes New Brunswick, Newfoundland and Labrador, Nova Scotia and Prince Edward Island; SD: standard deviation; Q1: the first quartile; Q3: the third quartile
Table 2. VoLP and WPAI outcomes

\begin{tabular}{|c|c|c|c|}
\hline \begin{tabular}{|l|} 
Variable \\
\end{tabular} & $\mathrm{N}$ & Mean (SD) & Median (Q1-Q3) \\
\hline \multicolumn{4}{|l|}{ VOLP outcomes } \\
\hline Employed* & $\begin{array}{c}277 \\
(72.51 \%)\end{array}$ & & \\
\hline \multicolumn{4}{|l|}{$\begin{array}{l}\text { Absenteeism due to caregiving } \\
\text { responsibilities in the past } 3 \\
\text { months }\end{array}$} \\
\hline \begin{tabular}{|l|} 
Number of absent workdays \\
\end{tabular} & 277 & $4.50(12.84)$ & $0(0-4.0)$ \\
\hline $\begin{array}{l}\text { Number of absent } \\
\text { workdays (absent workdays } \\
>0 \text { ) }\end{array}$ & 124 & $10.05(17.70)$ & $4.0(2.0-7.0)$ \\
\hline \% work time absent & 249 & $10.28(20.75)$ & $0(0-7.69)$ \\
\hline $\begin{array}{l}\% \text { work time absent (absent } \\
\text { workdays }>0 \text { ) }\end{array}$ & 124 & $20.63(25.55)$ & $7.93(4.51-25.89)$ \\
\hline $\begin{array}{l}\text { Caregivers who have worked in } \\
\text { the past } 7 \text { days }\end{array}$ & 232 & & \\
\hline \multicolumn{4}{|l|}{$\begin{array}{l}\text { Presenteeism due to caregiving } \\
\text { responsibilities in the past } 7 \\
\text { days }\end{array}$} \\
\hline$\%$ time loss while working & 232 & $8.86(16.41)$ & $0(0-13.41)$ \\
\hline $\begin{array}{l}\text { \% time loss while working } \\
\text { (time loss }>0 \text { ) }\end{array}$ & 81 & $25.36(18.81)$ & $20.0(12.5-33.3)$ \\
\hline \multicolumn{4}{|l|}{ WPAI outcomes } \\
\hline $\begin{array}{l}\text { Caregivers who were working } \\
\text { for pay }\end{array}$ & 262 & & \\
\hline \multicolumn{4}{|l|}{$\begin{array}{l}\text { Absenteeism due to caregiving } \\
\text { responsibilities in the past } 7 \\
\text { days }\end{array}$} \\
\hline \begin{tabular}{|l|} 
Number of absent workhours \\
\end{tabular} & 262 & $3.10(8.89)$ & $0(0-2.0)$ \\
\hline $\begin{array}{l}\text { Number of absent } \\
\text { workhours (absent } \\
\text { workhours }>0 \text { ) }\end{array}$ & 90 & $9.03(13.32)$ & $5(2.0-8.0)$ \\
\hline$\%$ work time absent & 262 & $11.29(23.55)$ & $0(0-8.62)$ \\
\hline $\begin{array}{l}\text { \% work time absent (absent } \\
\text { workhours }>0 \text { ) }\end{array}$ & 90 & $29.47(30.27)$ & $14.29(6.25-50.0)$ \\
\hline $\begin{array}{l}\text { Caregivers whose actual work } \\
\text { hour }>0 \text { in the past } 7 \text { days }\end{array}$ & 227 & & \\
\hline \multicolumn{4}{|l|}{$\begin{array}{l}\text { Presenteeism due to caregiving } \\
\text { responsibilities in the past } 7 \\
\text { days }\end{array}$} \\
\hline$\%$ time loss while working & 227 & $37.62(28.74)$ & $40.0(10.0-60.0)$ \\
\hline $\begin{array}{l}\% \text { time loss while working } \\
\text { (time loss }>0 \text { ) }\end{array}$ & 186 & $45.91(25.03)$ & $50.0(20.0-70.0)$ \\
\hline
\end{tabular}

(111.6) Effectiveness of a personalized health profile on specificity of self-management goals among people living with HIV in Canada: findings from a blinded pragmatic randomized controlled trial

Maryam Mozafarinia, MSc, Division of Experimental Medicine, McGill University, Montreal, Quebec, Canada; Fateme Rajabiyazdi, $\mathrm{PhD}$, System and Computer Engineering, Faculty of Engineering and Design, Carlton University, Ottawa, Ontario, Canada; Marie-Josée Brouillette, MD, Department of Psychiatry, McGill University Health Centre, Montreal, Quebec, Canada; Lesley K. Fellows, PhD, MD, Department of Neurology and Neurosurgery and Chronic Viral Illness service, Montreal Neurological Institute, Montreal, Quebec, Canada; Bärbel Knäuper, PhD, Department of Psychology, McGill University, Montreal, Quebec, Canada; Nancy E. Mayo, PhD, Division of Experimental Medicine, Department of Medicine and School of Physical and Occupational Therapy, McGill Uiversity, Montreal, Quebec, Canada 
Aims: To estimate among people living with HIV, to what extent providing feedback on their health outcomes, compared to no feedback, will affect number and specificity of patient-formulated selfmanagement goals. Methods: A personalized health profile (Dashboard) was produced for individuals enrolled in a Canadian HIV Brain Health Now cohort study at cohort entry and at the last recorded visit. Participants were randomized $(1: 1)$ to receive or not their Dashboard prior to a goal setting exercise. Self-defined goals in free text were collected through an online platform. Both groups received instructions on goal setting and tips to improve brain health. The primary outcomes were number and specificity of the goals. Specificity was measured by total number of specific words (matched a developed lexicon) per person words using text mining techniques and estimated by negative binomial regression. Results: Of 350 participants initially approached, 176 were eligible and enrolled between Jan 13 and July 14, 2020. A total of 110 survey responses, including 421 goals, received and analyzed. The average number of goals was similar for both feedback and control groups (3.7 vs 3.9). Number of specific words for feedback and control groups were 642 and 739 , respectively. Of total number of words used by both groups, 30\% were specific and represented goal criteria. Specific nouns and actionable verbs were present to some extent and "measurable" and "time-bound" words were mainly missing. Negative binomial regression showed no difference in goal specificity among groups $(\mathrm{RR}=0.93,95 \%$ CI $0.78-1.10)$. Cognitive ability did not affect goal specificity $(\mathrm{RR}=1.13,95 \% \mathrm{CI} 0.45,2.82)$. There was a weak correlation between goal specificity and education $(r=0.21,95 \% \mathrm{CI}$ $0.02,0.38)$ and cognition $(r=0.17,95 \% \mathrm{CI}-0.01,0.35)$. Goals set by both groups overlapped in 8 areas and had little difference in rank. Conclusion: Personal Brain Health Dashboard did not help with formulation of high-quality goals. Text mining has the potential to help with difficulties of goal evaluation outside of the face-to-face setting. With more data and use of learning models, automated answers could be generated to provide a more dynamic platform.

(111.7) Using Qualitative Interviews and Deliberative Dialogue to Triangulate Systematic Review Findings about Clinicians Use of Patient-Reported Outcome Measures (PROMs) in daily practice

Angela Wolff, PhD, RN, Associate professor School of Nursing, Trinity Western University, Langley, British Columbia, Canada; Nelly D. Oelke, PhD, RN, Associate professor University of British Columbia, Okanagan, Kelowna, British Columbia, Canada; Vidhi Thakkar, PhD, Postdoctoral Fellow, School of Nursing, Trinity Western University, Langley, British Columbia, Canada; Andrea Dresselhuis, MSN, RN, Research CoordinatOR School of Nursing, Trinity Western University, Langley, British Columbia, Canada

Aims: Individual patient-level data from PROMs are critical to support clinical decision making in a person-centered approach. A mixed-method systematic review (SR) was undertaken to synthesize the abundant research evidence (qualitative, mixed method, quantitative, quality improvement/knowledge translation projects) from eight databases about the needs of and factors influencing how healthcare providers (HCPs) implement PROMs in daily practice. We present the novel use of integrated knowledge translation (iKT) methods, particularly qualitative interviews and deliberative dialog (DD), to triangulate the SR findings. Methods: Knowledge translation approaches used throughout the entire project involved researchers, knowledge users, and patients as equal partners in the research team. The study consisted of three methods: systematic review (SR), qualitative interviews with HCPs, and deliberative dialogue (DD). In Phase 1, the SR of mixed evidence used a narrative synthesis approach (Popay et al., 2006) to synthesize findings from 167 articles. The second phase consisted of 20 clinician interviews that were analyzed using deductive content analysis. Finally, a DD forum (Phase 3) was designed and attended by 50 key stakeholders/knowledge users involved with or interested in PROMs use by HCPs. The data analysis in each phase was guided by implementation science frameworks (e.g., Theoretical Domains Framework). Results: Following the synthesis of the evidence and analysis of qualitative interviews, the DD forum allowed us to validate our findings, reach consensus on the essential needs of HCPs, and achieve agreement on factors influencing end-user behaviors. The analysis of the DD forum was integrated with data from Phases 1 and 2 to create guidelines on "how to" support optimal implementation of PCMs and highlight key strategies for sustained use in daily practice. Conclusion: The mixedmethod SR provides a comprehensive and integrated understanding of the issue at hand; however, the addition of qualitative interviews provides thick, rich, and detailed accounts of the phenomenon. Through DD, consensus was reached on what matters to clinicians to incorporate PROMS into their daily practice. By means of engaging clinicians, evidence generated using these three methods provides a robust understanding of what is meaningful to end-users who utilize PROMs in daily practice. These data were used to create a resource guide.

(111.8) The International Survey of People Living with Chronic Conditions (PaRIS survey): development of the patient questionnaire

Ian Porter, Health Services \& Policy Research Group, University of Exeter, Exeter, UK; Mieke Rijken, Nivel (The Netherlands Institute for Health Services Research), Utrecht, The Netherlands; Oliver Groene, Optimedis A.G., Hamburg, Germany; Rosa Suñol, Fundacion Avedis Donabedian Research Institute, Barcelona, Spain; Rachel Williams, Ipsos MORI, London, UK; Michael van den Berg, Health Division, OECD, Paris, France; Jimmy Martin Delgado, Health Services \& Policy Research Group, University of Exeter, Exeter, UK; Jon P Evans, Health Services \& Policy Research Group, University of Exeter, Exeter, UK; Marta Ballester, Fundacion Avedis Donabedian Research Institute, Barcelona, Spain; Janika Blömeke, Optimedis A.G., Hamburg, Germany; Laura Thomas, Ipsos MORI, London, UK; Chloe Thomas, Health Services \& Policy Research Group, University of Exeter, Exeter, UK; Peter Groenewegen, Nivel (The Netherlands Institute for Health Services Research), Utrecht, The Netherlands; Wienke Boerma, Nivel (The Netherlands Institute for Health Services Research), Utrecht, The Netherlands; Monique Heijmans, Nivel (The Netherlands Institute for Health Services Research), Utrecht, The Netherlands; Katherine De Bienassis, Health Division, OECD, Paris, France; Candan Kendir, Health Division, OECD, Paris, France; Niek Klazinga, Health Division, OECD, Paris, France; Dolf De Boer, Nivel (The Netherlands Institute for Health Services Research), Utrecht, The Netherlands; Jose M Valderas, Health Services \& Policy Research Group, University of Exeter, Exeter, UK

Aims: The Organization for Economic Cooperation and Development (OECD) PaRIS survey aims to support countries in improving care for people living with chronic conditions by collecting information on how these people experience the quality and performance of the primary and ambulatory care services they use in their country. We present the development of the patient questionnaire of this survey. Methods: Candidate scales and items were identified through a comprehensive and systematic process including a series of systematic literature reviews, engagement with international stakeholders including the PaRIS Patient Advisory Panel, Technical Advisory Community and National Project Managers of participating countries, 
and oversight by a Working Party of OECD member states representatives. Following a mapping exercise onto the conceptual framework, four instruments for each domain were shortlisted using predefined criteria. The psychometric performance of the candidate instruments was assessed using the EMPRO method. A subsequent modified Delphi procedure was implemented for selecting a core instrument for each domain and additional relevant scales/items. Further consultations took place with the relevant stakeholders to confirm the suitability of the proposed questionnaire, whose feedback resulted in a number of iterations until a final version was agreed. Results: 217 instruments were identified measuring one or more of the domains of the conceptual framework. The two-staged Delphi procedure resulted in consensus on a single core instrument for each of the four main domains, as well as additional questions to ensure comprehensiveness. The final version of the survey includes the following sections: "Your health" (18 items; PROMIS Global-10, WHO-5, and others), "Managing your health and health care" (26; Porter-Novelli and others), "Your experience of health care (P3CEQ and others)" (49), and About yourself (24). Conclusion: A comprehensive questionnaire has been constructed based on the PaRIS survey framework for people living with chronic conditions and following an inclusive approach. The current questionnaire will be evaluated through cognitive testing before being piloted in a Field Trial in participating countries. These subsequent stages will offer opportunities for improving the questionnaire, ensuring adequate performance and offering insights into how the questionnaire can be modified to reduce the burden of administration while balancing comprehensiveness and metric performance.

\section{2: Policy Application in Vulnerable Populations}

\section{(112.1) Tailored implementation of a narrative PREM in the disability sector}

Marjolein Van Rooijen, MSc, Research institute CAPHRI University of Maastricht, Maastricht, The Netherlands; Anneke van Dijk, Dr., Research institute CAPHRI University of Maastricht, Maastricht, The Netherlands; Stephanie Lenzen, Dr., Research Centre for Autonomy and Participation of Persons with a Chronic Illness, Zuyd University of Applied Sciences, Heerlen, The Netherlands; Ruth Dalemans, Dr., Research Centre for Autonomy and Participation of Persons with a Chronic Illness, Zuyd University of Applied Sciences, Heerlen, The Netherlands; Albine Moser, Dr., Research Centre for Autonomy and Participation of Persons with a Chronic Illness, Zuyd University of Applied Sciences, Heerlen, The Netherlands; Sandra Beurskens, Prof.dr., Research institute CAPHRI University of Maastricht, Maastricht, The Netherlands

Aims: Patient-Reported Experience Measures (PREMs) are used to gain insight into care users' experiences and to improve the quality of care. Implementation is challenging and complex especially in the disability sector due to communication vulnerability of the care users. This study aims to develop and evaluate tailored implementation strategies that support the integrated uptake of a narrative PREM in the disability sectOR while engaging all relevant stakeholders, including care users who are communication vulnerable. Methods: Using Participatory Action Research, first a context analysis was conducted to identify barriers in the current state of implementation. Next, identified barriers were prioritized, categorized, and reformulated into implementation goals and draft strategies were selected. Then, implementation strategies were developed in co-creation. Special attention was paid to involving care users who are communication vulnerable. This cyclic process, consisting of plan, act, observe, and reflect phases was repeated until consensus was met. Lastly, a process evaluation was conducted to get insight in the use, experiences and perceived impact of the developed implementation strategies. Results: The context analysis showed 28 barriers on the micro-, meso-, and macro-level. Four implementation goals were formulated: clear purpose and added value, being prepared, successful execution, and integrated use of results. In the development phase care users $(N=7)$, professionals $(N=3)$ and facility managers $(N=2)$ used 13 sessions to develop 10 strategies, e.g., a pocket booklet to prepare for the PREM, a refreshers PREM training and coaching on the job. In the process evaluation, strategy uptake, experiences, and impact were evaluated at three facilities. Care users $(N=35)$, professionals $(N=11)$, and facility managers $(N=3)$ participated. A majority of the participants attended and used the strategies. Factors experienced as contributing to PREM uptake were the strategies' multi-stakeholder and practical approach, and that strategies improve skills and awareness. The impact of the set of implementation strategies was mainly on micro-level: improving experienced clear purpose and added value, and preparation. Conclusion: The systematic development of tailored strategies to improve PREM uptake has been successful on micro-level. The engagement of all relevant stakeholders, including care users who are communication vulnerable, was needed to incorporate their specific needs for the development of strategies.

(112.2) Investigating the discriminatory sensitivity of the new EQ5D-5LPsychosocial multi-attribute utility instrument compared to the EQ-5D-5L for a large cohort of Australians with multiple sclerosis

Julie Campbell, PhD, University of Tasmania, Menzies Institute for Medical Research, Hobart, Australia; Hasnat Ahmad, MEc, University of Tasmania, Menzies Institute for Medical Research, Hobart, Australia; Gang Chen, PhD, Monash University, Melbourne, Australia; Suzi Claflin, PhD, University of Tasmania, Menzies Institute for Medical Research, Hobart, Australia; Bruce TaylOR PhD, University of Tasmania, Menzies Institute for Medical Research, Hobart, Australia; Ingrid van der Mei, PhD, University of Tasmania, Menzies Institute for Medical Research, Hobart, Australia; Richard Norman, PhD, Curtin University, Perth, Australia; Laura Laslett, $\mathrm{PhD}$, University of Tasmania, Menzies Institute for Medical Research, Hobart, Australia; Steve Simpson-Yap, PhD, The University of Melbourne, Melbourne, Australia; Glen Henson, BEc(Hons), University of Tasmania, Hobart, Australia; Andrew Palmer, MBBS BMedSCI University of Tasmania, Menzies Institute for Medical Research, Hobart, Australia

Aims: Multiple sclerosis (MS) is a neurodegenerative disease of the central nervous system leading to increasing disability over time and reduced health-related quality of life (HRQoL). Resourcing decisions are based on cost-utility analysis (CUA) for Health Technology Assessments (HTA). Several multi-attribute utility instruments (MAUIs) are available from which health state utilities (HSUs) can be derived for use in CUA. The EQ-5D-5L MAUI is used in over $63 \%$ of economic evaluations and recommended for CUA in over $85 \%$ of HTA guidelines worldwide. However, due to its limited domains of psychosocial health, it has been found to be deficient in capturing psychosocial health for people with complex and chronic diseases. We aimed to investigate the discriminatory sensitivity of the new 'EQ-5D-5LPsychosocial' MAUI compared to the EQ-5D-5L for people with MS in Australia. To our knowledge, this will be the first such comparison worldwide. Methods: We conducted a comprehensive HRQoL survey among Australian MS Longitudinal Study participants. Data were collected for the EQ-5D-5L and AQoL-8D MAUIs. MS-related disease severity and sociodemographic data were also collected. HSUs were generated for the EQ-5D-5L and EQ-5D- 
5LPsychosocial bolt-on (bolt-on adopts four questions from the AQoL-8D MAUI) both using Australian tariffs. Mean (standard deviation [SD]) HSUs were estimated and stratified by disability severity (classified as no, mild, moderate, and severe disability). Results: $N=1683$ completed the survey (67\% response). Mean age of the respondents was 58.6 years, $80 \%$ were female. Over half $(55 \%)$ of the respondents were in the moderate or severe disability categories, $20 \%$ had mild disability, and $24 \%$ had no disability. Overall, mean HSUs were EQ-5D-5L:0.58 \pm 0.32 and EQ-5D-5LPsychosocial $0.62 \pm 0.29$. Mean EQ-5D-5L/EQ-5D-5LPsychosocial HSUs across disability categories were no disability $0.85 / 0.79$; mild: $0.71 / 0.66$; moderate: EQ-5D-5L 0.52/0.53; severe: $0.18 / 0.50 . N=157(10 \%)$ reported perfect health (i.e. $\mathrm{HSU}=1.0$ ) on the EQ-5D-5L, and reported a mean HSU of $0.90 \pm 0.08$ on the alternative instrument. The ceiling effect reduced to $n=7(<1 \%)$ for the EQ-5D-5LPsychosocial. A floor effect for the EQ-5D-5L was also identified. Conclusion: The EQ-5D5LPsychosocial MAUI had better sensitivity than the prevalent EQ-5D-5L as people approach HSUs that reflect full health, in Australians with multiple sclerosis. This has implications for the choice of MAUI for HTA guidelines that prescribe the EQ-5D-5L.

Table 1: Respondents chacteristics

\begin{tabular}{|c|c|}
\hline & Respondents \\
\hline Characteristics & $(\mathrm{N}=1683)$ \\
\hline \multicolumn{2}{|l|}{ Age at the time of survey } \\
\hline Average in years $(\mathrm{n})$ & $58.6(1683)$ \\
\hline \multicolumn{2}{|l|}{ Sex } \\
\hline Male \%(n) & $20.4(343)$ \\
\hline Female $\%(n)$ & $79.6(1340)$ \\
\hline \multicolumn{2}{|l|}{ Age group } \\
\hline$<35 \%(\mathrm{n})$ & $1.8(31)$ \\
\hline $35-44 \%(n)$ & $9.3(157)$ \\
\hline $45-54 \%(n)$ & $24.7(415)$ \\
\hline $55-64 \%(\mathrm{n})$ & $32.1(540)$ \\
\hline $65+\%(n)$ & $32.1(540)$ \\
\hline \multicolumn{2}{|l|}{ State/Territory of usual residence } \\
\hline New South Wales \% (n) & $28.1(473)$ \\
\hline Victoria $\%(n)$ & $29.3(493)$ \\
\hline Queensland \% (n) & $12.4(209)$ \\
\hline South Australia \% (n) & $9.9(167)$ \\
\hline Western Australia \% (n) & $9.8(165)$ \\
\hline Australian Capital Territory \% (n) & $4.0(67)$ \\
\hline Tasmania \% (n) & $5.7(96)$ \\
\hline Northern Territory \% (n) & $<1.0(1)$ \\
\hline \multicolumn{2}{|l|}{ Education Level } \\
\hline Primary \% (n) & $<1.0(8)$ \\
\hline Secondary $\%(\mathrm{n})$ & $24.6(400)$ \\
\hline Occupation Certificate \% (n) & $33.5(545)$ \\
\hline University (bachelors) $\%(\mathrm{n})$ & $21.0(341)$ \\
\hline University (Postgrad) \% (n) & $16.7(272)$ \\
\hline Other $\%(\mathrm{n})$ & $3.7(60)$ \\
\hline \multicolumn{2}{|l|}{ *MS type } \\
\hline PPMS \% (n) & $11.3(190)$ \\
\hline RRMS \% (n) & $62.8(1,056)$ \\
\hline SPMS \% (n) & $14.2(239)$ \\
\hline PRMS \% (n) & $2.5(42)$ \\
\hline Unsure \% (n) & $8.0(134)$ \\
\hline \multicolumn{2}{|l|}{ Disability severity } \\
\hline No disability $\%(\mathrm{n})$ & $23.9(402)$ \\
\hline Mild disability \% (n) & $20.4(343)$ \\
\hline Moderate disability $\%(\mathrm{n})$ & $36.1(608)$ \\
\hline Severe disability \% (n) & $18.8(317)$ \\
\hline MS duration since diagnosis & \\
\hline
\end{tabular}

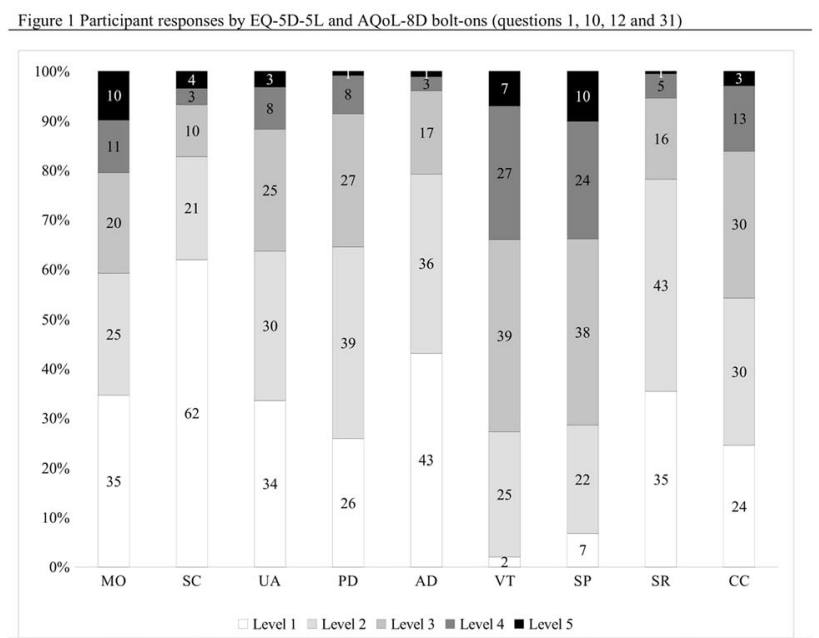

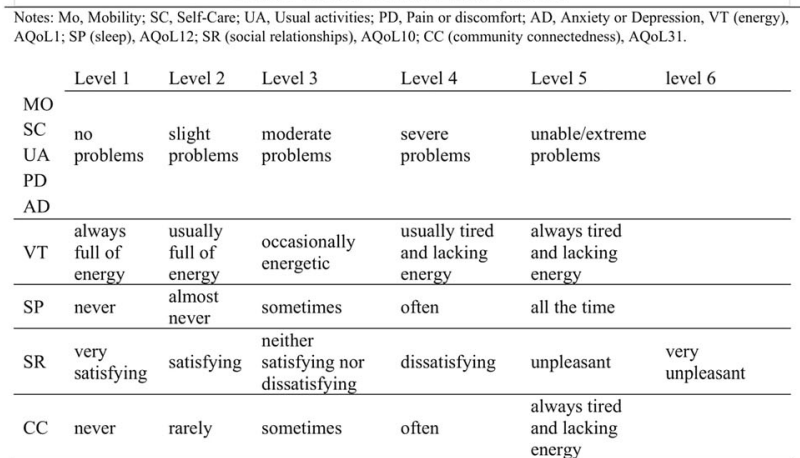

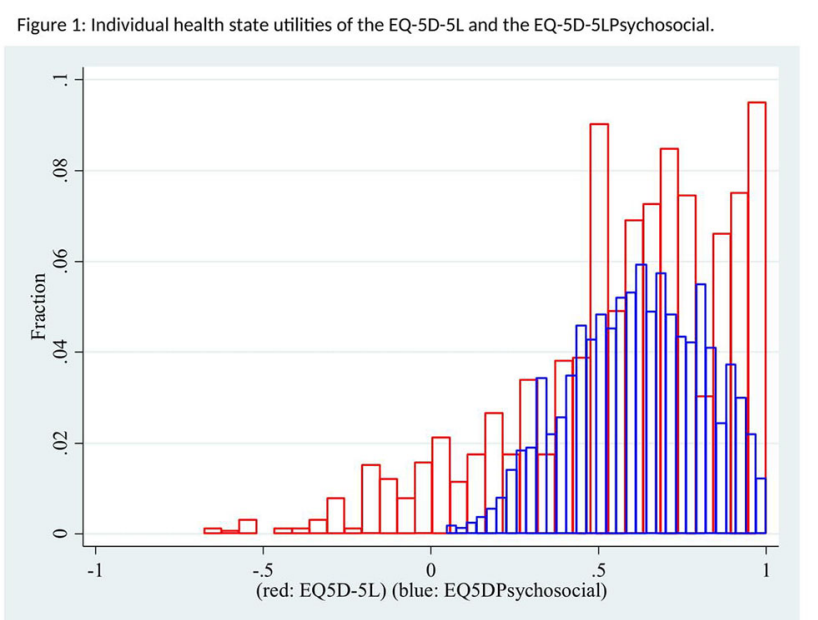

(112.3) Pooling trial data to examine quality of life in people with opioid use disorder

Thomas Patton, PhD, University of California San Diego, San Diego, California, USA; Jan Boehnke, PhD, University of Dundee, Dundee, UK; Annick Borquez, PhD, University of California San Diego, San Diego, California, USA 
Aims: Recent findings have shown that health-related quality-of-life (HRQoL) evidence for use in economic evaluations of interventions to address opioid use disorder (OUD) is limited. This study aims to extend the evidence base using structural equation modeling (SEM) methods to equate heterogeneous HRQoL data collected in studies funded by the National Institute on Drug Abuse (NIDA). Methods: Trial data were obtained from the NIDA website if they collected the following measures: generic measures of HRQoL (EQ-5D-3L, SF-12 Versions 1 or 2) and self-reported opioid use with the Addiction Severity Index. External evidence was used to equate different measures of HRQoL onto the scale of the EQ-5D using SEM techniques. A beta regression analysis was then performed on the resulting EQ-5D index values to disentangle variations in the type of opioid use, self-reported use of other substances, and demographic characteristics. Marginal means were calculated using regression outputs and holding opioid use covariates fixed in the sample to predict HRQoL for key health states. Results: Data from six trials were combined to produce a sample of 2,068 individuals, in whom $63 \%$ were male and the mean lifetime opioid use was nine years. Figure 1 shows EQ-5D values across studies. Marginal mean estimates were as follows: no opioid use within 30 days $=0.86$ $(\mathrm{SD}=0.03)$, daily injecting heroin use $=0.82(\mathrm{SD}=0.03)$, daily methadone use $=0.86(\mathrm{SD}=0.03)$, daily use of other opiates $=0.80$ (0.04). Conclusion: Estimates were higher than those in the existing literature (Connock et al. 2007; Wittenberg et al. 2016). One possible explanation is that previous estimates were elicited in participants for hypothetical vignettes, describing a range of defined OUD states, whereas our estimates are based on individuals' descriptions of their own 'experienced' health states. This reduces the risk of bias associated with the 'labelling' of health states. Limitations include questions over the generalizability of the sample due to the inclusion criteria for clinical trials. Nonetheless, these findings contribute to the evidence base for the economic evaluation of OUD interventions, such as the scale-up of medications for OUD, which is urgently needed in the US and other settings.

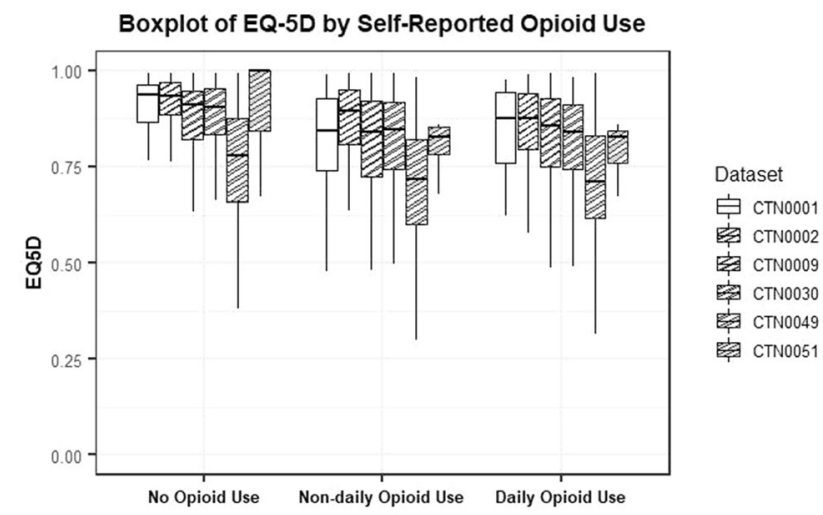

(112.4) A multi-instrument comparison study of six multiattribute utility instruments for a large cohort of Australians with multiple sclerosis: implications for resource allocation decision making
Julie Campbell, $\mathrm{PhD}$, University of Tasmania, Menzies Institute for Medical Research, Hobart, Australia; Hasnat Ahmad, MEc, University of Tasmania, Menzies Institute for Medical Research, Hobart, Australia; Suzi Claflin, PhD, University of Tasmania, Menzies Institute for Medical Research, Hobart, Australia; Bruce TaylOR MD, $\mathrm{PhD}$, University of Tasmania, Menzies Institute for Medical Research, Hobart, Australia; Ingrid van der Mei, PhD, University of Tasmania, Menzies Institute for Medical Research, Hobart, Australia; Richard Norman, PhD, Curtin University, Perth, Australia; Gang Chen, PhD, Monash University, Melbourne, Australia; Laura Laslett, $\mathrm{PhD}$, University of Tasmania, Menzies Institute for Medical Research, Hobart, Australia; Steve Simpson-Yap, PhD, The University of Melbourne, Melbourne, Australia; Glen Henson, BEc (Hons), University of Tasmania, Hobart, Australia; Andrew Palmer, MBBS BMedSCI University of Tasmania, Menzies Institute for Medical Research, Hobart, Australia

Aims: Multiple sclerosis (MS) is a neurodegenerative disease of the central nervous system leading to increasing disability over time and reduced health-related quality of life (HRQoL). Robust health economic evidence (including health state utilities [HSUs]) is critical for cost-utility analyses to ensure efficient allocation of scarce healthcare resources. This is especially relevant for complex, chronic diseases such as MS, the most common neurological condition of young adults, affecting over 2.8 million people worldwide. Several multiattribute utility instruments (MAUIs) are available from which HSUs can be derived. We aimed to determine the preferentially sensitive MAUI(s) for people living with MS. Methods: We conducted a comprehensive HRQoL survey among Australian MS Longitudinal Study (AMSLS) participants. The survey included six MAUIs (EQ5D-5L, EQ-5D-5LPsychosocial, AQoL-8D, SF-6D versions 1/2, PROMIS/Propr), a subjective wellbeing index, sociodemographic variables, MS-related disability severity, MS disease course and COVID-19 questions. Disability was assessed with the patient determined disease steps scale (PDDS), mapped against the goldstandard Expanded Disability Status Scale (EDSS) and classified as no disability (EDSS level: 0), mild (EDSS 1-3.5), moderate (EDSS 4-6) and severe (EDSS 6.5-9.5) disability. HSUs were generated from Australian tariffs for the EQ-5D-5L, EQ-5D-5LPsychosocial, SF-6Dv1 and AQoL-8D. Ceiling and floor effects were investigated. Bland-Altman plots and Shannon's Indices were examined. Minimal important differences (MIDs) and population norms were extracted from the literature. Results: $N=2513$ AMSLS participants were invited to take part and $n=1683$ completed the survey (67\% response rate). HSUs were derived for over $97 \%$ of respondents. Mean age was 58.6 years, $80 \%$ were female, $19 \%$ reported severe disease and $63 \%$ had relapsing-remitting MS. Mean (SD) HSUs ranged from $0.45 \pm 0.29$ (SF-6Dv1) to $0.63 \pm 0.22$ (AQoL-8D). The EQ-5D-5L revealed the highest ceiling effect, with $10 \%$ of respondents reporting perfect health $(\mathrm{HSU}=1.0)$. These participants reported a mean HSU that was at least 0.09 HSU units less than perfect health for the AQoL-8D, EQ-5D-5LPsychosocial and SF6Dv1 (exceeding the MID for any MAUI). Mean HSU decreased with increasing disability severity, ranging from 0.85 (no disability) to 0.18 (severe disability). Conclusion: Preliminary analyses using Australian tariff-derived HSU suggests that the EQ-5D-5L has the least discriminatory sensitivity for people living with MS in Australia compared to other MAUIs. 
Figure 1: Flow of patients into the study

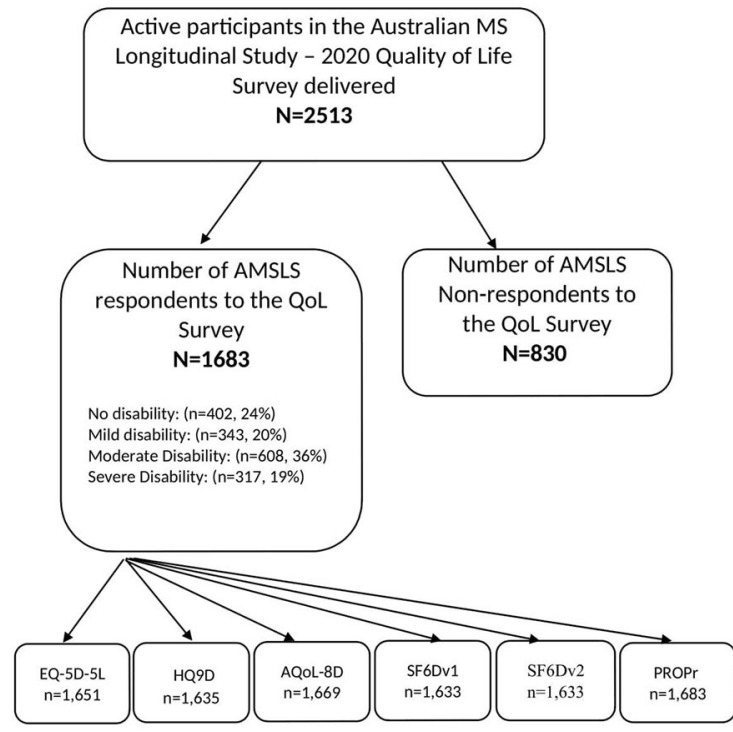

Note: No disability includes EDSS level 0, Mild includes EDSS levels 1-3.5, Moderate includes EDSS levels 4-6, and Severe includes EDSS levels 6.5-9.5. Disability status was not reported by 13 respondents.
Figure 2:Mean health state utilities for people with MS for the AQoL-8D, EQ-5D-5LPsychosocial, EQ-5D-5L and SF6D version 1 stratified by disability severity and using the Australian tariff.

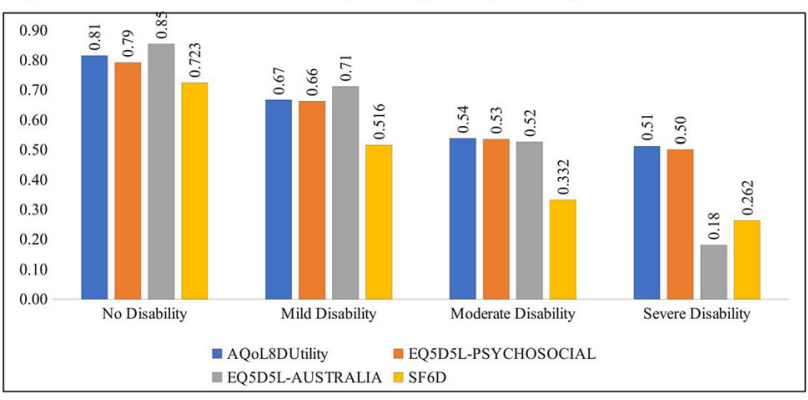

No disability includes EDSS level 0, Mild includes EDSS levels 1-3.5, Moderate includes EDSS levels 4-6, and Severe includes EDSS levels 6.5-9.5
Ltd., Shanghai, China; Xiaowen Zhang, Wuhan Neurophth Biological Technology Co.,Ltd., Wuhan, China; Kai Yoon Fan, Wuhan Neurophth Biological Technology Co.,Ltd., Shanghai, China; Linguo Li, Chinese Organization for Rare Disorders, Beijing, China; Yali Zhu, MPH, Happy Life Techonology Co.,Ltd., Beijing, China

Aims: Leber hereditary optic neuropathy (LHON) is a mitochondrial inherited optic neuropathy characterized by bilateral painless subacute failure of central vision. LHON often occurs in young males and likely impairs patients' health-related quality of life (HRQoL). This study aims to evaluate HRQoL and psychological burden in Chinese LHON patients. Methods: This is the first nation-wide cross-sectional survey among Chinese LHON patients from 28 provinces conducted between Sept. and Oct. 2020. Patients' socio-demographic characteristics were collected through online self-reported questionnaire. HRQoL were measured by EuroQol Five Dimensions Three Levels (EQ-5D-3L) utility and visual analog scale (EQ-5D-VAS) score. Psychological symptoms were measured by the total and subscale scores of Depression Anxiety Stress Scale (DASS-21), with a higher score indicating greater severity of psychological burden. Multivariable linear regression model was used to evaluate the associations of different factors (age, gender, marriage, education, employment, treatment, visual status, comorbidities, and annual household income) with HRQoL or psychological burden. Results: 539 patients (mean age $24.2 \pm 9.9$ years; males $87.9 \%$ ) were included in this study. The mean EQ-5D utility $(0.73 \pm 0.18)$ and EQ-VAS score $(60.8 \pm 28.9)$ were generally lower than those in normative (utility 0.80-0.96; EQVAS 77-86) and visual disorder population (utility 0.85 ) reported in previous studies. Overall, 354 (65.7\%), 299 (55.5\%), 298 (55.3\%), $153(28.4 \%)$, and 104 (19.3\%) reported difficulties in usual activities, anxiety/depression, mobilities, self-care, and pain/discomfort, respectively. The DASS-21 mean score was $21.5 \pm 14.3$, with 295 $(54.7 \%), 248(46.0 \%)$, and $189(35.1 \%)$ reported moderate-to-severe depression, anxiety, and stress symptoms. In multivariate analysis, unsatisfactory visual condition $(\beta=-0.147 ; \beta=5.334)$, unemployment/retirement $\quad(\beta=-0.059 ; \quad \beta=6.083), \quad$ and age $(\beta=-0.002, \beta=0.207)$ were significantly associated with lower EQ-5D utility and higher DASS-total score $(p<0.05)$. Unsatisfactory visual condition $(\beta=-11.896)$, unemployment $(\beta=-6.706)$, and non-treatment $(\beta=-11.488)$ were negatively associated with EQVAS score $(p<0.05)$. Conclusion: This study indicated that Chinese LHON patients had poor HRQoL and substantial psychological burden. We found that age, unsatisfactory visual condition, unemployment, and non-treatment were significant associate for HRQoL or psychological burden. Our findings would provide scientific evidence in guiding effective strategies to improve HRQoL of LHON patients in China.

\section{3: Theoretical and Methodological Research}

\section{(113.1) Making valid decisions using five sources of validity} evidence

Melanie Hawkins, PhD, Swinburne University of Technology, Melbourne, Australia; Christina Cheng, $\mathrm{PhD}$, Swinburne University of Technology, Melbourne, Australia; Gerald R Elsworth, PhD, Swinburne University of Technology, Melbourne, Australia; Richard H Osborne, PhD, Swinburne University of Technology, Melbourne, Australia

Aims: Validity testing, like all good science, should be guided by a theoretical rationale and evidence-based practice to determine the appropriateness, meaningfulness, and usefulness (i.e., quality or validity) of data for making decisions. Yet, measurement in health lacks widespread use of modern validity testing theory to justify data- 
based decisions. Furthermore, many existing health measurement standards and guidelines do not reflect modern views that validity is about the interpretation and use of scores, not a static property of an instrument. To claim that "the instrument is valid" dangerously ignores the fact that the instrument might be used to justify (potentially) different decisions and in (potentially) different contexts than those in which it was developed or previously tested. Hence, there is a need to provide a validity argument for the inferences drawn from the data each time an instrument is used in a different context or for a different purpose. The aim of this research is to describe the five sources of validity evidence from the Standards for Educational and Psychological Testing, the authoritative reference for validity testing, and propose these as a useful and necessary theoretical rationale for making decisions based on patient-reported outcomes (PRO) data. Methods: The Standards requires a range of evidence that is based on five sources: (1) test content, (2) response (i.e., cognitive) processes, (3) internal structure, (4) relations to other variables, and (5) the consequences of testing as related to validity. The Standards' theoretical rationale puts the onus on developers (and users) of PRO instruments to qualitatively and quantitatively test and report on aspects of validity from instrument development through to the use (or misuse) of data for specific decisions. Results: The five sources of validity evidence will be outlined and examples for different measurement purposes highlighted. Conclusion: The Standards' five sources of validity evidence provides a theoretical rationale-which is lacking and therefore necessary in PRO measurement-that promotes an argument-based approach to validation to justify the extent to which a PRO instrument generates appropriate, meaningful, and useful data that result in valid healthcare decisions.

\section{(113.2) Components of a behavioural model drive quality of life in a community of older persons}

Nancy Mayo, McGill University, Montreal, Quebec, Canada; Kedar Mate, PhD, Research Institute McGill University Health Center, Montreal, Quebec, Canada; Olayinka Akinrolie, MSc, Applied Health Science Program, University of Manitoba, Winnipeg, Manitoba, Canada; Hong Chan, MSc, Applied Health Science Program, University of Manitoba, Winnipeg, Manitoba, Canada; Ruth Barclay, $\mathrm{PhD}$, Applied Health Science Program, University of Manitoba, Winnipeg, Manitoba, Canada; submitted on behalf of "HRQL-OPAL Research Team"

Aims: There is a strong modern bias towards medicalizing aging and this is manifested in the content of current health-related quality of measures. The world-wide movement towards active aging lacks a measurement framework that could be used to evaluate the success of active aging programs, a gap that needs to be filled. The purpose of this study was to ascertain from older persons participating in an outdoor walking program, drivers of quality of life (QOL), information to be used to inform a measurement approach for older persons to engage in active living. Methods: Data collected using the Patient Generated Index (PGI) from people enrolled in the Getting Older Adult OUTdoors (GO-OUT) trial were used. The areas nominated were mapped to the World Health Organization's International Classification of Functioning, Disability and Health (ICF). Results: A total of 934 text threads were identified by 204 participants which mapped to 460 unique ICF codes. The areas nominated grouped into components of a well-known behavior change model linking capability, opportunity, and motivation to behaviors that could define active living (COM-B model). Figure 1 shows the categories nominated as classified under the components of the COM-B model. Areas related to capability were nominated the most frequently ( $42 \%$ of all nominations) with mobility the most frequently nominated (20\%) and sleep the least $(3 \%) ; 14 \%$ of all nominations related to opportunities and 5\% related to motivational factors. Four types of activities were classified as behaviors. Conclusion: The findings suggest that active living for older people could be defined in terms of physical, household, social, and cognitive activities. To realize these behaviors, people require specific capabilities, opportunities to allow these behaviors to happen, and motivational factors to initiate and sustain the behaviors. Areas nominated as relevant to QOL among a community-based sample of older persons were compatible with a behavioral change model suggesting that the COM-B model could be used as a measurement framework for developing a measure of HRQL for older persons for active living.

Figure 1: Areas of QOL Concern to Older Persons in an Active Living Program (Number \& \% of Mentions)

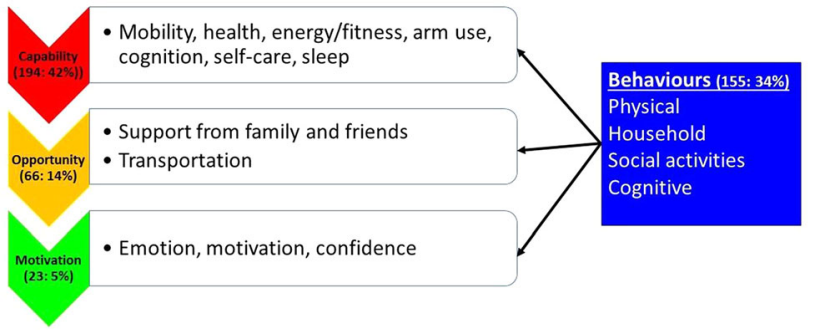

(113.3) Validity evidence regarding the use of the VR-12 and the EQ-5D-5L in emergency departments

Jae-Yung Kwon, University of Victoria, Victoria, British Columbia, Canada; Lena Cuthbertson, BC Ministry of Health Office of PatientCentred Measurement, Vancouver, British Columbia, Canada; Richard Sawatzky, Trinity Western University, Langley, British Columbia, Canada

Aims: Two generic patient-reported outcome measures (PROMs; VR12 and EQ-5D-5L) were fielded across Emergency Departments (EDs) in British Columbia (BC) to inform the selection of a PROM for understanding the health status of patients over time and across organizations. The study objectives were to use the BC PROMs data to compare the validity evidence, including their (1) distributional properties, (2) concordance, and (3) discriminant ability in differentiating levels of comorbidity burden as well as self-reported physical and emotional health status (including mental health diagnoses). Methods: This was a retrospective cohort study using the 2018 ED Sector Surveys in BC and administrative health data. Discriminant validity was assessed by comparing the extent to which the VR-12 and EQ-5D distinguish groups of patients defined by different comorbidity levels and self-reported physical and emotional health status. Multivariate logistic regression was used to evaluate the extent to which the measures distinguish mental health diagnoses. Results: The mean age of respondents $(N=5876)$ was 57.4 (SD 19.9) years with the majority being female $(57.4 \%)$. For distributional properties, the VR-12 items and summary scores of the Physical and Mental Health Scale (PCS and MCS) showed relatively more normal distribution compared to the EQ-5D items and index score (majority reported no to slight problems for all items ranging from 65 to $91 \%$ ). The VR-12 utility and EQ-5D index scores were highly correlated $(r=0.77)$, whereas the correlations of the EQ-5D with the PCS and MCS were much smaller ( $r=0.66$ and 0.48 , respectively). The VR12 PCS produced the largest effect size (ES) for discriminating comorbidity levels. ESs for discriminating self-reported physical health were largest for the EQ-5D Visual Analog Scale and the PCS, whereas the VR-12 MCS produced the largest ES for discriminating self-reported mental health (Fig. 1). Having a mental health diagnosis was predominantly distinguished by the MCS, which accounts for 
$58 \%$ of the explained variance (28\% for the EQ-5D) (Fig. 2). Conclusion: Results suggest that the EQ-5D predominantly reflects physical health status. The VR-12 PCS and MCS are recommended for distinguishing patients with different levels of comorbidity and mental health diagnoses in ED surveys.
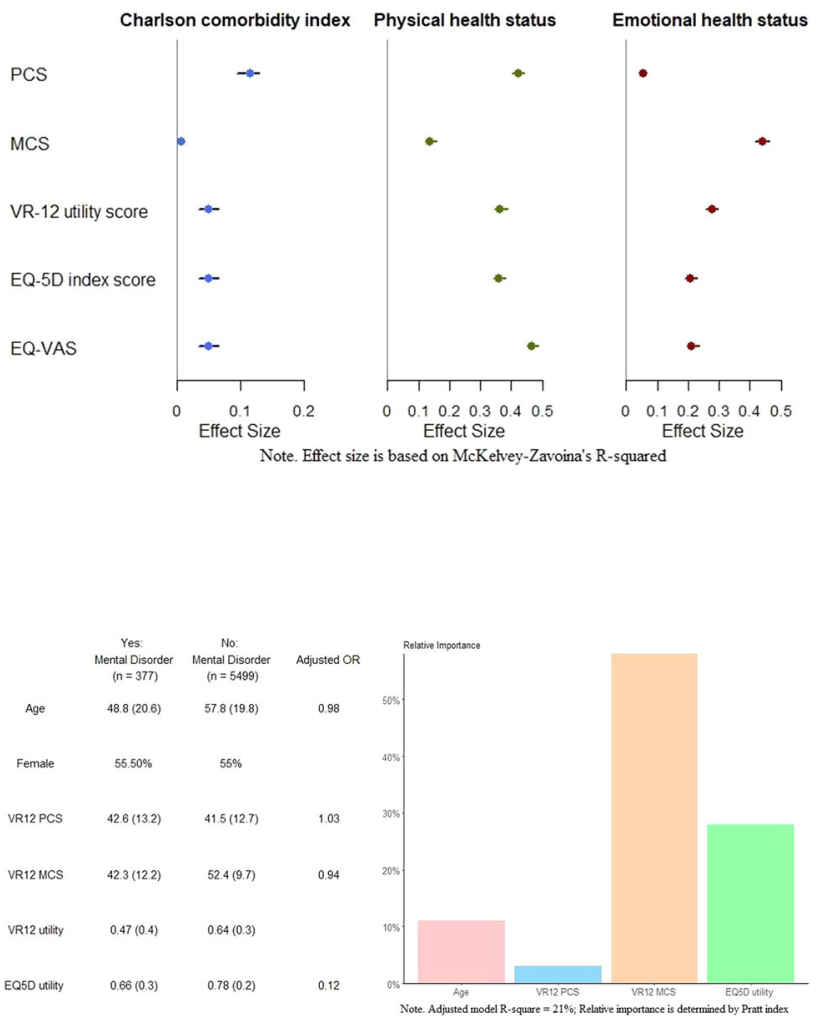

(113.4) Using an argument-based approach to examine validity evidence of the eHealth Literacy Questionnaire (eHLQ)

Christina Cheng, doctor of Philosophy, Swinburne University of Technology, Hawthorn, Australia; Gerald Elsworth, PhD, Swinburne University of Technology, Hawthorn, Australia; Richard Osborne, $\mathrm{PhD}$, Swinburne University of Technology, Hawthorn, Australia

Aims: Robust evidence of a patient-reported outcome measure (PROM) is essential to justify the use of the tool for improving healthcare. This study aimed to use an argument-based approach to validity testing by examining five sources of evidence of an eHealth literacy measurement tool, and the eHealth Literacy Questionnaire (eHLQ). The evidence was used to evaluate the interpretation and use of the scores of the eHLQ as a tool to promote access and usage of eHealth services for health equity in the Australian community health settings. Methods: An interpretation/use argument was first established to describe the intended interpretation and proposed use of the eHLQ scores. Using a mix-method approach, five sources of validity evidence including test content, response process, internal structure, relations to other variables, and consequences of testing were collected and evaluated to construct a validity argument for the eHLQ. Data were collected at three community health sites in Victoria, Australia $(n=530)$. Results: Items were understood as intended and no evidence of differential item functioning (DIF) for different administration formats was found. A 7-factor Bayesian confirmatory factor analysis model, using small-variance priors for cross-loadings and residual covariances demonstrated satisfactory fit. All items loaded well on their relevant factors (factor loading range: 0.36-0.94) with no significant cross-loadings. All scales were found to have good internal reliability (composite reliability range: $0.73-0.90$ ). However, discriminant validity among five of the seven dimensions was not clearly established. IRT analysis found no disordered threshold, and items represented a range of difficulty and good discrimination of different ability. No or ignorable DIF was found for settings, age, sex, education, and technology use. Known-group validity results were mixed due to limited strong hypotheses from previous studies. Using the eHLQ led to insights into the eHealth literacy needs of participants and generation of digital and non-digital health intervention ideas for users. Conclusion: This study demonstrated that a rigorous assessment of the interpretation and use of PROM score using an argument-based approach to validity is feasible. The evidence can be used to support decisions for choosing the appropriate PROMs for improving quality of healthcare.

\section{4: Policy Application for Different Health Conditions}

(114.1) The International Survey of People Living with Chronic Conditions (PaRIS survey): development of the conceptual framework

Ian Porter, Health Services \& Policy Research Group, University of Exeter, Exeter, UK; Mieke Rijken, Nivel (The Netherlands Institute for Health Services Research), Utrecht, The Netherlands; Oliver Groene, Optimedis A.G., Hamburg, Germany; Rosa Suñol, Fundacion Avedis Donabedian Research Institute, Barcelona, Spain; Rachel Williams, Ipsos MORI, London, UK; Jimmy Martin Delgado, Health Services \& Policy Research Group, University of Exeter, Exeter, UK; Marta Ballester, Fundacion Avedis Donabedian Research Institute, Barcelona, Spain; Laura Thomas, Ipsos MORI, London, UK; Michael van den Berg, Health Division, OECD, Paris, France; Katherine De Bienassis, Health Division, OECD, Paris, France; Candan Kendir, Health Division, OECD, Paris, France; Niek Klazinga, Health Division, OECD, Paris, France; Dolf de Boer, Nivel (The Netherlands Institute for Health Services Research), Utrecht, The Netherlands; Jose M Valderas, Health Services \& Policy Research Group, University of Exeter, Exeter, UK

Aims: The Organization for Economic Cooperation and Development (OECD) PaRIS survey aims to support countries in improving care for people living with chronic conditions by collecting information on how these people experience the quality and performance of the primary and ambulatory care services they use in their country. We present the development of the conceptual framework underpinning the survey. Methods: Starting from the OECD Health Care Quality Indicators framework (2015), we identified frameworks and conceptual models relevant to the needs of people receiving primary and ambulatory care for chronic conditions using bespoke structured searches in PubMed. A draft framework was developed though narrative review and comparison of each of the domains and subdomains in the identified frameworks with consideration of: overlap; support in the literature, minimization of the number of domains (without significantly compromising comprehensiveness); and alignment with the previous recommendations from a taskforce. An iterative process followed, based on sustained engagement with an international Patient Advisory Panel (PaRIS-PP) and supported by an international and multidisciplinary Technical Advisory Community (TAC) and oversight by a Working Party of OECD member states representatives. International virtual patient co-development workshops were organized for revisiting the scope, identifying gaps, minimizing overlap, and ensuring consistency of terminology with patient preferences. Results: 78 frameworks were identified. Each iteration of feedback from PaRIS-PP and TAC and the international patient codevelopment workshops contributed to the final framework, which 
identifies the following domains (subdomains): patient-reported outcomes (symptoms, functioning, self-reported health status, healthrelated quality of life); patient-reported experiences of care (access, comprehensiveness, continuity, coordination, safety, people-centred care (individualization of care, decision making, interacting with health professionals), self-management support, trust, overall perceived quality of care); health and health care capabilities; health behaviours (physical activity, diet, tobacco use, alcohol use); individual and sociodemographic factors (demographic, biometric, and morbidity factors; socioeconomic factors); delivery system design (clinic, main health care professional); health system design, policy, and context. Conclusion: This conceptual framework has been developed through a systematic, replicable, and inclusive process. It will support the design of a patient and a provider survey for the development and implementation of patient-reported indicators of health system performance.

\section{(114.2) Evaluating the impact of patient engagement} in transforming person-centered outcome research

Tamara McCarron, PhD, University of Calgary, Calgary, Alberta, Canada; Deborah Marshall, PhD, University of Calgary, Calgary, Alberta, Canada; Tracy Wasylak, MSc, Strategic Clinical Networks, Alberta Health Services, Calgary, Alberta, Canada; Nancy Marlett, $\mathrm{PhD}$, University of Calgary, Calgary, Alberta, Canada

Aims: We created the Patient and Community Engagement Researcher (PaCER) Program after testing the feasibility of training patients to conduct peer-led research as an approach to patient engagement. PaCER was established as a partnership between Alberta Health Services Alberta Strategic Clinical Networks ${ }^{\mathrm{TM}}$ (SCNs) and patients. We report on the impact of this innovative partnership. Methods: We evaluated impact using two approaches: (1) applying the Canadian Academy of Health Sciences (CAHS) impact framework that identified indicators for assessing research impact within five domains: (i) advancing knowledge; (ii) building capacity; (iii) informing decision making; (iv) health impacts; and (v) broad socioeconomic impacts and (2) an external evaluation, consisting of two surveys, which were independently analyzed by researchers. Results: Indicators were found within four domains of the CAHS framework: (1) advancing knowledge included grant applications, researcher's engagement, and peer-reviewed publications; building capacity included students who graduated from the training program (PaCER), patient-led research proposals, and SCNs engaged in research activities; informing decisions included individuals who served on provincial committees and on research review panels; and health impacts included quality improvement recommendations and secured research contracts. The external evaluation identified considerations to improve the overall sustainability of the program including the development of proper infrastructure, improving ongoing research efforts, and creating additional quality improvement opportunities for individuals after graduation. Conclusion: SCNs were created to improve outcomes and health care sustainability with integrated teams to provide an innovative approach to health transformation. Partnerships among patients and health systems can shift health culture. Success requires a partnership with health leaders responsible for system transformation and willingness to implement findings into practice, investment in building the knowledge and skills of patients and providing opportunities for researchers, patients, and clinicians to work productively together to support positive change in the health system.
(114.3) Second-hand tobacco smoke exposure in children: a neglected driver of health disparities and reduced HRQOL in Nigeria

Afolabi Oyapero, BDS, MPH, FMCDS, TTS., Lagos State University College of Medicine, Ikeja, Nigeria; Olubukola Olatosi, BDS, MPH, FMCDS, Dept. of Child Dental Health, Faculty of Dental Sciences, College of Medicine, University of Lagos, Lagos, Nigeria

Aims: The adverse effects of second-hand smoke (SHS) exposure has been reported in the literature, but data on SHS exposure among children in Nigeria are virtually non-existent. We estimated the prevalence of SHS exposure to tobacco among school children in Nigeria and to assess its association with Child-HRQOL. Methods: Data from three school-based descriptive studies in Lagos, Nigeria were utilized and socioeconomic characteristics and smoking behaviour in the households were obtained using validated questionnaires. Parental and relatives smoking habit were utilized as the indicators of SHS exposure. Child HRQOL was determined with the Pediatric Quality of Life Physical Functioning Scale (PedsQL ${ }^{\mathrm{TM}}$ ). Prevalence rates were calculated and chi-squared tests, ANOVA and logistic regression analysis were conducted to determine significant associations between SHS exposure and HRQoL. Significance was set at $p<0.05$. Results: Within the preceding month, 37.6\% of the children were regularly exposed to SHS; $15.5 \%$ had parents and family members who smoked tobacco indoors while $7.9 \%$ were daily exposed to SHS; $19.7 \%$ of the children daily experienced cough, nasal congestion, throat, or eye irritation. Compared with unexposed children $(78.0 \pm 6.0)$, those regularly exposed $(66.0 \pm 8.0)$ had significantly lower mean HRQOL scores. In the regression analysis, the likelihood of SHS exposure was significantly higher in those with parents that smoke regularly (OR 2.62; 95\% CI 1.50-5.27; $p=0.042$ ), lower levels of paternal education (OR 1.72; 95\% CI $0.61-5.27 ; p=0.021)$ who live in cramped accommodations (OR $1.79 ; 95 \%$ CI $1.02-5.67 ; p=0.044)$ and those from households with $\geq 5$ children (OR 1.51; 95\% CI 0.90-2.56; $p=0.003$ ). HRQOL scores were lower among SHS-exposed children and significant for nasal congestion (OR 1.83; CI 1.25-3.42) and cough (OR 2.37; CI 1.77-4.13) in them. Conclusion: There was significant association between Child-HRQOL and regular exposure to SHS, especially among those with illiterate parents, with large families who live in cramped accommodations. Tobacco control policies should emphasize protection of children through the adoption of smoke-free homes and increase parental awareness on the impact of SHS exposure on child HRQOL.

\section{(114.4) Development of consumer-reported outcome measure (CROM) standards for the tobacco industry with respect to psychometric CROM using a consortium-based approach: methodology and scope}

Esther Afolalu, PhD, Philip Morris International R\&D, Neuchatel, Switzerland; Stacey McCaffrey, JUUL Labs, Inc, Washington DC, District Of Columbia, USA; Ryan Black, JUUL Labs, Inc, Washignton DC, District Of Columbia, USA; Christelle Chrea, Philip Morris International R\&D, Neuchatel, Switzerland; Geoffrey Curtin, RAI Services Company, Winston-Salem, North Carolina, USA; Lesley Giles, Japan Tobacco International, Geneva, Switzerland; Daisuke Nishihara, Japan Tobacco International, Geneva, Switzerland; Krishna Prasad, British American Tobacco, London, UK; Mandara Shetty, British American Tobacco, London, UK; submitted on behalf of "CORESTA CROM Task Force WG02"

Aims: In November 2018, CORESTA approved the formation of a new Task Force (TF) to establish best practices and guidelines for the 
integration of consumer-reported outcome measures (CROM) in tobacco regulatory research. The primary objective of the CROM TF is to provide guidance on how to identify, develop, and validate CROM and to provide access to CROM for evaluating tobacco and nicotine-containing products for pre-market and post-market purposes. Here, we describe the research completed by one of the TF working groups, WG02, with the purpose of developing CROM standards for tobacco regulatory research with respect to psychometric CROM (i.e., CROM intended to measure underlying psychological attributes-e.g., reinforcing effects). Methods: WG02 comprises 11 researchers from the CROM TF, representing 7 different tobacco companies and with experience in psychometrics, patient-reported outcomes (PRO), survey methodology, and product use behavior. WG02 members collaboratively drafted an operational definition of psychometric CROM and devised an approach for developing the standards. Results: Consistent with approaches taken by other outcomes research organizations, WG02 adopted a consensus-based approach for drafting the standards, which includes (1) review of literature and (2) an iterative peer-review process leveraging various sources, such as conference presentation and review by subject matter experts from tobacco, PRO, and related fields. Through literature review and WG02 member input, the scope was defined and initial content for the 5 components of the standards was drafted. These components include: (1) appropriate content, (2) development process and validation, (3) adaptation/modification of existing CROM, (4) application/implementation and interpretation of CROM, and (5) linguistic/cultural translation. Conclusion: This research represents the development of draft standards for psychometric CROM for tobacco regulatory research, with the intention to complement the 2020 FDA draft guidance issued by the Food and Drug Administration Center for Tobacco Products. The initial draft content is being disseminated for peer review to gather feedback.

\section{5: Cancer Methods for Different Populations}

\section{(115.1) Can missing PRO data be handled appropriately by last observation carried forward (LOCF)?}

Lene Nielsen, Quality of Life Research Center, Department of Haematology, Odense University Hospital \& Research Unit for Multimorbidity, Department of Internal Medicine and Cardiology, Viborg Regional Hospital, Viborg, Denmark, Odense, Denmark; Rebecca Mercieca-Bebber, PhD, Research Fellow, NHMRC Clinical Trials Centre, University of Sydney, Sydney, Australia; Sören Möller, Statistician, PhD, OPEN, Open Patient data Explorative Network, Odense University Hospital \& Department of Clinical Research, University of Southern Denmark, Odense, Denmark; Louise Redder, MD, PhD student, Quality of Life Research Center, Department of Haematology, Odense University Hospital, Odense, Denmark; Mary Jarden, professor Department of Haematology, Copenhagen University Hospital, Copenhagen, Copenhagen, Denmark; Christian Lykkegaard Andersen, MD, professor Department of Haematology, Copenhagen University Hospital, Copenhagen \& Research Unit for General Practice and Section of General Practice, Department of Public Health, University of Copenhagen, Copenhagen, Denmark; Henrik Frederiksen, professor Quality of Life Research Center, Department of Haematology, Odense University Hospital, Odense, Denmark; Henrik Gregersen, MD, PhD, Department of Haematology, Aalborg University Hospital, Aalborg, Denmark; Anja Klostergaard, MD, Department of Haematology, Aarhus University Hospital, Aarhus, Denmark; Morten Saaby Steffensen, MD, Department of Haematology, Regional Hospital West Jutland, Holstebro, Denmark; Per Trøllund Pedersen, MD, Department of Haematology, South West Jutland Hospital, Esbjerg, Denmark; Maja Hinge, MD, PhD,
Department of Haematology, Vejle Hospital, Vejle, Denmark; Mikael Frederiksen, MD, Department of Haematology, Hospital of Southern Jutland, Aabenraa, Denmark; Bo Amdi Jensen, MD, Department of Haematology, Zealand University Hospital, Roskilde, Denmark; Carsten Helleberg, MD, Department of Haematology, Copenhagen University Hospital, Copenhagen, Denmark; Anne Kærgaard Mylin, $\mathrm{MD}, \mathrm{PhD}$, Department of Haematology, Copenhagen University Hospital, Copenhagen, Denmark; Niels Abildgaard, MD, professor Quality of Life Research Center, Department of Haematology, Odense University Hospital, Odense, Denmark; Madeleine King, professor University of Sydney, Faculty of Science, school of Psychology \& Quality of Life Research Center, Department of Haematology, Odense University Hospital, Sydney, Australia

Aims: Non-response (NR) to patient-reported outcomes (PRO) questionnaires can lead to loss of study power, reduced precision and bias if not appropriately handled. Last observation carried forward (LOCF) is a simple strategy for handling missing data, but may lead to bias if NRs are missing not at random. This study aimed to investigate the relationship between reasons for intermittent NR and LOCF. Methods: Data originated from the ongoing survey "Quality of Life in Danish Multiple Myeloma patients", where patients with multiple myeloma complete EORTC QLQ-C30, QLQ-MY20, QLQCIPN20 and SF12v2 questionnaires, totaling 22 domains, at enrolment and at 12 pre-scheduled follow-up time points. Questionnaires completed within seven days of the scheduled date are considered "on-time responses"; otherwise they are defined as NR and a study nurse contacts the patient, document the reason for NR from a list and invite the patient to complete the questionnaire (denoted "salvage response"). Data were analysed with linear regression with clustered standard errors. In total and divided by reasons for NR, the differences between LOCF and salvage scores were estimated. Differences with $p<0.05$ were considered significant. Results: By December 31, 2020, the 619 included patients had completed 5,829 of the 6,074 (96\%) scheduled follow-up questionnaires, of which 618 (11\%) were salvage responses. Total mean salvage scores were significantly worse than the LOCF mean score in 5/22 domains. Divided into reason for $\mathrm{NR}$, the mean salvage scores due to hospital admission, or mental or physical reason were significantly worse than the LOCF mean score in $11 / 22$ domains. Mean salvage score due to technical difficulties/procedural errors was significantly better in $1 / 22$, and mean salvage scores due to overlooked/forgotten questionnaires were significantly worse in $3 / 22$ domains. Conclusion: NRs due to hospital admission, or mental or physical reasons were missing not at random for $50 \%$ of the domains, so LOCF would cause bias if used to handle missing PRO data. LOCF was largely suitable for handling missing PRO data due to technical difficulties/procedural errors or overlooked/forgetting to complete, as only few domains had a significantly different mean salvage score compared to the LOCFimputed value.

\section{(115.2) The Use of BWS, DCE, SG, and TTO in children} and adolescents: A case study of cancer patients and survivors

Minghui Li, PhD, University of Tennessee Health Science Center, Memphis, Tennessee, USA; Pingyu Chen, PhD, University of Tennessee Health Science Center, Memphis, Tennessee, USA; I-Chan Huang, PhD, St. Jude Children's Research Hospital, Memphis, Tennessee, USA

Aims: Different direct preference-based measures of quality of life (QoL), including the best-worst scaling (BWS), discrete choice experiment (DCE), standard gamble (SG), and time trade-off (TTO) have been tested and used in various adult populations. However, little is known about the feasibility of implementing these measures in 
children and adolescents. This study aimed to examine the feasibility of implementing the BWS, DCE, SG, and TTO methods in pediatric cancer patients and survivors. Methods: This study included a random sample of 440 cancer patients and survivors aged 8-12 years (children) and 13-18 years (adolescents) who were recruited from a community-based survey panel. After receiving consent from their parents or guardians, eligible individuals were invited to complete an online survey. Each participant was assigned to one of the methods (BWS, DCE, SG, or TTO) based on quota sampling in five characteristics (patient/survivOR age, sex, race/ethnicity, and cancer diagnosis). Each measure contained 10 randomly ordered tasks. The feasibility was evaluated by perceived difficulty to complete the task (a Likert-type scale) and the logic issues (defined as selecting the same response in all tasks). Kruskal-Wallis tests were used to compare perceived difficulties and logic issues among BWS, DCE, SG, and TTO methods. Results: Among participants, 227 (51.6\%) were children and $213(48.4 \%)$ were adolescents. A higher percentage of BWS participants reported difficulty in completing the tasks, compared to the DCE, SG, and TTO participants (12.9\% vs. 20.2 vs. $21.3 \%$ vs. $18.7 \%, \mathrm{p}=0.025)$. Among BWS participants, a higher percentage of cancer survivors reported difficulty in completing the task, compared to cancer patients $(16.0 \%$ vs. $10.2 \%, p=0.002)$. In addition, a higher percentage of BWS participants had logic issues, compared to DCE, SG, and TTO participants ( $0.9 \%$ vs. $25.5 \%$ vs. $19.1 \%$ vs. $23.6 \%, p<0.001)$. No other differences were observed across different characteristics in BWS participants ( $p$ 's $>0.05$ ). Conclusion: Among direct measures of QoL, pediatric cancer patients and survivors demonstrated the BWS as the least difficult method to use and possessed the fewest logic issues compared to the DCE, SG, and TTO methods. BWS is a promising direct measure for assessing QoL in pediatric cancer populations.

(115.3) Change in a single-item indicator of treatment tolerability in cancer is associated with early treatment discontinuation

John Peipert, PhD, Northwestern University, Chicago, Illinois, USA; Fengmin Zhao, PhD, Dana-Farber Cancer Institute, Boston, Massachusetts, USA; Ju-Whei Lee, MS, Dana-Farber Cancer Institute, Boston, Massachusetts, USA; Fanxgin Hong, PhD, Dana-Farber Cancer Institute, Boston, Massachusetts, USA; Shu-En Shen, MS, Northwestern University Feinberg School of Medicine, Chicago, Illinois, USA; Edward Ip, PhD, Wake Forest School of Medicine, Winston-Salem, North Carolina, USA; Nathaniel O'Connell, PhD, Wake Forest School of Medicine, Wake Forest, North Carolina, USA; Noah Graham, MS, Dana-Farber Cancer Institute, Boston, Massachusetts, USA; Mary Lou Smith, JD, MBA, Research Advocacy Network, Chicago, Illinois, USA; Ilana Gareen, PhD, Brown University Center for Statistical Sciences, Providence, Rhode Island, USA; Ruth Carlos, MD, MS, University of Michigan Comprehensive Cancer Center, Ann ArobOR Michigan, USA; Samilia Obeng-Gyasi, MD, MPH, The Ohio State University Comprehensive Cancer Center, Columbus, Ohio, USA; Shaji K. Kumar, MD, Division of Hematology, Mayo Clinic, Rochester, Minnesota, USA; Kathy Miller, MD, Indiana University Comprehensive Cancer Center, Indianapolis, Indiana, USA; Ann Partridge, MD, MPH, Dana Farber Cancer Institute, Boston, Massachusetts, USA; Tait Shanafelt, MD, Stanford School of Medicine, Stanford, California, USA; Joseph A. Sparano, MD, Albert Einstein Cancer Center, Bronx, New York, USA; Keith Stewart, MBChB, MRCP, FRCPC, MBA, Princess Margaret Cancer Centre, Toronto, Ontario, Canada; Ahmad Tarhini, MD, PhD, Moffitt Cancer Center, Tampa Bay, Florida, USA; Mary Thomas, U.S. Department of Veterans Affairs, Washington DC, District of Columbia, USA; Mattias Weiss, MD, PhD, ThedaCare Cancer Center, Appleton, Wisconsin, USA; David Cella, Northwestern University
Feinberg School of Medicine, Chicago, Illinois, USA; Robert Gray, Harvard TH Chan School of Public Health, Boston, USA; Lynne I. Wagner, Wake Forest School of Medicine, Winston Salem, North Carolina, USA

Aims: To amplify the patient's voice in tolerability assessment for cancer trials, we must include patient reports in addition to clinicianassessed adverse events (AE). One promising approach is using a single item from the Functional Assessment of Cancer Therapy (FACT) system, GP5: "I am bothered by side effects of treatment." We examined whether increases in side effect bother per GP5 during the early cycles of 3 ECOG-ACRIN clinical trials were associated with subsequent early treatment discontinuation (ETD) due to AEs in the induction treatment setting. Methods: Data from the induction step of 3 phase III trials were used: ECOG-ACRIN E1A06 (multiple myeloma), E1912 (chronic lymphocytic leukemia), and E1A11 (multiple myeloma). In each trial, GP5 was administered at baseline and an "early" post-baseline timepoint: E1A06 $=7$ months postbaseline; $\mathrm{E} 1912=3$ months; $\mathrm{E} 1 \mathrm{~A} 11=5.5$ months. GP5 has 5 response options: "Not at all" (0), "A little bit" (1), "Somewhat" (2), "Quite a bit" (3), "Very much" (4). We calculated change in GP5 as the difference between baseline and post-baseline responses, which was then categorized as: $>1$ category increase (yes vs. no) $;>2$ category increase (yes vs. no). We used unadjusted Cox regression models to estimate the hazard ratio (HR) of ETD (GP5 change yes vs. no). The event for these models was completing less than the prespecified number of treatment cycles due to an AE. Patients were followed until they exited the study or to the end of induction. Results: In E1A06, increase of $>1$ [HR 1.49 (95\% CI 0.89, 2.49)] and $>2$ GP5 response categories (HR $1.72(0.98,3.01)$ was as positively associated with ETD. (Figure) In E1912, these effects were $>1$ category HR $2.60(95 \%$ CI $1.49,4.53)$ and $>2$ category HR $2.22(1.22,4.05)$. Finally, in E1A11, these effects were $>1$ category HR $2.01(95 \%$ CI 1.18, 3.44) and > 2 category HR $2.33(1.52,3.61)$. Conclusion: Increase in GP5-indicated side effect bother early postbaseline may be an efficient way to indicate cancer treatment tolerability. It may have specific utility for signaling patients who experience early, severe toxicity impact and who are at risk for not completing treatment.

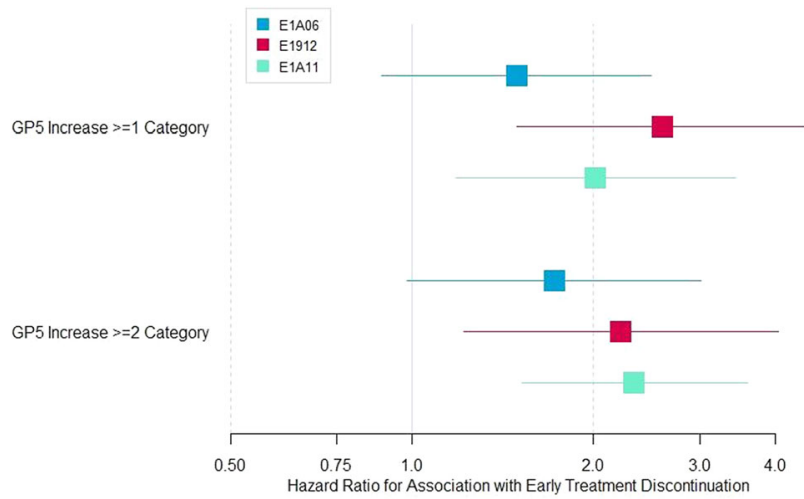

(115.4) Stakeholder perceptions of key aspects of high-quality cancer care to assess with Patient-reported Outcomes: a systematic review

Rachel Kurtzman, MSPH, Department of Health BehaviOR University of North Carolina at Chapel Hill, Chapel Hill, North Carolina, USA; Jennifer Bissram, MLS, Health Sciences Library, University of North Carolina at Chapel Hill, Chapel Hill, North Carolina, USA; Kea Turner, PhD, MPH, MA, Moffitt Cancer Center, Tampa, Florida, USA; Thomas Atkinson, PhD, Memorial Sloan Kettering Cancer 
Center, New York, New York, USA; Jennifer Jansen, MPH, Lineberger Comprehensive Cancer Center, Chapel Hill, North Carolina, USA; Philip Carr, MPH, Lineberger Comprehensive Cancer Center, Chapel Hill, North Carolina, USA; C. Tyler Ellis, MD, University of Louisville, Louisville, Kentucky, USA; Ashley Freeman, MD, FRCPC, British Columbia Cancer Agency, Vancouver, British Columbia, Canada; Ethan Basch, MD, Lineberger Comprehensive Cancer Center, Chapel Hill, North Carolina, USA; Angela Stover, $\mathrm{PhD}$, Department of Health Policy and Management, University of North Carolina at Chapel Hill, Chapel Hill, North Carolina, USA

Aims: We conducted a systematic review to identify areas of cancer care delivery that are important to patients and other stakeholders when considering quality of care and are amenable to performance evaluation with patient-reported outcome measures (PROMs). Methods: Standard review methodology was used, consistent with PRISMA (Preferred Reporting Items for Systematic Reviews and Meta-Analyses). MEDLINE/PubMed, EMBASE, and the Cochrane Library were searched to identify relevant studies through August 2020 (no beginning date was specified). Two independent coders reviewed abstracts and double-coded full text of potentially eligible articles. Conflicts were resolved by a third coder. Eligible studies involved key-stakeholder perceptions of what constitutes high-quality care for cancer, including symptoms, toxicities, physical function, and other similar topics. Stakeholders included cancer patients, caregivers, clinicians, and healthcare leaders. We excluded studies that used pediatric/adolescent populations, examined efficacy of treatment regimens, comparative effectiveness, or studies using existing PROMs (without mention of stakeholder perceptions). Results: Systematic searches yielded 1,813 articles after de-duplication. Of those, 1,310 articles were coded as irrelevant during abstract review, and 467 were excluded during full-text review, leaving 36 articles for extraction. The patient perspective was represented in nearly all studies (30/36 studies), 20/36 studies were restricted to patients, and $10 / 36$ studies assessed perspectives of both patients and another group (clinicians or family members). Interviews were the most common form of data collection. Half of studies (18/36) were conducted outside the USA (mostly in Europe). Additionally, half of eligible articles were published between 2016 and 2020, indicating increasing work in this area over time. Important aspects of high-quality cancer care that can be assessed with PROMs included specific physical symptoms (e.g., nausea, fatigue, gastrointestinal issues, pain, sleep issues) and psychosocial symptoms (e.g., anxiety, depression). Other important aspects included symptom management, social functioning, financial toxicity, and quality of life. Articles also mentioned satisfaction with care, which are already commonly collected as a performance measure. Conclusion: This systematic review showed that patients and other stakeholders perceive high-quality cancer care to be adequate control of physical and psychosocial symptoms. Results were used to inform feasibility testing of PROMs as performance measures in six U.S. cancer centers.

(115.5) Adoption of the Patient-Reported Outcomes Version of the Common Terminology Criteria for Adverse Events (PROCTCAE) and Arising Descriptive Methods in Cancer Clinical Trials: A Landscape Review and Illustration with a Multiple Myeloma Study

Laurie Eliason, MPH, GlaxoSmithKline, Upper Providence, Pennsylvania, USA; Boris Gorsh, GlaxoSmithKline, Upper Providence, Pennsylvania, USA; Sandhya Sapra, GlaxoSmithKline, Upper Providence, Pennsylvania, USA; Angély Loubert, Modus Outcomes, Lyon, France; Kristin Creel, Modus Outcomes, Lyon, France; Randy Davis, GlaxoSmithKline, Upper Providence, Pennsylvania, USA; Linda Nelsen, GlaxoSmithKline, Upper Providence, Pennsylvania,
USA; Anna Cardellino, GlaxoSmithKline, Upper Providence, Pennsylvania, USA; Antoine Regnault, Modus Outcomes, Lyon, France

Aims: The PRO-CTCAE is a patient-reported outcome measure of symptomatic toxicity for use in oncology trials, designed to complement the physician's grading tool for adverse events (AEs), CTCAE criteria. PRO-CTCAE uptake and use have not been uniformly assessed; understanding how to best analyze and present PROCTCAE data is important. We reviewed literature on PRO-CTCAE use recommendations and clinical trials reporting the PRO-CTCAE to ascertain its use and analytical methods to inform future analyses. Methods: A review of the literature and ongoing oncology trials using PubMed, oncology and outcomes research conferences, and ClinicalTrials.gov was completed in November 2020. Selected literature included guidance on item selection, analytical methods, and PROCTCAE endpoint use in clinical trials. Analytical and visualization methods from the literature informed descriptive PRO-CTCAE analyses conducted on data from the DREAMM-2 (NCT03525678) trial of single-agent belantamab mafodotin (BLENREP), a B-cell maturation antigen-binding antibody-drug conjugate, in relapsed/refractory multiple myeloma. Results: After screening $(n=197), 45$ articles were reviewed: 13 related to recommendations for use/analysis, 7 to item selection, and 25 to examples of PRO-CTCAE use. Overall, 118 completed/ongoing trials reported PRO-CTCAE use. Most $(92 / 118,78 \%)$ trials included the measure as a secondary endpoint. The number of trials using the PRO-CTCAE increased from 3 in 2015 to 30 in 2020. PRO-CTCAE data in the literature were reported descriptively using tables (maximum post-baseline ratings, mean change over time, ratings higher than a predefined cutoff) and graphs (line graphs, stacked bar charts, heatmaps). Descriptive analyses were applied to DREAMM-2 data to illustrate patient-reported AEs in different groups at one time point or dynamics over time, and production of an aggregated summary of each patient-reported $\mathrm{AE}$ occurrence throughout follow-up, controlled or not controlled for baseline ratings. Conclusion: The importance of tolerability and symptomatic AEs for patients is increasingly recognized in oncology, particularly for new therapies. This landscape review demonstrates recent growth in interest and adoption of PRO-CTCAE. Furthermore, this research increases understanding of existing methods and further evaluates ways of analyzing and presenting PRO-CTCAE data over time. Additional research exploring innovative methods to analyze PRO-CTCAE data and address its challenges is needed.Funding Source: GlaxoSmithKline (Study 212225).

\section{Oral Briefs}

\section{1: Clinical Research for Different Health Conditions}

(B201.1) Content validity and measurement properties of the PROFAD-SSI-SF: A disease-specific patient-reported outcome measure for patients with primary Sjögren's Syndrome

Kimberly Raymond, QualityMetric, Johnston, Rhode Island, USA; Stephen Maher, QualityMetric, Johnston, Rhode Island, USA; Cory Saucier, QualityMetric, Johnston, Rhode Island, USA; Meaghan O'ConnOR QualityMetric, Johnston, Rhode Island, USA; Aaron Yarlas, QualityMetric, Johnston, Rhode Island, USA; Mark Kosinski, QualityMetric, Johnston, Rhode Island, USA; Wen-Hung Chen, GlaxoSmithKline, Collegeville, Pennsylvania, USA; Kerry Gairy, GlaxoSmithKline, Brentford, Middlesex, UK

Aims: Primary Sjögren's Syndrome (pSS) is a systemic autoimmune disease characterized by mucosal tissue dryness (e.g., ocular, oral). The Profile of Fatigue and Discomfort-Sicca Symptoms Inventory Short Form (PROFAD-SSI-SF) is a 19-item patient-reported outcome 
(PRO) measure developed to assess pain, fatigue, and dryness in patients with pSS. This analysis aimed to evaluate the content validity and measurement properties of the PROFAD-SSI-SF. Methods: Qualitative and quantitative analyses (GSK Studies 208,396 and 213,253 ) were used to evaluate the content validity and measurement properties of the PROFAD-SSI-SF. The qualitative analysis included: (1) secondary analysis of data from an online concept elicitation (CE) discussion forum with 46 patients with pSS to inform disease model development; (2) telephone interviews with 6 key opinion leaders (KOLs) to confirm the conceptual model; and (3) cognitive debriefing (CD) telephone interviews with 20 patients with pSS to explore patient understanding and relevance of the PROFAD-SSI-SF items. The quantitative analysis included post-hoc analysis of data from a Phase 2 randomized, double-blind, placebo-controlled study (NCT02631538) in patients with pSS to evaluate measurement properties of the PROFAD-SSI-SF. Results: Per the CE forum, sicca (ocular, 74\% [ $n=34]$; oral, $87 \%$ [ $n=40]$; cutaneous, 37\% [ $n=17]$; vaginal, $24 \%[n=11]$; nasal, $15 \%[n=7]$; otic, $7 \%[n=3])$, fatigue $(87 \%[n=40])$, and pain $(89 \%[n=41])$ were the most commonly reported symptoms, affecting almost all aspects of functioning and well-being. This finding highlights the importance of assessing these concepts to evaluate disease burden and treatment benefit in patients with pSS. A disease model was developed based on CE findings; KOLs confirmed its accuracy and comprehensiveness. Participants in the CD interviews described the PROFAD-SSI-SF items as easily understood $(100 \%, n=19 / 19)$, with $14 / 19$ items considered relevant by $\geq 18 / 20$ participants $(\geq 90 \%$ ). The quantitative study confirmed acceptable fit of the factor structure of the PROFAD-SSI-SF, as well as good internal consistency, construct validity, and responsiveness to change in patients with pSS. Conclusion: Findings from this study support the content validity and measurement properties of the PROFAD-SSI-SF, a fit-for-purpose PRO measure appropriate for use among patients with pSS in clinical trials supporting drug development.Study funding: GSK.

(B201.2) Improving the structural validity of legacy instruments in common hand conditions: exploratory factor analysis, structural equation modelling, and Rasch measurement analysis of the QuickDASH in Dupuytren's disease and carpal tunnel syndrome

Conrad Harrison, University of Oxford, Oxford, UK; Paul Stirling, Queen Margaret Hospital, Dunfermline, UK; Jane McEachan, Queen Margaret Hospital, Dunfermline, UK; Jeremy Rodrigues, Warwick Clinical Trials Unit, Warwick Medical School, University of Warwick, Coventry, UK

Aims: The shortened version of the Disabilities of the Arm, Shoulder, and Hand (QuickDASH) is one of the most commonly used patientreported outcome measures (PROMs) in hand conditions, but it was not developed to contemporary psychometric standards. By assuming its ordinal scores behave as continuous and unidimensional data, clinically relevant changes may not be detected, or implied incorrectly. We aimed to evaluate and improve the structural validity of the QuickDASH in two of the most common hand conditions: Dupuytren's disease and carpal tunnel syndrome (CTS), using exploratory factor analysis (EFA), structural equation modelling (SEM), and Rasch measurement theory (RMT). Methods: We separately analyzsd the preoperative QuickDASH responses of 750 patients undergoing surgery for Dupuytren's disease and 1916 patients undergoing surgery for CTS at a single centre. EFA was carried out in R, with weighted least-squares regression and Oblimin rotation. For each condition,
SEM was performed in two, independent samples of 200 randomly selected participants. Model fit was assessed using the X2 test, comparative fit index (CFI), Tucker-Lewis index (TLI), root-meansquare error of approximation (RMSEA) and standardized root-meansquare residuals (SRMR). Informed by this, relevant items were retained and fitted to the Rasch model. Close or disordered response thresholds were collapsed and RMT analysis was repeated. Itemperson plots and general additive models were used to determine the appropriateness of item targeting. Results: EFA and SEM suggested a two-factor structure, in both conditions, with items 1-6 measuring 'function' and items 9-11 measuring 'hand symptoms' (for Dupuytren's disease: $X^{2} p$ value 0.243 , CFI 1.000, TLI 0.999, RMSEA 0.027, SRMR 0.050; for CTS: $X^{2} p$ value 0.167, CFI 0.999, TLI 0.999, RMSEA 0.032, SRMR 0.046). In the Dupuytren's cohort, the two most negative response thresholds for each item were close or disordered. After collapsing these response options, the 'function' items demonstrated good Rasch model fit. CTS responses demonstrated Rasch fit without modification. Item targeting was more appropriate for CTS than Dupuytren's disease. Conclusion: We propose a new scoring system for QuickDASH 'function' items (1-6), that provides better-quality measurement of hand function in patients with Dupuytren's disease and CTS.

(B201.3) Satisfaction with treatment in patients with coronary artery disease following coronary revascularization

Mubasiru Lamidi, University of Calgary, Calgary, Alberta, Canada; Meng Wang, University of Calgary, Calgary, Alberta, Canada; Danielle A. Southern, University of Calgary, Calgary, Alberta, Canada; Oluwagbohunmi Awosoga, University of Lethbridge, Calgary, Alberta, Canada; Matthew T. James, University of Calgary, Calgary, Alberta, Canada; Bryan Har, University of Calgary, Calgary, Alberta, Canada; Colleen M Norris, University of Alberta, Calgary, Alberta, Canada; Stephen B. Wilton, University of Calgary, Calgary, Alberta, Canada; Tolulope T. Sajobi, University of Calgary, Calgary, Alberta, Canada

Aims: Coronary artery bypass graft (CABG) and percutaneous intervention (PCI) are widely used revascularization procedures for managing coronary artery disease (CAD). Current recommendations regarding the optimal treatment options are largely based on clinical outcomes with limited considerations for patients' preferences and post-treatment satisfaction with care. This study evaluates differences in patient-reported satisfaction with treatment among patients who had coronary revascularization. Methods: Data are from the Alberta Provincial Project on Outcomes for Coronary Heart Disease $(\mathrm{APPROACH})$ registry, a population-based registry of individuals with heart disease in Alberta, Canada. Our cohort consists of adult patients with CAD who received a coronary revascularization procedure. Satisfaction with treatment was measured using the treatment satisfaction subscale of the 19-item Seattle Angina Questionnaire at two weeks and 1 year following coronary angiogram. Differences in patients' demographic and clinical characteristics of patients who received either treatment procedure were evaluated using analysis of variance or chi square tests. Mixed-effects regression analysis with inverse probability of treatment weighing based on a propensity score was used to evaluate the association between type of revascularization procedure and change in patient-reported satisfaction with treatment over a 1-year follow-up. Results: Of the 1456 patients included in this analysis, 331(22.7\%) were treated with CABG, 260 (17.9\%) were females, and $707(48.6 \%)$ had acute coronary syndrome. Patients who received either $\mathrm{CABG}$ or PCI differed with respect to disease indi- 
cation $(p<0.001)$, age $(p<0.01)$, sex $(p<0.02)$, smoking status $(p<0.02)$, ejection fraction $(p=0.01)$, comorbid hypertension $(p<0.01)$, diabetes $(p<0.01)$, and dyslipidemia $(p<0.04)$. Propensity-weighted analysis revealed that individuals who had CABG expressed significantly lower satisfaction than those who had PCI following revascularization at baseline (2 week). But CABG patients reported significantly large improvement in perceived satisfaction than PCI patients over the one-year period. Conclusion: Patient treated with CABG expressed significantly larger improvement in perceived satisfaction with their treatment than those who received PCI over a one-year period. This highlights the need for heart teams and other care providers to consider patients' satisfaction as key outcome, in addition to other clinical outcomes, when evaluating optimal revascularization procedure for managing CAD.

(B201.4) Shorter treatment duration could benefit patientreported outcomes in patients with chronic hepatitis $\mathbf{C}$ infection

Nancy Reau, Rush University Medical Center, Chicago, Illinois, USA; Wei-Han (Wendy) Cheng, AbbVie Inc., North Chicago, Illinois, USA; Oscar Hayes, AbbVie Inc., North Chicago, Illinois, USA; Steve Marx, AbbVie Inc., North Chicago, Illinois, USA; Carol Bao, AbbVie Inc., North Chicago, Illinois, USA; Jordan Feld, Toronto Centre for Liver Disease, Toronto, Ontario, Canada

Aims: Glecaprevir/pibrentasvir (G/P) is the first 8-week pan-genotypic treatment approved for all treatment-naïve patients with chronic hepatitis $\mathrm{C}$ virus $(\mathrm{HCV})$ without or with compensated cirrhosis. Treatment of HCV with G/P is associated with improved patientreported outcomes (PROs). We compared PRO scores and proportion of patients achieving meaningful clinically important difference (MCID) in treatment-naïve, non-cirrhotic HCV patients treated with G/P for 8 vs 12 weeks. Methods: This post-hoc study included pooled PRO data from 7 registrational G/P trials, of all non-cirrhotic randomized patients with $\mathrm{HCV}$, on $\geq 1$ dose of $\mathrm{G} / \mathrm{P}$ and had a baseline and $\geq 1$ post-baseline efficacy assessment. Patients were categorized into 2 groups: G/P treatment for 8 weeks and for 12 weeks. PROs were collected at baseline, Week 4, end of treatment (8 or 12 weeks), and post-treatment Week 12. PROs included Short Form 36 Health Survey (SF-36) measured as Physical Component Summary (PCS), Mental Component Summary (MCS) and 8 domain scores, and the Fatigue Severity Scale (FSS) questionnaire. Mean PRO scores were calculated in patients on G/P for 8 and 12 weeks. Proportion of patients achieving MCID at specific visits for each PRO was also determined. Differences between groups were evaluated using t tests and chi-square tests for continuous and categorical variables, respectively. Results: A total of 2,171 patients (852 and 1319 treated with G/P for 8 weeks and 12 weeks, respectively) were analyzed. In general, patients treated with $\mathrm{G} / \mathrm{P}$ for 8 weeks had similar or greater improvements in mean PRO scores compared with patients treated for 12 weeks, with score improvement differences slightly more pronounced at end of treatment versus post-treatment Week 12 (Figure). Higher proportions of patients achieved MCID in 8-week vs 12-week treatment group (with statistically significant difference in SF-36 social functioning, role physical, bodily pain, and general health domains and FSS at end of treatment). Differences in PRO improvements between 8 vs 12 weeks of G/P remained significant after adjusting for baseline patient characteristics. Conclusion: This post-hoc analysis of registrational trial data collected in patients with chronic HCV infection shows that comparable or even better level of improvement in PROs can be achieved with shorter G/P treatment duration.

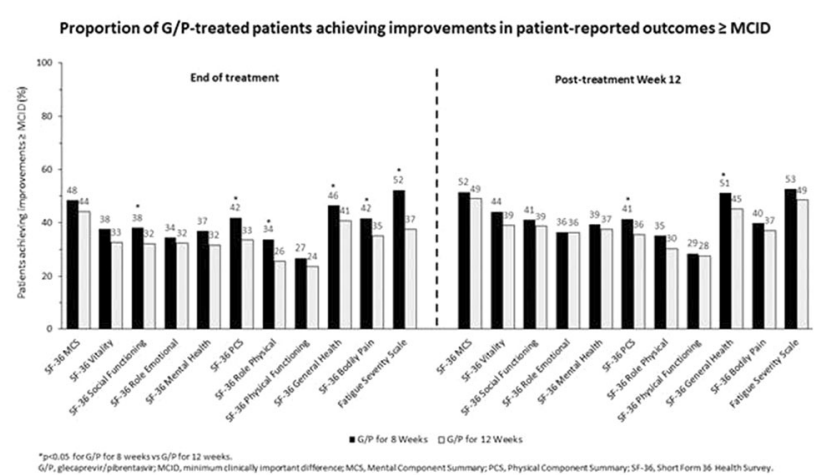

(B201.5) Prediction of postoperative pain score of USgHIFU in the treatment of benign uterine diseases

Guowei Sang, State Key Laboratory of Ultrasound in Medicine and Engineering, Chongqing Medical University, Chongqing, China; Wei Xu, doctor School of Public Health and Management, Chongqing Medical University, Chongqing, China; Yang $\mathrm{Pu}$, doctor School of Public Health and Management, Chongqing Medical University, Chongqing, China; Hongfan Yu, School of Public Health and Management, Chongqing Medical University, Chongqing, China; Qingsong Yu, School of Public Health and Management, Chongqing Medical University, Chongqing, China; Xin Zhang, State Key Laboratory of Ultrasound in Medicine and Engineering, Chongqing Medical University, Chongqing, China; Qiuling Shi, State Key Laboratory of Ultrasound in Medicine and Engineering, Chongqing Medical University, Chongqing, China;

Aims: Ultrasound-guided high-intensity focused ultrasound (USgHIFU) ablation treatment for benign diseases of uterus is rapidly gaining popularity as a treatment modality. Patients treated with USgHIFU will generally be uncomfortable and painful after operation. We aimed to observe the trajectory of postoperative pain score in patients treated with USgHIFU for benign uterine diseases, the use of postoperative analgesics, and the intraoperative pain score within $2 \mathrm{~h}$ after operation, so as to predict the change of postoperative pain and the use of analgesics. Methods: In this study, conducted from June 2020 until March 2021, a total of 670 patients were treated with USgHIFU, including 504 patients with uterine fibroids and 164 with adenomyosis. Patients were using the standardised hospital protocol for sedation and analgesia. Pain were evaluated every half hour after operation until the NRS pain score was less than 4. Patients were asked to recall intraoperative pain severity on a $0-10$ scale at $2 \mathrm{~h}$ after surgery. Postoperative analgesics within $2 \mathrm{~h}$ after operation were recorded. Results: The number of patients with hysteromyoma reporting 4 or greater pain score decreased to $36.17 \%$ by the 2 nd hour after surgery, while the number of patients with adenomyosis decreased to $36.59 \%$ at $2.5 \mathrm{~h}(36.59 \%)$. The use of postoperative analgesics in patients with hysteromyoma was $27.47 \%$, and that in patients with adenomyosis was $43.90 \%$. The Markov prediction model showed that there was a high probability that the patients with hysteromyoma were in mild pain $2 \mathrm{~h}$ before, and nurses stopped recording $2 \mathrm{~h}$ after. However, adenomyosis had a high probability of mild pain before $2.5 \mathrm{~h}$, and the nursing staff stopped recording after $2.5 \mathrm{~h}$. Conclusion: According to the trajectory of postoperative pain score and the results of Markov prediction model, we should pay close attention to the pain of patients with hysteromyoma within $2 \mathrm{~h}$ after operation and to monitor the pain of patients with adenomyosis within $2.5 \mathrm{~h}$ after operation, and give analgesic treatment when necessary. 
(B201.6) Do two frequently used patient-reported outcome measures capture the important issues for people living with ocular surface disease?

Anita Slade, PhD, Centre for Patient Reported Outcomes Research, University of Birmingham, Birmingham, UK; Alberto Recchioni, $\mathrm{PhD}$, Institute of Inflammation and Ageing University of Birmingham, Birmingham, UK; Laura Nice, PhD, University of Birmingham, Birmingham, UK; Elizabeth Dancey, Institute of Inflammation and Ageing, University of Birmingham, Birmingham, UK; Melanie Calvert, PHD, Centre for Patient Reported Outcomes Research, University of Birmingham, Birmingham, UK; Saaeha Rauz, PhD, FRCOphth, Institute of Inflammation and Ageing, University of Birmingham, Birmingham, UK

Aims: Ocular surface disease (OSD) and autoimmune diseases cause dry eye symptoms (DES) including fatigue, dryness, irritation, pain, watery eyes and poor vision. Patient-reported outcome measures (PROMs) can capture the impact of DES on health-related quality of life. A systematic review using COSMIN guidelines identified two PROMs that would be suitable for use in research and clinical practice. These included the Impact of Dry Eye Disease on Everyday Life (IDEEL) and Ocular Surface Disease Index (OSDI). However, it was not clear if the PROMs captured experiences that were important to people living with OSD. Therefore, we set out to develop a conceptual framework reflecting individual's experiences of OSD symptoms, identify their importance, and map them against the OSDI and IDEEL. Methods: Group concept mapping (GCM) is a semi-quantitative mixed method that captures and structures participants' experiences of living with OSD. GroupwisdomTM concept mapping online software was used. Participants were recruited though OSD support networks in the UK. Ethical approval was given and participants consented to take part.GCM consist of 3 stages: statement generation, statement sorting, and individual statement rating (importance/relevance). Each stage was completed before moving to the next stage. Multidimensional scaling and hierarchical cluster analysis produces visual representations of participants' experiences as a points and cluster map, including average rating of individual items across and within clusters. Highly rated important and relevant statements were mapped against items included in the OSDI and IDEEL. Results: 39 participants consented to take part. 125 items and 6 clusters were generated. Cluster analysis suggested a commonality of themes including Environmental Impacts, Pain and discomfort, Eye Treatments (all 3 were rated highest for importance and relevance) Daily living impacts, Psychosocial, and Miscellaneous issues. Mapping the highest rated statements against the OSDI and IDEEL suggested not all the important or relevant areas were being captured by these PROMs. Conclusion: Additional items may be required for clinical trials and research depending on the study requirements. GCM is a useful tool for mapping important and relevant key domains and items against PROMs being used in clinical practice and research.

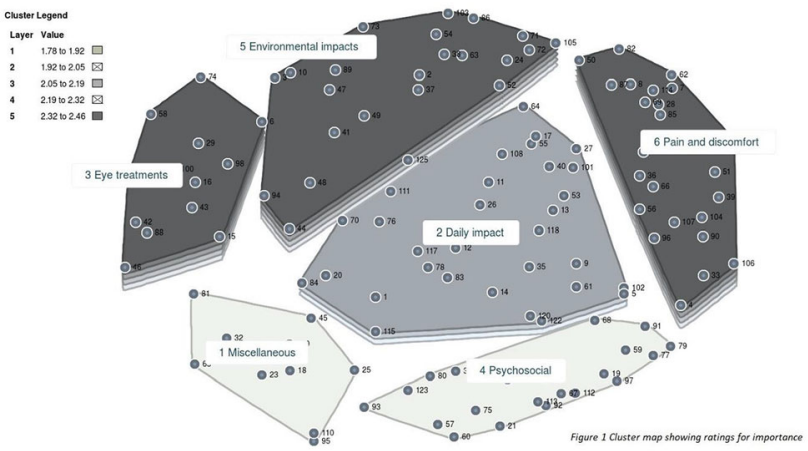

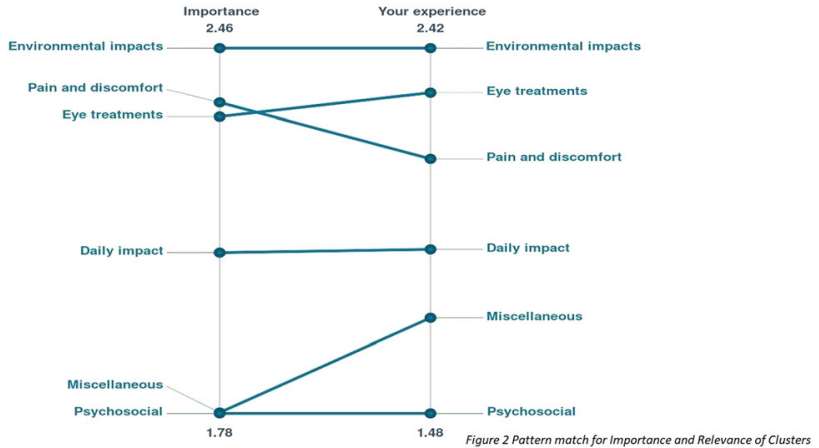

(B201.7) Persistent sleep disturbance after spine surgery is associated with failure to achieve meaningful improvements in pain and health-related Quality of Life

Majd Marrache, MD, Johns Hopkins University, Baltimore, Maryland, USA; Varun Puvanesarajah, MD, Johns Hopkins University, Baltimore, Maryland, USA; Micheal Raad, MD, Johns Hopkins University, Baltimore, Maryland, USA; David Cohen, MD, Johns Hopkins University, Baltimore, Maryland, USA; Lee Riley, MD, Johns Hopkins University, Baltimore, Maryland, USA; Brian Neuman, MD, Johns Hopkins University, Baltimore, Maryland, USA; Khaled Kebaish, MD, Johns Hopkins University, Baltimore, Maryland, USA; Amit Jain, MD, Johns Hopkins University, Baltimore, Maryland, USA; Richard L. Skolasky, ScD, Johns Hopkins University, Baltimore, Maryland, USA

Aims: Sleep hygiene is an important component of well-being and is closely linked to mental health; however, little is known about how sleep disturbance (SD) in spine surgery patients influences healthrelated quality-of-life (HRQOL) or recovery after surgery. We aim to determine prevalence of SD among spine surgery patients; correlation between SD and HRQOL; and association between SD resolution and HRQOL recovery. Methods: Retrospective review of 508 adult spine surgery patients at an academic medical center: mean age 60 years (SD 13); 98\% non-Hispanic; 73\% white. Participants completed an assessment before surgery (age, sex, race/ethnicity, education, household income, relationship status, comorbidity, and current opioid use). We recorded intraoperative and/or postoperative complications. Before and after surgery, assessments were Oswestry Disability Index (ODI) or the Neck Disability Index (NDI) and PROMIS-29 profile.Using PROMIS SD score, we grouped participants as no SD $(<55)$, mild $(55-60)$, moderate $(60-70)$, or severe $(>70)$. Pearson correlation tests were used to assess correlations between SD and HRQOL. Regression analysis was used to estimate the effect of resolved or continuing SD on HRQOL and likelihood of meaningful improvements in HRQOL. Results: Preoperative SD was reported by 127 participants (25\%). SD was significantly correlated with worse ODI/NDI values and worse scores in all PROMIS health domains (all, $p<0.001$ ). At the immediate postoperative assessment, SD had resolved in 80 of 127 participants $(63 \%)$. Compared with participants who reported no preoperative SD, those with ongoing SD were significantly less likely to achieve clinically meaningful improvements in Pain Interference (odds ratio [OR[, 0.49; 95\% confidence interval [CI], 0.28, 0.84), Physical Function (OR 0.32; 95\% CI 0.13, 0.82), and Satisfaction with Participation in Social Roles (OR 0.57; 95\% CI 0.37, 0.80). Conclusion: One quarter of spine surgery patients reported at least moderate SD. Poor preoperative sleep quality and ongoing sleep disturbance were associated with 
worse HRQOL. These results highlight the importance of addressing patients' sleep disturbance before and after surgery.

\begin{tabular}{|c|c|c|c|c|}
\hline \multirow{3}{*}{ Measure } & \multirow{3}{*}{$\begin{array}{c}\text { Sleep } \\
\text { Disturbance } \\
\text { Correlation }\end{array}$} & \multirow{2}{*}{\multicolumn{2}{|c|}{$\begin{array}{l}\text { Preoperative Sleep } \\
\text { Disturbance* }\end{array}$}} & \multirow{3}{*}{$P$} \\
\hline & & & & \\
\hline & & No $(n=239)$ & Yes $(n=93)$ & \\
\hline Neck Disability Index & 0.50 & $34 \pm 18$ & $60 \pm 14$ & $<0.001$ \\
\hline Oswestry Disability Index & 0.63 & $27 \pm 19$ & $51 \pm 15$ & $<0.001$ \\
\hline \multicolumn{5}{|l|}{ PROMIS-29 health domain } \\
\hline Pain Interference & 0.45 & $60 \pm 8.9$ & $68 \pm 6.6$ & $<0.001$ \\
\hline Physical Function & -0.34 & $39 \pm 7.9$ & $33 \pm 6.5$ & $<0.001$ \\
\hline $\begin{array}{l}\text { Satisfaction with } \\
\text { Participation in Social } \\
\text { Roles }\end{array}$ & -0.45 & $45 \pm 9.4$ & $36 \pm 7.0$ & $<0.001$ \\
\hline
\end{tabular}

(B201.8) The predictive value of PROs and baseline characteristics on patient's adherence to maintenance medication in asthma

Jamie Rees, MSc, GSK, London, UK; Tom Keeley, PhD, GSK, London, UK; Alison Moore, PhD, GSK, London, UK

Aims: Adherence to asthma maintenance medication is an important factor which contributes to a patient's asthma control. The primary study investigated the effect of a connected inhaler system (CIS) on a patient's adherence. The objective of this exploratory investigation was to understand whether adherence at baseline (month prior to randomisation), months $4-6$, or change in adherence could be predicted by participants' baseline patient-reported outcome (PRO) scores and other baseline characteristics. This analysis informs whether it is possible to identify those who could benefit from additional interventions to increase their maintenance medication adherence. Methods: In the primary study, participants received fluticasone furoate/vilanterol ELLIPTA inhalers $(N=437)$ and were randomised to one of five CIS study arms, receiving varying levels of feedback from the sensors on their adherence. PROs were collected during the study at various timepoints (Screening, Randomisation, Months 1 and 6) including: ACT, ASUI, BMQ, MARS-A, PAM-13 and SGRQ. For this analysis, stepwise regression models were fitted for the following dependent variables of adherence responder: adherence rate $\geq 80 \%$ at baseline, $\geq 80 \%$ at months $4-6$, change from baseline $\geq 15 \%$ and the composite at months 4-6. Predictor variables considered were demographic characteristics (including age, gender and BMI) and PRO scores at screening. Results: Based on the selection method (5\% significance level) and Akaike's information criterion (AIC), age was a significant predictor for all models, suggesting that older patients have higher odds of being adherent to their maintenance therapy. MARS-A was a significant predictor in the adherence rate $\geq 80 \%$ at baseline and months 4-6, meaning participants with higher MARS-A scores at screening have higher odds of being adherent in the short and long term. Other significant predictors included BMQ General Benefit, ASUI and ACT, suggesting participants who have better scores in these PROs have higher odds of being adherent. Conclusion: In this analysis of patients' PRO scores and baseline characteristics, age was the most consistent predictor of adherence across all models, along with MARS-A, ACT, ASUI and BMQ general benefit scores at screening. This suggests those who are younger and have worse scores in these PROs may require further support in their asthma care.

References:

[1] Moore A, Preece A, Sharma R, et al. A randomised controlled trial of the effect of a connected inhaler system on medication adherence in uncontrolled asthmatic patients. Eur Respir J 2020; in press (https://doi.org/10.1183/13993003.03103-2020)

(B201.9) Validity evidence in meso-level decision making using patient-reported outcomes in hospice palliative care: a dialogue
Dominique Duquette, MN, University of Alberta, Edmonton, Alberta, Canada

Aims: A contemporary view of measurement validity suggests that an evaluative judgement is based on theoretical rationales and the accumulation of five types of evidence, namely: test content, response processes, internal structure, relations to other variables and consequences of testing. Yet, validation practices seldom establish evidence from the consequences of testing. If a sound judgement of validity requires integrating multiple types of evidence, credible evidence on the consequences of using PRO for meso-level decision making should be established. PRO results used for decision making have consequences (intended and unintended, negative and positive) ensuing from various users' interpretations of instruments and results. Decision makers are responsible for ensuring that unintended consequences are critically examined to promote a valid interpretation of PRO data for the intended use and intended population. Methods: What are the necessary conditions of appropriate and adequate mesolevel decisions based on PRO results for the hospice palliative care population? This question will be addressed through a dialogue as a method to gain skills and insights. The proposed dialogue will be inspired by the Socratic method. Such dialogic examination into assumptions and values will stimulate critical thinking and reasoning while exposing potentially adverse consequences for vulnerable populations. The dialogue will be facilitated through four phases, including an in-preparation activity to engage participants in a reflective process about a personal experience and a synchronous case study as a device for philosophizing. Results: The insights gained from the dialogue will attune decision makers to their responsibility towards unintended consequences. This liability can lead to furthering validity practices in evaluating the soundness of interpretations and uses of PRO data for specific populations and strengthening validity evidence for reasonable decisions using PRO. Furthermore, a Socratic Dialogue promotes critical thinking and argumentation skills necessary for evaluative judgement and decision making. Conclusion: Using PRO data in meso-level decision making is fundamental to a patient-centred healthcare system. To hold confidence in the adequacy and appropriateness of the decisions made, credible evidence for validity, including the consequences of tests, need to be established. Moreover, decision makers require robust critical thinking and reasoning skills to articulate sound arguments that justify their decisions.

\section{2: Methodological Research}

(B202.1) Methods for determining how much missing data are tolerable when scoring a patient-reported outcome measure

P. Cristian Gugiu, Clinical Outcomes Solutions, Chicago, Illinois, USA; Stacie Hudgens, MA (AbD), Clinical Outcomes Solutions, Tucson, Arizona, USA; Ashley F Slagle, Aspen Consulting, Steamboat, Colorado, USA; Heli Kapadia, Clinical Outcomes Solutions, Chicago, Illinois, USA; Cheryl Coon, Outcometrix, Salida, Colorado, USA

Aims: Patient-reported outcome measures vary in terms of response scales, items, and scoring methodologies. Missing data parameters, necessary for scoring, may be defined using complete case or prespecified levels of missingness based on the total number of items. Historically, the $50 \%$ rule has been applied to measures without evaluating the appropriateness of this approach. This work considers methodologies for determining tolerable levels of missingness in scoring PRO measures. Methods: The PROMIS depression dataset was used for this comparison of methods. In one approach, the statistical test of equivalence approach was applied to generate Cronbach's alpha by missing data rate as well as Standard Error of 
Measurement (SEM) for weighted sums by missing rate. A bootstrap 95\% confidence interval (CI) was computed for the missing rate of $0 \%$ (tolerance margin comparison). Using this dataset, 1,000 simulated datasets were generated. Values were randomly selected and set to missing (Missing Completely at Random). For the 10-80\% missing rates, $90 \%$ CIs were generated. If the simulated CI was contained within the margins, results were equivalent to the condition of no missing data. In the second approach, a series of Cronbach's alphas were produced as items were deleted (i.e., missing) including steps where the most reliable item is set to missing and alpha recomputed; with a simulation of the variability of mean scores as items are randomly set to missing. Results: 753 subjects with non-missing, itemlevel data were included. Results suggest that Cronbach's alpha is less sensitive to the impact of missing data. In each evaluation, Cronbach's alpha remained stable $(>0.70)$ whereas the SEM increased proportionally to the amount of missing data. Missing data tolerance was considered as $60 \%$ for the full, calibrated item bank, $30 \%$ for the short-form Depression scale, and 20\% calibrated items Depression scale. Conclusion: Missing item level data impacts the ability to score a PRO measure resulting in missing data for interpreting patient outcomes. Results suggest that the $50 \%$ rule may not be appropriate, and that reliance on this rule may undermine the reliability parameters and, thus, the interpretability of scores. These results highlight the importance of evaluating missing data tolerances for all PRO measures.

(B202.2) Daily Diary Data: averaging over multiple days of data increases and homogenizes inter-item correlations and factor loadings

Stephanie Rhoten, PhD, IQVIA, Patient-Centered Solutions, San Francisco, California, USA; Pip Griffiths, BSc, PhD, IQVIA, PatientCentered Solutions, Saint-Herblon, France; Cristina Ivanescu, PhD, IQVIA, Patient-Centered Solutions, Amsterdam, The Netherlands

Aims: To our knowledge, there is currently no firm guidance on how patient-reported outcome (PRO) data collected daily should be psychometrically assessed, with some researchers examining item-level properties using a single day and others averaging items over several days prior to analysis. The current aim is to investigate whether itemlevel analyses on daily diary PRO measures, specifically inter-item correlations and factor analyses, should be performed on single-day raw item scores or weekly item averages. Methods: Analysis was performed on simulated daily data including 1000 patients. Each patient had seven days of simulated data for a hypothetical PRO with 20 items and three subdomains. Inter-item correlations and exploratory factor analysis (EFA) were conducted for each single day as well as item averages across the seven days to explore differences using the estimated scores. Results: Results for the inter-item correlations demonstrated that the correlations using average item scores were consistently around 0.2 higher than the correlations between each pair of items. For items that share a domain, the relationships across the correlation matrix also became more homogenized, or converged on similar values, when assessed at the weekly average level. EFA was able to recover all three simulated factors when both single-day and weekly average data were used. However, similar to the inter-item correlations, the factor loadings at the weekly average level were larger and homogenized compared to the daily level. Conclusion: Although a similar pattern of results may emerge when analyzing data with respect to domain structure at both the single-day and weekly average level, there are also important differences. Increased correlations and factor loadings at the weekly average level may obscure weaker item relationships and potentially give researchers misplaced confidence in the strength of the relationship between the items.
Homogenized inter-item correlations and factor loadings may also lead to erroneous interpretations of how the items work together to inform a single scale. Overall, item-level analysis on weekly item averages may result in difficulty detecting less informative items when creating scales and summary scores, and analysis on daily-level item scores is recommended for daily diary data. Future research should also evaluate these results in multi-level models.

(B202.3) Establishing the content validity of LIMB-Q Kids: a new patient-reported outcome measure for children with lower limb deformities

Harpreet Chhina, PhD, BC Children's Hospital, Vancouver, British Columbia, Canada; Anne Klassen, D.Phil., McMaster University, Hamilton, Ontario, Canada; Jacek Kopec, MD, MSc, PhD, University of British Columbia, Vancouver, British Columbia, Canada; John Oliffe, $\mathrm{PhD}, \mathrm{RN}$, University of British Columbia, Vancouver, British Columbia, Canada; Anthony Cooper, FRCS, MBChB, BC Children's Hospital, Vancouver, British Columbia, Canada

Aims: To establish the content validity of a new patient-reported outcome measure for children with lower limb deformities. Methods: LIMB-Q kids consist of 11 independently functioning scales that measure physical function, symptoms (leg, hip, knee, ankle, and foot), appearance, leg-related distress, social, school, and psychological function. LIMB-Q Kids was developed based on an international qualitative study conducted by the authors. In order to evaluate the comprehensibility, comprehension, and relevance of the content of the LIMB-Q Kids, individual cognitive debriefing interviews were conducted with children with lower limb deformities from Australia, Canada, and the USA. Interviews were done with the clinical experts including the orthopaedic surgeons, physiotherapists, occupational therapists, nurses, psychologists, and prosthetists from Australia, Canada, Ethiopia, India, UK, and the USA. All interviews were recorded and transcribed verbatim. The analysis included closely examining the transcribed interviews, summarising edits after each interview, and editing the items. Results: Forty interviews were conducted (17 patients ages $8-17$ years, 4 parents, and 19 experts). A total of five rounds of interviews were conducted. The first draft of LIMB-Q Kids had 124 items. After the first, second, and third round of interviews, $91 \%, 82 \%$, and $94 \%$ of the items remained unchanged, respectively. Some items needed minor edits to improve the comprehension. During round 4, only the Leg Symptoms Scale was reviewed for any additional items that need to be added for children with amputations. Three items were added to the Leg Symptom Scale at this time. During round 5, the final version of LIMB-Q Kids had 158 items. No further changes were required based on patient feedback at this time. In total, there were 37 new items added. However, $89 \%$ of the items were added for the individual symptom scales. Conclusion: Interviews with children and clinical experts allowed us to identify items that required re-wording, as well as developing new items. This process was helpful for refining the items included in LIMB-Q Kids before field testing in a larger sample of children. Overall feedback from children and experts showed that the new PROM is comprehensive, understandable by the target patient population, and has relevance to this patient population.

(B202.4) A blinded approach to ensure integrity of interpreting secondary health-related quality of life (HRQL) endpoints when the primary endpoint is known: A practical example from the CARFI study—a Nordic Myeloma Study Group trial 
Henrik Eshoj, PhD, Quality of Life Research Center, Department of Haematology, Odense University Hospital, Odense, Denmark; Madeleine T King, professor University of Sydney, School of Psychology, Sydney Quality of Life Office, Australia, Sydney, Australia; Niels Abildgaard, professor MD, Quality of Life Research Center, Department of Haematology, Odense University Hospital, Odense, Denmark; Lene Kongsgaard Nielsen, PhD, Research Unit for Multimorbidity, Department of Internal Medicine and Cardiology, Viborg Regional Hospital, Viborg, Denmark; Henrik Gregersen, Ass. Clin Prof., MD, Department of Haematology, Aalborg University Hospital, Aalborg, Denmark

Aims: Patient-reported outcomes (PRO) are important secondary endpoints in cancer trials. However, because primary trial endpoint is often known by the time PROs are analysed, the interpretation of PROs may be influenced. We present a method for conducting blinded interpretation of secondary PRO endpoints in cancer trials. Methods: In the CARFI trial, relapsed multiple myeloma patients underwent salvage stem cell transplantation before being randomized to Carfilzomib + Dexamethasone maintenance therapy $(\mathrm{Kd})$ or observation alone (Obs). The primary trial endpoint was time to progression (TTP). Secondary PRO endpoints were assessed with EORTC QLQ-C30 at baseline and every second month. The primary PRO endpoint was the QLQ-C30 Summary Score (QLQ-C-Sum), an overall health-related quality-of-life (HRQL) score that includes 13/15 QLQ-C30 domains. The 13 individual domains of the QLQ-CSum were supportive secondary PRO endpoints. PRO data were analysed with mixed models, with $p<0.05$ and $p<0.01$ indicating statistically significant difference for the primary and secondary PRO endpoints, respectively. Between-group minimal important difference of 10 points was used for QLQ-C-Sum, and domain-specific MIDs were used for the 13 domain scores. Two blinded interpretations of the findings were made, one assuming that group A received $\mathrm{Kd}$ (1) and another assuming that group A received Obs (2). Results: In total, 168 patients were randomised. TTP was significantly prolonged with 8 months for patients allocated to Kd. For the QLQ-C-Sum, a difference of 3.7 (95\% CI -7.6 to $0.3, p=0.068$ ) was found. For the individual domains, clinically meaningful differences, however, not statistically significant, were found in $7 / 13$, all in favour of group A. Interpretation 1; Kd does not detrimentally impact overall HRQL more than Obs. In conjunction with the prolonged TTP, $\mathrm{Kd}$ is the preferred treatment choice. Interpretation 2; Obs does not detrimentally impact overall HRQL more than Kd. However, as Obs leads to shorter TTP, is cheaper and less burdensome to patients (= no treatment visits at hospitals), this trial supports shared decision to meet patient preference between progression-free survival and quality of life. Conclusion: This is the first cancer trial to use a blinded interpretation approach, which ensures integrity of interpretation of secondary PRO endpoints analysed after primary endpoint results are known.

\section{(B202.5) Estimand considerations for time-to-event analysis of patient-reported-outcomes}

Libby Floden, PhD, MPH, Clinical Outcomes Solutions, Chicago, Arizona, USA; Konstantina Skaltsa, PhD, IQVIA, Barcelona, Spain; Rachael Lawrance, PhD, Adelphi Values, Cheshire, UK

Aims: There is growing emphasis on the importance of the patient experience in evaluating treatments and assessing the overall burden of disease. In many trials including in oncology, patients are assessed repeatedly over the course of treatment, and beyond, including when crossing over treatment arms, after progressive disease and treatment discontinuation. Clinical endpoints are often analyzed as a time-toevent, e.g., progression-free survival. Similarly, important patient- reported outcomes (PROs) can be obtained by modeling time-to-event data to estimate, for example, median time to meaningful deterioration. In the regulatory setting, the lack of clear PRO research objectives and inconsistency in how PRO data are analyzed makes it difficult to interpret results both within and across trials. The estimand framework provides a structure to align a research question with study design, conduct, and statistical analysis. The aim of this work is to highlight considerations when applying the estimand framework for time-to-event analyses of PRO data, including defining events, censoring rules, and interpretation. Methods: We apply the five attributes of estimands as defined in the International Committee on Harmonisation ICH E9(R1) addendum (ICH 2019) to two time-to-PROdeterioration research questions. We identify four types of events that can occur post-randomization impacting the meaning of the data, termed intercurrent events (ICEs): disease progression, death, treatment discontinuation, and treatment arm crossover. We present various strategies to handle ICEs and how these affect the interpretation of the estimand. Results: The hypothetical strategy where patients are envisioned and analyzed as if they have not experienced the ICE is operationalized by censoring at the time of the ICE, such as disease progression. Results using this strategy may be biased if we cannot assume that those who are censored have the same probability of experiencing an event as those who are not. The composite strategy where the ICE, such as patient death, is incorporated into the event definition can be interpreted more easily and may be more relevant in some settings. Conclusion: Different estimands may be relevant for different stakeholders. Careful consideration of the trial setting, the scientific question of interest, and the likely impact of intercurrent events is necessary to design and interpret time-to-event PROs.

\section{(B202.6) Recommendations for including or reviewing patient- reported outcome endpoints in grant applications}

Claire Snyder, PhD, Johns Hopkins, Baltimore, Maryland, USA; Alexandra Gilbert, Leeds Cancer Centre, Leeds, UK; David Moher, Ottawa Hospital Research Institute, Ottawa, Ontario, Canada; Derek Kyte, PhD, University of Worcester, Worcester, UK; Ellie Daniels, American Cancer Society, Atlanta, Georgia, USA; Madeleine King, University of Sydney, Sydney, Australia; Melanie Calvert, University of Birmingham, Birmingham, UK; Ronald Chen, University of Kansas Cancer Center, Kansas City, Missouri, USA; Michael Brundage, Queen's Cancer Research Institute, Kingston, Ontario, Canada; Submitted on behalf of "The PROTEUS Consortium"

Aims: Grant applicants and grant reviewers require guidance on the key information that should be included in funding applications to demonstrate rigorous patient-reported outcome (PRO) methods. PRO experts from the international PROTEUS-Trials Consortium developed practical prioritized recommendations to inform grant applicants preparing research strategies and grant reviewers evaluating applications. Methods: A multidisciplinary team $(n=9)$ developed recommendations through a four-step informal consensus development process: (1) identifying candidate recommendations, (2) rating candidate recommendations (from $1=$ always include to $4=$ not necessary), (3) ranking candidate recommendations based on the ratings, and (4) refining the recommendations. The candidate recommendations were drawn from existing guidance documents, including the SPIRIT-PRO recommendations for writing protocols and the SISAQOL recommendations for PRO data analysis. Results: Forty items were considered for recommendation (37 from SPIRITPRO, 3 from SISAQOL). From the rating exercise, the average ratings ranged from 1.1 to 3.2 , which were then ranked as follows. Five items with average ratings of 1.1-1.4 were preliminarily categorized as "always include"; 18 items with average ratings of $1.5-2.2$ were 
preliminarily categorized as "always include when PRO is primary endpoint or if a second paragraph of PRO content can be included in the grant application"; and 17 items with average ratings $>2.2$ were preliminarily categorized as "helpful if space allows." After discussion and refinement, including combining some items, 6 topics were deemed to be essential: rationale, research question, outcomes of interest, timepoints for assessment, data collection plan, and analysis methods. Inclusion of an additional 10 topics was supported when the PRO is a primary endpoint or if space allows inclusion of more PRO content. These topics provide more detail regarding the PRO study's background and rationale, data collection and management, and analysis. The remaining 15 PRO topics may also be helpful to include in grant applications if space allows. Conclusion: Following the above recommendations positions research teams to demonstrate appropriate PRO methods in their grant applications and grant reviewers to evaluate proposed PRO studies effectively. By "beginning with the end in mind," these recommendations are intended to help both grant applicants and grant reviewers ensure the rigor and relevance of PRO research studies.

\section{(B202.7) Using Mixed Methods to Evaluate Differential Item Functioning on the Kansas City Cardiomyopathy Questionnaire (KCCQ) Physical Limitations Domain}

Theresa Coles, Duke University, Durham, North Carolina, USA; Nicole Lucas, Duke University, Durham, North Carolina, USA; Li Lin, Duke University, Durham, North Carolina, USA; Molly McFatrich, Duke, Durham, North Carolina, USA; Debra Henke, Duke University, Durham, North Carolina, USA; Amy Corneli, Duke University, Durham, North Carolina, USA; Jennifer Ridgeway, Mayo Clinic, Rochester, Minnesota, USA; Emma Behnken, Mayo Clinic, Rochester, Minnesota, USA; Fraser Bocell, FDA, Silver Spring, Maryland, USA; Heidi Dohse, Tour de Heart, Hailey, Idaho, USA; Shannon Dunlay, Mayo Clinic, Rochester, Minnesota, USA; Lindsay Emanuel, Mayo Clinic, Rochester, Minnesota, USA; Ileana Pina, FDA, Silver Spring, Maryland, USA; Brittany Caldwell, FDA, Silver Spring, Maryland, USA; Anindita Saha, FDA, Silver Spring, Maryland, USA; John Spertus, University of Missouri-Kansas City, Kansas City, Missouri, USA; Michelle Tarver, FDA, Silver Spring, Maryland, USA; Bryce Reeve, Duke University, Durham, North Carolina, USA; Kevin Weinfurt, Duke University, Durham, North Carolina, USA

Aims: Differential item functioning (DIF) analysis examines the validity of outcome comparisons by population subgroups, an important method as we strive towards equity in health outcomes. The purpose of this presentation is to describe a mixed methods (MM) approach to evaluating DIF on the Kansas City Cardiomyopathy Questionnaire (KCCQ-23) Physical Limitations (PL) domain items. This DIF evaluation was driven by the overarching premise that women and men may interpret KCCQ-23 items differently due to different lived experiences of heart failure (HF), and because of a historic lack of representation of women in HF studies. Methods: Our approach was a three-step convergent sequential MM study. First, we sought expert insight from 12 cardiology clinicians to examine each of the six KCCQ-23 PL items and hypothesize why men or women may interpret specific items or response choices differently. Second, we conducted a quantitative DIF evaluation using two methods: Wald chi-square test within an IRT framework and ordinal logistic regression (OLR). Data were from HF-ACTION $(n=2331)$, a clinical trial designed to test the safety and efficacy of aerobic exercise training in medically stable outpatients with HF. The magnitude of DIF was evaluated using weighted area between the expected score curves and comparing $-2 \log$ likelihood between OLR models. Third, we conducted qualitative semi-structured interviews with 25 women and men with HF to explore their interpretations of the KCCQ-23 items to further evaluate our quantitative findings. Interviews were audio recorded, transcribed, and analyzed using descriptive qualitative analytical approaches by gender to describe similarities and differences in how women and men interpreted questions and response options on the KCCQ-23. Results: Three KCCQ-23 items were hypothesized by clinicians to exhibit potential DIF (dressing yourself; showering/bathing; doing yardwork, housework or carrying groceries) due to cultural/societal expectations for men and women. Statistically significant DIF was identified on two items via OLR only (climbing a flight of stairs without stopping, hurrying or jogging), and the magnitude of DIF was negligible. The qualitative study is ongoing and results will be presented at the conference. Conclusion: The MM design provides context to sensitive quantitative methods and describes patient and clinician input on why items may exhibit DIF.

\section{(B202.8) Positive and negative predictive values for continuous test scores}

Felix Fischer, PhD, Charite - Universitaetsmedizin Berlin, Berlin, Germany

Aims: Patient-reported outcome measures (PROMs) are frequently used as diagnostic screening tools. Positive and negative predictive values (PPV/NPV) help to put estimates of accuracy of a diagnostic test in clinical context and provide risk estimates for a given patient taking into account baseline prevalence in the population. Usually, the test results are dichotomized at a prespecified cutoff, which results in a loss of information. This study presents an approach to calculate PPV and NPV for each possible PROM score. Methods: Probabilities of test results such as theta or T-scores are modeled in a Bayesian framework stratified by disease status. Application of Bayes' theorem allows to obtain probabilities of disease status conditional on test result. The method is applied to PROMIS Wave 2 data. 187 patients completed the PROMIS Emotional Distress Depression CAT and clinical diagnosis of major depressive disorder served as reference standard (53 diseased). Results: PROMIS T-scores were normally distributed in particpants with (mean: 64.7, sd: 7.9) and without (mean: 51.7, 6.9) diagnosis of depression. Given an expected prevalence of $15 \%$, this results in a $21 \%(16-26 \%)$ probability of depression if the actual observed score is 60 and an $80 \%(63-93 \%)$ probability if the actual score is 70 . These scores differ considerably from the PPV using the optimal cutoff 58.9, which is average probability of depression at $46 \%$ (95\% CI 36-58\%). Conclusion: Calculating the PPV for each potential score allows a more finegrained assessment of risk of disease. The method has some advantages over the use of logistic regression, e.g., that the baseline prevalence can be represented by a flexible prior distribution, acknowledging uncertainty about baseline risk. The method, therefore, has the potential to improve interpretation and understanding of PROM scores in screening applications.

(B202.9) Validity and reliability of the PROMIS-29v2 and SF$12 \mathrm{v} 2$ for assessing health-related quality of life outcomes in cardiac patients

Dion Candelaria, MN, BSc, RN, Susan Wakil School of Nursing and Midwifery, Faculty of Medicine and Health, The University of Sydney, Sydney, Australia; Laila Ladak, PhD, RN, The Aga Khan University, Karachi, Pakistan; Sue Randall, PhD, RN, Susan Wakil School of Nursing and Midwifery, Faculty of Medicine and Health, The University of Sydney, Sydney, Australia; Ann Kirkness, RN, 
Royal North Shore Hospital, Northern Sydney Local Health District, Sydney, Australia; Kellie Roach, RN, Ryde Hospital, Northern Sydney Local Health District, Sydney, Australia; Judith Fethney, BA, Susan Wakil School of Nursing and Midwifery, Faculty of Medicine and Health, The University of Sydney, Sydney, Australia; Robyn Gallagher, PhD, RN, Susan Wakil School of Nursing and Midwifery, Faculty of Medicine and Health, The University of Sydney, Sydney, Australia

Aims: The 29-item Patient-Reported Outcomes Measurement Information System v2.0 (PROMIS-29v2) is a promising health-related quality of life (HRQL) measure for use in cardiac patients but has not been fully evaluated in this population. The aim of the current study is to examine the validity, reliability, and sensitivity to change of the PROMIS-29v2 in patients with coronary heart disease (CHD), in comparison to the well-established Short-form Health Survey v2.0 (SF-12v2). Methods: HRQL data were collected via electronic surveys from patients at four cardiac rehabilitation sites. Analyses included: intercorrelations on summary scores between questionnaires, General Linear Model to examine discriminant validity for age, sex, and referral diagnosis, item analysis to assess internal consistency reliability, and a Linear Mixed Model, paired t-tests, and effect size (ES) comparisons to determine sensitivity to change. Results: Patients $(n=89)$ had a mean (SD) age of $66.92(9.25)$ years, $83.1 \%$ were male. The PROMIS-29v2 and SF-12v2 summary scores were strongly correlated: physical $(r=0.602, p<0.01)$, mental $(r=0.506, p<0.01)$. The PROMIS-29v2 demonstrated discriminant validity for sex and referral diagnosis: women reported worse physical and mental health, and patients who had elective percutaneous coronary intervention (PCI) reported better physical health than other diagnostic groups. Good internal consistency was established with Cronbach's alpha 0.782 to 0.957 . Both questionnaires were sensitive to change in physical health (PROMIS-29v2 ES 0.51 vs SF-12v2 ES 0.66), but the PROMIS-29v2 demonstrated better sensitivity to changes in mental health than the SF-12v2 (ES 0.70 vs 0.25 ). Conclusion: The PROMIS-29v2 was valid, reliable, and sensitive to changes in HRQL in cardiac patients. In many respects, the PROMIS$29 \mathrm{v} 2$ is comparable to the well-established SF-12v2. The symptomrelated items in the PROMIS-29v2 provide additional useful clinical information not explicitly captured by the SF-12v2. We also demonstrated that electronic data capture was feasible, which makes PROMIS-29v2 a suitable tool in assessing HRQL in various CR settings. Future work should focus on establishing minimal clinically important difference (MCID) benchmarks for PROMIS-29v2 to quantify changes felt by patients in response to cardiac events and treatments and to allow for more direct comparisons with other HRQL measures.

\section{(B202.10) Mixed methods for clinician-reported outcome (ClinRO) development: photographic sorting, consensus analysis, and semi-structured interviews}

Selam Shah, MPH, ICON plc, Boston, Massachusetts, USA; Adilson Dacosta, MD, MSc, PhD, Sun Pharmaceutical, Princeton, New Jersey, USA; James Stansbury, PhD, ICON plc, Gaithersburg, Maryland, USA; Siu-Long Yao, MD, Sun Pharmaceutical, Princeton, New Jersey, USA; Olga Moshkovich, MPH, ICON plc, San Francisco, California, USA; Alan Nagle, ICON plc, Limerick, Ireland; Renan Minotto, MD, MSc, PhD, Dermatology Department, Hospital Santa Casa de Porto Alegre, UFCSPA, Porto Alegre, Brazil; Claudio L F Souza, Licenciatura Profissional, Medicina, Dermatologia, UNIFENAS, Alfenas, MG, Brazil; submitted on behalf of "SUN ClinRO Development Group"
Aims: Development of clinical outcome assessment (COA) measure content often relies wholly on the use of semi-structured interviews in concept elicitation. While the approach can ensure inclusion of valid content that matters to respondents, concerns about rating scale boundaries or measure reliability often remain unexplored in early work. We combined sorting and ranking tasks with interviews in developing an illustrated ClinRO for nail psoriasis to address these concerns. Methods: One-hour-long mixed-methods interview sessions $(N=16)$ were conducted with a global roster of dermatologists. Concept elicitation (CE) interviews explored professional insights on nail pathology assessment and incorporated photographic pile sorting and ranking exercises. The photo sorts were analyzed with cultural consensus analysis and multi-dimensional scaling. The sorts and rankings allowed for deriving an optimal consensus (a "correct" answer) around severity ratings to inform original illustrations for the new ClinRO. A two-dimensional representation of aggregate sort information helped refine rating-scale category boundaries, clarifying sometimes contradictory statements from the qualitative interviews. The approach allowed for largely confirmatory cognitive debriefing interviews (CDI, $N=5$ ) focused on draft measure usability, including a preliminary test of reliability. Results: The CE interviews yielded qualitative data informing scale category descriptions and instrument design reflecting expert dermatologists' typical practice. The consensus analyses verified that "high competence" dermatologists (i.e., task consensus drivers, tending to avoid differential diagnosis) shared a common approach to severity evaluation $(\mathrm{ER}=5.1$ and 16.8 for sorts and full ranking, respectively), reflected in high average "competence" scores (xrits $=0.60(95 \%$ CI $0.57-0.63$ ) and $0.81(95 \%$ CI 0.78-0.85)). Use of the scaling diagram-aided decisions around category descriptors. The CDI verified scale usability, suggested refinements, with promising preliminary reliability $(\mathrm{ICC}=0.739$, 95\% CI 0.34-0.84) when evaluating photos showing variation in earlier sorting. Conclusion: The addition of sorting and ranking tasks to early measure development engaged respondents and enhanced confidence in rating scale construction, including descriptor boundaries. Measuring professional consensus, even with small samples from concept elicitation work, adds assurance that the scale will be used reliably by clinical investigators. The approach appears particularly apt for developing well-defined and reliable ClinRO instruments with illustrations and category descriptors for global use.

\section{(B202.11) Using multiple sources of evidence to choose between severity and interference-based response options for a patient-reported outcome measure}

Manraj Kaur, PT, PhD, Brigham and Women's Hospital/Harvard Medical School, Boston, Massachusetts, USA; Andrea Pusic, Brigham and Women's Hospital/Harvard Medical School, Boston, Massachusetts, USA; Feng Xie, McMaster University, Hamilton, Ontario, Canada; Louise Bordeleau, Juranvinski Cancer Center, Hamilton, Ontario, Canada; Toni Zhong, University of Toronto, Toronto, Ontario, Canada; Stefan Cano, Modus Outcomes, London, UK; Elena Tsangaris, Brigham and Women's Hospital/Harvard Medical School, Boston, Massachusetts, USA; Trisia Breitkopf, McMaster University, Hamilton, Ontario, Canada; Anne Klassen, McMaster University, Hamilton, Ontario, Canada;

Aims: Selecting appropriate response options for patient-reported outcome measures (PROMs) is fundamental to ensuring that the PROM measures what it intends to measure. However, little guidance is available on how to choose the most appropriate set. This results in a variety of response sets being used, such as severity (e.g., none to severe), interference (e.g., not at all to very much), or a combination of the two (e.g., moderate pain that limits some activities), without 
any justification regarding the choice. This study aimed to empirically determine the optimal response set to use in the BREAST-Q Utility module, a preference-based measure for breast cancer (BC). Methods: As part of the development of the BREAST-Q Utility module, interviews were conducted with 57 adult women with BC from hospitals in Canada and the USA. The interviews were coded, and participants' words were used to draft items and response options that measure "severity" and "interference with daily activities" for 6 constructs-fatigue, pain, emotional distress, usual activities, arm mobility, and unpleasant symptoms. Items and response options were pilot tested with nine patients and 27 experts, followed by a field test with women with BC recruited through Love Research Army, USA. Data were analyzed for: (1) missingness, (2) distribution by demographic (age, education), clinical (BC stage, time since diagnosis), and treatment (on versus off) characteristics, (3) test-retest reliability (TRT), and (4) construct validity with EQ-5D and EORTC-QLQ-C30. Data from interviews, the pilot test, and field-test were triangulated to select the best response options for the aforementioned constructs. Results: The field test included 1717 women aged $46 \pm 10$ years. Most women were diagnosed with primary BC stage 0-2 (84\%) within the last decade (52\%). Items measuring interference for fatigue, pain, emotional distress, and unpleasant symptoms were found to capture higher levels of variability (by demographic, clinical, and treatment characteristics) and had marginally higher TRT and construct validity than items measuring severity. For arm mobility and usual activities, this difference was not substantial. When all data sources were considered, interference items were found to better capture patient experience. Conclusion: This study highlights the value of transparent, empirical, and patient-driven decisions when choosing response options for PROMs.

\section{3: Clinical Research of COVID-19}

(B203.1) The impact of COVID-19 pandemic on health status, based on the EQ-5D-5L, of adults visiting emergency departments and primary care clinics in Alberta, Canada

Fatima Al Sayah, University of Alberta, Edmonton, Alberta, Canada; Roland Simon, Health Quality Council of Alberta, Calgary, Alberta, Canada; Benjamin Higgins, Health Quality Council of Alberta, Calgary, Alberta, Canada; Markus Lahtinen, Health Quality Council of Alberta, Calgary, Alberta, Canada; Arto Ohinmaa, University of Alberta, Edmonton, Alberta, Canada; Jeffrey A. Johnson, University of Alberta, Edmonton, Alberta, Canada

Aims: The aim of the study is to examine the impact of the COVID19 pandemic on health-related quality of life (HRQL), based on the EQ-5D-5L, of adults visiting emergency departments (ED) and primary care (PC) clinics in Alberta, Canada; and explore whether the impact of the pandemic is disproportionate across groups of the population. Methods: We used data from two repeated cross-sectional population-based surveys and examined EQ-5D-5L dimensions and index and visual analogue scale (VAS) scores in the "pre-COVID19" period (September 2019-February 2020: ED survey $N=5927$; PC survey $N=317$ ), and throughout "wave 1" (March 2020-August 2020: ED survey $\mathrm{N}=4781$; PC survey $N=375$ ), and "wave 2" (September 2020-January 2021: ED survey $N=4443$; PC survey $N=327$ ), in the overall samples and by population sub-groups. Results: In the ED sample, there were no differences in the level of problems reported in any EQ-5D-5L dimension, the index or VAS scores pre and during COVID. Small to moderate differences were observed in the PC sample, whereby a higher proportion of respondents reported more problems during the pandemic compared to preCOVID, especially in the pain/discomfort and anxiety/depression dimensions. There were no significant differences in reporting problems on EQ-5D-5L dimensions, or in the index or VAS scores across age subgroups, gender, and income levels in the ED survey; however, differences were observed in the PC survey: for instance, adults aged 25-44 years and those in the middle-income category reported slightly less problems during COVID, and a higher proportion of adults aged 45-64 years and females reported more problems during-COVID compared to pre-COVID. Index and VAS scored were the lowest for those in the highest income category during COVID. Overall, the samples in both surveys had lower HRQL compared to the general Alberta population except for the anxiety/depression dimension. Conclusion: In adults seeking ED and PC services in Alberta, the impact of the COVID-19 pandemic on HRQL was minimal in the former and more pronounced in the latter especially in the anxiety/depression dimension, with noticeable variability across population sub-groups whereby younger respondents and females were among the most affected. It is imperative to consider the most affected groups in the population in developing policies around COVID-19.

(B203.2) Application of electronic patient-reported outcomebased symptom monitoring for asymptomatic patients of SARSCoV-2: a feasibility study

Qingsong Yu, Postgraduate, School of Public Health and Management, Chongqing Medical University, Chongqing, China; Wei Xu, doctor School of Public Health and Management, Chongqing Medical University, Chongqing, China; Hongfan Yu, Postgraduate, School of Public Health and Management, Chongqing Medical University, Chongqing, China; Yang Pu, doctor School of Public Health and Management, Chongqing Medical University, Chongqing, China; Qiuling Shi, doctor School of Public Health and Management, State Key Laboratory of Ultrasound in Medicine and Engineering,Chongqing Medical University, Chongqing, China

Aims: With the development of the epidemic situation, improving the management of asymptomatic patients with coronavirus disease 2019 (COVID-19) has become one of the priorities in the gradual return to normality. We aimed to evaluate the feasibility, usability and acceptability of e-PRO (electronic patient-reported outcome) platform in potential symptom monitoring and early alerting during the period of centralized management for asymptomatic-infected patients with COVID-19. Methods: Data extracted from a prospective cohort study of 64 asymptomatic-infected patients. Symptoms were assessed by symptom inventory generated by Delphi Technique with daily symptom assessment via smartphone APP for 30 days. The symptom measure used a $0-10$ scale and a $24-\mathrm{h}$ recall period. Data were collected and recorded into the REDcap database. Patient compliance and data integrity were examined. Results: Sixty-four patients with complete 30-day symptom measurement and records were included in this analysis. During the 30 days of monitoring, over $80 \%$ of asymptomatic-infected patients complete all 21 symptom items. The finishing rate of symptom less than 80 percent on the ninth day $(71.9 \%)$ and thirtieth day $(78.1 \%)$. Of them, the percentage of each item missing was low (range 1.7-3.7\%). Missing rate of symptom scale items per day was below $5 \%$ except in the fifth day (6.5\%), tenth day $(9.3 \%)$ and 29 th day $(8.9 \%)$. More than $90 \%$ of all symptoms were scored on a scale of $0-4$. Symptoms reported 4 or greater included sleep disturbance $(2.8 \%, 50 / 1761)$, anxiety $(1.2 \%, 21 / 1762)$, and lack of appetite $(0.45 \%, 8 / 1759)$. Conclusion: Symptom monitoring based on e-PRO has revealed high feasibility, acceptability, and compliance in asymptomatic-infected populations, and it can also identify potential symptoms. 
Tablel Number of patients completed symptom inventory per day (21 symptom items were all finished )

\begin{tabular}{|c|c|c|c|}
\hline $\begin{array}{l}\text { Time } \\
\text { point }\end{array}$ & $\begin{array}{c}\text { Actual number of } \\
\text { patients }\end{array}$ & $\begin{array}{l}\text { Number of patients that } \\
\text { should be filled }\end{array}$ & $\%$ \\
\hline 1 & 61 & 64 & 95.3 \\
\hline 2 & 59 & 64 & 92.2 \\
\hline 3 & 60 & 64 & 93.8 \\
\hline 4 & 59 & 64 & 92.2 \\
\hline 5 & 56 & 64 & 87.5 \\
\hline 6 & 55 & 64 & 85.9 \\
\hline 7 & 57 & 64 & 89.1 \\
\hline 8 & 56 & 64 & 87.5 \\
\hline 9 & 46 & 64 & 71.9 \\
\hline 10 & 53 & 64 & 82.8 \\
\hline 11 & 58 & 64 & 90.6 \\
\hline 12 & 56 & 64 & 87.5 \\
\hline 13 & 55 & 64 & 85.9 \\
\hline 14 & 57 & 64 & 89.1 \\
\hline 15 & 55 & 64 & 85.9 \\
\hline 16 & 58 & 64 & 90.6 \\
\hline 17 & 58 & 64 & 90.6 \\
\hline 18 & 60 & 64 & 93.8 \\
\hline 19 & 59 & 64 & 92.2 \\
\hline 20 & 60 & 64 & 93.8 \\
\hline 21 & 58 & 64 & 90.6 \\
\hline 22 & 58 & 64 & 90.6 \\
\hline 23 & 58 & 64 & 90.6 \\
\hline 24 & 54 & 64 & 84.4 \\
\hline 25 & 57 & 64 & 89.1 \\
\hline 26 & 55 & 64 & 85.9 \\
\hline 27 & 58 & 64 & 90.6 \\
\hline 28 & 54 & 64 & 84.4 \\
\hline 29 & 54 & 64 & 84.4 \\
\hline 30 & 50 & 64 & 78.1 \\
\hline
\end{tabular}

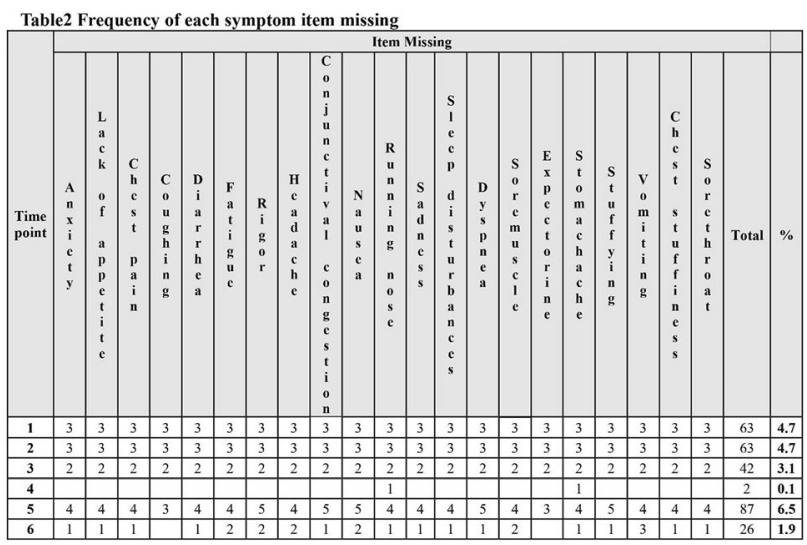

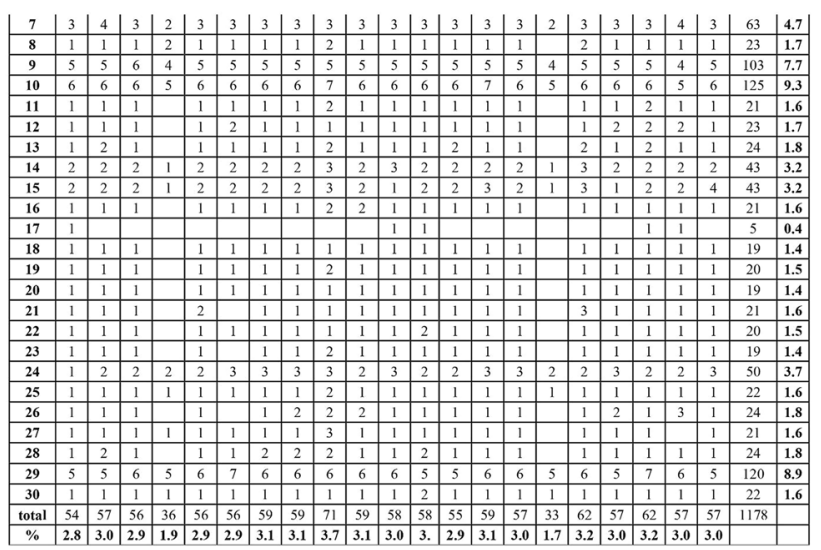

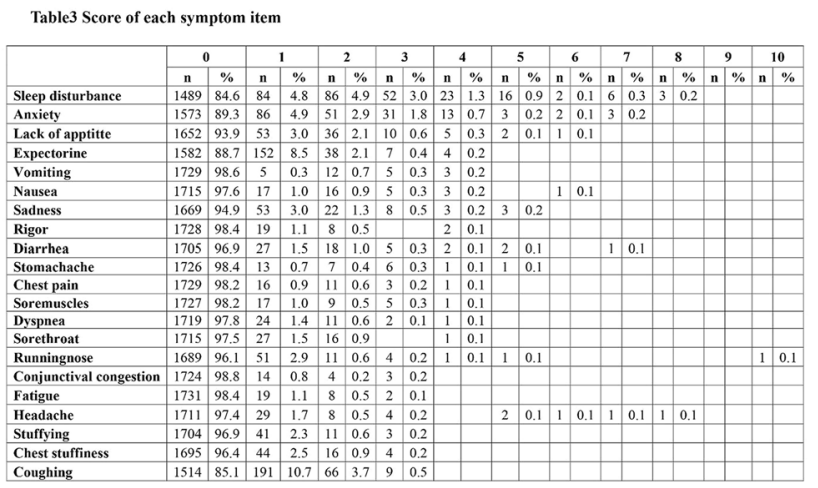

(B203.3) Work-related quality of life of night-shift healthcare workers at the time of COVID-19: a survey in Parisian public hospital networks (AP-HP ALADDIN)

Martin Duracinsky, MD, PhD, Partient-Centered Outcomes Research, AP-HP, Paris, France; Vincent Di Beo, Aix Marseille Univ, INSERM, IRD, SESSTIM, Sciences Economiques \& Sociales de la Santé \& Traitement de l'Information Médicale, Marseille, France; Lorraine Cousin, Unité de Recherche Clinique en Economie de la Santé (URCECO), AP-HP, Hôpital Hôtel-Dieu, Paris, France; Olivier Chassany, Unité de Recherche Clinique en Economie de la Santé (URC-ECO), AP-HP, Hôpital Hôtel-Dieu, Paris, France; Patrizia Carrieri, Aix Marseille Univ, INSERM, IRD, SESSTIM, Sciences Economiques \& Sociales de la Santé \& Traitement de l'Information Médicale, Marseille, France; Fabienne Marcellin, Aix Marseille Univ, INSERM, IRD, SESSTIM, Sciences Economiques \& Sociales de la Santé \& Traitement de l'Information Médicale, Marseille, France

Aims: Hospital healthcare workers (HCW) are on the frontline during the COVID-19 pandemic, a stressful period with both human and organizational challenges. Among them, night-shift HCW are exposed to health deterioration due to an alteration of the circadian rhythms. Work-related quality of life (WRQoL) remains poorly documented in this population at the time of COVID-19.This study aims at documenting determinants of WRQoL among night-shift HCW in Parisian public hospitals (AP-HP) during the first wave of the COVID-19 pandemic. Methods: The AP-HP ALADDIN observational cross-sectional online survey (15 June to 15 September 2020) included 1387 night-shift HCW of the AP-HP hospitals, and collected data on HCW's socio-economic, behavioral, and work-related 
characteristics as well as their perceptions about the quality of their working life (WRQoL scale). Survey data were weighed (margin calibration) to ensure their representativeness of the entire population of 12,000 HCW in AP-HP hospitals regarding sex, age, and professional category. Linear regression model was used to identify correlates of the WRQoL global score (range 0 to 115 , higher values denote better WRQoL). Results: Median [interquartile range] WRQoL score was 71 [63-78]. The model was adjusted for socioeconomic characteristics, professional category, perceived health, physical activity, and history of harassment at work. A recent change for night shift during the COVID epidemic (coefficient [95\% confidence interval]: $-12.56[-23.81 ;-1.31], p=0.029)$, difficulties in getting screened for SARS-CoV-2 infection (-2.95 [-4.25; $-1.65], p<0.001)$, and considering protective measures inadequate $(-2.09[-3.52 ;-0.65], p=0.004)$ were independently associated with lower WRQoL. Perceiving under-estimation of night-shift work by day-shift colleagues, reporting night work as a source of tension with friends, or feeling more irritable since working at night also impaired WRQoL. By contrast, satisfaction regarding information received on COVID from the employer (4.67 [3.26; 6.07], $p<0.001$ ) and feeling valued by the general population during the epidemic $(1.41[0.13 ; 2.70], p=0.031)$ were independently associated with higher WRQoL. Conclusion: Lack of or insufficient access to screening, information, and protective measures impaired WRQoL of night-shift HCW during the COVID-19 first wave. Social and professional recognition of night-shift work appear as key determinants of WRQoL in this population.

(B203.4) Qualitative interviews with COVID-19 patients: content validity of the FLU-PRO Plus for use in COVID-19 clinical research

Kimberly Raymond, QualityMetric, Johnston, Rhode Island, USA; Tom Keeley, GSK, Uxbridge, UK; Helen Birch, GSK, Brentford, UK; Sacha Satram, Vir Biotechnology, San Francisco, California, USA; Carolina Reyes, Vir Biotechnology, San Francisco, California, USA; Cory Saucier, QualityMetric, Johnston, Rhode Island, USA; Craig Tipple, GSK, Brentford, UK; April Foster, QualityMetric, Johnston, Rhode Island, USA; Andrew Lovely, QualityMetric, Johnston, Rhode Island, USA; Mark Kosinski, QualityMetric, Johnston, Rhode Island, USA

Aims: The Coronavirus Disease-2019 (COVID-19) pandemic presents an urgent challenge to understand this novel disease from the patient perspective and identify a fit-for-purpose patient-reported outcome (PRO) measure of symptoms suitable for efficacy analyses of COVID-19 treatments. This qualitative, non-interventional study identified symptoms and impacts important to COVID-19 patients and assessed whether the Influenza Patient Reported Outcome Plus (FLUPRO Plus) — an augmented version of the FLU-PRO — is content valid for assessing the symptoms of COVID-19. Methods: Thirty adults, residing in the US, with a recently confirmed positive COVID-19 laboratory test, were recruited for participation. The sample included individuals with and without comorbidities, who had mild, moderate, or severe COVID-19 symptoms. Hybrid concept elicitation/cognitive debriefing interviews were conducted, via telephone or webcam, by trained qualitative interviewers using a semi-structured guide. Results: Participants experienced numerous symptoms during their COVID-19 illness. Symptoms reported across participants varied. Commonly reported symptoms included fatigue $(n=30,100 \%)$, nasal congestion $(n=25,83 \%)$, cough $(n=24,80 \%)$, loss of taste $(n=23,77 \%)$, loss of smell $(n=21,70 \%)$, and more debilitating respiratory symptoms such as chest congestion $(n=15,50 \%)$ and difficulty breathing $(n=17,57 \%)$. Despite the heterogeneity of symptomatic experiences, all items of the FLU-PRO Plus received high levels of endorsement across the 30 interviews and mapped directly to symptoms reported by participants. Study participants shared positive feedback indicating that the FLU-PRO Plus is easy to understand and complete, with instructions, items, recall period, and response options appropriate for capturing their experience of COVID-19. Conclusion: Findings from this study provide valuable knowledge of illness trajectories and impacts and identify symptoms most important to measure in clinical research. Results support FLUPRO Plus as appropriate, understandable, comprehensive, and relevant. Coverage of symptoms included in this instrument is appropriate and necessary for capturing the diversity of symptoms and align with participants' descriptions of COVID-19, demonstrating comprehensiveness and appropriateness of the instrument's content. The results of this study support the content validity of the FLU-PRO Plus, providing evidence that it is well suited for use in individuals with COVID-19. Funding: GSK (Study 215032).

(B203.5) Measuring the impact of COVID-19 on the quality of life of the survivors, partners and family members: A cross-sectional international online survey

Rubina Shah, Cardiff University, Cardiff, UK; Faraz M Ali, Cardiff University, Cardiff, UK; Stuart J Nixon, MS society, Cardiff, UK; John R Ingram, Cardiff University, Cardiff, UK; Sam M Salek, University of Hertfordshire, Hatfield, UK; Andrew Y Finlay, Cardiff University, Cardiff, UK

Aims: This study aimed to measure the impact of COVID-19 on the quality of life (QoL) of survivors and of their partners and family members. Methods: A prospective cross-sectional global online survey was conducted using social media from June to August 2020 aimed at COVID-19 patients and partners or family members (age $\geq 18$ years). The EQ-5D-3L was used to measure the QoL of survivors of COVID-19, and the Family Reported Outcome Measure (FROM-16) was used to assess the impact on their partner/family member's QoL. Results: The survey was completed by 735 COVID19 survivors (mean age $=48$ years; females $=563$ ) at a mean of 12.8 weeks after diagnosis and by 571 partners and 164 family members $(n=735$; mean age $=47$ years; females $=246)$ from Europe $(50.6 \%)$, North America $(38.5 \%)$ and rest of the world $(10.9 \%)$. The EQ-5D mean score for COVID-19 survivors was 8.65 (SD = 1.9, median $=9$; range 6-14). $81.1 \%(596 / 735)$ reported pain and discomfort, 79.5\% (584/735) problems with usual activities, 68.7\% (505/ 735) anxiety and depression and 56.2\% (413/735) problems with mobility. Hospitalised survivors $(20 \%, n=148)$ and survivors with existing health conditions $(30 \%, n=227)$ reported significantly more problems with mobility and usual activities $(p<0.05)$, with hospitalised also experiencing significant impact on self-care $(p \leq 0.001)$.Among 735 partners and family members, the mean FROM-16 score (maximum score $=$ highest impact $=32$ ) was 15 $($ median $=15$, range $0-32) .93 .6 \%(688 / 735)$ reported being worried, $81.7 \%(601 / 735)$ frustrated, $78.4 \%$ (676/735) sad, $83.3 \%(612 / 735)$ reported impact on their family activities, $68.9 \%$ (507/735) on sleep and $68.1 \%(500 / 735)$ on their sex life. Conclusion: The lives of the partners and other family members of COVID-19 survivors are severely affected by their family member having COVID-19. COVID-19 survivors report a major persisting impact on their physical and psychosocial functional behaviours. There is a need for a holistic support system sensitive to the needs of COVID-19 survivors and of their family members who experience a major "secondary burden". 


\section{4: Clinical Application Studies of Children and Vulnerable Populations}

(B204.1) Contextual factors affecting participation of children with low vision or blindness

Deepak Bagga, PhD, L V Prasad Eye Institute, Hyderabad, Telangana, India, Hyderabad, India; Vijaya Gothwal, PhD, L V Prasad Eye Institute, Hyderabad, India, Hyderabad, India; Jill Keeffe, L V Prasad Eye Institute, Hyderabad, India, Melbourne, Australia; Serge Resnikoff, University of New South Wales, Sydney, Australia, Geneva, Switzerland

Aims: The International Classification of Functioning, Disability and Health (ICF), describes participation as an outcome of interactions among an individual's body functions and contextual factors that includes environmental and personal factors. Hence, assessing influence of contextual factors on participation of children with low vision or blindness is important to plan their Low Vision Rehabilitation (LVR) interventions. The aim of this study was to identify the relevant and important contextual factors affecting participation of school-age children (8-17 years) with low vision or blindness. Methods: A total of 541 individuals including children with low vision or blindness $(n=188)$, their parents $(n=81)$, children with normal vision $(n=208)$, teachers $(n=52)$, and LVR service providers $(n=12)$ participated in brainstorming sessions or semistructured interviews to generate ideas related to contextual factors and participation. Brainstorming sessions were audio recorded and transcribed. This was followed by identification of relevant concepts that were linked with the ICF codes to verify the comprehensiveness of content and idea synthesis. The concept mapping methodology was used to identify domains of contextual factors from the perspectives of participants. Results: A total of 2267 statements were generated from 58 brainstorming sessions. The synthesis of statements provided 123 unique contextual factors. Cluster analyses revealed 7 domains of environmental factors: support and attitudes of family members, support and attitudes of teachers, bullying, society, products and technology, lighting, and services, systems and policies; and 3 domains of personal factors: child's feelings, confidence, and interests. Thirty-eight percent (47/123) of the contextual factors were rated above average relevance and importance for participation of children with low vision or blindness. Perceived importance assigned to the contextual factors by children with low vision or blindness had moderate to strong and positive correlation with the ratings of their parents and teachers; on the other hand, it had poor correlation with the perceived importance assigned by children with normal vision and low vision rehabilitation service providers. Conclusion: The key findings were (1) school-age children can identify the relevant and important domains of contextual factors that can affect their participation and (2).the perception regarding contextual factors affecting participation of children with low vision or blindness differs among stakeholders.
(B204.2) Effectiveness of ePRO Alert Intervention on Symptom Burden and Readiness for Discharge in Patients Undergoing Lung Cancer Surgery: Secondary Analysis of A Randomized Controlled Study

Yaqin Wang, Sichuan Cancer Hospital, Chengdu, China; Wei Dai, Sichuan Cancer Hospital, Chengdu, China; Jia Liao, Sichuan Cancer Hospital, Chengdu, China; Xiaoqin Liu, Sichuan Cancer Hospital, Chengdu, China; Hongfan Yu, Chongqing Medical University, Chongqing, China; Qiuling Shi, Chongqing Medical University, Chongqing, China

Aims: This study aimed to investigate the effect of the electronic patiented-reported outcome (ePRO) early warning intervention program on symptom burden and readiness for discharge of patients undergoing lung cancer surgery at the time of discharge, and to provide a reference for the development and implementation of future ePRO precision intervention programs. Methods: This study was a secondary analysis of data from a randomized controlled trial $(\mathrm{CN}$ PRO-Lung 2), which conducted in three tertiary hospitals between November 2019 and August 2020. The inclusion criteria for the study population were had a clinical diagnosis of primary lung cancer, planed to receive surgical treatment, aged $\geq 18$ years, and willing to participate in this study. Patients were randomized in a 1:1 ratio to the intervention group (ePRO threshold warning) or the control group. The study instruments used were the MD Anderson Symptom Inventory-Lung Cancer and the Discharge Readiness Scale. Results: The final intervention group included 51 patients and the control group included 55 patients. There were no statistically significant differences between the two groups in terms of age, body mass index, postoperative hospital days, gender, smoking history, surgical approach, surgical type, tumor pathological type, and tumor pathological stage. The top five symptom burdens on the day of discharge were pain, fatigue, shortness of breath, coughing, and constipation. The symptom burden in the intervention group was lower than the control group. The differences in fatigue and coughing were statistically significant. The total score and the score of personal status, knowledge, coping ability, and expected support were high, and the differences between the test groups were not statistically significant differences. Core symptoms are negatively correlated with readiness for discharge in some ways $(-0.199 \leq r \leq-0.445)$. Conclusion: ePRO threshold warning shows significant benefits in reducing patient symptom burden. The core symptoms are negatively correlated with readiness for discharge, suggesting that further researches may be needed to see if readiness for discharge is appropriate in the era of minimally invasive surgery. 
Table 1. Demographics and clinical characteristics

\begin{tabular}{|c|c|c|c|c|}
\hline Variables & $\begin{array}{c}\text { ePRO intervention group } \\
\qquad(\mathbf{n}=\mathbf{5 1})\end{array}$ & $\begin{array}{l}\text { ePRO control group } \\
\qquad(\mathrm{n}=55)\end{array}$ & $t / X^{2}$ & $\mathbf{P}$ \\
\hline Age, mean (SD), yr & $51.14+11.65$ & $51.09+10.21$ & 0.319 & 0.98 \\
\hline Body mass index, mean (SD) & $22.85 \pm 2.60$ & $22.24 \pm 3.13$ & 0.541 & 0.27 \\
\hline $\begin{array}{l}\text { Postoperative hospital days } \\
\text { mean (SD) }\end{array}$ & $5.22+1.93$ & $5.16+2.00$ & 0.022 & 0.892 \\
\hline Gender & & & 0.248 & 0.618 \\
\hline Male & 20 & 19 & & \\
\hline Female & 31 & 36 & & \\
\hline Highest level of education & & & 0.001 & 0.971 \\
\hline Middle school and below & 23 & 25 & & \\
\hline Above middle school & 28 & 30 & & \\
\hline Smoking history & & & 2.939 & 0.568 \\
\hline None & 40 & 46 & & \\
\hline Light smoking & 0 & 1 & & \\
\hline Moderate smoking & 1 & 2 & & \\
\hline Heavy smoking & 7 & 5 & & \\
\hline $\begin{array}{l}\text { Quitting smoking } \quad \text { ( } 66 \\
\text { months) }\end{array}$ & 3 & 1 & & \\
\hline Surgical approach & & & 0.297 & 0.586 \\
\hline Minimal invasive surgery & 48 & 53 & & \\
\hline Open surgery & 3 & 2 & & \\
\hline Surgical type & & & 3.044 & 0.550 \\
\hline Sublobar resection & 13 & 18 & & \\
\hline Lobectomy & 35 & 30 & & \\
\hline bilobectomy & 1 & 2 & & \\
\hline Extended resection & 2 & 5 & & \\
\hline Tumor pathological type & & & 2.137 & 0.144 \\
\hline Adenocarcinoma & 47 & 54 & & \\
\hline Others & 4 & 1 & & \\
\hline Tumor pathological stage & & & 1.355 & 0.508 \\
\hline I & 45 & 49 & & \\
\hline II & 4 & 2 & & \\
\hline III & 2 & 4 & & \\
\hline Postoperative complications & & & 0.553 & 0.457 \\
\hline No & 46 & 47 & & \\
\hline Yes & 5 & 8 & & \\
\hline
\end{tabular}

Table 2. Comparison of symptom burden on the day of discharge

\begin{tabular}{|c|c|c|c|c|}
\hline \multirow{2}{*}{ Variables } & \multicolumn{2}{|c|}{ Mean \pm SD } & \multirow{2}{*}{$\mathbf{Z}$} & \multirow{2}{*}{$\mathbf{P}$} \\
\hline & ePRO intervention group ( $n=51$ ) & ePRO control group ( $\mathrm{n}=55)$ & & \\
\hline Pain & $2.04 \pm 1.40$ & $2.76 \pm 1.90$ & 1.817 & 0.069 \\
\hline Fatigue & $2.20 \pm 1.78$ & $3.05 \pm 1.98$ & 2.547 & 0.011 \\
\hline Nausea & $0.49 \pm 0.76$ & $0.96 \pm 0.1 .61$ & 0.963 & 0.336 \\
\hline Disturbed sleep & $1.67 \pm 1.60$ & $2.64 \pm 2.20$ & 2.318 & 0.020 \\
\hline Distress & $1.49 \pm 1.74$ & $2.05 \pm 1.87$ & 1.709 & 0.087 \\
\hline Shortness of breath & $2.41 \pm 2.13$ & $3.00 \pm 1.95$ & 1.699 & 0.089 \\
\hline Difficulty & $1.33 \pm 1.44$ & $1.60 \pm 1.58$ & 0.752 & 0.452 \\
\hline \multicolumn{5}{|l|}{ remembering } \\
\hline Lack of appetite & $1.49 \pm 1.64$ & $2.16 \pm 2.09$ & 1.643 & 0.100 \\
\hline Drowsiness & $1.69 \pm 1.69$ & $2.11 \pm 1.73$ & 1.444 & 0.149 \\
\hline dry mouth & $1.57 \pm 1.46$ & $2.24 \pm 1.98$ & 1.570 & 0.116 \\
\hline Sadness & $1.37 \pm 1.93$ & $1.60 \pm 1.84$ & 0.829 & 0.407 \\
\hline vomiting & $0.31 \pm 0.74$ & $0.62 \pm 1.37$ & 1.183 & 0.237 \\
\hline Numbness or tingling & $0.63 \pm 1.02$ & $1.15 \pm 1.56$ & 1.697 & 0.090 \\
\hline Coughing & $2.53 \pm 1.75$ & $3.45 \pm 2.24$ & 2.090 & 0.037 \\
\hline Constipation & $2.10 \pm 2.19$ & $3.24 \pm 3.20$ & 1.599 & 0.111 \\
\hline Sore throat & $1.43 \pm 1.47$ & $1.76 \pm 2.04$ & 0.457 & 0.648 \\
\hline
\end{tabular}

Table 3. Comparison of readiness for hospital discharge between the two groups

\begin{tabular}{|c|c|c|c|c|}
\hline \multirow{2}{*}{ Variables } & \multicolumn{2}{|c|}{ Mean \pm SD } & \multirow{2}{*}{$\mathbf{z}$} & \multirow{2}{*}{$\mathbf{P}$} \\
\hline & ePRO intervention group $(n=51)$ & ePRO control group ( $\mathbf{n}=\mathbf{5 5}$ ) & & \\
\hline Total Score & $155.35 \pm 45.93$ & $160.05 \pm 31.02$ & -0.013 & 0.990 \\
\hline Personal status & $49.76 \pm 13.11$ & $50.13 \pm 9.75$ & -0.234 & 0.815 \\
\hline Knowledge & $51.53 \pm 23.91$ & $51.93 \pm 20.96$ & -0.399 & 0.690 \\
\hline Coping ability & $22.14 \pm 6.82$ & $23.80 \pm 4.86$ & 1.026 & 0.305 \\
\hline Expected support & $31.92 \pm 8.39$ & $34.20 \pm 6.42$ & 1.308 & 0.191 \\
\hline
\end{tabular}

Table 4. Analysis of the correlation between core symptoms and readiness for discharge

\begin{tabular}{|c|c|c|c|c|c|}
\hline & Pain & Fatigue & Shortness breath & Coughing & Constipation \\
\hline Pain & 1 & $.742^{* *}$ & $.397^{* *}$ & $.566^{* *}$ & $.212^{\circ}$ \\
\hline Fatigue & $.742^{* *}$ & 1 & $.644^{* *}$ & $.629^{*}$ & $.295^{*}$ \\
\hline Shortness breath & $.397^{* *}$ & $.644^{* *}$ & 1 & $.629^{* *}$ & $.441^{* 4}$ \\
\hline Coughing & $.566^{* *}$ & $.629^{\prime \prime \prime}$ & $.629^{n *}$ & 1 & $.409^{* \prime}$ \\
\hline Constipation & $.212^{*}$ & $.295^{* *}$ & $.441^{* *}$ & $.409^{* *}$ & 1 \\
\hline Total Score & -0.133 & $-.296^{*}$ & $-.314^{*}$ & -0.185 & $-.232^{\circ}$ \\
\hline Personal status & $-.211^{*}$ & $-.445^{* *}$ & $-.400^{* *}$ & $-.246^{*}$ & $-.214^{\circ}$ \\
\hline Knowledge & -0.043 & -0.156 & $-.222^{*}$ & -0.126 & $-.199^{\circ}$ \\
\hline Coping ability & -0.085 & $-.304^{* *}$ & $-.291^{* *}$ & -0.108 & $-.231^{*}$ \\
\hline Expected support & -0.174 & -0.150 & -0.124 & -0.122 & -0.101 \\
\hline
\end{tabular}

Abbreviations: *** means $\mathrm{P} \leqslant 0.01,{ }^{*}$ means $\mathrm{P} \leqslant 0.05$.

(B204.3) Association between malocclusion and Oral Healthrelated Quality of life among population-based sample children in SalvadOR Brazil 
Mercia Santos, Master, UFBA, SalvadOR Brazil; Tatiana de Almeida, doctor in Public Health, School of Dentistry, Federal University of Bahia - UFBA, SalvadOR Brazil; Jéssica Lima, Undergraduate Student, School of Dentistry, Federal University of Bahia - UFBA, SalvadOR Brazil; Lucililian Silva, Undergraduate Student, School of Dentistry, Federal University of Bahia - UFBA, SalvadOR Brazil; Maria Beatriz Cabral, doctor in Public Health, School of Dentistry, Federal University of Bahia - UFBA, SalvadOR Brazil; Maria Isabel Vianna, doctor in Public Health, School of Dentistry, Federal University of Bahia - UFBA, SalvadOR Brazil; Maria Cristina Cangussu, doctor in Public Health, School of Dentistry, Federal University of Bahia - UFBA, SalvadOR Brazil

Aims: In Brazil, malocclusion prevalence on preschoolers presents regional patterns that may be related to social inequalities and environmental factors. As the oral health-related quality of life (OHRQoL) is also mediated by these factors, the present study aimed to measure the association between malocclusion and OHRQoL in a population-based sample of 1 to 6-year-old children living in the city of SalvadOR Bahia, Brazil. Methods: 1566 children/parents were recruited from randomly drawn health units and municipal daycare centers in SalvadOR Brazil. Parents filled out a self-administered questionnaire to obtain socio-demographic and general health data, behaviors aspects, and OHRQOL measurements. Previously calibrated examiners performed children's dental examinations. Malocclusion was scored according to the Foster and Hamilton Index. OHRQoL was measured with the Brazilian version of the Early Childhood Oral Health Impact Scale (B-ECOHIS). Malocclusion, covariates, and general, child, and family OHRQOL impact were dichotomized for analysis. Statistical analysis involved Pearson's chisquare test and binomial logistic regression considering a significance level of $5 \%$. Results: The prevalence of mild to moderate/severe malocclusion was $26 \%$. The most frequent conditions were open bite $(13.9 \%)$ and deep overbite $(11.1 \%)$. The negative impact on OHRQoL was reported by $32.8 \%$ of those parents, with child impact $(27.1 \%)$ higher than the family OHRQoL impact (20.0\%). Child domains of symptom (20.9\%) and function (17.1\%), and family domain of parental distress $(17.9 \%)$ were the most frequently impacted ones. In the bivariate analysis, malocclusion was associated with general impact ( $p=0.008)$, child impact $(p=0.066)$, and family impact $(p=0.001)$. In the crude association, only general OHRQOL impact (OR $0.7195 \%$ CI $0.56-0.92$ ) and family OHRQOL impact (OR 0.59 95\% CI $0.43-0.80$ ) maintained statistical significance. However, after adjusting for covariates, none of the outcomes remained significant. Conclusion: In that representative sample of children and their families in the city of SalvadOR Brazil malocclusion was not associated with a negative impact on OHRQoL.

\begin{tabular}{|c|c|c|c|c|}
\hline Outcomes & Crude OR $(95 \% \mathrm{CI})$ & pValue & Ajustaded OR $(95 \% \mathrm{CI})$ & pValue \\
\hline General OHRQDL & $0,71(0,56-0,92)$ & 0,008 & $1,12(0,74-1,69)^{3}$ & 0,584 \\
\hline Child impact & $0,78(0,68-1,02)$ & 0,066 & $0,93(064-1,35)^{0}$ & 0,697 \\
\hline Familiar Impact & $0,59(0,43-0,80)$ & 0,001 & $0,65(0,41-1,03)^{6}$ & 0,064 \\
\hline
\end{tabular}

(B204.4) Utilization of patient-reported outcomes instruments in pediatric asthma trials and product labels

Ember Lu, PharmD, GSK, Philadelphia, Pennsylvania, USA; AnneSophie Michel, Mapi Research Trust, Lyon, France; Simona Boccaletti, GSK, Brentford, UK; Anna Richards, MD, GSK, Brentford, UK; Tom Keeley, PhD, GSK, Brentford, UK
Aims: Pediatric asthma (age 1-12) causes substantial burden to patients. There are several patient-reported outcome (PRO) instruments used in clinical trials to measure the impact of the disease. The aim of this review is to identify the most frequently used PRO instruments in clinical trials for pediatric asthma and their subsequent use in product labels. Methods: A targeted literature review of MEDLINE (via Pubmed) and EMBASE was conducted using PRISMA methodology to identify PRO instruments validated for use in paediatric asthma. For each identified PRO, we determined their frequency of use in pediatric clinical trials on ClinicalTrials.gov, with a first post date of January 2015 to December 2020. Finally, we searched for PROs labelling claims in FDA or EMA approved products in pediatric asthma using the Mapi Research Trust PROLABELSTM database. Results: The three most frequently used PROs in pediatric clinical trials were the Asthma Control Questionnaire (ACQ), Childhood Asthma Control Test (C-ACT), and Pediatric Asthma Quality of Life Questionnaire (PAQLQ). We identified three product labels (mepolizumab, tiotropium bromide, budesonide/ formoterol) containing these PROs. After a manual search of the labels, it was confirmed that the label for budesonide/formoterol was the only one to list a specific PRO (PAQLQ) in reference to the pediatric population. ACQ was only referenced for the adult population and $\mathrm{C}-\mathrm{ACT}$ was not included in any labels. As an exploratory analysis, we reviewed the phase III pediatric trials of the three products to identify which PROs were assessed. Mepolizumab included ACQ and C-ACT, tiotropium bromide included ACQ and PAQLQ, and budesonide/formoterol included PAQLQ. All pediatric phase III outcomes measured with the mentioned PRO tools did not reach statistical significance. Conclusion: Pediatric-specific PRO tools (ACQ, C-ACT, PAQLQ) were utilized in clinical trials for pediatric asthma, but results did not reach statistical significance and, except for budesonide/formoterol, do not appear in the product label. These findings raise awareness of the challenges in assessing PROs in pediatric asthma programs and provides support for further assessment of how PROs can be optimized to capture patients' symptomatic improvement and holistic experience. Funding source: GSK.

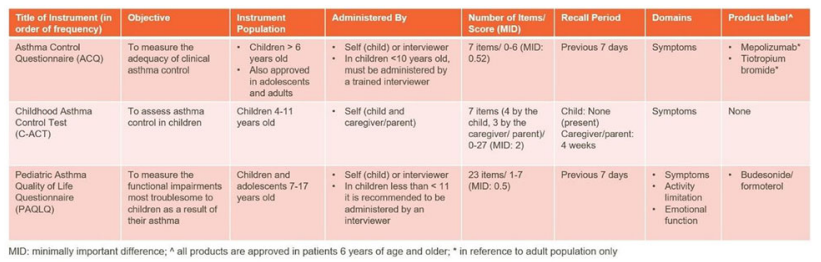

(B204.6) Compliance on drug storage among young-old, middleold and old-old adults

Lori Suet Hang Lo, MSc, BN, The Open University of Hong Kong, Hong Kong, Hong Kong; Linda Yin King Lee, PhD, MA, MN,BN, The Open University of Hong Kong, Hong Kong, Hong Kong; Irene Yuen Fung Wong, MHM,BN, The Open University of Hong Kong, Hong Kong, Hong Kong; Yuen Yan Lai, The Open University of Hong Kong, Hong Kong, Hong Kong; Hoi Tong Au, The Open University of Hong Kong, Hong Kong, Hong Kong; Tsz Ying Wong, The Open University of Hong Kong, Hong Kong, Hong Kong; Chui Ping Cheung, The Open University of Hong Kong, Hong Kong, Hong Kong; Wai Lam Shek, The Open University of Hong Kong, Hong Kong, Hong Kong; Choi Yi Wong, The Open University of Hong Kong, Hong Kong, Hong Kong; Hoi Lam Chan, The Open University of Hong Kong, Hong Kong, Hong Kong; Hon Sze Cheuk, The Open University of Hong Kong, Hong Kong, Hong Kong; Nga Hing Mak, The Open University of Hong Kong, Hong Kong, Hong Kong; Shu Ching Wong, The Open University of Hong Kong, Hong Kong, Hong Kong; Wing Yan Cheung, The Open University of Hong Kong, Hong Kong, Hong Kong 
Aims: Storing drug at home is common globally. Adopting proper environment and method to store drug are necessary to avoid negative consequences. Compared with younger adults, studies indicated that the older adults had higher prevalence of managing drugs at home by themselves but were less compliant with the recommendations on drug storage. However, previous findings were inconclusive to show the association between the compliance on drug storage and the age of the older adults. This study aimed to examine the compliance on drug storage between older adults in different old-age groups. Methods: This cross-sectional study recruited 117 older adults who aged 65 years or above and managed drugs at home. Data were collected during home visits. Participants' demographic information was collected via questionnaires, and their compliance on drug storage was assessed using the In-home Drug Storage Checklist. The checklist assessed participants' compliance in two areas: environment and method for storing drug. The overall compliance on drug storage was evaluated, and the compliance rate (\%) was calculated. Kruskal-Wallis Test was used to compare the difference in compliance rates between subjects in the three age groups, which were young-old (65 to 74 years), middle-old ( 75 to 84 years), and old-old ( 85 years or above). Results: The old-old adults demonstrated a lower drug storage compliance rate $(47.61 \%)$ than the middleold adults $(50.36 \%)$ and the young-old adults $(63.61 \%)(\mathrm{p}=0.041)$. The common non-compliance problems were storing drugs at wrong temperatures, not keeping drugs in locked cabinet, discarding primary packaging, and keeping expired drugs. Conclusion: This study revealed that the older adults, especially the old-old adults, demonstrated unsatisfactory compliance on storing drug at home. Deteriorated visual and cognitive functions at advanced age posed a challenge to older adults on following the instructions of proper drug storage. We recommend improving the readability of instructions on drug packaging, implementing educational program, and providing regular monitoring via home visit to identify and rectify older adults' problems. Findings from this study provided insight into the support for in-home drug storage of older adults.

\section{(B204.7) Measuring the symptom burden of lung surgery patients: the validity and utility of the Perioperative Symptom Assessment for Lung Surgery (PSA-Lung)}

Hongfan Yu, BSc, School of Public Health and Management, Chongqing Medical University, Chongqing, China; Qingsong Yu, BSc, School of Public Health and Management, Chongqing Medical University, Chongqing, China; Li Tang, M.D., State Key Laboratory of Biomedical Engineering, Chongqing Medical University, Chongqing, China; Wei Dai, M.M., Department of Thoracic Surgery, Sichuan Cancer Hospital \& Institute, Sichuan Cancer Center, School of Medicine, University of Electronic Science and Technology of China, Sichuan, China; Xing Wei, M.M., Department of Thoracic Surgery, Sichuan Cancer Hospital \& Institute, Sichuan Cancer Center, School of Medicine, University of Electronic Science and Technology of China, Sichuan, China; Yaqin Wang, M.M., Department of Thoracic Surgery, Sichuan Cancer Hospital \& Institute, Sichuan Cancer Center, School of Medicine, University of Electronic Science and Technology of China, Sichuan, China; Jia Liao, BSc, Department of Thoracic Surgery, Sichuan Cancer Hospital \& Institute, Sichuan Cancer Center, School of Medicine, University of Electronic Science and Technology of China, Sichuan, China; Wei Xu, M.M., School of Public Health and Management, Chongqing Medical University, Chongqing, China; Yang Pu, BSc, School of Public Health and Management, Chongqing Medical University, Chongqing, China; Qiuling Shi, PhD, School of Public Health and Management, Chongqing Medical University, Chongqing, China

Aims: Using patient-reported outcomes (PROs) in perioperative care with longitudinal assessment is increasingly common. However, lack of special inventory for surgery patients. We established the psychometric properties of a inventory of the Perioperative Symptom Assessment for patients with lung surgery (PSA-Lung). Methods: Our process included: (1) generating specific candidate items pool from qualitative interviews with surgery patients, followed by input into two round of expert panel; (2) dropping items that lacked strong clinical relevance; (3) validating psychometric properties (reliability, validity) of the resulting PSA-Lung; and (4) conducting cognitive debriefing interviews with patients to confirm ease of comprehension, comfortable, and acceptability. Results: Qualitative interviews with 39 patients and investigation with 42 experts generated 9 symptom items (pain, coughing, shortness of breath, hoarse, disturbed sleep, fatigue, drowsy, anxious, distressed) and 2 function items (activity, walking) that formed the PSA-Lung draft. A total of 223 patients (single port $=119$, multiple port or open surgery $=104)$ participated in the cohort; $98.66 \%(220 /$ 223) provided retest PROs data. Dropped items (hoarse and anxious) that reflected lowest mean scores. Internal consistencies were 0.912 for all items and 0.885 for symptoms and 0.887 for function items. Construct validity revealed a two-factor structure. Test-retest reliability was 0.794 for all items. Criterion validity were 0.876 for symptom with MD Anderson Symptom Inventory core subscale and 0.764 for function items with MDASI interference subscale. Known-group validity was supported by the detection of significant differences by surgical type (single port vs. others) in all items (Mean \pm SD, $5.05 \pm 2.01$ vs. $5.69 \pm 1.95, P=0.0175)$, symptom $(5.06 \pm 1.99$ vs. $5.58 \pm 1.88$, $P=0.0463)$, and function scores $(5.02 \pm 2.59$ vs. $6.06 \pm 2.80$, $P=0.0042$ ). Cognitive debriefing with $95.91 \%$ patients demonstrated that the PSA-Lung is an easy-to-use and understandable tool. Conclusion: The PSA-Lung is a valid, reliable, understandable, and concise tool for measuring symptom severity and functional status in patients undergoing lung surgery and can be useful in assessing perioperative symptom burden via PROs. 

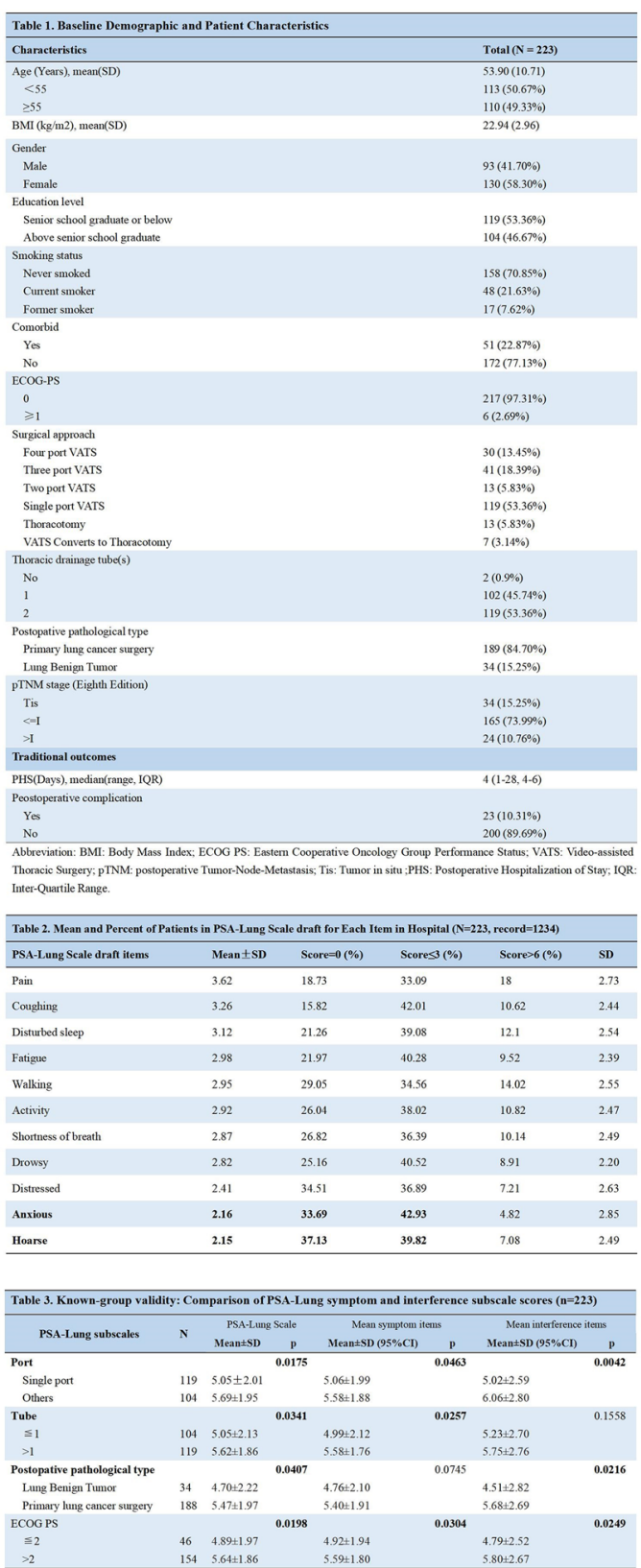

(B204.8) Validation of the electronic Patient Benefit Index for Psoriasis

Marina Otten, PhD, University Medical Center Hamburg-Eppendorf, Hamburg, Germany; Anastasia Fleyder, University Medical Center Hamburg-Eppendorf, Hamburg, Germany; Patrick Reinders, University Medical Center Hamburg-Eppendorf, Hamburg, Germany; Natalia Kirsten, University Medical Center Hamburg-Eppendorf, Hamburg, Germany; Matthias Augustin, University Medical Center Hamburg-Eppendorf, Hamburg, Germany

Aims: Due to the increased use of electronic applications, the collection of electronic patient-reported outcomes is also becoming more important. The Patient Benefit Index (PBI) for psoriasis is a valid instrument to capture treatment goals and benefits from patients' perspective. The aim of this study was to test the validity of two versions of the electronic PBI (ePBI) for psoriasis. Methods: 210 patients with psoriasis participated in this prospective, non-interventional crossover study (according to ISPOR). Half completed the paper questionnaire and the app version of the ePBI, and the other half completed the paper questions and the web version of the ePBI (order alternated). Participants then completed questionnaires on usability and acceptability of the three available versions, as well as on demographics. Data were analyzed descriptively using SPSS, subgroup analyses were conducted, comparisons were made between the paper-based and electronic versions of the PBI as well as between the app and the web version. Results: Participants were 44.6 years old on average (Med: 44.0 ), $58.6 \%$ were male. The ePBI took an average of $6.06 \mathrm{~min}$ to complete by self-assessment (Med: 5.00), and the paper PBI $7.55 \mathrm{~min}$ (Med: 6.00). Usability and acceptance of the ePBI proved to be high: e.g., $91.0 \%$ of participants agreed that it was easy to use (PBI: 90.9\%), 53.8\% could imagine filling out the ePBI regularly, $25.2 \%$ were unsure. In contrast, $45.2 \%$ would fill out the paper PBI regularly, $29.0 \%$ were unsure. $63.3 \%$ preferred the electronic version, $10.0 \%$ the paper version, and $21.9 \%$ would not care. The mean PBI score was 2.92 and that of the ePBI was 2.88 on a scale of $0-4$. The intraclass correlation between the two versions was 0.93 , which corresponds to a significant and valid agreement. Conclusion: The electronic version of the PBI is a valid instrument with high usability and acceptance. In the future, the electronic versions will facilitate data collection in studies as well as in routine care. Thus, patient-reported outcomes can be more efficiently recorded, documented, and subsequently made usable.

\section{(B204.9) Evolution and determinants of quality of life in older} people in Portugal, Spain and Sweden (2011-2015)

Antonio González Herrera, National School of Public Health, Carlos III Health Institute, Madrid, Spain; Carmen Rodríguez-Blázquez, National Epidemiology Centre, Carlos III Institute of Health, Madrid, Spain; Alba Ayala-García, University Institute of Gender Studies, University Carlos III, Madrid, Spain; Maria João Forjaz, National Epidemiology Centre, Carlos III Institute of Health, Madrid, Spain

Aims: To analyze the change in quality of life (QoL) of older people and the influence of determinants such as health and socio-economic situation, in Portugal, Spain and Sweden between 2011 and 2015. Methods: Longitudinal study with a representative sample of people aged 50 years or more from Portugal, Spain, and Sweden. The data were extracted from waves 4 (2011) and 6 (2015) of the Survey of Health, Ageing and Retirement in Europe (SHARE) with a sample size of 7589 in each wave. QoL was assessed with the CASP-12 scale. Other variables were perceived health, depression, social participation, lifelong learning, income level, as well as sociodemographic factors. A multiple linear regression model with the CASP-12 score difference between the two waves as the dependent variable was performed. Health, depression, social participation, lifelong learning, and wealth were independent variables, controlling by sex, age, and country. Results: Mean CASP-12 in W4 was 35.9 (standard deviation, $\mathrm{SD}=6.1)$ and 35.7 in W6 $(\mathrm{SD}=6.5 ; p=0.007)$. This change in $\mathrm{QoL}$ levels after 4 years is determined by the country in the case of Portugal with respect to Sweden $(\beta=-1.312, p=0.008)$, since the values obtained for Spain are not statistically significant. Regarding sociodemographic variables, only age influences the difference in QoL, decreasing QoL for older people $(\beta=-0.114, p<0.001)$. Depression and perceived poor health were also negatively associated to change in QoL $(\beta=-0.387, p<0.001$ and $\beta=-1.102$, $p<0.001$, respectively). However, social participation $(\beta=0.023$, $p=0.025)$, lifelong learning $(\beta=0.024, p=0.036)$, and the income level $(\beta=0.211, p=0.045)$ are variables that favor the increase in QoL between waves. Conclusion: The change in QoL over 4 years is 
negatively associated to age, perceived poor health and depression and positively to social participation, lifelong learning, and income level. These aspects should be considered when proposing policies that seek to improve QoL of older people.

(B204.10) Inhalation technique and treatment adherence in pediatric patients with asthma: ARCA Study (Asthma Research in Children and Adolescents)

Catalina Lizano-Barrantes, PharmD, IMIM- Hospital del Mar Medical Research Institute, Barcelona, Spain; Karina Mayoral, IMIMHospital del Mar Medical Research Institute, Barcelona, Spain; María Teresa Guerra, Centro de Salud de Jerez Sur, Cádiz, Spain; Alberto Bercedo, Centro de Salud Dobra, Torrelavega, Spain; Manuel PraenaCrespo, Centro de Salud La Candelaria, Sevilla, Spain; María Ángeles Carrasco, Consultorio Sevilla la Nueva, Madrid, Spain; Eva Tato, Hospital Universitario Araba, Álava, Spain; Àngels Pont, IMIMHospital del Mar Medical Research Institute, Barcelona, Spain; Olatz Garin, IMIM- Hospital del Mar Medical Research Institute, Barcelona, Spain; Montse Ferrer, IMIM- Hospital del Mar Medical Research Institute, Barcelona, Spain; submitted on behalf of "the Arca Group"

Aims: The objective of the study is to evaluate inhalation technique and treatment adherence in pediatric patients with asthma according to their age. Methods: Participants aged 6 to 14 years old from ARCA, a multicenter, observational, longitudinal, and prospective study of patients with persistent asthma were included. The information was collected in the telephone interview at baseline. The inhaler technique questionnaire has 5 items: 1 - Breath out fully before, 2-Close lips tightly, 3-Breath in deeply, 4-Hold breath after, and 5-Breathe out slowly; with answer options on a 5-point Likert scale (Always, Often, Sometimes, Rarely, Never). The Medication Intake Survey-Asthma (MIS-A) measures key complementary adherence properties (taking adherence, therapeutic coverage, correct dosing, drug holidays, overdosing). Differences between groups according to age (6-7 years-answered by parent/guardian-; 8-11 years and adolescents $12-14$ years old) were evaluated with ANOVA. Results: Of the 249 participants recruited, 230 answered the inhalation techniques questionnaire. There were significant differences by age in 2 items: 'Breath in deeply' (Always in $84.5 \%$ of adolescents, $73.1 \%$ in $8-11$ years and $62.7 \%$ in $6-7$ years, $\mathrm{p}=0.014$ ), and 'Breathe out slowly' (Always in 47,9\% adolescents, $34,3 \%$ in $8-11$ years and $23,5 \%$ in $6-7$ years, $p=0.017)$. The MIS-A was answered by 224 participants without statistically significant differences in adherence in the last week $(58.0 \%, 57.0 \%$ y $58.2 \%$, $p=0.816)$. Conclusion: Adolescents report that they always perform two of the inhalation technique steps more frequently, while age does not affect adherence to inhalers.Funding: Instituto de Salud Carlos III FEDER: Fondo Europeo de Desarrollo Regional (PI15/00449 and FI16/00071), Universidad de Costa Rica (OAICE-85-2019).

\section{5: Cancer Clinical Research Applications}

(B205.1) Urinary incontinence and Quality of Life after radical prostatectomy: results of an observational study

Monica Pinto, MD, Istituto Nazionale Tumori - IRCCS - Fondazione G. Pascale, Napoli, Italy; Alessandra Filice, PT (Physical Therapist), Istituto Nazionale Tumori - IRCCS - Fondazione G. Pascale, Napoli, Italy; Maura Tracey De Bellis, RN (Research Nurse), Istituto Nazionale Tumori - IRCCS - Fondazione G. Pascale, Napoli, Italy
Aims: Urinary incontinence is a frequent and disabling condition after treatment of prostate cancer. The aims of this observational study were to investigate the Quality of Life and the severity of urinary incontinence after radical prostatectomy to better satisfy rehabilitation needs of cancer patients. Methods: We enrolled 141 prostate cancer patients from the outpatients rehabilitation unit at the National Cancer Institute for urinary incontinence after radical prostatectomy from January 2nd, 2019 until March 30th, 2021. Inclusion criteria were over 18 years of age, no metastasis, no severe comorbidities, and no other cancer. All patients gave a freely given informed consent for the anonymous use of personal and clinical data. Patients were divided into 2 groups according to the average number of pads used per day in the 3 day urinary diary: Group 1 included 83 patients using from 0 to 3 pads; Group 2 included 58 patients using 4 pads or more. We evaluated the Health-Related Quality of Life with the Medical Outcomes Study Short-Form 12-Item Health Survey (SF-12) using both Physical Component Summary (PCS) and Mental Component Summary (MCS) scores in all patients. We collected age, number of the pads used per day, and scores of PCS and MCS for each patient and calculated mean values \pm Standard Deviation (SD) in each group. Results: The results are summarized in Table 1. In Group 1, the mean values are age $71.61(\min 54, \max 87)$, number of pads/day 1.88 $(\mathrm{SD} \pm 1.14), \mathrm{SF}-12$ PCS 35.43 (SD \pm 10.24$)$, SF-12 MCS 45.39 $(\mathrm{SD} \pm$ 9.55).In Group 2 the mean values are: age $69.87(\min 47, \max$ 88), number of pads/day 5.52 (SD 2.66) SF-12 PCS 37.25 (SD \pm 10.36), and SF-12 MCS 45.09 (SD \pm 10.32 ). Conclusion: In both groups, the mean value of PCS scores are lower than those of MCS of similar size despite the significantly different number of pads used; we can assume that incontinence itself affects quality of life and pelvic floor rehabilitation is recommended also in mild cases.

\begin{tabular}{|l|l|l|}
\hline & Group $1=<3$ pads/day & Group $2=>4$ pads/day \\
\hline No. patients & 59 & 83 \\
\hline Age & $71.61(\min 54, \max 87)$ & $69.87(\min 47, \max 88)$ \\
\hline SF 12: & & \\
\hline PCS & $35.43($ SD 10.24$)$ & $37.25($ SD 10.36$)$ \\
\hline MCS & $45.39($ SD 9.55$)$ & $45.09($ SD 10.32$)$ \\
\hline No. Pads/day & $1.88($ SD 1.14$)$ & $5.52($ SD 2.66$)$ \\
\hline
\end{tabular}

(B205.2) Higher dispositional optimism predicts better healthrelated quality of life after esophageal cancer surgery

Yangjun Liu, MD, Karolinska Institutet, Stockholm, Sweden; Erik Pettersson, Karolinska Institutet, Stockholm, Sweden; Anna Schandl, Karolinska Institutet, Stockholm, Sweden; Sheraz Markar, Imperial College London, London, UK; Asif Johar, Karolinska Institutet, Stockholm, Sweden; Pernilla Lagergren, Karolinska Institutet, Stockholm, Sweden;

Aims: To assess whether dispositional optimism affects health-related quality of life (HRQL) after esophageal cancer surgery. Methods: Data for this longitudinal study were drawn from a prospective, ongoing Swedish-nationwide cohort study entitled OSCAR (Oesophageal Surgery on Cancer patients-Adaptation and Recovery). The exposure was dispositional optimism measured at 1 year post-surgery using the Swedish version of Life Orientation Test-Revised (LOT-R). Patients were categorized into four subgroups (very low, moderately low, moderately high, and very high dispositional optimism) based on the quartile of the LOT-R sum score. The outcome was HRQL measured at 1, 1.5, and 2 years post-surgery using the European Organization for Research and Treatment of Cancer Quality of Life Questionnaire-Core 30 (EORTC QLQ-C30) and disease site-specific (Esophago-Gastric) module EORTC QLQ-OG25. Linear mixed effects models, adjusted for potential 
confounders, were used to examine the mean score difference (MSD) with $95 \%$ confidence interval of HRQL among the four patient subgroups. Statistical significance of adjusted MSD was tested only if it had clinical relevance based on evidence-based guidelines. Results: In total, 192 patients were included in the analysis. Compared to patients with very low and moderately low dispositional optimism, patients with moderately high dispositional optimism reported clinically relevantly and statistically significantly better global quality of life (MSD, 10; 95\% CI 4 to 17) and less severe diarrhea (MSD, $-9 ; 95 \%$ CI - 18 to - 1). Compared with patients with very low, moderately low, and moderately high dispositional optimism, patients with very high dispositional optimism reported clinically relevantly better global quality of life, emotional function and social function (MSDs range, 10 to 16), and less severe symptoms of pain, dyspnea, diarrhea, anxiety, dry mouth, trouble with taste, worry about weight loss and self-doubt about body image (MSDs range, -9 to -22 ). In the eating difficulty subscale, the predictive effect of dispositional optimism on HRQL varied over time. Conclusion: Higher dispositional optimism predicts better HRQL after esophageal cancer surgery. The predictive value of dispositional optimism may help identify high-risk patients with poor HRQL recovery after esophageal cancer surgery, leading to timely and tailored interventions to improve postoperative HRQL.

\section{(B205.3) Feeling better versus feeling good: interpreting pain relief using MCID and PASS for patients with lung cancer surgery}

Qiuling Shi, Chongqing Medical University, Chongqing, China; Wei Xu, School of Public Health and Management, Chongqing Medical University, Chongqing, China; Hongfan Yu, School of Public Health and Management, Chongqing Medical University, Chongqing, China; Xin Wei, Department of Thoracic Surgery, Sichuan Cancer Hospital, School of Medicine, University of Electronic Science and Technology of China, Sichuan, China; Wei Dai, Department of Thoracic Surgery, Sichuan Cancer Hospital, School of Medicine, University of Electronic Science and Technology of China, Sichuan, China; Qiang Li, Department of Thoracic Surgery, Sichuan Cancer Hospital, School of Medicine, University of Electronic Science and Technology of China, Sichuan, China

Aims: Using patient-reported outcome (PRO) to evaluate pain relief requires an adequate reduction of the numeric score (feeling better), defined by the Minimal Clinically Important Difference (MCID). Besides, patients perceive benefits when pain score decreasing below a threshold (feeling good), defined by the Patient Acceptable Symptom State (PASS). We aimed to examine how those two thresholds, MCID and PASS, performed when applied in perioperative settings. Methods: We conducted a secondary analysis using data from a prospective cohort of patients under lung cancer surgery. MD Anderson Symptom Inventory (MDASI, 0-10 scale) was used to assess pain daily from day 1 after surgery (POD1) to discharge, then weekly after discharge up to 4 weeks. Patients reported 4 or greater pain on POD1 were included. Time to 'feeling better' was defined as when pain score decreasing by $30 \%$ from POD1, while time to 'feeling good' as when pain score decreasing below 4 . Factors related to time to 'feeling better' or 'feeling good' were identified by cox models. Median days between surgical approaches were compared by log-rank tests. Results: Among 364 patients analyzed, $80.5 \%$ (293/364) were under video-assised thoracoscopic surgery (VATs) and 19.5\%(71/364) under thoracotomy. On the day of discharge, $77.8 \%$ (283/364) of patients 'felt better,' while $68.7 \%$ 'felt good.' Thoracotomy (HR $=1.4(95 \%$ CI 1.1-1.9)) and older-than$55(\mathrm{HR}=1.3(95 \%$ CI $1.1-1.7))$ were related to a delayed 'feeling better.' Delayed 'feeling good' was associated with thoracotomy $(\mathrm{HR}=1.6(95 \%$ CI $1.2-2.1))$ and $\mathrm{ASA}>1 \quad(\mathrm{HR}=1.3(95 \%$ CI 1.0-1.6)). Patients with thoracotomy took 5(95\% CI 3-7) days to feel better and 6(95\% CI 5-12) days to feel good, which were longer than did patients with VATs (3(95\% CI 3-4)days for 'feeling better' and 4(95\% CI 4-5) for 'feeling good') (all $\mathrm{p}<0.05$, Fig. 1). Conclusion: Both MCID and PASS are able to differentiate surgical approaches in terms of PRO-measured pain relief. Compared with 'feeling better,' 'feeling good' was more challenging for postoperative care. The clinically relevant interpretation of MCID and PASS makes them potential endpoints for perioperative researches on new surgical techniques.
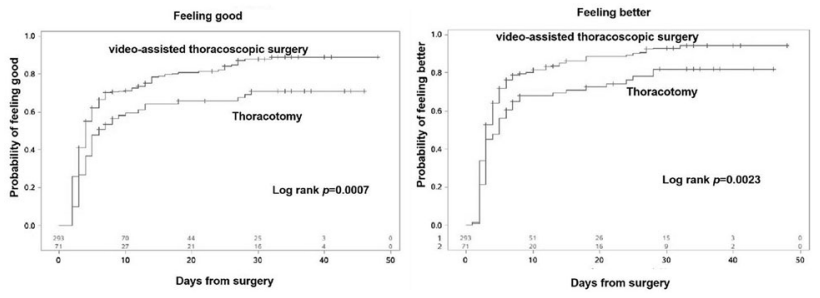

(B205.4) Early postoperative patient-reported outcomes after thoracoscopic segmentectomy versus lobectomy: a multicentre longitudinal study

Wei Dai, Department of Thoracic Surgery, Sichuan Cancer Hospital, School of Medicine, University of Electronic Science and Technology of China, Chengdu, China; Cecilia Pompili, Section of Patient Centred Outcomes Research, Leeds Institute for Medical Research at St James's, University of Leeds, Leeds, UK; Bin Qiu, Department of Thoracic Surgery, National Cancer Center, National Clinical Research Center for Cancer, Cancer Hospital, Chinese Academy of Medical Sciences and Peking Union Medical College, Beijing, China; Xing Wei, Department of Thoracic Surgery, Sichuan Cancer Hospital \& Institute, Sichuan Cancer Center, School of Medicine, University of Electronic Science and Technology of China, Chengdu, China; Cheng Shen, Department of Thoracic Surgery, West China Hospital, Sichuan University, Chengdu, China; Shuai Chang, Department of Thoracic Surgery, West China Hospital, Sichuan University, Chengdu, China; Qiuling Shi, Center for Cancer Prevention Research, Sichuan Cancer Hospital \& Institute, Sichuan Cancer Center, School of Medicine, University of Electronic Science and Technology of China, Chengdu, China; Qiang Li, Department of Thoracic Surgery, Sichuan Cancer Hospital \& Institute, Sichuan Cancer Center, School of Medicine, University of Electronic Science and Technology of China, Chengdu, China; Zhong Wu, Department of Cardiovascular Surgery, West China Hospital, Sichuan University, Chengdu, China; Guowei Che, Department of Thoracic Surgery, West China Hospital, Sichuan University, Chengdu, China; submitted on behalf of "CN-PRO-Lung"

Aims: Patient-reported outcomes are critical for obtaining valuable patient insight into different surgical treatment options with comparable clinical outcomes. This study aimed to compare early postoperative patient-reported symptoms and functioning between thoracoscopic segmentectomy and lobectomy for peripheral non-small-cell lung cancer (NSCLC) $\leq 2$ $\mathrm{cm}$. Methods: This study included 110 patients who underwent thoracoscopic segmentectomy or lobectomy for peripheral NSCLC $\leq 2 \mathrm{~cm}$ between 2017 and 2020 in a multicentre prospective longitudinal study (CN-PRO-Lung 1). Symptom severity, functional status, and short-term clinical outcomes were compared between groups. Symptom severity and functional status were measured using the MD Anderson Symptom Inventory-Lung Cancer at baseline, daily during postoperative hospitalisation, and weekly post-discharge. Both the proportion of moderate-tosevere scores and mean scores on a $0-10$ scale were compared between groups. Results: Overall, 48 and 62 patients underwent thoracoscopic segmentectomy and lobectomy, respectively. The mean patient age in both groups was 53 years, and more than $60 \%$ of the patients in each group were female. The MDASI-LC completion rates at baseline, during the 6-day postoperative hospitalisation, and during the 4 weeks post-discharge were $100 \%, 94.3-99.1 \%$, and $73.4-80.9 \%$, respectively. No 
significant between-group differences were found in the severity of the top five symptoms (coughing, shortness of breath, pain, fatigue, and disturbed sleep) or in the impairment of all six function items (work, walking, general activity, enjoyment of life, mood, and relations with others) during both the 6-day postoperative hospitalisation or the 4-week post-discharge. After adjustment for the type of lymphadenectomy and the number of chest tubes, the results were similar. Both the symptom recovery time of the top five symptoms and functional recovery time of the six function items were comparable. The effect size across all the top five symptoms and six function items between the groups at discharge and 4 weeks postdischarge was $<0.2$. Short-term clinical outcomes of postoperative hospital stay $(p=0.14)$, operative time $(p=0.67)$, drainage time $(p=0.10)$, postoperative morphine equivalent dose $(p=0.22)$, and complication rate $(p=0.94)$ were also comparable. Conclusion: In patients with peripheral NSCLC $\leq 2 \mathrm{~cm}$, thoracoscopic segmentectomy and lobectomy may produce comparable levels of symptom burden and functional impairment during the early postoperative period.

\begin{tabular}{|c|c|c|c|c|c|}
\hline \multirow[t]{2}{*}{ Item } & \multicolumn{2}{|c|}{ Segmentectomy $(n=48)$} & \multicolumn{2}{|c|}{ Lobectomy $(n=62)$} & \multirow[t]{2}{*}{ P-value } \\
\hline & $\begin{array}{l}\text { No. of patients } \\
\text { recovered }\end{array}$ & $\begin{array}{l}\text { Median recovery } \\
\text { time }(95 \% \mathrm{Cl})-\mathrm{d}\end{array}$ & $\begin{array}{c}\text { No. of patients } \\
\text { recovered }\end{array}$ & $\begin{array}{l}\text { Median recovery } \\
\text { time }(95 \% \mathrm{Cl})-\mathrm{d}\end{array}$ & \\
\hline Coughing & $13(72.2 \%)$ & $13(4,27)$ & $16(64.0 \%)$ & $4(2,-)$ & 0.92 \\
\hline $\begin{array}{l}\text { Shortness of } \\
\text { breath }\end{array}$ & $17(70.8 \%)$ & $4.5(2,27)$ & $26(68.4 \%)$ & $3.5(2,21)$ & 0.90 \\
\hline $\begin{array}{l}\text { Disturbed } \\
\text { sleep }\end{array}$ & $19(67.9 \%)$ & $8.5(3,29)$ & $18(75.0 \%)$ & $3(2,13)$ & 0.29 \\
\hline Pain & $23(76.7 \%)$ & $4(3,14)$ & $35(81.4 \%)$ & $4(3,6)$ & 0.47 \\
\hline Fatigue & $20(80.0 \%)$ & $4(4,18)$ & $30(76.9 \%)$ & $4(3,11)$ & 0.72 \\
\hline
\end{tabular}

\begin{tabular}{|c|c|c|c|c|c|}
\hline \multirow[t]{2}{*}{ Item } & \multicolumn{2}{|c|}{ Segmentectomy $(n=48)$} & \multicolumn{2}{|c|}{ Lobectomy ( $n=62$ ) } & \multirow[t]{2}{*}{ P-value } \\
\hline & $\begin{array}{l}\text { No. of patients } \\
\text { recovered }\end{array}$ & $\begin{array}{l}\text { Median recovery } \\
\text { time }(95 \% \mathrm{Cl})-\mathrm{d}\end{array}$ & $\begin{array}{l}\text { No. of patients } \\
\text { recovered }\end{array}$ & $\begin{array}{l}\text { Median recovery } \\
\text { time }(95 \% \mathrm{Cl})-\mathrm{d}\end{array}$ & \\
\hline Activity & $13(39.4 \%)$ & $-(25,-)$ & $19(40.4 \%)$ & $-(13,-)$ & 0.74 \\
\hline Mood & $10(40.0 \%)$ & $-(18,-)$ & $12(57.1 \%)$ & $14.5(4,-)$ & 0.22 \\
\hline Work & $14(46.7 \%)$ & $-(14,-)$ & $19(41.3 \%)$ & $-(14,-)$ & 0.77 \\
\hline Walking & $12(37.5 \%)$ & $-(13,-)$ & $27(56.2 \%)$ & $19(4,-)$ & 0.09 \\
\hline $\begin{array}{l}\text { Relations } \\
\text { with others }\end{array}$ & $9(52.9 \%)$ & $13(3,-)$ & $9(47.4 \%)$ & $-(3,-)$ & 0.81 \\
\hline $\begin{array}{l}\text { Enjoyment } \\
\text { of life }\end{array}$ & 12 (44.4\%) & $-(5,-)$ & $11(32.4 \%)$ & $-(18,-)$ & 0.52 \\
\hline
\end{tabular}

$\mathrm{Cl}$ : confidence interval.

${ }^{\circ}$ F Functional recovery time was defined as days to return to two consecutive scores $<2$ from scores $\geq 2$ on postoperative day 1 .

(B205.5) Systematic nurse-led consultations based on electronic patient-reported outcome among women with gynecological cancer during chemotherapy (The CONNECT study)

Mille Christiansen, MSc, PhD Student, Copenhagen University Hospital, Rigshospitalet, Department of Oncology, Copenhagen, Denmark; Karin Piil, MHScN, Ph D, Associate professor Copenhagen University Hospital, Rigshospitalet, Department of Oncology, Copenhagen, Denmark; Mary Jarden, MScN, PhD, professor Copenhagen University Hospital, Rigshospitalet, Department of Haematology, Copenhagen, Denmark; Mansoor Mirza, MD, Chief Physician, Copenhagen University Hospital, Rigshospitalet, Department of Oncology, Copenhagen, Denmark; Helle Pappot, MD, DMSc,
Figure 1: Proportion of patients with moderate-to-severe score of the top five symptoms during the 6-day postoperative hospitalisation
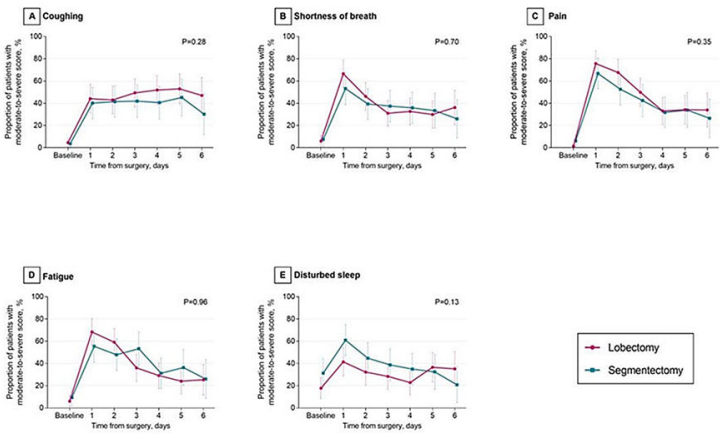

Figure 2: Proportion of patients with moderate-to-severe score of the six function items during the 6-day postoperative hospitalisation
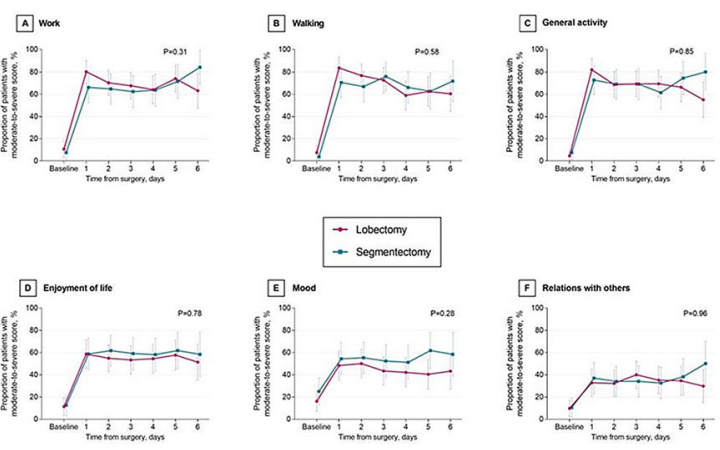

Figure 3: Proportion of patients with moderate-to-severe score of the top 5 symptoms during the 4-week post-discharge
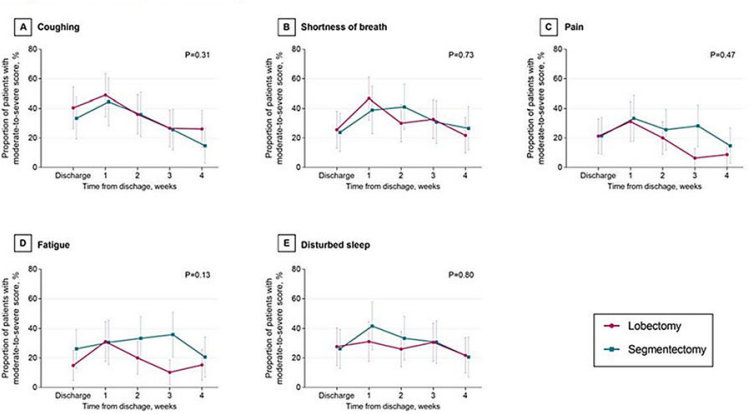

Figure 4: Proportion of patients with moderate-to-severe score of the 6 function items during the 4-week post-discharge
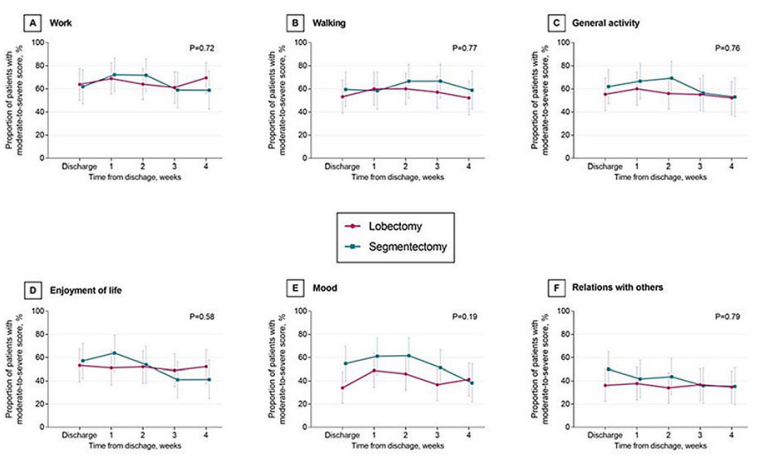


\begin{tabular}{|c|c|c|c|}
\hline Variables & Segmentectomy $(n=48)$ & Lobectomy $(n=62)$ & P-value \\
\hline Age (years), mean $\pm S D$ & $53 \pm 11.0$ & $53 \pm 9.5$ & 0.96 \\
\hline Sex, n (\%) & & & 0.45 \\
\hline Male & $18(37.5)$ & $19(30.6)$ & \\
\hline Female & $30(62.5)$ & $43(69.4)$ & \\
\hline Educational level, $n(\%)$ & & & 0.24 \\
\hline$<$ High school & $24(50.0)$ & $38(61.3)$ & \\
\hline 2High school & $24(50.0)$ & $24(38.7)$ & \\
\hline $\mathrm{BMI}\left(\mathrm{kg} / \mathrm{m}^{2}\right)$, mean $\pm \mathrm{SD}$ & $22.8 \pm 2.8$ & $23.0 \pm 2.6$ & 0.60 \\
\hline Smoking status, $\mathrm{n}(\%)$ & & & 0.46 \\
\hline Never & $41(85.4)$ & $51(82.3)$ & \\
\hline Current & $3(6.3)$ & $8(12.9)$ & \\
\hline Former & $4(8.3)$ & $3(4.8)$ & \\
\hline ASA-PS classification, $n(\%)$ & & & 0.58 \\
\hline s1 & $25(52.1)$ & $29(46.8)$ & \\
\hline$>1$ & $23(47.9)$ & $33(53.2)$ & \\
\hline Charlson Comorbidity Index, $n(\%)$ & & & 0.98 \\
\hline 0 & $20(41.7)$ & $26(41.9)$ & \\
\hline$\geq 1$ & $28(58.3)$ & $36(58.1)$ & \\
\hline FEV1\%, median (range), \% & $97.6(72.2-197.1)$ & $94.3(50.4-128.4)$ & 0.12 \\
\hline DLCO\%, median (range), \% & $95.1(56.2-159.1)$ & $95.4(22.6-138.8)$ & 0.56 \\
\hline \multicolumn{4}{|c|}{$\begin{array}{l}\text { ASA-PS: American Society of Anesthesiologists physical status; BMI: body mass index; FEV1\%: } \\
\text { percentage of predicted forced expiratory volume in } 1 \text { second; DLCO\%; percentage of predicted } \\
\text { diffusion capacity of carbon monoxide. } \\
\text { 'Four patients in the segmentectomy group and three in the lobectomy group with data unavaliable. }\end{array}$} \\
\hline Table 2: Nodule and operative ch & aracteristics & & \\
\hline Variables & Segmentectomy $(n=48)$ & Lobectomy $(n=62)$ & P-value \\
\hline Nodule Diameter (cm), mean $\$$ SD & $1.1(0.4)$ & $1.3(0.4)$ & 0.01 \\
\hline Nodule type, $\mathrm{n}(\%)$ & & & 0.02 \\
\hline$G G O(C T R=0)$ & $38(79.2)$ & $31(50.0)$ & \\
\hline $\begin{array}{l}\text { Non-invasive part solid } \\
(0<C T R \leq 0.5)\end{array}$ & $3(6.3)$ & $6(17.7)$ & \\
\hline Invasive part solid $(0.5<C T R<1)$ & $4(8.3)$ & $11(9.7)$ & \\
\hline Solid (CTR=1) & $3(6.3)$ & $14(22.6)$ & \\
\hline Nodule location, $n(\%)$ & & & 0.85 \\
\hline Right Upper Lobe & $20(41.7)$ & $23(37.1)$ & \\
\hline Right Lower Lobe & $6(12.5)$ & $8(12.9)$ & \\
\hline Left Upper Lobe & $15(31.2)$ & $18(29.0)$ & \\
\hline Left Lower Lobe & $7(14.6)$ & $13(21.0)$ & \\
\hline Surgical approach, n (\%) & & & 0.52 \\
\hline Uniportal VATS & $33(68.8)$ & $39(62.9)$ & \\
\hline Multiportal VATS & $15(31.2)$ & $23(37.1)$ & \\
\hline Type of Iymphadenectomy, $n(\%)$ & & & $<0.001$ \\
\hline Systematic Iymph node dissection & $2(4.2)$ & $29(46.8)$ & \\
\hline Lymph node sampling & $46(95.8)$ & $33(53.2)$ & \\
\hline Number of chest tubes, $n(\%)$ & & & $<0.001$ \\
\hline One tube & $42(87.5)$ & $35(56.4)$ & \\
\hline Two tubes & $6(12.5)$ & $27(43.6)$ & \\
\hline Nodule pathological stage, $n$ (\%) & & & 0.03 \\
\hline 0 & $5(10.4)$ & $1(1.6)$ & \\
\hline IA1 & $24(50.0)$ & $23(37.1)$ & \\
\hline IA2 & $19(39.6)$ & $37(59.7)$ & \\
\hline III & $0(0.0)$ & $1(1.6)$ & \\
\hline Nodule histology, $n(\%)$ & & & NA \\
\hline Adenocarcinoma & $48(100.0)$ & $62(100.0)$ & \\
\hline Type of segmentectomy $y^{3}, \mathrm{n}(\%)$ & & & NA \\
\hline Simple segmentectomy & $13(27.1)$ & NA & \\
\hline Complex segmentectomy & $35(72.9)$ & NA & \\
\hline Resected pulmonary segment, $n(\%)$ & & & NA \\
\hline 1 segment & $32(66.7)$ & NA & \\
\hline 2 segments & $12(25.0)$ & NA & \\
\hline 3 segments & $4(8.3)$ & NA & \\
\hline
\end{tabular}

CTR: consolidation-to-tumour ratio; GGO: ground-glass opacity; SD: standard deviation; VATS: video-assisted thoracoscopic surgery.

"Simple segmentectomy was defined as the resection of the segment 6 , the lingular segment, or the left superior segment, while complex segmentectomy was defined as the resection of other segments.

\begin{tabular}{|c|c|c|c|}
\hline Variables & Segmentectomy $(n=48)$ & Lobectomy $(n=62)$ & P-value \\
\hline Operative time (min), median (range) & $139(60-275)$ & $144(65-250)$ & 0.67 \\
\hline $\begin{array}{l}\text { Postoperative hospital stay (days), } \\
\text { median (range) }\end{array}$ & $6(3-26)$ & $6(3-14)$ & 0.14 \\
\hline Drainage time (days), median (range) & $3(2-26)$ & $4(2-10)$ & 0.10 \\
\hline $\begin{array}{l}\text { Postoperative morphine equivalent } \\
\text { dose ( } \mathrm{mg} \text { ), median (range) }\end{array}$ & $219(5-555)$ & $265(0-960)$ & 0.22 \\
\hline Complications $\mathbf{s}^{3}, \mathrm{n}(\%)$ & $8(16.7)$ & $10(16.1)$ & 0.94 \\
\hline
\end{tabular}

professor Copenhagen University Hospital, Rigshospitalet, Department of Oncology, Copenhagen, Denmark

Aims: To develop a model of care for systematic nurse-led consultations based on ePRO facilitating symptom management and to investigate how these consultations can be introduced into a multidisciplinary treatment regimen for patients with ovarian- or endometrial cancer exploring the effect on quality of life compared to standard care. Methods: A quasi-experimental four-phase, sequential cohort research design with comparisons between non-equivalent groups. This study will examine: (1) the frequency and severity of clinician-reported symptoms and adverse events (CTCAE), HRQoL (EORTC QLQ-C30 + OV-28/EN-24), levels of anxiety and depressive symptoms (HADS), and self-efficacy (SES6G) among women with ovarian- or endometrial cancer receiving standard care, (2) development of a new model of nurse-led care, (3) test the feasibility of systematic nurse-led consultations based on ePRO, and (4) estimate the effect of the ePRO based model on frequency and severity of nurse-reported symptoms and adverse events (CTCAE), HRQoL (EORTC QLQ-C30 + OV-28/EN-24), HADS, and SES6G among women with ovarian- or endometrial cancer compared to standard care. The primary outcome will be the difference in global HRQOL (EORTC QLQ-C30) at 9 months. Further, we will conduct qualitative individual and focus-group interviews to explore experiences and satisfaction among patients, nurses, and physicians. Results: Throughout the research phases, we will engage a patient advisory that will be invited to provide the research team with feedback, comments on written materials, and collaborate in the development of the research. The theoretical perspective for the nurse-led consultations is the evidence-based and participatory, patient-focused process for advanced practice nursing role development, implementation, and evaluation-the PEPPA framework. Moreover, we will create algorithms in the ePRO platform to stratify the patient's response of symptom severity into three levels and each level activates different actions as self-management advice or contacts the oncological Department. The results of this study will be available in 2024 . Conclusion: We hypothesize that proactive use of ePRO in nurse-led consultations may contribute to enhanced quality of life, symptom management, self-management, and an improved CONNECTion between patients and healthcare professionals.

(B205.6) Association between demographic and clinical variables and subgroups of breast cancer patients with similar severity of fatigue and sleep disturbance while receiving chemotherapy

Meagan Whisenant, PhD, APRN, The University of Texas Health Science Center at Houston, Houston, Texas, USA; Jessica Jones, MD, The University of Texas Health Science Center at Houston, Houston, Texas, USA; Mazi Rasulnia, PhD, Pack Health, Birmingham, Alabama, USA; Brett Wisse, BBA, Pack Health, Birmingham, Alabama, USA; Valerie Shelton, BS, The University of Texas MD Anderson Cancer Center, Houston, Texas, USA; Kelly Brassil, PhD, RN, Pack Health, Houston, Texas, USA

Aims: The objective of this study was to identify subgroups of breast cancer patients receiving chemotherapy and experiencing similar severity of fatigue and sleep disturbance. Methods: In a secondary analysis of data collected from 166 newly diagnosed breast cancer patients receiving chemotherapy in a larger eHealth coaching study, subgroups of women experiencing similar symptom severity were identified. Symptom severity scores were self-reported at day 30 following initiation of chemotherapy on the 11-point ( $0=$ no symptom, $10=$ as bad as can be imagined) MD Anderson Symptom Inventory (MDASI), a valid, reliable measure of symptom burden. Symptom severity scores for fatigue and sleep disturbance were 
included in a Latent Profile Analysis (LPA) to categorize patients into subgroups. Demographic and clinical factors related to subgroup membership were explored using binomial logistic regression. Differences in functioning (measured using MDASI interference scores) and mental and physical health (measured using PROMIS GH-10) were evaluated using independent samples t tests. Results: Mean age was 53.9 years $(\mathrm{SD}=11.4), 16.3 \%$ were Black, $10.8 \%$ were Hispanic, $54.2 \%$ were employed full time. $83.1 \%$ had non-metastatic breast cancer. Two subgroups of women with distinct symptom severity were identified: low (56.0\% of women) and high (44.0\% of women). Women in the low severity subgroup had an average fatigue severity of $2.17(\mathrm{SD}=1.27)$ and sleep disturbance severity of 2.25 $(\mathrm{SD}=2.13)$ and in the high severity subgroup had an average fatigue severity of $7.03(\mathrm{SD}=1.31)$ and sleep disturbance severity of 4.99 $(\mathrm{SD}=3.14)$. Demographic and clinical variables were not related to class membership. Women in the high subgroup reported greater symptom interference with functioning $(p=0.000)$ and lower mental $(p=0.000)$ and physical health $(p=0.000)$ when compared to the low subgroup. Conclusion: Two subgroups of distinct fatigue and sleep disturbance were identified for breast cancer patients receiving chemotherapy. The absence of association between subgroup membership and demographic and clinical variables suggests unknown biological interactions and epigenetic factors that should be explored in future studies. Identification of women at risk for high symptoms during treatment supports proactive symptom management thereby potentially mitigating poor outcomes and functional impairments.

\section{(B205.7) Comparison of upper extremity lymphedema} after sentinel lymph node biopsy and axillary lymph node dissection: patient-reported outcomes in 3044 patients diagnosed with lymphedema after breast cancer treatment

Lotte Poulsen, MD, PhD, Research Unit for Plastic Surgery, Odense University Hospital, Odense, Denmark; Manraj Kaur, PhD, Department of Plastic Surgery, Brigham and Womens Hospital, Boston, Massachusetts, USA; Amalie L Jacobsen, Medical student, Research Unit for Plastic Surgery, Odense University Hospital, Odense, Denmark; Mie P Bjarnesen, MD, Research Unit for Plastic Surgery, Odense University Hospital, Odense, Denmark; Anna P Bjarnesen, MD, Research Unit for Plastic Surgery, Odense University Hospital, Odense, Denmark; Anne Klassen, DPhil, PhD, Department of Pediatrics, McMaster University, Hamilton, Ontario, Canada; Claire de Vries, MD, PhD, Department of Plastic Surgery, Catharina Hospital, Eindhoven, The Netherlands; Jens A Sorensen, MD, PhD, Research Unit for Plastic Surgery, Odense University Hospital, Odense, Denmark

Aims: A limited number of studies have examined the impact of type of axillary lymph node surgery on the health-related quality of life (HRQOL) or incidence of breast cancer-related lymphedema (BRCL) from the patient's perspective. The objective of this study was to assess the impact of sentinel lymph node dissection (SLND) and axillary lymph node dissection (ALND) on the HRQOL in women diagnosed with BRCL using the validated, UE-lymphedemaspecific PROM, and the LYMPH-Q Upper extremity (UE) module. Methods: Adult women diagnosed with BRCL were identified from the Danish National Health Data Authority database for the period January 2008 to January 2020. Women were invited to participate in the study using a secure electronic mailbox and provide consent for participation in the online survey and chart review. Women who agreed were directed to an online REDCap survey that included questions about demographics, cancer diagnosis and treatment, and BRCL. Following this, women were asked to complete four LYMPH-Q UE module scales measuring symptoms, function, distress, and arm appearance.
Information pertaining to axillary surgery was obtained from an online pathology repository. Multivariable linear regression, adjusted for statistically significant confounding variables, was used to examine differences in the SLND and ALND groups on the LYMPHQ UE scale scores. Results: 3044 women with BRCL were included in the analysis. The mean age of the participants was $63.7 \pm 10.6$ years (range $23-100$ years), and the mean follow-up duration was 8.6 years \pm 5.15 years (range $0-36$ years). The majority of participants underwent ALND $(n=2805,92.1 \%)$, and only $7.9 \%(\mathrm{n}=239)$ received SLND. The mean number of lymph nodes removed in the SLND group was $2.2 \pm 1.4$. A statistically significant difference between the SLND and ALND groups was found for type of breast procedure, cancer stage, and years since the first surgery. The prevalence of infection and seroma was higher in the ALND group. No statistically significant difference was found in the two groups on the LYMPH-Q UE scale scores. Conclusion: There is no difference in the long-term HRQOL of women undergoing SLND or ALND when assessed using LYMPH-Q UE module scales.

(B205.8) Leveraging patient-reported outcomes to measure frailty in relapsed/ refractory multiple myeloma

Meena N. Murugappan, PharmD, MPH, PhD(C), ORISE Fellow, Office of Oncologic Diseases, Center for Drug Evaluation and Research (CDER), U.S. Food and Drug Administration (U.S. FDA), Silver Spring, Maryland, USA; Bellinda L. King-Kallimanis, PhD, Oncology Center for Excellence (U.S. FDA), Silver Spring, Maryland, USA; Vishal Bhatnagar, MD, Oncology Center for Excellence (U.S. FDA), Silver Spring, Maryland, USA; Bindu Kanapuru, MD, Center for Drug Evaluation and Research (CDER), U.S. Food and Drug Administration (U.S. FDA), Silver Spring, Maryland, USA; Ting-Yu Chen, PhD, ORISE Fellow, Office of Oncologic Diseases, Center for Drug Evaluation and Research (CDER), U.S. Food and Drug Administration (U.S. FDA), Silver Spring, Maryland, USA; Erica G. Horodniceanu, MPH, Oncology Center for Excellence (U.S. FDA), Silver Spring, Maryland, USA; Harpreet Singh, MD, Oncology Center for Excellence (U.S. FDA), Silver Spring, Maryland, USA; Paul G. Kluetz, MD, Oncology Center for Excellence (U.S. FDA), Silver Spring, Maryland, USA

Aims: Frailty is defined as the accumulation of aging-associated diseases and disabilities, leaving patients in a state of increased vulnerability to adverse outcomes like hospitalization and death. While several frailty measures exist for multiple myeloma patients, they primarily rely on clinician assessment or Electronic Health Records. Benefits to using frailty measures based on patient self-report include ease-of-use and patient-centricity. Our aim was to leverage PatientReported Outcome (PRO) data from relapsed/ refractory multiple myeloma (RRMM) clinical trials submitted to the U.S. Food and Drug Administration (FDA) for regulatory review, to map PRO questions to an existing frailty model. Methods: Baseline patient responses to the EORTC QLQ-C30 were pooled from nine RRMM trials submitted to FDA between 2010 and 2020. Items from the QLQ-C30 were selected to closely match criteria from the Fried frailty model (Table 1). Spearman's correlation coefficients were generated between selected QLQ-C30 items. Two approaches to dichotomization of patient responses were explored: (1) 'not at all'/ 'a little' $=0$ and 'quite a bit'/ 'very much' $=1$ vs. (2) 'not at all' $=0$ and 'a little'/ 'quite a bit'/ 'very much $=1$. Both approaches were used to generate Patient-Reported Frailty Phenotype (PRFP) scores to explore differences in the proportion of patients categorized as Frail, Pre-frail, and Fit. Results: 5,544 patients completed all selected QLQ$\mathrm{C} 30$ items at baseline. Spearman's correlation coefficients between items ranged from 0.36 to 0.64 . Two Fried Frailty criteria (exhaustion 
and physical activity) had multiple candidate QLQ-C30 items (Table 1). Items 10 and 6 were selected due to higher correlations. Using dichotomization approach \#1, we found that $54.2 \%$ of patients were classified as Fit, $24.7 \%$ as Pre-frail, and $21.1 \%$ as Frail. Approach \#2 yielded considerably different results: $10.7 \%$ were classified as Fit, $26.6 \%$ as Pre-frail, and $62.7 \%$ as Frail. Conclusion: In our preliminary conceptual model, we mapped items from the QLQ-C30 to corresponding Fried frailty criteria. Our model requires further testing and validation to select an appropriate dichotomization approach or explore other mechanisms to determine the thresholds for Fit, Pre-frail, and Frail. Next steps include exploring alternate proxy items from the QLQ-C30 and comparing proposed models through Confirmatory Factor Analyses and Known-groups analyses.

\begin{tabular}{|c|c|c|}
\hline $\begin{array}{l}\text { Fried Phenotype of } \\
\text { Frailty Criteria }\end{array}$ & $\begin{array}{c}\text { Fried Frailty } \\
\text { Score } \\
(0=\text { absent } \\
1=\text { present }) \\
\end{array}$ & Matching EORTC QLQ-C30 Item for PRFP \\
\hline weight loss & 0 or 1 & Have you lacked appetite? (Q13) \\
\hline exhaustion & 0 or 1 & $\begin{array}{l}\text { Did you need to rest? (Q10) } \\
\text { [Alternate item: Were you tired? (Q18)] }\end{array}$ \\
\hline weakness & 0 or 1 & Have you felt weak? (Q12) \\
\hline slowness & 0 or 1 & $\begin{array}{l}\text { Do you have any trouble taking a short walk outside of the } \\
\text { house? (Q3) }\end{array}$ \\
\hline $\begin{array}{l}\text { Physical activity } \\
\text { (as measured by the } \\
\text { Minnesota Leisure } \\
\text { Time Activity } \\
\text { Questionnaire) } \\
\end{array}$ & 0 or 1 & $\begin{array}{l}\text { Were you limited in doing either your work or other daily } \\
\text { activities? (Q6) } \\
\text { [Alternate item: Were you limited in pursuing your hobbies or } \\
\text { other leisure time activities? (Q7)] }\end{array}$ \\
\hline \multicolumn{3}{|c|}{$\begin{array}{c}\text { Sum of Fried Frailty Scores } \\
0-\text { Fit } \\
1 \text { or } 2-\text { Pre-frail } \\
\geq 3-\text { Frail }\end{array}$} \\
\hline
\end{tabular}

(B205.9) Development of a PROMIS® short form for assessing physical function in lung cancer patients

Steven Blum, MBA, MA, Bristol-Myers Squibb, Lawrenceville, New Jersey, USA; Mona Martin, RN, MPA, Evidera I PPD, Bethesda, Maryland, USA; Donald Bushnell, MA, Evidera I PPD, Bethesda, Maryland, USA; Larissa Stassek, MPH, Evidera I PPD, Bethesda, Maryland, USA; Sejin Lee, BPharm, MBA, MA, University of North Carolina, Chapel Hill, North Carolina, USA; James Shaw, PhD, PharmD, MPH, Bristol Myers Squibb, Lawrenceville, New Jersey, USA; Donald Patrick, MSPH, PhD, University of Washington, Seattle, Washington, USA

Aims: Decline and improvement in physical function (PF) may vary between different cancer types and cancer treatments. PROMIS ${ }^{\circledR}$ (Patient-Reported Outcome Measurement Information System) offers the opportunity to select existing items from established item banks which may be relevant and meaningful to a given population. These items can be administered using computer-adaptive testing or for ease of administration as a static short-form questionnaire. A short form or questionnaire tailored for use in a specific cancer-type may be more sensitive to changes in PF that may be less discernible when using more generic questionnaires. We describe the development of a new PF short form optimized for use with a lung cancer population. Methods: Starting with the full PROMIS and PROMIS-Cancer PF item banks, a modified Delphi process was used to identify and remove items with problematic construction or relevance. Remaining items were then evaluated during qualitative interviews with patients treated for either non-small cell lung cancer (NSCLC) or small-cell lung cancer (SCLC). The interviews included open-ended questions and a nested card-sort exercise designed to address: concept relevance (important and meaningful concepts that patients actually engaged in; both with-and-without difficulty); understandability; and the potential sensitivity of the items. Interviews were conducted both in-person and remotely and were audio recorded, transcribed, and analyzed. All participants also self-completed the full-set of unduplicated PROMIS $\mathrm{PF}$ items to provide quantitative data to use in a mixed-methods analysis to select a subset of PF items relevant to patients with lung cancer. Results: The Delphi panel evaluated 169 PROMIS PF items, voting to drop 93 and retain 76 items for further evaluation in the qualitative interviews. Interviews were conducted with 30 adult patients from 6 different sites in the US with a clinician-confirmed diagnosis of NSCLC $(n=23)$ or SCLC $(n=7)$. Ultimately, a final set of 21 unmodified PROMIS items were selected based on the assessment of the relevance and importance of the items to lung cancer patients along with the quantitative assessment of the items. Conclusion: The resulting short-form contains 21 PROMIS Physical Function items identified as being highly relevant and important to patients with lung cancer and represent a full spectrum of concepts within the over-arching construct of physical function.

\section{6: Clinical Care Applications}

(B206.1) Comparing goal attainment scaling versus PROMs for measuring patient goals

Sarah Hudson Scholle, DrPH, MPH, National Committee for Quality Assurance, Washington, District Of Columbia, USA; Catherine Clair, MHS, Johns Hopkins Bloomberg School of Public Health, Baltimore, Maryland, USA; Shelby Mixson, MSc, National Committee for Quality Assurance, Washington, District Of Columbia, USA; Theresa Hwee, MPH, National Committee for Quality Assurance, Washington, District Of Columbia, USA; Caroline Blaum, MD, MS, National Committee for Quality Assurance, Washington, District Of Columbia, USA; Lee Jennings, MD, MSHS, University of Oklahoma Health Sciences Center, Oklahoma City, Oklahoma, USA; Erin Giovannetti, $\mathrm{PhD}$, MedStar Heath Research Institute, Baltimore, Maryland, USA;

Aims: Both patient-reported outcome measures (PROM) and goal attainment scaling (GAS) can be used to set and monitor patient goals. To date, no studies have compared these methods in clinical care nor explored how sites and clinicians select a method. Methods: Four-site implementation study using Computer-Adaptive Testing (CAT) PROMs and GAS to measure goal achievement for patients with functional disabilities. After identifying "what matters most" to an individual, the clinician and patient could create a goal using either a PROM or GAS, a structured approach for specifying outcomes on a 5-point scale from "better than expected" to "worse than expected". A tablet-based application was offered for clinicians to record GAS and PROMs. We describe each site's goal setting approach and results. We also report on semi-structured interviews with 15 of the 33 participating clinicians. We coded interview transcripts using a coding scheme based on an interview guide with dual coders. Results: Site 1 chose GAS alone because it could be more easily documented in its electronic health record. The other sites used the tablet app. In Site 2, the two clinicians chose GAS alone. In Site 3, three out of nine clinicians used both methods and PROMs were recorded for $55 \%$ of patients, compared to Site 4 where all five clinicians used both methods and PROMs were recorded for $74.7 \%$. The rate of goal achievement was similar for patients using GAS and PROM approaches (range $40.0 \%$ to $81.8 \%$ for GAS, $54.5 \%$ to $75.5 \%$ for PROMs). Clinicians reported that PROMs were short, easy to understand, and facilitated by existing workflows using PROMs. They described GAS as more flexible and less intimidating for patients, however, identifying the "much worse than expected" field was challenging. Clinicians noted that PROMs were easier to administer for complex patients and GAS was better with less medically complex patients who were engaged in their care. Conclusion: Sites and clinicians selected a goal-setting approach based on existing electronic systems and workflows, clinician preferences, and patient factors. Both PROMs and GAS offer promising methods for goal setting, and 
implementation should consider ways to target approaches to meet patient needs.

\section{(B206.2) Digital patient-reported outcomes and emotional distress}

Amalie Søgaard Nielsen, Master of Arts, University of Copenhagen, Copenhagen, Denmark; Richard H. Osborne, Ph.D., Faculty of Health, Arts and Design, Swinburne University of Technology, Melbourne, Australia; Lars Kayser, MD, Ph.D., Department of Public Health, University of Copenhagen, Copenhagen, Denmark; Sanne Jensen, The Danish Health Data Authorities, Copehagen, Denmark; Lisa Hanna, Ph.D., School of Health and Social Development, Deakin University, Melbourne, Australia; Birgit Furstrand Larsen, MD, Ph.D., Diagnostic Centre, University Research Clinic for Innovative Patient Pathways, Silkeborg Regional Hospital, Department of Clinical Medicine, Aarhus University, Silkeborg, Denmark; Charlotte Weiling Appel, Ph.D., Diagnostic Centre, University Research Clinic for Innovative Patient Pathways, Silkeborg Regional Hospital, Department of Clinical Medicine, Aarhus University, Silkeborg, Denmark

Aims: Digital Patient-Reported Outcome (PRO) concepts have been introduced in the routine clinical outpatient care of people with Inflammatory Bowel Disease (IBD) as well as in many other chronic conditions. The purpose of digital PROs in this context is to replace or supplement face-to-face follow-up sessions through symptom screening, and to inform follow-up sessions through questions about health-related quality of life. However, not all patients use digital PROs as intended, and little is known about the determinants of engagement. This study aimed to map differences between users and non-users of a PRO system for people with IBD as well as exploring the mechanisms behind engagement, use, and non-use. Methods: A mixed method study was conducted. First, a questionnaire including the Readiness and enablement index for Health technology (ReadHy) and the Service User Technology Acceptability Questionnaire (SUTAQ) was sent to all IBD patients $(n=827)$ registered at a Danish Regional Hospital by electronic, secure e-mail $(n=784)$ or on paper to their home address $(n=43)$. Participants were also recruited purposively for interviews $(n=16)$ based on their responses to the survey. Results: The users and non-users groups differed with regard to level of emotional distress based on questionnaire responses. Patients with high level of emotional distress were not enrolled in the digital PRO system as often as patients with low emotional distress. However, interviews revealed that patients with severe IBD symptoms and a higher degree of emotional distress related to their disease had a tendency to focus on the holistic aspects of care, and recognized that digital Patient-Reported Outcomes might lead to increased focus on mental health and extra-intestinal symptoms. The patients trusted that clinicians read and react to the knowledge they receive via PRO. Conclusion: This study shows a willingness among patients to engage in digital PRO despite, or even because of, a high level of emotional distress. These data will inform health systems' approach to the enrolment of patients into digital PRO systems.

(B206.3) Assessing burden of treatment at the point-of-care: development and feasibility of the PETS-Now web-based clinical tool

Kathleen Yost, PhD, Mayo Clinic, Rochester, Minnesota, USA; Jennifer Ridgeway, PhD, Mayo Clinic, Rochester, Minnesota, USA; Bayly Bucknell, MS, Mayo Clinic, Rochester, Minnesota, USA; Mike Wambua, Hennepin Healthcare Research Institute, Minneapolis, Minnesota, USA; Natalie Erbs, MD, Mayo Clinic, Rochester,
Minnesota, USA; Summer Allen, MD, Mayo Clinic, Rochester, Minnesota, USA; Elizabeth Rogers, MD, University of Minnesota Medical School, Minneapolis, Minnesota, USA; Mark Linzer, MD, Hennepin Healthcare, Minneapolis, Minnesota, USA; Roger Anderson, $\mathrm{PhD}$, University of Virginia School of Medicine, Charlottesville, Virginia, USA; David Eton, PhD, Mayo Clinic, Rochester, Minnesota, USA

Aims: Burden of treatment (BoT) is the workload of health care, its impact on patient functioning and well-being, and stressors that exacerbate it like financial concerns about medical expenses. The Patient Experience with Treatment and Self-management (PETS) is a validated measure of patient-reported BoT for people with multiple chronic conditions (MCC). We aimed to (1) adapt PETS to capture and report patients' BoT to providers at the point-of-care, and (2) assess the feasibility of using the tool in clinical practice. Methods: We engaged MCC patients and providers from diverse primary care settings in two rounds of focus groups. In round 1, participants identified content from the PETS to include in a brief, web-based version ("PETS-Now"). In round 2, we obtained feedback on the tablet version of PETS-Now and on the presentation of results in a summary report. A quasi-experimental design was used to evaluate acceptability and feasibility of practice implementation. Results: Seventeen patients and 25 providers participated in focus groups between May 2017 and June 2018. PETS domains retained for inclusion in PETS-Now were Monitoring Health, Taking Medicine, Getting Health Information, Following a Healthy Lifestyle, Medical Expenses, Personal Relationships, Access to Care, and Medical Equipment. The tool instructs the patient to select the domain of greatest concern and then identify specific challenges within that domain. The patient also completes the role limitations and physi$\mathrm{cal} /$ mental exhaustion scales from the PETS to assess the impact of BoT on functioning and well-being. Sixteen primary care providers and 170 patients ( 85 control, 85 PETS-Now intervention) participated in the feasibility study. Some protocol modifications were made due to the coronavirus pandemic. Based on post-encounter surveys in the PETS-Now arm, physicians $(72 \%)$ were more likely than patients (49\%) to acknowledge reviewing the summary report during the encounter. Providers who did not review the PETS-Now report noted a lack of time or forgetting about it. Most patients (97\%) and providers $(72 \%)$ reported being "quite" or "very" willing to use the tool at their next visit. Conclusion: We demonstrated the feasibility of implementing the PETS-Now in diverse primary care practices. Future research will assess the efficacy of the PETS-Now at reducing BoT.

\section{(B206.4) Self and Proxy Completion of Quality of Life Assessment in Dementia: A Review of Reviews}

Autumn Beemer, Honors Bachelor of Science in Nursing, University of Alberta, Edmonton, Alberta, Canada; Iva Piplica, Honors Bachelor of Science in Nursing, University of Alberta, Edmonton, Alberta, Canada; Rick Sawatzky, Trinity Western University, Langley, British Columbia, Canada; Kara Schick-Makaroff, University of Alberta, Edmonton, Alberta, Canada

Aims: Dementia frequently impacts the quality of life (QOL) of individuals living with this illness. QOL assessment invites the perspectives of these individuals to be shared with family caregivers and health care providers. A review of reviews was conducted with the aim to describe synthesized evidence regarding self- and proxy reports of QOL for individuals living with dementia. Methods: Searches were conducted in CINAHL, EMBASE, PsycINFO, and MEDLINE, not limited by date, and yielded a total of 266 results. A screening process included titles and abstracts, followed by full texts, 
as well as citation chaining of included articles. Articles were included if they addressed self- or proxy-rated patient-reported outcome measures (PROMs), focused on any type of dementia in adults, and were a review or synthesis published in English. Articles were appraised for quality, and relevant data were extracted. Results: Twenty-five syntheses were included. Individuals with dementia are largely able to self-report QOL, and this is considered to be the "gold standard." Proxy-reports have often been incongruent with self-reports. Various patient factors (e.g., increased neuropsychiatric symptoms) and proxy factors (e.g., increased caregiver burden) have been found to be related to incongruence between self- and proxyreported QOL. Proxy-reports may vary significantly from self-reports, depending on the proxy's relationship to the patient. Closer proximity and higher frequency of contact with the patient tends to lead to higher agreement between self- and proxy reports of QOL. Some authors have suggested using both caregiver and health care provider proxy reports of patient QOL given that they have different perspectives. Conclusion: Using a combination of self- and proxy reports when assessing QOL for people living with dementia is consistently recommended. Although proxy reports are not an ideal substitute for self-reports, it is still better to collect proxy-reported QOL data than to forgo collecting QOL data altogether.

\section{(B206.5) An academic center's initial experience on facilitators and barriers for implementing patient-reported outcome measures in clinical care}

Marzyeh Amini, Center for Medical Decision Making, Department of Public Health, Erasmus MC University Medical Center, Rotterdam, The Netherlands; Arvind Oemrawsingh, Center for Medical Decision Making, Department of Public Health, Erasmus MC University Medical Center, Rotterdam, The Netherlands; Lisanne M. Verweij, Erasmus MC University Medical Center, Rotterdam, The Netherlands; Hester F. Lingsma, Center for Medical Decision Making, Department of Public Health, Erasmus MC University Medical Center, Rotterdam, The Netherlands; Jan A. Hazelzet, Center for Medical Decision Making, Department of Public Health, Erasmus MC University Medical Center, Rotterdam, The Netherlands; Frank Eijkenaar, Erasmus School of Health Policy and Management, Erasmus University Rotterdam, Rotterdam, The Netherlands; Nikki van Leeuwen, Center for Medical Decision Making, Department of Public Health, Erasmus MC University Medical Center, Rotterdam, The Netherlands

Aims: Successful implementation of patient-reported outcome measures (PROMs) in a clinical setting is crucial to be able to learn from PROMs when using them in clinical practice in hospitals and healthcare organizations and potentially supporting decision making. The aim of this cross-sectional study was to explore the perspectives of healthcare providers and researchers in a large academic hospital on facilitators and barriers for implementing PROMs in clinical care. Methods: A customized web-based questionnaire was developed based on literature study and an expert panel consensus procedure. It was disseminated to healthcare providers (e.g. physicians, nurses, paramedics) and researchers across multiple medical departments implementing PROMs in clinical care for 38 multidisciplinary "disease teams". The number of statements included in the questionnaire was dependent on the implementation stages that respondents were currently in, including "Purpose and Intake", "Designing", "Preparing", "Commencing", "Reflecting and Developing". Questionnaire statements were rated using a 5-point Likert scale ranging from "strongly agree" to "strongly disagree". In addition, 8 openended questions were included allowing respondents to mention additional facilitators and barriers for implementing PROMs.
Descriptive statistics were used to summarize results. Results: In total, 61 participants from both surgical and non-surgical departments completed the survey. Most respondents $(51 \%)$ were medical specialists and the median employment duration was 14 years. Frequently reported facilitators were the presence of a PROM coordinator in the (outpatient)clinic $(85 \%)$ and the integration of PROMs in the electronic health record $(81 \%)$. In open-ended questions, nine respondents $(15 \%)$ across most implementation stages reported that motivation/enthusiasm/interest within the disease team is needed for successfully implementing PROMs. Commonly reported barriers across most stages were language barriers $(76 \%)$, IT issues $(\mathrm{N}=17$ open responses), time constraints $(\mathrm{N}=14)$. Other reported barriers were challenges in multidisciplinary collaboration due to conflicting interests, schedules, and task division, and excessive duration of the implementation process ( $\mathrm{N}=3$ for every barrier). Conclusion: For the successful implementation of PROMs in clinical practice for the valid decision in care, it is imperative that prior to and during the implementation, healthcare organizations consider accommodating motivated healthcare professionals, involving PROMs coordinators, and investing in an adequate IT-infrastructure and removal of language barriers.

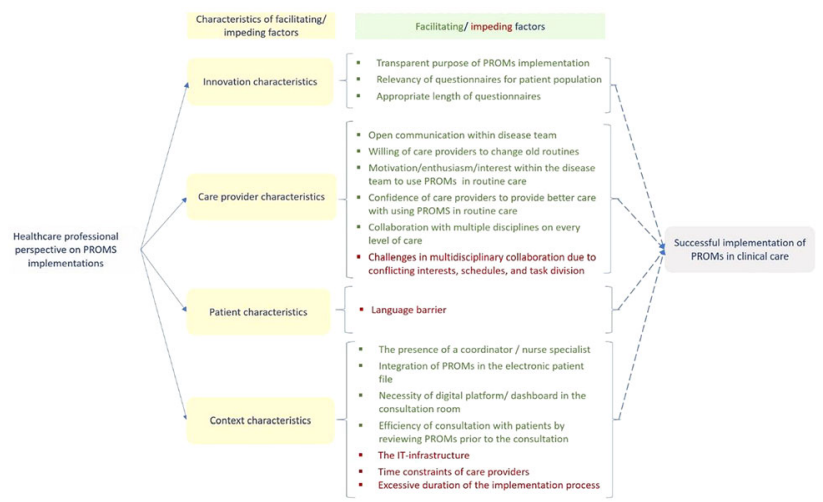

(B206.7) Feasibility of using the Paediatric Measure Yourself Medical Outcome Profile (P-MYMOP) at a paediatric outpatient department

Sana Ishaque, MBBS, MSc (Clinical Epi), PhD, The University of Adelaide, Adelaide, Australia; Amy Salter, PhD, School of Public Health University of Adelaide, Adelaide, Australia; Jonathan Karnon, $\mathrm{PhD}$, Flinders University, Adelaide, Australia; Rachel Roberts, $\mathrm{PhD}$, School of Psychology University of Adelaide, Adelaide, Australia; David Thomas, MBBS, Department of General Medicine, Women's and Children's Hospital Adelaide, Adelaide, Australia

Aims: The measurement of patient-reported outcomes provides a means of integrating opinions and values of patients in the process of their clinical care. This study was designed to identify the barriers and facilitators to the use of the Paediatric Measure Yourself Medical Outcome Profile (P-MYMOP), a tool developed for children 7 to 11 years old in a paediatric outpatient department (OPD). Methods: Participating nurses introduced the P-MYMOP to children 7-11 years old and their parents attending the OPD and recommended that they take the completed tool to their consultation. Participating doctors discussed the completed P-MYMOP form with child-parent pairs. Six participants, (three nurses and three doctors) were interviewed about their experience of the process. The resulting data were analysed qualitatively using deductive content analysis. Results: The study ran for 19 afternoons in the OPD. Over the study period, 77 eligible children attended the OPD. Forty-two P-MYMOP forms were distributed and approximately $70 \%$ patients completed them. 
Participating nurses felt able to distribute the forms and OPD doctors found the P-MYMOP simple and straightforward to interpret. Both nurses and doctors stated that they would be willing to continue this practice in future. Barriers identified to the implementation of the P-MYMOP included: misunderstanding of patients and nurses about intended use of the P-MYMOP (some patients left their completed forms at the front desk and parent proxy completion instead of children's self-completion, the impact of the busy clinic workload on nurses' ability of distribute the P-MYMOP, and that eligible children constituted only a small fraction of the total clinic population. Facilitators identified included: identification and communication with a clinic leader supporting the implementation of the P-MYMOP, advanced signposting of the potentially eligible patients on appointment lists by clinic nurses, and printed copies available for distribution. Conclusion: This feasibility study collected evidence on the barriers and facilitators of implementing the P-MYMOP in a clinical practice. Participating nurses and doctors were able to implement the tool within their routine clinical consultations with relative ease. The study has demonstrated feasibility and acceptability of the tool which can now be incorporated as routine practice and further evaluated for its psychometric properties.

(B206.8) Exploring the use of patient-reported outcomes in adult social care: A scoping review

Sarah E Hughes, MHSc, Centre for Patient Reported Outcomes Research, University of Birmingham, Birmingham, UK; Olalekan Lee Aiyegbusi, $\mathrm{PhD}$, Centre for Patient Reported Outcome Research, University of Birmingham, Birmingham, UK; Daniel S Lasserson, PhD, Warwick Medical School, University of Warwick, Coventry, UK; Christel McMullan, Centre for Patient Reported Outcome Research, University of Birmingham, PhD, Birmingham, UK; Grace M Turner, PhD, Centre for Patient Reported Outcome Research, University of Birmingham, Birmingham, UK; Samantha Cruz-Rivera, $\mathrm{PhD}$, Centre for Patient Reported Outcome Research, University of Birmingham, Birmingham, UK; Phil Collis, Patient Partner, Centre for Patient Reported Outcome Research, University of Birmingham, Birmingham, UK; Jon Glasby, PhD, Department of Social Work and Social Care, University of Birmingham, Birmingham, UK; Melanie Calvert, $\mathrm{PhD}$, Centre for Patient Reported Outcome Research, University of Birmingham, Birmingham, UK

Aims: Patient-reported outcome measures (PROMs) are used widely in healthcare to capture individuals' perspectives of their health, wellbeing, and quality of life. However, the use of PROMs, or personreported outcomes, in adult social care, where service users may have complex long-term health conditions, is currently unclear. This scoping review aimed to explore the evidence relating to use of PROMs in adult social care. Methods: The electronic databases Medline (Ovid), PsychInfo (Ovid), ASSIA (ProQuest), Social Care Online (SCIE), Web of Science, HMIC, and EMBASE (Ovid) were searched with terms relating to "social care setting", "social care populations", "care type", "patient-reported outcomes", and "implementation" in September 2020 to identify eligible studies published since 2010. No restrictions on study design or language of publication were applied. Quality appraisal was undertaken using the CASP checklists. Data were extracted to tables and the Framework Method used for qualitative synthesis of the included sources. Two reviewers completed screening independently and double extraction was completed for ten percent of the included studies. Results: Electronic database searching in September 2020 located 2744 records. Following deduplication, title/abstract and full-text screening, 179 records were included in the review. Studies were found to originate primarily from high-income countries, were diverse in their aims, target population, study design, and methods, and used PROMs to measure a range of constructs. Most studies utilised PROMs to evaluate a health-focussed intervention delivered within a social care setting or with a population utilising social care services. Studies with a purely social care focus were found to use PROMs within service/ economic evaluations or surveys exploring service users' experience of care. Few studies used PROMs as a social care intervention. Conclusion: The use of PROMs in adult social care does not yet appear to be widespread. Evidence of PROM utilisation to support the routine provision of adult social care services was found to be limited. Overall, PROM deployment remains oriented towards the outcomes of healthcare interventions when these are delivered in a social care context. Further research that explores the feasibility of using PROMs in adult social care, including barriers and enablers to their use, is recommended.

(B206.9) How do family caregivers of older adults with dementia perceive the meaning of quality of life?

Afeez Hazzan, PhD, SUNY Brockport, Brockport, New York, USA; Jason Dauenhauer, Ph.D., MSW, SUNY Brockport, Brockport, New York, USA; Kristin Heffernan, Ph.D., LMSW, SUNY Brockport, Brockport, New York, USA; Joyce Hazzan, MBA, Liberty University, Brockport, New York, USA

Aims: Family caregivers of older adults living with dementia are relatives, friends, or neighbors who provide assistance related to this illness, but who are unpaid for the services they provide. Studies have shown that caring for older adults who have dementia can be challenging, particularly as the disease progresses. Compared to family caregivers of people with other conditions, family caregivers of older adults with dementia generally suffer from higher levels of burden and depression, and this often results in significant impact on their quality of life. While there are validated instruments available to measure quality of life, evidence from family caregivers regarding what constitutes quality of life is scarce. The current study presents findings of focus group and one-on-one interviews with family caregivers of older adults with dementia regarding what quality of life means in the context of dementia family caregiving. Methods: Purposeful sampling was used in selecting 23 family caregivers of older adults with dementia to participate in focus groups or interviews. A semi-structured interview guide was utilized during the interviews to raise pertinent open-ended questions for the participants to address. The interview and focus group transcripts were analyzed using qualitative description methodology to identify themes that are pertinent to the phenomenon under investigation. Results: Regarding what quality of life means to them, the following themes were derived from the analysis of focus group and interviews with family caregivers of older adults with dementia: (1) availability of a strong support system that includes family, friends, and paid help; (2) financial security and ability to afford the necessities of life; (3) companionship and socialization; and (4) health, emotional well-being, and quality sleep. Conclusion: Findings from this study suggest that family caregivers of older adults with dementia have clear and consistent opinion on what constitutes quality of life in the context of dementia family caregiving. This study highlights the need for the provision of much needed support to help family caregivers of older adults with dementia improve their quality of life.

(B206.10) Implementation of an in situ developed PatientReported Experience Measure in rural Ecuador 
Jimmy Martin, MD, Universidad Católica de Santiago de Guayaquil, Guayaquil, Ecuador; Aurora Mula, BsC, Foundation for the Promotion of Health and Biomedical Research, Sant Joan, Spain; Mercedes Guilabert, PhD, Universidad Miguel Hernandez, Elche, Spain; Gustavo Ramírez, MsC, Universidad Católica de Santiago de Guayaquil, Guayaquil, Ecuador; José Mira, PhD, Foundation for the Promotion of Health and Biomedical Research, Sant Joan, Spain

Aims: The global diabetes prevalence in 2019 in adults is estimated to be $9.3 \%$ (463 million people). In EcuadOR, diabetes is the second cause of death, only after ischemic heart disease. The objective of this study was to develop in Ecuador for the first time a PREM instrument, the EPD Questionnaire. This study was conducted involving all stakeholders (including national health authorities) as part of an international cooperation project. Methods: A mixed-methods study was conducted during May 2019 and March 2020. Patients, professionals, and policymakers were involved collecting their views in qualitative preliminary phase for item development. Second, a paperbased study was done. To be eligible, participants had to be 18 years or older, been diagnosed with type 2 diabetes, and be able to understand the questions. Setting of the study was primary care health centers from rural and urban areas of Ecuador. The hypothesis that scores on the instrument would be higher in urban areas compared to rural areas was tested using the Mann-Whitney $U$ test. This is due to that in urban areas, it exists a higher education level and improved access to health. Results: A total of 486 patients from urban (57\%) and rural areas (43\%) from coastal and highlands participated. $58 \%$ were women, mean age of participants was 59.2, and years in treatment were 8 . The PREM instrument had 8 items and measured the following dimensions, information (items $3,5,6$ ), patient-centered care (items 2, 4, 7) and care delivery (items 1,8). Responses are rated in a four-point Likert scale ( $1=$ hardly ever $-4=$ everyday). Overall patients from rural areas reported worse experiences in two of the three factors $(\mathrm{p}<0.05)$, patient-centered care $(7.2$ vs 7.7$)$, and care delivery (5.2 vs 5.6). Conclusion: A tool to incorporate the voice of patients as an outcome measure has been validated. Patients experience of urban and rural areas must be taken into consideration, particularly in Ecuador and other Latin American countries, in which rural and native communities pose different characteristics, related to access to health, income, and education. In general, individuals located in urban areas show better results indicating a flaw of the national health system.

\section{Outstanding Poster Award Finalists}

\section{(1001) The Swedish and Portuguese translations of the Person- centred Practice Inventory-Staff (PCPI-S) instrument and the effect of social desirability on measurement}

John Chaplin, $\mathrm{PhD}$, Sahlgrenska Academy at University of Gothenburg, Gothenburg, Sweden; Carolina Lunde, PhD, Department of Psychology, University of Gothenburg, Gothenburg, Sweden; Filipa Ventura, PhD, Nursing School of Coimbra, Coimbra, Portugal; Jeanette Melin, PhD, RISE Research Institutes of Sweden, Gothenburg, Sweden

Aims: Theoretically derived instruments are needed to assess perceptions of person-centred practice (PCP) which can facilitate the implementation of patient-reported outcomes (PROs). The Personcentred Practice Inventory (PCPI) can be used for this purpose. The aim of this study was to translate, validate, evaluate and compare the Swedish and Portuguese translations for assessing perceptions of PCP. Methods: The translation process followed guidelines established by the International Society for Pharmacoeconomics and Outcome Research (ISPOR). The process included a review by a multinational expert panel. The Swedish and Portuguese instruments were further compared with the Norwegian, French and German versions. Exploratory Factor Analysis examined the factor structure (secondary analysis Norwegian version, $\mathrm{n}=258$ ) and compared this to the three domains of the Gothenburg Person-Centred Care (GPCC) model of Partnership, Narrative and Documentation. Results: All 59 items were translated and adapted for use in multi-healthcare settings. Issues related to cultural adaptation in the two target languages were minimal and resolved via panel discussions. Three-, four- and fivefactor solutions were tested on Norwegian data from a nursing population. The four-factor solution (56 items satisfied EFA criteria) provided the best option, explaining $39 \%$ of the total variance. The first eigenvalue (22.5) of the correlation matrix was more than three times larger than the second (7.4), suggesting the presence of a dominant, higher-order general factor which corresponded with the GPCC Partnership domain (20 items; $22 \%$ of variance). The other factors related to a supportive environment, self-reflection and work engagement. Extensive ceiling effects were noted, which the panel assessed to be due to social desirability (SD) response bias. Conclusion: The PCPI can be used to assess readiness for person-centred care and the implementation of PROs. In this study, SD was identified as a potential threat to scale construction. It is recommended that a measure of SD is included in future use of the PCPI and items reworded to allow neutral responses to be explored. This could be achieved by either setting a time frame or a situational reference. Further testing is being conducted. This paper will discuss the use of the PCPI-S and the effect of SD on scale construction.

(1002) Clinician workload and patient satisfaction of patientreported outcome-based symptom management for patients undergoing lung cancer surgery: a multicentre, randomised controlled trial

Wei Dai, Department of Thoracic Surgery, Sichuan Cancer Hospital, School of Medicine, University of Electronic Science and Technology of China, Chengdu, China; Wenhong Feng, Department of Thoracic and Cardiovascular Surgery, Jiangyou People's Hospital, Jiangyou, China; Yuanqiang Zhang, Department of Cardiothoracic Surgery, Zigong First People's Hospital, Zigong, China; Yaqin Wang, Department of Thoracic Surgery, Sichuan Cancer Hospital, School of Medicine, University of Electronic Science and Technology of China, Chengdu, China; Jia Liao, Department of Thoracic Surgery, Sichuan Cancer Hospital, School of Medicine, University of Electronic Science and Technology of China, Chengdu, China; Yangjun Liu, Department of Molecular Medicine and Surgery, Karolinska Insitutet, Stockholm, Sweden; Wei Xu, School of Public Health and Management, Chongqing Medical University, Chongqing, China; Shaohua Xie, Department of Thoracic Surgery, Sichuan Cancer Hospital, School of Medicine, University of Electronic Science and Technology of China, Chengdu, China; Xing Wei, Department of Thoracic Surgery, Sichuan Cancer Hospital, School of Medicine, University of Electronic Science and Technology of China, Chengdu, China; Cecilia Pompili, Section of Patient Centred Outcomes Research, Leeds Institute for Medical Research at St James's, University of Leeds, Leeds, UK; Qiang Li, Department of Thoracic Surgery, Sichuan Cancer Hospital, School of Medicine, University of Electronic Science and Technology of China, Chengdu, China; Qiuling Shi, Center for Cancer Prevention Research, Sichuan Cancer Hospital \& Institute, Sichuan Cancer Center, School of Medicine, University of Electronic Science and Technology of China, Chengdu, China; submitted on behalf of "CN-PRO-Lung"

Aims: Patient-reported outcome (PRO)-based symptom management is an ideal model for high-quality patient-centered care. This study aimed to profile the clinician workload, clinician acceptability, and 
patient satisfaction of this model in patients undergoing lung cancer surgery. Methods: This multicenter trial (CN-PRO-Lung 2) was conducted in three tertiary hospitals between November 2019 and August 2020. The trial randomly assigned patients with an initial diagnosis of lung cancer and scheduled to undergo surgery to two groups (PRO-based symptom management or usual care) in a 1:1 ratio. Real-time alerts were sent to the clinicians when patients in the PRO-based symptom management group reported the 5 target symptom scores reaching the preset threshold (score $\geq 4$ on a $0-10$ scale). The clinicians managed patients' symptoms within $24 \mathrm{~h}$. Patients in the usual care group received symptom management as usual, and the clinicians did not receive any alerts relating to their patients' symptom. Symptoms were assessed using the MD Anderson Symptom Inventory-Lung Cancer at baseline, daily post-surgery, and twice a week after discharge for up to 4 weeks. The trial registration number was ChiCTR1900020846. Results: Of the 166 patients randomized, 65 in the PRO-based management group who completed the protocol were analyzed. Clinicians spent a median time of 2 min (range, 1-17 $\mathrm{min}$ ) to handle a symptom threshold event, and $11 \mathrm{~min}$ (range, 2-88 min) per patient throughout the study period. The acceptability of the PRO-based symptom management model and the ePRO system among the 5 participating surgeons was high, with a score ranging from 7 to 10 on a $0-10$ scale (higher scores indicating better outcomes). In the PRO-based symptom management group, $96.4 \%$ of the patients thought that the PRO-based symptom management model was helpful in their postoperative recovery. The median score of patient satisfaction was 9 (range, 5-10). Patients reported that this model was necessary (median score: 10) and that it did not interfere with their life (median score: 0). Conclusion: PRObased symptom management after lung cancer surgery showed low clinician burden, high clinician acceptability, and high patient satisfaction, suggesting that it is feasible after major surgery.

\begin{tabular}{lcc}
\hline Table 1. Clinician workload.* & & \\
\hline Type & Median Time (range) - min & Mean Time (SD) - min \\
\hline Time spent per event & $2(1-27)$ & $3.03(2.58)$ \\
Time spent per patient & $11(2-88)$ & $16.1(15.5)$ \\
Time spent per day & $35(1-169)$ & $45.8(43.6)$ \\
\hline
\end{tabular}

* Recording handling time for symptom threshold events was not compulsive. In total, 63 (96.9\%) patients reported symptom threshold events, and $68.5 \%(302 / 441)$ of the events handling time was recorded to identify the doctor's burden precisely in this trial. The handling time for 4 events was incorrectly recorded and excluded from the final analysis.

\begin{tabular}{|c|c|c|}
\hline \multirow{3}{*}{$\begin{array}{l}\text { Question } \\
\text { 1. To what extent do you know PRO? }\end{array}$} & Before Trial & After Trial \\
\hline & \multicolumn{2}{|c|}{ Median (range) score } \\
\hline & $10(9-10)$ & $10(9-10)$ \\
\hline $\begin{array}{l}\text { 2. Do you think the PRO-Based Symptom Care model is } \\
\text { necessary? }\end{array}$ & $10(8-10)$ & $10(8-10)$ \\
\hline $\begin{array}{l}\text { 3. Would you like to recommend this model (PRO-Based } \\
\text { Symptom Care) to other patients? }\end{array}$ & $10(9-10)$ & $10(8-10)$ \\
\hline $\begin{array}{l}\text { 4. Would you like to recommend this model (PRO-Based } \\
\text { Symptom Care) to other doctors? }\end{array}$ & $10(8-10)$ & $8(7-10)$ \\
\hline 5. Do you think the introduction of SMARS is necessary? & $9(9-10)$ & $10(9-10)$ \\
\hline 6. What is your level of satisfaction with the SMARS? & $8(7-10)$ & $9(8-10)$ \\
\hline 7. Do you think using SMARS adds burden on patients? & $4(1-10)$ & $3(2-4)$ \\
\hline 8. Do you think using SMARS adds burden on doctors? & $4(2-7)$ & $3(2-6)$ \\
\hline $\begin{array}{l}\text { * Response rates were } 100 \% \text { for all questions. Higher scor } \\
0-10-\text { point scales for questions } 1-6 \text {, and lower scores repr } \\
\text { scales for questions 7-8. PRO denotes patient-reported ou } \\
\text { monitoring, alerting and response system. }\end{array}$ & $\begin{array}{l}\text { nt better outc } \\
\text { d SMARS sym }\end{array}$ & -10-point \\
\hline
\end{tabular}

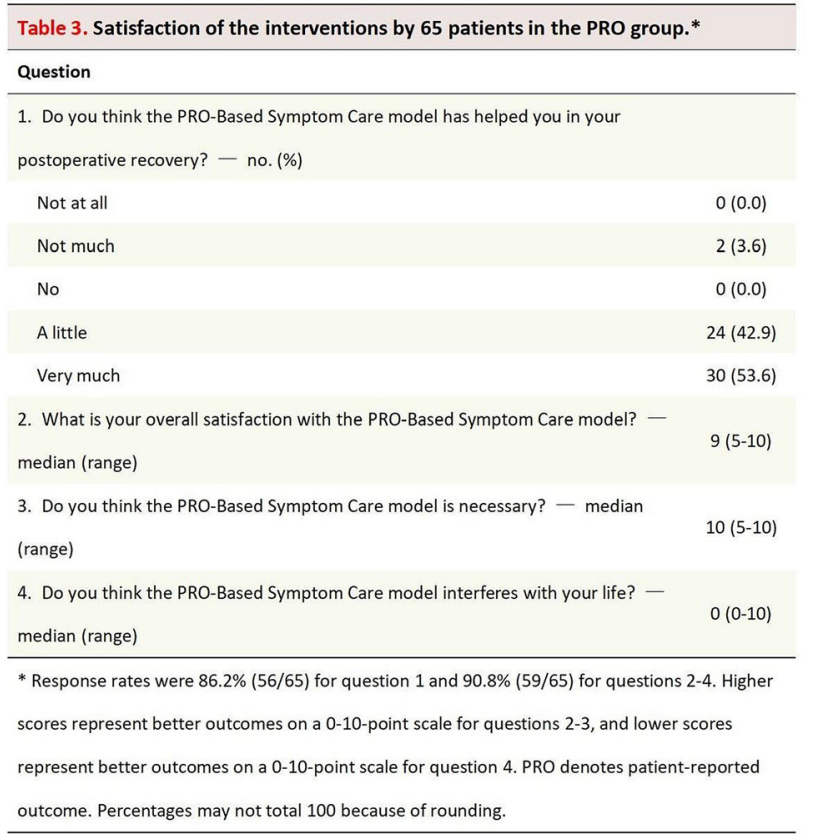

(1003) Contextualizing qualitative analysis with quantitative data on the symptoms and side effects associated with melanoma treatment in the real world: the value of patient registries

Akosua Ofori, Master of Public Health, Vitaccess, Oxford, UK; Harshitha Ravindra, MTech, Vitaccess, Oxford, UK; Katie Hall, MPH, Vitaccess, Oxford, UK; Catherine Bottomley, PhD, Vitaccess, Oxford, UK; Casey Quinn, PhD, Vitaccess, Oxford, UK; Mark Larkin, PhD, Vitaccess, Oxford, UK; Maggie Heinrich, PhD, Vitaccess, Oxford, UK

Aims: The Melanoma UK digital registry, founded in collaboration with Melanoma UK, explores real-world patient-reported impacts of melanoma using a mobile application (app). Alongside measures of 
quality of life (QoL), the app includes 11 PRO-CTCAE questions about symptoms and side effects. This study's objective was to explore patients' experience of symptoms and side effects during treatment. Methods: Four registry participants took part in a focus group, primarily held to better understand fever as a treatment side effect; fever is not included in the PRO-CTCAE instrument. A thematic analysis was conducted to identify prominent themes from the discussion.Participants' experiences were contextualized using one patient from the registry receiving dabrafenib + trametinib and another receiving ipilimumab + nivolumab as reference cases. Five registry participants were matched by treatment and age or disease stage. Demographics, side effects, EQ-5D-5L and QLQ-C30 data were compared. Results: Focus group participants were aged 33-66 years; all had Stage 4 melanoma. Two reported receiving immunotherapy, with one specifying ipilimumab + nivolumab; two received dabrafenib + trametinib. Participants most commonly reported experiencing shivering, shaking, dehydration, and poor appetite with fever. Fatigue and sensitivity to cold were reported as long-term side effects.Participants also reported treatment changes due to side effects and the dilemma of continuing with treatment to remain in remission while experiencing life-limiting side effects. Matching the dabrafenib + trametinib reference case with an identical patient who was 10 years younger showed higher QoL in the younger patient. Several identical patients with Stage 3 melanoma experienced a mixed number of side effects, and lower QoL. The reference case for ipilimumab + nivolumab was compared to a matched Stage 3 patient. The QoL was higher in the reference patient despite greater side effects. Conclusion: In a focus group on side effects in patients participating in a digital registry, we identified the most prevalent symptoms occurring with fever and the longer-term effects of treatment. By combining qualitative data with data from the registry, we showed the value in using one data source to enrich the other, while also demonstrating the impact on a focus group if different participants had opted in and provided data, which can only be explored with matchable, quantitative data available.
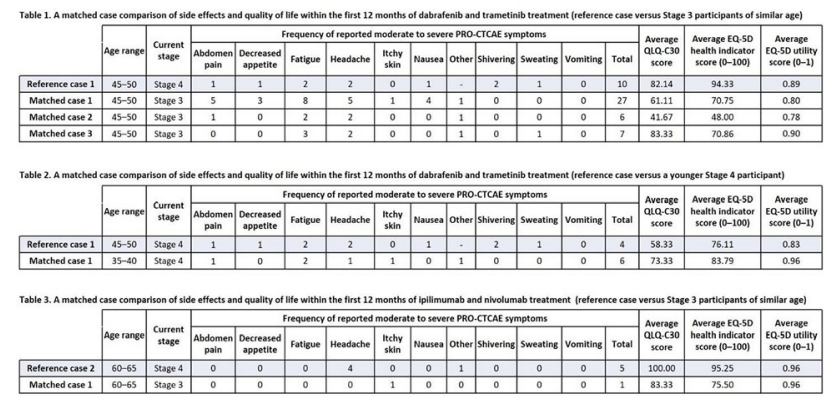

\section{(1004) Vaccine for COVID-19: what are the preferences of the general public in Quebec}

Thomas Poder, Ph.D., CR-IUSMM, Montréal, Quebec, Canada; Gabin Morillon, M.Sc., CERDI, Clermont-Ferrand, France

Aims: Expectations toward a vaccine for COVID-19 are very high. However, the effectiveness of its adoption will highly depends on the acceptance of the population. To improve this acceptance, we must know the preferences of the public for the main characteristics of the vaccine. This will help to design an appropriate strategy. Methods: A discrete choice experiment (DCE) was conducted in the province of Quebec, Canada, between October and November 2020. The survey was conducted online using quota sampling. Using a fractional factorial design, we produced a DCE with 360 choice cards. Each choice card described two scenarios with a vaccine and an opt-out option for no vaccine. Choice cards were divided in 30 blocks, and each respondent was randomly allocated to one block containing 12 choice cards. Scenarios with a vaccine were described with 7 attributes using 3 to 6 levels: country producing the vaccine, effectiveness (30\% to $95 \%$ ), side effects, duration of effectiveness ( 3 to 24 months), population group with priority for access (general public, health professionals, 65 years or older), waiting time to be vaccinated when the vaccine will be available ( 2 to 8 months), health institution recommending the vaccine (WHO or Quebec public health agency or both). A mixed logit model was used to estimate the relative importance of each attribute. A latent class model was used to determine different groups of behavior. Results: 1980 participants were recruited and 1599 were usable for the analysis. About $7.4 \%$ always chose the opt-out option for no vaccine. Results indicated that effectiveness and side effects were the two most important attributes in the decision of the general public to be vaccinated (i.e., relative importance between 23 and 29\%). Duration of effectiveness, health institution recommending the vaccine, and country producing the vaccine were the following three most important attributes (12-17\%). These were followed by the waiting time to be vaccinated (4\%) and group priority (3\%). The latent class model found 4 groups, including pro- and anti-vaccine groups. Conclusion: Vaccination strategy needs to be appropriately designed to better fit the preferences of the public and improve its acceptance, particularly the choice of the vaccine and its distribution.

(1005) Self-esteem as partial mediator of mental health outcomes in young people with epilepsy

Peter T. Cahill, M.Sc., McMaster University, Hamilton, Ontario, Canada; Mark A. Ferro, University of Waterloo, Waterloo, Ontario, Canada; Wenonah N. Campbell, McMaster University, Hamilton, Ontario, Canada; Gabriel M. Ronen, McMaster University, Hamilton, Ontario, Canada

Aims: Self-esteem is a potentially modifiable predictor of mental health and, consequently, quality of life for young people with epilepsy. In this study, we evaluated whether self-esteem mediates the impacts of environmental and epilepsy-specific factors on mental health outcomes. Methods: A prospective cohort of 480 young people with epilepsy and their families participated in 5 visits over 28 months. Data were collected on clinical seizure burden, cognitive comorbidity, peer and parental support, self-esteem, and mental health symptoms using predominately child-reported outcomes. Structural equation modeling was used to specify and test relationships between these constructs simultaneously, using exogenous variables from the baseline visit and endogenous variables from subsequent visits. We completed a mediation analysis using biascorrected bootstrapped tests of direct, indirect, and total effects, focusing on the statistical significance of indirect effects. Results: The results indicated that self-esteem mediated the effect of peer support $(\beta=-0.15$, standard error $[\mathrm{SE}]=0.06)$ and clinical seizure burden $(\beta=0.23, \mathrm{SE}=0.10)$ on mental health. In contrast, no mediating effects were found in the relationships of parental support $(\beta=-$ $0.07, \mathrm{SE}=0.04)$ and cognitive comorbidity $(\beta=-0.01, \mathrm{SE}=0.01)$ on mental health. Conclusion: Results were consistent with self-esteem mediating the impact that both peer support and clinical seizure burden have on mental health outcomes in young people with epilepsy. However, the findings were not consistent with self-esteem mediating the impact of parental support or cognitive comorbidity. We interpret these results as preliminary support indicating that assessment of and interventions targeting self-esteem may be appropriate to support the mental health and thereby the quality of life of young people with epilepsy. Supporting self-esteem may mitigate negative influences on mental health caused by resistant epilepsy or 
low peer support. We encourage further research to confirm whether this statistically significant mediation is also clinically important.

\section{(1006) Factors influencing patient treatment decision-making in early breast cancer}

Alessandra Gennari, Università Degli Studi Del Piemonte Orientale Scuola di Medicina, Novara, Italy; Chikako Shimizu, Comprehensive Cancer Center, National Center for Global Health and Medicine, Tokyo, Japan; Susan McCutcheon, AstraZeneca, Cambridge, UK; Emuella Flood, AstraZeneca, Gaithersburg, Maryland, USA; Graceanne Wayser, Kantar Health, New York, New York, USA; Kathleen Beusterien, Kantar Health, New York, New York, USA; Emily Mulvihill, Kantar Health, New York, New York, USA; Christian Jackisch, Klinikum Offenbach GmbH, Offenbach Am Main, Germany; Stella Mokiou, AstraZeneca, Cambridge, UK

Aims: New therapies being investigated for the systemic treatment of early breast cancer (eBC) in the neoadjuvant and adjuvant settings will expand treatment choice. We identified key treatment pathway attributes influencing patient treatment decision-making in $\mathrm{eBC}$ in Germany, Italy and Japan. Methods: Patients diagnosed with HER2negative, stage I-IIIa breast cancer (BC) after 2014 engaged in a 45 -min telephone interview in local language (25 Sep to 24 Oct 2020). A semi-structured interview guide was used, including openended questions about how patients value different treatment pathways for $\mathrm{eBC}$ and which attributes influence patients' choice of neoadjuvant and adjuvant eBC treatments. Results: Overall, 24 patients $(n=8$ per country; stage I, $n=10$; stage II-IIIa, $n=14)$ participated. At diagnosis, median (range) age was 48 (33-59) years. All had received prior surgery and chemotherapy. Many patients $(n=14)$ were given only 1 treatment option; patients offered $>1$ treatment option were given choices (e.g. modality) at specific treatment steps $(n=9)$. Most patients preferred a flexible treatment plan with involvement in treatment choice $(\mathrm{n}=17)$ to a set plan $(n=5)$; reasons included: opportunity to evaluate options $(n=12)$, avoid unnecessary treatment and associated side effects $(n=10)$ and personalise treatment $(n=7)$. Many patients were notified of their risk status by their physician $(n=14)$. Final treatment decisions were often made by patients alone with their physician $(n=18)$; some consulted family $(n=4)$, a psychologist $(n=4)$ or other patients with $\mathrm{BC}(\mathrm{n}=3)$. When asked to identify important attributes in the context of treatment pathways, patients prioritised efficacy (no signs/symptoms of cancer 2 years post-treatment; $\mathrm{n}=18$ ), followed by risk of a serious adverse event $(n=15)$, anaemia $(n=14)$, fatigue $(n=13)$ and nausea $(n=10)$. A third of patients $(n=4 / 12)$ who gave feedback on the minimal meaningful percentage difference in 2-year efficacy stated that a 5\% difference would drive treatment choice. Conclusion: Patients value a treatment plan tailored to their disease characteristics and treatment preferences. Efficacy attributes drive treatment decisions and small differences affect choice. This qualitative research will inform a larger quantitative patient preference study. Funding: AstraZeneca, as part of an alliance between AstraZeneca and Merck Sharp \& Dohme Corp., a subsidiary of Merck \& Co., Inc., Kenilworth, NJ, USA.

\section{New Investigator Poster Award Finalists}

(2001) Leveraging artificial intelligence to analyze unstructured PRO data for pediatric cancer survivors: a validation study on psychological stress and meaning/purpose domains

Zhaohua Lu, PhD, St. Jude Children's Research Hospital, Memphis, Tennessee, USA; Jin-ah Sim, PhD, St. Jude Children's Research
Hospital, Memphis, Tennessee, USA; Jade Xiaoqing Wang, PhD, St. Jude Children's Research Hospital, Memphis, Tennessee, USA; Kumar Srivastava, PhD, St. Jude Children's Research Hospital, Memphis, Tennessee, USA; Leslie Robison, PhD, St. Jude Children's Research Hospital, Memphis, Tennessee, USA; Melissa Hudson, MD, St. Jude Children's Research Hospital, Memphis, Tennessee, USA; Justin Baker, MD, St. Jude Children's Research Hospital, Memphis, Tennessee, USA; I-Chan Huang, PhD, St. Jude Children's Research Hospital, Memphis, Tennessee, USA

Aims: Having in-depth conversations with cancer survivors about psychological stress and meaning/purpose may facilitate interventions to improve quality of life. However, methods to analyze qualitative PROs are understudied. We aimed to leverage natural language processing (NLP) and machine learning (ML) techniques to abstract interview-based psychological stress and meaning/purpose perceived by child/adolescent cancer survivors and tested the validity of NLP/ ML algorithms using expert-coded findings as the gold standard. Methods: We recruited 51 survivors (aged $8-17.9$ years; $\geq 5$ years off-therapy) from the St. Jude Lifetime Cohort Study and their caregivers and performed semi-structured interviews focusing on psychological stress and meaning/purpose related to cancer experiences. After verbatim transcription, two content experts systematically coded and semantically labeled the data by different attributes (anger, controllability, and fear attributes for psychological stress domain; goal, optimism, and purpose attributes for meaning/ purpose domain). Analyzable sample sizes were 245 and 513 semantic units abstracted from the psychological stress and meaning/ purpose interviews. Two NLP/ML methods were used to validate the semantic attributes: 1) Word2vec with Extreme Gradient Boosting (XGBoost) and 2) Bidirectional Encoder Representations from Transformers (BERT). The accuracy, receiver operating characteristic (ROC), and precision-recall (PR) curves metrics were used to compare the validities of NLP/ML methods. Results: Compared to Word2vec/XGBoost, BERT demonstrated superior accuracy (cutpoint: $>0.8$ ) on both PRO domains, including 0.89 (95\% CI 0.86 , $0.94)$ and 0.85 (95\% CI $0.80,0.89)$ for anger and fear attributes on the psychological stress domain, and 0.86 (95\% CI 0.83, 0.89), 0.86 (95\% CI $0.83,0.89)$, and 0.84 (95\% CI $0.81,0.87)$ for goal, optimism, and purpose attributes on the meaning/purpose domain. Additionally, BERT yielded significantly higher areas under the PR curves, with differences by 0.13 (95\% CI $0.01,0.26$ ), 0.14 (95\% CI $0.04,0.23$ ), and $0.15(0.01,0.28)$ for anger, controllability, and fear attributes on the psychological stress domain, and differences by $0.23(95 \% \mathrm{CI}$ $0.14,0.31), 0.14$ (95\% CI $0.02,0.25)$, and 0.05 (95\% CI $0.02,0.16$ ) for goal, optimism, and purpose attributes on the meaning/purpose domain. Conclusion: The BERT model has superior performance in characterizing psychological and meaning/purpose PROs for pediatric cancer survivors than Word2vec/XGBoost. Further studies are warranted to validate NLP/ML methods for unstructured PRO big data (e.g., electronic health records).

(2002) Development and psychometric evaluation of the Symptom Burden Questionnaire for Long COVID (SBQ-LC)

Sarah Hughes, MHSc, Centre for Patient Reported Outcome Research, University of Birmingham, Birmingham, UK; Olalekan Lee Aiyegbusi, PhD, Centre for Patient Reported Outcome Research, University of Birmingham, Birmingham, UK; Samantha Cruz-Rivera, $\mathrm{PhD}$, Centre for Patient Reported Outcome Research, University of Birmingham, Birmingham, UK; Christel McMullan, PhD, Centre for Patient Reported Outcome Research, University of Birmingham, Birmingham, UK; Elin Haf Davies, PhD, Aparito Limited, Wrexham, UK; Chris Frost, Aparito Limited, Wrexham, UK; Gary Price, Centre for Patient Reported Outcome Research, University of Birmingham, 
Birmingham, UK; Karen Matthews, LongCovidSOS, Birmingham, UK; Jenny Camaradou, Citizen Partner (UK), COVID-END (COVID19 Evidence Network to support Decision-making), Hamilton, Ontario, Canada; Joht Singh Chandan, PhD, Institute for Applied Health Research, University of Birmingham, Birmingham, UK; Shamil Haroon, PhD, Institute for Applied Health Research, University of Birmingham, Birmingham, UK; Melanie Calvert, $\mathrm{PhD}$, Centre for Patient Reported Outcome Research, University of Birmingham, Birmingham, UK; submitted on behalf of "the Therapies for Long COVID (TLC) Study Group"

Aims: The symptoms of Coronavirus Disease 2019 (COVID-19) usually resolve within 12 weeks of infection. However, for approximately 1 in 10 individuals, symptoms can persist. Symptoms persisting beyond 12 weeks are known collectively as Long COVID. Complex and not yet well understood, Long COVID can have a significant impact on an individual's mental and physical functioning and quality of life. The Symptom Burden Questionnaire for Long COVID (SBQ-LC) is a new patient-reported outcome (PRO) measuring symptom burden and interference in Long COVID. It has been developed as part of the Therapies for Long COVID (TLC) Study for use in research and as a clinical tool for use with individual patients. This study aims to develop and validate the SBQ-LC. Methods: The results of a systematic literature review were used to develop an initial item library of symptoms across nine domains. Cognitive interviews involving individuals with Long COVID and item review by experts established the SBQ-LC's content validity. The item library was field tested in a sample of individuals with Long COVID recruited online and via Long COVID support networks. Rasch measurement theory (RMT) and classical test theory methods (CTT) were used to refine the SBQ-LC and evaluate its psychometric measurement properties. All development and validation work were carried out within an electronic patient-reported outcome (ePRO) environment using the Aparito Atom5 $5^{\mathrm{TM}}$ platform. Results: This study is ongoing. This presentation will report on item development, content validation, and initial psychometric evaluation including assessment of rating scale functioning, unidimensionality and local dependence, item calibration, targeting, reliability, and construct validity. The implementation of a bespoke ePRO platform for instrument development will be discussed from a methodological perspective. Conclusion: The SBQLC is a new PRO measuring symptom burden in Long COVID. Further psychometric evaluation in a large, UK-based community sample of individuals with Long COVID $(n=2000)$ is planned. The measure will be used with clinical data to identify symptom clusters and characterise Long COVID syndromes. The measure will also be used to assess the effectiveness of therapies in reducing symptom burden and improving quality of life to support the care of individuals living with Long COVID.

\section{(2003) Low and moderate grade adverse events are important contributors to patient-reported treatment side-effect bother}

Nathaniel O'Connell, PhD, Wake Forest School of Medicine, Winston Salem, North Carolina, USA; Fengmin Zhao, PhD, Dana Farber Cancer Institute, Cambridge, Massachusetts, USA; Ju-Whei Lee, Dana Farber Cancer Institute, Cambridge, Massachusetts, USA; Fanxgin Hong, PhD, Dana Farber Cancer Institute, Cambridge, Massachusetts, USA; Shu-En Shen, Northwestern University, Chicago, Illinois, USA; Edward Ip, PhD, Wake Forest School of Medicine, Winston Salem, North Carolina, USA; John Peipert, $\mathrm{PhD}$, Northwestern University, Chicago, Illinois, USA; Noah Graham, Dana-Farber Cancer Institute, Cambridge, Massachusetts, USA; Mary Lou Smith, JD, Research Advocacy Network, Plano, Texas, USA; Llana Gareen, PhD, Brown University, Providence, Rhode Island, USA; Ruth Carlos, MD, University of Michigan, Ann
ArbOR Michigan, USA; Samilia Obeng-Gyasi, MD, The Ohio State University, Columbus, Ohio, USA; Kathy Miller, MD, Indiana University School of Medicine, Indianapolis, Indiana, USA; Ann Partridge, MD, Dana-Farber Cancer Institute, Cambridge, Massachusetts, USA; Tait Shanafelt, MD, Stanford University, Stanford, California, USA; Joseph Sparano, MD, Montefiore Medical Center and Albert Einstein College of Medicine, Bronx, New York, USA; Keith Stewart, MB, Mayo Clinic, Scottsdale, Arizona, USA; Ahmad Tarhini, MD,PhD, Moffit Cancer Center, Tampa, Florida, USA; Mary Thomas, US Department of Veterans Affairs, Washington, District of Columbia, USA; Matthias Weiss, MD, Mayo Clinic, La Crosse, Wisconsin, USA; David Cella, PhD, Northwestern University, Chicago, Illinois, USA; Lynne Wagner, Wake Forest School of Medicine, Winston Salem, North Carolina, USA; Robert Gray, Dana-Farber Cancer Institute, Cambridge, Massachusetts, USA

Aims: In cancer trials, adverse events (AEs) are graded from 1 to 5 in terms of increasing severity, corresponding to mild, moderate, severe, life-threatening, and death, respectively. To simplify data collection, mild and moderate AEs are often not reported in phase III trials. This practice may inhibit our ability to understand patient toxicity burden. In this study, we analyze the relationship between grade of AEs experienced and patient-reported side-effect bother in 3 ECOGACRIN trials. Methods: We analyzed data from 2 phase III trials in myeloma and 1 in leukemia, two of which had limited reporting of mild/moderate AEs, and one with AE data captured across all grades. The FACT-GP5 item, "I am bothered by side effects of treatment" ("Not at all," "A little bit," "Somewhat," "Quite a bit," "Very much") was used to define side-effect bother. At each patient's last GP5 assessment prior to end of treatment, we summarized patients reporting "somewhat" or more side effect bother by percentage of grade $3+$ AEs experienced in their current and preceding treatment cycle. In the trial with complete $\mathrm{AE}$ data, we used logistic regression to model a binary response "side-effect bother" by the count of grade- 1 and grade- 2 AEs in the current and preceding cycles, controlling for baseline GP5 and count of grade-3 and 4 AEs. Results: Among patients who were at least "somewhat" bothered by side effects, only $12 \%$ (7 of 58), 17\% (47 of 271), and 50\% (39 of 78) in each of the 3 trials reported a grade- $3+\mathrm{AE}$ in the current or preceding treatment cycle. In the trial with complete AE data, count of grade-1 AEs (Odds Ratio 1.08; 95\% CI [1.02, 1.13], $p=0.005$ ) and grade-2 AEs (Odds Ratio 1.25; 95\% CI [1.10, 1.41], $\mathrm{p}<0.001$ ) were significantly associated with increased odds of side-effect bother. Conclusion: Few patients with moderate to high side-effect bother had severe AEs documented in corresponding cycles. Furthermore, grade-1-2 AEs were associated with side-effect bother. Collectively, our findings suggest that limiting AE capture to grade 3 or higher misses important contributors to treatment burden, thus, does not adequately represent patients' experiences.

\section{Student Poster Award Finalists}

\section{(2004) Hematological patients' usage and perception of a web- based patient portal for routine care}

Jens Lehmann, MSc, University Hospital of Psychiatry II, Medical University of Innsbruck, Innsbruck, Austria; Petra Buhl, MSc, Oncotyrol-Center for Personalized Cancer Medicine, Innsbruck, Austria; Johannes M Giesinger, PhD, University Hospital of Psychiatry II, Medical University of Innsbruck, Innsbruck, Austria; Lisa M Wintner, Mag., University Hospital of Psychiatry II, Medical University of Innsbruck, Innsbruck, Austria; Monika Sztankay, PhD, University Hospital of Psychiatry II, Medical University of Innsbruck, Innsbruck, Austria; Lucia Neppl, MSc, University Hospital of Psychiatry II, Medical University of Innsbruck, Innsbruck, Austria; 
Wolfgang Willenbacher, PhD, Oncotyrol—Center for Personalized Cancer Medicine, Innsbruck, Austria; Roman Weger, Mag., Oncotyrol-Center for Personalized Cancer Medicine, Innsbruck, Austria; Walpurga Weyrer, MD, Comprehensive Cancer Center Innsbruck, Innsbruck, Austria; Gerhard Rumpold, PhD, University Clinic of Medical Psychology, Medical University of Innsbruck, Innsbruck, Austria; Bernhard Holzner, PhD, University Hospital of Psychiatry II, Medical University of Innsbruck, Innsbruck, Austria

Aims: Patient portals offer the possibility to assess patient-reported outcomes (PROs) remotely and past research has demonstrated their potential benefits. In this study, we evaluated patients' use of a webbased patient portal where patients can complete questionnaires and receive self-management advice based on their results. The portal complements an existing PRO monitoring at our outpatient clinic. Methods: We included patients with multiple myeloma and chronic lymphocytic leukemia who were already participating in the PRO monitoring. Patients were introduced to the portal and asked to complete questionnaires prior to their next hospital visits (Fig. 1 gives an overview of the procedure and the portal's features). We analyzed system access logs gathered during the study period and conducted three semi-structured interviews during each patients' consecutive visits (baseline at inclusion; two following visits) to evaluate their usage and perception of the portal. Results: Between July 2017 and August 2020, we approached $\mathrm{N}=122$ patients for participation in the study, of whom $\mathrm{n}=102(83.6 \%)$ consented to use the patient portal. Patients were on average 60 (SD 10.4) years old. Of $\mathrm{n}=71$ patients providing data at all three study time points, $\mathrm{n}=26(36.7 \%)$ patients consistently used the portal prior to their hospital visits. During an average session, patients viewed 5.3 different pages and spent $9.4 \mathrm{~min}$ on the portal. Feedback from interviews was largely positive with no patients reporting difficulties navigating the survey and $50 \%$ of patients valuing the self-management tools provided in the portal. Regarding the portal content, patients were interested in reviewing their own results and reported high satisfaction with the dynamic selfmanagement advice, which were, respectively, among the most viewed portal pages. Conclusion: In our study, the majority of our patients showed initial interested in using the portal. The few technical complaints and the average time spent in the portal demonstrate the feasibility of our patient portal to be used in patients' everyday lives. Our data give insight into why long-term usage of the portal dropped over time, and we aim to improve several aspects of the portal in a next step (e.g., including a reminder in-between visits and closer PRO symptom monitoring via an onco-nurse).

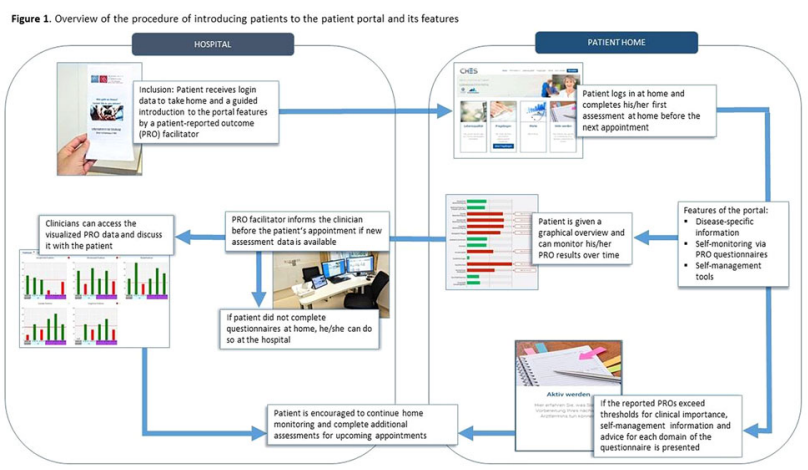

(2005) Mental health and insomnias during the COVID-19 first pandemic wave among Parisian public hospital night shift workers (AP-HP)

Lorraine Cousin, PhDc, Unité de Recherche Clinique en Economie de la Santé (URC-ECO), AP-HP, Hôpital Hôtel-Dieu, F-75004, Paris, France; Vincent Di Beo, Master Degree, Aix Marseille Univ,
INSERM, IRD, SESSTIM, Sciences Economiques \& Sociales de la Santé \& Traitement de l'Information Médicale, Marseille, France; Fabienne Marcellin, Aix Marseille Univ, INSERM, IRD, SESSTIM, Sciences Economiques \& Sociales de la Santé \& Traitement de l'Information Médicale, Marseille, France; Olivia Rousset Torrente, Unité de Recherche Clinique en Economie de la Santé (URC-ECO), AP-HP, Hôpital Hôtel-Dieu, F-75004, Paris, France; Olivier Chassany, professor Unité de Recherche Clinique en Economie de la Santé (URC-ECO), AP-HP, Hôpital Hôtel-Dieu, F-75004, Paris, France; Patrizia Carrieri, Aix Marseille Univ, INSERM, IRD, SESSTIM, Sciences Economiques \& Sociales de la Santé \& Traitement de l'Information Médicale, Marseille, France; Martin Duracinsky, MD, Unité de Recherche Clinique en Economie de la Santé (URC-ECO), AP-HP, Hôpital Hôtel-Dieu, F-75004, Paris, France

Aims: Epidemic and health crises cause significant stress and anxiety in health professionals who manage at risks patients. Within the population of hospital workers, night-shift healthcare workers (HCW) are particularly exposed to health risks because of the alteration of circadian rhythms which may lead to high level of stress, depressive symptoms, and to sleep disturbance. The aims were to describe nightshift HCW's mental health, sleep quality and post-traumatic stress levels and to identify associated factors. Methods: We used data from the online ALADDIN survey (15 June to 15 September 2020) among 1,387 night-shift HCW working in the large Parisian public hospital network (AP-HP). After a margin calibration of data on sex, age, and professional category to ensure their representativeness of the whole population of $12,000 \mathrm{HCW}$ in Paris public hospitals, we performed multiple linear regression models to identify factors affecting mental health and insomnia scores, assessed using the hospital anxiety and depression (HAD) scale, the Insomnia Severity Index, and the Impact of Event Scale-Revised (IES-R). Results: The prevalence of severe anxiety (HAD score $\geq 11$ ), severe depression (HAD score $\geq 11$ ), and post-traumatic stress (IES-R score $\geq 37$ ), was $18.9 \%, 7.6 \%$, and $11.5 \%$, respectively. Only $24.0 \%$ of HCW did not report insomnia. Multivariable analysis showed that being part of the floating pool $(\mathrm{AOR}[\mathrm{CI} 95 \%]=0.18[0.05-0.63])$, being older and having a longer career $(0.94[0.92-0.97])$, practicing sport $(0.52[0.37-0.75])$, and being satisfied of the information on COVID given by hierarchy $(0.48[0.32-0.73])$ were protective factors for the development of mental health issues and insomnia. By contrast, reporting financial difficulties (4.68[2.31-9.47]), having a history of mental health issues (4.19[2.10-8.35]), of harassment at work (2.85[1.87-4.36]), or of COVID infection (2.02[1.10-3.69]), reporting stigmatization and undervalue of night-shift work (2.10[1.16-3.82]), being afraid of transmitting the COVID-19 to one's relatives (2.12[1.31-3.44]), alternating work between days and nights (1.95[1.16-3.28]) were risk factors for mental health and insomnia. Conclusion: Targeted interventions such as financial reward or reduction of night/day work alternation must be considered to improve the mental health and sleep of night-shift hospital workers, which have been severely affected during the COVID-19 first epidemic wave.

(2006) Iterative testing of alternative stopping rules for the NIH toolbox emotion battery

Saki Amagai, Northwestern University, Chicago, Illinois, USA; Rina Fox, Ph.D., M.P.H., Northwestern University, Chicago, Illinois, USA; Aaron Kaat, Ph.D., Northwestern University, Chicago, Illinois, USA; Michael Kallen, Ph.D., M.P.H., Northwestern University, Chicago, Illinois, USA; Benjamin Schalet, Ph.D., Northwestern University, Chicago, Illinois, USA; Cindy Nowinski, M.D., Ph.D., Northwestern University, Chicago, Illinois, USA; Richard Gershon, Ph.D., Northwestern University, Chicago, Illinois, USA 
Aims: The current stopping rules for the NIH Toolbox Emotion Battery (NIHTB-EB) CATs are effective for some test takers, but the assessments can be burdensome for high-functioning individuals. Simultaneously, they do not yield adequate reliability for some clinical populations. We evaluated potential stopping rules for CATs and evaluated which minimized burden while maximizing precision for clinical use. Methods: We conducted simulations to compare four potential CAT stopping rules to the current rules for 13 NIHTB-EB item banks for simulated general and clinical adult samples. The current rules terminate the test if $\geq 4$ items have been administered (minimum), the standard error (SE) of the EAP score estimate is < 0.3 , or 12 items have been administered (maximum). The potential rules included a SE-change rule, six- and eight-item fixed-length CAT rules, and a reduced maximum rule. The $\mathrm{SE}$ threshold for interim stopping was reduced to 0.224 for the SE change and reduced maximum rules. We compared the reliability achieved by each set of rules to the reliability of the current rules, by grouping simulees [Reliability $<0.85,0.85$ Reliability $<0.90,0.90$ Reliability $<0.95,0.95$ Reliability]. We also compared the number of items administered. Results: Although the SE change rule increased the proportion of simulations achieving empirical reliability $>0.95(+22.8 \%$ general, $+29.9 \%$ clinical) compared to the current rules, the average percentage of simulations achieving empirical reliability $<0.85$ also notably increased $(+4.2 \%$ general, $+2.2 \%$ clinical $)$. Similar patterns were observed for the six-item CAT. Relative to the current rules, the eight-item CAT also increased the percentage of simulations achieving reliability $>0.95(+27.5 \%$ general, $+36 \%$ clinical $)$. Importantly, this did not excessively increase the percentage of simulations achieving reliability $<0.85(+1.6 \%$ general, $+0.7 \%$ clinical). Finally, the reduced maximum rule maintained reliability comparable to the eight-item CAT, while decreasing burden by not always requiring eight items. The mean number of items administered was only 1.13 greater than the current rules for general ( 7.53 vs 6.40 ) and 1.83 greater for clinical (7.26 vs 5.43). Conclusion: The reduced maximum rule best balanced burden and precision for clinical use. We recommend our simulation methodology as a way to optimize rules of existing health-related quality of life item banks.

\section{COVID-19 and Quality of Life}

\section{(3001) Health beliefs towards COVID-19 vaccination and intention to receive COVID-19 vaccine in individuals}

Linda Yin King Lee, PhD, The Open University of Hong Kong, Kowloon, Hong Kong; Kit Ying Chu, The Open University of Hong Kong, Kowloon, Hong Kong; Max Hin Wa Chan, The Open University of Hong Kong, Kowloon, Hong Kong; Chloe Tsz Ching Wong, The Open University of Hong Kong, Kowloon, Hong Kong; Heidi Po Ying Leung, The Open University of Hong Kong, Kowloon, Hong Kong; Issac Chun Wing Chan, The Open University of Hong Kong, Kowloon, Hong Kong; Crystal Kit Ying Ng, The Open University of Hong Kong, Kowloon, Hong Kong; Rachel Yuen Shan Wong, The Open University of Hong Kong, Kowloon, Hong Kong; Angel Lok Ching Pun, The Open University of Hong Kong, Kowloon, Hong Kong; Yaki Hoi Ying Ng, The Open University of Hong Kong, Kowloon, Hong Kong; Joe Ka Chun Ng, The Open University of Hong Kong, Kowloon, Hong Kong

Aims: The ongoing COVID-19 pandemic results in a tremendous health and economic impact to countries worldwide. This impact intensifies the need for an effective vaccine to contain the pandemic. Since late 2020, many countries and regions have started their territory-wide COVID-19 vaccination program. However, hesitation or reluctance among the individuals in receiving the vaccine may compromise the overall effectiveness of the vaccine in containing the pandemic. With the understanding that health beliefs are important factors that explain the update of health preventive behaviOR, this study aimed to examine the correlation between health beliefs towards COVID-19 vaccination and intention to receive COVID-19 vaccine in individuals. Methods: Guided by the Health Belief Model, this cross-sectional survey was conducted on a quota sample of 800 adult individuals in Hong Kong in February 2021 before the commencement of the COVID-19 vaccination program. Health beliefs towards COVID-19 vaccination were measured by the Health Belief Model Scale-COVID-19. A higher score indicated a higher degree of agreement. Intention to receive COVID-19 vaccine was measured by one question using a four-point Likert Scale (from definitely unindent to definitely intend). Spearman correlation test was used to examine the correlation between variables. Results: Less than one third $(28.9 \%)$ of the subjects intended to receive the COVID-19 vaccine. Subjects were neutral about their perceived susceptibility to COVID-19 $($ mean $=11.1 / 20.0$ ), perceived benefits of receiving the vaccine $($ mean $=11.9 / 20.0)$, perceived barriers of receiving the vaccine $($ mean $=19.6 / 32.0)$, and cures to action $($ mean $=17.0 / 32.0)$. However, they perceived that COVID-19 was a serious disease $($ mean $=15.3 / 20.0)$. Four domains of health beliefs were significantly correlated with intention to receive COVID-19 vaccine: perceived susceptibility ( $\mathrm{rs}=0.306, \mathrm{p}<0.001$ ); perceived benefits ( $\mathrm{rs}=0.511$, $\mathrm{p}<0.001$ ); perceived barriers ( $\mathrm{rs}=-0.427, \mathrm{p}=<0.001$ ); cues to action $(\mathrm{rs}=0.409, \mathrm{p}<0.001)$. Conclusion: Despite the individuals perceived that COVID-19 was a serious disease, their perceived severity of COVID-19 was not correlated with their intention to receive COVID-19 vaccine. Effective promotion strategies should work on the other domains of health beliefs.

(3002) The newly developed symptoms evolution of COVID-19 (SE-C19) patient-reported outcome instrument: psychometric properties and defining clinical responders

Diana Rofail, PhD, CPsychol, MBA, Regeneron Pharmaceuticals, Inc., Tarrytown, New York, USA; Pip Griffiths, IQVIA, Reading, UK; Giulio Flore, IQVIA, Amsterdam, the The Netherlands;

Mohamed Hussein, Regeneron Pharmaceuticals Inc., Tarrytown, New York, USA; Sumathi Sivapalasingam, Regeneron Pharmaceuticals Inc., Tarrytown, New York, USA; Anna Podolanczuk, Weill Cornell Medical College, New York, New York, USA; Ana María Rodríguez, IQVIA, Reading, UK; Vera Mastey, Regeneron Pharmaceuticals Inc., Tarrytown, New York, USA; Chad Gwaltney, Gwaltney Consulting, Westerly, Rhode Island, USA

Aims: To explore the psychometric properties of the SE-C19 and define responder definition using Regeneron's R10933-10,987-COV2067 study in outpatients with COVID-19. Methods: The SE-C19 is a 23-item scale with four response categories (0- "none" to 3- "severe") and a 24-h recall period. Analyses were conducted on blinded data. For each item, test-retest reliability was examined between consecutive days, known-groups validity was assessed by comparing scores of high- and low-severity patients using the Patient Global Impression of Severity (PGIS), and responsiveness was explored by comparing the change in scores between Baseline, Day 15, and Day 29 in improved and non-improved patients. Rasch analysis was used to understand the severity range of the items and appropriateness of the response scale. A responder definition was developed in line with available FDA guidance and prior precedence in diseases with similar expression. Results: Generally, item test-retest reliability was acceptable (intraclass correlations $[\mathrm{ICC}]>0.50)$ to $\operatorname{good}(>0.75)$. "Rash," "Chest pain," "Confusion," "Vomiting" were used too infrequently for a calculable test-retest reliability value on some pairs of examined days. "Red or watery eyes," "Stomachache," and "Dizziness" had variable reliability, with some pairs of days showing 
reliability estimates above acceptable thresholds, and others not.At baseline, patients with higher PGIS scores reported more severe SEC19 item scores. In most cases, differences between PGIS severity groups were statistically significant. Moderate or severe patients rated an average of 1.37 symptoms on the SE-C19 as "Severe," while only 0.20 were rated as "Severe" among patients with no or mild severity ( $\mathrm{P}<0.0001)$.In most cases, SE-C19 item values decreased over time among patients who reported improvement on the PGIS or the Patient Global Impression of Change with the exceptions of: "Confusion," "Rash," "Red or watery eyes." This, combined with Rasch analysis suggested a responder definition based on 19 items (excluding sneezing, rash, vomiting, and confusion), merging mild/moderate response categories, and allowing for non-severe cough, fatigue, and headache. Conclusion: Results support the reliability and validity of the SE-C19's items as useful for tracking onset, severity, and recovery of symptoms. A symptom resolution responder definition was defined and implemented in the Phase 3 efficacy analysis.

\section{(3003) Using patient-reported outcome measures to assess psychological well-being in the US general population during the COVID-19 pandemic}

Elena Tsangaris, Harvard University, Boston, Massachusetts, USA; Manraj N Kaur, Brigham and Women's Hospital, Boston,

Massachusetts, USA; Tanujit Dey, Brigham and Women's Hospital, Boston, Massachusetts, USA; Shelby Deibert, Department of Pediatrics, Boston, Massachusetts, USA; Janina Kueper, Brigham and Women's Hospital, Boston, Massachusetts, USA; Maria Edelen, Brigham and Women's Hospital, Boston, Massachusetts, USA; Andrea L Pusic, Brigham and Women's Hospital, Boston, Massachusetts, USA

Aims: The impact of the COVID-19 pandemic on psychological wellbeing will likely be long lasting. Efforts directed towards monitoring the onset and progression of distress and mental health disorders are needed to identify and prioritize at-risk populations. This study assesses the psychological well-being of the USA (US) general population during the COVID-19 pandemic using validated patientreported outcome measures (PROMs). Methods: A cross-sectional study design was used. Adults (18 years or older) who could read and write in English were recruited through Prolific in May 2020. Participants completed a REDCap survey including demographic and health-related questions and three PROMs measuring global health (PROMIS-10 Global Health), anxiety (Generalized Anxiety Disorder Scale-7 (GAD-7)), and depression (Patient Health Questionnaire-9 (PHQ-9)). Stepwise multiple linear regression was used to identify factors associated with worse psychological well-being. Results: Mean age of the 2023 participants was $31.92 \pm 11.57$ years (range, $18-82)$. Participants were mainly White $(64.7 \%, \mathrm{n}=1309)$, female $(52.2 \%, \mathrm{n}=1057)$, working full-time before the pandemic $(43.5 \%$, $\mathrm{n}=879)$, and completed a college, trade, or university degree $(40.7 \%$, $\mathrm{n}=823$ ). Most participants reported mild to severe anxiety $(57.3 \%$, $\mathrm{n}=1158)$ and depression $(60 \%, \mathrm{n}=1276)$ on the GAD-7 and PHQ-9, respectively. Worse psychological well-being was associated with participants aged 18-49 years, Asian, who identified as ciswomen or gender diverse, had higher BMI $(\geq 25)$, and lost their job or were unable to work. Conclusion: PROMs can be used to assess and monitor psychological well-being during the COVID-19 pandemic and to inform planning and delivery of targeted public health interventions to support at-risk populations.

(3004) Development and content validation of a COVID-19 patient-reported symptom questionnaire (SE-C19)
Diana Rofail, Regeneron Pharmaceuticals, Inc., Tarrytown, New York, USA; Nadine McGale, Modus Outcomes, Letchworth Garden City, UK; Joseph Im, Regeneron Pharmaceuticals, Inc., Tarrytown, New York, USA; Alissa Rams, Modus Outcomes, Letchworth Garden City, UK; Krystian Przydzial, Modus Outcomes, Letchworth Garden City, UK; Vera Mastey, Regeneron Pharmaceuticals Inc., Tarrytown, New York, USA; Sumathi Sivapalasingam, Regeneron Pharmaceuticals Inc., Tarrytown, New York, USA; Anna Podolanczuk, Weill Cornell Medical College, New York, New York, USA

Aims: At the onset of the COVID-19 pandemic, there was limited understanding of symptom experience and disease progression. We developed and validated a fit-for-purpose disease-specific instrument to assess symptoms in patients with COVID-19 to inform endpoints in an interventional trial for non-hospitalized patients (Study: R1093310,987-COV-2067). Methods: The initial drafting of the 23-item Symptoms Evolution of COVID-19 (SE-C19) Instrument was developed based on the Centers for Disease Control and Prevention (CDC) symptom list (CDC, 2020) and available published literature specific to patients with COVID-19 (Michelen, 2020) as of Spring 2020. The measurement principles outlined in the Food and Drug Administration (FDA) patient-reported outcomes (PRO) Guidance (FDA, 2009) and the four methodological Patient-Focused Drug Development Guidances (FDA, 2020) were also considered. Following initial development, semi-structured qualitative interviews were conducted with a purposive sample of 30 non-hospitalized COVID-19 patients. Interviews involved two stages: (1) concept elicitation, to obtain information about the symptoms experienced as a result of COVID-19 in patients' own words, and (2) cognitive debriefing, for patients to describe their understanding of the SE-C19 instructions, specific symptoms, response options, and recall period to ensure the content of the SE-C19 is relevant and comprehensive. Five clinicians treating COVID-19 outpatients were also interviewed to obtain their insights on symptoms experienced by patients and provide input on the SE-C19. Results: Patients reported no issues regarding the relevance or appropriateness of the SE-C19 instructions, including the 24-h recall period. The comprehensiveness of the SEC19 was confirmed against the conceptual model developed in the qualitative research. Minor conceptual gaps were revealed to capture nuances in the experience of nasal and gustatory symptoms, systemic manifestations of sickness, and other symptoms. Almost all items were endorsed by patients as being appropriate, well understood, and easy to respond to. The clinicians largely approved all items, response options, and recall period. Conclusion: The qualitative research provided supportive evidence of the content validity of the SE-C19 to assess the symptoms of outpatients with COVID-19, and its use in clinical trials to evaluate the benefit of treatment. Minor changes may be considered to improve conceptual clarity and ease of responding.

\section{(3005) Psychometric validation of the FLU-PRO Plus among patients with COVID-19: measurement properties, challenges, and opportunities}

Tom Keeley, PhD, GSK, Brentford, UK; Heather L Gelhorn, Evidera, Bethesda, Maryland, USA; Haylee Andrews, Evidera, Bethesda, Maryland, USA; Wen-Hung Chen, GSK, Collegeville, Pennsylvania, USA; Helen Birch, GSK, Brentford, UK; Sacha Satram, Vir Biotechnology, San Francisco, California, USA; Carolina Reyes, Vir Biotechnology, San Francisco, California, USA; Amanda Lopuski, GSK, Collegeville, Pennsylvania, USA

Aims: The inFLUenza-Patient-Reported Outcome (FLU-PRO) measure was developed in 2015-2016 to assess the core symptoms of respiratory viral diseases. Several clinical trials evaluating treatments for COVID-19-rapidly initiated in 2020 to meet the needs of 
patients-have included a COVID-19 adapted version of the FLUPRO as a measure of symptoms. The FLU-PRO Plus contains the original 32 FLU-PRO items, with two additional items on the loss of smell and taste (yes/no) included. The purpose of this study was to evaluate the measurement properties, responsiveness, and responder definition of the FLU-PRO Plus for use in outpatients with COVID19. Methods: FLU-PRO Plus data from a randomized, double-blind, multi-center, placebo-controlled trial of a treatment for COVID-19 were used for the evaluation. Participants completed the FLU-PRO Plus daily during the first three weeks following enrollment into the trial and then intermittently thereafter. Participants also completed the SF-12 measure and the Work Productivity and Activity Impairment questionnaire productivity measure at key timepoints. Clinical outcomes included oxygen saturation, hospitalization, and supplemental oxygen use. The factor structure of the FLU-PRO Plus was tested using confirmatory factor analyses. Internal consistency and testretest reliability were assessed. Construct validity was evaluated through correlations with other trial measures. Known-groups validity was assessed through ANOVA with oxygen saturation, supplemental oxygen use, hospitalization, and highest setting of care. Responsiveness was assessed using hospitalization, productivity, and supplemental oxygen use. Finally, a prespecified responder definition was evaluated. Results: Specific psychometric results and reflections from working in this novel disease area will be shared. Challenges in developing the analysis plan included limited availability of PRO measures to use as criterion variables, a customized response scale for the added items, pre-defining responders based on input from regulators, and rapid timelines. Conclusion: For critical global health crises, the rapid selection, modification, and validation of appropriate PROs for use in evaluating novel treatments is challenging but feasible. The results of this study will inform whether the FLU-PRO Plus is fit for use as a measure of COVID-19 symptoms. Funding: GSK (Study 215,031).

\section{(3006) Tobacco use and alcohol consumption among night-shift hospital healthcare workers during the first wave of the COVID- 19 pandemic in Paris hospitals (AP-HP ALADDIN survey)}

Lorraine Cousin, PhDc, Unité de Recherche Clinique en Economie de la Santé (URC-ECO), AP-HP, Hôpital Hôtel-Dieu, F-75004, Paris, France; Vincent Di Beo, Aix Marseille Univ, INSERM, IRD, SESSTIM, Sciences Economiques \& Sociales de la Santé \& Traitement de l'Information Médicale, Marseille, France; Fabienne Marcellin, Aix Marseille Univ, INSERM, IRD, SESSTIM, Sciences Economiques \& Sociales de la Santé \& Traitement de l'Information Médicale, Marseille, France; Olivia Rousset Torrente, Unité de Recherche Clinique en Economie de la Santé (URC-ECO), AP-HP, Hôpital Hôtel-Dieu, Paris, France; Olivier Chassany, professor Unité de Recherche Clinique en Economie de la Santé (URC-ECO), AP-HP, Hôpital Hôtel-Dieu, Paris, France; Patrizia Carrieri, Aix Marseille Univ, INSERM, IRD, SESSTIM, Sciences Economiques \& Sociales de la Santé \& Traitement de l'Information Médicale, Marseille, France; Martin Duracinsky, MD, Unité de Recherche Clinique en Economie de la Santé (URC-ECO), AP-HP, Hôpital Hôtel-Dieu, Paris, France

Aims: During the COVID-19 pandemic, night-shift hospital healthcare workers $(\mathrm{HCW})$ were particularly solicited to take care of a large number of patients. Addictive behaviors can develop during these difficult times for front-line caregivers. Among HCW, night-shift workers are particularly vulnerable since they are exposed to many risks factors related to the alteration of the circadian rhythms.The aim of this study was to describe the prevalence of tobacco use and alcohol consumption among night-shift HCW during the first wave of the COVID-19 pandemic in a large Paris public hospital network
(Assistance Publique-Hôpitaux de Paris: AP-HP). Methods: The AP-HP ALADDIN cross-sectional online survey (15 June to 15 September 2020) included 1387 night-shift HCW of the AP-HP hospitals, and collected data concerning their current behaviors and their potential changes since the beginning of the COVID-19 pandemic. Psychoactive substances consumption was assessed using a series of items documenting level, frequency, and changes in consumption as well as the Fagerström and AUDIT-c scales to identify dependence to tobacco and alcohol, respectively. Prevalence of use was estimated after weighting survey data (margin calibration) to ensure their representativeness of the whole population of 12,000 HCW in Parisian public hospitals regarding sex, age, and professional category. Results: The weighted estimated prevalence of daily smoking and daily alcohol drinking was $21.4 \%$ and $1.3 \%$, respectively. The prevalence of daily smokers was consistent with the one found in general population (daily smokers prevalence in general population $=24 \%$ ) whereas prevalence of daily drinkers was lower in our sample (daily drinkers prevalence in general population $=7.1 \%$ ). Among smokers, $66.0 \%$ were dependent to nicotine. None of the alcohol drinkers was dependent to alcohol but $42.5 \%$ presented hazardous alcohol use. During the COVID-19 pandemic, tobacco use increased for $26.7 \%$ of smokers and alcohol consumption increased for $8.6 \%$ of drinkers. Conclusion: In this study, prevalence of daily tobacco use was similar as in the general population, while daily alcohol consumption was lower. These results also show an increase in tobacco use and alcohol consumption since the beginning of the COVID-19 pandemic for a non-negligible proportion of night-shift $\mathrm{HCW}$ that must be monitored to protect their health.

\section{(3007) Supportive care in pancreatic and oesophagogastric cancers: a qualitative investigation}

Nadia Khan, BBiomedSc(Hons), Monash University, Melbourne, Australia; Ashika Maharaj, Monash University, Melbourne, Australia; Sue Evans, Monash University, Melbourne, Australia; Charles Pilgrim, Alfred Health, Melbourne, Australia; John Zalcberg, Monash University, Melbourne, Australia; Wendy Brown, Alfred Health, Melbourne, Australia; Paul Cashin, Monash Health, Melbourne, Australia; Daniel Croagh, Monash Health, Melbourne, Australia; Natasha Michael, Cabrini Health, Melbourne, Australia; Jeremy Shapiro, Cabrini Health, Melbourne, Australia; Liane Ioannou, Monash University, Melbourne, Australia

Aims: Pancreatic and oesophagogastric (OG) cancers have a dismal prognosis and high symptom burden, with supportive care forming an integral component of cancer management. This study aimed to explore the supportive care experiences of people living with pancreatic and OG cancers and identify opportunities for improvement. Methods: Semi-structured interviews were conducted with people living with pancreatic and OG cancers, and their caregivers, across Victoria, Australia during 2020. Results: Thirty patients and 11 caregivers were interviewed. Themes included: (i) supportive care needs across the cancer journey, (ii) management of supportive care needs, (iii) quality of supportive care, (iv) impact of COVID-19, (v) role of the caregiver, and (vi) opportunities for improvement. Predominant supportive care needs included diet-related issues, weight loss, pain, and difficulties with activities of daily living, with support provided to varying levels of satisfaction. Many patients did not access supportive care services due to COVID-19. Caregivers played a critical role in supporting patients and desired support for themselves. Greater awareness of and access to available services, availability of a cancer care coordinator and higher quality support were perceived as opportunities for improvement. Conclusion: Unmet needs are prevalent across the pancreatic and OG cancer journey, with supportive care provided to varying levels of satisfaction. 
Policymakers and health services should promote and enable access to high-quality multidisciplinary support services for patients and their caregivers.

\section{(3008) COVID-19-related Clinical Outcome Assessments (COAs)—A review}

Celine Desvignes-Gleizes, Mapi Research Trust, Lyon, France; Florian Aubert, Mapi Research Trust, Lyon, France; Laure-Lou Perrier, Mapi Research Trust, Lyon, France; Sonia Bothorel, Mapi Research Trust, Lyon, France

Aims: Following the COVID-19 pandemic, industry may encounter challenges in identifying methods to assess the numerous and heterogeneous-related symptoms but also the impact of COVID-19 on the world population when designing trials. Since the beginning of the outbreak, numerous COVID-19-related COA were developed and identified in this review. Methods: A literature review was performed in PubMed, Embase, PsychInfo and in grey literature in March 2021 and all identified COAs were described in the unique COA database, PROQOLID ${ }^{\mathrm{TM}}$. Results: 95 COAs were identified and described in PROQOLID, including 68 Patient-Reported Outcomes (PROs) (e.g. COVID-19 Anxiety Syndrome Scale), 12 Clinician-Reported Outcome (ClinRO) (e.g. Simon Fraser University COVID-19 impact scale) and composite measures (ClinRO with biomarker, e.g. BresciaCovid Respiratory Severity Scale). Concepts measured are mainly psychological functioning $(n=39)$, symptoms $(n=21)$ and social functioning $(n=6)$. One PRO assesses quality of life (QOL) (COVID-19-Impact on QOL scale). PROs were mainly developed in adults, except for in adults and adolescents (Fear of COVID-19 Scale and FDA COVID-19-Related Symptoms Assessment). One ObserverReported Outcome has been developed for a paediatric population (CoRonavIruS health and Impact Survey-Parent/Caregiver Version for Children and Adolescents-Baseline \& Follow-up versions). The FDA also released a guideline in September 2020 to assist sponsors, which provides a set of 14 common COVID-19-related symptoms to assess in clinical trials (including sore throat, shortness of breath and cough). Conclusion: The use of COAs targeting all aspects of COVID-19's impact supports the continued efforts of industry in the development of effective treatments for COVID-19.

\section{(3009) Alone together: Socializing behaviours, psychosocial health, and wellbeing following the COVID-19 lockdown in six European countries.}

Laura Altweck, PhD, Greifswald University, Greifswald, Germany; Samuel Tomczyk, Dr, Greifswald University, Greifswald, Germany

Aims: Background. How we socialise and interact with others varies greatly across European countries, for instance, Southern European countries are considered more family oriented whereas Northern European countries are perceived as rather distant. Regulations to contain the spread of COVID-19 limited the public's social interactions, which may have affected European populations differently. Therefore, this study examines the relationship between psychosocial health and social behaviour after the lockdown period in six European countries of varying degrees of collectivism. Methods: Methods. The special COVID19 survey of the Survey of Health, Ageing and Retirement in Europe panel data was employed to examine differences in face-to-face and virtual contact respectively with four contact groups (children, parents, other relatives, others: friends/neighbours/colleagues) in six European countries (low collectivism $=$ Sweden $\&$ The The Netherlands, moderate $=$ Germany \& Spain, high $=$ Greece \& Portugal; $\mathrm{N}=11,622$; age: $\mathrm{M}=70.6, \mathrm{SD}=9.1 ; 56.3 \%$ female). Regression analyses were used to examine associations between sociodemographic background, psychosocial health (self-rated health before the lockdown, its improvement or deterioration, nervousness, depression, and loneliness), and social contact. Results: A trend was seen where more collectivist countries reported greater contact with relatives, but the reverse was seen with others. Greater loneliness was related to fewer social contacts in almost all contact groups and mediums. Similarly, greater nervousness was associated with less face-to-face social contact with other relatives and others, but with more virtual contact with children. Worse self-rated health before the COVID-19 outbreak was related to less contact to others, while change in self-rated health was not significantly related to social contact. Conclusion: Collectivism and psychosocial health indicators were differentially related to social contact: nervousness was linked to less face-to-face contact and collectivism with more frequent social contact with relatives. Most notably-even after lockdown regulations were lifted-loneliness was most strongly related to face-to-face and virtual social contact (and isolation). Loneliness in older people has been linked to a variety of conditions like heart disease, cognitive decline, and even death. In the coming years, it is likely that adverse long-term health consequences are yet to manifest.

\section{(3010) The Impact of COVID-19 on the Wellbeing of People with Food Allergy}

Gang Chen, PhD, Monash University, Melbourne, Australia; Audrey Dunngalvin, PhD, University College Cork, Cork, Ireland; Dianne E. Campbell, PhD, The Children's Hospital at Westmead, Sydney, Australia

Aims: The COVID-19 pandemic has substantial impacted people's daily lives. The exogenous shock and social isolation may have imposed additional stressors for people with food allergy. This study aimed to empirically explore to what extent have COVID-19 pandemic impacted the quality of life and life satisfaction of people with food allergy. Methods: An online cross-sectional survey was conducted in Australia with targeting respondents consisted of parents/caregivers of children (1 to 17 years) and adults with a selfreported clinician diagnosis of IgE-mediated food allergy. Respondents proxy assessed for their children or self-reported their own quality of life and life satisfaction (including both global and life domain satisfaction), how their lives been impacted by the COVID-19 pandemic. The proxy version of the Assessment of Quality of Life (AQoL)-6D and the Child Health Utility 9D (CHU9D) were used in this study for children and adolescents, whilst the AQoL-8D was used for adults. Furthermore, the Food Allergy Quality of Life Questionnaire, Parent Form 10 (FAQLQ-PF10) and the FAQLQ, Adult Form 10 (FAQLQ-AF10) were also included. Regression analyses were conducted to investigate the associations between perceived COVID19 impacts and wellbeing, controlling for a rich set of personal characteristics. Results: A total of 247 respondents participated in the parent-proxy survey for food-allergic children (mean age, 8 years) and 75 adult respondents self-reported (mean age, 42 years). By regression analyses, those respondents who perceived their own/their children's lives had been negatively influenced by COVID-19 had mean decrements of health state utility scores between -0.09 to 0.15 . Global life satisfaction the mean decrements were around 0.96 to -0.98 (on a $0-10$ scale). Associations between negative COVID-19 impacts and food allergy specific quality of life were significant for children but not for adults. Among life domains, the impacts associated with the reduced global life satisfaction were greatest with perceived negative impacts on personal relationships and future security among children/adolescents and on standard of living among adults. Conclusion: The perceived negative impact from the COVID-19 pandemic was significantly associated with poorer 
generic health-related quality of life and life satisfaction, whilst the effects on food allergy specific quality of life observed in children, but not adults.

\section{(3011) Is HIV a Burden or Resource in Covid19 Care Paths for People Living with HIV (PLWHIV) during the first wave in France?}

Guillaume Roucoux, PhD candidate, PROQOL Team - Inserm UMR 1123, Paris, France; Frédérique Thonon, PhD, PROQOL Team Inserm UMR 1123, Paris, France; David Zucman, MD, Foch Hospital, Suresnes, France; David Rey, MD PhD, Trait d'Union University Hospitals of Strasbourg, Strasbourg, France; Diane Bollens, MD PhD, Saint Antoine Hospital, Paris, France; Fatoumata Waggeh, Kremlin Bicêtre Hospital, Kremlin Bicêtre, France; Katia Bourdic, Kremlin Bicêtre Hospital, Kremlin Bicêtre, France; Antoine Chéret, MD PhD, Kremlin Bicêtre Hospital, Kremlin Bicêtre, France; Martin Duracinsky, MD PhD, PROQOL Team - Inserm UMR 1123; Kremlin Bicêtre Hospital, Paris, France

Aims: In 2020, the French government developed access to Covid19 screening, guidelines for treatment and prevention, while its infected population was growing. By focusing on PLWHIV's care paths, we aim to highlight the influence of living with HIV in light of Covid19. Methods: Qualitative study with semi-structured interviews on the perception of vulnerability, symptoms, diagnosis, screening, and treatment for Covid19. Eight hospitals recruited participants between June and September 2020. Individual interviews were conducted over the telephone $(n=28)$ or face-to-face $(n=6)$. Thematic coding was achieved with Sonal software. Results: The study involved 34 PLWHIV, 20 were COVID-19 positive, 7 negative, and 7 non-tested: 24 men (18 gay or bisexual men) and 10 women, mostly born in France $(\mathrm{n}=24)$ and employed $(\mathrm{n}=18)$; median age: 55 . All participants were diagnosed with HIV between 1983 and 2019. Most of the participants understood that being HIV positive was not a risk factor for COVID-19 severe complications. Having "strong immune defenses," "thanks to the protective treatment," increased perception of non-vulnerability. Only one patient felt more vulnerable to COVID-19 due to his HIV status. With one other patient, they called themselves "viruses' collectors." No symptoms were specific to PLWHIV. The fever reminded 3 patients of their HIV seroconversion. Only 4 participants were diagnosed for COVID-19 by their infectious disease specialist. Due to comorbidities, 2 patients received incorrect diagnoses for bronchitis and thyroid disorder, respectively. One patient coped better with HIV than COVID-19 diagnosis. Several became resilient against COVID-19 thanks to HIV: "I have survived a deadly disease; I'm not going to let myself be bothered by a deadly little virus now." Being monitored for HIV made (free) screening access easier. One participant stressed general reluctancy to being screened for both viruses. Among testing barriers: fears that the testing inoculates COVID-19 or reveals HIV, and non-concomitant appointment for HIV and COVID-19. Being COVID-19 positive reminded one participant of his "HIV story." One patient said her ART "helped" her to recover from COVID-19. Conclusion: Living with HIV improved COVID-19 care paths including non-vulnerability, resilience, and more accessible screening. Rare negative side effects included frustration regarding postponed HIV appointments, bad memories, and fears regarding blood sampling.

(3190) Health-related quality of life, health literacy, and COVID19-related worries of 16-17-year-old adolescents and parents, one year into the pandemic
Hilde T. Mikkelsen, Department of Health and Nursing, Faculty of Health and Sport Sciences, University of Agder, Kristiansand, Norway; Siv Skarstein, Department of Nursing and Health Promotion, Faculty of Health Sciences, Oslo Metropolitan University, Oslo, Norway; Sølvi Helseth, Department of Nursing and Health Promotion, Faculty of Health Sciences, Oslo Metropolitan University, Oslo, Norway; Milada Cvancarova Småstuen, Department of Nursing and Health Promotion, Faculty of Health Sciences, Oslo Metropolitan University, Oslo, Norway; Kristin Haraldstad, Department of Health and Nursing, Faculty of Health and Sport Sciences, University of Agder, Kristiansand, Norway; Gudrun Rohde, Department of Health and Nursing, Faculty of Health and Sport Sciences, University of Agder, Kristiansand, Norway

Aims: To describe health-related quality of life (HRQoL) of 16-17year-old adolescents and parents of adolescents, their health literacy (HL) and COVID-19-related worries, about one year into the pandemic. Further, to assess the strength of associations between gender, HL, COVID-19-related worries, and HRQoL. Methods: A crosssectional study was conducted in 215 adolescents and 320 parents. We analyzed HRQoL, HL, COVID-19-related worries, and sociodemographic variables. KIDSCREEN-10 and RAND-36 were used to measure HRQoL. Data were analyzed using bivariate methods, multiple linear regression, and robust regression. Results: The adolescents' HRQOL scores were notably lower compared to scores from previous Norwegian studies and European norms. Parents' HRQoL scores were comparable to Norwegian normative data. Adolescents and parents reported moderate to high levels of $\mathrm{HL}$ and a high degree of COVID-19-related worries. In both groups, females reported significantly lower scores on HRQoL and more worries compared to males. The majority of adolescents and parents reported a negative change in life due to the pandemic. In adolescents, higher HL was significantly associated with higher levels of HRQoL. COVID-19related worries were not associated with HRQoL. In parents, higher $\mathrm{HL}$ in the domain "understand health information" was significantly associated with higher levels of HRQoL for the mental component sum score (MCS) and with lower levels of HRQoL for the physical component sum score (PCS). Being worried a lot about infecting others, was significantly associated with higher MCS compared to being a little worried. Being worried a lot about family/friends becoming sick, was significantly associated with lower MCS compared to being a little worried. COVID-19-related worries were not significantly associated with PCS. Conclusion: Our results indicate that the pandemic has a major negative impact on adolescents' HRQoL. Parents' HRQoL remained unchanged and comparable to previous studies. Our study demonstrate that HL, gender and COVID19-related worries are significantly associated to adolescents' and parents' HRQoL, indicating that efforts aimed to increase their HL might indirectly affect their HRQOL as well, and that gender specific interventions or strategies could be beneficial. We highlight the need for strategies to enhance adolescents' HRQoL and mental health during and after the pandemic.

\section{(3199) Ethnic differences in post-traumatic resiliency among COVID-19 survivors}

Alexandra Howell, MD/MPH Candidate, UT Health San Antonio Long School of Medicine, San Antonio, Texas, USA; Chen-Pin Wang, Ph.D, UT Health San Antonio, Population Health Sciences, San Antonio, Texas, USA; Kathleen Case, Ph.D, UT Health San Antonio Center for Research to Advance Community Health (ReACH), San Antonio, Texas, USA; Sarah Lill, UT Health San Antonio Center for Research to Advance Community Health (ReACH), San Antonio, Texas, USA; Daniel MacCarthy, UT Health San Antonio, Population Health Sciences, San Antonio, Texas, USA; 
Joel Tsevat, MD, MPH, UT Health San Antonio Center for Research to Advance Community Health ( $\mathrm{Re} A C H)$, San Antonio, Texas, USA

Aims: As the COVID-19 pandemic evolves, there is a need to understand the lasting impacts of the disease, both salutary and negative. To date, no study has investigated the ethnic differences in salutary outcomes of COVID-19 infection. In this study, we investigated how post-traumatic resiliency (PTR) differs between Latinx and non-Latinx survivors of COVID-19. Furthermore, we sought to examine whether health status-in particular mental health-and social determinants of health $(\mathrm{SDH})$ moderate ethnic differences in post-traumatic resiliency following COVID-19 infection. Methods: From December 2020 to May 2021, we surveyed (in English and Spanish) survivors of COVID-19 infected between March-September $2020(\mathrm{n}=343)$. Key measures included 1) the PROMIS- $29+2$ health profile (health status) and 2) the CAIR Pandemic Impact Questionnaire (C-PIQ). Responses to 4 C-PIQ items (hospitalization, lost job/income, difficulty getting food/medications/medical help/ other necessities, negative impact on relationships with friends or family) served as measures for SDH impacts of COVID-19. Responses to 5 C-PIQ items were summed into a total growth score (range 0-20), which served as our outcome measure for PTR. Bivariate analyses included chi-square tests, Wilcoxon rank-sum tests, and t-tests; generalized linear models with suitable distributions were conducted for multivariable analyses. Results: Overall, survivors of COVID-19 $(\mathrm{n}=261$; [mean $\{\mathrm{SD}\}$ age $42\{15\}$ years, $66 \%$ female, $83 \%$ Latinx) had compromised health status on all domains and a mean [SD] CAIR growth score $=7.5$ [4.8]. Bivariate analyses showed significantly higher CAIR growth scores among Latinx compared with non-Latinx (7.8[4.7] vs. 5.3[4.6], $\mathrm{p}=0.001)$. Health status and C-PIQ items addressing SDH were not associated with a difference in CAIR growth scores. In multivariable analyses, Latinx respondents had significantly higher PTR scores $(2.25[\mathrm{SE}=0.75] \mathrm{p}=0.003)$ after adjusting for age, sex, depression, anxiety, and SDH. Ethnic difference in PTR was modestly moderated by health status (ethnic difference in CAIR growth score increased by $0.52[\mathrm{SE}=0.28]$ per 0.1 unit increase in PROMIS-29 +2 utility, $p=0.06$ ). Conclusion: Latinx COVID-19 survivors reported greater post-traumatic resiliency than non-Latinx. Ethnic differences in PTR may be impacted by health status.

\section{(3200) Knowledge, Attitude, and Adherence to Standard Precautions Among Front-Line Clinical Physiotherapists During the COVID-19 Pandemic: A Cross-Sectional Survey}

Charles I. Ezema, PT, PhD, Department of Medical Rehabilitation, Faculty of Health Sciences and Technology, College of Medicine, University of Nigeria, Nsukka, Enugu, Alberta, Nigeria; Ogochukwu K. Onyeso, MSc, PT, Faculty of Health Sciences, University of Lethbridge, Lethbridge, Alberta, Canada; Udoka A. OkafOR PT, $\mathrm{PhD}$, Department of Physiotherapy, Faculty of Clinical Sciences, College of Medicine, University of Lagos, Lagos, Nigeria; Lance M. Mabry, DPT, Department of Physical Therapy, High Point University, High Point, North Carolina, USA; Mishael E. Adje, MSc, PT, Department of Therapeutic Sciences, Trier University of Applied Sciences, Trier, Germany; Jessica Shiraku, MSc, PT, Department of Physical Medicine and Rehabilitation, The Nairobi Hospital, Nairobi, Kenya; Jean-Michel Brismée, PT, Sc.D, Department of Rehabilitation Sciences, Center for Rehabilitation Research, Texas Tech University Health Sciences Center, Lubbock, Texas, USA; Frédéric Froment, PT, Sc.D, Department of Rehabilitation Sciences, Center for Rehabilitation Research, Texas Tech University Health Sciences Center, Lubbock, Texas, USA; Oluwagbohunmi Awosoga, $\mathrm{PhD}$, MBA, Faculty of Health Sciences, University of Lethbridge, Lethbridge, Alberta, Canada
Aims: Physiotherapists are at high risk of contracting the severe acute respiratory syndrome coronavirus 2 (SARS-CoV-2) because their job often involves direct patient contact. The aim of this study was to investigate the knowledge of pathology and prevention of the novel coronavirus (COVID-19), attitude towards clinical practice, and adherence to standard precautions among clinical physiotherapists. This survey was administered at the critical time of the pandemic to help the participants examine the behavioral and environmental risks associated with their duty as frontline $\mathrm{HCW}$, and to explore alternative non-contact clinical practices such as telehealth physiotherapy during the COVID-19 pandemic. Methods: The cross-sectional survey utilized snowballing method to obtain study participants. A sevensection questionnaire was developed and validated to obtain information about the demographics, knowledge of COVID-19 pandemic, attitude, standard precautions, and nature of physiotherapy practices. Data were analyzed using descriptive statistics, Cronbach's Alpha, Pearson's correlation, and ANOVA. Results: Surveys were collected from 53 countries resulting in 2550 valid responses. All respondents $(\mathrm{n}=2550,100 \%)$ were aware of and practiced during the COVID-19 pandemic. However, only $(\mathrm{n}=312,12.2 \%)$ were actively involved in the management of confirmed cases. The percentage score of participants' knowledge regarding COVID-19 pathology, prevention, attitude, and safe practices averaged $77.73 \pm 10.11,89.70 \pm 9.26$, $77.44 \pm 7.04$, and $61.59 \pm 16.63$, respectively. Knowledge about COVID-19 pathology differed across demographic variables (each time $\mathrm{p}<0.001, \eta 2 \leq 0.07)$. Most participants $(\mathrm{n}=1936,75.9 \%)$ reported an inadequate supply of personal protective equipment (PPE). Conclusion: Respondents had good knowledge of COVID-19 pathology and a positive attitude towards safe clinical practice. Governmental support of physiotherapy telehealth policies may reduce the risk of COVID-19 transmission while facilitating efficacious use of limited PPE resources. Governments should pursue legislation to support telehealth in physiotherapy by technological support, telehealth reimbursement policies, and curriculum adjustment.Keywords: Infectious Disease Transmission, Personal Protective Equipment, Physiotherapy, Telehealth, Universal Precautions.

\section{(3219) Addressing quality of life needs in an underserved, urban community during COVID-19}

Michelle D'Abundo, PhD, MSH, CHES, Seton Hall University, Belmar, New Jersey, United States; Paschal Nwako, PhD, Camden County Health Department, Camden, New Jersey, United States; Robert Weaver, PhD, Rowan University, Glassboro, New Jersey, United States; Nicole Vaughn, PhD, Rowan University, Glassboro, New Jersey, United States; Paul Franco, PhD, Seton Hall University, Nutley, New Jersey, United States

Aims: While COVID-19 affected the lives of all people, negative impacts to quality of life were often more severe for residents living in underserved urban communities. The CDC (2020) identified the five areas of social determinants contributing to racial and ethnic minority groups being disproportionately affected by COVID-19 including neighborhood and physical environment, health and health care, occupation and job conditions, income and wealth, education. One community affected was Camden City, NJ where $36.4 \%$ of residents live in poverty and $12.7 \%$ are uninsured. This diverse community is primarily composed of Hispanic or Latino (51\%), and Black (41.4\%) residents (US Census Bureau, 2019). The digital divide is evident in Camden City with $63.9 \%$ (US Census Bureau, 2019) of households having a broadband internet subscription compared to the National Average of $82.7 \%$ (ACS, 2016). In November 2020, Camden City experienced a surge of COVID-19 cases, which was higher than other parts of the county. Methods: To address the 
spike in COVID-19 cases, Camden County Health Department worked with stakeholders to connect residents to resources offered by public, private, and charitable organizations. To address the digital divide and technology issues, a blended contact approach was used including door-to-door outreach, phone calls, and text messaging. To address concerns related to trust, community members were hired and trained to contact residents. Results: In total, 4,052 residents responded to a five-item survey including the following question "Do you need help with food, economic, health, shelter, COVID-19 or other resources?" When residents identified an issue, they were immediately connected to available resources. Residents were also asked, "Would you be interested in sharing your thoughts about the COVID-19 vaccine that is coming soon?" The responses were used to understand residents' perspectives and to plan messaging about vaccination initiatives. Conclusion: After the project concluded, researchers collaborated with the Camden County Health Department to assist with understanding the implications for future disaster preparedness. While community needs assessments are common, this initiative was unique by including community stakeholders, multiple points of contact, and providing immediate connection to resources to support and promote quality of life through addressing the needs of Camden City residents during COVID-19.

\section{Poster Presentations}

\section{(3012) Patient interpretations vary for PROMIS (Patient- Reported Outcomes Measurement Information System) upper extremity questions}

Chao Long, MD MPH, The Johns Hopkins Hospital and The Curtis National Hand Center, Baltimore, Maryland, USA; Laura Beres, PhD, MPH, Johns Hopkins Bloomberg School of Public Health, Baltimore, Maryland, USA; Albert Wu, MD, MPH, Johns Hopkins Bloomberg School of Public Health, Baltimore, Maryland, USA; Aviram Giladi, MD, MS, The Curtis National Hand Center, MedStar Union Memorial Hospital, Baltimore, Maryland, USA

Aims: The Patient-Reported Outcomes Measurement Information System Upper Extremity (PROMIS-UE) Short Form (SF) 7a includes seven items from the PROMIS-UE Item Bank v2.0, which includes items selected from the Physical Functioning Item Bank via consensus review and quantitative evaluation. We conducted a qualitative evaluation of the PROMIS-UE SF in patients with hand or upper extremity conditions. We sought to detect potential variability or challenges in comprehension, decision processes, response processes, and recall. Methods: We conducted cognitive interviews with adult, English-speaking patients purposively sampled for mixed literacy from a hand and upper extremity clinic. Interviews were conducted in-person or by phone (based on patient preference in the COVID-19 era), audio recorded, and documented in analytic memos. Interviews used a combination of "think aloud" and verbal probing techniques for each question (numbered PFA14r1, PFA34, PFA36, PFB13, PFB28r1, PFB34, PFM16). We utilized framework analysis to identify themes related to our goals. To ensure the credibility of our findings, we collected additional interviews that included memberchecking of initial findings. Results: Twelve patients 20-81 years old were interviewed. For PFA14r1 and PFB28r1 on lifting/carrying, patients were unsure how much " 10 pounds $/ 5 \mathrm{~kg}$ " weighed and tried to imagine household items of that weight. For PFA14r1, PFA34, PFB13, PFB28r1, and PFB34, patients were unsure whether to respond with their ability to perform the task with the injured extremity alone, with either the injured or healthy extremity, or with both extremities. For PFA14r1, PFA34, and PFM16, patients indicated their response depends on specifics not mentioned in the question. For PFB28r1 and PFM16, patients struggled to recall performing these tasks with their injuries. Patients interpreted PFA36 as difficulty fitting splints into sleeves rather than functional limitations. Patients noted their responses vary depending on time of day. One patient noted uncertainty regarding whether she should answer questions based on learned adaptive techniques. The last five interviews included member checking and confirmed the authenticity of our results. Conclusion: Patients' cognitive processes and interpretations varied for each question of the PROMIS UE SF. This may undermine instrument reliability and validity. Two potential solutions include rewording questions or incorporating visuals to improve the instrument's ability to communicate question intent.

(3013) Using Natural Language Processing to Identify Health and Life Areas that matter to people living with obesity across the globe

Ana Maria Moga, PT, MsC, PhD candidate, Faculty of Medicine, School of Physical \& Occupational Therapy, McGill University, Montreal, Quebec, Canada; Laurie Twells, Associate professor PhD, School of Pharmacy \& Faculty of Medicine, Memorial University, St. John's, Newfoundland and LabradOR Canada; Mary Forhan, Associate professor $\mathrm{PhD}$, OT, Faculty of Rehabilitation Occupational Therapy, University Alberta, Edmonton, Alberta, Canada; Nancy E. Mayo, professor PhD, Faculty of Medicine, School of Physical \& Occupational Therapy, McGill University, Montreal, Quebec, Canada

Aims: Generic measures are often used for comparative- and costeffectiveness studies of weight-management intervention as many have utility-based indices. However, they may inaccurately estimate the quality of life (QoL) impact as they do not cover obesity-relevant dimensions. Conversely, obesity-specific measures include such content but lack an index value across dimensions. To fill this measurement gap, our overall goal is to develop an obesity-specific preference-based measure that incorporates the unique concerns of individuals living with obesity. This study aimed to identify the most important health and life areas from the perspective of people living with obesity and quantify the extent to which those areas are included in existing measures common in obesity research and management. Methods: We administered a cross-sectional survey via an online panel between April-August 2020 to individuals living with obesity in five countries (Canada, India, Australia, UK, and the USA). Using the Patient-Generated Index (PGI) - an individualized QOL measure-we asked respondents to nominate five areas (free text) of their life and health affected by their weight. We transformed the free (unstructured) text answers to structured ICF codes (International Classification of Functioning, Disability and Health Framework Codes) using a Natural Language Processing Python algorithm. Then, we identified the areas of life and health (ICF Codes) that matter the most to people living with obesity. Data assessment is ongoing, and the presentation will summarize all results. Results: A total of 900 people living with obesity completed the survey (BMI: $38.8 \pm 7.0 \mathrm{~kg} / \mathrm{m} 2$; age: $45 \pm 6.0$ years; $64 \%$ women). Table 1 illustrates the most frequent areas nominated and their inclusion in generic measures. Of the 12 most nominated areas, utility measures consistently cover only "walking," "pain," and "socializing" (30\%), whereas the Impact of Weight on Quality of Life includes half of the areas critical to people living with obesity (50\%). Conclusion: There is a clear gap in the content coverage of obesity concerns in existing measures. Not only is it essential to identify the areas of QoL concern from those with obesity when developing new measures, but the people impacted should also have a voice as to their relative importance to overall QoL. 


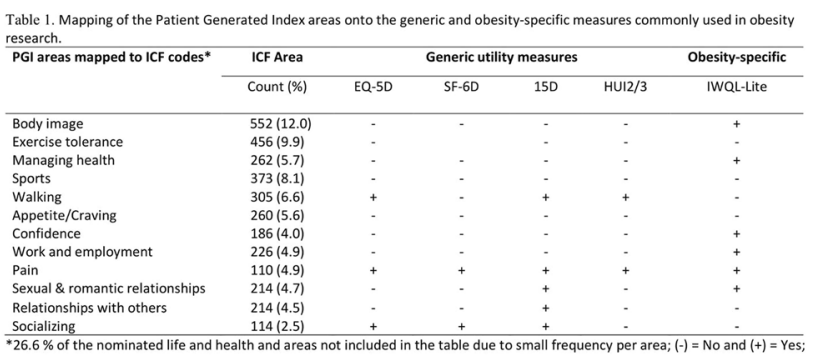

(3014) Development of LYMPH-Q upper extremity scales measuring worry, work impact, and burden of conservative treatments

Manraj Kaur, PhD, Brigham and Women's Hospital, Harvard Medical School, Boston, Massachusetts, USA; Sylvie Cornacchi, MSc., Department of Pediatrics, McMaster University, Hamilton, Ontario, Canada; Anne Klassen, PhD, Department of Pediatrics, McMaster University, Hamilton, Ontario, Canada; Louise Bordeleau, MD, Department of Oncology, McMaster University/Juravinski Cancer Centre, Hamilton, Ontario, Canada; Maureen Sawyer, BHSc(PT), Maureen Sawyer Physiotherapy, Hamilton, Ontario, Canada; Andrea Pusic, MD, Brigham and Women's Hospital, Harvard Medical School, Boston, Massachusetts, USA

Aims: Breast-cancer-related lymphedema (BCRL) affects $14-21 \%$ of women post-cancer treatment and can have a substantial impact on health-related quality of life. Our team developed the LYMPH-Q Upper Extremity to provide a comprehensive patient-reported outcome measure for this patient population. Interviews were conducted with 15 patients and used to form six scales that measure arm symptoms, function, appearance, psychological function, and satisfaction with both information, and arm sleeves, which were field tested with over 3000 women. During expert consultations, the need for additional scales measuring worry, work impact, and burden of lymphedema treatment was identified. The aim of this study was to elicit additional in-depth information on the impact of BCRL on the aforementioned concepts for scale development. Methods: The original and supplementary $(\mathrm{n}=12)$ semi-structured interviews were conducted with women diagnosed with BCRL recruited from hospitals in Canada and USA. Interviews were audio-recorded, transcribed verbatim, and coded using line-by-line approach. Constant comparison was used to refine the codes and develop an item pool for scale development. Relevant codes from the new interviews that pertained to lymphedema worry, work impact, and burden of conservative treatments were added to the original interview data. Interviews continued until data saturation was achieved. Results: Interviews were conducted with 27 women aged 40 to 76 years. All women described lymphedema worry in terms of their experience (e.g., fear of worsening symptoms) and impact on usual activities (e.g., stopped certain chores). Most women reported making several accommodations to their workspace (e.g., elevated desk) and work schedule (e.g., frequent breaks). Few women reported changing jobs or stopping work. All women described burden of ongoing symptom management including wearing and taking care of compression garments, exercise routine, and accessing community care. The role of caregiver in management of symptoms was also described (e.g., help with driving), as was the financial burden of BCRL. Conclusion: Qualitative data confirmed the importance of the three concepts suggested by expert consultation. Next steps will involve the development of three new LYMPH-Q scales and refinement with women with BCRL and clinician experts and a field-test study. Following which, the scales will be made available for clinical research and practice.
(3015) Patient-Reported Outcomes Research in Trauma: testing the usability of an ePRO system (PRiORiTY)

Christel McMullan, PhD, Centre for Patient Reported Outcomes Research, Institute of Applied Health Research, University of Birmingham; NIHR Surgical Reconstruction and Microbiology Research Centre (SRMRC), University of Birmingham; Birmingham Health Partners Centre for Regulatory Science and Innovation, Birmingham, UK; Sally Bradshaw, Centre for Patient Reported Outcome Research, Institute of Applied Health Research, University of Birmingham, Birmingham, UK; Ameeta Retzer, Centre for Patient Reported Outcome Research, Institute of Applied Health Research, University of Birmingham; Birmingham Health Partners Centre for Regulatory Science and Innovation; National Institute for Health Research (NIHR) Applied Research Centre (ARC)—West Midlands, Birmingham, UK; Anita Slade, Centre for Patient Reported Outcomes Research, Institute of Applied Health Research, University of Birmingham; NIHR Surgical Reconstruction and Microbiology Research Centre (SRMRC), University of Birmingham; Birmingham Health Partners Centre for Regulatory Science and Innovation, Birmingham, UK; Belli Antonio, Institute of Inflammation and Ageing, College of Medical Sciences, University of Birmingham, Birmingham, UK; Elin Haf Davies, Aparito Ltd, Wrexham, UK; Gary McNamara, Aparito Ltd, Wrexham, UK; Luke Flavell, Patient partner, Birmingham, UK; Jackie Flavell, Patient partner, Birmingham, UK; Melanie Calvert, Centre for Patient Reported Outcomes Research, Institute of Applied Health Research, University of Birmingham; NIHR Surgical Reconstruction and Microbiology Research Centre (SRMRC), University of Birmingham; Birmingham Health Partners Centre for Regulatory Science and Innovation; National Institute for Health Research (NIHR) Applied Research Centre (ARC) - West Midlands; NIHR Birmingham Biomedical Research Centre, University of Birmingham, Birmingham, UK; Grace Turner, Centre for Patient Reported Outcome Research, Institute of Applied Health Research, University of Birmingham; NIHR Surgical Reconstruction and Microbiology Research Centre (SRMRC), University of Birmingham; Birmingham Health Partners Centre for Regulatory Science and Innovation, Birmingham, UK

Aims: Over 50 million people have a traumatic brain injury (TBI) each year and global incidence is rising. Improvements in the clinical management of TBI have resulted in improved survival rates; however, this means that more people are living with life changing injuries and reduced quality of life. Electronic assessment of patientreported outcomes (PROs) post-TBI may facilitate early identification of ongoing issues, support shared-decision making, and help improve long-term outcomes.The aim of the PRiORiTy study is to explore the feasibility and acceptability of using an electronic PRO (ePRO) system for patients with a TBI. Methods: The PRiORiTy study includes three stages: a qualitative study, a usability study, and a feasibility study. The usability study consists of (1) the design of an ePRO system and (2) cognitive interviews with eight people with a TBI to test this platform in a non-clinical setting. Results: The qualitative study conducted by the authors showed that patients and clinicians were supportive of the development and use of an ePRO system and identified a number of key features to be included in an ePRO system.These findings were used to develop an ePRO system application in Atom5TM by Aparito Ltd as part of the usability study. Four questionnaires were programmed onto the Atom5TM system (PHQ-2, GAD-2, Abbreviated PCL-C, TBI-QOL-Ability/Satisfaction with social roles and activities SF). Preliminary results from the cognitive interviews show that participants find the Atom5TM ePRO system easy to use and navigate, and would be happy to use this app to report their symptoms regularly if it was possible. Comments regarding font size and the wording of some questions were made. Conclusion: Positive attitudes towards ePROs demonstrate the potential to capture PROs electronically in routine clinical practice 
and research. The next steps are to refine the ePRO system based on the results of the usability study, and test the feasibility and acceptability of the app by TBI patients and clinical team to inform care. It is anticipated that the PRiORiTy study will facilitate identification of TBI patients requiring clinical intervention/support as well as informing system development in other areas of trauma research.

(3016) Does change in health-related quality of life scores (QLQC30) add prognostic value beyond clinical measures and improve prognostic accuracy in cancer RCTs?

Abigirl Machingura, European Organization for Research and Treatment of Cancer (EORTC), Brussels, Belgium; Mekdes Taye, European Organization for Research and Treatment of Cancer (EORTC), Brussels, Belgium; Jammbe Musoro, European Organization for Research and Treatment of Cancer (EORTC), Brussels, Belgium; Madeline Pe, European Organization for Research and Treatment of Cancer (EORTC), Brussels, Belgium; Corneel Coens, European Organization for Research and Treatment of Cancer (EORTC), Brussels, Belgium; Francesca Martinelli, European Organization for Research and Treatment of Cancer (EORTC), Brussels, Belgium; Dongsheng Tu, Canadian Cancer Trials Group, Queen's University, Kingston, Ontario, Canada; Ethan Basch, Lineberger Comprehensive Cancer Center, Chapel Hill, North Carolina, USA; Jolie Ringash, Princess Margaret Cancer Centre and The University of Toronto, Toronto, Ontario, Canada; Yvonne Brandberg, Karolinska Institutet, Solna, Sweden; Morgens Groenvold, University of Copenhagen, Copenhagen, Denmark; Alexander Eggermont, Princess Máxima Center and University Medical Center Utrecht, Utrecht, The Netherlands; Fatima Cardoso, Champalimaud Clinical Center, Lisbon, Portugal; Jan Van Meerbeeck, Antwerp University Hospital, Edegem, Belgium; Winette Van der Graaf, The Netherlands Cancer Institute-Van Leeuwenhoek, Amsterdam, The Netherlands; Martin Taphoorn, Universiteit Leiden/McHaaglanden, Leiden, The Netherlands; Jaap C. Reijneveld, Amsterdam University Medical Centre, Amsterdam, The Netherlands; Riccardo Soffietti, Universita di Torino, Torino, Italy; Galina Velikova, Leeds Institute of Medical Research at St James's, Leeds, UK; Henning Flechtner, Otto-von-Guericke-University Magdeburg, Magdeburg, Germany; Andrew Bottomley, European Organization for Research and Treatment of Cancer (EORTC), Brussels, Belgium; submitted on behalf of "the EORTC Quality of Life Group, Brain tumour, Breast Cancer, Melanoma Group, Lung Cancer, Soft Tissue and Bone Sarcoma, Radiation Oncology, Lymphoma, Gastrointestinal Tract Cancer, Head and Neck Cancer, Genito-Urinary Cancers, and Gynecological Cancer Groups”

Aims: Several studies have demonstrated that baseline health-related quality of life (HRQoL) scores are prognostic for cancer patient survival. Fewer studies have investigated the added prognostic value of change over time in HRQoL scores. A study by Ediebah et al. (2014) using data from an EORTC lung cancer trial showed that change in HRQoL predict survival. This study aims to validate these findings using a large pooled dataset that includes several trials and disease sites. Methods: Published clinical trial HRQoL data using the EORTC QLQ-C30 from EORTC and Canadian Cancer Trials Group were pooled across several cancer sites. Two types of change scores were considered: change from baseline to 3 months from treatment start ( 3 months change) and change from baseline to end of treatment (end of treatment change). The prognostic significance of the change scores for each QLQ-C30 scale was assessed using Cox regression on overall survival adjusting for baseline score, study, socio-demographic, and clinical variables. Covariates were checked for suitability and intercorrelation. Model selection was based on stepwise selection using 5\% significance level, and bootstrapping was used to evaluate the stability of the final models. C-index was used to check model accuracy. Results: Data were pooled from 10,203 patients in 28 closed trials with 7,707 and 6,639 patients available for the analysis of 3 months change and end of treatment change, respectively. At 3 months, improvement in global health status, physical functioning, nausea/vomiting, pain, dyspnoea, and appetite loss were significantly associated with survival. At end of treatment, worsening in cognitive functioning and improvement in global health status, physical functioning, and appetite loss were significantly associated with survival. Adding HRQoL variables improved the model's prognostic accuracy by $10 \%$ for 3 months change and $16 \%$ for end of treatment change. Bootstrap evaluation did not reveal major instability issues. Conclusion: For both 3 months and end of treatment HRQoL changes from baseline, improvements in global health status, physical functioning, and appetite loss significantly lowered the risk of death. These findings support that in addition to clinical measures, change in HRQoL scores (as measured by the EORTC QLQ-C30) predicts survival among cancer patients.

(3017) Diet and physical activity patterns and their association with symptom burden in patients with cancer treated in earlyphase clinical trials

Goldy George, PhD, The University of Texas MD Anderson Cancer Center, Houston, Texas, USA; Alan Kim, BS, University of Chicago, Chicago, Illinois, USA; Melat Gebremeskel, BS, University of Chicago, Chicago, Illinois, USA; Meryna Manandhar, MBBS, MPH, The University of Texas MD Anderson Cancer Center, Houston, Texas, USA; Harsha Pradeep, MPH, The University of Texas MD Anderson Cancer Center, Houston, Texas, USA; Sarina Piha-Paul, MD, The University of Texas MD Anderson Cancer Center, Houston, Texas, USA; Filip Janku, MD, PhD, The University of Texas MD Anderson Cancer Center, Houston, Texas, USA; Vivek Subbiah, MD, The University of Texas MD Anderson Cancer Center, Houston, Texas, USA; Jordi Rodon, MD, PhD, The University of Texas MD Anderson Cancer Center, Houston, Texas, USA; Charles Cleeland, $\mathrm{PhD}$, The University of Texas MD Anderson Cancer Center, Houston, Texas, USA; David Hong, MD, The University of Texas MD Anderson Cancer Center, Houston, Texas, USA

Aims: Our goal was to examine diet and physical activity patterns and their association with patient-reported symptom burden in patients treated in early-phase oncology clinical trials. Methods: Patients with performance status (ECOG-PS) of 0 or 1 starting an early-phase clinical trial were recruited into a longitudinal cohort design, with assessments at baseline trial entry and at the end of cycle 1 . Dietrelated practices were measured using a diet questionnaire previously pilot tested in oncology clinical trial patients, exercise practices with the validated Godin Physical Activity Questionnaire, and symptom severity and symptom interference with functioning with the validated MD Anderson Symptom Inventory. Statistical tests included Spearman correlations. Results: Most (93\%) early-phase clinical trial patients $(\mathrm{N}=80$; mean age $=56 \mathrm{y} ; 51 \%$ male; $82 \%$ ECOG-PS $=1$; $35 \%$ in trials including immune checkpoint inhibitors, $65 \%$ in targeted therapy trials) believed that diet was important even while on a clinical trial, and $87 \%$ were interested in eating healthfully. Patients rated their diet as moderately/quite healthful (mean $=3$ on $0-5$ scale). However, $28 \%$ of patients felt that study medications had impacted their diet while on a clinical trial, including via increased loss of appetite, nausea, and poor taste sensation. Greater healthfulness of diet during their clinical trial was associated with less pain $(P=0.002)$, fatigue ( $P=0.017)$, nausea $(P=0.035)$, and drowsiness $(\mathrm{P}=0.045)$, and reduced symptom-related interference with activityrelated functioning $(\mathrm{P}=0.020)$ at end of cycle. Furthermore, $85 \%$ of 
patients felt that they could get at least small amounts of exercise while on a clinical trial. Patients spent, on average, $14 \mathrm{~min} / \mathrm{d}$ in mildintensity, $5 \mathrm{~min} / \mathrm{d}$ in moderate-intensity, and $2 \mathrm{~min} / \mathrm{d}$ in strenuousintensity exercise. Greater total minutes spent in any-intensity exercise at trial baseline was associated with less fatigue $(\mathrm{P}=0.022)$ and drowsiness $(\mathrm{P}=0.037)$ at trial baseline, and with less pain $(\mathrm{P}=0.026)$ at end of cycle. Conclusion: Despite having advanced, metastatic disease, patients with advanced cancer on early-phase clinical trials strive to maintain healthful diets and obtain at least some physical activity. Diet and physical activity may impact, and conversely, also be impacted by symptom burden in these patients. Diet and physical activity prescriptions for Phase I patients may need to be individualized for patients and should consider patients' symptom severity profiles.

(3018) Content sort of patient-reported outcome measures used in patients with nail conditions: a systematic review

Leah Algu, BHSc, BScN candidate, Lawrence S. Bloomberg Faculty of Nursing, University of Toronto, Toronto, Ontario, Canada; Khizar Abid, BHSc, MD Candidate, Temerty Faculty of Medicine, University of Toronto, Ajax, Ontario, Canada; Rakhshan Kamran, BSc, MD Candidate, Michael G. DeGroote School of Medicine, McMaster University, Hamilton, Ontario, Canada; Cameron Leveille, MD, Michael G. DeGroote School of Medicine, McMaster University, Hamilton, Ontario, Canada; Claire Stewart, MD, Department of Dermatology, Weill Cornell Medical College, New York, New York, USA; Shari Lipner, MD, PhD, Department of Dermatology, Weill Cornell Medical College, New York, New York, USA; Trisia Breitkopf, MSc, Department of Pediatrics, McMaster University, Hamilton, Ontario, Canada; Charlene Rae, MSc, PhD Candidate, Department of Pediatrics, McMaster University, Hamilton, Ontario, Canada; Anne Klassen, D. Phil, Department of Pediatrics, McMaster University, Hamilton ON, Hamilton, Ontario, Canada

Aims: Nail conditions can be congenital, acquired, or signs of systemic disease. These conditions can cause patients physical, psychological, and social challenges in their daily life. Patient-reported outcome measures (PROMs) can be used to capture these constructs, and aid in clinical decision making and treatment evaluations. The objective of this study was to identify nail-specific PROMs and describe the concepts measured. Methods: A systematic review was conducted following PRISMA guidelines. MEDLINE and EMBASE were searched from inception to February 2021 to identify PROMs used in patients with nail conditions. Content analysis was conducted on PROMs designed specifically for nail conditions, by qualitatively coding the items to identify the concepts covered and classifying content phrasing as positive or negative. Results: For this review, 209 of 4251 articles were screened. After full-text screening, seven nail-specific PROMs were identified. Nail-specific PROMs were targeted for use in onychomycosis or nail psoriasis. Nail-specific PROMs included 175 items that were sorted into a total of 194 concepts of interest. These were categorized into 5 domains, 18 subdomains, and 67 unique health concepts. Psychological wellbeing was the most frequently measured domain with a total of 50 items $(25.8 \%)$. A limited number of items measured nail appearance $(\mathrm{n}=32,16.5 \%)$, physical function $(\mathrm{n}=19,9.8 \%)$, and nail care $(\mathrm{n}=17,8.6 \%)$. A total of $68.6 \%(\mathrm{n}=120)$ of items had negative content or phrasing. Conclusion: In individuals with nail conditions, treatments can impact nail appearance, physical function, activities of daily living, and body image. This review highlights important gaps in the content captured in currently used PROMs designed for nail conditions. There is opportunity for a new nail-specific PROM to be developed which captures the concepts that matter to patients.
(3019) Creating a natural history study through comprehensive stakeholder engagement and approaches to regulatory science for rare diseases

Josey Hensley, National Organization for Rare Disorders, Danbury, Connecticut, USA; Pamela Gavin, National Organization for Rare Disorders, Danbury, Connecticut, USA; Stephanie Christopher, MA, National Organization for Rare Disorders, Danbury, Connecticut, USA; Amelia Robert, National Organization for Rare Disorders, Danbury, Connecticut, USA; Hussein Ezzeldin, USFDA, Silver Spring, Maryland, USA; Sarah Stothers, USFDA, Silver Spring, Maryland, USA; Ting-Hsuan Lee, USFDA, Silver Spring, Maryland, USA

Aims: Rare-disease therapeutic development is challenged by the ineffective standard of care concurrent controls in randomized clinical trials (RCTs) for populations of patients that are, by nature, limited in size and facing rapid disease progression. The National Organization for Rare Disorders (NORD) developed a pilot study, the Natural History Of Metachromatic Leukodystrophy (HOME) study. Through comprehensive stakeholder engagement, this study was designed to inform good research practices for designing and conducting a natural history study (NHS) with high quality and highly usable data that can serve as external controls to increase statistical power of future RCTs. Methods: Comprehensive stakeholder involvement leverages knowledge from patients, caregivers, patient-advocate organizations, clinicians, industry, and regulators to ensure this pilot study maintains patient centricity, scientific rigOR, and clinical relevance. Patient and caregiver-reported outcomes via online and mobile platforms are captured in conjunction with virtual tele-health assessments conducted by a clinical study coordinator. The study will shed light on effective methods of remote study participation and use of NHS data to augment RCTs. Study visits are conducted at baseline, and three-, six-, nine-, and twelve-months. Results: Early study engagement with the Family and Caregiver Advisory Committee $(n=5)$, the Industry and Clinician Advisory Committee $(\mathrm{n}=10)$, and FDA resulted in a study design that aims to decrease participant burden while ensuring rigorous collection of primary and secondary clinical endpoints. NORD's IAMRARE ${ }^{\mathrm{TM}}$ Registry Program hosts the online patient and caregiver-reported outcome questions, while a mobile applicationSurvey of Health and Patient Experience (SHAPE) — was developed to allow participants to report episodic health events. Video-based telehealth assessments enable clinical evaluation of endpoints at scheduled intervals. This innovative approach to NHS data collection engages participants in clinical research from the comfort of home. Conclusion: The pilot HOME Study explores innovative methods for study participation and data collection to address an unmet need in rare disease therapeutic development. Through the engagement of a multi-stakeholder group, the study team designed a novel approach to collect NHS data to serve as external controls in RCTs, not only for MLD, but other rare disorders as well.

(3020) International experts Delphi panel to gain consensus on health and functioning concepts impacted in users of tobacco and nicotine products

Esther Afolalu, PhD, Philip Morris International R\&D, Neuchatel, Switzerland; Vivienne Law, Zebra26 Ltd, Cheshire, UK; Lee Wemyss, Dash-Global Ltd, Cheshire, UK; Andrew Turner, DashGlobal Ltd, Cheshire, UK; Linda Abetz-Webb, Patient-Centered Outcomes Assessments, Ltd, Cheshire, UK; Christelle Chrea, Philip Morris International R\&D, Neuchatel, Switzerland

Aims: To gain consensus and feedback from international experts on the most important health and functioning concepts to be considered when evaluating a switch from smoking cigarettes to using smoke- 
free tobacco and nicotine products (sfTNP) (e.g., e-cigarettes, heated tobacco products, and smokeless tobacco). Methods: Experts with professional experience in tobacco cessation treatment and tobacco research/policy completed three rounds of an online survey combining quantitative (MaxDiff ranking) and qualitative assessments to elicit ranking, consensus, and feedback on concepts. Experts $(\mathrm{N}=105$; men, 67$)$ were recruited in Europe $(\mathrm{n}=41)$, North America $(\mathrm{n}=20)$, Central/South America $(\mathrm{n}=15)$, Asia $(\mathrm{n}=17)$, Africa $(n=6)$, and the Middle East $(n=6)$. The majority $(n=79)$ worked in hospitals or other clinical settings, mostly as specialists (e.g., oncologists, dentists, pulmonologists, and cardiologists) or general care practitioners; 26 worked in advocacy/policy, counselling, or research settings. All experts participating in Round I completed Round II, and 100 completed Round III. Results: In Round I, the experts evaluated 69 concepts and rated health impact as the most important and esthetics as the least important; thus, 36 concepts were carried forward to Round II. Round II results reinforced the importance of concepts relating to physical health (e.g., cardiovascular and respiratory symptoms) and worries about health impact. This led to elimination of 16 concepts, and 20 concepts were ranked in Round III. The experts reached a consensus that all 20 concepts were important to measure as part of a switch, though four concepts (cardiovascular symptoms, shortness of breath, chest pain, and worry about smokingrelated diseases and impact on general health) made up 50\% of the total score in the MaxDiff ranking analysis. Latent subgroup analysis also showed that the relative importance of each concept differed among the experts, depending on professional specialty and region. Conclusion: This three-round expert Delphi panel identified and reached consensus on a ranked list of important concepts for supporting the development of a new instrument for assessing the selfreported impact of switching to sfTNPs on health and functioning. The next step is to assess the importance of the concepts in sfTNP users to aid the development of appropriate questionnaire items for the instrument.

\section{(3021) Empowerment in patient-provider interactions: from theory to generic questionnaire}

Emilie Haarslev Schroeder Marqvorsen, RN, PhD student, Research Unit Women's and Children's Health, Rigshospitalet University Hospital, Copenhagen, Denmark; Line Lund, PhD, Research Unit, Department of Palliative Medicine, Bispebjerg Hospital, Copenhagen, Denmark; Anne Sophie Mathiesen, RN, PhD, Department of Endocrinology, Center for Cancer and Organ Diseases, Rigshospitalet University Hospital, Copenhagen, Denmark; Kirsty Winkley, RN, PhD, Department of Adult Nursing, Kings College London, London, UK; Bodil Rasmussen, RN, PhD, professor School of Nursing and Midwifery, Deakin University, Melbourne, Australia; Thordis Thomsen, RN, PhD, professor Department of Clinical Medicine, Herlev-Gentofte Hospital, Herlev, Denmark; Frans Pouwer, PhD, professor Department of Psychology, Southern University Denmark, Odense, Denmark; Mette Rothmann, RN, PhD, Department of Endocrinology and Department of Rheumatology,

Steno Diabetes Center Odense, Odense University Hospital, Odense, Denmark; Rikke Jørgensen, RN, PhD, Department of Clinical Medicine, Faculty of Medicine, Aalborg University, Aalborg, Denmark; Mette Linnet Olesen, RN, PhD, Research Unit Women's and Children's Health, Rigshospitalet University Hospital, Copenhagen, Denmark; Sigrid Biener, MScPH, Research Unit Women's and Children's Health, Rigshospitalet University Hospital, Copenhagen, Denmark; Mette Due-Christensen, RN, PhD, Department of Adult Nursing, Kings College London, London, UK; Gitte Husted, RN, PhD, Steno Diabetes Center Copenhagen, Gentofte, Denmark; Janne Weis, RN, PhD, Department of Neonatology, Rigshospitalet University Hospital, Copenhagen, Denmark; Vibeke
Zoffmann, RN, PhD, professor Research Unit Women's and Children's Health, Rigshospitalet University Hospital, Copenhagen, Denmark

Aims: Empowerment is an important alternative to paternalistic concepts like adherence and compliance and may be facilitated through interactions between patients and healthcare providers (HCPs). However, empowerment can be difficult to realize in clinical practice. To fully be able to evaluate current care and compare the effectiveness of interventions across the healthcare system, a generic patient-reported outcome (PRO) measure is needed. Here, we present the development of the EMPOWER-UP questionnaire, measuring empowerment in patient-HCP interactions. Methods: The development process follows the most recent recommendations (1). The domains of EMPOWER-UP were determined a priori based on four inter-related grounded theories (2-5). To generate items, we reviewed findings and the analysis codebook from these studies, and findings from a qualitative evaluation of an intervention study (6). Together with an expert panel, we reviewed all items to ensure simple, generic wording, and pre-tested them in cognitive interviews with chronically ill people from Denmark (DK), the UK (UK), and Australia (AUS). Data from a main sample of 720 chronically ill people $(\mathrm{DK}=360$, $\mathrm{UK}=240$, AUS $=120$ ), and two sub-samples of 224 people with type 2 diabetes from a randomized trial and up to 1260 people from chronic illness rehabilitation courses in municipalities in DK will help finalize the questionnaire and test its psychometric properties. Results: The domains are barriers to 1) achieve a collaborative relationship, 2) resolve a life-versus-disease conflict, 3) accomplish shared decision making, and 4) achieve a person-specific empowering evidence. We included 41 items for pre-testing in 29 interviews in DK, the UK, and AUS. The process is ongoing and has reached step four (Fig. 1), where data from the main sample are collected. Data in the two sub-samples will be collected through 2021-2023. Conclusion: EMPOWER-UP is founded on a comprehensive theoretical foundation of empowerment, life skills theory, self-determination theory, and four inter-related grounded theories. Findings from these theories have been recognized in multiple disease contexts, thus, emphasizing their generic value. The development and validation process are ongoing. Once finished, EMPOWER-UP will enable measurements of barriers to empowerment in patient-HCP interactions across the entire health system.

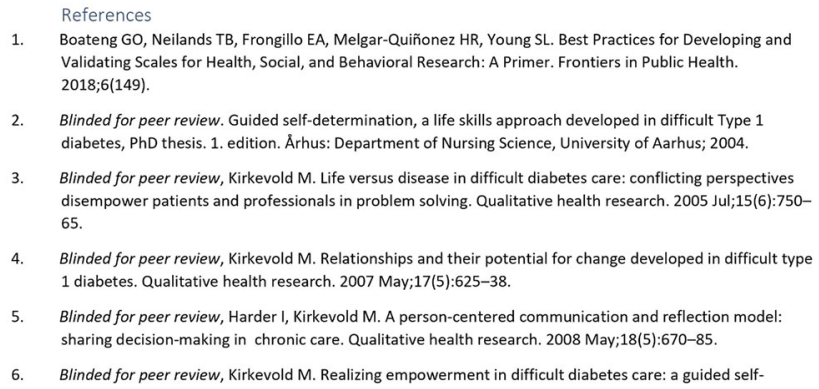

Empowerment in patient-provider interactions: from theory to generic questionnaire - Figure 1

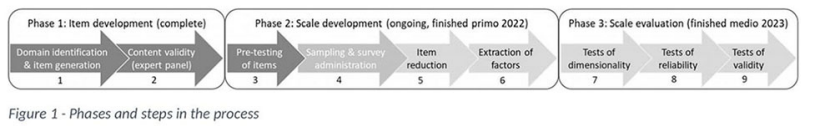

(3022) A novel Self-Regulation Assessment (SeRA) for a rehabilitation population 
Tanja Mol, MSc, Center of Excellence for Rehabilitation Medicine, UMC Utrecht Brain Center, University Medical Center Utrecht, and De Hoogstraat Rehabilitation, Utrecht, The Netherlands; Coen van Bennekom, MD, PhD, Heliomare Rehabilitation Center, Research and Development., Wijk aan zee, The Netherlands; Eline Scholten, $\mathrm{PhD}$, Center of Excellence for Rehabilitation Medicine, UMC Utrecht Brain Center, University Medical Center Utrecht, and De Hoogstraat Rehabilitation, Utrecht, The Netherlands; Marcel Post, PhD, Center of Excellence for Rehabilitation Medicine, UMC Utrecht Brain Center, University Medical Center Utrecht, and De Hoogstraat Rehabilitation, Utrecht, The Netherlands

Aims:Self-regulation refers to self-management and self-control, with or without disability. Self-regulation is recognized as an important factor in medical rehabilitation and outcome measurement would be desirable. Prior research revealed a conceptual model of self-regulation, based on aspects identified by former rehabilitation patients. A subsequent systematic review of self-regulation measures revealed many measures, but no measure was identified which fitted this model. Therefore, we aimed to construct a novel Patient-Reported Outcome Measure of self-regulation. Methods: Items were collected, generated and revised based on the previously developed model. These items were discussed with experts from the field, and cognitive interviews with former patients were conducted. A list of 22 items was distributed in a cross-sectional online survey. Exploratory factor analyses and internal consistency analysis were used to define scales and select items. Results:A total of 563 former rehabilitation patients completed the survey. The mean age of the respondents was 56.5 years old, the largest included diagnostic groups were Spinal Cord Injury and Acquired Brain Injury, and the majority of the respondents followed a rehabilitation trajectory 4 to 2 years ago. Exploratory factor analyses resulted in a four-factor solution, which were in agreement with the previously developed model. The factors were labelled as 'insight in (your) condition', 'insight in (your) abilities', 'apply self-regulation' and 'organization of help'. Item reduction was conducted based on item-total correlations and item content. This resulted in a 12 -item patient-reported outcome measure with a four-factor structure. Cronbach's Alpha of the total scale was 0.89. Cronbach's Alpha for the subscales based on the defined factors were, respectively, 0.83, 0.76, 0.79, and 0.85. Conclusion:A novel self-regulation assessment, called SeRA, was developed. This PatientReported Outcome Measure holds a four-factor solution including 12 items.

\section{(3023) Trajectories in insomnia symptoms over three years among premenopausal breast cancer survivors}

Chloe M. Beverly Hery, MS, The Ohio State University, Columbus, Ohio, USA; Sarah A. Janse, The Ohio State University, Columbus, Ohio, USA; Kimberly J. VanZee, Memorial Sloan Kettering Cancer Center, New York, New York, USA; Elizabeth Z. Naftalis, Health Texas Community Health Services, Dallas, Texas, USA; Michelle J. Naughton, The Ohio State University, Columbus, Ohio, USA

Aims: Sleep disruptions are very common in cancer survivors and can negatively impact quality of life. Young breast cancer survivors face unique challenges and may have distinct sleep pattern subgroups. We examined trajectories of sleep quality/insomnia over three years among premenopausal breast cancer survivors. Methods: 835 women, $\leq 45$ years old with stage I-III breast cancer, were recruited to the Menstrual Cycle Maintenance and Quality of Life After Breast Cancer Study within 8 months of a breast cancer diagnosis. Sleep patterns were assessed at 6-month intervals from baseline through three years post-recruitment, using the Women's Health Initiative Insomnia Rating Scale (WHIIRS), a measure of sleep quality and insomnia symptoms. Higher scores (range 0-20) indicate more sleep disruption, with scores $\geq 9$ compatible with a clinical diagnosis of insomnia. Group-based trajectory modeling was used to identify distinct trajectories of insomnia symptoms. The final model was chosen by comparing the average posterior probability of assignment (PPA) for each group and examining the distinctiveness and interpretability of the trajectories and group sizes. Key quality of life, demographic, and clinical characteristics were assessed as predictors of group membership. Results: The mean age of participants was 38 years (range: 20-46 years) at enrollment. Three distinct sleep trajectories were identified: no insomnia $(\mathrm{n}=262,31.3 \%)$, moderate insomnia $(\mathrm{n}=451,53.9 \%)$, and high insomnia symptoms $(\mathrm{n}=123$, $14.7 \%$ ). Average PPA was between $87-89 \%$ for each group. WHIIRS scores were relatively stable over time. Insomnia symptoms were worse at baseline but did improve for the no insomnia and moderate insomnia groups (see Fig. 1). Older age $(\mathrm{p}=0.05)$, lower education $(\mathrm{p}=0.04)$, having received chemotherapy $(\mathrm{p}<0.0001)$, hot flashes $(\mathrm{p}<0.0001)$, night sweats $(\mathrm{p}<0.0001)$, and lower SF-12 physical $(\mathrm{p}<0.0001)$ and lower SF-12 mental health component scores $(\mathrm{p}<0.0001)$ were associated with the moderate and high insomnia groups. Conclusion: Insomnia symptoms are problematic for some breast cancer survivors. We identified three distinct sleep trajectories and factors associated with more sleep problems. Analyses such as this enable us to identify high-risk patients that may need and benefit from sleep interventions in order to address this important issue in survivorship.

Figure 1. Three-group trajectory plots of WHIIRS scores over 36 months, with $95 \%$ confidence intervals:

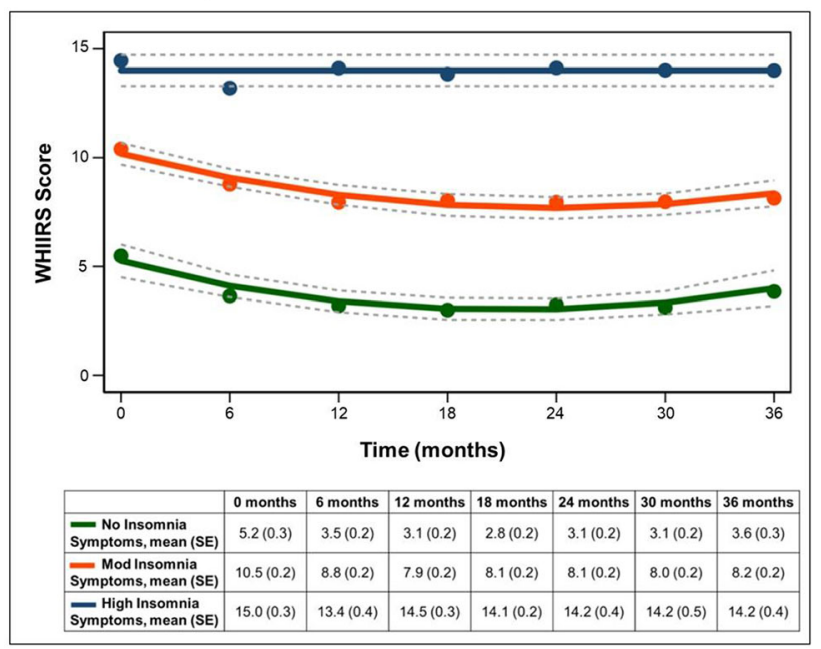

(3024) Beyond vision: visual functioning and quality of life in children operated for childhood cataract

Vijaya Gothwal, PhD, L V Prasad Eye Institute, Hyderabad, India; Anil Kumar Mandal, MD, Jasti V Ramanamma Children's Eye Care Centre, L V Prasad Eye Institute, Hyderabad, India; Akshay Badakere, FRCS, Jasti V Ramanamma Children's Eye Care Centre, L V Prasad Eye Institute, Hyderabad, India

Aims: Cataracts are responsible for $5-20 \%$ of childhood blindness globally making it a major cause of preventable blindness in children. It is recommended that children with congenital cataracts should be operated when the child is between 6 and 8 weeks to reach an optimal outcome. The majority of the studies have focused on visual acuity outcomes and given this, there is paucity of data regarding visual functioning (VF) and vision-related quality of life (VRQoL) in children operated for cataract. The purpose of our study was to investigate the VF and VRQoL in children operated for cataract during early childhood. Methods: 106 children aged 8-17 years (mean age, 
11.3 years $)$ operated for developmental $(n=68)$ or congenital $(\mathrm{n}=38)$ cataract (median duration since surgery, 4 years) at our centre completed two validated instruments measuring VF (L V Prasad- Functional Vision Questionnaire II, LVP-FVQ II) and VRQoL (Impact of Vision Impairment for Children, IVI-C) during their routine clinic visit. Rasch analysis was used to optimize the measurement properties of LVP-FVQ II and IVI-C. Rasch-calibrated scores from both these instruments were used in linear regression models to identify predictors associated with VF and VRQoL. Results: The mean (SD) VRQoL score was 1.57 (SD 1.64) logits and the mean VF score was -2.78 (2.61) logits. Better visual acuity in better-seeing eye was the only significant predictor of higher VF and VRQoL. Children operated for developmental cataract reported significantly better VF and VRQoL compared to congenital cataract group. Children operated for unilateral cataract reported significantly better VRQoL compared to bilateral cataract group. Copying small letters from board was most difficult, and watching television was reported to be the least difficult activity by the children. Conclusion: Lower visual acuity in the better-seeing eye was the strongest predictor of lower VF and VRQoL in children operated for cataract in early childhood. Children operated for congenital cataract are more likely to have lower VF and VRQoL in the long term. Therefore, parents/caregivers and all eye care professionals should pay more attention towards stimulating visual functioning in these children in the early developmental years following surgery.

\section{(3025) A psychometric comparison of three approaches to chemotherapy-induced peripheral neuropathy assessment: Patient report, clinical grading, and sensory function}

Tiffany Li, The University of Sydney, Sydney, Australia; Hannah C. Timmins, BSc (Hons), The University of Sydney, Sydney, Australia; Terry Trinh, PhD, The University of New South Wales, Sydney, Australia; David Mizrahi, PhD, The University of New South Wales, Sydney, Australia; Lisa G. Horvath, MBBS, FRACP, Chris O'Brien Lifehouse, Sydney, Australia; Peter Grimison, MBBS, FRACP, Chris O'Brien Lifehouse, Sydney, Australia; Michelle Harrison, MBBS, FRACP, Chris O'Brien Lifehouse, Sydney, Australia; Claudia Rutherford, PhD, The University of Sydney, Sydney, Australia; Madeleine King, PhD, The University of Sydney, Sydney, Australia; Matthew C. Kiernan, MBBS, FRACP, The University of Sydney, Sydney, Australia; David Goldstein, MBBS, FRACP, The University of New South Wales, Sydney, Australia; Susanna B. Park, PhD, The University of Sydney, Sydney, Australia

Aims: Worldwide cancer survivor numbers are steadily increasing, leading to a need for research to focus on survivor's quality of life, and reduction of treatment residual adverse events. Chemotherapyinduced peripheral neuropathy (CIPN) is a significant and pervasive side effect of numerous chemotherapies, often impacting cancer survivor's long-term quality of life. Early identification of CIPN is crucial in preventing long-lasting nerve damage. However, this is complicated by a lack of a gold standard CIPN outcome measure. This study aimed to evaluate the validity and responsiveness of different approaches to CIPN assessment in order to identify optimal outcome measures. Methods: 859 cancer patients ( $54 \pm 13$ years) treated with various neurotoxic chemotherapies (taxanes, platinums, vinca-alkaloids, bortezomib, immunomodulatory drugs) underwent comprehensive nerve assessment with patient-reported outcome measures (PROMs; EORTC QLQ-CIPN20, FACT/GOG Ntx-13, and PRO-CTCAE), clinical grading (National Cancer Institute NCI grade, Total Neuropathy Score (TNSc), nerve conduction studies), and sensory functional measures (monofilaments, 2-point discrimination, grating orientation, grooved pegboard). Convergent validity was assessed as a Spearman's correlation coefficient of $>0.7$ between each measure and NCI grade. Discriminant validity compared measures using $\mathrm{t}$ tests between NCI grade $\leq 1$ (non-significant) and grade $\geq 2$ (significant) CIPN. Responsiveness was evaluated in a subset of 356 patients who were prospectively assessed, comparing pre-treatment to mid-treatment scores with effect sizes (ES). Results: PROMs demonstrated convergent validity with high correlations to NCI grade $(\mathrm{r}=0.74-0.88, \mathrm{P}<0.01)$. Sensory functional measures and other clinical grading scales did not achieve acceptable correlations (all $\mathrm{r}<0.7, \mathrm{P}<0.01$ ). Discriminant validity was demonstrated in all measures, indicating that all measures were able to differentiate between non-significant and significant CIPN (all P $<0.01$ ). PROMs were overall the most responsive outcome measures $(\mathrm{ES}=$ $0.63-1.63)$. Sensory functional measures and other clinical grading scales did not demonstrate high responsiveness $(\mathrm{ES}=0.06-0.27)$ with the exception of the Total Neuropathy Score (TNSc), a composite neurological grading scale $(\mathrm{ES}=1.04)$. Conclusion: PROMs demonstrated superior validity and responsiveness compared to clinical grading and sensory functional measures in CIPN, despite their subjectivity. This present series suggests that the use of PROMs in combination with a responsive objective measure such as the TNSc provides most optimal evaluation of CIPN.

\section{(3026) Real-Time Symptom Monitoring: Developing a Disease- specific Patient-Reported Outcomes (PROs) Tool for Patients with Upper-Gastrointestinal Cancer}

Tennille Lewin, BSN, Monash University, Melbourne, Australia; Jonathan Kim, Monash University, Melbourne, Australia; Sue Evans, Victorian Cancer Registry, Melbourne, Australia; Charles Pilgrim, Alfred Health, Melbourne, Australia; Kate White, Sydney University, Sydney, Australia; John Zalcberg, Monash University, Melbourne, Australia; Lisa Brady, Monash University, Melbourne, Australia; Jessy Hansen, Monash University, Melbourne, Australia; Darshini Ayton, Monash University, Melbourne, Australia; Afaf Girgis, University of New South Wales, Sydney, Australia; Madeleine King, Sydney University, Sydney, Australia; Ashika Maharaj, Monash University, Melbourne, Australia; Rachel Neale, Berghofer Medical Research Institute, Brisbane, Australia; Jennifer Philip, University of Melbourne, Melbourne, Australia; Claudia Rutherford, Sydney University, Sydney, Australia; Arul Earnest, Monash University, Melbourne, Australia; Liane Ioannou, Monash University, Melbourne, Australia

Aims: To use co-design methodology with patients, carers, and Health Professionals (HP) to develop a disease-specific symptom monitoring tool for patients with Upper Oesophagogastric (UGI) cancer. The codesigned tool will be used to report real-time Patient-Reported Outcomes (PROs) within the intervention arm of the registry-based randomised controlled trial, PRO patient. Methods: The development of the tool occurred across multiple phases. (1) Validated cancer Health-Related Quality of Life (HRQoL) questionnaires were mapped to identify a list of PROs (i.e. symptoms and concerns); (2) Identified PROs were presented to focus groups including health professionals (clinicians, allied health and nurses) and consumers (patients and care givers) to determine their perceptions on the relevance, impact and action-ability of each symptom/concern; (3) Focus group results were used to develop a questionnaire to be sent to patients on the UGI cancer registry (UGICR), requiring patients to identify and rank their ten most commonly experienced symptoms/concerns from the list of PROs; and 4) Frequency and ranking of participant responses to the PRO items were analysed and using Stata to finalise the tool. Results: A total of 65 UGI cancer-specific PROs were identified from validated disease-specific questionnaires and presented to HP and consumer focus groups. All focus groups agreed that each of the symptoms/concerns was relevant, actionable, and impacted patients 
HRQoL; however, they felt the length of the tool would be burdensome for patients to complete. Based on the results of the focus groups, two PRO items were considered to be similar to other PRO items and were combined. This meant the final list of PROs presented to patients in the questionnaire was 63. A total of 159 out of 519 completed questionnaires were received, giving a response rate of $30 \%$. Frequency and ranking of the symptoms/concerns were undertaken to identify the symptoms/concerns to be included in the final tool. Conclusion: This study has developed a co-designed diseasespecific PROs tool for patients with UGI cancer to report their symptoms and concerns. This will allow for the provision of real-time feedback to HPs in order to guide clinical and supportive care to improve HRQoL for these patients.

\section{(3027) Identifying predictors of propensity for response shift using latent class analysis}

Richard Sawatzky, Trinity Western University, Langley, British Columbia, Canada; Lara Russell, PhD, Trinity Western University, Langley, British Columbia, Canada; Tolulope Sajobi, PhD, University of Calgary, Calgary, Alberta, Canada; Juxin Liu, PhD, University of Saskatchewan, Saskatoon, Saskatchewan, Canada; Lisa Lix, PhD, University of Manitoba, University of Manitoba, Manitoba, Canada; Bruno Zumbo, PhD, University of British Columbia, Vancouver, British Columbia, Canada

Aims: Understanding individuals' propensity for response shift (RS) can improve the measurement of patient-reported outcomes in diverse populations. Our aim was to identify individual characteristics associated with a propensity for RS. Specifically, our objectives were to a) distinguish latent classes of people who experienced RS in physical and mental health versus those who did not and b) identify demographic and health-related characteristics that predict the latent classes. Methods: Analyses were based on a random sample of 10,000 participants from the Medicare Health Outcomes Survey Cohort 19 (2016-2018) in the USA who completed the Veterans Rand 12 item health survey (VR-12) at baseline and after two years $(\mathrm{N}=245,475)$. A longitudinal 2-parameter multidimensional item response theory (IRT) model was used to measure change in latent physical and mental health status based on the VR-12 items at the two time points. Latent class analysis was used to distinguish people who experienced RS by specifying two classes: 1) a "no-RS class" with time invariant IRT model parameters and 2) a "RS class" where the IRT model parameters varied over time (Fig. 1). Model fit was assessed based on relative improvements in information criteria and the Vuong-LoMendell-Rubin likelihood ratio test. Multivariable logistic regression analysis and purposeful variable selection procedures were used to determine which demographic and health-related variables predict latent class membership. Results: The sample included predominantly older adults $(86 \%>65)$, females $(59 \%)$, and people classified as "white" (81\%). The 2-class model had superior fit relative to a conventional IRT model assuming no RS. The RS class represented $14 \%$ of the sample (entropy $=0.74$ ). RS was predicted by (a) demographic variables, including age, gender, race, education, marital status, and language spoken at home; (b) several diagnoses, notably stroke and diabetes; (c) several other health indicators, including amount of sleep and having experienced a fall during the past 12 months; and (d) survey completion by self or proxy (Fig. 2). Conclusion: Propensity for RS was associated with various demographic and clinical characteristics. This research demonstrates the exploratory value of latent class models for RS to generate hypotheses about patient characteristics that may contribute to RS.
Figure 1: Latent class response shift model of the VR-12
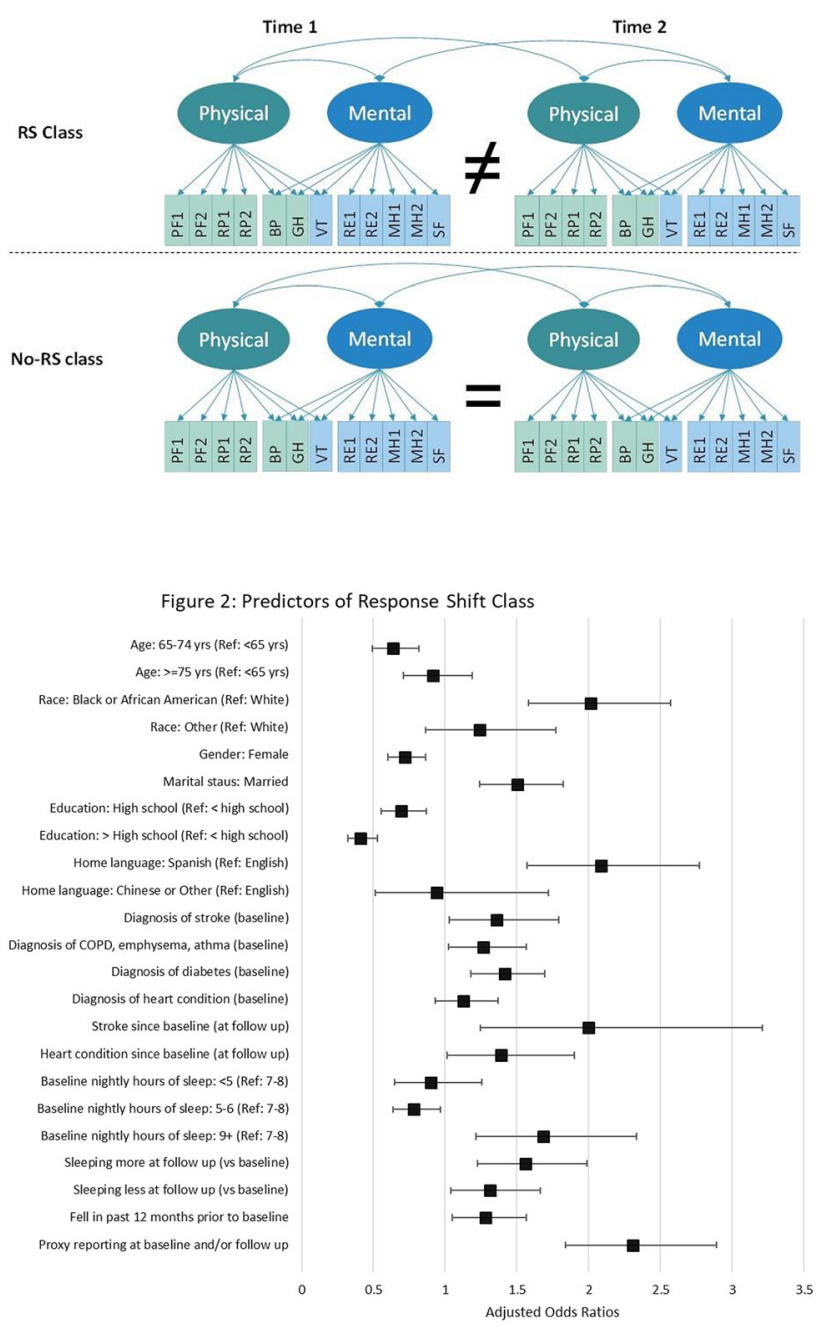

(3028) Issues in the practice of quality-of-life assessment research: A qualitative study for use in considering education methods

Kikuko Miyazaki, PhD, Kyoto University, Kyoto, Japan; Tatsuto Nishigori, MD, PhD, Kyoto University, Kyoto, Japan; Nobuichiro Tamura, MD, PhD, Kurashiki Central Hospital, Kurashiki, Japan; Rika Hayashida, RN, MS, University of Nagasaki, Siebold, Nagasaki, Japan; Shinichi Noto, PhD, Niigata University of Health and Welfare, Niigata, Japan; Kojiro Shimozuma, MD, PhD, Ritsumeikan University, Kusatsu, Japan; Shinya Saito, MD, PhD, Okayama University, Okayama, Japan; Yoshimi Suzukamo, PhD, Tohoku University, Sendai, Japan

Aims: To clarify challenges arising in the practice of quality-of-life (QOL) assessment research in order to consider education methods useful in training QOL assessment researchers. Methods: We conducted semi-structured interviews, using an interview guide, with researchers conducting QOL assessment research. Participants were QOL assessment research practitioners who provided written consent to participate in the study. Interviewers received standardized training concerning interview research methods. The interview guide included the following items: (1) summary of ongoing QOL assessment research, (2) how the researchers arrived at the idea for (1), (3) reason 
why QOL assessment is necessary, (4) issues in conducting QOL assessment research, and (5) participant background information. Interviews were recorded with permission, verbatim transcripts were created from the audio data, and the transcript data were used for analysis. Data underwent qualitative content analysis using the data analysis software NVivo 10. Multiple researchers examined the data during the analysis process to prevent arbitrary results. (Tohoku University Ethics Review 2016-858.) Results: A total of 30 QOL/ PRO researchers were interviewed $(\operatorname{men}=19$, women $=11)$. Concerning age, participants were primarily between the ages of 40-49 $(\mathrm{n}=12), 30-39(\mathrm{n}=8)$, and $50-59(\mathrm{n}=6)$, while primary occupations were doctor $(n=11)$, nurse $(n=7)$, therapist $(n=5)$, and dentist $(n=3)$. The average duration of interviews was $53 \mathrm{~min}$. Seven core categories and 25 categories were generated through analysis. The core categories were divided into challenges unique to QOL research ("complexity of QOL research," "clinical applications of QOL research," "QOL scales," "QOL assessment learning methods") and challenges common to all clinical research ("research resources," "costs of study implementation," "basic knowledge of clinical research"). Conclusion: We found that several challenges common to all clinical studies, such as basic knowledge of clinical research, arise before one can conduct QOL assessment research. This suggests the need to consider different education methods for researchers with different levels of clinical research proficiency. It is also essential to create an environment that facilitates the implementation of QOL assessment research, while simultaneously considering strategies for the implementation and dissemination of QOL assessment research in clinical settings.

\section{(3029) Electronic visualizations of patient-reported outcome measure data: a systematic review}

Karen DiValerio Gibbs, MSN MPH RN, University of Texas Health Science Center at Houston, Houston, Texas, USA; Meagan

Whisenant, PhD APRN, University of Texas Health Science Center at Houston, Houston, Texas, USA; Ann Maliackal, University of Texas Health Science Center at Houston, Houston, Texas, USA; Elizabeth Pawelek, University of Texas Health Science Center at Houston, Houston, Texas, USA; Stacey Crane, PhD RN CPON, University of Texas Health Science Center at Houston, Houston, Texas, USA

Aims: Electronic patient-reported outcome measure (PROM) systems provide visualizations of PROM data that highlight trends in patient outcomes and can help clinicians' evaluate the effectiveness of medical interventions. However, visualizations of PROM data have not been extensively explored in the literature. The aim of this systematic review was to determine the content and design elements that have been used in electronic visualizations of PROM data for patients and clinicians, and to identify which elements are most effective in enhancing understanding and system use. Methods: We conducted a rigorous systematic review (PROSPERO 2020 CRD42020209076) to identify studies that used an electronic system for the collection of individual PROMs and included a data visualization component for clinicians or patients. Two authors independently screened and extracted data from the included articles. Research quality was assessed using the Mixed Methods Appraisal Tool. Results: We identified 42 articles that met inclusion criteria; 34 articles provided 29 unique visualizations from PROM systems; 8 articles compared different visualization design elements and/or electronic PROM systems. The visualizations were largely designed for use in cancer care $(59.5 \%, \mathrm{n}=25)$ in the outpatient or home setting. Most visualizations used line graphs $(48.3 \%, \mathrm{n}=14)$ or bar graphs $(27.6 \%, \mathrm{n}=8)$; none used icons or body maps. Almost all displayed data over time and allowed for simultaneous display of different PROM data for comparison. Frequently used design elements were judicious use of colors and shading to represent numerical quantities, grid lines on graphs, reference lines or shading to highlight goals or population averages, and the ability to view data details on demand. Few visualizations were adapted for patients with visual impairments and/or colorblindness, or offered customizable graphics. The research articles that compared the effectiveness of design elements were limited in scope and primarily used line graphs, bar graphs, and pictograph faces. Conclusion: It is essential to involve patient and clinician stakeholders in the design of PROM visualizations, to enhance understanding of PROM data and use of electronic PROM systems in clinical care. Further research that includes novel visualization designs is needed to determine which design elements are most effective in enhancing understanding and system use.

\section{(3030) Preliminary validation of the Patient-Generated Index in people with COPD}

Ava Mehdipour, MSc, McMaster University, Hamilton, Ontario, Canada; Sachi O’Hoski, PT, McMaster University, Hamilton, Ontario, Canada; Marla Beauchamp, PT, PhD, McMaster University, Hamilton, Ontario, Canada; Ayse Kuspinar, PT, PhD, McMaster University, Hamilton, Ontario, Canada

Aims: The Patient-Generated Index (PGI) is a well-known individualized measure of health-related quality of life (HRQoL). The PGI is unique in that it allows the participant to nominate the areas of life impacted by their health condition, enabling patients' perspectives to be captured. Chronic obstructive pulmonary disease (COPD) is a heterogenous condition in which symptoms, prognosis, and cause vary between patients. Each patient experiences the disease differently, therefore, having a measure that can capture this heterogeneity can provide researchers and clinicians with an accurate representation of patients' HRQoL. The PGI can be a promising tool for capturing COPD patients' HRQoL; however, there is limited evidence with regard to its validity. Therefore, the aim of this study was to evaluate the validity of the PGI in individuals with COPD. Methods: Data were obtained from a cross-sectional study where individuals with COPD were recruited from healthcare centers in Ontario, Canada. Convergent validity of the PGI was evaluated by assessing the correlation between PGI and RAND-36 subscale scores. Spearman's correlation coefficients were calculated, and moderate correlations (0.5) were hypothesized. Known-groups validity was evaluated by comparing PGI scores between individuals with moderate, severe, and very severe airflow limitation. An analysis of variance was performed, with a post-hoc Sidak correction, to assess the difference in PGI scores between severity groups, and the significance level was set at $<0.05$. Results: Sixty-six participants (mean age $=70$; median FEV1\% predicted $=36.5$ ) were enrolled in the study with a mean PGI score of 34.55 (out of 100). Correlations with RAND-36 subscale scores ranged from 0.11 (emotional well-being subscale) to 0.44 (physical function subscale). There was a significant difference in PGI scores between disease severity groups $(\mathrm{F}(2,52)=5.56$, $p=0.0065)$ and scores between moderate and very severe groups significantly differed $(p=0.008)$. Conclusion: Small to moderate correlations between the PGI and the RAND-36 do not imply that the PGI has low validity, but that it is providing unique information not captured by the RAND-36. Moreover, the PGI was able to discriminate between individuals with different COPD manifestations. Further studies are warranted to establish the PGI's responsiveness in individuals with COPD. 


\section{(3031) The complexity of HIV-related frailty: a structural equation modelling approach}

Mehmet Inceer, PhD, Mcgill University, Montréal, Quebec, Canada; Jan Boehnke, University of Dundee, Dundee, UK; Marie-Josee Brouillette, McGill University, Montreal, Quebec, Canada; Lesley Fellows, McGill University, Montreal, Quebec, Canada; Jose Morais, McGill University, Montreal, Quebec, Canada; Nancy Mayo, McGill University, Montreal, Quebec, Canada

Introduction: HIV has become a chronic manageable condition and the underlying reasons for mortality in people living with HIV are no longer directly caused by the virus. This means that a larger group of people will be aging with HIV and face age-related conditions such as frailty. Frailty is a multifactorial syndrome with causes originating from morbidities, genetics, lifestyle, and the environment. Consequently, frailty manifests on physiological, physical, emotional, cognitive, and social dimensions of health. The interconnectedness between frailty constructs is of interest to understand the manifestation of frailty in HIV. Objective: The objective is to estimate the structure and relationships between and among physiological, physical, emotional, cognitive, and social frailty constructs and their relationship with co-morbidity, personal factors, environmental factors, health perception, and quality of life in people living with HIV. Methods: The project uses longitudinal questionnaire data from the Positive Brain Health Network (BHN) cohort. The BHN is a prospective study that involved 856 persons (aged $>35$ ) living with HIV recruited since 2014 from five clinics across Canada and followed over four annual visits. The BHN dataset allows a comprehensive characterization of participants aging with HIV. A longitudinal conceptual model was developed to translate the notion of a HIV-specific frailty phenotype into empirical tests via structural equation models. The data analysis is currently underway, and the results will be ready to present for the conference. Implications: Investigating whether different frailty constructs share common contributors and consequences is important because frailty is likely to result in the emergence of disabilities eventually limiting mobility and independence. Focusing on an HIV cohort is likely to broaden our theoretical understanding of frailty, because frailty can appear as early as 50-year-old in people living with HIV, disconnecting the frailty from age.

\section{(3032) Pilot Implementation of Weekly Collection of ePROMs for Patients with Head and Neck Cancer during Radiotherapy}

Lydia Pascal, Guy's and St Thomas' NHS Foundation Trust, London, UK; Mieke Van Hemelrijck, King's College London, London, UK; Teresa Guerrero Urbano, Guy's and St Thomas' NHS Foundation Trust, London, UK; Mary Lei, Guy's and St Thomas' NHS Foundation Trust, London, UK

Aims: Head and neck cancer (HNC) and its treatment can have significant functional consequences that impair quality of life (QoL). Electronic patient outcome reported measures (ePROMs) enable close monitoring of QoL throughout a patient's cancer journey. A pilot is being conducted to assess the feasibility and acceptability of delivering an ePROMS programme to patients with HNC during radiotherapy (RT) and the follow-up period thereafter. This interim analysis aims to assess feasibility of weekly collection of ePROMs during RT. We report on patients who have completed the course of RT so far specifically compliance rates, completion times, and patient feedback of use. Methods: This pilot was registered as a service evaluation project. Following institutional approval eligible patients were approached after the initial oncology consultation and consented to take part. Patients completed the University of Washington v4.1 and MD Anderson Dysphagia Inventory (MDADI) questionnaires at baseline and weekly during RT accessed via a web link sent to the patient's phone via text message. Inclusion Criteria: English speakers, undergoing radical $\mathrm{RT}$ for $\mathrm{HNC}$, access to and able to use smart phone or home device. Results: To date, 11 patients have completed ePROMs over a 6 week course of RT (Dec 2020-April 2021). The median completion time reduced during the course of RT: $11 \mathrm{~min}$ (726) at baseline, $10 \min (30-7)$ in week 2 and remained between 6 and 7 min during the other weeks. $78 \%$ (60/77) of questionnaires were completed. Compliance reduced during the course of RT: $64 \%$ in week 2 and $55 \%$ in week 6 compared to $100 \%$ at baseline. Those with lower individual median compliance $(57-71 \%)$ had longer completion times (11-27 min). From patient feedback, at baseline $80 \%(\mathrm{n}=10)$ found ePROMS easy/very easy to complete, by week 6 this was $100 \%(n=7)$. Conclusion: The implementation of ePROMs appears feasible during RT. The majority of the patients completed ePROMs throughout RT. Lower compliance was associated with higher completion times indicating that this could be a barrier to use. Compliance was also consistently lower in the latter weeks of RT, in keeping with the time points of increased toxicity from RT.

\section{(3033) Participation in social activities and subjective well-being} among older adults on chronic pain medications

Irene Nsiah, MPH, University of Mississippi, Oxford, Mississippi, USA; Hyllore Imeri, Mr.Ph., University of Mississippi, Oxford, Mississippi, USA; John P Bentley, PhD, University of Mississippi, Oxford, Mississippi, USA; Sujith Ramachandran, PhD, University of Mississippi, Oxford, Mississippi, USA

Aims: The objective of this study was to examine the impact of gender on the relationship between social participation and subjective well-being among older adults using pain medications. Methods: A retrospective study was conducted using the 2019 (Round 9) National Health and Aging Trends Study (NHATS) data, a nationally representative longitudinal sample of Medicare beneficiaries aged 65 years and older. For the current study, individuals were included if they reported taking pain medications for five days or more per week over the last month. Individuals with Alzheimer's and/or dementia, as well as surveys completed by proxies, were excluded. Social participation was operationalized using the sum score of four items which include visiting family and friends, going out for enjoyment, attending religious services, and participation in other organized activities. Subjective well-being was operationalized as a latent variable using four items reflecting positive and negative emotions, and three items reflecting self-evaluation. Structural equation modeling was used to test the relationship between social participation and subjective wellbeing, as well as the moderating effect of gender, while adjusting for covariates. Results: A total of 964 participants were included in the study. A majority of participants were female $(61.3 \%)$, White $(81.0 \%)$, and community-dwelling $(94.9 \%)$ older adults. In addition, $44.0 \%$ were $65-74$ years, $40.9 \%$ were aged $75-84$ years, $15.1 \%$ were 85 years and older, and $48.8 \%$ were married or living with a partner. $26.6 \%$ of participants rated their health as excellent or very good, $37.2 \%$ as good or fair, and $36.2 \%$ as poor. Confirmatory factor analysis showed appropriate fit for subjective well-being. Social participation had a statistically significant association with subjective well-being ( $\beta=0.145 ; 95 \%$ CI $0.080,0.210 ; \mathrm{p}<0.001)$ after adjusting for demographic and clinical covariates. However, the relationship was not moderated by gender $(\mathrm{p}=0.737)$. Conclusion: Social participation is positively and significantly associated with subjective well-being among older adults on chronic pain medications. Interventions aimed at improving subjective well-being among 
the elderly should consider incorporating a social activities component, regardless of gender.

\section{(3034) Developing a Brief Index of the Health-Aspects of Quality of Life in Older People Living with HIV}

Kedar Mate, BSc (PT), MSc. PhD., Mayo Clinic/McGill University Health Centre, Montreal, Quebec, Canada; Bertrand Lebouché, MD. $\mathrm{PhD}$, Family Medicine, McGill University, Montreal, Quebec, Canada; Nancy Mayo, PT. PhD, McGill University, Montreal, Quebec, Canada

Aims: A challenge with current measures of health-related quality of life (HRQL), generic or disease specific, is that they are of the profile type, in that there are multiple domains each with multiple items leading to multiple scores. There are many advantages to having one score to represent multiple domains particularly when gains in one domain may be offset by losses in another domain, e.g., less pain, more fatigue. Creating an index requires a weighting system for the domains. An optimal weighting system would be to use preferences from the population of concern. In the context of HIV, only profile HRQL measures are available. Preference-based measures typically have one item per domain, and this "best" item represents the dimension. The global aim is to develop a short, HIV-specific, HRQL measure with a scoring system based on patient preferences for the different dimensions. To judge whether such an approach would yield interpretable results, the first step was to develop a prototype measure based on weights derived from health impact. Methods: The data came from the Canadian Positive Brain Health Now cohort $(n=854$; mean age 53 years) that used the Wilson-Cleary model for the measurement framework. Both standard format and individualized measures were available. The latter identified the important areas of life that are affected by HIV and items from the standard format measures were mapped to these areas and formed the domains. Rasch analysis was used to identify the best performing item to represent each dimension. Each item was then regressed on self-rated health (scored 0 to 100), and the regression parameters were used as weights to form an index score for the prototype measure. Results: The areas identified (9 impairment domains and 5 quality of life domains) from the individualized measure are listed in Table 1 along with the "best" item and the impact weight $(\beta)$ for each response option. Linearity across response options was observed for all items. Conclusion: The domains identified split between health-aspects of quality of life ( 9 impairment dimensions) and social aspects of quality of life (5 dimensions). This suggests that HRQL in HIV would be best represented by two separate measures.
Title: Developing a Brief Index of the Health-Aspects of Quality of Life in Older People Living with HIV

Table 1: Items representing dimensions and impact weights

\begin{tabular}{|c|c|c|c|c|}
\hline Health-aspect of quality of life & Response options & Levels & $\beta$ (SE) & SE \\
\hline \multicolumn{5}{|l|}{ Pain } \\
\hline \multirow[t]{3}{*}{ Pain/discomfort } & None & 1 & Referent & \\
\hline & Moderate & 2 & -9.68 & 1.04 \\
\hline & Extreme & 3 & -26.56 & 2.19 \\
\hline \multicolumn{5}{|l|}{ Depression } \\
\hline \multirow[t]{3}{*}{ Anxiety / Depression } & None & 1 & Referent & \\
\hline & Moderate & 2 & -9.51 & 1.04 \\
\hline & Extreme & 3 & -25.44 & 2.32 \\
\hline \multirow[t]{4}{*}{ How much do you enjoy life? } & An extreme amount & 1 & Referent & \\
\hline & A little & 2 & -6.75 & 1.71 \\
\hline & A moderate amount & 3 & -13.98 & 1.8 \\
\hline & Very much or greater & 4 & -23.65 & 2.01 \\
\hline \multicolumn{5}{|l|}{ Fatigue } \\
\hline \multirow[t]{4}{*}{ Did you have a lot of energy? } & None of the time & 1 & \begin{tabular}{|l} 
Referent \\
\end{tabular} & \\
\hline & Some of the time & 2 & -5.18 & 2.34 \\
\hline & Most of the time & 3 & -13.83 & 2.26 \\
\hline & All of the time & 4 & -23.42 & 2.35 \\
\hline \multicolumn{5}{|l|}{ Exercise tolerance } \\
\hline \multirow{3}{*}{$\begin{array}{l}\text { Vigorous activities, such as } \\
\text { running, lifting heavy objects, } \\
\text { participating in strenuous sports }\end{array}$} & Not limited at all & 1 & Referent & \\
\hline & Limited a little & 2 & -7.97 & 1.15 \\
\hline & Limited a lot & 3 & -20.04 & 1.36 \\
\hline \multicolumn{5}{|l|}{ Memory } \\
\hline \multirow{3}{*}{$\begin{array}{l}\text { Miss appointments and meetings } \\
\text { you had scheduled? }\end{array}$} & Never & 1 & Referent & \\
\hline & Rarely & 2 & -7.47 & 1.09 \\
\hline & Sometimes or more & 3 & -19.71 & 2.61 \\
\hline \multicolumn{5}{|l|}{ Perception of self/body image } \\
\hline \multirow[t]{3}{*}{$\begin{array}{l}\text { How satisfied are you with } \\
\text { yourself? }\end{array}$} & $\begin{array}{l}\text { One or more areas of my } \\
\text { body that I don't like }\end{array}$ & 1 & Referent & \\
\hline & Neither 1 nor 3 & 2 & -6.32 & 1.27 \\
\hline & $\begin{array}{l}\text { I am happy with my } \\
\text { bodily appearance }\end{array}$ & 3 & -16.99 & 1.74 \\
\hline \multicolumn{5}{|l|}{ Sleep } \\
\hline $\begin{array}{l}\text { Do you feel rested when you } \\
\text { wake up? }\end{array}$ & $\begin{array}{l}\text { Little or none of the } \\
\text { time }\end{array}$ & & Referent & \\
\hline
\end{tabular}

\begin{tabular}{|c|c|c|c|c|}
\hline & Some of the time & & -6.05 & 1.66 \\
\hline & All or most of the time & & -15.84 & 1.8 \\
\hline Cognition - concentration & & & & \\
\hline Have trouble concentrating on & Never & 1 & Referent & \\
\hline what people are saying during a & Rarely & 2 & -5.45 & 1.44 \\
\hline & Sometimes or more & 3 & -14.69 & 1.93 \\
\hline Anxiety & & & & \\
\hline HADS: worrying thoughts go & A great deal of the time & 1 & Referent & \\
\hline through my mind & A lot of the time & 2 & -4.63 & 1.32 \\
\hline & Not too often or never & 3 & -12.91 & 1.29 \\
\hline Participation & & & & \\
\hline Usual activities & None & 1 & Referent & \\
\hline & Moderate & 2 & -15.64 & 1.18 \\
\hline & Extreme & 3 & -31.39 & 5.06 \\
\hline Leisure & & & & \\
\hline To what extent do you have the & Completely & 1 & Referent & \\
\hline activities? & Mostly & 2 & -7.84 & 1.46 \\
\hline & Moderately or more & 3 & -19.06 & 1.72 \\
\hline Lonely & & & & \\
\hline Do you find yourself feeling & Almost never & 1 & Referent & \\
\hline & \begin{tabular}{|l|} 
Sometimes \\
\end{tabular} & 2 & -5.97 & 1.19 \\
\hline & Quite often & 3 & -13.63 & 1.56 \\
\hline Apathy/motivation & & & & \\
\hline Do you have plans and goals for & Not at all & 1 & Referent & \\
\hline the future? & Some & 2 & -5.13 & 1.89 \\
\hline & A lot & 3 & -12.75 & 1.87 \\
\hline Resilience & & & & \\
\hline How much do you fear for the & Not at all & 1 & Referent & \\
\hline & A little & 2 & -4.31 & 1.32 \\
\hline & \begin{tabular}{|l|}
$\begin{array}{l}\text { A moderate amount or } \\
\text { more }\end{array}$ \\
\end{tabular} & 3 & -11.29 & 1.44 \\
\hline Social support & & & & \\
\hline About how many times did you & Less than once a week & 0 & Referent & \\
\hline talk to someone (friends, & Once a week & 1 & -1.77 & 1.21 \\
\hline telephone in the past week? & $\begin{array}{l}\begin{array}{l}\text { Almost every day or } \\
\text { daily }\end{array} \\
\end{array}$ & 2 & -10.55 & 1.57 \\
\hline
\end{tabular}




\section{(3035) Estimation of meaningful change thresholds} for the EORTC QLQ-C30 and QLQ-LC13 in patients with nonsmall cell lung cancer (NSCLC)

Jennifer Beaumont, MS, Clinical Outcomes Solutions, Tucson, Arizona, USA; Hailin Yu, Clinical Outcomes Solutions, Tucson, Arizona, USA; Huamao M Lin, Millennium Pharmaceuticals, Inc., a wholly owned subsidiary of Takeda Pharmaceutical Company Limited, Cambridge, Massachusetts, USA; Erin Goodman, Millennium Pharmaceuticals, Inc., a wholly owned subsidiary of Takeda Pharmaceutical Company Limited, Cambridge, Massachusetts, USA; Shoshana Hecht, Xcenda L.L.C., Carrollton, Texas, USA; Kimberly Le, Xcenda L.L.C., Carrollton, Texas, USA; Heather Romero, Millennium Pharmaceuticals, Inc., a wholly owned subsidiary of Takeda Pharmaceutical Company Limited, Cambridge, Massachusetts, USA; Stacie Hudgens, Clinical Outcomes Solutions, Tucson, Arizona, USA

Aims: To further inform interpretation of HRQoL associated with brigatinib in anaplastic lymphoma kinase-positive (ALK+) NSCLC, this analysis aimed to derive meaningful change thresholds (MCTs) for all domains of the European Organisation for Research and Treatment of Cancer Quality of Life Questionnaire Cancer-30 item (QLQ-C30) and 13-item lung cancer module (QLQ-LC13) in patients with ALK+ advanced lung cancer. This analysis also assessed the measurement validity of a 4-item dyspnea scale created from these 2 measures. Methods: Based on data from ALTA-1L (NCT02737501), a Phase 3 multicenter open-label study of brigatinib versus crizotinib in patients with ALK+ advanced lung cancer, and Rasch analysis was used to assess measurement and discriminant validity of the newly created 4-item dyspnea scale. Then, the QLQ-C30 items for overall health and quality of life were used as anchors to estimate MCTs for all domains. Polyserial correlation coefficients were calculated between changes in domain scores from Baseline to Cycle 5 and changes in anchor variables. Where adequate correlation $(>0.40)$ was observed, within-group change scores were examined with respect to the standardized effect size, paired t-test for the change from Baseline, and consideration of the confidence intervals associated with the no change and improved/worsened groups. Results were triangulated across anchors to determine estimated MCTs. Results: The 4-item dyspnea scale adequately assessed the latent symptom severity continuum per Rasch analyses. The anchors had correlations $>0.40$ with all functioning domains, fatigue, pain, appetite loss, and all dyspnea scores, indicating adequacy for these analyses. Correlations between both anchors and remaining symptom domains were $<0.40$, so within-group results were not examined. MCTs suggested by withingroup analyses are summarized in Table 1, along with raw score categorical change equivalents. A two-category change was found to be meaningful for physical and emotional functioning and the multiitem dyspnea scales. A single categorical change was found to be meaningful for the remaining scales. Conclusion: MCTs were derived for the QLQ-C30 and QLC-LC13 domains, including a newly created composite dyspnea endpoint using anchor-based techniques, enhancing the interpretation of these outcomes for evaluating the HRQoL benefit from brigatinib treatment among patients with ALK + NSCLC.
Table 1. Correlations between anchors and EORTC QLQ-C30 and LC13 scores and recommended MCTs

\begin{tabular}{|l|c|c|c|c|}
\hline & $\begin{array}{c}\text { Polyserial correlation for change from } \\
\text { Baseline to Cycle 5 }\end{array}$ & \multicolumn{2}{c|}{ MCT recommendation } \\
\hline Domain & $\begin{array}{c}\text { Anchor }=\text { overall } \\
\text { health }\end{array}$ & $\begin{array}{c}\text { Anchor }=\text { quality } \\
\text { of life }\end{array}$ & 0 to 100 score & $\begin{array}{c}\text { Raw score } \\
\text { category change }\end{array}$ \\
\hline Physical functioning & 0.62 & 0.67 & 8 to 13 & 2 \\
\hline Role functioning & 0.58 & 0.64 & 8 to 13 & 1 \\
\hline Emotional functioning & 0.56 & 0.52 & 11 to 17 & 2 \\
\hline Cognitive functioning & 0.46 & 0.45 & 7 to 12 & 1 \\
\hline Social functioning & 0.52 & 0.58 & 11 & 1 \\
\hline Fatigue & -0.66 & -0.67 & 6 to 7 & 1 \\
\hline Pain & -0.50 & -0.51 & 12 to 14 & 1 \\
\hline Appetite loss & -0.50 & -0.45 & 11 to 20 & 1 \\
\hline 1-item dyspnea (QLQ-C30) & -0.44 & -0.46 & 11 to 15 & 1 \\
\hline 3-item dyspnea (QLQ-LC13) & -0.48 & -0.53 & 13 to 15 & 2 \\
\hline 4-item dyspnea (combined) & -0.50 & -0.54 & 13 to 15 & 2 \\
\hline
\end{tabular}

(3036) Qualitative methods for research in response shift: development of methodological guidelines

Antoinette Davey, University of Exeter, Health Service and Policy Research Group, Exeter, UK; Bernice Gulek-BakirCI Washington State University, Redmond, Washington, USA; Kathryn Lasch, Patient Voice Matters Consulting LLC, Newton, Massachusetts, USA; Nikki Ow, McGill University, The University of British Columbia, Vancouver, British Columbia, Canada; Kara SchickMakaroff, University of Alberta, Edmonton, Alberta, Canada; Stine Thestrup Hansen, Zealand University Hospital, Roskilde, Denmark

Aims: Response shift (RS) occurs in Patient-Reported Outcomes Measures (PROM) when circumstances arise over time that make people change their evaluation of the underlying construct. Qualitative methodologies may be used to determine the presence of RS. However, there are no reporting guidelines for qualitative studies to use when reporting RS. The aim of this study was to develop methodological guidelines for qualitative research in RS. Methods: A literature search in PubMed was undertaken, along with hand searching of research conducted by the Qualitative Response Shift Group, to identify RS articles that used qualitative approaches. A framework analytical method to analysis allowed for qualitative themes to be organized into a matrix highlighting areas of RS. COREQ, an existing reporting guideline for qualitative studies, was used to not only analyze included texts, but also suggest additional elements of reporting in qualitative RS studies. Schwartz et al.'s theoretical model of RS further informed analysis. Extracted data from texts (e.g. approach, questions, analytical methods) were used to support recommendations and provide in-depth information on how to conduct, analyze, and report qualitative research on RS. Results: Six articles were identified and analyzed using COREQ and methodological guidelines for each element of qualitative approaches to studying RS were created, from study design to analysis. Three studies used a longitudinal mixed methodological approach. Sample sizes ranged from 19 to 50 . To detect RS, two studies used the cognitive think-aloud method and the then-test, one used vignettes, and the rest used narratives. Thematic and content analysis was used in all studies. Two studies used the Sprangers and Schwartz's model in designing and analyzing study results. Questions used in the qualitative element of the studies were designed to explore what informed RS at the time of completion. Overall, the use of a theoretical model of RS to guide qualitative research and a longitudinal design appeared to be the most often used. Conclusion: Recommendations in each stage of the research process for qualitative results of RS studies are provided. Guidelines on how to conduct, analyse, and report on RS using qualitative methods will provide PROM researchers with a framework to confidently follow. 
(3037) Measuring Duchenne muscular dystrophy impact: Development of a proxy-reported measure derived from PROMIS item banks

Roland Stark, M.Ed., DeltaQuest Foundation, Concord, Massachusetts, USA; Katrina Borowiec, M.A., Boston College Lynch School of Education, Chicopee, Massachusetts, USA; David Cella, $\mathrm{PhD}$, Northwestern University, Chicago, Illinois, USA; Katherine Gooch, PhD, Sarepta Pharmaceuticals, Cambridge, Massachusetts, USA; Ivana Audhya, M.Sc., Sarepta Pharmaceuticals, Cambridge, Massachusetts, USA; Carolyn Schwartz, Sc.D, DeltaQuest Foundation, Concord, Massachusetts, USA

Aims: Person-reported outcomes (PRO) measurement development for rare diseases has lagged behind that of more common diseases. The present work aimed to create a condition-specific proxy-report measure for Duchenne Muscular Dystrophy (DMD) by drawing relevant items from available item banks and supplementing as needed. Methods: This web-based study recruited DMD caregivers via Rare Patient Voice, patient-advocacy groups, and word of mouth. Recruitment was stratified by age of the caregiver's child with DMD: $2-7,8-12,13-17$, and $>18$. Telephone interviews were conducted with DMD parent-caregivers to pretest possible measures for content validity. The web-based study utilized an algorithm to categorize respondents' ambulatory status for tailored administration of PRO Measurement Information System (PROMIS) Parent-Proxy items as well as some new items developed based on caregiver interviews. Psychometric analyses were implemented according to item response theory (IRT) standards. Results: The study sample included 521 DMD caregivers representing equally the four age strata. The caregiver sample had a mean age of 41.5, was drawn from the contiguous USA, and was $76 \%$ female. The new measure included the following domains: fatigue impact, strength impact, cognitive function, upper extremity function, positive affect, negative affect, sleep-device symptoms, and mobility. The first five domains had strong psychometric characteristics (unidimensionality; acceptable model fit; strong standardized factor loadings; high marginal reliability). Negative Affect fit a bifactor model, which was expected since its items were drawn from varied item banks covering anger, anxiety, depressive symptoms, and psychological stress. The General factor from this bifactor model had good model fit, high marginal reliability, and strong factor loadings. Sleep-device symptoms were not unidimensional, and mobility did not have a simple structure due to residual correlations among items at opposite end of the mobility-disability continuum. These two domain scores were retained as clinimetric indices (i.e., uncalibrated scales), to achieve the overall goal of having a content-valid DMD-specific measure across all stages of disease severity. Conclusion: The present study created a DMD-specific proxyreport measure derived from PROMIS item banks and supplemental items that could potentially be utilized across all stages of DMD. Future research will focus on assessing the responsiveness and validity of the measure over time.

\section{(3038) PROMIS-29 and EORTC QLQ-C30-an empirical investigation towards a common conception of health}

Claudia Hartmann, Charité - Universitaetsmedizin Berlin, Berlin, Germany; Felix Fischer, PhD, Department of Psychosomatic Medicine, Center of Internal Medicine and Dermatology, Charité Universitätsmedizin Berlin, Berlin, Germany, Berlin, Germany; Mathias Rose, Prof., Department of Psychosomatic Medicine, Center of Internal Medicine and Dermatology, Charité-Universitätsmedizin Berlin, Berlin, Germany, Berlin, Germany; Maria M. Karsten, PhD, MD, Department of Gynecology with Breast Center, Charité University Hospital Berlin, Berlin, Germany, Germany
Aims: The assessment of health-related quality of life (HRQOL) has become a key component not only in clinical trials but also in clinical routine worldwide. PROMIS-29 as generic and EORTC QLQ-C30 as disease-specific patient-reported outcome measures share a similar concept of HRQOL and report outcomes on a comparable T-metric anchored to general population samples. In this study, we investigate the empirical agreement of these underlying concepts. Methods: We collected PROMIS-29 and QLQ-C30 data from 2,379 female patients at a breast cancer outpatient center. We calculated descriptive statistics and Pearson correlations between PROMIS-29 and EORTC-QLQ-C30 scales. We performed exploratory (EFA) and confirmatory factor analysis (CFA) in randomly split subsamples in order to assess the underlying psychometric structure of both instruments. Results: The only female cohort (mean age $=47.27, \pm 14.5$ )-reported mean HRQOL scores were comparable between the corresponding subscales of both instruments and similar to general population reference values. Correlation between the corresponding subscales of both instruments ranged between 0.66 (Social Functioning) and 0.79 (Physical Functioning). A six-factor EFA and model-driven CFAs provided further support for conceptual agreement of the scales. Conclusion: EORTC QLQ-C30 and PROMIS-29 showed similar scores and satisfactory agreement in clinical routine care. This suggests that the underlying conceptualization of health is reasonably close. Hence, the development of score transformation algorithms or calibration of both instruments on common scales could prospectively increase comparability of clinical and research PRO data collected with either instrument.

(3039) Successful incorporation of electronic patient-reported outcomes using PROMIS-29 into standard of care for lymphoma patients receiving CD19-directed chimeric antigen receptor $T$ (CAR T) cell therapy

Gunjan L. Shah, MD MS, Memorial Sloan Kettering Cancer Center, New York, New York, USA; Michael Scordo, MD, Memorial Sloan Kettering Cancer Center, New York, New York, USA; Craig Sauter, MD, Memorial Sloan Kettering Cancer Center, New York, New York, USA; Connie Batlevi, MD PhD, Memorial Sloan Kettering Cancer Center, New York, New York, USA; Parastoo B. Dahi, MD, Memorial Sloan Kettering Cancer Center, New York, New York, USA; Richard Lin, MD, Memorial Sloan Kettering Cancer Center, New York, New York, USA; M.lia Palomba, MD, Memorial Sloan Kettering Cancer Center, New York, New York, USA; Miguel-Angel Perales, MD, Memorial Sloan Kettering Cancer Center, New York, New York, USA; Sergio A. Giralt, MD, Memorial Sloan Kettering Cancer Center, New York, New York, USA

Aims: CD19 directed chimeric antigen receptor T (CAR T) cell therapy is a novel treatment for patients with relapsed/refractory diffuse large B cell lymphoma with great clinical benefit, but potentially significant toxicities. Using our center's electronic platform, we aimed to integrate the collection of patient-reported outcomes into standard of care to improve these patients' clinical experience. Methods: Patients received the PROMIS-29 survey through the patient portal. Baseline surveys were automatically generated by a chemotherapy order, and follow-up surveys on Day 0 (infusion), weekly for the first month (partly inpatient), monthly for the first year, and every 3 months for the second year were generated by the cell infusion order. Patients were censored from further surveys at time of relapse or progression of disease. Survey responses in preidentified severe ranges prompted instructions to call the clinician's office and for the clinical team to call the patient. Results: From May 2019 to December 2020, 65 English-speaking patients received commercially available CAR T cells (31 tisagenlecleucel, 34 axicabtagene ciloleucel), with median age of 65 (range 24-83) and 34\% 
female. $46 \%$ patients were not censored, and $72 \%$ were alive as of $12 / 31 / 20$. IT issues around survey appearance in the portal were resolved by 3 months after the initiation of the surveys. Compliance was similar across age groups when divided by decade. The survey response rate at the baseline time point was $55 \%$, decreased while the patients were admitted, and improved to $40-50 \%$ of eligible patients at most later timepoints (Fig. 1). Conclusion: We demonstrate the feasibility of incorporating patient-reported outcomes into standard of care practice after CAR T cell therapy. Missing surveys were more common while inpatient which may be due to toxicity or less use of the patient portal while admitted. Additional work is ongoing to improve the response rates at all time points. Importantly, the electronic system allowed us to capture later timepoints when patients have less frequent visits and may be followed locally. Full survey results will also be presented.

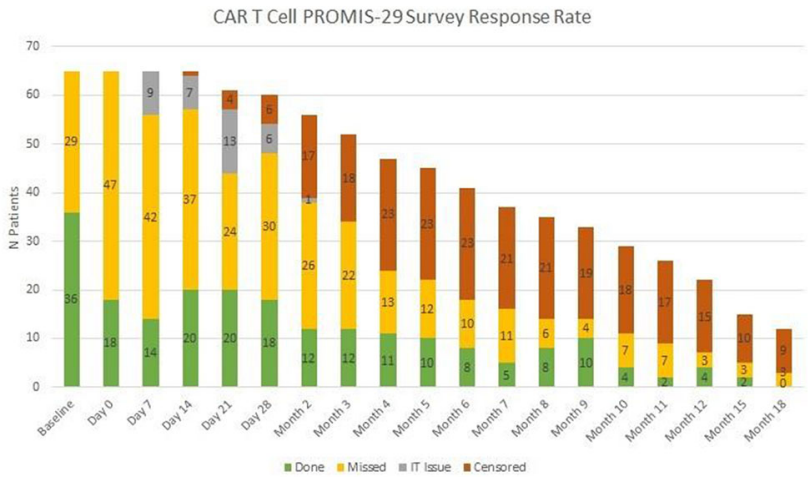

(3040) Using PROMIS-preference (PROPr) Scores to Measure Changes in Quality of Life After Tyrosine Kinase Inhibitor Discontinuation

Aaron Winn, PhD, MPP, Medical College of Wisconsin, Milwaukee, Wisconsin, USA; Ehab Atallah, MD, Medical College of Wisconsin, Milwaukee, Wisconsin, USA; Li Lin, PhD, Duke University, Durham, North Carolina, USA; James Thompson, MD, MS, Roswell Park Comprehensive Cancer Center, Buffalo, New York, USA; Joseph Moore, MD, Duke University, Durham, North Carolina, USA; Richard Larson, MD, The University of Chicago, Chicago, Illinois, USA; Jorge Cortes, MD, Augusta University, Augusta, Georgia, USA; Michael Mauro, MD, Memorial Sloan Kettering Cancer Center, New York City, New York, USA; Javier Pinilla, MD, PhD, Moffitt Cancer Center, Tampa, Florida, USA; Kathryn Flynn, PhD, Medical College of Wisconsin, Milwaukee, Wisconsin, USA

Aims: Trials suggest that discontinuation of tyrosine kinase inhibitor (TKI) therapy is safe for patients with chronic myeloid leukemia (CML) who have a sustained deep molecular response. The Life After Stopping TKIs (LAST) study was the first to evaluate patient-reported outcomes (PROs) after TKI discontinuation among U.S. patients. It found that TKI discontinuation was associated with modest improvements in PROMIS PROs, while for patients who restarted a TKI for disease recurrence, PROs worsened again. This study examines if similar results are seen when the PRO scores are converted to a preference-based measure, which will also be used in future health economic evaluations. Methods: PROs were measured up to 14 times over 3 years using PROMIS computerized adaptive tests. PROMIS preference (PROPr) scores reflecting health utilities were computed based on published crosswalks. We estimated two models. The first model included patients who had stopped and remained off of TKIs, and the second model included only observations after patients restarted TKIs. To account for repeated observations, we used generalized estimating equations with a $\log$ link and Gaussian distribution. For both models, we treated each follow-up visit as an indicator variable. We present model-generated predicted values. Results: After patients discontinued TKIs, their PROPr scores improved from baseline results. Compared to baseline, we immediately see an increase at 1 month after discontinuation (coefficient $0.13,95 \%$ CI $0.07,0.20$ ), which is sustained among patients that remain off TKI until the final observation at 36 months (coefficient $0.20,95 \%$ CI $0.13,0.28$ ), predicted values shown in Fig. 1. After restarting TKIs, there were no statistically significant difference in follow-up months. However, compared to month 2 PROPr scores we see a non-significant decline in months 4 (coefficient $-0.23,95 \% \mathrm{CI}-0.87,0.41$ ) and 5 scores (coefficient -0.46 , $95 \%$ CI $-1.13,0.21)$ Conclusion: TKI discontinuation results in a $20 \%$ improvement in health utilities as estimated using PROPr mapping. These data can help patients understand that stopping TKIs generally improves health-related quality of life. Moreover, these results can be used for future cost-effectiveness analysis.

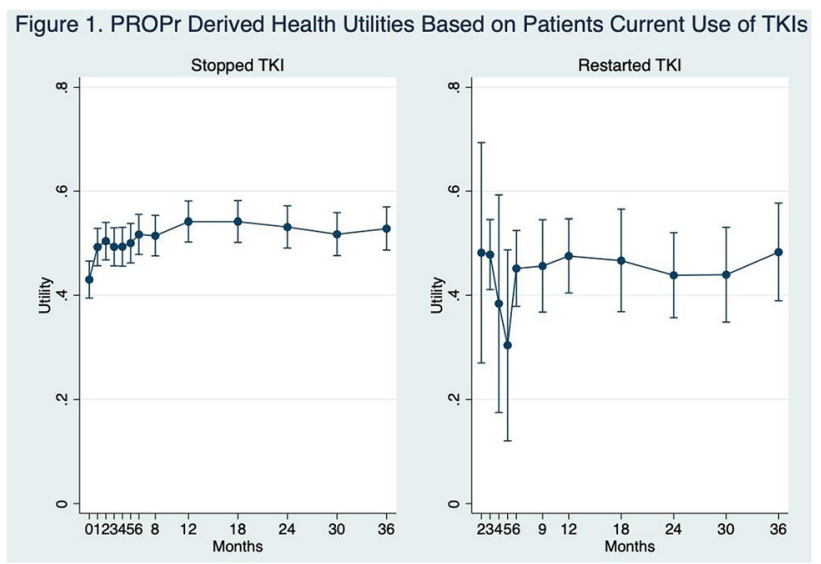

(3041) Psychometric properties of the WHOQOL-BREF in Mexican older adults

Ana L. Gonzalez-Celis, PhD, National University of Mexico, Ciudad de Mexico, Mexico; Juana Gomez-Benito, PhD, University of Barcelona, Barcelona, Spain; Karina Reyes-Jarquin, PhD, National University of Mexico, Mexico, Mexico; Leticia Bautista-Diaz, PhD, National University of Mexico, Mexico, Mexico; Ana G. MagallanesRodriguez, PhD, University of Baja California, Baja California, Mexico; Margarita Chavez-Becerra, Master, National University of Mexico, Mexico, Mexico; Edgardo Ruiz-Carrillo, PhD, National University of Mexico, Mexico, Mexico

Aims: The main aim of this study was to examine the psychometric properties of the WHOQOL-BREF as a generic instrument for assessing the quality of life among Mexican older adults. Methods: A total of 847 adults aged $60-90$ years $($ mean $=69.38, \mathrm{SD}=6.87), 490$ $(57.9 \%)$ women volunteered to participate and gave informed consent for application of the questionnaires: WHOQOL-BREF, Philadelphia Geriatric Center (PGC) Morale Scale, and Multifaceted Inventory of Loneliness (MIL).They had a medium-to-low economic level, with average schooling of six years. Results: There was no evidence of ceiling or floor effects for any of the WHOQOL-BREF items. Nonnormal distributions were found for all domains, but not for the WHOQOL-Total. Less than $1 \%$ of data was missing for all items, except for the item referring to sexual activity (2\%).The theoretical four-factor structure was confirmed, and internal consistency was shown to be acceptable (Cronbach's alpha of 0.868 for the 24 items).All domain scores, except that for the Psychological domain, indicated discriminant validity between women and men. Likewise, all domains of the WHOQOL discriminated well between sick and healthy respondents, with the effect size for total quality of life being 
moderate $(d=0.550)$. There were significant associations between the domains of the WHOQOL, between WHOQOL and PGC Morale Scale, and MIL. Conclusion: The World Health Organization (WHO) has developed the WHOQOL- BREF to assess the quality of life. Although this instrument has been studied in several countries the literature is scarce in Latin America, especially about older people. The fact that higher scores were obtained on the Social Relationships and Psychological domains than on the Physical and Environment domains highlights the importance of the social and psychological dimensions when assessing the quality of life. These results may also reflect the significance which these Mexican elders ascribe to different aspects of their lives and serve to remind us of the relevance of these dimensions when conducting cross-cultural research on the quality of life. The adaptation of the WHOQOL-BREF for use with Mexican older adults shows good psychometric properties, and it can be regarded as a reliable instrument for assessing their quality of life.

\section{(3042) Measurement properties of patient-reported outcome measures (PROMs) in hyperhidrosis: a systematic review}

Michaela Gabes, Otto-von-Guericke University Magdeburg, Magdeburg, Germany; Helge Knüttel, University of Regensburg, Regensburg, Germany; Gesina Kann, University of Regensburg, Regensburg, Germany; Christina Tischer, Bavarian Health and Food Safety Authority, Bad Kissingen, Germany; Christian Apfelbacher, Otto-von-Guericke-University, Magdeburg, Germany

Aims: To evaluate the methodological quality of studies reporting measurement properties of patient-reported outcome measures (PROMs) in hyperhidrosis by applying the COnsensus-based Standards for the selection of health Measurement INstruments (COSMIN) Risk of Bias checklist. Thereby, we are able to give a recommendation for the use of PROMs in future clinical trials in hyperhidrosis. Methods: Studies which evaluated, described, or compared measurement properties of patient-reported outcome measures were considered as eligible. We performed a systematic literature search in MEDLINE, EMBASE, and Web of Science. The methodological quality of each study was assessed using the COSMIN Risk of Bias checklist. Furthermore, predefined quality criteria for good measurement properties were applied, and the quality of the evidence was graded. Results: Twenty-three articles reporting on 13 patientreported outcome measures were included. Three instruments can be further recommended for use. They showed evidence for sufficient content validity and moderate to high quality evidence for sufficient internal consistency. The methodological assessment showed existing evidence gaps for nine other PROMs, which therefore require further validation studies to make an adequate decision on their recommendation. The short-form health survey with 36 items (SF-36) is the only questionnaire not recommended for use in patients with hyperhidrosis due to moderate to high quality evidence for insufficient measurement properties. Conclusion: Three PROMs, the Hyperhidrosis Quality of Life Index (HidroQoL), the Hyperhidrosis Questionnaire (HQ), and the Sweating Cognitions Inventory (SCI) can be recommended for use in future clinical trials in hyperhidrosis. Results obtained with these three instruments can be seen as trustworthy. Nevertheless, further validation of all three PROMs is desirable.

\section{(3043) Do existing outcome measures capture the burden of caring for an individual with neurogenic orthostatic hypotension? A qualitative concept mapping study}

Katy Gallop, Acaster Lloyd Consulting Ltd, London, UK; Ngan Pham, Theravance Biopharma US, Inc., South San Francisco, California, USA; Grant Maclaine, Theravance Biopharma Ireland
Limited, Dublin, Ireland; Emma Saunders, Multiple System Atrophy Trust, London, UK; Bonnie Black, Vanderbilt University Medical Center, Nashville, Tennessee, USA; Sarah Acaster, Acaster Lloyd Consulting Ltd, London, UK

Aims: This study explored the health-related quality of life (HRQoL) impact experienced by informal caregivers of individuals with neurogenic orthostatic hypotension $(\mathrm{nOH})$ and either Parkinson's disease (PD), multiple system atrophy (MSA), pure autonomic failure (PAF) or dementia with Lewy bodies (DLB) and evaluated the content validity of several existing outcome measures used to capture caregiver burden. Methods: Semi-structured, qualitative telephone interviews were conducted with informal caregivers of individuals with a confirmed diagnosis of $\mathrm{nOH}$ and either PD, MSA, PAF or DLB. Data were analysed using thematic analysis and a conceptual model was developed using the concepts identified in the analysis. Concept mapping compared the concepts reported in the interviews to concepts measured in five instruments: EQ-5D, Burden Scale for Family Caregivers-short (BSFC-s), Zarit Burden Interview-12 (ZBI-12), CarerQoL, and the Hospital Anxiety and Depression Scale (HADS). Results: Twenty participants (mean age 58 years) were interviewed (August-September 2020), 70\% were female of whom 65\% cared for their spouse and $70 \%$ cared for an individual with PD. Most care recipients $(70 \%)$ were prescribed pharmacologic treatment for their $\mathrm{nOH}$. All participants reported an impact on their time, particularly patient supervision needed to prevent falls. Many also reported negative emotional impacts, including worry or anxiety about falls, stress, and depression. Other areas of caregiver impact included physical well-being, work, independence, relationships, and social/leisure activities. Concept mapping showed that all concepts measured in the CarerQoL and most EQ-5D concepts were reported in the interviews. Several concepts in the BSFC-s and ZBI-12 were not reported, however, some (e.g. 'feeling torn') may overlap with concepts caregivers reported (e.g. stress). Anxiety and depression were reported spontaneously, however, it was largely related to the risk of the patient falling or being injured, which is not included in any of the outcome measures. Other concepts reported and not included in the measures were lack of freedom, loneliness, and frustration. Conclusion: This is the first qualitative study exploring the impact of caring for an individual with $\mathrm{nOH}$ and highlights the wide-ranging negative impact of $\mathrm{nOH}$ on caregivers. Concept mapping indicates existing measures may not fully capture caregiver burden in $\mathrm{nOH}$.

\section{(3044) Translation challenges associated with the use of variables} in eCOA software development

Rachna Kaul, MDS, MPA, RWS Life Sciences, East Hartford, Connecticut, USA; Alisa Heinzman, MFA, RWS Life Sciences, Broomfield, Colorado, USA; Carolyn Schulz, BA, RWS Life Sciences, East Hartford, Connecticut, USA; Christian Lyon, MBA, RWS Life Scinces, Broomfield, Colorado, USA; Tim Poepsel, PhD, RWS Life Sciences, East Hartford, Connecticut, USA; Allyson Nolde, MPP, RWS Life Sciences, Chicago, Illinois, USA; Rebecca Nolde, MS, RWS Life Sciences, Boston, Massachusetts, USA; Brittanie Newton, BA, RWS Life Sciences, East Hartford, Connecticut, USA; Elizabeth McCullough, MA, RWS Life Sciences, East Hartford, Connecticut, USA; Shawn McKown, MA, RWS Life Sciences, East Hartford, Connecticut, USA;

Aims: Languages differ in their linguistic requirements, and words or phrases cannot always be cleanly substituted in the process of translation. For example, variables like " $x$ " seem reasonable in the English phrase "I had $x$ headaches last week", where " $x$ " stands for a number of two or more. However, if this phrase is translated, the linguistic requirements of the target language may prohibit a simple substitution and necessitate a different approach to retain the 
grammatical accuracy of the translation. The aim of this research was to assess the translation challenges that arise when variables are inserted into the programming of electronic Clinical Outcomes Assessments (eCOA) undergoing translation. Methods: We reviewed 23 translations from 3 eCOA linguistic validation -projects in which variables were used to replace words or phrases and identified 3 categories of disruption: body part variables, recall periods variables, and numeric/ordinal variables. Results: In English, lexical inflection (i.e., changing the structure of a word) based on its placement within a sentence is less common than in other languages. Due to this the same English word for a body part (e.g., knee) may need to be translated differently in different sentences in order to be grammatically correct. Similarly, varying /recall periods, like past two weeks or last month, can be substituted for one another in English sentences. However, other languages require consideration of gender and/or number. Sentences that use numeric/ordinal variables also need to be translated separately, for languages that express grammatical number differently than English. Table 1 gives examples of some of the variable errors encountered. Conclusion: Ensuring the proper display of global languages on an electronic device is an ongoing industry challenge. Use of variables in text for translation can introduce translation inaccuracy and timeline delays due to additional software development work or rounds of review. To overcome these hurdles, we recommend omitting variables from eCOA software builds whenever possible, or storing sentence-level versions of all iterations to remove the possibility of linguistic problems. Taking these linguistic variations into consideration during the software build process for eCOAs is critical to ensuring a positive patient experience.

Table 1: Examples of variable issues in $\mathrm{eCOA}$ translations

\begin{tabular}{|c|c|c|c|c|}
\hline \multicolumn{5}{|c|}{ Body part variable issue } \\
\hline Source & Language & $\begin{array}{l}\text { Translation } \\
\text { created by the } \\
\text { use of } \\
\text { variables }\end{array}$ & $\begin{array}{l}\text { Description of } \\
\text { the issue }\end{array}$ & $\begin{array}{l}\text { Corrected } \\
\text { translation }\end{array}$ \\
\hline \multirow{2}{*}{$\begin{array}{l}\text { Think about } \\
\text { your (Left } \\
\text { Knee) Joint } \\
\text { when } \\
\text { answering the } \\
\text { questionnaire. } \\
\text { Indicate the } \\
\text { severity of } \\
\text { your pain and } \\
\text { stiffness and } \\
\text { the difficulty } \\
\text { you have in } \\
\text { doing daily } \\
\text { activities that } \\
\text { you feel are } \\
\text { caused by the } \\
\text { arthritis in } \\
\text { your (Left } \\
\text { Knee Joint). }\end{array}$} & $\begin{array}{l}\text { Estonian- } \\
\text { Estonia }\end{array}$ & $\begin{array}{l}\text { Küsimustikule } \\
\text { vastates mõelge } \\
\text { oma vasakule } \\
\text { põlvele. } \\
\text { Näidake, kui } \\
\text { tugevat valu, } \\
\text { liigese jäikust } \\
\text { vôi füüsilist } \\
\text { võimetust olete } \\
\text { tundnud artroosi } \\
\text { (liigesehaiguse) } \\
\text { tôttu oma } \\
\text { vasakule põlvele }\end{array}$ & \multirow{2}{*}{\begin{tabular}{|l|} 
In Estonian- \\
Estonia and \\
German- \\
Germany, nouns \\
like different \\
body parts, \\
change based \\
on their \\
placement within \\
a sentence. The \\
source just \\
shows (the \\
selected joint) \\
text or "Left \\
Knee Joint" in \\
this example, \\
without \\
indicating that it \\
is the variable. \\
Therefore, the \\
same translated \\
varibale can't be \\
used in place of \\
the noun in \\
different parts of \\
the sentence.
\end{tabular}} & $\begin{array}{l}\text { Küsimustikule } \\
\text { vastates mõelge } \\
\text { oma vasakule } \\
\text { pölvele. } \\
\text { Näidake, kui } \\
\text { tugevat valu, } \\
\text { liigese jäikust } \\
\text { vôi füüsilist } \\
\text { võimetust olete } \\
\text { tundnud artroosi } \\
\text { (liigesehaiguse) } \\
\text { tõttu oma } \\
\text { vasakus põlves. }\end{array}$ \\
\hline & $\begin{array}{l}\text { German- } \\
\text { Germany }\end{array}$ & $\begin{array}{l}\text { Bei der } \\
\text { Beantwortung ... } \\
\text { bitte nur an Ihr } \\
\text { Linkes Knie... }\end{array}$ & & $\begin{array}{l}\text { Bei der } \\
\text { Beantwortung ... } \\
\text { bitte nur an Ihr } \\
\text { Linken Knie... }\end{array}$ \\
\hline \multicolumn{5}{|c|}{ Recall period variable issues } \\
\hline Source & Language & $\begin{array}{l}\text { Translation } \\
\text { created by the } \\
\text { use of } \\
\text { variables }\end{array}$ & $\begin{array}{l}\text { Description of } \\
\text { the issue }\end{array}$ & $\begin{array}{l}\text { Corrected } \\
\text { translation }\end{array}$ \\
\hline $\begin{array}{l}\text { Over the past } \\
\text { (timeframe) }\end{array}$ & \multirow[t]{2}{*}{ Russian-Latvia } & $\begin{array}{l}\text { В течение } \\
\text { последних } \\
\text { (временные } \\
\text { рамки) }\end{array}$ & \multirow{2}{*}{$\begin{array}{l}\text { Unlike in } \\
\text { English-US, the } \\
\text { insertion of a } \\
\text { variable for } \\
\text { particular time } \\
\text { frame, for } \\
\text { example } 2 \\
\text { months or } 7 \\
\text { days in the } \\
\text { context of "over } \\
\text { the past..." and } \\
\text { "in the past..." } \\
\text { differs in } \\
\text { Russian-Latvia. }\end{array}$} & $\begin{array}{l}\text { В течение } \\
\text { последней / } \\
\text { последнего / } \\
\text { последних } \\
\text { (временные } \\
\text { рамки) }\end{array}$ \\
\hline $\begin{array}{l}\text { In the past } \\
\text { (timeframe) }\end{array}$ & & $\begin{array}{l}\text { За последние } \\
\text { (временные } \\
\text { рамки) }\end{array}$ & & $\begin{array}{l}\text { За последний / } \\
\text { последнюю / } \\
\text { последние / } \\
\text { прошлый / } \\
\text { прошлую / }\end{array}$ \\
\hline
\end{tabular}

Table 1: Examples of variable issues in eCOA translations

\begin{tabular}{|c|c|c|c|c|}
\hline & & & $\begin{array}{l}\text { Using the same } \\
\text { translation in } \\
\text { both these } \\
\text { contexts will } \\
\text { introduce } \\
\text { grammatical } \\
\text { errors. }\end{array}$ & $\begin{array}{l}\text { прошлые } \\
\text { (временные } \\
\text { рамки) }\end{array}$ \\
\hline \multicolumn{5}{|c|}{ Numeric/ordinal variable issues } \\
\hline Source & Language & $\begin{array}{l}\text { Translation } \\
\text { created by the } \\
\text { use of } \\
\text { variables }\end{array}$ & $\begin{array}{l}\text { Description of } \\
\text { the issue }\end{array}$ & $\begin{array}{l}\text { Corrected } \\
\text { translation }\end{array}$ \\
\hline $\begin{array}{l}\text { For your \%f } \\
\text { poop, how hard } \\
\text { did you push? } \\
\text { (\%f - is for } \\
\text { each number of } \\
\text { poop. For } \\
\text { example, if the } \\
\text { subject reported } \\
\text { they did } 10 \\
\text { poops since } \\
\text { bedtime last } \\
\text { night till now, } \\
\text { then \% } \text { would } \\
\text { be first poop, } \\
\text { second poop, ... } \\
\text { tenth poop) }\end{array}$ & $\begin{array}{l}\text { Serbian - } \\
\text { Serbia }\end{array}$ & $\begin{array}{l}\text { Када си } \\
\text { имао/ла } \\
\text { данашњу прва } \\
\text { каку }\end{array}$ & $\begin{array}{l}\text { When inserting } \\
\text { the translation of } \\
\text { the variable } \\
\text { string "first" in } \\
\text { the source, the } \\
\text { word "npвa"is } \\
\text { not } \\
\text { grammatically } \\
\text { correct in } \\
\text { context, and } \\
\text { needs to be } \\
\text { modified to } \\
\text { "пpвy" to be the } \\
\text { correct } \\
\text { grammatical } \\
\text { form of the } \\
\text { Serbian word } \\
\text { "first" based on } \\
\text { its placement in } \\
\text { the sentence. }\end{array}$ & $\begin{array}{l}\text { Када си } \\
\text { имао/ла } \\
\text { данашњу прву } \\
\text { каку }\end{array}$ \\
\hline
\end{tabular}

(3045) Longitudinal associations between PROMIS®-29 anxiety and depression symptoms and pain Impact Stratification Scores in a sample of U.S. military service members

Michael Dunbar, PhD, RAND Corporation, Pittsburgh, Pennsylvania, USA; Anthony Rodriguez, PhD, RAND Corporation, Boston, Massachusetts, USA; Maria Edelen, PhD, RAND Corporation, Boston, Massachusetts, USA; Ron Hays, PhD, UCLA, Los Angeles, California, USA; Ian Coulter, PhD, RAND Corporation, Santa Monica, California, USA; Daniel Siconolfi, PhD, RAND Corporation, Pittsburgh, Pennsylvania, USA; Patricia Herman, PhD, RAND Corporation, Santa Monica, California, USA

Aims: The Impact Stratification Score (ISS) has been proposed as a standardized measure of the impact of chronic low back pain (LBP) based on items from the PROMIS ${ }^{\circledR}-29$ profile that capture pain intensity, physical functioning, and pain interference. LBP is highly prevalent among military service members and is known to correlate with psychological distress in cross-sectional samples. However, no studies have examined the ISS or its association with psychological symptoms over time in military samples. This study examines longitudinal associations between psychological symptoms and the impact of LBP among USA (U.S.) military service members. Methods: The study involved secondary data analysis of a sample of U.S. military service members ages 18-50 with LBP $(n=733)$. Participants completed items from the PROMIS ${ }^{\circledR}-29$ profile at three time points during treatment: baseline (T1), week 6 of treatment (T2), and week 12 of treatment (T3). Impact of LBP was quantified using the ISS (ranging from $8=$ least impact to $50=$ greatest impact). Psychological symptoms were assessed as PROMIS ${ }^{\circledR}-29$ anxiety scores, depression scores, and a total emotional distress (ED) score (mean of anxiety and depression scores). Separate autoregressive cross-lagged (ARCL) models examined reciprocal associations of ISS with anxiety, depression, and ED scores from T1 to T3. Results: Within each timepoint, ISS was significantly and positively correlated with anxiety, depression, and ED. In all ARCL models, anxiety, depression, and ED scores predicted ISS at the next time point. Associations between anxiety and subsequent ISS and depression and subsequent ISS were similar in magnitude (e.g., Anxiety T2 to ISS T3: $\mathrm{b}=0.12, \mathrm{p}<0.001$; Depression T2 to ISS T3: $\mathrm{b}=0.12, \mathrm{p}<0.001$ ). ISS did not predict future depression or ED scores at any time point, but ISS at T2 was significantly, positively associated with anxiety scores at $\mathrm{T} 3(\mathrm{~b}=0.07, \mathrm{p}=0.04)$. Conclusion: Psychological symptoms consistently and prospectively predict impact of LBP as measured by ISS 
among service members undergoing pain treatment. ISS may also be associated with future anxiety, but not depression. PROMIS ${ }^{\circledR}$ anxiety and depression items may be important adjunctive measures to consider when quantifying future pain impact in treatment for LBP.

\section{(3046) Assessing the impact of open-label designs on patient- reported outcomes: a cross-trial investigation in metastatic melanoma}

Jennifer Lord-Bessen, PhD, Bristol Myers Squibb, Princeton, New Jersey, USA; James Signorovitch, Analysis Group, Inc., Boston, Massachusetts, USA; Min Yang, Analysis Group, Inc., Boston, Massachusetts, USA; Ibou Diey, Analysis Group, Inc., Boston, Massachusetts, USA; Jessica Roydhouse, University of Tasmania, Tasmania, Australia

Aims: Systematic reviews suggest that knowledge of treatment assignment may affect PROs and subsequent interpretation. This is of particular concern in oncology, given the preponderance of single-arm and open-label trials. We sought to measure the magnitude of open-label bias using propensity scores to compare PROs for similar patient groups using data from melanoma trials with different degrees of concealment. Methods: Two trials of ipilimumab for the treatment of metastatic melanoma (MDX010-20 and CA184-022) had comparable designs and used the EORTC QLQ-C30 to measure quality of life, symptoms, and function. Patients were aware of the drug they received in CA184-022 ("open-label"), but not in MDX010-20 ("blinded"). Mean changes from baseline in these domains at week 12 were compared between the $3 \mathrm{mg} / \mathrm{kg}$ ipilimumab arms of these trials. Propensity scores were used to balance patient clinical and sociodemographic characteristics and baseline PRO domain scores. Overall survival (OS) was compared to assess cross-trial similarity. Results: After propensity score adjustment, all baseline characteristics were balanced between the blinded $(\mathrm{N}=125)$ and open-label groups $(\mathrm{N}=69)$, and OS was similar (logrank test 0.9 ), along with similar proportions of questionnaire completion at week 12 (66\% vs. 64\%, respectively). Differences in change scores from baseline (mean \pm standard error) between the open-label vs. blinded groups ranged from $-5.2 \pm 5.2$ (role functioning; slightly worse for open-label) to $+2.2 \pm 4.1$ (emotional functioning; slightly better for open-label) (Table). Similar results were seen for the symptom scales with a range from $-8.3 \pm 4.6$ (diarrhea; slightly better for openlabel) to $6.2 \pm 4.1$ (pain; slightly worse for open-label). Differences were not statistically significant, and all were smaller than their respective half of the baseline standard deviation. Conclusion: Changes in QLQ-C30 domain scores were similar between patients receiving ipilimumab with different levels of concealment. Numerical differences were not consistent in direction and did not indicate clinically or statistically significant bias favoring the open-label group. This study adds to the growing body of evidence that concerns regarding open-label reporting bias may be minOR and should not prohibit the interpretation of large and meaningful treatment effects on PROs.

\begin{tabular}{|c|c|c|c|c|}
\hline \multicolumn{5}{|c|}{$\begin{array}{l}\text { Mean change } \\
\text { from baseline to week } 12 \pm S D\end{array}$} \\
\hline & $\begin{array}{c}\text { MDX010-20 } \\
\text { Blinded } \\
N=83\end{array}$ & $\begin{array}{c}\text { CA184-022 } \\
\text { Open-label } \\
N=44\end{array}$ & $\begin{array}{c}\text { Mean } \\
\text { Difference } \pm \\
\text { SE }\end{array}$ & P-value \\
\hline \multicolumn{5}{|l|}{ Quality of Life } \\
\hline Global health status & $-9.75 \pm 21.98$ & $-11.10 \pm 19.48$ & $-1.35 \pm 3.80$ & 0.74 \\
\hline \multicolumn{5}{|l|}{ Functional scales } \\
\hline Physical functioning & $-6.65 \pm 21.59$ & $-9.97 \pm 19.57$ & $-3.32 \pm 3.78$ & 0.44 \\
\hline Role functioning & $-13.71 \pm 31.75$ & $-18.93 \pm 25.72$ & $-5.22 \pm 5.21$ & 0.37 \\
\hline Emotional functioning & $-5.17 \pm 22.65$ & $-2.99 \pm 21.63$ & $2.18 \pm 4.10$ & 0.6 \\
\hline Cognitive functioning & $-6.99 \pm 22.25$ & $-5.85 \pm 17.09$ & $1.14 \pm 3.55$ & 0.78 \\
\hline Social functioning & $-8.34 \pm 29.84$ & $-8.64 \pm 26.84$ & $-0.30 \pm 5.21$ & 0.95 \\
\hline
\end{tabular}

(3047) Minimal clinically important differences for patientreported outcome measures of symptoms and health-related Quality of Life in patients with uterine fibroid

Wei Xu, School of Public Health and Management, Chongqing Medical University, Chongqing, China; Li Tang, Shaoxing second hospital, Zhejiang, China; Jingyu Zhang,

State Key Laboratory of Ultrasound in Medicine and Engineering, Chongqing Medical University, Chongqing, China; Yuxian Nie, State Key Laboratory of Ultrasound in Medicine and Engineering, Chongqing Medical University, Chongqing, China; Xueyao Su, School of Public Health and Management, Chongqing Medical University, Chongqing, China; Jiayuan Zhang, School of Public Health and Management, Chongqing Medical University, Chongqing, China; Qiuling Shi, School of Public Health and Management, Chongqing Medical University, Chongqing, China

Aims: To investigate the minimal clinically important difference (MCID) of Uterine Fibroid Symptom and Health-related Quality of Life Questionnaire (UFS-HRQoL) in patients with uterine fibroid using the largest array of methods Methods: Data were extracted from a prospective cohort study of patients with High Intensity Focused Ultrasound (HIFU) or myomectomy for uterine fibroids. Patients completed 6 and 12-month follow-up were used to compute the MCIDs. We divided the patients to two data sets, randomly, one for development, the other for validation. We established MCID of Uterine Fibroid Symptom (UFS) and HRQoL, respectively. The pooled MCID was estimated by calculating the arithmetic weighted mean, resulting from the combination of anchor-based (weight, twothirds) and distribution-based (weight, one-third) methods. Correlations between the potential anchors and each PROM were explored by using Pearson or point-biserial correlation coefficients. correlations $(r \geq 0.3)$ were established as criteria to proceed with the calculation of the MCIDs by using the anchor-based methods. To estimate MCIDs, we used mean change, and receiver-operating characteristic curves, for anchor-based approaches, and $0.5 \mathrm{SD}, \mathrm{SE}$ of measurement, 1.96 SE of measurement, and minimal detectable change for distribution-based approaches. Results: A total of 1939 patients were included. In the analysis, sixty-nine percent female with uterine fibroid $(41.3 \pm 5.1$ years of age $)$ underwent HIFU, others ( $40.9 \pm 5.0$ years of age) underwent myomectomy. Anchors were "rate your health in general, compared to one year ago" of SF-36, and physical functioning subscales of SF-36. Pooled MCIDs were 11.4 for UFS, and 17.1 for HRQoL. The MCID for quality of life were validated using SF-36 other subscales except physical functioning, UFS and HRQoL change scores categorize to improvement group (difference $>=$ MCID), and without improvement group (difference $<$ MCID). UFS and HRQoL were significantly concordant with bodily pain, general health and vitality in SF-36 (all $\mathrm{P}<0.05)$. Conclusion: We determined anchor-based and distribution-based approaches to established MCIDs of UFS and HRQoL from the largest cohort of patients with uterine fibroid ever. The improvement of 11.4 of UFS and 17.1 of HRQoL could be interpreted according to each patient's specificities and incorporated into clinical practice to guide different stakeholders in the decision-making process. 
A

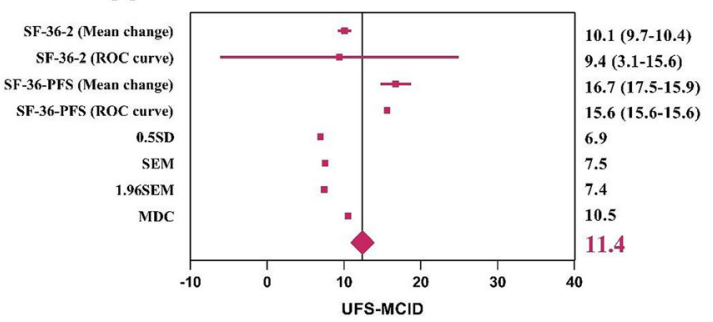

B

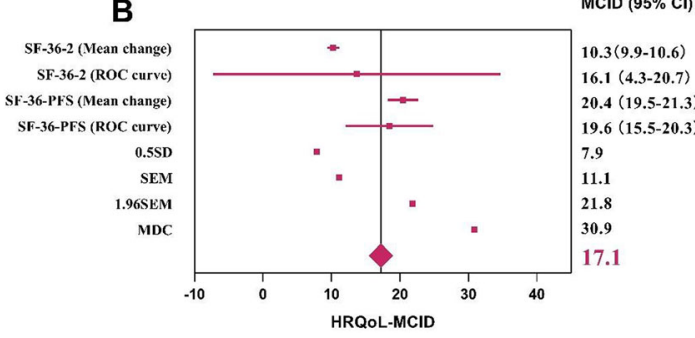

Table 1 . The difference quality of life in SF-36 between two group using the MCID to define the improvement after surgery 12 month

\begin{tabular}{|c|c|c|c|c|}
\hline UFS & $\begin{array}{l}\text { Not improved } \\
(\mathrm{n}=866)\end{array}$ & $\begin{array}{l}\text { Improved } \\
(\mathrm{n}=787)\end{array}$ & $\mathrm{t}$. & P. \\
\hline Role-Physical. & $91.45 \cdot(21.48)$ & $93.07 \cdot(17.51)$ & -1.67 & 0.09 \\
\hline Bodily Pain & $88.42 \cdot(13.23)$ & 91.16.(11.9)。 & -4.41 . & $<0.001$ \\
\hline General-Health. & $71.66 \cdot(17.02)$ & $74.91 \cdot(14.78)$ & -4.12 . & $<0.001$ \\
\hline Vitality & $75.75 \cdot(14.44)$ & 77.9-(13.66) & -3.11 . & 0.002 \\
\hline Social-Functioning . & $86.62 \cdot(14.28)$ & $87.5 \cdot(13.81)$ & -1.27 & 0.21 \\
\hline Role-Emotional. & $90.8 \cdot(23.26)$ & $91.66 \cdot(21.12)$ & -0.78 & 0.43 \\
\hline Mental Health & $73.51 \cdot(14.61)$ & 74.77.(13.2) & -1.82 . & 0.07. \\
\hline HRQoL. & $\begin{array}{l}\text { Not improved } \\
(n=1074)\end{array}$ & $\begin{array}{l}\text { Improved. } \\
(\mathrm{n}=579)\end{array}$ & $\mathrm{t}$ & P. \\
\hline Role-Physical. & $91.45 \cdot(21.48)$ & $93.07 \cdot(17.51)$ & -0.72 & 0.47 \\
\hline Bodily Pain & $88.42 \cdot(13.23)$ & $91.16 \cdot(11.9)$ & -4.22 & $<0.001$ \\
\hline General-Health & $71.66 \cdot(17.02)$ & $74.91 \cdot(14.78)$ & -2.26 & $<0.001$ \\
\hline Vitality. & $75.75 \cdot(14.44)$ & 77.9.(13.66) & -3.87 & $<0.001$ \\
\hline Social-Functioning . & $86.62 \cdot(14.28)$ & 87.5.(13.81) & -1.71 & 0.09 \\
\hline Role-Emotional. & $90.8 \cdot(23.26)$ & $91.66 \cdot(21.12)$ & -3.9 & $<0.001$ \\
\hline Mental Health. & $73.51 \cdot(14.61)$ & 74.77.(13.2) & -3.35 . & $<0.001$ \\
\hline
\end{tabular}

(3048) Challenges and solutions in the implementation of clinical trial exit interviews to bring greater patient centricity to future trial procedures

Yanfen Guan, M.S., Merck \& Co., Inc., Kenilworth, New Jersey, USA; Felipe Arbelaez, Merck \& Co., Inc., Kenilworth, New Jersey, USA; Allison Nguyen, Merck \& Co., Inc., Westpoint, Pennsylvania, USA; Samantha Wratten, Adelphi Values, Bollington, UK; Charlotte Panter, Adelphi Values, Bollington, UK; Rob Arbuckle, Adelphi Values, Bollington, UK; Jessica Weaver, Merck \& Co., Inc., Kenilworth, New Jersey, USA

Aims: A stand-alone exit interview study was conducted in the context of a Phase 2a clinical trial of endometriosis-related pain to obtain patient feedback on electronic Patient-Reported Outcome (ePRO) feasibility and trial experience. Described here are proposed solutions for operational challenges that may commonly arise in implementing exit interviews. Methods: Fifteen participants (female; mean age 35.4 years [range: 25-48]) fluent in English $(n=3)$ or Spanish $(n=12)$ from 6 clinical trial sites in the USA and Puerto Rico participated in a 60-min, 1:1, semi-structured telephone interview within 2 weeks of their final clinical site visit. The interview explored feasibility and usability of an ePRO (used as part of the trial) and collected responses to Section C of the Transcelerate Study Participant Feedback Questionnaire. Qualitative analysis was performed on verbatim transcripts. Results: Three main challenges were addressed proactively during study planning and flexibly throughout implementation:Clinical team concerns: prior to study start, sponsor's clinical trial team buy-in to the benefits of the exit interview and process alignment was critical; processes were agreed for key procedures such as timing of informed consent and Adverse Event reporting to avoid possibly impacting the trial endpoint assessments. Complex logistics: timelines and roles and responsibilities were clearly outlined in standardized site training; clear and frequent communication between the clinical sites and interviewers was planned, especially when participants were transitioned from clinical trial to the exit interview stage. Slow recruitment: As the exit interview study was not part of the overall trial procedure, recruitment challenges were encountered due to lack of interest and resources from clinical site investigators, slow trial recruitment, and COVID-19 pandemic. These were addressed by prioritizing sites with high enrollment, resources/capacity, and central IRB oversight, and continuous site monitoring. Appropriate compensations for sites and participants were prespecified at onset to encourage engagement. Conclusion: The key for successfully conducting an exit interview study requires early engagement and continuous collaboration with the clinical trial team, site investigators and coordinators, and qualitative interviewers. Flexibility and perseverance in overcoming logistical challenges is worthwhile to obtain valuable patient input to support ePRO refinement and future trial design.

\section{(3049) Patient-reported outcomes of wedge resection versus segmentectomy for lung disease: A longitudinal cohort study}

Xing Wei, Department of Thoracic Surgery, Sichuan Cancer Hospital \& Institute, Sichuan Cancer Center, School of Medicine, University of Electronic Science and Technology of China, Chengdu 610,041, China., Chengdu, China; Hongfan Yu, BSc, School of Public Health and Management, Chongqing Medical University, Chongqing, China; Wei Dai, MD, Department of Thoracic Surgery, Sichuan Cancer Hospital \& Institute, Sichuan Cancer Center, School of Medicine, University of Electronic Science and Technology of China, Chengdu, Sichuan, China; Qiuling Shi, MD; PhD, Center for Cancer Prevention Research, Sichuan Cancer Hospital, School of Medicine, University of Electronic Science and Technology of China, Chengdu, Sichuan, China

Aims: Wedge resection and segmentectomy are important methods of sub-lobectomy, which were widely used to treat primary tumors, metastases tumors and benign diseases of lungs. Studies about the effects of wedge resection and segmentectomy with respect to patientreported outcomes (PROs) are rare. This study performed a longitudinal PRO assessment to compare the effects of these surgeries for lung diseases from the patients' perspective. Methods: We investigated lung diseases patients from a previous prospective multicentre study. Longitudinal data of clinical characteristics and PROs were collected. PROs were obtained preoperatively, daily in the hospital postoperatively, and weekly up to 4 weeks after discharge or the beginning of postoperative adjuvant therapy. Symptoms and their impact on daily functioning and quality of life (QOL) were assessed using the MD Anderson Symptom Inventory for lung cancer and a single-item QOL scale. Trajectories of PROs over the investigation period were compared. Results: Overall, 140 primary lung disease patients who underwent video-assisted thoracoscopic surgery, including 56 and 84 patients who underwent wedge resection and segmentectomy, respectively, were included. During postoperative hospitalization, wedge resection patients reported milder disturbed sleep $(\mathrm{p}=0.026)$, dry mouth $(\mathrm{p}=0.0063)$, coughing $(\mathrm{p}=0.006)$, constipation $(p=0.0058)$, sore throat $(p=0.0317)$, and interference 
of activity $(\mathrm{p}=0.0394)$ and QOL $(\mathrm{p}=0.0486)$. Post-discharge, VATS patients had milder shortness of breath $(\mathrm{p}=0.0051)$, problems of remembering things $(\mathrm{p}=0.026)$, and interference of activity $(p=0.0327)$. Conclusion: From the patients' perspective, wedge resection benefited the recovery process. This supports shared decision-making regarding surgery type for lung diseases.

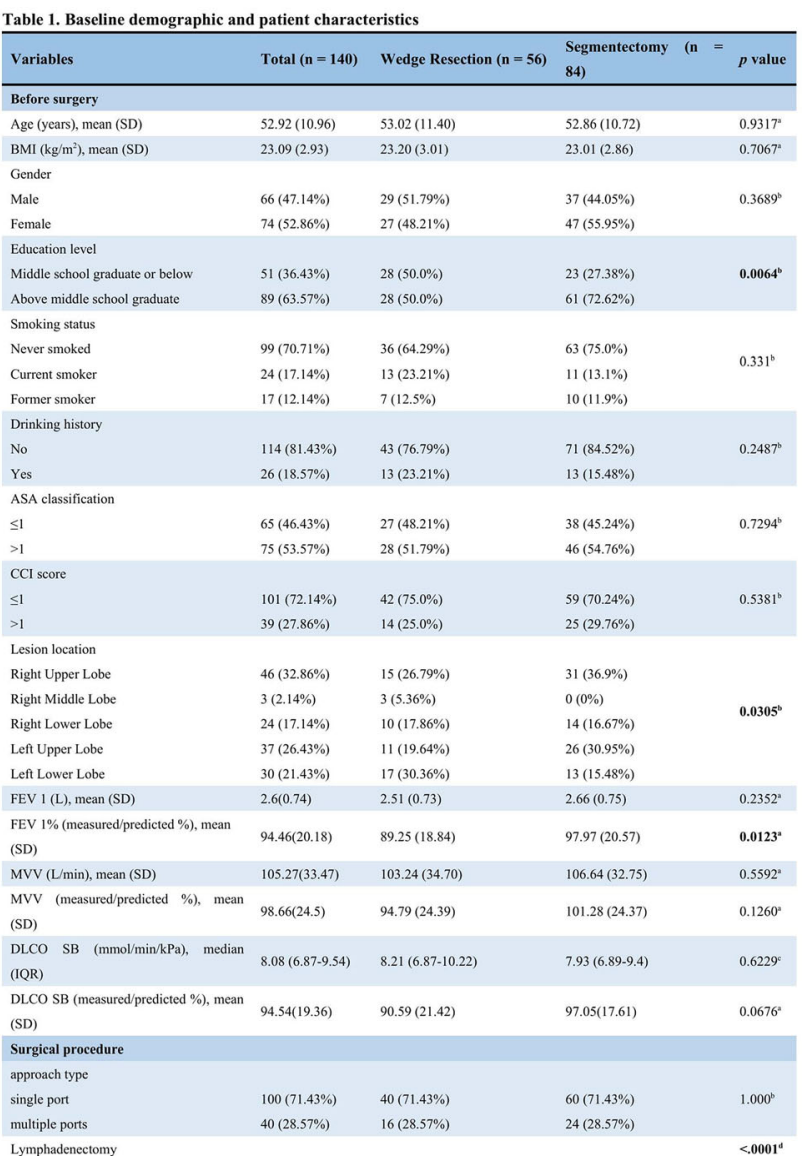

\begin{tabular}{|c|c|c|c|c|}
\hline Systematic lymph node dissection & $4(2.86 \%)$ & $0(0 \%)$ & $4(4.76 \%)$ & \\
\hline Selective lymph node sampling & $78(55.71 \%)$ & $8(14.29 \%)$ & $70(83.33 \%)$ & \\
\hline Not performed & $58(41.43 \%)$ & $48(85.71 \%)$ & $10(11.9 \%)$ & \\
\hline \multicolumn{5}{|l|}{ Thoracic drainage tube(s) } \\
\hline 1 & $103(88.03 \%)$ & $43(97.73 \%)$ & $60(82.19 \%)$ & \multirow{3}{*}{$0.0064^{\circ}$} \\
\hline 2 & $14(11.97 \%)$ & $1(2.27 \%)$ & $11(17.81 \%)$ & \\
\hline \multicolumn{4}{|l|}{ missing data $(\mathrm{n}=23)$} & \\
\hline Operative time (min), mean (SD) & $114.17(52.20)$ & $79.70(35.74)$ & $137.15(48.79)$ & $<.0001^{\circ}$ \\
\hline \multicolumn{5}{|l|}{ After surgery (pathology) } \\
\hline pTNM stage (Eighth Edition) & & & & \multirow{4}{*}{$0.0534^{4}$} \\
\hline not application & $50(35.71 \%)$ & $33(58.93 \%)$ & $17(20.24 \%)$ & \\
\hline$\leq \mathrm{I}$ & $62(44.29 \%)$ & $7(12.5 \%)$ & $55(65.48 \%)$ & \\
\hline$>1$ & $28(20.0 \%)$ & $13(28.57 \%)$ & $12(14.29 \%)$ & \\
\hline \multicolumn{5}{|l|}{ pathological type } \\
\hline Adcnocarcinoma & $83(96.51 \%)$ & $19(86.36 \%)$ & $64(100 \%)$ & \multirow{4}{*}{$0.0109^{b}$} \\
\hline Squamous cell carcinoma & $2(2.33 \%)$ & $2(9.09 \%)$ & $0(0 \%)$ & \\
\hline & $1(1$ & & & \\
\hline Others & $.16 \%)$ & $1(4.55 \%)$ & $0(0 \%)$ & \\
\hline \multicolumn{5}{|l|}{ missing data (n=54) } \\
\hline \multicolumn{5}{|l|}{ Traditional outcomes } \\
\hline LOS (days), median (IQR) & $11.5(9-14)$ & $11.00(8.50-13.50)$ & $12.0(9.0-14.0)$ & $0.1658^{\circ}$ \\
\hline PHS (days), median (IQR) & $5(4-6)$ & $4.00(3.00-6.00)$ & $6.00(5.00-7.00)$ & $<.0001^{\circ}$ \\
\hline \multicolumn{5}{|c|}{ Perioperative complication, Clavien-Dindo Classification } \\
\hline none & $116(82.86 \%)$ & $33(58.93 \%)$ & $67(79.76)$ & \multirow{3}{*}{$0.1059^{4}$} \\
\hline <grade 2 & $11(7.86 \%)$ & $7(12.5 \%)$ & $6(7.14 \%)$ & \\
\hline zgrade 2 & $13(9.29 \%)$ & $16(28.57 \%)$ & $11(13.10 \%)$ & \\
\hline $\begin{array}{l}\text { Postoperative morphine equivalent dose), } \\
\text { median (IQR) }\end{array}$ & $215(117.5-274.0)$ & $210(102-241.5)$ & $233(122-310)$ & $0.0565^{\circ}$ \\
\hline
\end{tabular}

Data are median (IQR) or n (\%). ${ }^{\mathrm{a}} \mathrm{T}-\mathrm{Test} ;{ }^{\mathrm{b}} \mathrm{Chi}-\mathrm{Square}$ Test; ${ }^{\mathrm{c}} \mathrm{Mann}$-Whitney U test; ${ }^{\mathrm{d}} \mathrm{Mantel}-$ Haenszel ChiSquare Test

BMI, body mass index; ASA, American Society of Anesthesiologists; CCI, Charlson Comorbidity Index; FEV1, forced expiratory volume in 1 second; MVV, maximal voluntary ventilation; DLCO, diffusion capacity of carbon monoxide. TNM, tumour node metastasis; LOS, length of stay; PHS, postoperative hospital stay

Table 2. In-hospital Patient-reported outcome of Wedge Resection vs. Segmentectomy patients $\left(1^{\text {t }}\right.$ to $7^{\text {th }}$ day after surgery)

\begin{tabular}{|c|c|c|c|}
\hline \multirow{3}{*}{ Items } & \multicolumn{3}{|c|}{ Wedge Resection ( $n=56$ ) vs. Segmentectomy ( $n=84$ ) } \\
\hline & \multicolumn{3}{|c|}{ Group } \\
\hline & Estimate & $\mathbf{S E}$ & $\mathbf{p}$ \\
\hline \multicolumn{4}{|c|}{ MDASI-LC Symptoms Part I. Core items } \\
\hline Pain & -1.2595 & 0.6832 & 0.0658 \\
\hline Fatigue (tiredness) & -0.9759 & 0.6767 & 0.1499 \\
\hline Nausea & -0.4812 & 0.5419 & 0.3749 \\
\hline Disturbed sleep & -1.7127 & 0.7673 & 0.026 \\
\hline Distressed (upset) & -1.0928 & 0.7273 & 0.1336 \\
\hline Shortness of breath & -0.9238 & 0.6243 & 0.1395 \\
\hline Problems of remembering things & -0.5039 & 0.602 & 0.4029 \\
\hline Lack of appetite & -1.0915 & 0.6553 & 0.0964 \\
\hline Drowsy (sleepy) & -0.4967 & 0.7062 & 0.4822 \\
\hline Dry mouth & -2.0494 & 0.7466 & 0.0063 \\
\hline Sadness & -0.1904 & 0.6418 & 0.7668 \\
\hline Vomiting & 0.06334 & 0.4697 & 0.8928 \\
\hline Numbness or tingling & -0.3037 & 0.473 & 0.5212 \\
\hline \multicolumn{4}{|c|}{ MDASI-LC Symptoms PART I. Lung Cancer-Specific Items } \\
\hline Coughing & -1.6956 & 0.6147 & 0.006 \\
\hline Constipation & -2.3393 & 0.8448 & 0.0058 \\
\hline Sore throat & -1.335 & 0.62 & 0.0317 \\
\hline \multicolumn{4}{|c|}{ MDASI-LC Symptoms PART II. Interfered Items } \\
\hline Activity & -1.6203 & 0.7845 & 0.0394 \\
\hline Mood & -0.6992 & 0.684 & 0.3071 \\
\hline Work & -1.1749 & 0.7823 & 0.1337 \\
\hline Relations with other people & -0.5442 & 0.7158 & 0.4474 \\
\hline Walking & -0.8769 & 0.8614 & 0.3091 \\
\hline Enjoyment of life & -1.5532 & 0.9177 & 0.0911 \\
\hline Single item QOL & 1.3811 & 0.6986 & 0.0486 \\
\hline
\end{tabular}

Mixed model adjusted variables: Education level, Lesion location , FEV 1\% (measured/predicted \%), Lymphadenectomy, Thoracic drainage tube(s), Operative time, PHS (days)

MDASI-LC, MD Anderson Symptom Inventory-Lung Cancer module; QOL, quality of life; SE, standard error; FEV1, forced expiratory volume in 1 second; PHS, postoperative hospital stay 
Table 3. Patient-reported outcome of Wedge Resection vs. Segmentectomy patients after discharge (discharge day to week 4 after discharge)

\begin{tabular}{|c|c|c|c|}
\hline \multirow{3}{*}{ Items } & \multicolumn{3}{|c|}{ Wedge Resection ( $n=56$ ) vs. Segmentectomy $(n=84)$} \\
\hline & \multicolumn{3}{|c|}{ Group } \\
\hline & Estimate & SE & $\mathbf{p}$ \\
\hline \multicolumn{4}{|c|}{ MDASI-LC Symptoms Part I. Core items } \\
\hline Pain & -0.5411 & 0.5196 & 0.2984 \\
\hline Fatigue (tiredness) & -0.8749 & 0.5143 & 0.0897 \\
\hline Nausea & -0.626 & 0.366 & 0.088 \\
\hline Disturbed sleep & -1.363 & 0.6705 & 0.0427 \\
\hline Distressed (upset) & -0.3948 & 0.6013 & 0.5118 \\
\hline Shortness of breath & -1.5262 & 0.5422 & 0.0051 \\
\hline Problems of remembering things & -1.117 & 0.5 & 0.026 \\
\hline Lack of appetite & -0.9939 & 0.5138 & 0.0538 \\
\hline Drowsy (slecpy) & -0.9456 & 0.5181 & 0.0687 \\
\hline Dry mouth & -1.2811 & 0.5047 & 0.0115 \\
\hline Sadness & -0.2522 & 0.5614 & 0.6535 \\
\hline Vomiting & 0.00149 & 0.3162 & 0.9962 \\
\hline Numbness or tingling & -0.4236 & 0.4125 & 0.305 \\
\hline \multicolumn{4}{|c|}{ MDASI-LC Symptoms PART I. Lung Cancer-Specific Items } \\
\hline Coughing & -0.2016 & 0.5341 & 0.7061 \\
\hline Constipation & -0.7783 & 0.5231 & 0.1376 \\
\hline Sore throat & -0.07649 & 0.4583 & 0.8675 \\
\hline \multicolumn{4}{|c|}{ MDASI-LC Symptoms PART II. Interfered Items } \\
\hline Activity & -1.2805 & 0.5974 & 0.0327 \\
\hline Mood & -0.2307 & 0.5897 & 0.6958 \\
\hline Work & -0.4573 & 0.544 & 0.4011 \\
\hline Relations with other people & -0.4969 & 0.6354 & 0.4347 \\
\hline Walking & -0.5228 & 0.6063 & 0.389 \\
\hline Enjoyment of life & -0.119 & 0.6372 & 0.8519 \\
\hline Single item QOL & 0.6215 & 0.6348 & 0.3282 \\
\hline
\end{tabular}

Lymphadenectomy, Thoracic drainage tube(s), Operative time, PHS (days)

MDASI-LC, MD Anderson Symptom Inventory-Lung Cancer module; QOL, quality of life; SE, standard error; FEV1, forced expiratory volume in 1 second; PHS, postoperative hospital stay
Figure 1. In-hospital Patient-reported outcome of Wedge Resection vs. Segmentectomy patients (1st to 7th day after surgery)
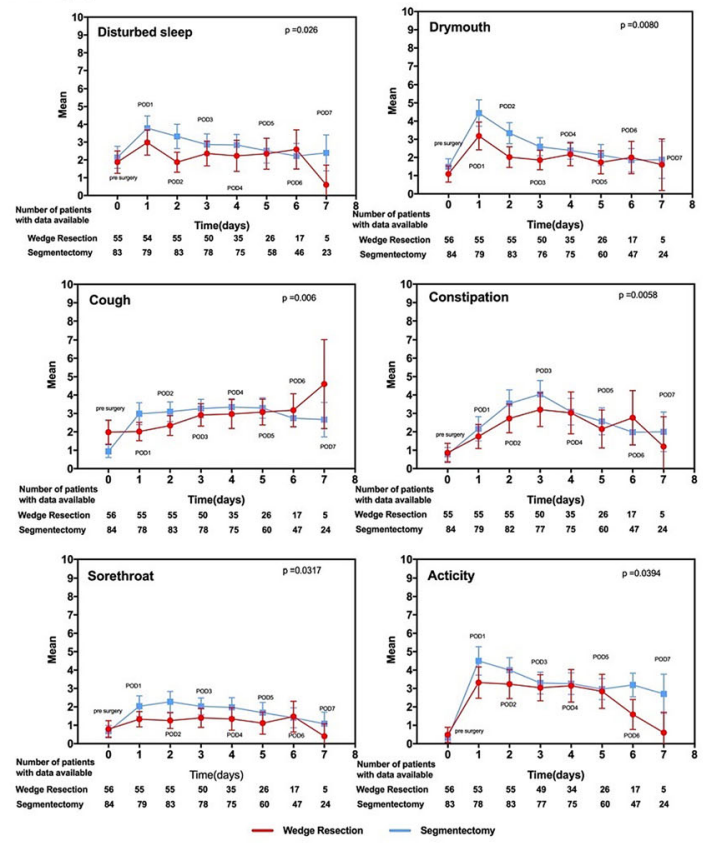

Figure 2. Patient-reported outcome of Wedge Resection vs. Segmentectomy patients after discharge (discharge day to week 4 after discharge)

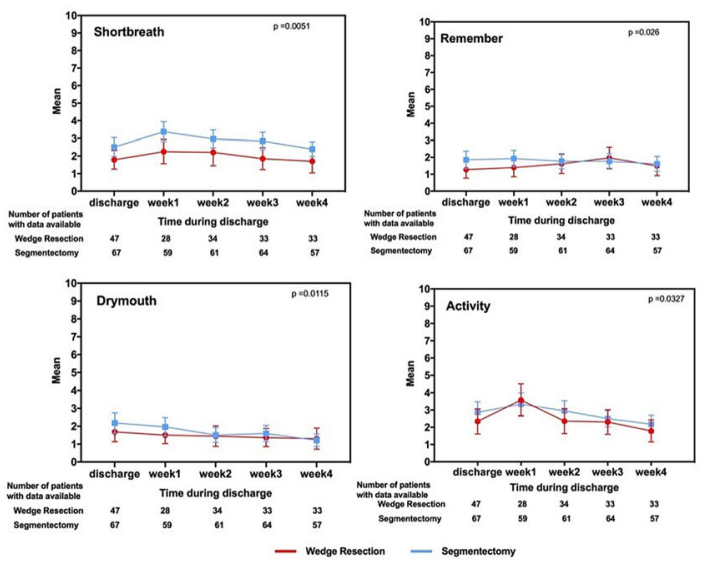

Figure 3. Quality of life of Wedge Resection vs. Segmentectomy patients in hospital and after discharge (1st to 7 th day after surgery and discharge day to week 4 after discharge)

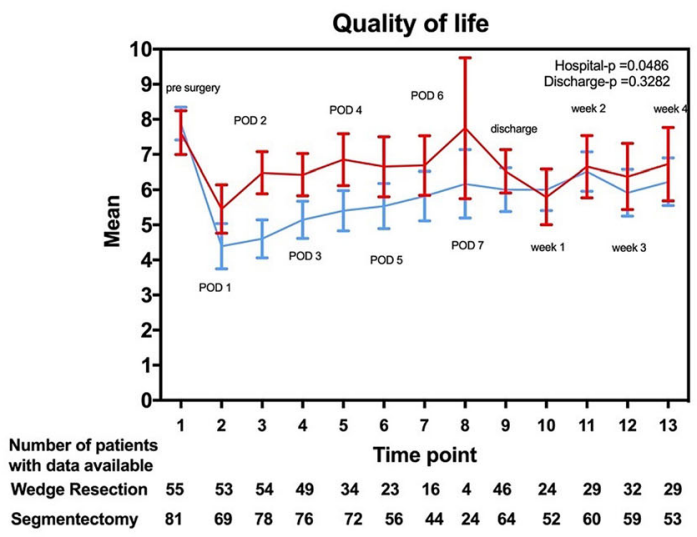


(3051) Why no effect?: Identifying appropriate outcomes for supported employment programs for youths with mental health conditions using a program evaluation approach

Nikki Ow, PhD, The University of British Columbia, Vancouver, British Columbia, Canada; Steve Mathias, M.D., The University of British Columbia, Vancouver, British Columbia, Canada; Skye Barbic, PhD., The University of British Columbia, Vancouver, British Columbia, Canada

Aims: Supported employment programs (SEP) have been primarily used for the past decade to support adults with mental illness in obtaining competitive employment. The main outcome for these programs is employment; however, often, barriers to obtaining and maintaining a job are often system level, outside the influence of SEP. Hence, success of SEP should not only be measured by employment alone. A broader approach to the measurement of outcomes of SEP is needed. The purpose of this study was to identify appropriate outcomes for SEP using data from two supported employment programs. Methods: Data from this study came from two 16-week supported employment programs of youths with mental health conditions. Qualitative and quantitative data were collected. Outcomes included in both studies were employment status, the Canadian Personal Recovery Outcome Measure (CPROM), the Generalized Anxiety Disorder scale (GAD-7), MyLifeTracker scale, the Satisfaction with Life scale (SWL) and the Patient Health Questionnaire (PHQ-9). Qualitative data was mapped onto Donabedian's Structure-Process-Outcome model to identify gaps in meaningful outcomes. Results: Using Donabedian's model, qualitative and quantitative analyses identified mental health outcomes related to job readiness and outcomes of the program (see Fig. 1). Other variables that should be monitored regularly include staff knowledge/training, access to social services, follow-up schedule of outcome measurement. The employment trend of participants last year also mirrored the Covid-19 lockdown restrictions in Canada (see Fig. 2). When there are external restrictions on employment opportunities, it is recommended that program duration be lengthened with the inclusion of more training courses or self-care strategies and to regularly conduct follow up with participants. Conclusion: SEP outcomes should reflect the elements of the program, the context, and the voices of the young people participating in the program. Many participants in our studies benefitted and obtained many skills that are likely to last beyond the 16-week intervention, but employment status alone did not reflect this. Having stronger mental health is an important step towards job readiness. To improve the quality of measuring SEP programs, attention should be paid towards enhancing the voices of young people through co-design of measurement approaches that optimize youth-centred outcomes and experiences.

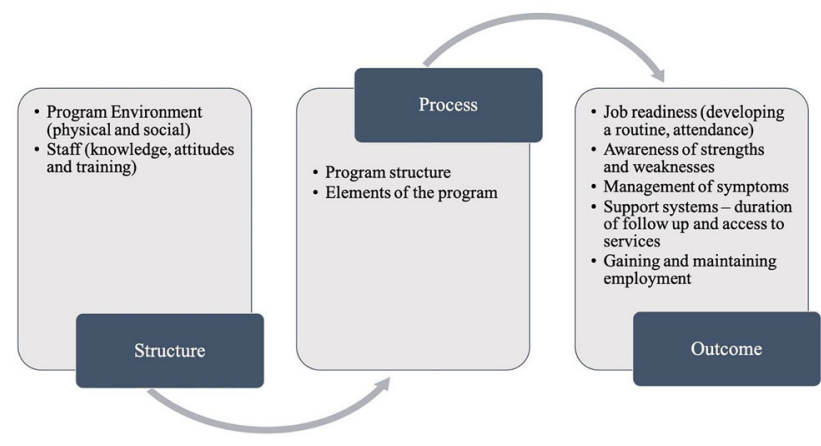

Figure 2 Number of participants employed from January 2020 to March 2021

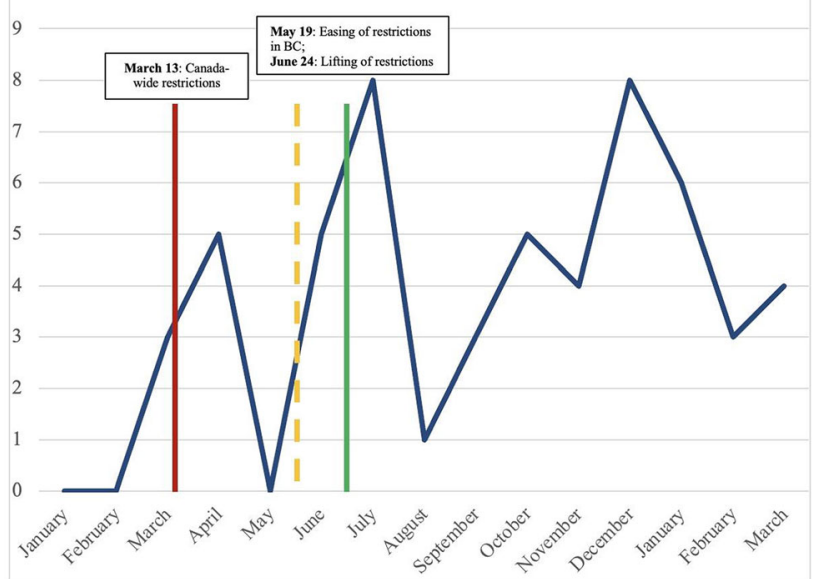

(3052) Using the EQ-5D-5L to investigate health-related quality of life impacts for people living with MS in New Zealand

Suzi Claflin, PhD, Menzies Institute for Medical Research, University of Tasmania, Hobart, Australia; Julie Campbell, PhD, Menzies Institute for Medical Research, Hobart, Australia; Richard Norman, Curtin University, Perth, Australia; Deborah Mason, New Zealand Brain Research Institute, Christchurch, New Zealand; Tomas Kalincik, University of Melbourne, Melbourne, Australia; Steve Simpson-Yap, University of Melbourne, Melbourne, Australia; Helmut Butzkueven, Monash University, Melbourne, Australia; William Carroll, Perron Institute, Nedlands, Australia; Andrew Palmer, Menzies Institute for Medical Research, University of Tasmania, Hobart, Australia; C. Leigh Blizzard, Menzies Institute for Medical Research, University of Tasmania, Hobart, Australia; Ingrid van der Mei, Menzies Institute for Medical Research, University of Tasmania, Hobart, Australia; Bruce TaylOR Menzies Institute for Medical Research, University of Tasmania, Hobart, Australia

Aims: MS is a neurodegenerative disease leading to increasing disability over time and reducing health-related quality of life (HRQoL). New Zealand's (NZ) Pharmaceutical Management Agency guidelines recommend the use of the EQ-5D-5L. However, the EQ-5D-5L has been found deficient in capturing psychosocial health. Here, we aimed to evaluate HRQoL among people with MS in NZ using the EQ-5D$5 \mathrm{~L}$ and the instrument's discriminatory sensitivity for a NZ MS cohort. Methods: Participants were recruited from the NZ MS Prevalence Study (NZMSPS) study. Participants self-completed a detailed 45-min online survey that included: EQ-5D-5L/EQ-VAS; disability (Expanded Disability Status Scale); Fatigue Severity Scale; Hospital Anxiety and Depression Scale; and socioeconomic/demographic/clinical data including disease-modifying therapy (DMT) use. Disability severity was classified into mild/moderate/severe. In the absence of a EuroQoL NZ tariff, HSUs were derived from an Australian tariff. Participant characteristics and outcome variables, including HSUs, were reported as frequencies/percentages, and means \pm standard deviations (SD). We evaluated associations between HSUs and participant characteristics with linear regression models. Results: $\mathrm{N}=254$ participants entered the study. Most participants were middle-aged and female $(79.5 \%), 70 \%$ held a Bachelor's degree, and about half were employed. Mean (SD) EQ5D-5L HSU was 0.58 (0.33). Mean HSUs for disability categories were: mild $0.80(0.17)$, moderate $0.57(0.21)$ and severe $0.14(0.32)$. Twelve percent reported HSU $=1.0$, two of which had moderate disability (mean EQ-VAS of 77.5). Our final regression model indicated an association between HSU and disability category, DMT use, 
fatigue and depression. Participants who had ever used a DMT had 1.1 times lower mean HSU compared to those who had never used a DMT (0.54 compared to 0.63). Fatigue and depression scores were negatively associated with HSU. Conclusion: HRQoL for people with MS in NZ was lower than comparable countries, including Australia. People with severe MS in NZ reported a much lower HSU than people with terminal cancer. The EQ-5D-5L revealed a reduced ceiling effect for this cohort than reported in the broader literature (12\% compared to $30 \%$ ), and the instrument was sensitive to disease severity. This suggests that the instrument can be used for NZ MS reimbursement decisions. We suggest a comparison with the new EQ5D-5LPsychosocial.

(3053) Patient usability and acceptability of longitudinal digital symptom reporting in radiation oncology: A pilot study

Pia Krause Møller, MPH, PhD student, Odense University Hospital, Odense NV, Denmark; Helle Pappot, MD, DMSc, professor Department of Oncology, Rigshospitalet, University Hospital of Copenhagen and Department of Clinical Medicine, University of Copenhagen, Copenhagen, Denmark; Tine Schytte, MD, PhD, Associate professor Department of Oncology, Odense University Hospital; Department of Clinical Research, University of Southern Denmark, Odense, Denmark; Uffe Bernchou, MSc, PhD, Associate professor Laboratory of Radiation Physics, Odense University Hospital; Department of Clinical Research, University of Southern Denmark, Odense, Denmark; Karin Brochstedt Dieperink, RN, PhD, Associate professor Department of Oncology, Odense University Hospital, AgeCare, Academy of Geriatric Cancer Research, Odense University Hospital, Odense, Denmark and Department of Clinical Research, University of Southern Denmark, Odense, Denmark

Aims: Patient-reported outcomes (PRO) are not an integral part of clinical practice in radiation oncology today. When evaluating novel treatment technologies like online MR-guided radiotherapy (MRgRT) a longitudinal collection of PRO may enhance the accuracy of symptom grading and provide new knowledge about treatment toxicity. In a pilot study, a systematic implementation process of a tailored PRO item set was initiated. The objectives presented here are patient usability and initial acceptance of digital symptom reporting during radiotherapy. Methods: Patients referred for pelvic radiotherapy with a curative intent were eligible. They were to respond a PRO item set, developed for this specific purpose, weekly during their four to eight week course of radiotherapy and four weeks following. In addition, health-related quality of life (HRQoL) was collected (Fig. 1). The patients used their own device from home to enter the app or website My Hospital transferring data directly to the patient chart. In this pilot study, clinicians were not allowed to enter the PRO responses, and the patients were informed about this ahead of the study. All patients evaluated usability and acceptance by completing a Patient Feedback Form on paper. Results: 41 cancer patients were enrolled; 32 prostate, 8 cervical and 1 bladder (median age 68(36-76)). The acceptance rate was high (87\%) and 93\% did electronic reporting. 38/41(93\%) responded to the Patient Feedback Form. Patients found the PRO item set useful and easy to complete (100\%), agreed on the frequency of weekly reporting (92\%) and found the time spent appropriate $(97 \%)$. Since no clinician feedback was provided, patients experienced their responses not being used (83\%), and communication with clinicians $(79 \%)$ or quality of care $(76 \%)$ did not improve. Despite this, the majority wanted to continue responding and recommend it to others (97\%) as it helped remembering symptoms $(79 \%)$ and increased their control of care (62\%)(Fig. 2). Conclusion: The acceptance rate of weekly PRO reporting during radiotherapy was high and the patients found the frequency and time spent appropriate. Clinician feedback must be provided for the patients to experience their responses being used and the possibility of improved communication and quality of care.

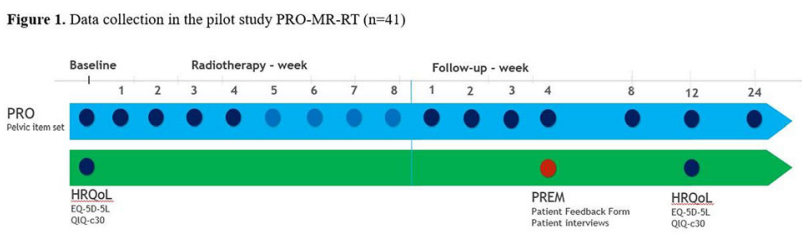

Figure 2. Patient Feedback Form evaluating ePRO in the pilot study PRO-MR-RT ( $\mathrm{n}=38$ )

\begin{tabular}{|c|c|c|}
\hline Questions & \multicolumn{2}{|c|}{ Response (\%) } \\
\hline \multirow[b]{2}{*}{ 1. Time it took to complete } & Too short & Too long \\
\hline & $97 \%$ & $3 \%$ \\
\hline \multirow{3}{*}{ 2.Number of times completing } & $\begin{array}{l}\text { Not often } \\
\text { enough }\end{array}$ & Too often \\
\hline & $92 \%$ & $8 \%$ \\
\hline & Strongly agree/agree & Disagree/strongly disagree \\
\hline 3.Easy to complete & $100 \%$ & 0 \\
\hline 4.Completing was useful & $100 \%$ & 0 \\
\hline 5.Easy to understand & $95 \%$ & $5 \%$ \\
\hline $\begin{array}{l}\text { 6.Easier to remember symptoms and side } \\
\text { effects }\end{array}$ & $79 \%$ & $21 \%$ \\
\hline 7.Improved discussions with clinician & $45 \%$ & $55 \%$ \\
\hline $\begin{array}{l}\text { 8. Clinicians used information for my } \\
\text { care" }^{\circ}\end{array}$ & $17 \%$ & $83 \%$ \\
\hline $\begin{array}{l}\text { 9. The quality of care improved because } \\
\text { of the questionnaire* }\end{array}$ & $24 \%$ & $76 \%$ \\
\hline $\begin{array}{l}\text { 10.Communication with clinician } \\
\text { improved }\end{array}$ & $21 \%$ & $79 \%$ \\
\hline 11. Made me more in control of care" & $62 \%$ & $38 \%$ \\
\hline 12.Recommend to other patients & $97 \%$ & $3 \%$ \\
\hline 13. Would like to continue responding & $97 \%$ & $3 \%$ \\
\hline
\end{tabular}

(3054) Measuring carer quality of life in Duchenne muscular dystrophy: A systematic review of the reliability and validity of self-report instruments using COSMIN

Philip Powell, PhD, University of Sheffield, Sheffield, UK; Jill Carlton, PhD, University of Sheffield, Sheffield, UK; Ruth Wong, $\mathrm{PhD}$, University of Sheffield, Sheffield, UK

Aims: Duchenne muscular dystrophy (DMD) is an inherited neuromuscular condition predominantly affecting boys. It is a progressive, life-limiting condition characterised by increasing muscle weakness, loss of ambulation and motor functioning, and cardiovascular and respiratory problems. DMD has a significant impact on the quality of life $(\mathrm{QoL})$ of people living with the condition and their informal carers. While many instruments are available to assess carer QoL in DMD, it is not clear which should be used. This work aimed to assess the relative psychometric properties of self-report instruments used to assess carer QoL in DMD. Methods: A systematic review methodology and COSMIN checklists were used. A two-stage search strategy of MEDLINE, Embase, CINAHL, PsycINFO, and the Cochrane Library was used to a) identify all articles using questionnaires to assess QoL in DMD carers; and b) identify articles reporting on the measurement properties of self-report instruments for DMD carers. Complementary searches were carried out on Google Scholar (to identify development papers) and via citation tracking. Two researchers independently assessed the search results against an a priori inclusion/exclusion criteria and extracted data on the measurement properties. Each measure was assessed against COSMIN standards. DMD carers were involved as collaborators in the assessment of questionnaire content validity. A review protocol has been registered on PROSPERO

https://www.crd.york.ac.uk/prospero/ 
display_record.php?ID=CRD42020200120). Results: In Stage 1 of the searches, 981 unique articles were identified, 106 remained after title/abstract sift and 34 after full text review, with 56 possible instruments. Of 56 potential instruments, 29 were taken forward (11 were inaccessible for duplicates and 17 were excluded following a review of the scale itself). Following additional searching in Stage 2, 14 articles on measurement properties were selected. Data extraction and assessment against COSMIN criteria is ongoing and will be completed by July 2021. Conclusion: Many instruments exist to assess QoL in carers of people with DMD. However, there is no available evidence on which tool is best suited to the job. Our ongoing review of 29 questionnaires used to assess QoL in DMD carers will provide evidence on the relative psychometric performance of these instruments, with the recommendation of which are best to use.

\section{(3055) English Translation and Cross-Cultural Adaptation of the Cost for Patients Questionnaire (CoPaQ)}

Thomas Poder, Ph.D., CR-IUSMM, Montréal, Quebec, Canada; Lucien Coulibaly, M.Sc., University of Sherbrooke, Sherbrooke, Quebec, Canada; Idriss Abakar, M.Sc., Laval University, Quebec, Quebec, Canada; Erin Strumpf, Ph.D., McGill University, Montreal, Quebec, Canada; Jason Guertin, Ph.D., Laval University, Quebec, Quebec, Canada; Maude Laberge, Ph.D., Laval University, Quebec, Quebec, Canada

Aims: Very few validated tools are available to measure health-related costs from a patient perspective. With the increase in the number of multinational and multicultural research project on the cost for patients, the need to adapt existing tools in other than the source language is essential. The objective of this study was to perform an English translation of the Cost for Patients Questionnaire (CoPaQ) that was conceptually equivalent to the original French version developed in Quebec. Methods: The translation was done using the cross-cultural translation techniques proposed by Vallerand in 1989. Five steps were considered: (1) Preparation; (2) Forward translation from French to English (two bilingual translators with English mother tongue); (3) Reconciliation (Research team); (4) Back-translation from English to French (two bilingual translators with French mother tongue); (5) Revision of all versions (Research team). Results: The translations were realized without any major difficulties. Five significant changes were made after the forward translation and seven after the back translation. The result of back translation was very similar to the original French version. Following the forward and back translation, most changes concerned unclear and confusion expressions that were difficult to translate. In these cases, the research team formulated an equivalent expression in the target version. Most changes were generally to change one word to better sounding in English. Conclusion: We produced an English translation of the Cost for Patients Questionnaire from the original French version. In the next stage, the researchers will complete the final stage of adaptation process by pretesting the prefinal version with subjects or patients from the target setting. Further researches are needed to ensure that the English version of the Cost for Patients Questionnaire demonstrates the measurement properties needed for the intended application.

\section{(3056) From patient-centred measures to patient-centred interventions: Capturing the heterogeneity of patient groups to inform intervention development}

Christina Cheng, doctor of Philosophy, Swinburne University of Technology, Hawthorn, Australia; Melanie Hawkins, PhD, Swinburne University of Technology, Hawthorn, Australia; Massuger
Wayne, Master of Nursing, Crohn's \& Colitis Australia, Camberwell, Australia; Leanne Raven, Master's in Nursing Studies, Crohn's \& Colitis Australia, Camberwell, Australia; Richard Osborne, PhD, Swinburne University of Technology, Hawthorn, Australia

Aims: Patient-centred measures should be used to inform development of patient-centred interventions. However, the traditional use of mean scores for interpretation will mask the heterogeneity of patient groups. The aim of this study was to identify subgroups among people living with inflammatory bowel disease (IBD) to better understand their varying health literacy needs to support tailored solutions. Methods: A nationwide cross-sectional survey of people with IBD was conducted in Australia (2020) using two patient-centred measures. The Health Literacy Questionnaire (HLQ) (9 scales) measures access and use of health information and services. Scale 5 (Motivated to engage with digital services) of the eHealth Literacy Questionnaire (eHLQ) was also included. A hierarchical cluster analysis using Ward's method for linkage was undertaken to identify subgroups with different health literacy patterns. A total of 3-20 cluster solutions were examined to find the optimal solution that best represents the various health literacy profiles of the cohort. Results: Among the 868 respondents who completed the survey, 866 were included for analysis. The analysis resulted in 13 clusters with different health literacy patterns. Two clusters-representing one fifth of the respondentsshowed patterns of generally higher scores across all 10 scales. Health literacy challenges were reported by almost $80 \%$ of respondents. Some respondents reported having good access to and understanding of health information but trouble engaging with healthcare services. Other respondents actively managed their health condition despite limited access to health information and services. Others did not have good access to information but did have good understanding of information when provided. About $7 \%$ of respondents had generally lower scores across all 10 scales. Conclusion: This study used cluster analysis to gain an in-depth understanding of the diverse health literacy needs of people living with IBD. The findings indicate that respondents experienced a range of challenges when accessing and using health information and services, while potential strengths were also identified. These data support consumer organisations and healthcare providers to tailor patient-centred interventions and implement service quality improvements to meet the varying needs of service users and to improve health equity.

(3057) Using machine learning to analyze patients' experiences in free text data as they use music therapy to ease the stress of hospitalization for cancer treatment

Thomas Atkinson, Ph.D., Memorial Sloan Kettering Cancer Center, New York, New York, USA; Nicholas Emard, MS, Memorial Sloan Kettering Cancer Center, New York, New York, USA; Kathleen Lynch, MS, MPH, Memorial Sloan Kettering Cancer Center, New York, New York, USA; Lior Gazit, MSc, Memorial Sloan Kettering Cancer Center, New York, New York, USA; Stefania Sokolowski, MA, Memorial Sloan Kettering Cancer Center, New York, New York, USA; Kevin Liou, MD, Memorial Sloan Kettering Cancer Center, New York, New York, USA; Jun Mao, MD, Memorial Sloan Kettering Cancer Center, New York, New York, USA; Yuelin Li, $\mathrm{PhD}$, Memorial Sloan Kettering Cancer Center, New York, New York, USA

Aims: Patient-reported outcomes measures are the gold standard for the capture of patient subjective health-related quality of life (HRQoL) experiences. Standard HRQoL questionnaires are typically written by researchers after immersion in patient-centered qualitative data. However, items are fixed in length and are, thus, limited in reproducing everchanging patient disease- and/or treatment-related experiences. Advances in machine learning may allow HRQoL to be 
captured verbatim from patient free text statements rather than the fixed words in questionnaires. To explore this, the present pilot study applied Bidirectional Encoder Representations from Transformers (BERT) to learn human-coded themes in patient semi-structured interview transcripts. Methods: Data from a quality improvement study of an Integrative Medicine service at a tertiary cancer center were included. Patients were referred to receive music therapy in the inpatient setting, followed by a brief one-on-one in-person semistructured interview. A qualitative methods expert coded the transcribed interviews for key themes, which were used to train the BERT model. A total of 259 free text entries from 10 patients were randomly divided into sets for training $(n=204)$, validation $(n=52)$, and testing $(\mathrm{n}=3)$. The learning and predictive accuracies of BERT were compared. Results: Expert qualitative analysts generated 35 specific codes (e.g., 'Relaxing', 'Lifting spirits', 'Calming Effect', and 'Significant/Meaningful Song'). Using optimized maximum learning and 10 epochs of training, the model was able to reach a 0.79 training accuracy but 0.29 accuracy during validation (i.e., $1 / 35$ if purely by chance). When prompted with a long sentence it had never encountered before, "She seemed like she was relaxed and she really enjoyed it ...", BERT predicted that the sentence belonged to the topic 'Relaxing' with a $71 \%$ probability, matching the topic given by expert coders. Conclusion: BERT provides a feasible option to process semistructured interview data on patients' experience and make reasonably accurate predictions even though the target texts contain words that BERT never encountered previously. However, BERT has limitations such that short text entries are prone to errors partly because of limited information. Use of BERT may be feasible for use to analyze patient free text reports of HRQoL and, thus, allow us to better understand their verbatim experiences.

(3058) Preference Study in Childhood Autism Spectrum Disorder (ASD) using the Childhood Autism Rating Scale (CARS2): A Feasibility Assessment

Kristina Hartl, MA, OPEN Health, Berlin, Germany; Nicholas Durno, MSc, OPEN Health, Oxford, UK; Ramona Schmid, MSc, PharmM, Les Laboratoires Servier, Suresnes, France; Marieke Heisen, PhD, OPEN Health, Rotterdam, The Netherlands; Olivier Ethgen, PhD, University of Liège, Liège, Belgium; Zsuzsanna Szilvasy, MSc, Autism-Europe, Budapest, Hungary; Evelyne Friedel, doctor in Law-Attorney at Law, Autism-Europe, Paris, France; Olivier Wong, MD, MSc, Medi-Qualité Omega, Paris, France; Tony Charman, PhD, King's College London, London, UK; Antonia San José Cáceres, $\mathrm{PhD}$, Hospital General Universitario Gregorio Marañón, Madrid, Spain; Axel Mühlbacher, PhD, Hochschule Neubrandenburg, Neubrandenburg, Germany; Ben Van Hout, PhD, OPEN Health, York, UK; John Brazier, PhD, The University of Sheffield, Sheffield, UK; Elly Stolk, PhD, EuroQoL Research Foundation, Rotterdam, The Netherlands

Aims: In childhood ASD, CARS2 is a condition-specific instrument to be filled out by clinicians, resulting in a score for diagnosis and severity. Our aim is to estimate a preference-based scoring of CARS2 to better understand the value of changes in the CARS2 score. Using caregivers and clinicians as proxies for autistic children, we first assessed the feasibility of establishing preferences for CARS2 attributes. Methods: Preparatory research was conducted to verify the suitability of CARS2 as the basis for a preference study in ASD, inform the study design, and establish specific feasibility criteria (Table 1). A committee of caregivers, clinicians, CARS2 experts, HTA experts and methodological experts in preference research advised on the project. The 15 CARS2 items were assessed regarding their relevance as attributes and the appropriateness of their wording for caregivers. Furthermore, choice task designs were developed: (1)
Best-Worst-Scaling (BWS) design presenting three different profiles of hypothetical autistic children; (2) Discrete Choice Experiment (DCE) design with two hypothetical profiles. The comprehensibility of the CARS2 attributes and different versions of choice tasks were tested in individual qualitative interviews with four caregivers, four clinicians, and two autistic adults. Results: Thirteen of the 15 CARS2 items were deemed appropriate attributes for the preference study, with the items 'level and consistency of intellectual response' deemed non-modifiable, and 'general impressions' having too much overlap with the other attributes. The attribute definitions and level descriptions were shortened and refined to ensure comprehension for nonexperts. The attributes were clear and understandable, caregivers and autistic adults identified with the attributes and perceived them as comprehensive, but the amount of information was a lot to process. The choice tasks were challenging for participants and needed further explanation. However, 9/10 respondents could make a choice for the presented profiles. Interviewees favored a DCE with two profiles (Fig. 1) over a BWS with three profiles. The feasibility assessment of interview findings (Table 1) did not identify any major issues that might impede the continuation to the quantitative research phase. Conclusion: A preference study in childhood ASD based on the CARS2 instrument is feasible with caregivers and clinicians as proxies for children's preferences.

\begin{tabular}{|c|c|}
\hline Criteria & Definition \\
\hline $\begin{array}{lll}\begin{array}{l}\text { Capturing relevant patient } \\
\text { experience }\end{array} & \end{array}$ & $\begin{array}{l}\text { Do the attributes, level descriptions and profiles resonate } \\
\text { with caregivers and clinicians? }\end{array}$ \\
\hline Choice task complexity & $\begin{array}{l}\text { Is the choice task question phrased in an understandable } \\
\text { manner? Does the proxy nature of the choice task make } \\
\text { sense to them? }\end{array}$ \\
\hline $\begin{array}{l}\text { Hypothetical nature of choice } \\
\text { tasks }\end{array}$ & $\begin{array}{l}\text { Is the hypothetical nature of the choice tasks clear and is } \\
\text { enough information provided to make an informed choice } \\
\text { in the hypothetical scenarios? }\end{array}$ \\
\hline $\begin{array}{l}\text { Intended meaning of attribute } \\
\text { definitions and levels }\end{array}$ & $\begin{array}{l}\text { Do the attributes and levels of the choice tasks retain the } \\
\text { same meaning as when CARS2 is used within a clinical } \\
\text { trial setting? }\end{array}$ \\
\hline Attribute dominance & $\begin{array}{l}\text { Are any attributes likely to exhibit attribute dominance, } \\
\text { i.e. do participants consistently choose those profiles } \\
\text { where a specific attribute shows a better level? }\end{array}$ \\
\hline $\begin{array}{l}\text { Cognitive burden and time } \\
\text { constraints }\end{array}$ & $\begin{array}{l}\text { Can the questions be designed such that caregivers and } \\
\text { clinicians can provide meaningful answers? Given the } \\
\text { survey time available, can background information } \\
\text { questions, warm up explanation/exercises, and a } \\
\text { sufficient number of choice tasks all be included within } \\
\text { an online survey? }\end{array}$ \\
\hline $\begin{array}{l}\text { Choice task and experimental } \\
\text { design feasibility }\end{array}$ & $\begin{array}{l}\text { What type of choice task presentation can be used to } \\
\text { minimize the cognitive burden subject to the constraint } \\
\text { that it also provides relevant data to estimate } \\
\text { preferences? Can an experimental design be developed } \\
\text { that provides the data required to model preferences for } \\
\text { the instrument considering the online survey duration and } \\
\text { target sample size? }\end{array}$ \\
\hline
\end{tabular}
necessary for the quantitative part of the preference study. 


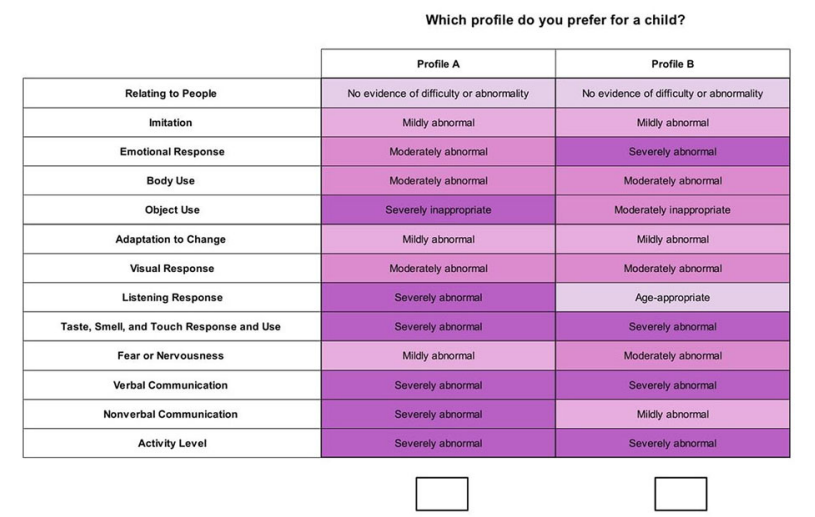

Figure 1 Choice Task Design Based on CARS2 (Standard Version) Following the Qualitative Interviews

\section{(3059) Fall Risk Factors in Adults with Obesity}

Ahmed Negm, MD, PhD, Department of Physical Therapy, Faculty of Rehabilitation Medicine, University of Alberta, Edmonton, Alberta, Canada; Gian Jhangri, MSc, School of Public Health, University of Alberta, Edmonton, Alberta, Canada; David Feeny, $\mathrm{PhD}$, 3.Department of Economics and Centre for Health Economics and Policy Analysis, McMaster University, Hamilton, Ontario, Canada; Mary Forhan, PhD, Department of Occupational Therapy, Faculty of Rehabilitation Medicine, University of Alberta, Edmonton, Alberta, Canada; Allyson Jones, PhD, Department of Physical Therapy, University of Alberta, Edmonton, Alberta, Canada

Aims: Falls are significant public health concerns. Every year, one in three older adults falls, and $10 \%$ of these falls cause serious injury or hospitalization. Although adults with obesity are at an increased risk of injury due to falls, little is known about the prevalence and risk factors of falls in this population. Risk factors such as restricted visual fields, use of an assistive walking device, medication use and fear of falling may predispose a person with obesity to falling. This study aimed to 1) estimate the prevalence of falls and 2) identify risk factors for falling that explain reported falls in adults with obesity. Methods: A cross-sectional survey of adults living with obesity was conducted recruiting participants from 1) Edmonton Bariatric Clinic (in-person survey) located in Edmonton, Alberta, and 2) the Obesity Canada website (online survey). Socio-demographic and medical characteristics fall history for the last 12 months, the activities-specific balance confidence (ABC) scale, and Health Utility Index 2 and 3 (HUI2; HUI3) were collected. The association of risk factors for falling (single and recurrent falls) was evaluated using multinomial regression models. A p $<0.05$ was considered for statistical significance. Results: Two hundred and ninety ( 150 Clinic; 140 online) participants completed the survey $(88 \%$ female, age mean $=47.1(\mathrm{SD}=11.8)$, body mass index $(\mathrm{BMI})=44.2(\mathrm{SD}=11.0)$. A hundred and twentyseven participants had no falls, 63 participants had one fall, and 100 participants had two or more falls. The mean score for ABC (fear of falling) was $79.2(\mathrm{SD}=18.7)$. The mean number of comorbidities was 3.3 ( $\mathrm{SD}=2.3$ ), with mental health issues $(43.0 \%)$ being the most prevalent condition. The univariate analysis identified BMI $(50+\mathrm{kg} /$ $\mathrm{m} 2$ ), fear of falling, using an assistive device, pain, neuropathy, hypertension, mental health, overall health status measured by HUI3, number of comorbidities, number of medications, depression, hypnotics, or sedative medications were associated with falls. Conclusion: More than half of adults with obesity from a bariatric specialty clinic and a National Obesity network reported at least one fall. However, most fall literature focuses on the older population; this study presents risk factors associated with falls in younger adults (mostly female) with obesity.

\section{(3060) Effectiveness of tools for transferring care in Intensive} Care Units: Systematic review

Andréa Van-dúnem, PhD Student, University of Guarulhos, São Paulo, Brazil; Noélle Freitas, PhD, University of Guarulhos, São Paulo, Brazil; Alfredo Pina-Oliveira, PhD, University of Guarulhos, São Paulo, Brazil; Rosa Áurea Fernandes, PhD, University of Guarulhos, São Paulo, Brazil; Meline Kron-Rodrigues, PhD, University of Guarulhos, Pindamonhangaba, Brazil

Aims: To evaluate the effectiveness of the use of tools to transfer care in patients admitted to an intensive care unit (ICU). Methods: Systematic review of the literature consulted in the electronic databases PubMed, Embase, Cochrane Library, LILACS until August 2020. Descriptors in Health Sciences (DECs) and Medical Subject Heading (MeSH) were used: "Patient Handoff", " Continuity of Patient Care “,” SBAR “,” ISBAR “,” SBAR-R “,” ISBARR “,” ISOBAR “,” Communication "and" Intensive Care Units ". There was no language restriction and only human studies were selected. Observational studies that reported the use of any tools for transferring care in patients admitted to any ICU were included. In this study, the risk of bias was assessed by Escala Newcastle-Ottawa (ENO). Results: The analysis included two studies of the total of 512 articles captured in the databases and showed that the impact on the performance of the health team suggests that the professionals' perceptions are positive and that there was an improvement in multidisciplinary communication, including nurses and doctors. As for the impact on patient care, the findings suggest that the use of transfer tools in care favors the complete filling of the medical record, as well as the notification of events in the medical records, thus, reducing the underreporting of adverse events. The bias analysis of the studies showed small methodological inaccuracies, especially regarding the possibility of a broad and comprehensive analysis of the study outcomes. Conclusion: The analysis indicated that there is an impact on the performance of the team and on patient care, but there is a notable lack of studies in the area, not reporting robust or high-quality evidence regarding the use of care transfer tools in ICUs of this institution. Therefore, it is recommended to develop studies and tools for transferring care in the ICU with the inclusion of large populations and well-designed drawings that analyze the use of tools for transferring care in the ICUs to determine their real effectiveness, patient safety and viability for the health team.

\section{(3061) Towards equity-based reporting of patient experiences in large-scale surveys: patterns of missing data by population group}

Lisa Corscadden, Office of Patient Centred Measurement, Vancouver, British Columbia, Canada; Rick Sawatzky, Trinity Western University, White Rock, British Columbia, Canada; Lena Cuthbertson, Office of Patient Centred Measurement, Vancouver, British Columbia, Canada; Sean Ho, Anchorlytics Consulting, Vancouver, British Columbia, Canada; Jae-Yung Kwon, University of Victoria, Victoria, British Columbia, Canada

Aims: Inequities in patient experiences can be better addressed with high quality information to inform change. The British Columbia Office of Patient Centred Measurement implements a provincial program to measure patient-reported experiences and health status. Interest amongst stakeholders for disaggregated reporting of results by equity-based stratifiers is growing. However, stratification across many population groups could lead to observed differences that are difficult to interpret or that may be a result of sampling, non-response, or measurement errors. Identifying differences in question non-response across population groups informs subsequent analyses and reporting. Methods: Validated questions and data from the 2018 BC 
Emergency Department (ED) Survey (EDPEC) $(n=14,008$, response rate $=36 \%$ ) were used as an exemplar to develop an analytical approach for equity-based reporting in combination with available social-demographic stratifiers of sex, age and self-reported ethnicity. Initial steps involved examining differences in self-selected question non-response across population groups, focusing on the percentage who said don't know/prefer not to answer (DK/PNTA). We present initial findings demonstrating differences in missing experience data by population group as motivation for further work. Results: The sample predominantly included older adults $(50 \%$ aged $55+)$ and females (54\%), and 65\% identified as White/European/North American. The other most common race/ethnic groups were Chinese (5\%), Indigenous (6\%) and South Asian (7\%). Questions with the highest percentage of DK/PNTA included questions on follow up care, pain and medication side effects (Fig. 1). Comparisons of patterns of DK/ PNTA across socio-demographic stratifiers revealed that females, those aged $70+$, and Chinese respondents had higher percentages of missing for most questions. South Asians tended to have a lower percentage missing. Conclusion: Early descriptive results show distinct patterns of question non-response across population groups. Failure to consider question non-response could result in misleading conclusions about inequities in experiences. These initial results provide an impetus for further analysis and stakeholder consultations in developing an equity-based approach to reporting on experience measures. Subsequent analyses will compare disaggregated results by population group with estimated experiences adjusted for missing data, differential item functioning, and case mix.

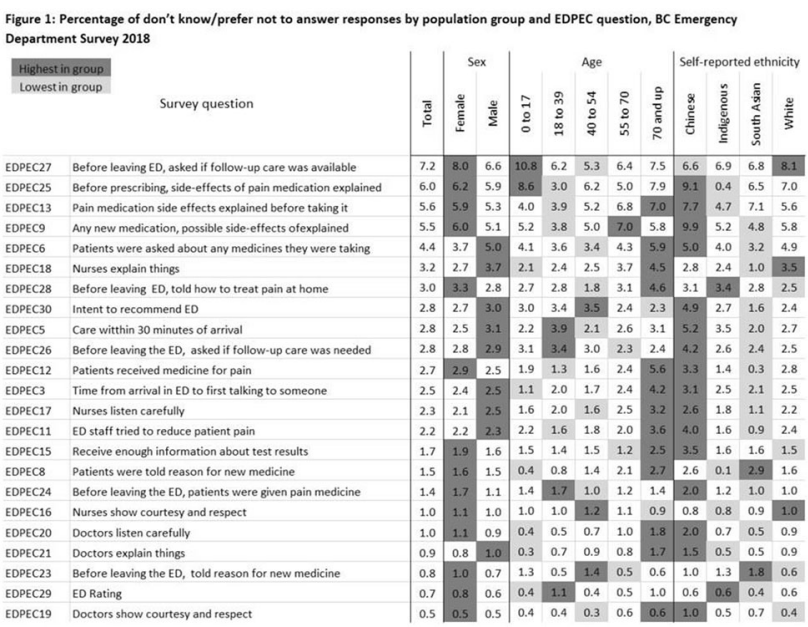

(3062) Patient and public involvement to gain direct insights into the burden of Fibrous Dysplasia/McCune-Albright Syndrome (FD/MAS) with FGF23-related hypophosphatemia

Sue Wood, Kyowa Kirin International, Marlow, UK; Claudine Woo, Ultragenyx, Novato, California, USA; Dori Beeler, Clinigma, Charlotte, North Carolina, USA; Rozanne Casper, Clinigma, Cape Town, South Africa; Samantha Mayberry, Kith Collective, Washington, District Of Columbia, USA; Kimberley McCleary, Kith Collective, Washington, District Of Columbia, USA; Angela Williams, Kyowa Kirin International, Marlow, UK

Aims: People with FD/MAS present with a variable spectrum of manifestations: skeletal features (bone deformity, fractures), as well as endocrine dysregulation (growth hormone excess, precocious puberty) and/or skin features (café au lait spots) due to a somatic mosaic mutation in the GNAS1 gene during embryogenesis. The dysplastic bone may produce excess amounts of the hormone FGF23, resulting in chronic hypophosphatemia that further exacerbates the musculoskeletal problems. FD/MAS is a rare, debilitating disease, and in people with FGF23-related hypophosphatemia, this condition exacerbates pain and limits function and mobility. A Patient and Public Involvement (PPI) exercise was conducted to ensure that there was direct patient and carer experience early in consideration of the design of a potential trial of a medicine to address FGF23-related hypophosphatemia in people with FD/MAS. Methods: Two approaches were used to gather insights from adults and carers of children with FD/MAS with/without FGF23-related hypophosphatemia identified via expert physicians. Semi-structured 1:1 interviews were conducted in Europe with three adults and parents of two adolescents. Expert physicians were consulted in development of the interview guide covering protocol considerations, disease trajectory, symptoms and disease burden. In addition, five parents of children and four adults participated in a facilitated live group discussion in the USA conducted via videoconferencing. Topics of discussion included diagnosis, burden of disease and perspectives on trial participation. Results: Both groups provided insights on trial duration, visit frequency and data collection methods to accommodate their commitments. All participants described skeletal, skin and endocrinerelated manifestations as well as pain and fatigue. Skeletal manifestations and pain were the most bothersome problems with grave need for better treatments. All participants indicated impaired physical functioning and mobility. Limitations of cognitive functioning and concentration were noted especially in children, as well as feelings of being sad and frustrated. Adults reported isolation, depression and physical impairment affecting family and interpersonal relationships. Conclusion: In this phenotypically variable disease, skeletal-related manifestations, pain and impact on function are consistently reported for people with FD/MAS with/without FGF23-related hypophosphatemia. These direct patient and carer insights reveal treatment priorities that can be incorporated into future trial design.

\section{(3063) Reliability and validity of the Colombian version of Kidscreen-27 for a children and adolescent scholar population from Bucaramanga (Colombia)}

Martha J Rodríguez, MSc, Universidad Santo Tomás, Bucaramanga, Colombia; Diana M Camargo, MSc, Universidad Industrial de Santander, Bucaramanga, Colombia; Luis C Orozco, MSc, Universidad Industrial de Santander, Bucaramanga, Colombia; Sonia C Concha, PhD, Universidad Santo Tomás, Bucaramanga, Colombia; Ethman Ariel Torres, MBA, Universidad Santo Tomás, Bucaramanga, Colombia

Aims: To assess the reliability and validity of the Colombian version of the Kidscreen-27 in school children and adolescents aged 8 to 18 years old in the city of Bucaramanga (Colombia). Methods: An evaluation of diagnostic technology was done with 1334 children and adolescents who filled out the Colombian version of the Kidscreen27. Both institutions and children were randomly selected, first by cluster sampling in 30 public and private schools and second, by simple random sampling. The test-retest reliability was assessed in 121 randomly selected students, two separate times, two weeks apart. The analyses were conducted on the entire sample as well as for age groups (8-11 years and 12-18 years), gender, and socioeconomic conditions (low and high). The internal consistency of the item responses was estimated via Cronbach's alpha coefficient as a measure of reliability of the Kidscreen scores; the test-retest reliability was evaluated by Intraclass Correlation Coefficient (ICC 2.1), values of 0.7 or higher were considered acceptable. The Rasch Rating Scale Model was used to establish the infit and outfit mean square, values between 0.6 and 1.4 indicated a good fit to the Rasch model. Differential Item Functioning (DIF) by age groups, gender and socioeconomic condition were identified. Stata version 14.0 and Winsteps software were used. Results: The mean age was 
$12.3 \pm 2.7$ years, $54.8 \%$ were women and $88.5 \%$ of the sample belonged to public schools. Cronbach's alpha ranged from 0.71 (Physical Well-Being) to 0.81 (Parents \& Autonomy) and ICC from 0.75 (Social Support \& Peers) to 0.85 (Parents \& Autonomy). Infit ranged from 0.78 to 1.32 and outfit from 0.74 to 1.45 for the five dimensions. One item (Have you been physically active?) showed uniform DIF by gender, and one (Have you had enough money to do the same things as your friends?) displayed by age groups. Nonuniform DIF was not observed. Conclusion: The Colombian version of the Kidscreen-27 showed good levels of reliability and satisfactory levels of validity considering that two items displayed DIF.

\section{(3064) Validity of the Colombian version of Kidscreen-27: A Rasch modelling approach}

Martha J. Rodríguez, MSc, Universidad Santo Tomás, Bucaramanga, Colombia; Diana M Camargo, MSc, Universidad Industrial de Santander, Bucaramanga, Colombia; Luis C Orozco, MSc, Universidad Industrial de Santander, Bucaramanga, Colombia; Sonia C Concha, PhD, Universidad Santo Tomás, Bucaramanga, Colombia

Aims: To validate the Colombian version of Kidscreen-27 using the Rasch measurement model establishing four out of six aspects of validity suggested by Messick and interpreted by Wolfe \& Smith. Methods: An evaluating diagnostic technology study was done. The Colombian version of the Kidscreen-27 was filled out by 1334 children and adolescents from third grade to high school graduation who were selected by cluster sampling from thirty public and private schools from Bucaramanga (Colombia). Gender, age groups (8-11 and 12-18 years) and socioeconomic conditions (low and high) were analyzed for the entire sample. With the use of Rasch Rating Scale Model, the substantive, content, structural and generalizability aspects of validity were evaluated. The rating scale structure, item quality, participant fit and ability, invariance structure and unidimensionality were analyzed. Invariance structure was tested via Differential Item Functioning (DIF) by gender, age groups, socioeconomic conditions and presence of functional limitation. Stata version 14.0 and Winsteps software were used for the analysis. Results: The mean age was $12.3 \pm 2.7$ years, $60 \%$ belonged to the $12-18$ years group, $54.8 \%$ were women. Five of the eight guidelines recommended by Linacre were fulfilled. Rasch item curves indicated disorder between the five response categories so it was necessary to collapse two of them resulting in three response categories. The twenty-seven items showed good fit (infit and outfit mean square 0.66 to 1.39). Uniform DIF was observed in seven items from three dimensions (Physical Well-Being, Parents \& Autonomy, and Social Support \& Peers) with regard to interest groups. Nonuniform DIF was not found. The Rasch measures explained $33.4 \%$ of the total variance with the first residual contrast accounting for $7.0 \%$. These 20 items did not target some participants, indicating that this sample had a better Health-Related Quality of Life than the questionnaire was able to evaluate. Conclusion: The resulted 20-item version of Kidscreen supported the substantive, content, structural and generalizability aspects of validity in this Colombian scholar population.

\section{(3065) No impact of Asian ethnicity on interpretation of EORTC QLQ-C30 scores: Group differences and differential item functioning in paroxysmal nocturnal hemoglobinuria}

Roland Stark, M.Ed., DeltaQuest Foundation, Concord, Massachusetts, USA; Katrina Borowiec, M.A., Boston College Lynch School of Education and Human Development, DeltaQuest

Foundation, Chicopee, Massachusetts, USA; Karl-Johan Myren, M.S.,
Alexion Pharmaceuticals, Stockholm, Sweden; Carolyn Schwartz, Sc.D., DeltaQuest Foundation, Concord, Massachusetts, USA

Aims: AimsParoxysmal nocturnal hemoglobinuria $(\mathrm{PNH})$ is a rare, life-threatening intravascular hematologic disorder with significant morbidity and premature mortality. There exists no disease-specific quality-of-life (QOL) measure for it. Its QOL effects resemble those of hematologic cancers, which supports the use of cancer-specific QOL measures in PNH clinical trials. The European Organisation for Research and Treatment of Cancer (EORTC) QLQ-C30 has published norms for many European and North American countries, but not for Asian countries. We investigated differences by Asian ethnicity in scores and item function on the EORTC QLQ-C30. Methods: MethodsThis secondary analysis focused on two non-inferiority pivotal PNH trials (NCT02946463 and NCT03056040) comparing eculizumab and ravulizumab $(n=441)$. Analysis of covariance examined the main effect of Asian ethnicity on baseline EORTC QLQ-C30 scores, after adjusting for propensity scores encompassing trial and demographic and clinical factors. Mixed modeling of longitudinal data compared subscale scores in Asian vs. non-Asian patients, after propensity adjustment. Differential item function (DIF) was examined using ordinal regression models at baseline and longitudinally, to predict item score from total score, ethnicity, and their interaction to test for uniform DIF (Main effect for Asian) and nonuniform DIF (Asian-by-total-score interaction). Finally, baseline Asian vs. non-Asian means by gender and age group were compared to aggregate general-population norms from Europe and North America to provide further context for interpreting ethnic differences. Results: ResultsOf the 15 baseline domains, Asians scored slightly better on role and emotional functioning and slightly worse on constipation and diarrhea $(0.22<$ Cohen's $\mathrm{d}<0.36)$. In longitudinal models, Asians reported slightly higher appetite loss, diarrhea, and financial difficulties than non-Asians $(\mathrm{R} 2$ increment $<0.0005)$. There was negligible uniform and non-uniform DIF, i.e., R2 0 to 0.018 , far below Zumbo's (1999) criterion of 0.13. On average there were larger differences from norms for Asians (mean $=0.05$, $\mathrm{sd}=0.44$ ) than non-Asians (mean $=-0.07, \mathrm{sd}=0.36$ ), but these differences' size and direction varied considerably by domain, age, and gender. Conclusion: ConclusionWhen compared to norms, Asians showed no systematic biases. DIF results supported this finding. We conclude that Asian ethnicity does not impact interpretation of EORTC QLQC30 scores.

(3066) Identifying biological mechanisms for the association between physical activities and poor Quality of Life (QOL): evidence from US National Health and Nutrition Examination Survey

Frederick Huang, Memphis University School, Memphis, Tennessee, USA; Jung-Hua Liu, PhD, Patient-Reported Outcomes Education Group, Germantown, Tennessee, USA; I-Chan Huang, PhD, St. Jude Children's Research Hospital, Memphis, Tennessee, USA

Aims: Previous studies have reported a significant association between a lower level of physical activity and poorer QOL; however, biological pathways behind this association are unclear. We tested the Clustered Cardiometabolic Risk (CCMR) factor as a potential mechanism to elucidate the relationship between physical activity and poor physical QOL. Methods: Study participants included 2,445 adults who participated in the US National Health and Nutrition Examination Survey in 2003-2006. Participants completed the CDC Healthy Days Questionnaire for assessing QOL, wore the ActiGraph accelerometer for assessing physical activity pattern (PAP), and completed laboratory tests for collecting biomarkers (triglyceride, glucose, insulin, waist circumference, blood pressure, HDL cholesterol) through which the CCMR factor was created. We measured 
QOL using the number of days where physical health was not good during the past 30 days, which was classified as poor ( $\geq 14$ days) or good ( $<14$ days) physical QOL. We classified PAP as moderate-tovigorous physical activity (MVPA), light-intensity physical activity (LIPA), and sedentary behavior (SB); we further classified PAP as unhealthy PAP (MVPA $<150$ min per week \& SB $>$ LIPA) or healthy PAP (MVPA $<150 \mathrm{~min}$ per week \& SB $<$ LIPA, or MVPA $\geq 150$ min per week). Logistic regressions analyzed the change in the associations of unhealthy PAP with poor physical QOL, adjusting for the CCMR factor and covariates (age, sex, education, smoking status). Results: Among participants, $51 \%$ had unhealthy PAP, and $11 \%$ had poor physical QOL. The mean Z-score for the CCMR factor was $-0.01(S D=0.61)$. Compared with having healthy PAP, individuals having unhealthy PAP had a higher risk of poor physical QOL (OR 1.92, p < 0.001). However, multivariate analysis revealed a significant association between the CCMR factor and poor physical QOL (OR 1.51, p = 0.001), whereas the association between unhealthy PAP and poor physical QOL became not significant (OR $1.35, \mathrm{p}=0.057$ ). Separate multivariable analyses focusing on the six variables underlying the CCMR factor found poor control in glucose (OR 1.20, p = 0.002), insulin (OR 1.26, p = 0.001), and waist circumference (OR 1.33, p < 0.001), instead of unhealthy PAP $(\mathrm{p}>0.05)$, was significantly associated with poor physical QOL. Conclusion: The CCMR factOR specifically glucose, insulin, and waist circumference, plays a mechanistic role to explain the association between physical activity and poor physical QOL.

\section{(3067) Measuring patient needs and treatment benefit in psoriatic arthritis: Development of a new tool}

Christine Blome, PhD, University Medical Center HamburgEppendorf, Hamburg, Germany; Helen Fedel, University Medical Center Hamburg-Eppendorf, Hamburg, Germany; Nesrine BenAnaya, MD, University Medical Center Hamburg-Eppendorf, Hamburg, Germany; Natalia Kirsten, MD, University Medical Center Hamburg-Eppendorf, Hamburg, Germany; Matthias Augustin, Prof. Dr., University Medical Center Hamburg-Eppendorf, Hamburg, Germany

Aims: Psoriasis is one of the most prevalent skin diseases. One out of four people with psoriasis is also affected by the inflammatory joint disease psoriatic arthritis (PsA). PsA causes additional impairments over and above those in patients without joint affection, resulting in specific treatment needs. Systematically assessing patients' treatment needs and benefits can support patient-centered care as it allows for targeted treatment planning and evaluation. For this purpose, the Patient Benefit Index (PBI) method has been developed, which so far only existed in a version for psoriasis and in a version rheumatoid arthritis (PBI-RA). This study aimed to develop an instrument for the measurement of patient-defined treatment goals and benefits in PsA treatment, the PBI-PsA. Methods: The instrument was developed on basis of the PBI-RA, which in turn had been developed based on a qualitative survey in 56 patients, an expert consensus including patients, and a cognitive debriefing. For the new PBI-PsA, we asked patients with PsA to describe their treatment goals using either an open questionnaire or semi-structured qualitative interviews. Patients completed the PBI-RA and gave feedback on how the questionnaire should be adapted in order to adequately cover treatment goals of people with PsA. We iteratively surveyed patients and adapted the instrument until interviewees did not express further need for changes so that saturation was achieved. Results: After surveying a total of 31 patients (11 with the open questionnaire, 20 in the interview), saturation was achieved. Changes made to the instrument included adapted wording as well as additional items. The resulting PBI-PsA includes 21 items (treatment goals), which cover the following areas: physical impairments (8 items), everyday and work life (4 items), social life (4 items), psychological burden ( 3 items), and burden due to treatment ( 2 items). Conclusion: People with PsA expessed specific treatment needs which are neither identical to those in people with psoriasis but without joint affection, nor to those of people with rheumatoid arthritis. This why the newly developed PBI-PsA may be preferrable to the existing PBI versions in this patient group. A psychometric evaluation of the PBI-PsA is currently ongoing.

\section{(3068) Using Fourier analysis to examine variations in outcome} scores for individuals with Meniere's Disease

Antoinette Davey, University of Exeter, Health Service and Policy Research Group, Exeter, UK; Gary Abel, University of Exeter Medical School, Exeter, UK; Jess Tyrrell, University of Exeter Medical School, Exeter, UK; Colin Green, University of Exeter Medical School, Exeter, UK; Jose Valderas, University of Exeter Medical School, Exeter, UK

Aims: Meniere's disease is an incurable, chronic disorder of the inner ear, with patients experiencing varying levels of severity in hearing loss, tinnitus, aural fullness and vertigo, affecting around 120,000 people in the UK. The symptoms experienced by patients significantly impact on patients' quality of life, with increased incidences of social isolation amongst sufferers. There has been a lack of literature on how time of the day affects Meniere's symptoms. Focusing on the fluctuating patterns of Meniere's symptoms may support clinical practitioners in better understanding, supporting and diagnosing patients. The aim is to examine the symptom severity profile over the course of a 24-h period and weekly period using a spectral analytical approach. Methods: A pre-existing dataset was provided which used the Meniere's Monitor mobile app to collect data from Meniere's sufferers on a daily basis including their level of severity in dizziness, aura fullness, tinnitus, and hearing loss. Other questions regarding stress, sleep quality and demographics were collected. Data was collected between 2015 and 2017 and a total of 853 individuals provided data. Variability of symptom severity over a $24-\mathrm{h}$ period was assessed with a multivariate mixed-effects regression model using Fourier components. Time transformations, using sine and cosine functions, were created and added to the four main symptoms. Adjustments were made to differentiate trends from demographic factors. Results: The majority of participants were female $(68.3 \%)$, and the mean age of the sample was 48.9 years. Over half of the participants were employed (58.3\%), and from Europe (54.2\%). There was variation in all four symptoms across a 24-h period. Peak aura fullness occurred between 4 and 8 pm compared to midnight. Tinnitus severity peaked at three points in the day from early morning (6am), midday $(12 \mathrm{pm})$, and $5 \mathrm{pm}$. Dizziness symptoms peaked at different points in the day, mainly $10 \mathrm{am}$ and $3 \mathrm{pm}$. Conclusion: Usage of fourier transformation enabled variability of Meniere's symptoms to be captured and analysed over a 24-h period demonstrating peaks of symptoms at different times of the day. This analytic method would be useful for patients in better understanding and managing the disease ultimately affecting their overall wellbeing.

(3069) Extracting Big Data from the Internet for Development of a New Patient-Reported Outcome Measure for Breast Implant Illness-a Proof of Concept study

Manraj Kaur, PT, PhD, Brigham and Women's Hospital/Harvard Medical School, Boston, Massachusetts, USA; Jinjie Liu, Harvard University, Boston, Massachusetts, USA; Sylvie Cornacchi, McMaster University, Hamilton, Ontario, Canada; Anne Klassen, McMaster University, Hamilton, Ontario, Canada; Andrea Pusic, 
Brigham and Women's Hospital/Harvard Medical School, Boston, Massachusetts, USA

Aims: Individuals with health conditions-especially rare and new conditions - often resort to online patient forums to share their experiences with illness/treatments, ask questions and seek resources. This large amount of rich patient data is freely available and has rarely been used in patient-reported outcomes (PRO) research. In this study, we will use the example of breast implant illness (BII) to demonstrate the feasibility of using big data from the internet to develop a BII-specific patient-reported outcome measure (PROM). Methods: BII is a term to describe a constellation of signs and symptoms experienced by women post-implant surgery (cosmetic/post-mastectomy). To-date, there are no diagnostic tests for BII or diagnostic codes; however, BII is being monitored by the US FDA-making it suitable for this proof of concept study. Nine publicly available BII-specific web forums were chosen post-consultation with BII-advocacy leaders. Web structures for web forums were reviewed and the Python Selenium package was used to automate extraction (known as "web scraping") of de-identified text from the individual posts/comments. Python pandas package was used to wrangle raw scraped data into a Microsoft Excel spreadsheet. Data were coded line-by-line and women's descriptions of BII experience were extracted. Constant comparison was used to refine codes and develop an item pool for PROM development. Results: Preliminary analysis revealed that fatigue, brain fog, pain (joints and breasts), anxiety, and hair loss were the most commonly reported symptoms. Many women also described emotional distress due to reduced participation in usual activities, fear of worsening symptoms, and lack of definitive diagnoses. Women seeking explant were concerned about appearance of breasts post-explant and long-term outcomes of en bloc versus total capsulectomy. Few women who had had explant surgery reported partial resolution of symptoms alongside improved physical and psychosocial well-being. Once analysis is complete, this data will be used to supplement in-depth interviews with women with BII, and develop BII-specific PROM. Conclusion: Web scraping is an innovative application of big data from the internet that allows a large amount of patient-reported data to be collected efficiently. This data can be used to inform the development of PROMs, understand key priorities and define patient-led research questions.

\section{(3070) Validation of a PROM for use in pediatric head and neck} cancer: The FACE-Q Craniofacial Module

Yi Wang, McMaster University, Hamilton, Ontario, Canada; Charlene Rae, PhD Candidate, McMaster University, Hamilton, Ontario, Canada; Karen Wong Riff, MD, PhD, The Hospital for Sick Children, Toronto, Ontario, Canada; Elena Tsangaris, PhD, Brigham and Women's Hospital, Boston, Massachusetts, USA; Marinka Hol, $\mathrm{MD}, \mathrm{PhD}$, Amsterdam UMC, Amsterdam, The Netherlands; Paul Nathan, MD, MSc, The Hospital for Sick Children, Toronto, Ontario, Canada; Eric Bouffet, MD, The Hospital for Sick Children, Toronto, Ontario, Canada; David Dix, MBChB, BC Children's Hospital Research Institute, Vancouver, British Columbia, Canada; Anne Klassen, DPhil, McMaster University, Hamilton, Ontario, Canada

Aims: Existing patient-reported outcome measures (PROMs) for patients with facial differences lack content validity, as few items address appearance and function issues. The FACE-Q Craniofacial Module is a new PROM developed to measure outcomes important to patients aged 8-29 years with a broad range of craniofacial conditions. This PROM has 27 scales and checklists that measure appearance, health-related quality of life, facial function and adverse effects. The aim of this study was to determine if FACE-Q content is relevant to patients with pediatric head and neck cancer (HNC), in order to include such patients in the FACE-Q international field-test. Methods: Cognitive interviews with patients with $\mathrm{HNC}$ aged 8 to 29 years $(n=15)$ were conducted and feedback from experts in pediatric oncology $(n=21)$ was obtained. Input was sought on all aspects of the FACE-Q content. The international field-test sample included patients from France, the The Netherlands, UK, and USA, aged 8 to 29 years and who were treated for a head and neck tumor when they were aged 0 to 18 years. The field-test data were analysed using Rasch Measurement Theory (RMT) analyses. Results: A small number of items $(n=6)$ were flagged for review for comprehensibility. Instructions, the time frame for responding, and response options were found to be comprehensible and appropriate by almost all patient and expert participants. Participants identified 10 missing items across the core scales, while experts identified swallowing/ dysphagia as an important item missing from the mouth function scale. For the international field-test, 112 participants with head and neck cancer were recruited. Psychometric analysis provided support for the reliability and validity of FACE-Q scales in a combined sample of 4743 patients with a broad range of cleft and non-cleft craniofacial conditions. Conclusion: The cognitive interviews with paediatric head and neck cancer patients showed that the FACE-Q Craniofacial Module has content validity for this patient population. Given that it can be hard to accrue a large sample to validate a PROM in rare conditions, the inclusion of a cohort of cancer patients in the field-test represents a strength of our study.

(3071) Rasch analysis of the brief Michigan Hand Questionnaire in patients with thumb osteoarthritis

Shannon Killip, MSc, McMaster University, Hamilton, Ontario, Canada; Joy MacDermid, PhD, PT, University of Western, London, Ontario, Canada; Tara Packham, PhD, OT Reg. (Ont.), McMaster University, Hamilton, Ontario, Canada; Robbert Wouters, PhD, Erasmus MC Rotterdam, Rotterdam, The Netherlands; Kathryn Sinden, PhD, RKin, Lakehead University, Thunder Bay, Ontario, Canada; Rebecca Gewurtz, PhD, OT Reg. (Ont.), McMaster University, Hamilton, Ontario, Canada; Ruud Selles, PhD, Erasmus MC Rotterdam, Rotterdam, The Netherlands; Harm Slijper, PhD, Erasmus MC Rotterdam, Rotterdam, The Netherlands; submitted on behalf of "The Hand-Wrist Study group collaborators"

Aims: The brief Michigan Hand Questionnaire (brief MHQ) is a 12-item self-reported measure of hand function for patients with hand disorders which has been validated using Classical Test Theory. The purpose of this Rasch analysis is to assess the psychometric properties of the brief MHQ for people with thumb osteoarthritis, and to make recommendation for improvements to the questionnaire if needed. Methods: The Michigan Hand Questionnaire and demographic data were collected from 923 thumb osteoarthritis patients treated nonsurgically or surgically in clinics for hand surgery and therapy in the The Netherlands. Rasch analysis was performed on the 12 items of the brief MHQ using RUMM 2030 to assess the fit of the brief MHQ to the Rasch model. To determine fit, analysis of fit summary statistics, individual person fit and individual item fit were assessed. Threshold distributions were assessed to identify if any items required rescoring. The Person Separation Index and Cronbach's alpha were calculated to measure reliability of the brief MHQ. Differential item functioning was assessed to identify item bias, and unidimensionality when combined with the Principal Component Analysis. Results: The brief MHQ showed misfit $(\chi 2=1312.5, \mathrm{df}=48, \mathrm{p}<0.0001)$ with 6 items having disordered thresholds and 9 items requiring rescoring. After deleting 3 of the rescored items due to significant item fit residuals, the brief MHQ has an acceptable reliability (Cronbach's alpha= 
$0.79)$, but misfit to the model $\left(\chi^{2}=49.6, \mathrm{df}=18, \mathrm{p}=0.0001\right)$, multidimensionality ( $10.2 \%$ of t-tests were significant), and item bias from non-uniform differential item functioning for 7 items across many person variables were found. Conclusion: Although no satisfactory solutions were found to correct the misfit to the Rasch model, it is recommended that the response options of the brief MHQ be rescored, and that items 6,9 and 10 be removed. The lack of unidimensionality indicates that the items do not represent the singular construct of hand disability and that totalling the scores of the brief MHQ does not provide a valid measure of hand disability for people with thumb osteoarthritis, which decreases the utility of the brief MHQ in clinical or research settings.

\section{(3072) Evaluating methods to assess thematic saturation in qualitative research for health outcomes: identifying a practical approach to evaluate sample adequacy}

Kayla Scippa, BA, Parexel International, Billerica, Massachusetts, USA; Ewelina Rzepa, PhD, Parexel International, Uxbridge, UK; Eva Brown Hajdukova, PhD, Parexel International, Prague, Czech Republic; Kavita Jarodia, PhD, Parexel International, Mohali, India; Katja Rudell, PhD, Parexel International, Uxbridge, UK

Aims: Thematic saturation is a principal research method used to determine sample sizes in qualitative studies. However, limited methodological descriptions of saturation are included in publications, often with little justification provided to support achievement in thematic saturation. There is a need for more consistent approach in describing rationale for qualitative sample adequacy. This research sought to compare common methods used to assess saturation in health outcomes research in order to identify the most practical and robust approach. Methods: A targeted literature review identified publications related to assessing health outcomes in qualitative research that included descriptions of operationalizing saturation. A shortlist of publications was generated based on the ability to recreate the saturation conditions using alternative data sets. Three simulation qualitative data sets, each $n=25$, were generated using an online database test data generator (generatedata.com). Three simulation data sets were generated in order to evaluate the reproducibility of results. Each saturation method on the data set was operationalized manually using Microsoft excel. Saturation results were then compared across methods and each data set. Results: Seven uniquely described saturation methods identified from the literature (Table 1) were operationalized using the test data sets. Results of the saturation experiment showed that in the first data set, methods 1 and 3 did not achieve saturation. In the second data set, methods 1,2 and 3 did not achieve saturation. In the final data set, all methods achieved saturation. The most prominent methods to reliably achieve saturation from our test datasets utilized cumulative comparison by chronologically evaluating novel conceptual information across the sample (methods 4, 5 and 7). Although method 6 achieved saturation in three data sets, this method is not deemed suitable to support health outcomes research as it pre-determines the number of prevalent themes to be identified from the data set hence imposes risk of missing other potentially important themes. Conclusion: Although saturation needs to be operationalized in a manner that is consistent with the research questions, assessing the cumulative occurrence of new concepts appears to be one of the most robust methods to determine the point at which data collection can be discontinued.

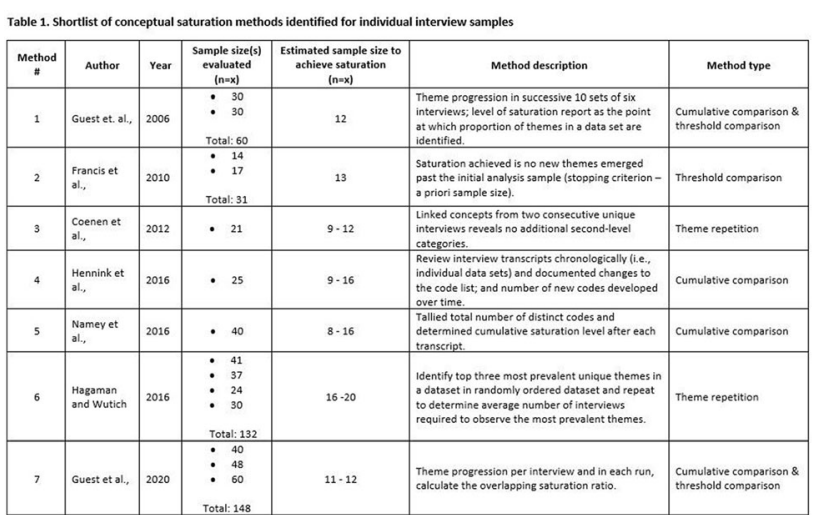

(3073) Vaporized nicotine use among current and former tobaccosmoking patients in primary HIV care: initiation, patterns of use, and perceived health effects

Rob Fredericksen, PhD MPH, University of Washington, Seattle, Washington, USA; Emma Fitzsimmons, University of Washington, Seattle, Washington, USA; Sharon Brown, University of Washington, Seattle, Washington, USA; Stephanie Loo, Fenway Community Health, Boston, Massachusetts, USA; Sarah Dougherty, University of Alabama, Birmingham, Alabama, USA; Lydia Drumright, University of Washington, Seattle, Washington, USA; John Pearce, University of Washington, Seattle, Washington, USA; Bridget Whitney, University of Washington, Seattle, Washington, USA; Robin Nance, University of Washington, Seattle, Washington, USA; Stephanie Ruderman, University of Washington, Seattle, Washington, USA; Kenneth Mayer, Fenway Community Health, Boston, Massachusetts, USA; Michael Mugavero, University of Alabama, Birmingham, Alabama, USA; Heidi Crane, University of Washington, Seattle, Washington USA; Andrew Hahn, University of Washington, Seattle, Washington USA

Aims: Little is known about the long-term health effects of using vaporized nicotine (VN). Recent deaths and illness in the general population have been traced to ingredients such as vitamin $\mathrm{E}$ acetate. Among people living with HIV (PLWH), there is a dearth of research elucidating perceptions of the health effects of $\mathrm{VN}$, patterns of $\mathrm{VN}$ use, and effectiveness of vaping as a tobacco cigarette cessation tool. Methods: We conducted in-depth, semi-structured qualitative interviews with PLWH that used VN in the past year at 3 geographically diverse U.S. HIV care clinics (AL, MA, WA). All were current or former tobacco cigarette smokers. We queried 1) reasons for starting $\mathrm{VN}$ use, 2) general knowledge regarding $\mathrm{VN}$ products, 3) user experience, 4) patterns of VN use, including concurrent use of other substances and tobacco cigarettes, 5) perceptions of $\mathrm{VN}$ health effects, 6) social dimensions of use, and 7) VN cessation attempts. Trained qualitative analysts coded transcripts for emergent themes within these areas. Results: All participants $(n=24,33 \%$ female, mean age $42,54 \%$ nonwhite) reported starting VN in order to reduce/ stop smoking tobacco cigarettes. VN was widely regarded as healthier and less carcinogenic than smoking tobacco cigarettes. However, participants had limited to no knowledge of vaping fluid ingredients; $21 \%$ falsely believed vaping to be nicotine-free. A quarter of participants that vaped had stopped smoking tobacco cigarettes entirely; $46 \%$ reduced smoking. However, $38 \%$ of those that vaped continued to routinely smoke tobacco cigarettes, often on the same day. Only one participant $(4.2 \%)$ completely stopped both vaping and smoking. Participants reported that vaping as an activity poorly replicates desired elements of the tobacco cigarette smoking ritual, and reported difficulty measuring intake levels and achieving satiety. Conclusion: VN was described by PLWH as having only marginal effectiveness as 
a tobacco cigarette cessation tool and was generally regarded as an inferior substitute. PLWH may be at risk for unanticipated health problems resulting from $\mathrm{VN}$, due to 1) concurrent use with tobacco cigarettes, 2) misperceptions of ingredients in vaping liquid, 3) perceptions of yet-unproven health benefits of vaping relative to smoking, and 4) difficulty measuring and tracking use.

(3074) Health-related quality of life among university professors from different fields of knowledge at a Colombian university

Juan J Sánchez, DDS, Universidad Santo Tomás, Bucaramanga, Colombia; Daniela L Sierra, DDS, Universidad Santo Tomás, Bucaramanga, Colombia; Martha J Rodríguez, MSc, Universidad Santo Tomás, Bucaramanga, Colombia; Ethman A Torres, MBA, Universidad Santo Tomás, Bucaramanga, Colombia

Aims: To assess the health-related quality of life (HRQL) among university professors from different areas of knowledge at a Colombian private university from October to November 2020. Methods: A cross-sectional study was conducted with 267 university professors who belonged to engineering, architecture, law, health, economics, basic science, foreign languages, and humanities areas using the Colombian abbreviated version of the World Health Organization Quality of Life (WHOQOL-BREF) questionnaire. Socio-demographic (sex, age, socioeconomic condition, educational level, marital status, chronic disease, physical activity, and medicine consumption), working situation (job title, weekly working hours, face-to-face/online classes, and teaching experience), and HRQL (physical health, psychological health, social relationships, and environment) variables were evaluated. A descriptive analysis was carried out, and Student's t-test and ANOVA were applied when appropriate. Also, a multiple linear regression analysis was performed. A p-value $<0.05$ was considered to be statistically significant. Results: The mean age was $44.3 \pm 10.8$ years, $51.3 \%$ were women, and $50.6 \%$ were married. Most participants $(62.6 \%)$ had a master's degree, 206 (77.2\%) had online classes due to the COVID-19 pandemic, and $70.4 \%$ worked $40 \mathrm{~h} /$ week at the university. Regarding their field of knowledge, $33.3 \%$ were from health areas, $24.0 \%$ from engineering and architecture areas, $21.0 \%$ from law areas, $13.1 \%$ from economics, and $8.6 \%$ belonged to other areas. Less than half $(39.7 \%)$ of the professors rated their HRQL as "very good", and 109 (40.8\%) were "very satisfied" with their health. Doing physical activity had a great influence on the physical health, psychological health, social relationships, and environment domains ( $\beta$ : 7.7 95\% CI 4.4-11.1/ $\beta$ : 7.1 95\% CI 3.7-10.4 / $\beta: 9.8$ 95\% CI 5.4-14.3 / $\beta: 6.7$ 95\% CI 3.4-10.1). Having face-to-face classes displayed a positive influence on physical health ( $\beta$ : 5.7 95\% CI 1.7-9.8), psychological health $(\beta: 5.395 \%$ CI $1.3-9.2$ ), and social relationships domains ( $\beta: 6.195 \%$ CI $0.9-11.3$ ). There was not any significant statistical difference regarding the field of knowledge teachers had. Conclusion: Doing physical activity and having face-to-face classes had an impact on HRQL.

\section{(3075) Screening for patient-reported distress and psycho- oncological treatment need in oncological routine: rapid implementation triggered by a global pandemic}

Monika Sztankay, PhD, University Hospital of Psychiatry II, Innsbruck, Austria; Lilo Fauser, BSc, University Innsbruck, Innsbruck, Austria; Anne Oberguggenberger, $\mathrm{PhD}$, University Hospital for Psychiatry II, Innsbruck, Austria; Monika Ladurner, Innsbruck University Hospital, Dept. Of Gynaecology and Obstetrics, Innsbruck, Austria; Barbara Pichler, Innsbruck University Hospital, Dept. Of Gynaecology and Obstetrics, Innsbruck, Austria; Daniel Egle, MD, Innsbruck University Hospital, Dept. Of Gynaecology and Obstetrics, Innsbruck, Austria; Christine Brunner, MD, Innsbruck University Hospital, Dept. Of Gynaecology and Obstetrics, Innsbruck, Austria; Florian Moritz, MD, Innsbruck University Hospital, Dept. Of Gynaecology and Obstetrics, Innsbruck, Austria; Alain Zeimet, Prof, Innsbruck University Hospital, Dept. Of Gynaecology and Obstetrics, Innsbruck, Austria; Petra Wiesbauer, MD, Innsbruck University Hospital, Dept. Of Gynaecology and Obstetrics, Innsbruck, Austria; Lisa Wintner, MSc, Medical University Innsbruck, Innsbruck, Austria; Bernhard Holzner, Prof, University Hospital for Psychiatry II, Innsbruck, Austria; Verena Meraner, Dr, University Hospital for Psychiatry II, Innsbruck, Austria

Aims: The assessment of patient-reported cancer-related distress and psycho-oncological treatment need is an evidence-based practice supported by clinical guidelines. Implementation in oncological routine remains, however, challenging. Restrictions due to the COVID pandemic lead to necessary changes in work procedures of psycho-oncological health care providers worldwide triggering the need for immediate action. Herein, we describe the rapid implementation of a standardized PRO assessment triggered by contextual factors (i.e. the start of the pandemic) in the workflow of a single oncological unit with very high patient fluctuation. Methods: Integration into clinical routine was adapted to contextual and system factors following a participatory approach involving all multi-professional stakeholders. An adapted version of the Hornheider Screening Instrument (HIS) was used for screening at each admission time point to the gynaeco-oncological inpatient-unit at the Innsbruck University Hospital, Dept. Of Gynaecology and Obstetrics, Austria. Feasibility evaluation was based on staff and patient participation and direct user-feedback. Results: In a one-year period, from 16th March 2020 to 17th March 2021, 523 patients (incl. two male patients) were screened with an overall of 1.066 assessment time points (completion rate of $93 \%$, assessment time points ranging from one to 17). Patients' median age was 60 years (range 20 to 97 years). Sixty-three per cent $(\mathrm{n}=332)$ of the patients had breast, 35\% $(\mathrm{n}=186)$ gynaecological cancer (eg. ovarian or cervical carcinoma). Initiation of screening completion was nurse-led, while psycho-oncologists $(n=3)$ performed data analyses, initiation of clinical action and data documentation. Screening results were fed back to the somatic health care team and discussed in the multi-disciplinary board. Feedback by staff emphasised the need for a multi-lingual instrument as well as the necessity of establishing linkage into the clinical information system to reduce administrative burden for the psycho-oncological treatment team. Conclusion: We report on the results of the rapid implementation of a standardized PRO assessment in the workflow of a single oncological unit with very high patient fluctuation. The screening procedure proved to be feasible and sustainable in clinical practice, while undergoing further adaption and improvement based on stakeholder feedback.

(3076) Validation of the French version of the Post-traumatic Growth Inventory in melanoma and breast cancer patients

Yseulys Dubuy, UMR INSERM 1246 SPHERE, Nantes, France; Véronique Sébille, professor UMR INSERM 1246 SPHERE, Nantes, France; Marianne Bourdon, PhD, UMR INSERM 1246 SPHERE, Nantes, France; Myriam Blanchin, PhD, UMR INSERM 1246 SPHERE, Nantes, France

Aims: The diagnosis of cancer is a traumatic event having negative consequences on patients' life. However, it may also favor positive psychological changes such as greater appreciation of life, spiritual growth, increased personal strength, new possibilities, and stronger relationships with others. These changes (that can arise when facing any traumatic event) have been acknowledged as "post-traumatic growth" and can be measured by the Post-Traumatic Growth 
Inventory (PTGI). Although the PTGI is available in several languages, data on the psychometric properties of the French-version PTGI are scarce. The current study was undertaken to provide a more comprehensive assessment of the reliability and validity of the French translation of the PTGI in a sample of patients with breast cancer or melanoma. Methods: The French translation of the PTGI is used as one of the assessment tools of the ELCCA study. ELCCA is a French cohort aiming to study socioeconomic, psychological, and healthrelated quality of life (QoL) changes following breast cancer or melanoma diagnosis (early stage, non-metastatic). Data collected one year post-diagnosis were used to assess the reliability and construct validity (i.e. factor structure, convergent and divergent validities, and known groups validity) of the PTGI. Reliability was assessed by Cronbach's alpha coefficients and Loevinger's scalability coefficients. Construct validity was evaluated using confirmatory factor analysis, item-rest score correlations, and comparisons between melanoma and breast cancer patients. Results: A total of 91 melanoma patients and 319 breast cancer patients participated in the oneyear post-diagnosis follow-up visit. The French translation of the PTGI supports the structure of the original version, with 21 items split into five domains: appreciation of life, spiritual growth, increased personal strength, new possibilities, and stronger relationships with others. This result was suggested by the good fit of the confirmatory factor analysis and the adequate reliability revealed by Cronbach's alpha coefficients and other psychometric indices. Conclusion: Integrating PTGI into studies is a crucial issue to evaluate positive psychological changes following traumatic life events. It would enable a better understanding of post-traumatic growth and could help identify situations where more personalized psychological support is needed.

\section{(3077) Translation and Linguistic Validation of the NFBrSI-24 for Use with Brain Cancer Patients Worldwide}

Lillian Savic, FACITtrans, Ponte Vedra, Florida, USA; Emily ParksVernizzi, MFA, FACITtrans, Ponte Vedra, Florida, USA; Benjamin Arnold, MA, FACITtrans, Ponte Vedra, Florida, USA; Jason Bredle, MFA, FACITtrans, Ponte Vedra, Florida, USA

Aims: The purpose of this study was to translate and linguistically validate the National Comprehensive Cancer Network/Functional Assessment of Cancer Therapy-Brain Cancer Symptom Index (NFBrSI-24), a 24-item patient-reported outcome (PRO) instrument designed for adult patients diagnosed with brain cancer. The instrument was translated/validated in 18 languages-Bengali, Bulgarian, Croatian, Gujarati, Hungarian, Japanese, Korean, Lithuanian, Malay, Marathi, Romanian, Serbian, Slovene, Tagalog, Tamil, Telugu, Urdu, and Vietnamese for use in Bulgaria, Croatia, Hungary, India, Japan, Lithuania, Malaysia, the Philippines, Romania, Serbia, Slovenia, South Korea, and Vietnam. Methods: The NFBrSI-24 was translated into each language based on the FACIT methodology consisting of two forward translations, one reconciled version of the two forward translations, one back-translation into English, independent review, harmonization across languages, and cognitive interviewing. 180 patients completed the translated NFBrSI-24 instrument in their native language and participated in an individual cognitive interview. The interviews evaluated the relevance, understandability, and appropriateness of the NFBrSI-24 translations, and qualitative analyses of patients' comments assessed the linguistic equivalence of each translated version. Results: Total study sample consisted of 180 brain cancer patients ( 79 women/101 men) with a mean age 52 (18 to 93 ). During the harmonization process, the phrases "find the right word(s) to say what I mean," "a change in my personality," and "I get frustrated" required alternative translation solutions to ensure cultural appropriateness and conceptual equivalence in some languages; and the revisions were endorsed by patients during cognitive interviews. Translations were revised as needed if patients' comments revealed misunderstanding of an item's intended meaning, e.g., "coordination," "seizures (convulsions)," and "difficulty expressing thoughts". Conclusion: The NFBrSI-24 has demonstrated linguistic validity across the 18 languages included in this study and is now available in a total of 35 languages. The translations are conceptually equivalent to the English source and are considered acceptable for PRO assessment in international research studies and clinical trials.

(3078) How can response shift be induced? Lessons from the realworld

Kedar Mate, BSc (PT), MSc. PhD., Mayo Clinic/McGill University Health Centre, Montreal, Quebec, Canada; Nikki Ow, OT. PhD., University of British Columbia, Vancouver, British Columbia, Canada; Nancy Mayo, PT. PhD., McGill University, Montreal, Quebec, Canada; Brittany Lapin, PhD. MPH, Cleveland Clinic, Cleveland, Ohio, USA; Richard Sawatzky, PhD. RN., Trinity Western University, Langley, British Columbia, Canada; Ruth Barclay, PT. PhD., University of Manitoba, Winnipeg, Manitoba, Canada

Aims: The experiences that surround the diagnosis, treatment, and recovery of a health condition can change how a person self-evaluates health outcomes over time. Response shift (RS) occurs when circumstances arise over time that make people change their evaluation of the underlying construct. These changes occur because people recalibrate, reprioritize, or reconceptualize the health outcomes under study or change the appraisal processes used to respond to the questions posed. Some therapeutic programs like psychotherapy and rehabilitation can induce a response shift by providing tools to shift focus. The objective of this project is to identify: (i) the specific elements of intervention or treatment that have the potential to induce response shift; and (ii) characteristics of the patient and/or social context that supports positive response shift or induces negative response shift. Methods: The members of Response Shift Special Interest Group (SIG) will be asked to self-identify or nominate experts whose area of clinical practice or research has the potential to induce a response shift. The interested persons will be invited to share opinions and experiences from clinical practice and research on how RS may influence outcomes and perceptions in their patients or study participants. A preliminary set of questions has been piloted with six experts and found to be feasible and relevant, with some revisions. Opinions will be sought using an open-ended survey. Results: Six experts from areas of psychiatry, psychology, addiction rehabilitation, and physical rehabilitation were interviewed. Areas of relevance that emerged were: revalue the importance of problem areas; prioritize areas that are under personal control; recalibrate the extent to which there is a problem; recognize blame; short term goal setting; vicarious learning; and peer-support. Lack of "safety net" factors prevented people from making a positive response shift. Conclusion: It seems that many interventions have elements in them that are targeted to inducing response shift even if not directly recognized. These elements identified from the survey will be combined with those from the literature to develop a response shift curriculum that could be used to educate health professionals on the power of response shift in healing.

(3079) Characterizing the quality-of-life impact of Duchenne Muscular Dystrophy on caregivers: a case-control investigation 
Carolyn Schwartz, ScD, DeltaQuest Foundation and Tufts University School of Medicine, Concord, Massachusetts, USA; Roland Stark, M.Ed., DeltaQuest Foundation, Concord, Massachusetts, USA; Ivana Audhya, M.Sc., Sarepta Therapeutics, Cambridge, Massachusetts, USA; Katherine Gooch, Ph.D., Sarepta Therapeutics, Cambridge, Massachusetts, USA

Aims: This study examined the impact of Duchenne Muscular Dystrophy (DMD) on family-member caregivers in terms of quality of life, life stress, and indirect costs, as compared to a stratified comparison group of parents of similar-age children without DMD. Methods: A web-based survey included DMD caregivers and a nationally representative comparison group of parents of children without DMD stratified by Child Age Group. Outcomes included quality of life, resilience, caregiver burden, stressful life events, financial strain, out-of-pocket costs, work productivity and unrealized ambitions. General linear models assessed the main effect of Caregiver Group and the interaction of Caregiver Group-by-Child-AgeGroup, after adjusting for demographic covariates. Results: Compared to parents without a DMD child, DMD Caregivers reported better physical health but worse mental health, positive affect/well-being, environmental mastery, difficulty paying bills, and more hours missed from work. Providing caregiving support for DMD teenagers was the most challenging. DMD caregivers curtailed their educational and professional ambitions, and modified their homes to accommodate the disability associated with DMD. Their non-DMD children had to make sacrifices as well. Nonetheless, in resilience and life stress, DMD caregivers were comparable to the comparison group, and showed consistent levels of positive emotions across the age of their DMD child. Conclusion: DMD caregivers fared worse on most outcomes and faced more hurdles in work life. They face constraints and hidden costs that impact their health and financial well-being. Caring for teenagers with DMD reported higher impact. Nonetheless, parents of DMD children of all ages maintained notable resilience and positivity.

\section{(3080) The use of pediatric PROMIS® itembanks in Dutch boys with haemophilia}

Jelmer Muis, Amsterdam MC, Amsterdam, The Netherlands; Isolde Kuijlaars, PhD Candidate, Van Creveldkliniek, University Medical Center Utrecht, Utrecht University, Utrecht, The Netherlands; Michiel Luijten, Phd Candidate, Amsterdam University Medical Centers, University of Amsterdam, Emma Children's Hospital, Child and Adolescent Psychiatry \& Psychosocial Care, Amsterdam Reproduction and Development, Amsterdam Public Health, Amsterdam, The Netherlands; Shannon van Hoorn, PhD Candidate, Erasmus MC, University Medical Center Rotterdam, Department of Public Health, Rotterdam, The Netherlands; Samantha Gouw, Paediatric haematologist, Amsterdam University Medical Centers, University of Amsterdam, Emma Children's Hospital, Child and Adolescent Psychiatry \& Psychosocial Care, Amsterdam Reproduction and Development, Amsterdam Public Health, Amsterdam, The Netherlands; Karin Fijnvandraat, professor Paediatric haematologist, Amsterdam University Medical Centers, University of Amsterdam, Emma Children's Hospital, Child and Adolescent Psychiatry \& Psychosocial Care, Amsterdam Reproduction and Development, Amsterdam Public Health, Amsterdam, The Netherlands; Kathelijn Fischer, Paediatric haematologist, Van Creveldkliniek, University Medical Center Utrecht, Utrecht University, Utrecht, The Netherlands; Marjon Cnossen, Paediatric haematologist, Erasmus MC, University Medical Center Rotterdam, Department of Pediatric Oncology and Hematology, Rotterdam, The Netherlands; Marjolein Peters, Paediatric haematologist (retired), Amsterdam University Medical
Centers, University of Amsterdam, Emma Children's Hospital, Child and Adolescent Psychiatry \& Psychosocial Care, Amsterdam Reproduction and Development, Amsterdam Public Health, Amsterdam, The Netherlands; Lotte Haverman, Projectleader PROM implementation / paediatric psychologist, Amsterdam University Medical Centers, University of Amsterdam, Emma Children's Hospital, Child and Adolescent Psychiatry \& Psychosocial Care, Amsterdam Reproduction and Development, Amsterdam Public Health, Amsterdam, The Netherlands

Aims: The most frequently used disease specific Patient-Reported Outcome Measures (PROMs) in pediatric haemophilia care can be experienced as a burden due to their length and sometimes irrelevant questions. Patient Reported Outcomes Measurement Information System (PROMIS ${ }^{\circledR}$ ) item banks using short forms (SF) or Computerized Adaptive Testing (CAT) could solve this problem. The objective of this study is to assess the psychometric properties and feasibility of eight PROMIS item banks within a clinical sample of boys with haemophilia (BWH). Methods: In this multicenter study, all BWH (aged 8-17 years) from six Dutch Haemophilia Treatment Centers will be invited to participate. For assessing convergent validity the PROMIS item bank T-scores will be compared to (subscales of) the Haemophilia Quality of Life Questionnaire for Children (HaemoQoL) and to the Pediatric Hemophilia Activities List (PedHAL) by using Pearson's $r$, at which $r \geq 0.70$ is considered acceptable (Table 1). To ensure a power of $>0.8$, a sample of $n \geq 64$ is needed. Reliability of the PROMIS item banks was expressed as standard error of theta $(\operatorname{SE}(\theta))$, at which an $\operatorname{SE}(\theta)<0.32$ corresponds to a reliability of 0.90 . Regarding feasibility, the number of completed items will be reported. Results: Regarding convergent validity, $r$ is hypothesized between $0.5-0.9$ for all correlations. Good reliability $(\operatorname{SE}(\theta)<0.32)$ is expected for all PROMIS item banks, based on Dutch studies in children from the general population and a clinical sample (Juvenile Idiopathic Arthritis). We expect the PROMIS item banks to be more feasible in terms of number of items completed. Conclusion: When the pediatric PROMIS item banks display good convergent validity with disease-specific legacy instruments, good internal consistency, and feasibility in a clinical sample of Dutch BWH, PROMIS can be used in research and clinical care in BWH and, thus, lower questionnaire-related burden.

\section{(3081) Have Digital Endpoints been used to support labelling} claims? A review

Marie Mc Carthy, ICON Clinical Research, Dublin, Ireland; Céline Desvignes-Gleizes, Mapi Research Trust, Lyon, France; Laure-Lou Perrier, Mapi Research Trust, Lyon, France

Aims: The use of digital health technologies (DHT) to generate endpoint data is becoming more commonplace. Questions remain about the value of digital endpoints (DGEP), their acceptance as primary and secondary endpoints, and their use to support labelling claims. This review was conducted to assess the maturity and credibility of DGEP in drug development studies. Methods: A comprehensive search was performed in ClinicalTrials.gov for interventional phase 3 and 4 completed studies (no time limit). Trials were selected if DHT were used as primary or secondary endpoints, using 41 terms 3 categorises: Medical Device and Vendor (e.g. Fitbit, Actiwatch), Generic terms (e.g. connected, home, remote), and Technology (e.g. actigraphy, wearable, Continuous Glucose Monitoring (CGM)). Labels and medical reviews (MR) of approved drugs used in the selected trials were reviewed. Results: Among the 917 reviewed trials, 109 described DGEP. 96 drugs' were identified, 26 were not yet approved. 3 drugs (2 EMA, 1 FDA) mentioned DGEP in both their labels and MR. 7 drugs only mentioned DGEP in MR (5 EMA, 2 FDA). Actigraphy and CGM were used as primary endpoints 
in 4 drug efficacy studies, but only one (Lyumjev, EMA) mentioned CGM in the label. In contrast, for Dayvigo (FDA approved), data from a device; ataxia meter was used to assess safety and mentioned in both label and MR. Circadin label (EMA) reports "sleep latency (as measured by objective and subjective means)" and the MR reports that "Efficacy evaluated by sleep quality monitored through wrist actigraphy". Conclusion: By focusing on completed phase 3 and 4 studies, we infer that there was sufficient regulatory support to justify the use of DHT in data collection to support study endpoints in over 100 studies. However, less than $3 \%$ of the DGEP progressed to specific labelling claims; this raises the need for further investigation into the rationale for not identifying specific DGEP in regulatory documents. This review demonstrates that the use of DGEP has reached a degree of maturity and evolved beyond their use as exploratory measures in phase 2 studies, and it is likely that DGEP will support more labelling claims into the future.

(3082) Efficient and Precise Ultra-QuickDASH Scale Measuring Lymphedema Impact Developed Using Computerized Adaptive Testing

Cai $\mathrm{Xu}, \mathrm{PhD}$, University of Texas MD Anderson Cancer Center, Houston, Texas, USA; Mark V. Schaverien, PhD, University of Texas MD Anderson Cancer Center, Houston, Texas, USA; Joani M. Christensen, MD, University of Texas MD Anderson Cancer Center, Houston, Texas, USA; Chris Gibbons, PhD, University of Texas MD Anderson Cancer Center, Houston, Texas, USA

Aims: This study aimed to evaluate and improve the accuracy and efficiency of the QuickDASH for use in assessment of limb function in patients with upper extremity lymphedema using modern psychometric techniques. Methods: We conducted confirmative factor analysis (CFA) and Mokken analysis to examine the assumption of unidimensionality for IRT model on data from 285 patients who completed the QuickDASH, and then fit the data to Samejima's graded response model (GRM) and assessed the assumption of local independence of items and calibrated the item responses for CAT simulation. Results: Initial CFA and Mokken analyses demonstrated good scalability of items and unidimensionality. However, the local independence of items assumption was violated between items 9 (severity of pain) and 11 (sleeping difficulty due to pain) (Yen's $\mathrm{Q} 3=0.46$ ) and disordered thresholds were evident for item 5 (cutting food). After addressing these breaches of assumptions, the GRM with the remaining 10 items were re-analyzed and achieved an improved fit to the data set. Simulation of CAT administration demonstrated a high correlation between scores on the CAT and the QuickDash $(\mathrm{r}=0.98)$. Items 2 (doing heavy chores) and 8 (limiting work or daily activities) were the most frequently used. The correlation among factor scores derived from the QuickDASH version with 11 items and the UltraQuickDASH version with items 2 and 8 was as high as 0.91 . Conclusion: By administering just these two best performing QuickDash items (items 2 and 8 ) we can obtain estimates that are very similar to those obtained from the fixed-length QuickDash without the need for CAT technology.

\section{(3083) What matters to children with cancer-A systematic review of qualitative studies and patient-reported outcome measures (PROMs)}

Maria Rothmund, Medical University Innsbruck, Innsbruck, Austria; Samantha C. Sodergren, University of Southampton, Southampton, UK; Gudrun E. Rohde, University of Agder, Agder, Norway; Teresa de Rojas, Accelerate, Brussels, Belgium; Gloria Paratico, University of Brescia, Brescia, Italy; Giorgia Albini, University of Brescia,
Brescia, Italy; Alessandra Majorana, University of Brescia, Brescia, Italy; Anne-Sophie Darlington, University of Southampton, Southampton, UK; David Riedl, Medical University Innsbruck, Innsbruck, Austria; submitted on behalf of "the EORTC Quality of Life Group"

Aims: Several patient-reported outcome measures (PROMs) for health-related quality of life (HRQOL) in children with cancer exist. However, these have been criticized for not accurately reflecting issues that matter to children and for seldom involving children in PROM development. We aimed to collect and compare the items assessed by existing PROMs and issues mentioned by children or parents in qualitative studies. Methods: A systematic literature search on PubMed, PsycInfo, COSMIN, and Cochrane was conducted. Studies were included if (a) children (8-14 years) with cancer undergoing treatment completed PROMs or (b) children or their parents provided qualitative information on HRQOL issues of the child. Issues identified in qualitative studies and PROM items were mapped onto the conceptual framework of HRQOL in children with cancer proposed by Anthony et al. (2014). Results: A total of 2551 records were identified of which 316 papers were included. Among the included studies, 78 collected qualitative data and 277 used PROMs (39 combined both methods). 142 different PROMs and more than 4000 individual items were identified. The major domains of physical, psychological, social, and general health proposed in the conceptual framework by Anthony et al. (2014) were confirmed and the majority of items mapped well onto the model. Most identified issues assessed physical function or symptoms while aspects of psychosocial functioning were often under-assessed in existing PROMs even though these emerged as key constructs in the qualitative studies. Conclusion: This study offers a comprehensive overview of HRQOL issues for children with cancer. While physical aspects of the disease are covered, most PROMs lack a comprehensive assessment of key psychosocial issues and, thus, overlook fundamental features of the cancer experience of children. This calls for the development of a more comprehensive PROM for children with cancer including items which children themselves have identified as relevant and important to them.

(3084) Statistical analysis of symptomatic adverse events over the course of oncology clinical trials: What are the appropriate methods for longitudinal PRO-CTCAE data?

Antoine Regnault, PhD, Modus Outcomes, Lyon, France; Angély Loubert, Modus Outcomes, Lyon, France; Randy Davis, GlaxoSmithKline, Upper Providence, Pennsylvania, USA; Boris Gorsh, GlaxoSmithKline, Upper Providence, Pennsylvania, USA; Stéphane Quéré, Modus Outcomes, Lyon, France; Linda Nelsen, GlaxoSmithKline, Upper Providence, Pennsylvania, USA; Laurie Eliason, GlaxoSmithKline, Upper Providence, Pennsylvania, USA

Aims: The Patient-Reported Outcomes version of the Common Terminology Criteria for Adverse Events (PRO-CTCAE) is increasingly used to assess symptomatic adverse events in oncology trials. Currently, no standard for longitudinal analysis of PRO-CTCAE data exists; we explored the performance of different statistical methods on longitudinal PRO-CTCAE data from an oncology clinical trial. Methods: The following statistical methods were identified as potentially appropriate to compare longitudinal PRO-CTCAE data between clinical trial groups: Toxicity over Time (ToxT) (includes repeated measurement model, time-to-event, area under the curve [AUC] analyses), Generalized Estimating Equations (GEE), and ordinal log-linear models (OLLM). ToxT methods treat PRO-CTCAE items as continuous, and GEE/OLLM as ordinal. Statistical methods were applied to longitudinal PRO-CTCAE data from 28 items in DREAMM-2, a Phase 2 trial of belantamab mafodotin in multiple 
myeloma (NCT03525678); the two dose groups (2.5 $\mathrm{mg}$ [ $\mathrm{n}=97]$, $3.4 \mathrm{mg}[\mathrm{n}=124]$ ) were compared. Results: Only two PRO-CTCAE items were flagged as showing a difference between dose groups: ToxT showed a difference in one PRO-CTCAE item (constipation) for only one of the many analyses (time-to-first-maximum postbaseline rating); GEE showed a difference for one item (chills); OLLM showed no difference in any item. The application of the different methods raised various challenges: selecting the parameters to define PRO-CTCAE-based events and a comparable timeframe for all patients in the AUC analysis for ToxT; the interpretation of results and parameter estimation for GEE; prespecification of the underlying scaling model for PRO-CTCAE ratings and some estimation issues for OLLM. All methods had to account for a high volume of missing data and multiple testing (driven by the number of items). Conclusion: The statistical methods tested with DREAMM-2 longitudinal PRO-CTCAE data led to similar conclusions when comparing dose groups. As expected, since analyses were comparing different doses of the same treatment, few differences between groups were observed. The ToxT approach may be a good compromise as more sophisticated analyses (GEE and OLLM, theoretically more appropriate for PROCTCAE item nature) did not lead to different conclusions. However, these results must be confirmed in other cases, especially comparative trials where more differences are expected. Funding: GSK (Study 212,225).

\section{(3085) Application of a Module of the MD Anderson Symptom Inventory in Chinese Patient after Lung Cancer Surgery (MDASI-LC)}

Li Tang,

State Key Laboratory of Ultrasound in Medicine and Engineering, Chongqing Medical University, Chongqing, China; Hongfan Yu, School of Public Health and Management, Chongqing Medical University, Chongqing, China; Wei Xu, School of Public Health and Management, Chongqing Medical University, Chongqing, China; Yaqin Wang, Department of Thoracic Surgery, Sichuan Cancer Hospital, School of Medicine, University of Electronic Science and Technology of China, Chengdu, China; Jia Liao, Department of Thoracic Surgery, Sichuan Cancer Hospital, School of Medicine, University of Electronic Science and Technology of China, Chengdu, China; Wei Dai, Department of Thoracic Surgery, Sichuan Cancer Hospital, School of Medicine, University of Electronic Science and Technology of China, Chengdu, China; Xin Shelley Wang, Department of Symptom Research, The University of Texas MD Anderson Cancer Center, Houston, Texas, USA; Qiuling Shi, State Key Laboratory of Ultrasound in Medicine and Engineering, Chongqing Medical University, Chongqing, China

Aims: Patient-reported outcomes (PROs) is of considerable value in perioperative care from the patient's perspective, but the PROs supported decision making has been limited because of the heterogeneity among patients. This study aims to provide a method to early identify the lung cancer patients who needs more intensive symptom managements after surgery according to the PROs-trajectories and the risk factors. Methods: The longitudinal data were extracted for lung cancer patients undergoing surgical treatments as part of the prospective cohort study. PROs were assessed with the MDASI-LC before surgery, daily after surgery and 4 weeks after discharge. We psychometrically examined the MDASI-LC's validity and reliability. Heterogeneities of the patients were profiled based on the symptom trajectories using the LCTM and NLME. The functional recovery outcomes were estimated using Kaplan-Meier analysis. Multivariate logistic regression were used to explore the risk factors. Results: A total of 424 patients were enrolled in this study. The MDASI-LC was proved to be a valid and reliable tool to capture symptom burden and functional interference in Chinese lung cancer patients after surgery. Cognitive debriefing demonstrated the MDASI-LC to be an understandable and easy-to-use assessment. Compared with pattern 1, patients of pattern 2 (severe to decline) and pattern 3 (severe remained) suffering a significantly increased score immediately after surgery were of younger age (OR 0.54), less likely to undergo single port VARTS (OR 1.59) and two chest tubes (OR 1.67). Compared with pattern 3, pattern 2 was associated with older age (OR 2.22). It was more quickly for pattern 1 (2.91 days) and pattern 2 (3.85 days) to recovered compared with pattern 3 (5.62 days). In 193 patients with 'single port VARTS', mild symptoms of POD1 (pattern 1) were related to older ages (OR 0.40) while this relationship was of younger age (OR 4.79). This bidirectional regulation effect of age has not been observed in the rest patients of 'multiple ports VARTS' or 'Open Surgery'. Conclusion: This study demonstrates the MDASI-LC a sensitive tool to profile the symptom trajectories of the lung cancer patients throughout the postoperative recovery period. Consequently, the heterogeneity and the target population called for intensive symptom managements could be recognized with PROs.

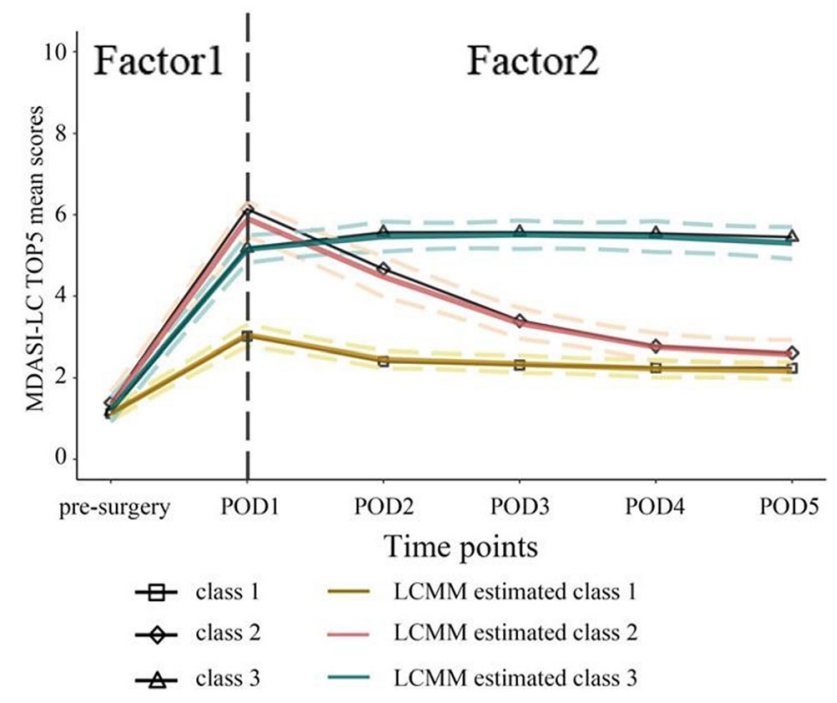




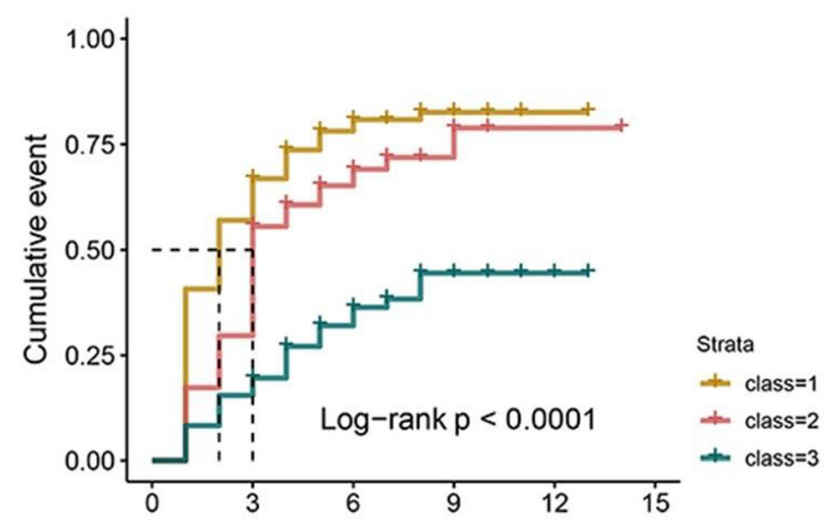

(3086) Item response theory analyses of MD Anderson Symptom Inventory for patients with lung cancer surgery

Jingyu Zhang,

State Key Laboratory of Ultrasound in Medicine and Engineering, Chongqing Medical University, Chongqing, China; Yubo Zhang,

State Key Laboratory of Ultrasound in Medicine and Engineering, Chongqing Medical University, Chongqing, China; Yuxian Nie, State Key Laboratory of Ultrasound in Medicine and Engineering, Chongqing Medical University, Chongqing, China; Qingsong Yu, School of Public Health and Management, Chongqing Medical University, Chongqing, China; Yang Pu, School of Public Health and Management, Chongqing Medical University, Chongqing, China; Yanyan Huang, School of Public Health and Management,

Chongqing Medical University, Chongqing, China; Xing Wei, Department of Thoracic Surgery, Sichuan Cancer Hospital, School of Medicine, University of Electronic Science and Technology of China, Chongqing, China; Qiuling Shi,

State Key Laboratory of Ultrasound in Medicine and Engineering, Chongqing Medical University, Chongqing, China;

Aims: To examine the metric properties of individual items of the MD Anderson Symptom Inventory(MDASI) core symptom items in perioperative patients with lung cancer by item response theory (IRT). Methods: We analyzed the data of MDASI core symptom items of perioperative patients with lung cancer who were recruited from one tertiary hospital and five community hospitals. MDASI was administrated before surgery and daily after surgery up to 12 days. Confirmatory factor analysis was run to evaluate the dimensionality of core items. The two-parameter graded response model was used to examine the category probability curves, location and discrimination parameters. IRT uses all available information, and thus, listwise deletion is unnecessary. Results: A total of 3853 MDASI core item records generated by 512 patients were included in the IRT analysis. Unidimensionality and local independence were satisfied. Item discrimination parameters(a) ranged from 1.36 to 2.35 . Item difficulty parameters(b) ranged from - 1.49 to 4.71. Except "pain," "fatigue," and "drowsy," the difficulty parameters b10 of the other items were greater than 3. The items of "remember," "vomiting," and "numbness" showed a high difficulty(b) in the high value part (values: 8,9 , 10). In the item characteristic curves(ICC), the threshold estimates for all values were presented in an increasing order, except value $=2$ which shows a low and flat characteristic curves in all items. Besides, value $=7$ and value $=8$ show a similar response options probability for "sleep," "appetite," "vomiting," and "numbness." Conclusion: The results of IRT analysis showed that most of the MDASI core items performed well in patients with lung cancer surgery, demonstrating a good discrimination and an acceptable difficulty distribution. The high difficulty in b10 may help avoid the ceiling effect of the symptom measurement. While the low probability of response to certain values of certain items also provides the clues to the item modification and threshold delineation in specific measurement tools developing for perioperative applications of lung cancer patients.

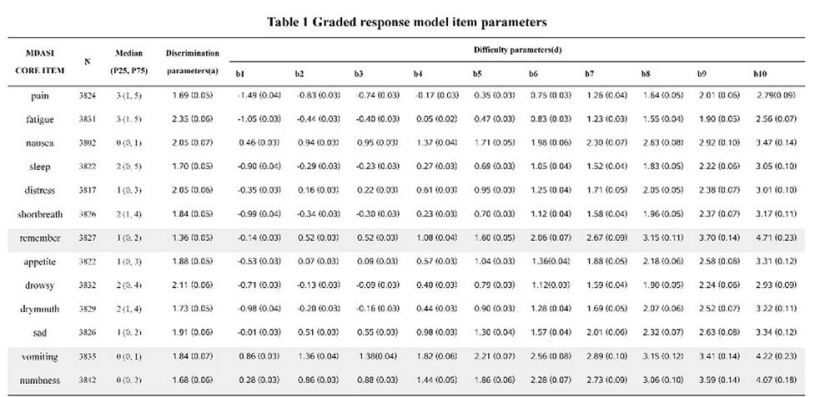

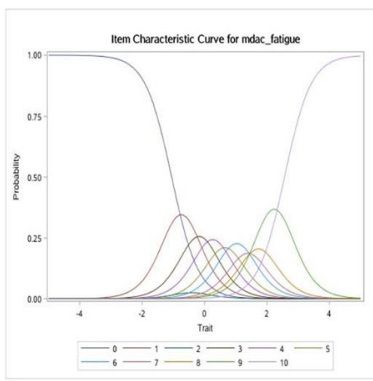

Figure 1. Item characteristic curve - fatigue

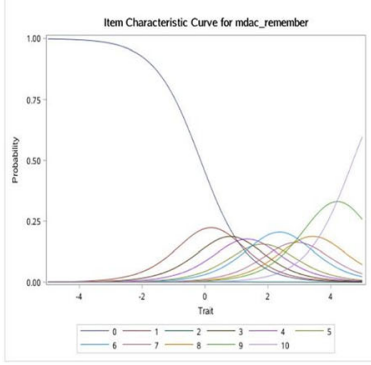

Figure 3. Item characteristic curves-remember

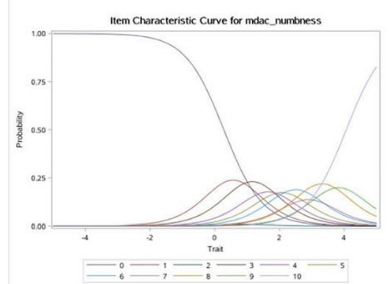

Figure 5. Item characteristic curves-numbness

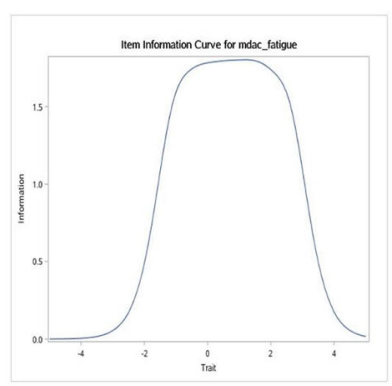

Figure 2. Item information curve-fatigue

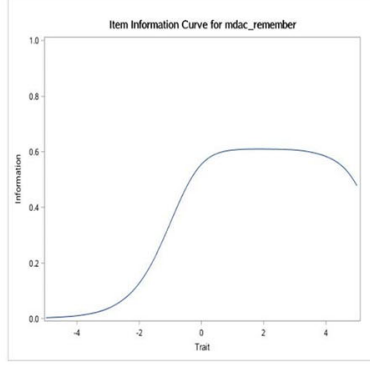

Figure 4. Item information curve-remember

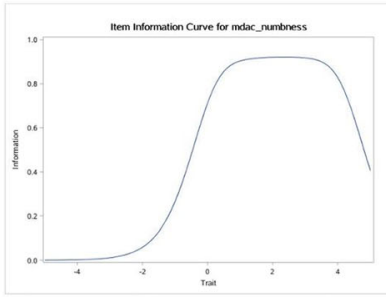

Figure 6. Item information curve-numbness
(3087) Analysis of patient-reported outcome compliance and risk factors in patients undergoing lung surgery during hospitalization

Jia Liao, Sichuan Cancer Hospital, School of Medicine, University of Electronic Science and Technology of China, Chengdu, China; Hongfan $\mathrm{Yu}$, School of Public Health and Management, Chongqing Medical University, Chongqing, China; Yaqin Wang, Department of Thoracic Surgery, Sichuan Cancer Hospital \& Institute, Sichuan Cancer Center, School of Medicine, University of Electronic Science and Technology of China, Chengdu, China; Wei Dai, Department of Thoracic Surgery, Sichuan Cancer Hospital \& Institute, Sichuan 
Cancer Center, School of Medicine, University of Electronic Science and Technology of China, Chengdu, China; Xiaoqin Liu, Department of Thoracic Surgery, Sichuan Cancer Hospital \& Institute, Sichuan Cancer Center, School of Medicine, University of Electronic Science and Technology of China, Chengdu, China; Qiuling Shi, Center for Cancer Prevention Research, Sichuan Cancer Hospital \& Institute, Sichuan Cancer Center, School of Medicine, University of Electronic Science and Technology of China, Chongqing, China

Aims: To identify the risk factors of patient-reported outcome (PRO) compliance in patients undergoing lung surgery during hospitalization and to provide a basis for the subsequent extensive clinical application of PRO to improve patient compliance. Methods: Data for this study were obtained from a prospective multicenter cohort study $(\mathrm{CN}$ PRO-Lung 1) from November 2017 to January 2020. The inclusion criteria for the study population were planning to undergo lung surgery, age $\geq 18$ years, and willingness to participate in the study. The instruments included the MD Anderson Symptom Inventory Lung Cancer module (MDASI-LC) and a single-item Quality of Life (QoL) scale. The time points for data collection included preoperative and daily postoperatively until 14 days. Patients whether filled out the questionnaires (MDASI and QoL) were used as dependent variables, and stepwise logistic regression analysis was used to identify the risk factors. Statistical analysis was performed using SAS 9.4 version software. Results: The mean age of the 512 patients in this study was $55.07 \pm 10.45$ years, and the median postoperative hospital stay was 5 days. Most patients were male $(53.71 \%)$, had an education level predominantly high above middle school graduate $(50.59 \%)$, had preoperative anesthesiologist assessment $>1$ in $55.66 \%$, underwent single-port video-assisted thoracoscopic surgery (50.98\%), were inserted one chest tube $(67.77 \%)$, and had tumor pathological stage I $(52.34 \%)$. Patient compliance rates of the MDASI-LC ranged from $59.38 \%$ to $100 \%$. The highest compliance collection point was at preoperative and the lowest compliance collection point was at the 14th postoperative day. The independent risk factor for MDASI-LC compliance was male [OR 1.578, 95\% CI (1.021-2.438), $\mathrm{P}=0.04$ ]. The independent risk factors influencing QoL compliance included nonsingle-port surgery [OR $1.562,95 \% \mathrm{CI}(1.052-2.32), \mathrm{P}=0.03$ ] and ASA $>1$ [OR 1.73, 95\% CI (1.164-2.572), $\mathrm{P}=0.007]$. Conclusion: Our study showed PRO compliance decreased over time. PRO compliance was lower in male patients, ASA > I, and nonsingle-port lung surgery, suggesting that more attention should be given to these patients in future studies.
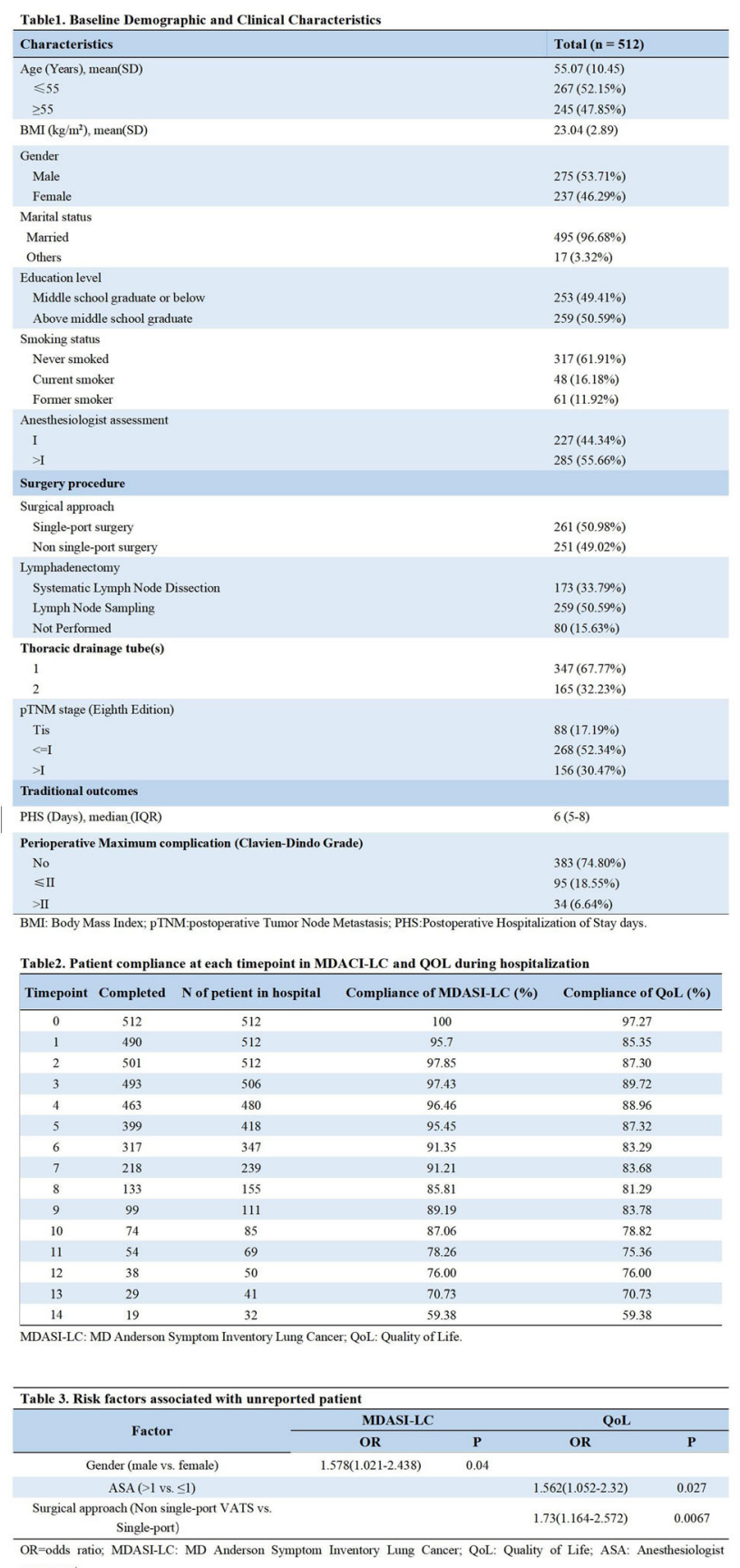

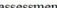

(3088) Global impressions of change for instrument validation: Adult problems in pediatric samples

Christina Zigler, PhD, MSEd, Duke University School of Medicine, Durham, North Carolina, USA; Z. Li, Duke University, Durham, North Carolina, USA; K Bahnson, Duke University, Durham, North Carolina, USA; A.V. Bennett, UNC, Chapel Hill, North Carolina, USA; A Gaultney, UCLA, Los Angeles, California, USA;

A Hernandez, Duke University, Durham, North Carolina, USA; R Randell, Duke University, Durham, North Carolina, USA; L. E. Schanberg, Duke University, Durham, North Carolina, USA; E Weitzman, Childrens Harvard, Boston, Massachusetts, USA; K Weinfurt, Duke University, Durham, North Carolina, USA; B Reeve, Duke University, Durham, North Carolina, USA 
Aims: Global impressions of change (GIC) are often used to evaluate the responsiveness and the minimally important differences of scores on patient-reported outcome measures (PROM). This approach has been critically evaluated in adults, ${ }^{1,2}$ however, data from pediatric samples are limited. This study aimed to evaluate the validity of domain-specific GICs as anchors in one pediatric sample. Methods: As part of a larger NIH-funded project, children with juvenile idiopathic arthritis (JIA) and systemic lupus erythematosus (SLE) were enrolled in a research registry via the Childhood Arthritis and Rheumatology Research Alliance (CARRA). From the CARRA registry database, we extracted scores on four PROMIS ${ }^{\circledR}$ pediatric measures (fatigue, mobility, pain interference, physical activity) completed at two visits about $\sim 6$ months apart (T1 and T2). At T2, participants also rated GICs on each domain on a 5-point scale ('much better now' to 'much worse now').We estimated correlations between the GIC ratings and the PROMIS change scores (T2-T1), scores at T1, and at $\mathrm{T} 2$. The stronger the correlation between a potential anchor and change score, the greater the confidence in using the anchor as the basis for interpreting the meaningfulness of changes in scores. ${ }^{3} \mathrm{We}$ also expected to see associations of equal magnitude between the GIC and scores at T1 and T2. ${ }^{1,2}$ Results: Correlations between GIC and PROMIS scores ranged from $0.03-0.55$ (Table 1). Generally, the strongest correlations for each domain and disease were between the GIC the change score. However, 5 of 8 were $<0.30$, with the highest correlation only corresponding to $25 \%$ shared variance. Almost all correlations were larger in magnitude at $\mathrm{T} 2$ compared to $\mathrm{T} 1$ (Table 1). Conclusion: Low correlations between GIC and change scores and stronger correlations between GIC and scores at $\mathrm{T} 2$ relative to $\mathrm{T} 1$ suggest that the patient's current state influenced their GIC response more than change in the domain. It is possible that the 6-month follow-up period used in this study presented a challenge for children's accurate recall of change in these domains. ${ }^{4}$ Before using a GIC as an anchor in pediatric samples, it is important for researchers to assess the validity of the GIC, just as for adults.

\section{References}

1. Norman, G.R., Stratford, P., \& Regehr, G. Methodological problems in the retrospective computation of responsiveness to change: The lesson of Cronbach. J Clin Epidemiol. 1997,50(8),869-879.

2. Guyatt GH, Norman GR, Juniper EF, Griffith LE. A critical look at transition ratings. J Clin Epidemiol. 2002 Sep;55(9):900-8. doi: 10.1016/s0895-4356(02)00435-3.

3. Revicki D, Hays RD, Cella D, Sloan J. Recommended methods for determining responsiveness and minimally important differences for patient-reported outcomes. J Clin Epidemiol. 2008 Feb;61(2):102-9. doi: 10.1016/j.jclinepi.2007.03.012. Epub 2007 Aug 3. PMID: 18177782.

4. Matza, L. S., Patrick, D. L., Riley, A. W., Alexander, J. J., Rajmil, L., Pleil, A. M., \& Bullinger, M. (2013). Pediatric Patient-Reported Outcome Instruments for Research to Support Medical Product Labeling: Report of the ISPOR PRO Good Research Practices for the Assessment of Children and Adolescents Task Force. Value in Health,16(4), 461-479. doi:10.1016/j.jval.2013.04.004

Table 1. Polyserial correlations between global impressions of change (GIC) and PROMIS T-scores in four domains (fatigue, mobility, pain, physical activity).

\begin{tabular}{|c|c|c|}
\hline GIC with & $\begin{array}{c}\text { JIA }\left(n=289^{1}\right) \\
\text { Correlation }(95 \% \mathrm{Cl})\end{array}$ & $\begin{array}{c}\text { SLE }\left(n=47^{1}\right) \\
\text { Correlation }(95 \% \mathrm{Cl})\end{array}$ \\
\hline Change in Fatigue (T2-T1) & $.29(0.19,0.40)$ & $.50(0.28,0.72)$ \\
\hline Fatigue $\mathrm{T} 2$ & $.26(0.15,0.37)$ & $.47(0.24,0.70)$ \\
\hline Fatigue T1 & $.03(-0.08,0.15)$ & $.10(-0.19,0.39)$ \\
\hline Change in Mobility (T2-T1) & $-.23(-0.34,-0.12)$ & $-.36(-0.62,-0.11)$ \\
\hline Mobility T2 & $-.37(-0.47,-0.27)$ & $-.02(-0.31,0.28)$ \\
\hline Mobility T1 & $-.10(-0.21,0.02)$ & $-.31(0.04,0.57)$ \\
\hline Change in Pain interference (T2-T1) & $.26(0.15,0.37)$ & $.41(0.17,0.65)$ \\
\hline Pain T2 & $.27(0.16,0.38)$ & $.55(0.34,0.76)$ \\
\hline Pain T1 & $.04(-0.08,0.16)$ & $.02(-0.27,0.32)$ \\
\hline Change in Physical Activity (T2-T1) & $-.22(-0.33,-0.11)$ & $-.28(-0.55,-0.01)$ \\
\hline Physical Activity T2 & $-.19(-0.30,-0.07)$ & $-.16(-0.45,0.12)$ \\
\hline Physical Activity T1 & $.03(-0.08,0.15)$ & $.14(-0.15,0.43)$ \\
\hline
\end{tabular}

(3089) Advances in measuring mental health in early childhood (1-5 years): The PROMIS Early Childhood measures for Anxiety, Depression, Irritability and Sleep Disturbance in the The Netherlands

Michiel Luijten, MSc., Amsterdam UMC, Amsterdam, The Netherlands; Marthe Egberts, PhD, Utrecht University, Utrecht, The Netherlands; Willemijn van Eldik, PhD, Utrecht University, Utrecht, The Netherlands; Martha Grootenhuis, Prof., Princess Máxima Center for Pediatric Oncology, Utrecht, The Netherlands; Raphaële van Litsenburg, PhD, Princess Máxima Center for Pediatric Oncology, Utrecht, The Netherlands; Trudy Mooren, Prof., Utrecht University, Utrecht, The Netherlands; Caroline Terwee, PhD, Amsterdam UMC, Amsterdam, The Netherlands; Lotte Haverman, PhD, Emma Children's Hospital, Amsterdam UMC, Amsterdam, The Netherlands

Aims: The American Psychiatric Association (APA) recently selected new level-2 DSM-V instruments for monitoring with shorter administration times, consisting of several Patient-Reported Outcomes Measurement Information System (PROMIS) measures for adults (aged 18+) and children and adolescents (aged 8-18). To increase the age range of these measures, the PROMIS initiative developed Early Childhood measures for measuring mental health in very young children (aged 1-5). These measures were translated to Dutch by forward and backward translations and cognitive debriefing. The objective of this study is to investigate the psychometric properties of four recently (2019) developed PROMIS Early Childhood (PROMIS EC) measures for assessing Anxiety, Depression, Irritability (anger) and Sleep Disturbance in the Dutch general population. Methods: Secondary data analyses will be performed on data collected in 2020-2021 from a study that assessed the consequences of the COVID-19 outbreak on very young children. The Anxiety, Depression, Irritability, and Sleep Disturbance complete item banks will be administered to parents of young (aged 1-5) children $(n=\sim 1300)$. To assess structural validity of each item bank a graded response model (GRM) will be fitted to the data after assessing the following assumptions: Unidimensionality through CFA (CFI $>0.95$, TLI $>$ 0.95 , RMSEA $<0.10)$, local independence by residual correlations $(\mathrm{r}<0.20)$ and monotonicity by Mokken analysis $(\mathrm{H}>0.50, \mathrm{Hi}>$ 0.30 ). Item fit of the GRM models will be inspected with $\mathrm{S}-\mathrm{X} 2$, where $\mathrm{p}<0.001$ indicates misfit. Additionally, percentage of participants reliably measured will be assessed using the standard error of measurement $(\mathrm{SEM})<0.32$ as a criterion (which equals a reliability of 0.90). If possible, differential item functioning (DIF) analyses will be performed between the Dutch and U.S. model. Results: Translations were successful. Validation results will be presented at the conference. Conclusion: After initial validation of these items banks, they can be implemented as CAT within the The Netherlands to provide new measures to reliably and validly assess mental health in very young children (aged 1-5).

\section{(3090) Recent trends in the use of wearable sensors to capture endpoint data in multiple sclerosis clinical trials.}

Laura Khurana, MPH, ERT, Medford, Massachusetts, USA; Lindsay Hughes, PhD, ERT, Medford, Massachusetts, USA; Fay Horak, PhD, ERT, Portland, Oregon, USA; Kristen Sowalsky, PhD, ERT, Portland, Oregon, USA

Aims: Wearable sensors capture objective, sensitive, and reliable digital outcome measures of human movement. Gait and balance characteristics quantified by wearable sensors are significantly correlated with clinical outcome assessments (COAs) that are commonly used to show treatment benefit in multiple sclerosis (MS) trials, including the Expanded Disability Status Scale (EDSS) and patient reported outcomes (MSWS-12 and ABC). These data also 
demonstrate more sensitivity to early-stage disease than the EDSS and gait speed, discriminate between MS/controls, and are related to the integrity of the neural pathways (refs 1-10). Given this evidence supporting the use of wearable sensors in MS clinical trials, our aim is characterize the endpoint strategy for clinical outcome assessments (COAs) in these trials including clinician-reported outcomes (ClinROs), patient-reported outcomes (PROs) and performance outcomes (PerfOs) and identify potential future directions and innovations for endpoint data collection. Methods: We reviewed MS clinical trial protocols with COA endpoints in ERT's database from 2018-2021, to determine the percentage of trials that also included wearable sensor data as primary, secondary, or exploratory endpoints. Results: All protocols $(\mathrm{N}=42)$ contained $\geq 1$ COAs as an endpoint, while only four protocols utilized wearable sensors $(9.5 \%)$. The breakdown of studies utilizing COAs as primary endpoints included: 16 ClinROs (38.1\%), 4 PROs (9.5\%), 10 PerfOs (23.8\%); secondary endpoints included: 29 ClinROs (69\%), 23 PROs (54.8\%), 20 PerfOs (47.6\%); exploratory endpoints included: 17 ClinROs (40.5\%), 16 PROs (38.1\%), 17 PerfOs $(40.5 \%)-4$ in which wearable sensors were utilized (9.5\%). Conclusion: Our results demonstrate a disparity in the use of wearable sensors to capture digital COA endpoints (PerfOs) in MS clinical trials over the past four years, relative to the evidence of their potential utility. We therefore have identified a large opportunity to leverage the use of wearable sensors to collect objective endpoint data in MS clinical trials, and encourage sponsors of these trials to consider including wearable sensor data in their endpoint strategy. Especially in combination with well-established COA endpoints, mobility data from wearable sensors have the potential to enable smaller sample sizes and earlier go/no-go decisions.

\section{(3091) Health-related quality of life in adults with multiple myeloma}

Matthew LeBlanc, BSN, Duke University, Durham, North Carolina, USA; Ashley Leak Bryant, PhD, University of North Carolina, Chapel Hill School of Nursing, Chapel Hill, North Carolina, USA; Thomas LeBlanc, MD, Duke University School of Medicine, Durham, North Carolina, USA; Qing Yang, PhD, Duke University School of Nursing, Durham, North Carolina, USA; Sophia Smith, $\mathrm{PhD}$, Duke University School of Nursing, Durham, North Carolina, USA

Aims: Despite impressive advances in treatment, multiple myeloma (MM) remains a chronic disease marked by multiple relapses, many lines of therapy, and poor quality of life. Much of the HRQoL evidence in MM comes from clinical trial reports which rarely report prevalence or severity of HRQoL outcomes, instead reporting only change from baseline, or differences between treatment arms. Large observational studies are better suited to describing HRQoL though few exists and almost none were conducted in the USA (US). The purpose of this study is to describe HRQoL in a US based sample of MM patients from across the disease trajectory. Methods: Surveys were mailed to MM patients identified through the Duke Cancer Institute Tumor Registry. HRQoL was assessed using Patient Reported Outcomes Measurement Information System (PROMIS) measures for global physical health (GPH), global mental health $(\mathrm{GMH})$, physical function, and ability to participate in social roles (i.e., social function). Point prevalence was estimated with and without nonresponse bias adjustments. Additionally, we used bivariate and multiple regression to identify patient characteristics significantly associated with HRQoL outcomes. Results: $690 \mathrm{MM}$ patients returned surveys for a response rate of $64.7 \%$. Mean GPH score, GMH score, and physical function score were all significantly worse than general population means. Mean social function was not significantly different from the general population. Greater than $50 \%$ had GPH and physical function one half standard deviation below the population mean, and $42.2 \%$ had GMH one half standard deviation below the population mean. Worse HRQoL across outcomes was associated with not being in remission, increased comorbidities, worse social support and past radiation therapy. Conclusion: Our study found that MM patients had significantly worse HRQoL than the general population. We also found that remission was a stronger predictor of HRQoL than current treatment status or time since diagnosis. Pursuing deep and durable remissions appears to improve not only survival but HRQoL as well. More research is needed to better describe the effects of MM treatment patterns (e.g., multiple lines of therapy, maintenance therapy) on HRQoL.

Table 1. Health-related quality of life in adults with multiple myeloma

\begin{tabular}{lccccc}
\hline & \multicolumn{2}{c}{ Unadjusted } & & \multicolumn{2}{c}{$\begin{array}{c}\text { Adjusted for non- } \\
\text { response bias }\end{array}$} \\
\cline { 2 - 3 } \cline { 5 - 6 } HRQoL Domain & mean & $95 \% \mathrm{Cl}$ & & mean & $95 \% \mathrm{Cl}$ \\
\hline Global physical health & $\mathbf{4 4 . 9 ^ { * * }}$ & $44.3-45.6$ & & $\mathbf{4 4 . 0 ^ { * * }}$ & $43.2-44.9$ \\
Global mental health & $\mathbf{4 7 . 5 ^ { * * }}$ & $46.8-48.2$ & & $\mathbf{4 6 . 1 ^ { * * }}$ & $42.6-49.6$ \\
Physical function & $\mathbf{4 4 . 1 ^ { * * }}$ & $43.4-44.8$ & & $\mathbf{4 3 . 2}^{\star *}$ & $41.9-44.5$ \\
Social function & 49.5 & $48.8-50.2$ & & $\mathbf{4 8 . 4}^{* *}$ & $46.0-49.9$
\end{tabular}

a. Confidence interval; b. Adjusted for age at enrollment, race, gender, partnered status and age at diagnosis using propensity scores; Means significantly worse than general population mean $(50),{ }^{*}=p<.05,{ }^{\star \star}=p<.001$.

(3092) Higher meaningfulness of sense of coherence (SOC) is associated with better mental well-being of the elderly people.

Junko Sakano, Ph.D., Okayama Prefectural University, Okayama, Japan; Hisashi Okata, Okayama Prefectural University, Okayama, Japan; Yoichi Sawada, Ph.D., Okayama Prefectural University, Okayama, Japan; Yuki Yajima, Ph.D., Niimi University, Okayama, Japan

Aims: The purpose of this study was to investigate which components of sense of coherence (comprehensibility, manageability, and meaningfulness) are strongly associated with mental well-being of the elderly people. Methods: A questionnaire survey was conducted for 567 Japanese community-dwelling adults aged $\geq 60$ years. The questionnaire included the Mental Health Continuum (MHC), Sense of Coherence Scale (13-item 5-point Likerts version), and Social Provisions Scale (SPS), as well as questions about respondent's sociodemographic characteristics (age, sex, education, presence of cohabitants, working style, and self-rating economic status) and physical health (chronic disease, locomotor and oral function, and nutrition status). A hierarchical multiple regression analysis was used to assess the relationships between three components of SOC and other socio-environmental variables and mental well-being. Results: The hierarchical multiple regression supported final steps model in which mental well-being was regressed on three components of SOC, social support, physical health, as well as socio-demographic variables $\quad(\mathrm{F} 14, \quad 528=16.73, \quad \mathrm{p}<0.001, \quad \mathrm{R} 2=0.307$, adjusted $\mathrm{R} 2=0.289)$. As a result, meaningfulness on SOC (std. beta $=0.401)$, social support (std. beta $=0.082$ ), self-rating economic status (std. beta $=0.085)$, and age (std. beta $=0.137)$ were significantly associated with mental well-being. Conclusion: The findings of this study suggests that sense of meaningfulness is the strongest predictor of older adults' well-being among the SOC components.

(3093) Quality of life and psychosocial outcomes in advanced colorectal cancer survivors: A qualitative exploration

Chloe Lim, PhD Candidate, The University of Sydney, Sydney, Australia; Rebekah Laidsaar-Powell, PhD, The University of Sydney, Sydney, Australia; Jane Young, professor The University of Sydney, 
Sydney, Australia; Michael Solomon, professor The University of Sydney, Sydney, Australia; Daniel Steffens, Associate professor Surgical Outcomes Research Centre, Royal Prince Alfred Hospital, Sydney, Australia; Cherry Koh, Associate professor Department of Colorectal Surgery, Royal Prince Alfred Hospital, Sydney, Australia; Nabila Ansari, doctor Department of Colorectal Surgery, Royal Prince Alfred Hospital, Sydney, Australia; David Yeo, doctor Department of UGI and Transplant Surgery, Royal Prince Alfred Hospital, Sydney, Australia; Prunella Blinman, Associate professor Concord Cancer Centre, Concord Repatriation General Hospital, Sydney, Australia; Philip Beale, Associate professor Concord Cancer Centre, Concord Repatriation General Hospital, Sydney, Australia; Bogda Koczwara, professor Department of Medical Oncology, Flinders Medical Center, Adelaide, Australia; Phyllis Butow, professor The University of Sydney, Sydney, Australia

Aims: Approximately 18\% of patients with colorectal cancer (CRC) are diagnosed with advanced cancer, while 30-40\% develop recurrent disease after treatment with curative intent. More modern treatment pathways enable longer survival for people with advanced CRC. These include: cytoreductive surgery and hypothermic intraperitoneal chemotherapy (CRS-HIPEC), pelvic exenteration, liver resection, and palliative chemotherapy without surgery. Yet, virtually no qualitative research has compared the experiences and perspectives of survivors of these different treatments. This study aims to explore the different needs and views of survivorship care of people who received the above treatments for advanced CRC. Methods: Adult survivors of CRC are being recruited 6 months to 2 years post-surgery from two major Australian hospitals. Approximately $\mathrm{N}=40$ survivors who have undergone CRS-HIPEC, pelvic exenteration, liver resection, or palliative chemotherapy without surgery will be recruited. All participants will complete the Functional Assessment of Cancer Therapy-Colorectal (FACT-C), Distress Thermometer, and Comprehensive Score for Financial Toxicity (COST) questionnaires. Participant demographic and clinical data are also collected. Questionnaire data will undergo descriptive analysis to characterise the sample. All participants will also participate in a qualitative semistructured telephone interview, analysed via the framework approach of thematic analysis. Qualitative interviews explore quality of life, employment and finances, stigma, supportive care needs, social support and functioning, perceptions of survivorship, and impacts of COVID-19 on survivorship. Results: Preliminary analysis of 30 interviews $(\mathrm{n}=10$ CRS-HIPEC, $\mathrm{n}=9$ pelvic exenteration, $\mathrm{n}=5$ liver resection, $\mathrm{n}=6$ palliative chemotherapy) reveals some advanced CRC survivors report post-surgical complications and chemotherapyinduced peripheral neuropathy, which can limit physical activity. Participants reportedly manage these through distraction, positive reframing, and contact with other CRC survivors. Most participants appeared satisfied with their cancer treatment teams. Some viewed their GPs as important coordinators in their health care. CRC survivors reported being more cautious about leaving the house due to COVID-19, and view the change to telehealth as less personal; however, rural/regional participants prefer the convenience of telehealth. Conclusion: The study findings will help guide development of interventions to improve the survivorship experience of patients who receive treatment for advanced CRC. This may include an information booklet, patient-reported outcome measure, clinical pathway, or targeted intervention.

\section{(3094) Effect of written expression on mental state and quality} of life of young breast cancer patients

Zhang Jiquan, master degree, Deyang People's Hospital, Deyang CIty, Sichuan Province, China; Fan Xu, master degree, Deyang People's Hospital, Deyang CIty, Sichuan Province, China; Lin Luo,
Deyang People's Hospital, Deyang CIty, Sichuan Province, China; Jia Wang, Deyang People's Hospital, Deyang CIty, Sichuan Province, China

Aims: The aims of this study was to analyze the effect of written expression on the mental state and quality of life of young breast cancer patients. Methods: According to the continuous enrollment method, Ninety-eight patients were recruited from a tumor center. 49 patients were assigned to the intervention group and 49 to the control group. All of the participates received routine care and the intervention group participated in written expression. Acceptance and Action Questionnaire II (AAQ-II), Symptom Checklist 90 (SCL-90), the Functional Assessment of Cancer Therapy-Breast Cancer (FACTB) were assessed before intervention (T1), immediately after the intervention (T2), and one month after the intervention (T3). Results: Ninety-eight patients completed the intervention program and followup. At T2, the AAQ-II score of the intervention group was $(20.39 \pm 6.13)$, the SCL-90 score was $(127.45 \pm 12.78)$, and the FACT-B score was $(112.58 \pm 11.66)$. For control group, the scores of AAQ-II, SCL-90 and FACT-B score were (26.05 \pm 5.19$)$, $(140.98 \pm 11.63)$ and $(98.13 \pm 11.54)$ respectively. The differences between the scores were statistical significance $(\mathrm{P}<0.05)$. At T3, the AAQ-II and SCL-90 scores of intervention group were lower than those of the control group $(\mathrm{P}<0.05)$ respectively, and FACT-B was higher $(\mathrm{P}<0.05)$. The repeated analysis of variance found that the scores of AAQ-II and SCL-90 of the intervention group were significantly improved after the intervention $(\mathrm{P}<0.05)$. Covariance analysis found that after excluding the time effect, the intervention program could significantly decrease the patient's AAQ-II and SCL90 scores, and increase the patient's score of FACT-B $(\mathrm{P}<0.05)$. Conclusion: The written expression intervention could improve the mental status and quality of life of young breast cancer patients. The written expression intervention was feasible and acceptable for young breast cancer patients, but future studies are needed to address whether more prolonged interventions can improve mental status and quality of life.

\section{(3095) Quality-of-life impact and perceived treatment efficacy among patients with spondyloarthritis}

Matthew Ferry, M.S.Ed., B.A., Health Union, LLC, Philadelphia, Pennsylvania, USA; Evan Krieg, MBA, Temple University-Fox School of Business and Management, Health Union, LLC, Philadelphia, Pennsylvania, USA; submitted on behalf of "Health Union, LLC"

Aims: Spondyloarthritis is a category of progressive rheumatic conditions with a high disease burden. Existing research is clinically focused and does not examine disease impact on patient quality of life and ability to work. We aim to determine how spondyloarthritis conditions affect patients' quality of life and employment, and to investigate awareness of patients' quality-of-life and employment priorities among healthcare professionals (HCPs). Methods: An online survey was conducted from March to July 2020 among patients living with spondyloarthritis conditions $(\mathrm{N}=1181)$ including ankylosing spondylitis $(n=787)$, axial spondyloarthritis $(n=105)$, and general spondyloarthritis $(n=289)$ to gain a better understanding of patient quality of life and perceived treatment efficacy. Respondents were recruited from the online health communities AnkylosingSpondylitis.net and AxialSpondyloarthritis.net, as well as from the sites' social media accounts. Survey questions measured quality and quantity of HCP interactions, treatment and care management plans, and symptoms. Responses were evaluated using descriptive statistics. Results: Among 1181 total spondyloarthritis patients, 69\% reported their work and/or careers being negatively affected by their conditions. Respondents experienced significant life changes, including 
$30 \%$ who went on social security and disability income, and $24 \%$ who made the choice to stop working. Only $6 \%$ of respondents feel their conditions are under control with their current treatment plans, and $80 \%$ of respondents actively use complementary alternative treatments. Overall, just $54 \%$ of respondents said their HCP understands the level of pain they experience, and $47 \%$ regularly discuss with their HCPs how their quality of life is affected by their diagnoses. Conclusion: Spondyloarthritis conditions have a high disease burden and adversely affect patient quality of life and employability. Patients can experience negative effects on quality of life but do not consistently have conversations about these effects with their HCPs. The majority of patients reported low perceived efficacy of their current treatment plans and frustration that HCPs do not understand the disease burden on patient quality of life. Patients and HCPs are not aligned in their expectations of treatment, and broader awareness is needed among practitioners regarding quality of life to ensure patients have adequate support to manage their spondyloarthritis.

(3096) Defining time to functional recovery and influencing factors among lung cancer patients after surgery

Ruoyan Gong, master, School of Public Health and Management, Chongqing Medical University, Chongqing, China; Wei Xu, doctor School of Public Health and Management, Chongqing Medical University, Chongqing, China; Qingsong Yu, School of Public Health and Management, Chongqing Medical University, Chongqing, China; Hongfan Yu, School of Public Health and Management, Chongqing Medical University, Chongqing, China; Yang Pu, School of Public Health and Management, Chongqing Medical University, Chongqing, China; Jingyu Zhang, State Key Laboratory of Ultrasound in Medicine and Engineering, College of Biomedical Engineering, Chongqing Medical University, Chongqing, China; Qiuling Shi, doctor School of Public Health and Management, Chongqing Medical University, Chongqing, China

Aims: After various type of lung cancer surgery, persistent weakness impairs symptoms and their interference on daily functioning. Time to functional recovery is often a neglected topic despite its clinical impact. The purpose of the present study was to describe the median recovery time from lung cancer surgery and to identify influencing factors of functional recovery among lung cancer patients. Methods: Data extracted from a prospective cohort study of 512 patients who had received lung cancer surgery (November 2017-November 2019). Symptoms and their interference on daily functioning were assessed by M.D. Anderson Symptom Inventory begin with admission and end with 2 years after discharge in patients,. patients completed questionnaires daily after surgery to discharge and weekly after discharge to 4 weeks. Kaplan-Meier analysis was used to estimate the proportion of function recovery and to compute the median time of recovery, and Cox proportional hazard regression analysis was performed to identify influencing factors. Functional recovery was defined as interference scores returning to presurgery levels (score $\leq 4)$ consecutive two days. Results: 512 patients with complete MDASI-LC scale were included in this analysis. Divided into six groups according to six items. At four weeks after discharge, the median recovery time of activity was $3 \mathrm{~d}$ with $95 \% \mathrm{CI}[3,4]$, walking was $4 d$ with $95 \%$ CI $[3,4]$, work was $7 d$ with $95 \%$ CI[5,13], relations was 4d with 95\% CI[3,5], enjoy_of_life was 4d with 95\% CI[3,5], and mood was $3 \mathrm{~d}$ with $95 \% \mathrm{CI}[2,3]$. The proportion of function recovery was computed by using length of stay in hospital at time of discharge and $1,2,3$, and 4 weeks after discharge. The proportion of function recovery was described as activity Range $64.29 \%$ (on discharge)$77.82 \%$ (on 4 weeks after discharge), walking (Range $62.46 \%$ $75.73 \%$ ), work (Range $47.42 \%-66.45 \%$ ), relations ( Range $60.5 \%$ $74.79 \%$ ), enjoy_of_life ( Range 58.52\%-73.36\%), and mood ( Range
67.06\%-80.59\%). By Cox proportional hazard regression analysis, fatigue $(\mathrm{P}<0.001)$ significantly impacted recovery of functional status. Conclusion: We identified time to interference function recovery after lung cancer surgery. Fatigue impacts the trajectory of functional recovery after lung cancer surgery. Further examination of these groups may assist in clinical trial design to tailor interventions to specific subgroups.

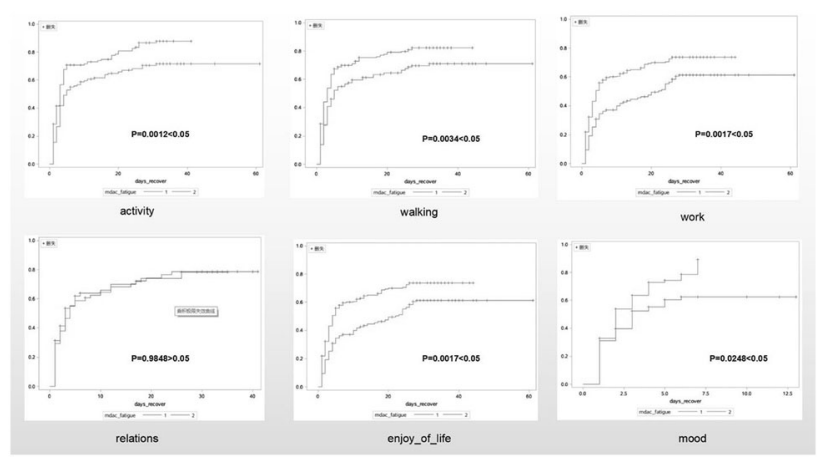

\begin{tabular}{|c|c|c|c|c|c|c|}
\hline \multirow[b]{2}{*}{ YDasI Foxctios } & \multirow{2}{*}{$\begin{array}{l}\text { XEDTAX TTEE TO } \\
\text { RECOVERY } \\
\text { (953CD) }\end{array}$} & \multicolumn{5}{|c|}{ RITE OF FNCTIOY RECOERY (s) } \\
\hline & & Discharge & $\begin{array}{c}\text { One-reck after } \\
\text { discharge }\end{array}$ & $\begin{array}{c}\text { Tro-meks after } \\
\text { discharge }\end{array}$ & $\begin{array}{c}\text { Thiree--eeks after } \\
\text { discharge }\end{array}$ & $\begin{array}{l}\text { Four- -reeks after } \\
\text { discharge }\end{array}$ \\
\hline Activity & $3[3,4]$ & 64.29 & 65.79 & 68.8 & 73.68 & 77.82 \\
\hline Kalking & $4[3,4]$ & 62.46 & 64.4 & 69.26 & 72.49 & 75.73 \\
\hline vork & $7[5,13]$ & 47.42 & 50.32 & 53.48 & 60.97 & 66.45 \\
\hline Relations & $4[3,5]$ & 60.5 & 62.18 & 68.07 & 72.27 & 74.79 \\
\hline Enjoy of life & $4[3,5]$ & 58.52 & 60.26 & 60.81 & 70.31 & 73.36 \\
\hline Yood & $3[2,3]$ & 67.06 & 69.41 & 75.29 & 78.82 & 80.59 \\
\hline
\end{tabular}

(3097) A Study on the Definition and Measurement of Quality Of Life in Occupational Therapy Intervention Research in Japan: A Scoping Review

Junichiro Muranaka, MS RegOT, Kanagawa University of Human Sciences, Nakagun, Japan; Satoshi Sasada, Ph,D,RegOT, Graduate Course of Health and Social Services, Kanagawa University of Human Services, Yokosuka, Japan; Masatoshi Gocyo, $\mathrm{Ph}, \mathrm{D}$, RegOT, Department of Occupational Therapy, Faculty of Health Science in Odawara, International University of Health and Welfare, Kanagawa, Japan; Takeru Mizuno, MS,RegOT, Showa University Medical Institute of Developmental Disabilities Research, Tokyo, Japan; Yuko Shigeta, MS, RegOT, Medical Corporation Association Kenreikai Fureai Hiratsuka Hospital, Kanagawa, Japan

Aims: The purpose of this study was to determine how quality of life has been defined and measured in domestic occupational therapy intervention studies and to examine issues that should be addressed in occupational therapy practice. Methods: A scoping review, proposed by Arksey and O'Malley and modified by Levac, was conducted on the literature retrieved using four databases for terms related to occupational therapy and QOL. The PRISMA Extension for Scoping Reviews (PRISMA-ScR) was used to ensure reproducibility in the process of literature adoption. Cross-reviews of the accepted articles were conducted with the co-authors, and then the accepted articles were analyzed according to the procedure for the definition of QOL and the measurement scale of QOL. Results: 19 articles were selected for inclusion. 17 articles were published in 2010 or later, and 2 articles were published before 2010. In terms of study design, 8 were randomized controlled trials, 1 was a modified randomized controlled trial, 3 were non-randomized controlled trials, 5 were comparative 
clinical trials, 1 was a cohort trial, and 1 was a single-system design. As for the definition of QOL, 5 studies had a clear definition of QOL, indicating that the definition of QOL was not consistent among studies. In addition, the QOL measurement scales used in the studies were based on measures that may not include Japanese patient circumstances or social and cultural background. Conclusion: Many of the QOL measurement scales used in this study were developed in other countries before 2000 , while these scales tended to be used more frequently after 2010 . Therefore, it was suggested that it is necessary to research and develop new QOL models and QOL measurement scales in Japan.

\section{(3098) Patient-Reported Outcome Measures for Youth Mental Health Services: A Scoping and Qualitative Review}

Mungunzul Megan Amarbayan, Master of Public Policy, The School of Public Policy, University of Calgary, Calgary, Alberta, Canada; Travis Carpenter, Medical doctor Temerty Faculty of Medicine, University of Toronto, Toronto, Ontario, Canada; Maria Santana, MPharm, PhD, Cumming School of Medicine, University of Calgary, Calgary, Alberta, Canada; Jennifer Zwicker, PhD, MPP, The School of Public Policy, University of Calgary, Calgary, Alberta, Canada

Aims: An estimated 10 to $20 \%$ of Canadian youth experience mental health concerns and value-based approaches to assessing youth mental health (YMH) care delivery are essential. Achieving valuebased YMH services requires an understanding of outcomes from youth's own perspective, as reported by Patient-Reported Outcome Measures (PROMs) and the Patient-Reported Outcome Measurement Information System (PROMIS). There is little consensus on which measures should be used in YMH services. We aimed to 1) map the literature on PROMs and PROMIS measures being used in YMH services and 2) describe youth perspectives on three of these measures. Methods: The scoping review was conducted according to the Arksey and O'Malley (2007) protocol. Searches were run in Medline, Embase, and PsycInfo and resulted in 571 titles and abstracts, of which 497 were excluded. From the remaining 66 title and abstracts, 29 studies moved to the full-text phase. PROMs and PROMIS were extracted from the studies. Three of the most common measures (Strength and Difficulties Questionnaire (SDQ), Goal Based Outcomes (GBOs), and the PROMIS depression measure) were discussed with a pilot focus group $(n=5)$ of youth and caregivers with experience accessing YMH services in Calgary, Alberta, Canada, to describe their perspectives on the measures. Results: We found 131 unique outcome measures being used for YMH services. From the focus group, it was found that there were few outcome measures currently being done in Alberta, and they are not done continuously. When participants were presented with the SDQ, GBOs, and PROMIS depression measure, common themes included concerns about the question types and the ability to capture meaningful progress with the measures. Overall, the GBOs had a more positive impression compared to the SDQ and PROMIS depression measure. Conclusion: Currently, Alberta has no policy for a province-wide set of outcome measures, particularly in YMH services. Despite the range of different measures available, there is little consensus or consistency on which PROMs or PROMIS measures are recommended for youth with mental health concerns. Further research on the strengths and limitations of these outcome measures for youth with mental health concerns is required for its utilization in the delivery of value-based care.

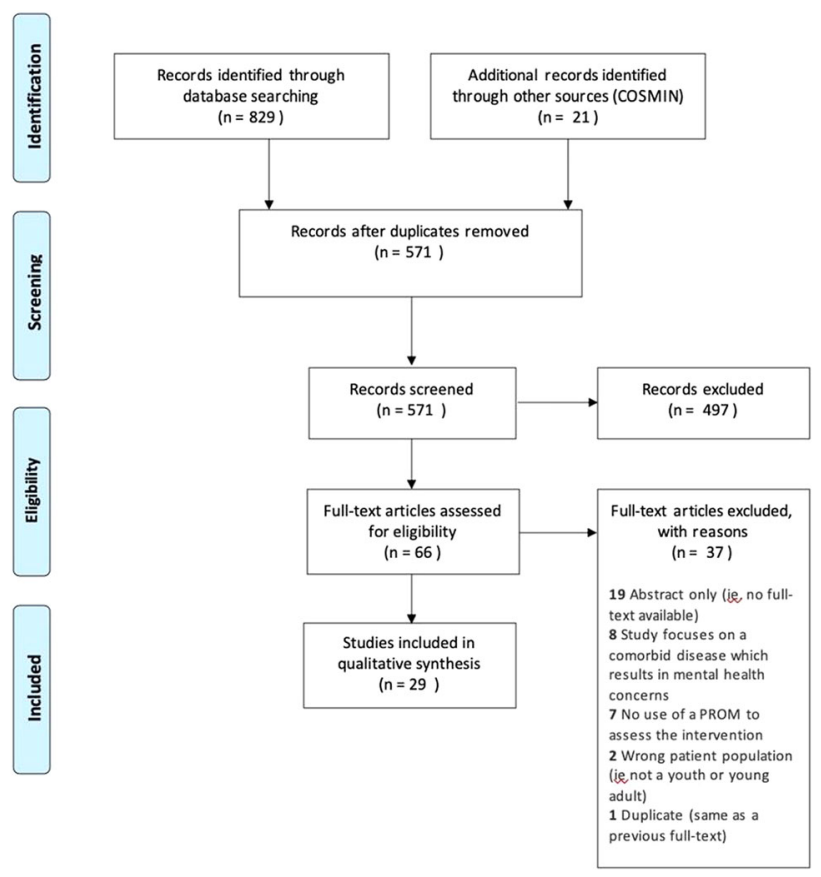

(3099) A systematic review of primary and secondary outcome reporting in randomised clinical trials of distal tibia and ankle fractures

Nathan Pearson, PhD, University of Warwick, Coventry, UK; Elizabeth Tutton, $\mathrm{PhD}$, University of Oxford, Oxford, UK; Alexander Joeris, AO ITC, Biel/Bienne, Switzerland; Stephen Gwilym, University of Oxford, Oxford, UK; Richard Grant, Patient Research Partner. National Institute for Health Research, Applied Research Collaboration, West Midlands, Coventry, UK; David Keene, University of Oxford, Oxford, UK; Kirstie Haywood, DPhil, University of Warwick, Coventry, UK; submitted on behalf of "COSTA Collaborators"

Aims: To examine primary and secondary outcome reporting in clinical trials of people experiencing distal tibia and ankle fractures. Methods: Five databases and three trial registries were searched from January 2000 to March 2021. NP screened all titles and abstracts and $\mathrm{KH}$ double-assessed a $10 \%$ subset. Studies were included if they were randomised controlled trials with patients with distal tibia (tibia, distal end segment type 43) and ankle fractures (malleolar segment type 44) as defined by the AO/OTA fracture classification. NP and KH (10\% subset) extracted data which included study reference, design, population and sample size, interventions studied, and outcomes reported (e.g., health, clinical, social, economic) and measurement approaches (e.g., patient or clinician-reported outcome). Primary and secondary outcomes were extracted and mapped to the ICF framework. Results: From 126 full text reviews, 83 studies were included covering 26 countries. Samples ranged from 16 to 321 . 46 studies evaluated two surgical interventions, seventeen evaluated post-surgical management, twelve evaluated rehabilitation interventions, five compared surgical and non-surgical interventions, and three evaluated pre-surgical management of injury. 105 primary outcomes were extracted. 65 outcomes focused primarily on Body Structure and Function (69\% of trials), 3 on Activities, 3 on Participation, 5 on Health-Related Quality of Life (HRQoL; 3-4\% of trials, respectively) and 17 on Processes of Care (22\% of trials). Reporting was inconsistent across trials. 342 secondary outcomes were extracted. 63 focused primarily on Body Structure and Function (38\% of trials), 20 on Activities (6\% of trials), 4 on Participation, 8 on HRQoL (9-15\% of trials, respectively) and 27 
on Processes of Care (32\% of trials). Conclusion: This review highlights the substantive heterogeneity in outcome selection and reporting. Reporting of primary and secondary outcomes was inconsistent in many studies, and multiple studies identified a range of primary endpoints (up to 8 in two studies). Consistently identified outcome domains such as pain were assessed using different tools. Inconsistency is detrimental to the completion of meta-analyses. Future work to develop a core outcome set for distal tibia and ankle fractures is needed to establish a minimum, standardised group of outcomes to be included in all trials.

\section{(3100) Quality-of-life changes following physiological pubertal hormone replacement therapy in boys 14 years or older with delayed puberty in a multicenter randomized clinical study: PRIBS}

John Chaplin, $\mathrm{PhD}$, Sahlgrenska Academy at University of Gothenburg, Gothenburg, Sweden; Martin Österbrand, MD, Department of Pediatrics, NU Hospital Group, Trollhättan, Sweden; Hans Fors, MD, PhD, Gothenburg Pediatric Growth Research Center, Sahlgrenska Academy at University of Gothenburg, Institute of Clinical Sciences, Department of Pediatrics, Queen Silvia Children's Hospital, Gothenburg, Sweden; Ensio Norjavaara, MD, $\mathrm{PhD}$, Gothenburg Pediatric Growth Research Center, Sahlgrenska Academy at University of Gothenburg, Institute of Clinical Sciences, Department of Pediatrics, Queen Silvia Children's Hospital, Gothenburg, Sweden

Aims: Data from different studies on the effects of testosterone replacement on quality of life are not consistent. The aim of the study was to examine the effects of physiological pubertal hormone replacement therapy in a follow-up of boys 14 years or older with delayed puberty. Methods: Boys with pubertal delay identified for hormone replacement therapy, over a four-year period, aged over 14 and under 17 years at inclusion, completed four questionnaires at baseline, 6 and 12 months. Quality of life was measured by a generic QoL instrument (Kidscreen-10 items) and a short-stature-specific QoL instrument (QoLiSSY-9 items). The DSRS-C, a measure of depression felt in the past week, and the Physical Activity Enjoyment Scale (PACES) were also completed. One-way repeated measures analysis of variance (ANOVA) was conducted to evaluate change in generic or short-stature QoL. Independent t-tests compared final follow-up with an age-matched reference population of growthhormone-treated short-stature boys. Results: Twenty-five boys completed the survey instruments $(n=25)$. The ANOVA test indicated a significant time effect on generic QoL (Wilks' Lambda $=0.769$ $\mathrm{F}(2,24)=3.606, \mathrm{p}<0.043$ ). Post hoc comparisons indicated a pairwise difference between baseline and 12 months $(\mathrm{P}<0.034)$. No significant differences were observed in short-stature QoL. Changes in emotional response were detected with a Friedman's test, showing a significant difference between time points $(\mathrm{X} 2 \mathrm{f}(2)=11.32$, $\mathrm{p}<0.003)$. Post-hoc tests adjusted for Bonferroni correction showed improvement between baseline and 6 months $(\mathrm{t}=0.680, \mathrm{p}<0.049)$ and at 12 months $(\mathrm{t}=0.880, \mathrm{p}<0.006)$. Enjoyment of physical activity did not change at follow-up. Compared to a reference population of age-matched short-stature boys $(n=62)$ at the 12 -month follow-up, there was no significant difference in the QoLiSSY score $(\mathrm{t}(84)=-1.17, \mathrm{p}=0.247)$. However, pubertal boys at the 12-month follow-up, scored significantly higher $(M=56.71, S D=12.7)$ on the measure of general QoL than the age-matched reference population of growth-hormone-treated boys $(\mathrm{M}=51.36, \mathrm{SD}=10.2)(\mathrm{t}(85)=2.06$, $\mathrm{p}=0.043$ ). Conclusion: There was a significant increase in Kidscreen scores over time, suggesting that testosterone replacement therapy increased participants' subjective level of generic QoL additionally there was a significant decrease in depression scores. However, no changes in relation to stature-specific QoL and enjoyment of physical activity were found.

(3101) Utilizing patient-reported outcomes at discharge to determine the symptom and physical recovery time after oncologic surgery for bladder carcinoma

Mona Kamal, MD, PhD, University of Texas, MD Anderson Cancer Center, Houston, Texas, USA; Xin Wang, University of Texas, MD Anderson Cancer Center, Houston, Texas, USA; Tsun-Hsuan Chen, University of Texas, MD Anderson Cancer Center, Houston, Texas, USA; Fang-yu Lin, University of Texas, MD Anderson Cancer Center, Houston, Texas, USA; Araceli Garcia-Gonzalez, University of Texas, MD Anderson Cancer Center, Houston, Texas, USA; Charles Cleeland, University of Texas, MD Anderson Cancer Center, Houston, Texas, USA; Neema Navai, University of Texas, MD Anderson Cancer Center, Houston, Texas, USA

Aims: Use patient-reported outcomes (PROs) at discharge to determine the symptoms and physical functioning recovery time after oncologic surgery for bladder carcinoma (BLC). Methods: In patients who underwent radical cystectomy for BLC in this prospective longitudinal study, using the MD Anderson Symptom Inventory for perioperative care for BLC patients (MDASI-PeriOp-BLC), we assessed PROs pre-surgery, at discharge and weekly for 11 weeks postoperatively. Moderate to severe symptom is defined as $\geq 4$ on $0-10$ scale. Kaplan-Meier and Cox regression analyses were used to calculate the symptoms and physical recovery time. Regression analysis was used to assess the association between given symptoms burden at discharge and time needed to recover to mild/no symptoms or functioning interferences. Results: Patients with PROs at least 3 time points were included in this report $(\mathrm{N}=52 / 62)$. Total $74 \%$ of sample without PRO missing data. The majority were men $(86 \%)$, white (94\%), and had good performance status (ECOG 0-1) (90\%). Most of the patients (97\%) received neoadjuvant chemotherapy, and $48 \%$ had 3 or more comorbidities. Average time of hospital stay was 6 (4-21) days, and $12 \%$ had postoperative complications. Pre-surgery, the worst reported symptoms were urinary frequency, sleep disturbance, urgent urination, fatigue, distress, and interference with general activities. At discharge, the top 6 severe symptoms were poor appetite, drowsiness, fatigue, pain, sleep disturbance, and abdominal tightness. Patients with moderate/severe fatigue, abdominal tightness, and sleep disturbance at discharge require the significant longer time to return to non/mild scores vs. others ((median 21 vs. 7 days), (median 15 vs. 7 days), and (median 21 vs. 7 days) (all $\mathrm{P}<0.05)$ ). Return of the sum scores of physical functioning interference (general activity, work, and walking interfering) to non/mild levels requires a significantly longer time in patients with moderate/severe scores at discharge vs. others (median $28(14-42)$ vs. $7(3-8)$ days, $\mathrm{P}<0.05$ ). Conclusion: Critical specific PROs at moderate to severe severity at discharge are clinically meaningful to determine the required time for symptoms and physical recovery in patients who underwent oncologic resection for BLC. The results support implementing PROs into routine patient care after oncologic resection for BLC for patientcentered perioperative care.

(3102) How the routine use of PROMs for hemodialysis care influences patient-clinician communication: a mixed methods study

Kara Schick-Makaroff, PhD, University of Alberta, Edmonton, Alberta, Canada; Lisa Wozniak, MA, University of Alberta, Edmonton, Alberta, Canada; Hilary Short, MSc, University of Alberta, Edmonton, Alberta, Canada; Sara Davison, MD, 
University of Alberta, Edmonton, Alberta, Canada; Scott Klarenbach, MD, University of Alberta, Edmonton, Alberta, Canada; Robert Buzinski, Patient Partner, Medicine Hat, Alberta, Canada; Jeffrey Johnson, PhD, University of Alberta, Edmonton, Alberta, Canada

Aims: Kidney failure requiring dialysis is associated with high symptom burden that negatively impacts quality of life. Unfortunately, symptoms are often under-recognized and under-treated by kidney care teams. Patient-reported outcome measures (PROMs) invite patients to self-report aspects of their quality of life, including symptoms, and have been reported to enhance communication with clinicians. We aimed to examine how the routine use of PROMs in incentre hemodialysis units influenced patient-clinician communication. Methods: A concurrent mixed-methods approach was employed. We used longitudinal data from a cluster randomized controlled trial (RCT) of 17 hemodialysis in northern Alberta that introduced a PROMs intervention. Patient-clinician communication was assessed using a modified Communication Assessment Tool (CAT). Using interpretative description, we also described patients' and nurses' perceptions of communication pertaining to routine PROM use. Through purposeful sampling, we interviewed 10 patients and 8 nurses, and conducted 6 observations in the dialysis units, which were documented in extensive field notes. We reviewed 510 open-ended survey responses from patients' responses to open-ended survey questions from RCT data. Qualitative data was managed using ATLAS.ti 8 and thematically analyzed. Results: Overall, we found PROM use did not improve patient-clinician communication. There was a small positive change in mean total CAT scores (range 1-5) from baseline to 12 -months in PROM use units $(0.25)$ but little difference from control group units that did not use PROMs (0.21). Possible reasons for why communication did not improve included patients reporting high satisfaction in communication at baseline prior to PROMs use. In addition, PROMs were not implemented as intended across units with considerable variation in how PROMs were used in care. Many nurses and patients viewed PROMs assessments as redundant to usual care, and there was a lack of understanding of the purpose of PROMs assessments. Many nurses conflated PROMs assessments with their clinical assessments, and patients were uncertain of what might happen with their PROM responses. Conclusion: Findings that PROMs did not improve communication is contradictory to the existing literature in other clinical settings. Yet qualitative data illuminated that PROM use was not enacted as intended, which may explain divergence.

(3103) The health-related quality of life of breast cancer survivors attending a nurse practitioner-led model of care for adjuvant endocrine treatment in Vancouver, Canada

Nicola Bai, BC Cancer, Vancouver, British Columbia, Canada; Tamara Shenkier, BC Cancer, Vancouver, British Columbia, Canada; Caroline Lohrisch, BC Cancer, Vancouver, British Columbia, Canada; Stephen Chia, BC Caner, Vancouver, British Columbia, Canada; Christine Simmons, BC Cancer, Vancouver, British Columbia, Canada; Anita Dotts, BC Women's Hospital + Health Centre, Vancouver, British Columbia, Canada, Helen McTaggart Cowan, Faculty of Health Sciences, Simon Fraser University, Burnaby, British Columbia, Canada

Aims: Breast cancer is the most prevalent cancer in women. The majority of breast cancers present in early stage, are potentially curable, and generally treated with adjuvant endocrine therapy (AET) for 5-10 years. The need for an oncologist's input may diminish after the first year treatment amongst many breast cancer survivors; however, the need for the expertise of other health care disciplines increases. As such, the After Breast Cancer (ABC) Service, a transitional, nurse practitioner-led clinic was implemented in Vancouver,
Canada. The service was aimed at optimizing the care of adjuvant hormone positive breast cancer patients with stages I-III. This study aimed to assess the health-related quality of life (HRQL) of women enrolled in the $\mathrm{ABC}$ Service. Methods: Breast cancer survivors completed the FACT-B +4 at the time of the first visit; follow-up measurements at 12 and 24 month were collected. They also provided details about employment status and household structure. Information about the survivors' clinical and treatment characteristics were extracted using electronic chart reviews. Descriptive statistics characterized the women enrolled in the ABC Service. Statistical tests of association evaluated changes in survivors' FACT-B +4 scores across the three timepoints. A multivariate linear regression assessed the factors that influenced the changes in the survivors' HRQL. Results: As of March 2021, baseline, 12 month, and 24 month responses to the FACT-B +4 were completed by 218,69 , and 44 survivors, respectively. The aggregate FACT-B +4 scores (mean $( \pm \mathrm{SD}))$ at baseline, 12 month, and 24 month were reported to be $109.1( \pm 21.4), 113.9( \pm 20.7)$, and 111.3 ( \pm 21.1$)$, respectively. When compared to the baseline subscale scores, statistically significant changes were observed for the physical wellbeing subscale for both follow-up timepoints, and for functional wellbeing for the 12 month timepoint. Initial analyses revealed that sociodemographic and clinical variables did not influence changes in HRQL. Conclusion: Preliminary findings revealed that the ABC Service appeared to address the unmet HRQL needs of breast cancer survivors. Breast cancer survivors enrolled the ABC Service reported an increase in FACT-B +4 scores after the initial visit; in particular, physical wellbeing significantly improved.

(3104) Determining construct validity of a new patient-reported outcome measure for patients with birthmarks

Charlene Rae, MSc, McMaster University, Hamilton, Ontario, Canada; Rakhshan Kamran, BSc(Hon), McMaster University, Hamilton, Ontario, Canada; Karen Wong Riff, MD, PhD, Hospital For Sick Children, Toronto, Ontario, Canada; Andrea Pusic, MD, Brigham and Women's Hospital, Boston, Massachusetts, USA; Anne Klassen, DPhil, McMaster University, Hamilton, Ontario, Canada

Aims: Birthmarks are visible skin lesions that present early in life, and are varied in location, appearance, neoplastic nature, and texture. Birthmarks can affect an individual's appearance, body image, and psychosocial functioning, concepts best measured by patient-reported outcome measures (PROMs). When treating birthmarks, the aim is to improve these outcomes. A new PROM called the FACE-Q Craniofacial Module includes a scale to measure the appearance of birthmarks. This scale has 8 items that ask how bothered someone is on a 4-point scale (not at all to very much). In addition, there are health-related quality of life (HRQOL) scales that measure Psychological, Social, School, and Appearance Distress. Scores on each of these scales range from 0 (Worst) to 100 (Best). The aim of this study was to examine construct validity of the Birthmark scale and to investigate HRQOL in individuals with birthmarks. Methods: Participants aged 8 to 29 years with a birthmark anywhere on the face or body were invited to complete relevant scales from the FACE-Q Craniofacial module between December 2016 and December 2019. Construct validity of the Birthmark scale was assessed by testing hypotheses of known group differences. Differences between groups were assessed using independent T-test and one way ANOVA. Pearson correlation co-efficient was used to examine relationships between scales, we expected moderate correlations $(0.3-0.5)$ because not all birthmarks were noticeable. Results: A total of 271 participants (mean age $=14$ ) were included. The majority of the sample were female $(60.1 \%)$, and had facial birthmarks $(62.3 \%)$. Age, gender, birthmark type, and treatment status were not associated with the 
Birthmark scale scores. Having a facial birthmark, reporting being bothered by the birthmark, and having more noticeable birthmarks were associated lower scores $(\mathrm{p}<=0.001)$. Scores on the Birthmark scale were moderately correlated with HRQOL, i.e., Appearance Distress $(r=0.4)$, Psychological $(r=0.3)$, Social $(r=0.3)$, and the School scale $(r=0.3)(p<0.001)$. Conclusion: This new PROM evidenced construct validity in a sample of patients with birthmarks, and can be used to assess the patient's perspective of appearance of birthmarks on any part of the body. Further work should focus on testing other psychometric properties such as test-rest and responsiveness.

(3105) The use of patient-reported outcome measures to describe a population of young people accessing integrated health and social service care centres

Kyle Dewsnap, The University of British Columbia, Vancouver, British Columbia, Canada; Steve Mathias, MD, University of British Columbia, Vancouver, British Columbia, Canada; Skye Barbic, $\mathrm{PhD}$, University of British Columbia, Vancouver, British Columbia, Canada

Aims: Approximately one in four young people in Canada are affected by a mental health challenges each year. In response, organizations across Canada have adopted a model of youth service that involves the integration of health care and social services into one easy to access location. The aims of the study were to (1) describe the clinical and sociodemographic characteristics of young people who access integrated youth services and (2) understand the extent to which clinical characteristics are different from data collected from youth in the general population. Methods: Foundry is a network of integrated youth health and social service centres across British Columbia, Canada. From six diverse Foundry centres (urban, suburban, rural), we asked young people requesting services to complete an assessment package which includes a demographic questionnaire and a battery of patient-reported outcome measures (PROMs) assessing levels of depression (PHQ-9), anxiety (GAD-7), and distress (Kessler Distress Scale/K10). We summarized the demographic (age, gender, ethnicity) and clinical characteristics (depression, anxiety, and distress) of young people who participated and compared their clinical to normative data available for age-matched youth. Results: We recruited 839 participants with mean age 20.6 years $(\mathrm{SD}=2.46$ years); $416(49.6 \%)$ participants identified as female, $60(7.2 \%)$ as non-binary (including two-spirited), and 296 (35.3\%) as male. Participants reported having a median of two mental health diagnoses [IQR $=0-4]$, with only $26(3.1 \%)$ of young people reporting no diagnoses. Compared against normative data, young people who accessed services scored significantly higher $(\mathrm{p}<0.05)$ on the GAD$7(\mathrm{M}=11.5, \mathrm{SD}=6.08)$, the PHQ-9 $(\mathrm{M}=13.3, \mathrm{SD}=7.16)$, and the $\mathrm{K} 10(\mathrm{M}=28.6, \mathrm{SD}=9.61)$. Conclusion: Our analysis suggests that young people who access Foundry services overwhelmingly report having been previously diagnosed with a mental illness and receive scores on PROMs that indicate abnormality high levels of depression, anxiety, and psychological distress. Our findings suggest that the use of PROMs can support the delivery and monitoring of integrated youth health services that match the intensity of the patient's presenting problem, as well as the preferences of the young person and their family.
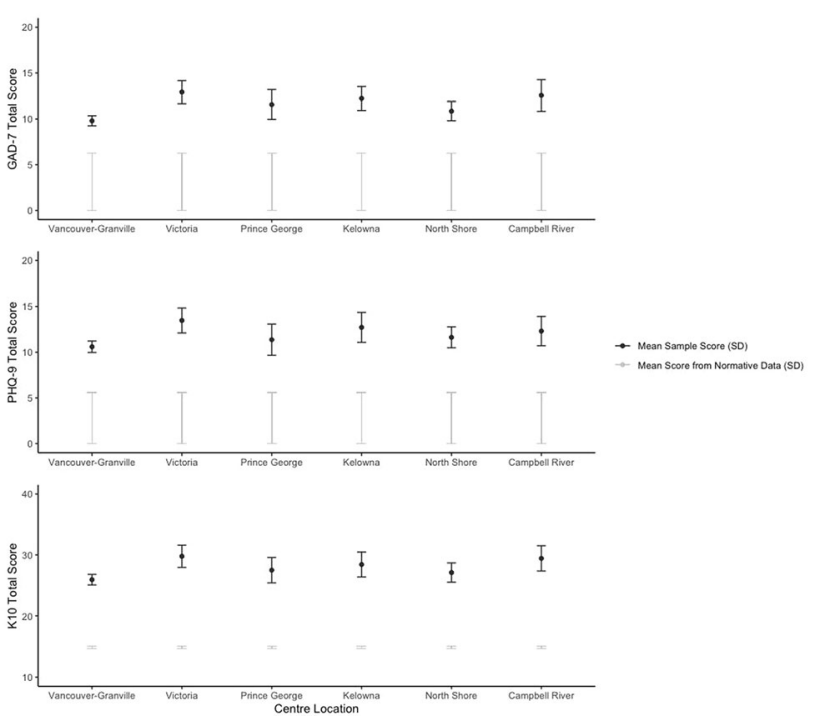


\begin{tabular}{|c|c|}
\hline & $\begin{array}{l}\text { Overall } \\
(\mathrm{N}=839)\end{array}$ \\
\hline \multicolumn{2}{|l|}{ Age (Years) } \\
\hline Mean (SD) & $20.6(2.46)$ \\
\hline Median [Min, Max] & $21.0[13.0,26.0]$ \\
\hline Missing & $27(3.2 \%)$ \\
\hline \multicolumn{2}{|l|}{ Gender } \\
\hline Female & $416(49.6 \%)$ \\
\hline Male & $296(35.3 \%)$ \\
\hline Non-Binary & $32(3.8 \%)$ \\
\hline Two-Spirit & $8(1.0 \%)$ \\
\hline Female (Transgender) & $10(1.2 \%)$ \\
\hline Male (Transgender) & $16(1.9 \%)$ \\
\hline Questioning & $6(0.7 \%)$ \\
\hline Declined to Answer & $2(0.2 \%)$ \\
\hline Option not Available & $14(1.7 \%)$ \\
\hline Multiple Options Selected & $34(4.1 \%)$ \\
\hline Missing & $5(0.6 \%)$ \\
\hline \multicolumn{2}{|l|}{ Ethnicity } \\
\hline South Asian & $32(3.8 \%)$ \\
\hline Black/African & $21(2.5 \%)$ \\
\hline Caribbean & $13(1.5 \%)$ \\
\hline Hispanic/Latin & $18(2.1 \%)$ \\
\hline First Natins/Metis/Inuit & $134(16.0 \%)$ \\
\hline Middle Eastern/North African & $22(2.6 \%)$ \\
\hline White & $446(53.2 \%)$ \\
\hline Other & $38(4.5 \%)$ \\
\hline Multiple Options Selected & $111(13.2 \%)$ \\
\hline Missing & $4(0.5 \%)$ \\
\hline \multicolumn{2}{|l|}{ Number of MH Dxs Reported } \\
\hline Mean (SD) & $2.08(1.20)$ \\
\hline Median [Min, Max] & $2.00[0,7.00]$ \\
\hline
\end{tabular}

\section{Overall}

( $\mathrm{N}=839)$

\section{GAD-7}

Mean (SD)

$11.5(6.08)$

Median [Min, Max] $12.0[0,21.0]$

Missing

$36(4.3 \%)$

K10

Mean (SD)

$28.6(9.61)$

Median [Min, Max]

Missing

$29.0[10.0,50.0]$

$37(4.4 \%)$

PHQ-9

Mean (SD)

$13.3(7.16)$

Median [Min, Max]

Missing

$14.0[0,27.0]$

$54(6.4 \%)$

PROM (Adjusted)

Mean (SD)

$17.1(6.21)$

Median [Min, Max] $17.0[0,30.0]$

Missing

$79(9.4 \%)$

IMRS

Mean (SD)

$39.3(8.30)$

Median [Min, Max] $39.0[13.0,65.0]$

Missing

$298(35.5 \%)$

BAI

Mean (SD)

$23.5(15.1)$

Median [Min, Max]

$23.0[0,63.0]$

Missing

$354(42.2 \%)$

\section{GAIN-SS}

Mean (SD)

Median [Min, Max]

$11.3(5.72)$

Missing

$11.0[0,23.0]$

$255(30.4 \%)$

(3106) Quality of life correlates with health conditions, absenteeism and turnover among professional caregivers in Alberta's continuing care facilities

Oluwagbohunmi Awosoga, PhD, MBA, Faculty of Health Sciences, University of Lethbridge, Lethbridge, Alberta, Canada; Ogochukwu 
Onyeso, MSc. PT., Department of Medical Rehabilitation, Faculty of Health Sciences and Technology, College of Medicine, University of Nigeria, Nsukka, Enugu, Nigeria; Adesola Odole, Ph.D,

Department of Physiotherapy, Faculty of Clinical Sciences, College of Medicine, University of Ibadan, Ibadan, Oyo, Nigeria; Christina Nord, MSc. PhD (Cand.), Faculty of Art and Sciences, University of Lethbridge, Lethbridge, Alberta, Canada; Ifeoma Nwosu, MSc. PT., Department of Medical Rehabilitation, Faculty of Health Sciences and Technology, College of Health Sciences, Nnamdi Azikiwe University, Awka, Anambra, Nigeria; Ezinne Ekediegwu, MSc. PT., Department of Medical Rehabilitation, Faculty of Health Sciences and Technology, College of Health Sciences, Nnamdi Azikiwe University, Awka, Anambra, Nigeria; Jon Doan, Ph.D. PEng., Faculty of Art and Sciences, University of Lethbridge, Lethbridge, Alberta, Canada; Sheli Murphy, Ph.D. RN, Rural Health, Professional Practice, Research and Libraries, Covenant Health, Edmonton, Alberta, Canada; Claudia Steinke, Ph.D. RN, Faculty of Health Sciences, University of Lethbridge, Lethbridge, Alberta, Canada

Aims: The population of older adults in Alberta continues to increase. Likewise, the number of assisted living (AL) and long-term care (LTC) facilities in Alberta are expanding, but little is known about the quality of life (QOL) of its caregivers. This study investigated the relationships among the QOL, physical health conditions, mental and emotional health (MEH), stress levels, and turnover and absenteeism (TAA) among professional caregivers in Alberta's AL and LTC facilities. Methods: The study was a cross-sectional survey using standardized questionnaires: Canadian Centre for Occupational Health and Safety, SF-36 QOL, and the Employment and Wellness Survey. Convenience sampling was used to select 39 AL and LTC facilities, and to recruit participants within the facilities. Ethical approval for the study was obtained from the University of Lethbridge. Data were analyzed using descriptive statistics, Pearson's correlation, and multivariate logistic regression. Results: Of 1385 surveys administered, 933 valid responses were received (response rate $=67.4 \%$ ). The majority of the participants were females $(90.8 \%)$ who were $\geq 35$ years $(73.6 \%)$, worked between 20 to $40 \mathrm{~h}$ weekly $(67.3 \%)$, and were satisfied with their overall health status $(68.1 \%)$. There were correlations between participants' QOL and each of MEH $(r=0.660)$, physical health $(r=0.491)$, health behaviors $(r=0.434)$, health condition $(r=0.382)$, stress levels $(r=-0.578)$, and TAA $(\mathrm{r}=0.308)$, all at $\mathrm{p}<0.001$. Results from the multivariate logistic regression will be presented at the conference. Conclusion: Increase in QOL corelates positively with healthy living and job satisfaction, while reduction in QOL correlates with increasing job stress, absenteeism and turnover. We deduced that improving the wellbeing and QOL of caregivers, may lead to staff retention. Keywords: Absenteeism, Continuing care, Job stress, Mental health, Physical health, Quality of life.

Pearson's correlations coefficients

\begin{tabular}{|c|c|c|c|c|c|c|}
\hline Variable & $\begin{array}{l}\text { Health } \\
\text { condition }\end{array}$ & $\begin{array}{l}\text { Mental and } \\
\text { emotional } \\
\text { health }\end{array}$ & $\begin{array}{l}\text { Stress } \\
\text { level }\end{array}$ & $\begin{array}{l}\text { Quality of } \\
\text { life }\end{array}$ & $\begin{array}{c}\text { Health } \\
\text { behavior }\end{array}$ & $\begin{array}{c}\text { Turnover } \\
\text { and } \\
\text { absenteeism }\end{array}$ \\
\hline $\begin{array}{l}\text { Physical } \\
\text { health }\end{array}$ & $0.626^{* *}$ & $0.606^{* *}$ & $0.398^{* *}$ & $0.491^{* *}$ & $0.387 * *$ & $0.278^{* * *}$ \\
\hline $\begin{array}{l}\text { Health } \\
\text { condition }\end{array}$ & - & $0.541^{* *}$ & $0.394^{* *}$ & $0.382^{* *}$ & $0.306^{* *}$ & $0.269 * *$ \\
\hline $\begin{array}{l}\text { Mental and } \\
\text { emotional } \\
\text { health }\end{array}$ & - & - & $0.560 * *$ & $0.660^{* *}$ & $0.405 * *$ & $0.398^{* *}$ \\
\hline Stress level & - & - & - & $-0.578^{* *}$ & $0.349 * *$ & $0.251^{* *}$ \\
\hline $\begin{array}{l}\text { Quality of } \\
\text { life }\end{array}$ & - & - & - & - & $0.434 * *$ & $0.308 * *$ \\
\hline $\begin{array}{l}\text { Health } \\
\text { behavior }\end{array}$ & - & - & - & - & - & $0.167^{* *}$ \\
\hline
\end{tabular}

Regression models

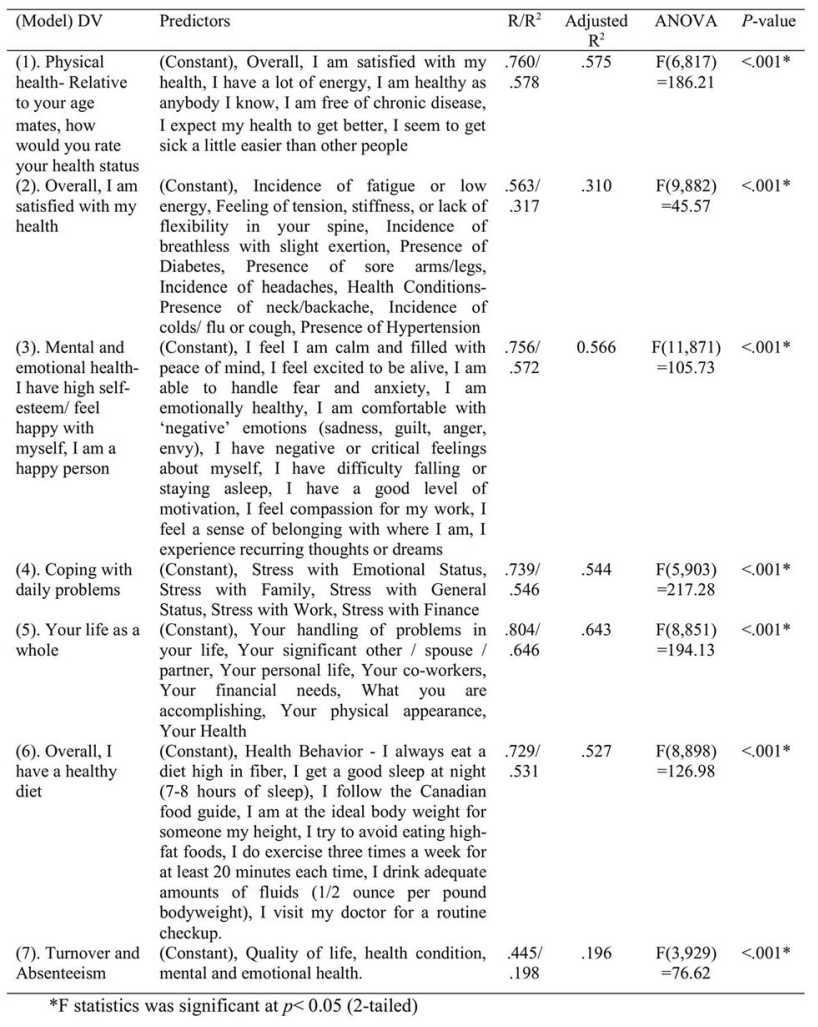

(3107) A longitudinal study of psychological adjustment trajectories for prostate cancer patients with radical prostatectomy

\section{Qingmei Huang, PhD, Fudan University, Shanghai, China}

Aims: For prostate cancer patients with radical prostatecomy, 1 year after surgery is the main recovery period for their physiological complications and psychological problems and maladaptation will seriously affect the quality of life during their survival period. Using longitudinal study design, this study was aimed to identify distinct trajectories of psychological adjustment of prostate cancer patients after radical prostatecomy. Methods: Using the Chinese version of MAX-PC scale as measurement, the psychological adjustment level of patients were assessed after diagnosis(T0), at about 1 month after surgery (T1), 3 month(T2), 6 month (T3) and 12 month after surgery(T4), respectively. Growth mixture models (GMM) were used to identify trajectory classes of psychological adjustment among different patients. Results: The results showed that the MAX-PC scores were highest at $\mathrm{T} 0$, while the scores had significant reduced from $\mathrm{T} 1$ to T4 after surgery. Repeated-measures ANOVA showed that there was statistically significant difference in the mean MAX-PC scores over time. By GMM analysis, the results suggested that the four-class model fitted best as for the lower BIC of 5317.19, the higher entropy value of 0.84 and the statistically significant result both for BLRT test and VLMR test. Overall, four distinct classes of trajectories patterns were identified for psychological adjustment in prostate cancer patients with radical prostatectomy, which were named as good adaptation class $(58.1 \%)$, positive adjustment class $(32.5 \%)$, negative adjustment class $(2.3 \%)$ and poor adjustment class $(7.0 \%)$. Conclusion: Although most of patients would achieve a good psychological adjustment outcome, some patients still couldn't adjust well, being with a poor psychological adjustment outcome. Future study should further explore the predictors for patients with poor psychological adjustment outcome. 


\section{(3108) Comparison of two methods for DIF analysis} among multiple groups: a simulation study

Odile Stahl, SPHERE, Université de Nantes, Nantes, France; Véronique Sébille, SPHERE, Université de Nantes, Nantes, France; Myriam Blanchin, SPHERE, Université de Nantes, Nantes, France

Aims: The perception and interpretation of items may vary according to patients' characteristics in Patient-Reported Outcomes data, a phenomenon known as Differential Item Functioning (DIF). The presence of DIF can lead to biased results and erroneous conclusions when comparing patients' groups. Various methods for DIF analysis have been proposed between two patients' groups [1]. However, few methods are available to analyze DIF between more than 2 patients' groups, in particular when using Rasch Measurement Theory (RMT).The aim of this study is to compare two methods for identifying DIF using RMT among 3 patients' groups in a simulation study. Methods: The two methods for DIF detection rely on partial creditmodels but differ in their process. The first method detects the overall presence of DIF in all items. If DIF is suspected, it then identifies items affected by DIF one-by-one starting from a model without DIF [2]. The second method identifies a group of items without DIF from a model with DIF on all items. This group of items is refined iteratively until a stable model is achieved [1]. Both methods were applied on responses simulated from 3 patients' groups with various sample sizes, number of items and answer categories, patterns of DIF (no DIF, uniform DIF, non-uniform DIF, DIF in only one group, DIF in 2 groups), with or without group effect.The performance of both algorithms was evaluated and compared regarding the rate of correct (DIF simulated) or incorrect detection (DIF not simulated) of DIF and regarding bias in the estimation of the latent trait difference between groups. Results: For both methods, the rate of incorrect detection of DIF should be lower than $5 \%$. However, the rate of correct detection of DIF, its type or the affected group is expected to be higher for the first method because DIF is identified item by item. Conclusion: Determining the most adequate method for DIF detection in more than 2 patients' groups will make possible to detect and account for DIF in studies comparing several treatment arms or countries.

References

[1] L. Tay, A. W. Meade, et M. Cao, « An Overview and Practical Guide to IRT Measurement Equivalence Analysis », Organ. Res. Methods, vol. 18, n 1, p. 3-46, janv. 2015, doi: 10.1177/1094428114553062.

[2] A. Guilleux et al., "RespOnse Shift ALgorithm in Item response theory (ROSALI) for response shift detection with missing data in longitudinal patient-reported outcome studies ", Qual. Life Res., vol. 24, n 3, p. 553-564, mars 2015, doi: 10.1007/s11136-014-0876-4.

(3109) Exploring Possible Interpretation Differences by Men and Women on the Kansas City Cardiomyopathy Questionnaire (KCCQ) via Qualitative Interviews.

Theresa Coles, Duke University, Durham, North Carolina, USA; Nicole Lucas, Duke University, Durham, North Carolina, USA; Molly McFatrich, Duke University, Durham, North Carolina, USA; Debra Henke, Duke University, Durham, North Carolina, USA; Jennifer Ridgeway, Mayo Clinic, Rochester, Minnesota, USA; Emma Behnken, Mayo Clinic, Rochester, Minnesota, USA; Fraser Bocell, FDA, Silver Spring, Maryland, USA; Heidi Dohse, Tour de Heart, Hailey, Idaho, USA; Shannon Dunlay, Mayo Clinic, Rochester, Minnesota, USA; Lindsay Emmanuel, Mayo Clinic, Rochester, Minnesota, USA; Ileana Pina, FDA, Silver Spring, Maryland, USA; Bryce Reeve, Duke University, Durham, North Carolina, USA; Brittany Caldwell, FDA, Silver Spring, Maryland, USA; Anindita Saha, FDA, Silver Spring, Maryland, USA; John Spertus, University of Missouri, Kansas City, Missouri, USA; Michelle Tarver, FDA, Silver Spring, Maryland, USA; Kevin Weinfurt, Duke University, Durham, North Carolina, USA; Amy Corneli, Duke University, Durham, North Carolina, USA
Aims: The Kansas City Cardiomyopathy Questionnaire (KCCQ-23) is a patient-reported outcome (PRO) measure used in clinical research to evaluate health-related quality of life (HRQOL) of patients with heart failure (HF). Studies show that women typically report worse quality of life than men with $\mathrm{HF}$, but the mechanism underlying this finding is not well understood. One potential reason is differences in how men and women interpret questions on the KCCQ. The purpose of this study is to evaluate gender-based differences in interpreting the KCCQ and to explore if there are aspects of HRQOL that are not captured by the KCCQ and are important to assess in men and/or women with HF. Methods: Patients of age $\geq 22$ years with cliniciandiagnosed $\mathrm{HF}$ and left ventricular ejection fraction $\leq 40 \%$ were recruited from two academic medical centers to participate in a semistructured concept elicitation and cognitive interview. Enrollment was stratified by patient-identified gender (half women/half men), variation in HF severity (as measured by New York Heart Association Functional Classification status: I-II/ III-IV), age ( $\leq 70 />70)$, and race (Black/White/Other). First, patients described their experiences with $\mathrm{HF}$, including symptoms they may have and how HF affects their daily activities. Participants then completed the KCCQ-23 on paper and were asked questions to explore their interpretation of the KCCQ23 items in cognitive interview format. At the end of the interview, patients were asked about any concepts that they thought are missing from the KCCQ-23. All interviews were conducted over the phone and audio recorded. Interviews were transcribed and descriptive qualitative analysis approaches were used to summarize the findings overall and by gender. Results: Twenty-five patients (56\% female) diagnosed with HF participated. The average participant age was 67 years (range: $25-88$ ), and the race distribution was $76 \%$ White, 24\% Black/African American. Average left ventricular ejection fraction was $30 \%$ (range: $15-40$ ). Few participants identified missing concepts on the KCCQ-23. Additional analyses are underway and further results will be presented at the conference. Conclusion: A presumption of PRO measures is that they measure health status consistently across subgroups, and this study provides insight on interpretation of KCCQ items by gender.

(3110) Self-reported emotional functioning of men undergoing active surveillance, radical prostatectomy, or radiotherapy in the Europa Uomo Patient-Reported Outcome Study

Lionne Venderbos, Department of Urology, Erasmus University Medical Center Rotterdam, Rotterdam, The Netherlands; Andre Deschamps, Europa Uomo, Antwerp, Belgium; Ernst-Günther Carl, Europa Uomo, Antwerp, Belgium; John Dowling, Europa Uomo, Antwerp, Belgium; Sebastiaan Remmers, Department of Urology, Erasmus University Medical Center, Rotterdam, The Netherlands; Monique Roobol, Department of Urology, Erasmus University Medical Center, Rotterdam, The Netherlands

Aims: Treatment for localized prostate cancer (PCa) is a preferencesensitive decision in which aspects like quality of life $(\mathrm{QoL})$ should be taken into account. Europa Uomo, representing the voice of European PCa-patients, initiated the patient-driven Europa Uomo Patient-Reported Outcome Study (EUPROMS) to inform their members about QoL post-PCa treatment. Here the PRO data on emotional functioning are presented for men who underwent active surveillance (AS), radical prostatectomy (RP), or radiotherapy (RT). Methods: An invitation to complete a cross-sectional online survey was distributed through Europa Uomo's network to European PCa patients who were undergoing or received treatment in the past. The survey included, amongst others, the EORTC-QLQ-C30 to measure cancer-related QoL [1]. The EORTC-QLQ-C30 emotional functioning scale scores and the items that comprise the scale are presented. Item proportions of the three treatment groups were compared with 
the proportion test. Results: A total of 2943 men completed the EUPROMS survey; median age was 71 years (IQR 65-75) and 82\% was living with a spouse. A total of $179(6.1 \%)$ men underwent AS, $1101(37.4 \%) \mathrm{RP}$, and $304(10.3 \%)$ RT. The median emotional functioning scale score is 91.7 for AS, RP, and RT with some variation in the IQRs. Looking at the single items of the emotional functioning subscale, proportionally there were slightly more men on AS that felt tense 'quite a bit' $(10.1 \%$ vs. $6.5 \%$ and $7.9 \%,(2)=3.4$, $\mathrm{p}=0.2)$, were worried 'quite a bit' $(15.6 \%$ vs. $10.9 \%$ and $9.2 \%$, $(2)=4.9, p=0.087)$, felt irritable 'quite a bit' $(12.3 \%$ vs. $7.5 \%$ and $6.3 \%,(2)=6.1, p=0.047)$, and felt depressed 'quite a bit' $(12.9 \%$ vs. $8.9 \%$ and $7.6 \%,(2)=4.0, p=0.14)$. Conclusion: Data from the EUPROMS study show that in terms of emotional functioning, AS, RP, and RT are well-tolerated treatments. On an individual level it will, however, be interesting to try to identify factors that are associated with positive and negative psychological responses to both diagnosis and treatment of localized $\mathrm{PCa}$, to optimize psychological outcomes also for those men that felt 'quite a bit' more tense, more worried, more irritable or depressed.

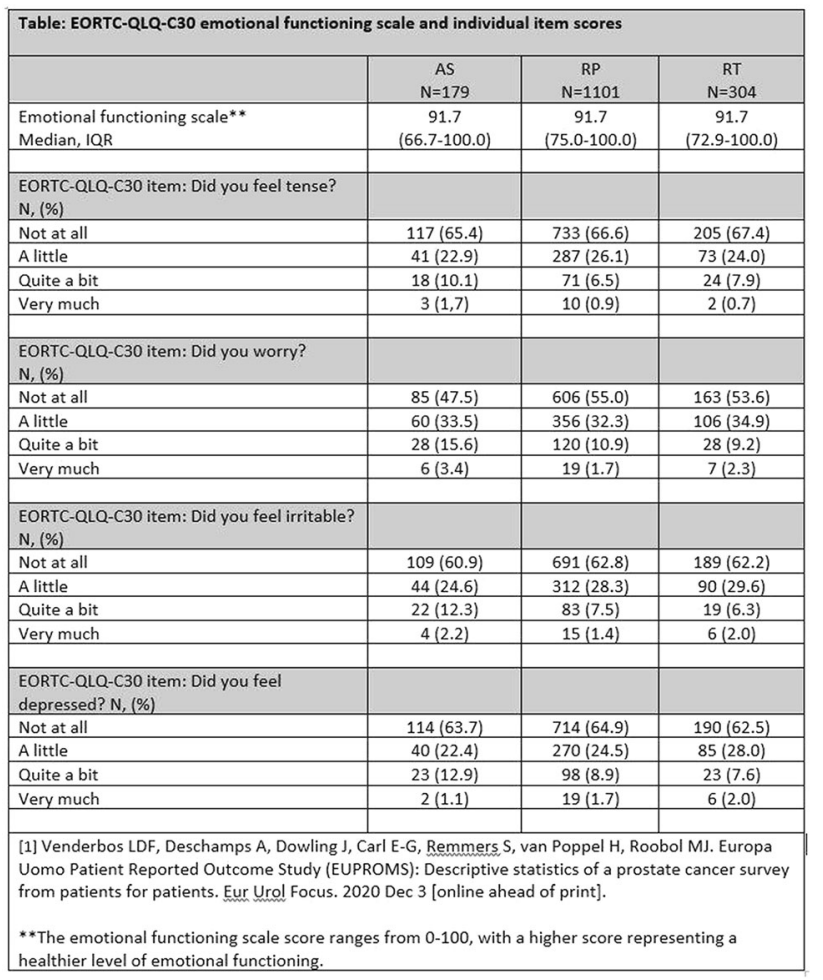

(3111) The importance of physical and psychosocial outcomes of bariatric surgery: a modified Delphi study

Alyssa Budin, Monash University, Melbourne, Australia; Priya Priya, Melbourne University, Melbourne, Australia; Wendy Brown, Monash University, Melbourne, Australia

Aims: Bariatric surgery, or weight loss surgery, is a long-term treatment for morbid obesity with an emerging trend of poor and deteriorating psychosocial health following surgery. The Australian and New Zealand Bariatric Surgery Registry (BSR) is currently working to develop a PROM specific to these outcomes, which is concise enough to be implemented within a registry setting. To facilitate considered selection from a larger list of potential physical, psychosocial, and quality of life outcomes, the current study aims to identify those outcomes that are most important or impactful on a patient's recovery, according to multiple stakeholder groups.
Methods: A modified Delphi methodology was used, including two rounds of a survey, to gauge the opinions of key stakeholders on the importance of a range of physical and psychosocial outcomes of bariatric surgery. Stakeholder groups included pre- and post-surgical patients, their friends and family, and a variety of healthcare practitioners including bariatric surgeons, general practitioners, nurses, dieticians, and psychologists. The second round of the survey facilitated participant insight into the collective views of other stakeholder groups, allowing for consideration and reassessment of outcome importance, as well as a final ranking of outcome domains. Results: The results of the first round of this study were presented at the 2021 ISOQOL-supported conference 'Using PROMs "Down Under". Survey data from the second round will aid in the definition of an outcome set, to be used as the focus of continued development towards a concise, new, bariatric-specific PROM. The differences in ratings between the two rounds, as well as between stakeholder groups, will give insight into the values of each group, and the impact of the opinions and preferences of other stakeholders. Conclusion: This study will culminate in a ranking of bariatric surgery outcomes by importance, as judged by key stakeholders. This will enable the BSR to refine their approach to PROM development by ensuring the items included in the PROM represent those outcomes that are most meaningful to patients and which are the most useful in clinical practice.

\section{(3112) Vision-related Quality of Life among Young Adults operated for Childhood Glaucoma}

Vijaya Gothwal, PhD, Brien Holden Eye Research Centre - PatientReported Outcomes Unit, L V Prasad Eye Institute, Hyderabad, India; Anil Kumar Mandal, MD, Jasti V Ramanamma Children's Eye Care Centre, L V Prasad Eye Institute, Hyderabad, India

Aims: Advances in surgical techniques have vastly improved the surgical success rates of childhood glaucoma (CG). Over the last few decades, several studies have reported the surgical outcomes in young children afflicted with CG. Given the chronic nature of the disease, life-long follow-up is crucial and as these children mature into adults, they are concerned about the long-term impact of CG on their quality of life $(\mathrm{QoL})$. There is lack of literature regarding the long-term visual outcomes from the perspectives of young adults who were operated for CG in their early developmental years. Therefore, the aim of this study was to investigate the vision-related quality (VRQoL) and its predictors among young adults operated for CG. Methods: 121 young adults (mean age, 24.9 years; 54\% male; 90\% bilateral CG) completed the VRQoL instrument, the Impact of Vision Impairment, in their clinic visit. Clinical data including visual acuity (VA) and visual fields were collated from medical records. Rasch analysis was used to optimize the psychometric properties of IVI. Linear regression analysis was used to examine associations between VRQoL, sociodemographic, and clinical variables. Results: Mean follow-up was 20.4 years. Median age at surgery was 12 months and $74 \%$ had bilateral primary congenital glaucoma. The IVI demonstrated optimal functioning when it was split into two scales-vision functioning (VF) and emotional well-being (EWB). The mean (SD) VF score was 2.67 (3.04) logits and EWB score was 2.67 (2.87) logits. In bilateral cases of CG, VA of better than 20/60 in the better-seeing eye was noted in $49 \%$ of patients. Better visual functioning and emotional well-being (VRQoL) were independently associated with better acuity in better-seeing eye. Unilateral CG reported better VRQoL than bilateral CG. Conclusion: Better seeing-eye mostly determines the VRQoL in young adults operated for glaucoma during childhood. Eye care professionals should focus on maximizing the visual potential of patients with CG so as to improve the VRQoL in the long-term. 
(3113) PictoQOL: a pictorial questionnaire for the assessment of health-related quality of life

Patrick Brzoska, MSc, EMPH, DrPH, Witten/Herdecke University, Faculty of Health, School of Medicine, Health Services Research, Witten, Germany; Fabian Erdsiek, Witten/Herdecke University, Faculty of Health, School of Medicine, Health Services Research, Witten, Germany; Tugba Aksakal, Witten/Herdecke University, Faculty of Health, School of Medicine, Health Services Research, Witten, Germany; Maria Mader, Bielefeld University, Bielefeld, Germany; Dennis Padberg, Witten/Herdecke University, Faculty of Health, School of Medicine, Health Services Research, Witten, Germany; Sabahat Ölcer, Dr., Witten/Herdecke University, Faculty of Health, School of Medicine, Health Services Research, Witten, Germany; Munzir Idris, Witten/Herdecke University, Faculty of Health, School of Medicine, Health Services Research, Witten, Germany; Yüce Yilmaz-Aslan, Witten/Herdecke University, Faculty of Health, School of Medicine, Health Services Research, Witten, Germany

Aims: Health-related quality of life (HRQOL) is commonly assessed by means of standardized self- or interviewer-administered questionnaires such as the SF-36 health survey. Such questionnaires usually need to be re-adapted prior to their application to migrant communities. In population-based research, this is usually not possible because of resource constraints. Consequently, some groups of migrants tend to be excluded from studies. If language- or culturespecific questionnaires are used, the comparability between the population groups may be limited because of poor measurement equivalence. Instead of using language-based instruments, one solution to these challenges may be the use of language-independent instruments based on pictograms/pictures. These can be applied to a large variety of population groups with different language skills and require no or only little re-adaptation. The aim of the present study was to develop such a pictorial questionnaire (PictoQOL) for the assessment of health-related quality of life and to examine its content validity across three exemplary population groups of different origin residing in Germany (non-migrants, Turkish migrants, and Syrian migrants). Methods: A participatory design combining qualitative and quantitative methods was used. Based on a scoping review of existing approaches to nonverbal assessment of psychological constructs and 6 focus group discussions with 9 health care users each, picture-based items along the dimensions of the SF-36 and WHOQOL were designed and piloted in cognitive interviews with 38 health care users. Non-migrants, Turkish migrants and Syrian-Arab migrants were interviewed. Results: The PictoQOL consists of a pictorial representation of a total of 20 different situations. Using a graphic Likert scale, respondents indicate how much each situation applies to them. Some representations proved to be culturally sensitive and were adapted. Respondents found the use of an additional graphic layer in the form of symbols in addition to pictures helpful for interpretation. Conclusion: The PictoQOL is considered to allow a more accessible assessment and better comparability of HRQOL across different population groups regardless of their literacy level. It is therefore considered to be superior to existing instruments for routine use in health research and practice. Future studies need to examine its convergent and factorial validity.

\section{(3114) Development of a Prototype Parkinson Health Profile Using Rasch Analysis}

Ayse Kuspinar, PT, PhD, McMaster University, Hamilton, Ontario, Canada; Stanley Hum, PhD, Montreal Neurological Institute, Montreal, Quebec, Canada; Nancy Mayo, PhD, McGill University, Montreal, Quebec, Canada
Aims: The purpose of this proposal is to develop the prototype for a new multi-dimensional disease-specific measure of health-related quality of life (HRQL) for people with Parkinson's Disease: The Prototype Parkinson Health Profile. Methods: The data comes from a patient registry, the McGill Brain Health Outcomes Platform (BHOP), to survey patient health status using a common core set of patientreported outcomes (PRO) and computerized cognitive tasks selected for neurological disorders based on their strong psychometric properties. Important domains were identified from the responses to the Patient Generated Index (PGI), an individualized measure of quality of life. BHOP PRO items were mapped onto each domain. The extent to which the items formed a uni-dimensional, linear construct was determined using Rasch analysis, and the best item was selected using the threshold map. Next polychoric correlation coefficients were calculated, and best-domain items that were highly correlated $(r>0.7)$ with each other were eliminated to increase structural independence. A Visual Analogue Scale of health rating was regressed onto each item, to provide estimates of how the response options were spread across the health construct. Regression coefficient values were expected to decrease between each response level. Results: The sample $(\mathrm{n}=103)$ was $39 \%$ women with an average age of $66 \pm 8$ years. The top 12 domains that patients identified on the PGI were included in the prototype Parkinson Health Profile. Rasch measurement models generally met the criteria for good fit, with nonsignificant Chi square values and high reliability, and mean item and person fit residuals close to zero with standard deviation of one. Items were not highly correlated with each other; correlations ranged from 0.11 to 0.62 . The final prototype Parkinson Health Profile included 12 items with 3 response levels each (dexterity, sleep, memory, concentration, tremors, rigidity, speech, urine control, swallowing, walking, fatigue, and mood). Regression coefficient values decreased between response levels. Conclusion: This study identified candidate items for inclusion in the Parkinson Health Profile. Next steps will involve conducting a series of individual interviews with patients in English and French to refine the items, and provide information on item clarity, response options and recall time.

\section{(3115) Professional satisfaction and burnout in a university hospital staff}

Pedro Ferreira, PhD, CEISUC/FEUC, Coimbra, Portugal; Vitor Raposo, PhD, CEISUC/FEUC, Coimbra, Portugal

Aims: Professional satisfaction has repercussions on the health and quality of life of workers, as well as on the productivity of organizations. The main objective of this study was to collect enough information to monitor the evaluation that hospital professionals make of the workplace and the care provided there. Methods: Of the 7,981 professionals, valid satisfaction data of 809 were obtained, corresponding to an overall response rate of $10.1 \%$. For data collection, the Professional Satisfaction Assessment Instrument (IASP) designed by CEISUC, the Copenhagen Burnout Inventory (CBI) and Maslach Burnout Inventory (MBI) questionnaires, and EQ-5D-5L were used. All questionnaires were completed online, respecting ethical considerations. Results: About $72.4 \%$ of the respondents were female, $43.0 \%$ nurses, $15.2 \%$ physicians, $15.1 \%$ administrative, $16.7 \%$ high technicians, and $13.3 \%$ clinical secretaries. The mean age was 45.7 ( \pm 10.1 ) years, ranging from 25 to 69 years. Regarding professional satisfaction, the average level found was 56.9, on a scale from 0 to 100 . The mean values of 54.0 were also found for the assessment of the quality of the unit as a workplace, with technological and financial resources being the subdimension evaluated in a relatively more negative way (42.9), especially maturity (25.6). The morale of the professionals was evaluated at 63.3. On the other hand, the quality of the unit as a service delivery organization was generally 
evaluated with a score of 62.7, and the approach to continuous quality improvement assessed with a score of 62.9.According to CBI, more than $36 \%$ and $8 \%$ of professionals presented, respectively, a moderate and high burnout in relation to personal aspects. On the other hand, according to the MBI, emotional exhaustion is present in more than half of the professionals (21.4\% with medium burnout and $31.0 \%$ with high burnout). Depersonalization occurred only in $36.2 \%$ of professionals $(23.4 \%$ with medium burnout and $12.8 \%$ with high burnout. Finally, just over $26.1 \%$ of the professionals presented problems of personal accomplishment at work and more than a third with high burnout. Conclusion: This study showed an average satisfaction rate among professionals. These showed worrying indicators of burnout.

\section{(3116) Is the treatment satisfaction questionnaire for medication} fit-for-purpose? A mixed-methods approach

Ana Maria Rodriguez-Leboeuf, PhD MSc PT, IQVIA, PatientCentered Endpoints; McGill University, Montreal, Quebec, Canada; Paul Williams, IQVIA, Paris, France; Alexandra Palmer, IQVIA, Boston, Massachusetts, USA

Aims: The Treatment Satisfaction Questionnaire for Medication (TSQM) is the most commonly used generic Patient-Reported Outcome (PRO) measuring treatment satisfaction. Its psychometric properties have been assessed in numerous intervention trials using Classical Test Theory (CTT). Given the heterogeneous treatment experience of people taking different medicines in routine health care, the psychometric properties of the TSQM may be challenged in noninterventional or patient preference research. The objectives were to determine the extent to which the psychometric properties of the TSQM are supported across various conditions in a real-world context Methods: Electronic versions of both the TSQM v1.4 and vII were administered to subjects of an online patient community managed by IQVIA (MediGuard) in the US, in the UK, in Spain, in France, and in Australia. CTT and Rasch measurement theory were used to determine, compare, and contrast the cross-sectional psychometric properties of the TSQM. Results: The survey was completed by 108 and 354 patients in the UK and in the US, respectively. Most patients who responded had university-level education $(67 \%)$, were female $(68 \%)$ and had a mean of $50( \pm 18.4)$ years of age. CTT analyses confirmed that the TSQM had excellent internal consistency and construct validity. High floor effects were seen for several items of the TSQM in some disease-level subgroups of patients (e.g. hypothyroidism). Rasch measurement theory (RMT) analyses showed that items were consistently ordered on an interval scale from low to high levels of treatment satisfaction. The Differential Item Functioning and Person-Item Threshold distribution suggests a potential alternate scoring structure that may be more generalizable across therapeutic areas in routine health care. Conclusion: The TSQM demonstrates generally high psychometric evidence for its use in a heterogeneous patient population in routine health care. However, the TSQM scoring could be revisited to improve its fit-for-purpose applicability.

\section{(3118) Development of an online training platform to facilitate the implementation of goal attainment scaling in clinical trials}

Kari Knox, Bachelor of Science in Nursing, Registered Nurse, Ardea Outcomes, Halifax, Nova Scotia, Canada; Susan Howlett, Ardea Outcomes, Halifax, Nova Scotia, Canada; Sanja Stanojevic, Ardea Outcomes, Halifax, Nova Scotia, Canada; Taylor Dunn, MSc, Ardea Outcomes, Halifax, Nova Scotia, Canada; Justin Stanley, Ardea Outcomes, Halifax, Nova Scotia, Canada; Chere Chapman, Ardea
Outcomes, Halifax, Nova Scotia, Canada; Kenneth Rockwood, Ardea Outcomes, Halifax, Nova Scotia, Canada

Aims: Goal attainment scaling (GAS) is a patient-centric outcome measure that can be used to measure individualized patient goals and the extent to which those goals are achieved following an intervention. Identifying Meaningful and Specific, Measurable, Achievable, Relevant/Realistic, and Time-bound (SMART) goals can be difficult and time consuming for naïve GAS interviewers. Training has long been an essential aspect to facilitate the implementation of GAS. Many established training programs require up to $8 \mathrm{~h}$ or sessions across multiple days, which is costly and constitutes a substantial time commitment. With the rapid switch to remote training due to COVID19 , our objective was to develop a comprehensive online training program to facilitate GAS use. Methods: We used a commercially available online platform, "Thinkific", to host and develop a comprehensive GAS training program. This platform allows for various adult learning methods to be employed, including video and written content, and also them to be utilized in an accessible format. The training content was developed to give the user a broad understanding of GAS and its implementation. Modules focused specifically on how to develop SMART, meaningful goals, which is a core competency for implementing GAS. Results: We developed a 1.5-h online training course for interviewers who wish to implement GAS as an endpoint in clinical trials. The course consists of five modules, each taking between 20 and $40 \mathrm{~min}$ to complete. The course content offers stepby-step instructions for conducting GAS visits as well as guidance and recommendations for best GAS practices. Skills and knowledge were evaluated with a quiz at the end of some modules. Various scenarios were provided to mimic real-life interactions. Conclusion: Training is a key component to ensuring patient-centric outcome measures, such as goal attainment scaling, are feasible in clinical trial settings. However, with the time and budgetary constraints that come with clinical trials, training needs to be time sensitive while still being effective. Here, we have developed a comprehensive online training that will facilitate GAS, allowing it to be used more readily in clinical studies, especially within the COVID-19 era.

(3119) Cognitive impairment is associated with greater preoperative disease activity and markedly reduced recovery following cervical and lumbar spine surgery

Richard L. Skolasky, Jr., ScD, Johns Hopkins University, Baltimore, Maryland, USA; Rachel Bronheim, MD, Johns Hopkins University, Baltimore, Maryland, USA; Emma Cotter, Johns Hopkins University, Baltimore, Maryland, USA; David Cohen, MD, Johns Hopkins University, Baltimore, Maryland, USA; Amit Jain, MD, Johns Hopkins University, Baltimore, Maryland, USA; Khaled Kebaish, MD, Johns Hopkins University, Baltimore, Maryland, USA; Sang Hun Lee, MD, Johns Hopkins University, Baltimore, Maryland, USA; Brian Neuman, MD, Johns Hopkins University, Baltimore, Maryland, USA; Lee Riley, MD, Johns Hopkins University, Baltimore, Maryland, USA

Aims: Previous studies have associated cognitive impairment with prolonged hospital stay, increased complication, and death; however, their role in disease activity and recovery following surgery are not well understood. We aimed to determine: prevalence of pre-operative cognitive impairment (CI) among spine surgery patients; association of CI on symptom burden and health-related quality of life (HRQOL); and effect of $\mathrm{CI}$ on achieving meaningful improvements in HRQOL. Methods: Prospective cohort study of 484 adult spine surgery patients at academic medical center (mean age 56, SD 19 years; $50 \%$ female; 95\% non-Hispanic; $88 \%$ white).Participants completed pre-operative assessment (age, sex, race/ethnicity, education, household income, relationship status, comorbidity, and 30-day opioid use). Before and 
after surgery, assessments were Pain Numeric Rating Scale (NRS) for back/leg and neck/arm, Oswestry Disability Index (ODI) or Neck Disability Index (NDI), and PROMIS-29 profile (Cognitive Abilities).Using Cognitive Abilities, we grouped participants as mild $(<40)$ and moderate $(<30)$ CI. We compared prevalence of CI to population rate. We compared NRS, O/NDI, and PROMIS-29 between groups. Likelihood of achieving MCID given presence of either mild or moderate CI was estimated using logistic regression. Results: Mild CI was reported by $94 / 484$ and moderate by $21 / 484$ ( $p>0.05$ ).Participants with mild $C I$ had greater back pain $(\mathrm{p}=0.003)$ and pain-related disability $(\mathrm{O} / \mathrm{NDI}, \mathrm{p}<0.001)$; worse HRQOL (Pain Interference(PI), Physical Function(PF), Fatigue(FA), Anxiety(AX), Depression(DP), Sleep Disturbance(SD), and Satisfaction with Ability to Perform Social Roles(SR), $p<0.001)$. Similar results for moderate $\mathrm{CI}$ (Table 1).At 6-months, mild CI was associated with reduction in likelihood of improvement in leg pain (OR 0.19), ODI (OR 0.35), and HRQOL (PI: OR 0.27; PF: OR 0.44; AX: OR 0.18; and SR: OR 0.35) (Table 2). Similar findings for moderate $\mathrm{CI}$ and for both at 12 months. Conclusion: One in five and one in 25 patients reported mild and moderate CI, respectively. Adjusting for the influence of age, comorbid condition, and opioid use, CI was associated with worse pain, pain-related disability, and HRQOL before surgery. Those with mild CI had roughly one-quarter to onethird likelihood of meaningful improvement after surgery. These results highlight the importance of addressing surgery patients' cognitive difficulties.

Table 1. Effects of cognitive impairment on pain, pain-related disability, and HROOL of 484 individuals presenting for pre-operative assessment of lumbar and cervical spine conditions.

\begin{tabular}{|c|c|c|c|c|c|c|}
\hline \multirow[t]{2}{*}{ PRO } & \multicolumn{2}{|c|}{ Mild CI } & \multirow{2}{*}{$\begin{array}{l}\text { p- } \\
\text { value }^{3}\end{array}$} & \multicolumn{2}{|c|}{ Moderate CI } & \multirow{2}{*}{\begin{tabular}{|l|} 
p- \\
value
\end{tabular}} \\
\hline & No & Yes & & No & Yes & \\
\hline \multicolumn{7}{|l|}{ Pain NRS } \\
\hline back $^{1}$ & $7.3(2.7)$ & $8.3(2.0)$ & .008 & $7.4(2.7)$ & $8.9(1.1)$ & .035 \\
\hline $\operatorname{leg}^{1}$ & $5.9(3.3)$ & $6.5(3.4)$ & .221 & $6.0(3.4)$ & $6.6(3.6)$ & .505 \\
\hline neck $^{2}$ & $6.2(3.0)$ & $7.1(3.0)$ & .092 & $6.3(3.0)$ & $8.9(1.6)$ & .017 \\
\hline $\mathrm{arm}^{2}$ & $5.0(3.5)$ & $5.5(3.0)$ & .415 & $5.1(3.4)$ & $5.3(2.8)$ & .934 \\
\hline \multicolumn{7}{|l|}{ Disability } \\
\hline $\mathrm{ODI}^{1}$ & $42.3(17.3)$ & $55.9(12.8)$ & $<.001$ & $43.0(17.3)$ & $60.0(11.5)$ & $<.001$ \\
\hline $\mathrm{NDI}^{2}$ & $36.5(17.4)$ & $49.0(14.0)$ & $<.001$ & $38.7(17.0)$ & $61.4(9.7)$ & $<.001$ \\
\hline \multicolumn{7}{|l|}{ PROMIS } \\
\hline Pain interference & $64.0(7.4)$ & $68.5(5.5)$ & $<.001$ & $64.6(7.3)$ & $71.4(4.5)$ & $<.001$ \\
\hline Physical function & $36.6(6.8)$ & $33.8(5.7)$ & $<.001$ & $36.3(6.8)$ & $31.6(4.6)$ & .002 \\
\hline Fatigue & $52.7(9.2)$ & $61.2(7.7)$ & $<.001$ & $53.9(9.3)$ & $64.0(9.3)$ & $<.001$ \\
\hline Anxiety & $51.2(9.2)$ & $57.7(11.7)$ & $<.001$ & $52.1(9.6)$ & $60.4(16.2)$ & $<.001$ \\
\hline Depression & $49.2(8.6)$ & $56.7(8.5)$ & $<.001$ & $50.2(8.9)$ & $60.4(7.1)$ & $<.001$ \\
\hline Sleep disturbance & $54.6(8.3)$ & $60.6(8.5)$ & $<.001$ & $55.5(8.5)$ & $62.4(9.9)$ & $<.001$ \\
\hline $\begin{array}{l}\text { Satisfaction with } \\
\text { Ability to Perform } \\
\text { Social Roles }\end{array}$ & $43.0(8.3)$ & $37.7(6.5)$ & $<.001$ & $42.3(8.2)$ & $33.4(5.0)$ & $<.001$ \\
\hline
\end{tabular}

(3120) The impact of symptom monitoring with patient-reported outcomes during treatment with immunotherapy: an investigation of the Quality of Life among melanoma patients

Lærke K. Tolstrup, RN, PhD, postdoc, Odense University Hospital, Odense, Denmark; Helle Pappot, MD, PhD, professor Onkologisk klinik, Rigshospitalet, Copenhagen, Denmark; Lars Bastholt, MD, senior consultant, Department of Oncology, Odense University Hospital, Odense, Denmark; Karin B. Dieperink, RN, PhD,

Department of Oncology, Odense University Hospital, Odense, Denmark

Aims: In a randomized controlled trial, we investigated if melanoma patients receiving immunotherapy had the number of severe immunerelated adverse events (irAEs) reduced by self-reporting their symptoms as an add-on to routine monitoring compared to standard care. This was not the case. However, as an explorative endpoint, we examined if there was a difference in the Quality of life (QoL) between the two groups, and if the patients who experienced severe irAEs had a poorer QoL compared to patients who had moderate, mild or no symptoms at all. Methods: The study population consisted of 138 patients at baseline. The patients completed the EuroQol EQ-5D Index and the FACT-M questionnaires at baseline, at week 24 and 48. We analyzed QoL from all the patients who had completed at least one questionnaire. For the FACT-M questionnaire, data was imputed in accordance with existing guidelines. Results: The control group had a significantly lower EQ-5D score (p-value 0.03 ) at week 48 compared to baseline, while patients in the intervention managed to maintain their QoL (p-value 0.81). Comparing the two groups, patients in the intervention group had a higher EQ-5D score at week 48 (p-value 0.05). With regard to the impact of grade 3 and 4 irAEs on QoL, there is a tendency that patients with serious irAEs had a lower QoL score compared to patients who had none (p-value 0.15 ) at week 48. In FACT-M, there was no difference between the two groups at baseline. At week 48, however, there was a tendency that patients in the intervention group had a higher score than patients in the control group (p-value 0.12 ). There was a tendency that patients who had no serious irAEs had a better QoL score than patients who did not have any (p-value 0.18 ) at week 48. Conclusion: The results from the QoL data for the melanoma patients in this study suggest that patients who self-reported their irAEs as a supplement to standard monitoring maintained their QoL life compared to patients who had received routine monitoring. Concerning the EQ-5D, patients in the intervention had a significantly better QoL than controls at week 48 .

(3122) A qualitative study to inform the development of a new PROM for nail conditions: the NAIL-Q

Trisia Breitkopf, MSc, McMaster University, Hamilton, ON, Ontario, Canada; Leah Algu, McMaster University, Hamilton, Ontario, Canada; Claire Stewart, Weill Cornell Medicine, New York, New York, USA; Charlene Rae, McMaster University, Hamilton, Ontario, Canada; Shari Lipner, Weill Cornell Medicine, New York, New York, USA; Maureen O'Malley, Ancaster Dermatology Centre, Ancaster, Ontario, Canada; Anne Klassen, McMaster University, Hamilton, Ontario, Canada

Aims: Nail conditions can negatively affect nail appearance and function as well as a patient's health-related quality of life. In a systematic review, our team identified 7 nail-specific patient-reported outcome measures (PROMs), but the quality of those PROMs was low based on COSMIN criteria, and covered only 2 nail conditions (onychomycosis, nail psoriasis) [1]. Often, generic measures were used in research studies. The aim of our study was to develop a scientifically sound nail-specific PROM that can be used for any type of nail condition. Methods: Development of NAIL-Q will involve a mixed methods multiphased study. In phase 1 (the focus of this presentation), our team used findings from the systematic review to develop an interview guide. We then conducted qualitative interviews with patients with nail conditions of the fingernails and/or toenails. Patients were recruited from dermatology clinics in Hamilton, Canada, and New York, USA. Interviews were conducted between Oct 2019 and Nov 2020 and were audio recorded, transcribed, and coded using a line-by-line approach. Results: We interviewed 23 patients (5 in Canada, 18 in USA) aged 21-87 years with a range of nail conditions (e.g., onychomycosis, brittle nails, lichen planus, etc.). Major concepts elicited from the interview data included nail appearance (e.g., color contour), nail care (e.g., time, cut), nail symptoms (e.g., pain, loosening), physical function (e.g., use of hands, accommodation), and psychosocial function (e.g., body image, appearance distress). Together these themes form a conceptual framework that will be used to inform the development of a new PROM. Conclusion: Nail conditions have an important impact on patients. In the next phase, our team will use the qualitative data to develop a PROM covering concepts elicited in the interviews. The 
PROM will then be field tested and analyzed using Rasch Measurement Theory. Kamran R, Algu L, Leveille CF, Stewart CR, Abid K, Lipner SR, Klassen AF, Rae C. Patient-reported outcome measures for patients with nail conditions: a systematic review of the psychometric evidence. Archives of Dermatological Research. 2021 Apr $5: 1-5$.

\section{(3123) Determining construct validity of scales from the FACE-Q craniofacial module for patients with craniosynostosis}

Rakhshan Kamran, BSc (Hons), MD (c), McMaster University, Ancaster, Ontario, Canada; Charlene Rae, MSc, Department of Pediatrics, McMaster University, Hamilton, Ontario, Canada; Karen W. Y. Wong Riff, MD, MSc, PhD, Division of Plastic and Reconstructive Surgery, The Hospital for Sick Children, Department of Surgery, University of Toronto, Toronto, Ontario, Canada; Andrea Pusic, MD, MHS, FACS, FRCSC, Division of Plastic and Reconstructive Surgery, Brigham and Women's Hospital, Department of Surgery, Harvard Medical School, Boston, Massachusetts, USA; Anne Klassen, BA (Hons), DPhil, Department of Pediatrics, McMaster University, Hamilton, Ontario, Canada

Aims: Craniosynostosis is a condition resulting from premature fusion of suture lines of the skull, resulting in a misshaped head. Craniosynostosis can impact appearance of the head, as well as both brain, and skull development. The FACE-Q Craniofacial Module is a new PROM designed for patients with craniofacial conditions, including Craniosynostosis. The FACE-Q includes an appearance scale (Head Shape) that can be used to evaluate surgical outcomes. In addition, there are health-related quality of life (HRQOL) scales that measure Psychological, Social, School, and Appearance Distress. Scores on each of these scales range from 0 (Worst) to 100 (Best).The aim of this study is to assess construct validity of the use of Head Shape and HRQOL scales in an international sample of patients with Craniosynostosis. Methods: Participants aged 8 to 29 years with a diagnosis of Craniosynostosis were recruited as part of the international FACE-Q Craniofacial module field-test. Construct validity was assessed by testing hypotheses of known group differences. Differences between groups were assessed using either the independent T-test or one way ANOVA. Pearson correlation co-efficient was used to examine relationships between scales, with strong correlations considered to be a co-efficient $>0.50$. Results: A total of 248 participants (mean age $=13$ ) were included. The majority were male $(58 \%)$, and from Canada (37\%). For the Head Shape scale, gender was not associated with scale score; however, lower scale scores were associated with older age $(\mathrm{p}<0.001)$, and with having a visibly different head shape $(\mathrm{p}=0.008)$. As hypothesized, scale scores were incrementally higher the more (not at all, a little, quite a bit, very much) patients reported that they liked their overall head shape $(p=0.008)$. Scores on the Head Shape scale were strongly correlated with HRQOL, i.e., Appearance Distress $(r=0.61)$, Psychological $(\mathrm{r}=0.58)$, and Social $(\mathrm{r}=0.55)$, and moderately with the School scale $(r=0.40)(p<0.001)$. Conclusion: This study provides further evidence of construct validity for the scales used to measure outcomes important to patients with Craniosynostosis. This is important because correction of head shape deformity is an aim of treatment for Craniosynostosis, which could be evaluated from the patient perspective using scales from the FACE-Q Craniofacial Module.

\section{(3124) Effect of 12: weeks aerobic exercise on quality of life of HIV infected persons}

Stanley Maduagwu, BSc PT, MSc Ortho and sports PT, PhD Exercise Physiology, Department of Medical Rehabilitation, Faculty of Health
Science and Technology, College of Health Sciences, Nnamdi Azikiwe University Awka, Nigeria, Nnewi, Nigeria; Ifeoma Blessing Nwosu, BMR (PT), MSc in orthopedic and sports Physiotherapy, Department of Medical Rehabilitation, Faculty of Health Science and Technology, College of Health Sciences, Nnamdi Azikiwe University Awka, Nigeria, Enugu, Nigeria; Michael Ogunlana, BSc.PT, MSc.Epid, PhD.PT, Postdoctoral Fellow, College of Health Sciences, University of KwaZulu-Natal Private Bag X54001, Durban, South Africa, Durban, South Africa

Aims: To determine the effect of 12 weeks moderate intensity aerobic exercise on quality of life persons infected with HIV: RandomizedControlled Trial. Methods: Using convenient sampling technique, 91 volunteer and willing HIV-infected female and male adults were enrolled to participate in 12-weeks moderate intensity aerobic exercise. By application of inclusion criteria, 82 participants eligibly qualified for the study and were randomly assigned to exercise and non-exercise groups. Research ANd Development (RAND) 36-item short form (SF-36) Health Survey Questionnaire was employed to assess the quality of life of the participants at baseline. The participants in the exercise group were subjected to 12-weeks moderate intensity aerobic exercise, three days per week and for $40 \mathrm{~min}$ duration for each day. The non-exercise group did not participate in any exercise, but rather took part in weekly lectures. At the end of the 12 weeks of the study, 32 participants were left in each group, giving dropout rate of $22 \%$. The quality of life of the 64 participants were reassessed using the same questionnaire. Descriptive statistic described the socio-demographic characteristics of the participants. Paired Student t-test and Student t-test for two independent samples analyzed the mean values of the scores of the questionnaire within and between groups to determine if any significant difference existed. Results: Insignificant difference ( $p>0.05$ ) existed in the mean ages of exercise $(40.84 \pm 10.05)$ and non-exercise $(39.38 \pm 10.03)$ groups. Paired Student t-test showed significant increment $(\mathrm{p}<0.05)$ in the mean scores between pre- and post-tests in the exercise group. In the non-exercise group, insignificant change $(\mathrm{p}>0.05)$ was found in the four domains of quality of life of the participants, and significant reduction $(\mathrm{p}<0.05)$ in the mean scores of the other four domains of the questionnaire between pre- and post-tests. For the mean scores between the exercise and non-exercise groups, Student t-test for two independent samples indicated significant difference $(\mathrm{p}<0.05)$. Conclusion: Engaging in moderate intensity aerobic exercise improves quality of life in persons infected with HIV.

(3125) Patient-reported anxiety and depression measures for use in Indian head and neck cancer populations: a psychometric evaluation

Chindhu Shunmuga Sundaram, The University of Sydney, Sydney, Australia; Haryana Dhillon, PhD, The University of Sydney, Sydney, Australia; Phyllis Butow, PhD, The University of Sydney, Sydney, Australia; Puma Sundaresan, PhD, The University of Sydney, Sydney, Australia; Mahati Chittem, IIT Hyderabad, Hyderabad, India; Niveditha Akula, IIT Hyderabad, Hyderabad, India; Surendran Veeraiah, Cancer Institute, WIA, Chennai, India; Claudia Rutherford, $\mathrm{PhD}$, The University of Sydney, Sydney, Australia

Aims: Head and neck cancers (HNC) are some of the most challenging cancers because they affect essential aspects of life such as speech, swallowing, and eating. Diagnosis and treatment of HNCs can be associated with considerable anxiety and depression. HNCs are common in India, with over 100,000 cases registered each year. However, suitable measures to assess anxiety and depression, in Indian languages, are lacking. We evaluated the psychometric properties of translated versions of Zung's self-rating Anxiety Scale (SAS) and the Patient health questionnaire-9 (PHQ-9) in Tamil, Telugu, and 
Hindi speaking Indian HNC populations. Methods: HNC patients were recruited from three tertiary cancer centres in India. Participants completed the translated versions of SAS and PHQ-9. We assessed targeting, scaling assumptions, construct validity (with exploratory and confirmatory factor analyses), convergent validity, and internal consistency reliability. Results: The study sample included 205 Tamil, 216 Telugu, and 200 Hindi speaking HNC patients. Exploratory and confirmatory factor analyses indicated a two-factor solution for PHQ-9 and a four-factor solution for SAS in all three languages. Cronbach's alpha coefficients ranged between 0.717 and 0.890 for PHQ-9 and between 0.803 and 0.868 for SAS, indicating good reliability. Correlations between hypothesized scales were as expected, providing evidence towards convergent validity. Conclusion: This comprehensive evaluation of the measurement properties of Tamil, Telugu, and Hindi versions of the SAS and PHQ-9 in large, Indian HNC populations supports their use as clinical assessment tools and research outcome measures across the disease and treatment continuum.

\section{(3126) Cognitive debriefing of the Adelphi Adherence Questionnaire (ADAQ $\odot$ ): a novel generic patient-reported measure of medication adherence drivers and behaviours}

Sarah Bentley, MSc, Adelphi Values, Bollington, UK; Lucy Morgan, MPhil, BA, Adelphi Values, Cheshire, UK; Elizabeth Exall, MSc, Adelphi Values, Cheshire, UK; Anna Roberts, PhD, Adelphi Values, Cheshire, UK; Rebecca Rossom, MD, MS, HealthPartners Institute, Minnesota, Minnesota, USA; Nicolas Roche, MD, PhD, APHP Centre University of Paris, Paris, France; Kamlesh Khunti, MD, PhD, FMedSCI University of Leicester, Leicester, UK; Victoria Higgins, BA (Hons), Adelphi Real World, Cheshire, UK; Rob Arbuckle, MSc, Adelphi Values, Cheshire, UK; James Piercy, MSc, Adelphi Real World, Cheshire, UK

Aims: There is no single gold standard method for measuring medication adherence. The Adelphi Adherence Questionnaire (ADAQ $\odot$ ) is a generic patient-reported outcome (PRO) assessment of medication adherence developed in line with best practice guidance for PRO development. The aim was to conduct cognitive debriefing (CD) interviews with patients taking prescription medication across a range of therapy areas and treatment modalities to assess content validity of the ADAQ $\odot$ Methods: The ADAQ $\subset$ was developed following a focussed literature/instrument review, concept elicitation interviews with individuals who were being prescribed medications, and involvement of three expert clinicians. Telephone CD interviews were conducted with 57 adults from the US $(n=21)$, Spain $(n=18)$, and Germany $(n=18)$ who were being prescribed medication for: hypertension $(\mathrm{n}=9)$, asthma $(\mathrm{n}=8)$, multiple myeloma $(\mathrm{n}=8)$, psoriasis $(n=8)$, diabetes $(n=7)$, depression $(n=7)$, multiple sclerosis $(n=7)$, and/or schizophrenia $(n=6)$. Recruitment quotas ensured a diverse sample of participants. Interviews were conducted in two rounds $(\mathrm{R} 1=28 ; \mathrm{R} 2=29)$ to explore the relevance and understanding of the item wording, instructions, recall period, and response options. Modifications to the ADAQ $\odot$ based on R1 interviews were further tested in R2. Verbatim transcripts were analysed in Atlas.Ti using thematic analysis. Results: The ADAQ $\odot$ includes items to assess adherence drivers and behaviours for multiple therapy modalities. The instructions and items were well understood and relevant to participants. Modifications following R1 included revising the instructions to refer to current medication(s) for one condition to reduce cognitive burden, removing items with lower relevance (i.e., running out of medication, social discouragement), adding examples to an item to improve understanding, and adding a response option for participants to indicate if they had stopped taking medication. Minor wording modifications were made following $\mathrm{R} 2$ to improve comprehension. Subgroup differences in item relevance were observed based on clinical characteristics (e.g. diagnosis, participant perception of severity of condition, mode of administration). Cost of medication was more relevant amongst US participants. Conclusion: This study supports the content validity of the ADAQ(C) in a demographically and clinically diverse sample with a range of conditions with varying severities, modes of medication administration, nationalities, ages, races and education levels. Future research will include psychometric validation of the ADAQ $\odot$.

\section{(3127) European normative data for interpreting the BODY-Q}

Farima Dalaei, Department of Plastic Surgery, Odense University Hospital and University of Southern Denmark, Odense, Denmark; Claire de Vries, MD, PhD, Department of Plastic Surgery, Brigham Women's Hospital, Boston, MA, USA, Boston, Massachusetts, USA; Lotte Poulsen, MD, PhD, Department of Plastic Surgery, Odense University Hospital and University of Southern Denmark, Odense, Denmark; Amalie Lind Jacobsen, B.Sc., Department of Plastic Surgery, Odense University Hospital and University of Southern Denmark, Odense, Denmark; Anne Klassen, BA, DPhil, professor Department of Pediatrics, McMaster University, Hamilton, Ontario, Canada; Jens Ahm Sørensen, MD, PhD, professor Department of Plastic Surgery, Odense University Hospital and University of Southern Denmark, Odense, Denmark; Andrea L. Pusic, MD, PhD, professor Department of Plastic Surgery, Brigham Women's Hospital, Boston, Massachusetts, USA

Aims: The BODY-Q is a comprehensive patient-reported outcome measure used to evaluate outcomes in patients undergoing bariatricand body contouring surgery. It consists of 27 independently functioning scales, measuring appearance, and health-related quality of life (HR-QL). Normative values for the BODY-Q have not been established therefore limiting current data interpretation. The aim of this study was to generate and evaluate normative scores of the general population for the BODY-Q in European countries where the BODY-Q has been translated. Methods: Participants were recruited through the survey-platform Prolific and Amazon Mechanical Turk (MTurk). Participants were invited to complete the BODY-Q through a REDCap link provided within Prolific and MTurk. Following countries were included: Denmark, The Netherlands, Sweden, England, Finland, Belgium, France, Poland, Italy, and Germany. Inclusion criteria were men and women aged 18 years or older. The analysis included descriptive statistics of patient demographics (age, gender, body mass index (BMI) race, educational level, marital status, employment status, and comorbidities) with a multivariable linear regression analysis. The Rasch converted mean scores of all scales for each country were compared with a one-way ANOVA multiple comparison with Post hoc Bonferroni test. Results: The BODY-Q was completed by 2,001 participants from the included countries; Denmark $(n=159)$, The Netherlands $(n=208)$, Sweden $(n=209)$, England $(\mathrm{n}=206)$, Finland $(\mathrm{n}=206)$, Belgium $(\mathrm{n}=179)$, France $(n=213)$, Poland $(n=211)$, Italy $(n=204)$ and Germany $(n=206)$. The mean age of participants ranged between $24 \pm 7$ to $36 \pm 14$ years. Mean BMI varied between $23.3 \pm 4.77$ and $26.0 \pm 5.85$. The findings of the European normative BODY-Q data will be presented for the first time at ISOQOL in October 2021. Conclusion: The BODY-Q normative scores show, how the BODY-Q scores of the general population differ between countries and cultures and, hence, the need for normative data. These data contribute with comparative values for interpreting earlier and future pre- and postoperative BODY-Q data in bariatric- and body contouring surgery in a clinical context and research perspective. 
(3128) The relationship between dry mouth and morphine equivalent dose in patients with lung cancer surgery

Lijun Zhang, School of Public Health and Management, Chongqing Medical University, Chongqing, China; Wei Xu, School of Public Health and Management, Chongqing Medical University, Chongqing, China; Yanyan Huang, School of Public Health and Management, Chongqing Medical University, Chongqing, China; Qiuling Shi, School of Public Health and Management, Chongqing Medical University, Chongqing, China

Aims: Dry mouth is a highly prevalent and significant symptom experienced by patients with lung cancer surgery and influences quality of life. Pain control medication is the most common cause of dry mouth. We conducted a study to examine the relationship between analgesic prescription and dry mouth, aiming to identify risk factors of dry mouth among postoperative lung cancer patients. Methods: Data collected in a cohort study in the department of thoracic surgery of a tertiary cancer center. MDASI-LC(The MD Anderson Symptom Inventory for Lung Cancer) was used to collect dry mouth symptoms daily during hospitalization. Latent class mixed mold was used to identify patient clusters with distinct dry mouth trajectories via the R package LCMM. Wilcoxon analysis was used to study the interaction between dry mouth and morphine equivalent doses(MED). Results: Of 424 postoperative patients with lung cancer enrolled, 404 had record of dry mouth in MDASI-LC. There were three patient clusters according Latent class mixed model. At least, $17.08 \%(69 / 404)$ patients are classified into high symptom score cluster, 48.78\%(189/404)patients are classified into low symptom score cluster. On univariate analysis higher MED was found in the higher dry mouth cluster, compared to low dry mouth cluster $(P=0.019)$. and BMI was higher in high dry mouth cluster than that in low symptom score cluster(22.7 vs 23.4; $\mathrm{p}=0.03$ ). Conclusion: We identified two trajectories of dry mouth symptoms over the course of recovery after lung surgery. The morphine equivalent dose was found to be related to higher level of dry mouth. Management strategies for dry mouth should be considered when opioids applied for the peroperative patients.

\section{Class-specific mean predicted trajectory}

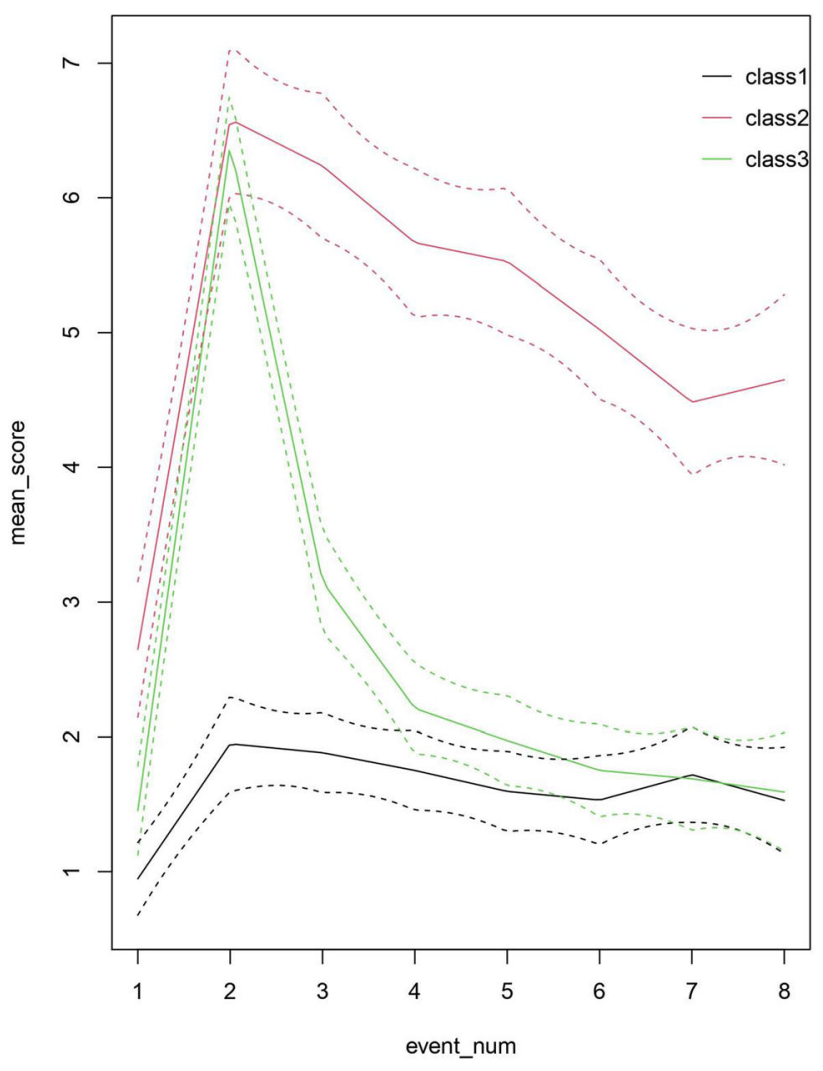

(3129) Silver lining of a pandemic: accelerated electronic integration of PROMs within chronic disease management

Karen Ruggles, Alberta Health Services, Lethbridge, Alberta, Canada; Andrew Frank-Wilson, PhD, Alberta Health Services, Lethbridge, Alberta, Canada; Kerri Colquhoun, Alberta Health Services, Lethbridge, Alberta, Canada

Aims: The integration of patient-reported outcomes has long been an aspiration of Chronic Disease Management (CDM) within Alberta Health Services (AHS). It is foundational to our growth as a learning health system and informs our collective understanding of success. The pandemic presented a transformational opportunity for innovation forcing adoption of virtual platforms by both clinicians and patients and enabled the use of Research Electronic Data Capture (REDCap) for the collection of EQ-5D-5L Health-Related Quality of Life and patient-reported experience measures. Methods: Prior to the pandemic clinicians would provide the EQ-5D-5L as a paper copy to patients at the start of the program for completion of a baseline, and again at the end of the 12 week program. Data would then be manually entered into the electronic medical record as a free text field and provided as only an overall summary. A copy of the summary was provided to both the patient and the referring physician. Post pandemic, a link to complete the electronic version of the EQ-5D-5L is provided to the patients at the same time intervals, and the information is automatically available for viewing by the clinicians, and ready for secondary use in program evaluation. Results: One year into the electronic integration 136 patients have trialed our new system. Currently follow-up measures have only been completed for 36 , but not all persons eligible have completed their program. We are noting fewer EQ-5DL's completed by patients at the end of their program, but the immediate availability of patient-reported measures allows us to readily evaluate the impact of program on patient health-related 
quality of life, and a similar process is now being considered for integration into a provincial Alberta Health Services framework. Conclusion: The potential benefits of the use of an electronic integrated patient-reported outcome measure tool include the reduction in transcription error with manual entry, potential for reduced bias as the tool is completed independently and not in the presence of a clinician, and allows for ready and widespread comparison. However, new barriers to patient uptake need to be better understood and mitigated.

\section{(3130) What is the minimum number of goals required} per subject for goal attainment scaling trials: a simulation study

Taylor Dunn, MSc, Ardea Outcomes, Halifax, Nova Scotia, Canada; Justin Stanley, BScE, Ardea Outcomes, Halifax, Nova Scotia, Canada; Kari Knox, BScN, Ardea Outcomes, Halifax, Nova Scotia, Canada; Sanja Stanojevic, PhD, Dalhousie University, Halifax, Nova Scotia, Canada; Susan Howlett, PhD, Dalhousie University, Halifax, Nova Scotia, Canada; Kenneth Rockwood, MD, Dalhousie University, Halifax, Nova Scotia, Canada

Aims: Goal attainment scaling (GAS) is a patient-centric outcome measure that captures meaningful change through personally identified goals. Traditionally, a minimum of three goals per subject has been recommended. Depending on the study population and time constraints, however, it may not be feasible to set this minimum number. Our aim was to investigate the effect of number of goals per subject on statistical power using data simulation techniques. Methods: We employed a probabilistic model introduced by Urach et al. (2019) for generating GAS data. This model allows for adjustment of many study parameters. For this analysis we varied the total number of subjects $\mathrm{m}$ (from 20 to 120), treatment effect size $\delta$ (from 0.4 to 1.0), and the parameter of interest: number of goals per subject $n$ (from 1 to 7). For each set of parameters, 1000 parallel group trials were simulated and a two-sided t test was performed on standardized T-scores. Power was computed as the percentage of simulations detecting a statistically significant treatment effect at $\alpha=0.05$. Results: The gain in statistical power ranged from 8 to $20 \%$ when 2 goals were set per subject, compared to 1 goal. The difference between 3 and 2 goals per subject was less pronounced, with power increases ranging from 2 to $11 \%$. This pattern of diminishing returns continued for more than 3 goals, e.g. $0-8 \%$ gains from 4 vs 3 goals per subject. With 3 goals per subject, the minimum sample size required to reach $80 \%$ was found to be $120,60,40$ and 30 subjects for effect sizes $\delta=0.4,0.6,0.8$ and 1.0, respectively. Conclusion: The power to detect a treatment effect increased with increasing number of goals per subject. The most substantial increase was from 1 to 2 goals per subject. For the moderate-large effect sizes considered here, the traditional 3 goal minimum reached $80 \%$ power with sample sizes ranging from 30 to 120 subjects. The 3 goal minimum is a sensible rule of thumb, though a 2 goal minimum may be suitable in studies with high sample sizes and/or hypothesized effect sizes.

(3131) A computer-assisted format of the adjusted DSWAL-QoL questionnaire for dysphagic patients with additional language and/or cognitive impairment: evaluation of its psychometric properties

Ingeborg Simpelaere, PhD, MSc, VIVES University College, Bruges, Belgium; Jessie Lemmens, PhD student, MSc, Zuyd University of Applied Sciences, Heerlen, The Netherlands; Ruth Dalemans, PhD, Zuyd University of Applied Sciences, Heerlen, The Netherlands

Aims: The computer-assisted format of the adjusted DSWAL-QoL questionnaire (CAFaDSWAL-QoL) has been developed to (1) facilitate self-report by dysphagic patients with additional language and/or cognitive impairment (DysLC), and (2) to meet benefits of health information technology applications. The CAFaDSWAL-QoL is based on previous validation studies, in which both modern item response theory (i.e., item analysis by the Rasch model) and classical test theory methods were applied. However, this patient-reported outcome (PRO) instrument was further communication-friendly adapted based on recent insights resulting from a content and needs assessment study that carefully examined what kind of adaptations (e.g., type of visual support) could improve the feasibility of the PRO. The recent CAFaDSWAL-QoL contains a preceding training program to become familiar with the line of questioning and response options. Different configuration options can be selected conform the patient's preference. This PRO also provides appropriate visual and auditory support that can be easily switched off and a final report that can be retrieved at any time (e.g., during a subsequent clinical consultation). Content validity of the CAFaDSWAL-QoL has been established in another study. The primary purpose of this study was to examine its psychometric properties. We will report on internal consistency and test-retest reliability. Methods: We included 50 patients based on the following inclusion criteria: (1) native Dutch speakers, (2) adults ( $\geq 18$ years old), (3) suffering from neurogenic or mechanical dysphagia during at least one month and (4) having additional language and/or cognitive impairments. All patients completed the CAFaDSWAL-QoL two times, with an interval of 2 weeks between test and retest. We will assess the CAFaDSWAL-QoL in terms of reliability using international accepted quality criteria. All statistical analyses will be performed using SPSS 27.0. Results: We will discuss in detail the resulting psychometric data and will present the adaptations that were made to the CAFaDSWALQoL. Conclusion: We hope the CAFaDSWAL-QoL will show good reliability. Using adapted communication-friendly digital questionnaires may enhance self-report among DysLC patients and prevent them from being excluded from health-related quality of life measurement studies due to failure in completing incomprehensible PROs. Such adaptations can also enhance the validation of these scales.

(3132) Feasibility of a patient-reported outcome measure-based symptom monitoring and management intervention to improve adherence to breast cancer adjuvant endocrine therapy

Kathryn Flynn, PhD, Medical College of Wisconsin, Milwaukee, Wisconsin, USA; Elizabeth Weil, Froedtert Hospital, Milwaukee, Wisconsin, USA; Nicole Fergestrom, Medical College of Wisconsin, Milwaukee, Wisconsin, USA; Melinda Stolley, Medical College of Wisconsin, Milwaukee, Wisconsin, USA; Sailaja Kamaraju, Medical College of Wisconsin, Milwaukee, Wisconsin, USA; Carolyn Oxencis, Froedtert Hospital, Milwaukee, Wisconsin, USA; Aaron Winn, Medical College of Wisconsin, Milwaukee, Wisconsin, USA; Purushottam Laud, Medical College of Wisconsin, Milwaukee, Wisconsin, USA; Joan Neuner, Medical College of Wisconsin, Milwaukee, Wisconsin, USA

Aims: Adjuvant endocrine therapy (AET) after estrogen-positive breast cancer reduces mortality, but one-third to one-half of patients discontinue it early or are nonadherent. Symptoms are important contributors to AET nonadherence. We developed an intervention for patients with evidence of early nonadherence to AET that used patient-reported outcome measures (PROM) to monitor symptoms with clinical pharmacist-led guideline-concordant management to address symptoms and improve AET adherence. Methods: In a single-arm, pre-post feasibility study, we used prescription fill records from an embedded all-payer, all-pharmacy claims database in the electronic health record to identify patients showing AET nonadherence and invited study participation. Reminders were sent by email or text with a REDCap link to symptom monitoring assessments (14 PRO-CTCAE items) weekly for 1 month and monthly until 6 months. Clinical oncology pharmacists used guideline-based 
symptom management to address severe symptoms reported on the assessments (e.g., joint pain: exercise, acupuncture; menopausal symptoms: gabapentin, SNRIs; fatigue: exercise). Switching to a different AET was also an option. Feasibility outcomes included completion of symptom reports and pharmacist recommendations. We measured changes in adherence (obtaining $<80 \%$ of all expected daily scheduled doses for the 6-month period) and PROMs (PROMIS computerized adaptive tests). Results: Of 38 eligible patients from 3 clinics, 20 consented and 18 participated, with 14 completing all assessments and no patient missing $>3$. All patients reported at least 1 of 4 main symptom types (musculoskeletal, vasomotOR urogenital, fatigue) during the 6 -month study period, and $>50 \%$ reported attempting pharmacist recommendations for addressing their symptoms. Mean pharmacist time spent was $88 \mathrm{~min}$ (SD $55 \mathrm{~min}$ ) per patient over 6 months. At baseline all patients were non-adherent; at 6-months $44 \%$ were adherent. Patientreported physical, mental, and social health improved, as did self-efficacy (Table). Because of small sample size, we did not include any statistical evaluation of adherence or PROMs; however. both outcome categories showed clinically significant improvements. Conclusion: A novel intervention to utilize pharmacists in an oncology practice to systematically monitor and manage symptoms shows promise to reduce symptoms, enhance self-efficacy, and improve adherence to AET.

Table. Mean patient-reported outcome scores at baseline and over time
\begin{tabular}{|l|c|c|c|}
\hline PROMIS Domain & Baseline & 3 months & $\mathbf{6}$ months \\
\hline \multicolumn{1}{|c|}{ Functioning (higher scores represent better functioning) } \\
\hline Self-efficacy to Manage Medications & 46.3 & 48.5 & 50.7 \\
\hline Self-efficacy to Manage Symptoms & 47.2 & 51.6 & 51.9 \\
\hline Physical Function & 46.7 & 48.5 & 51.0 \\
\hline $\begin{array}{l}\text { Ability to Participate in Social Roles } \\
\text { and Activities }\end{array}$ & 51.2 & 54.8 & 56.7 \\
\hline Emotional Support & 53.4 & 55.3 & 55.4 \\
\hline Instrumental Support & 55.8 & 58.0 & 61.6 \\
\hline Informational Support & 55.3 & 59.4 & 57.5 \\
\hline \multicolumn{1}{|c|}{ Symptoms (higher scores represent worse symptoms) } \\
\hline Pain Interference & 55.2 & 50.1 & 48.1 \\
\hline Fatigue & 55.9 & 51.4 & 49.9 \\
\hline Depression & 50.5 & 47.7 & 47.7 \\
\hline Anxiety & 52.8 & 48.4 & 48.5 \\
\hline Sleep Disturbance & 53.3 & 51.9 & 50.3 \\
\hline Sleep-Related Impairment & 54.3 & 53.4 & 47.8 \\
\hline
\end{tabular}

(3133) The experience of spirituality among Chinese breast cancer survivors: a qualitative study

Haozhi Xia, Fudan University, Shanghai, China; Libin Gu, Nanjing University of Chinese Medicine, Nanjing, China; Tingting Cai, Fudan University, Shanghai, China; Qingmei Huang, Fudan University, Shanghai, China; Yang Yang, Fudan University Shanghai Cancer Center, Shanghai, China; Changrong Yuan, Fudan University, Shanghai, China

Aims: Since spirituality is multidimensional and individual natural and varieties depending on culture and life experience, which is often explored together with religion, this study aimed to explore the experience of spirituality among breast cancer survivors in mainland China, who mostly have no religion belief. Methods: A qualitative approach guided by phenomenology was used. Sixteen participants were recruited using purposive sampling. In-depth interviews were conducted to collect data. Transcribed interview data were analyzed by two researchers according to Colaizzi' 7 steps phenomenological approach. Results: In this study, Chinese breast cancer survivors described spirituality as an indispensable component of the experience of fighting against cancer. Six key themes emerged: Maintaining dignity, Having peace in mind, Searching for meaning in life, Gaining strength from social supports, Cherishing life, and Living without regret. The dignity in this context was considered as self-control of the body change, maintaining the social roles and autonomy in decision making. Survivors tried to keep peace in mind by accepting the life with cancer, accomplishing self-transcendence, and facing death. They searched for meaning in life by taking responsibility for the family, helping others, and appreciating the past life. Conclusion: When facing life-threatening events, like the diagnosis of cancer, individuals come to issues on spirituality, which affect the quality of life. The study provides a detailed description of how spirituality is experienced by breast cancer survivors in mainland China. Besides the inner strength of survivors' beliefs and attitudes towards life, the outside factors, such as social supports and interaction with others, also play important roles in spirituality for the survivors. These findings will help health providers' education and practice to improve spiritual care for Chinses breast cancer survivors with non-religious interventions.

(3134) Work ability and its association with quality of life and burnout in pediatric dentists

Paul Ionov, MD, Pavlov First St.Petersburg State Medical University, St Petersburg, Russia

Aims: Pediatric dentists are considered to be the group of specialists most exposed to occupational stress. Their health status, quality of life (QoL), and working conditions may have impact on their work ability and in doing so on quality of child oral care. Decreased work ability and high level of burnout have been reported among dentists. By now data about work ability, QoL and burnout syndrome in pediatric dentists is lacking. The aim was to evaluate work ability among dentists pediatric dentists working in municipal dental clinics, and to identify its association with quality of life and burnout. Methods: 120 dentists from 8 municipal dental clinics of St. Petersburg that provide dental care for children were included in the study. All the dentists completed Work Ability Index, RAND SF-36, Maslach Burnout Inventory (MBI), and a checklist consisting of 10 questions regarding demographic characteristics. Emotional exhaustion, depersonalization, and personal accomplishment were evaluated by MBI. We used Mann-Whitney U test and linear regression for analysis. Results: 93\% of participants were female, mean age -47.2 years \pm 11.3 , mean work experience-19 years. According to specialization the distribution was as follows: pediatric dentists $-n=83$, orthodontic dentists $-\mathrm{n}=25$, dental surgeons- $\mathrm{n}=12.79 \%$ dentists exhibited decreased work ability; among them $11 \%$ - low work ability. Social, emotional functioning, mental health, and vitality in dentists were lower than in control group $(\mathrm{p}<0,05)$. Most dentists experienced professional burnout. High degree of professional burnout was found in 7,5\% of pediatric dentists according to all three scales; $62,5 \%$ had high degree of emotional exhaustion and depersonalization with an average or low level of reduction of professional achievements; $10 \%$ - high degree of burnout according to one scale. Decreased work ability was associated with higher age, lower physical functioning, presence of emotional exhaustion and depersonalization $(\mathrm{p}<0,001)$. Conclusion: The majority of pediatric dentists exhibit decreased work ability, reduced social and mental well-being, and vitality as well as reported symptoms of burnout syndrome. Work ability was associated with age, physical functioning, emotional exhaustion, and depersonalization. The data obtained emphasize the necessity for prophylactic measures to maintain work ability and quality of life among pediatric dentists.

(3135) The association between nocturnal sleep duration and time of sleep with quality of life 
Yuan He, Master of Science, Université Paris-Saclay, Paris, France; Yanbo Zhu, doctor of Medicine, Beijing University of Chinese Medicine, Beijing, China; Qian Zhang, Beijing University of Chinese Medicine, Beijing, China; Ooh Chye Tan, Beijing University of Chinese Medicine, Beijing, China; Yisha Xie, Beijing University of Chinese Medicine, Beijing, China; Wenqiong Liu, Beijing University of Chinese Medicine, Beijing, China; Xinrui Wu, Beijing University of Chinese Medicine, Beijing, China; Fanghui Ma, Beijing University of Chinese Medicine, Beijing, China

Aims: To explore the nocturnal sleep duration and the timing of sleep and their influence on quality of life (QOL). Methods: Cross-sectional survey method was adopted among participants from Beijing and Shijiazhuang city of Hebei Province from March 2015 to December 2016. SF-36 questionnaire was used to evaluate the QOL. PSQI was used to investigate the duration of sleep at night and the timing of sleep. The duration of sleep at night was divided into $<6.0 \mathrm{~h}$, $6.0-8.0 \mathrm{~h}$, and $>8.0 \mathrm{~h}$. The timing of sleep was classified into $<$ 24:00 and $\geq 24: 00$. Logistic regression analysis was used to explore the influence of the duration of sleep and the timing of sleep on QOL. Results: 1886 valid adult samples were included in the analysis. Logistic regression analysis showed that the duration of sleep of the group of 6.0-8.0 h was taken as the reference group, the group of $<6.0 \mathrm{~h}$ illustrated risk factors for physiological fields [OR 1.391, $95 \% \mathrm{CI}(1.107,1.749), \mathrm{P}<0.05]$ and psychological fields [OR 1.390, $95 \%$ CI $(1.104,1.749), \mathrm{P}<0.05]$.The timing of sleep for those $<$ 24:00 was taken as the reference group, those sleep after $\geq 24: 00$ showed risk factors for physiological fields [OR 1.284, 95\% CI (1.013, 1.628), $\mathrm{P}<0.05]$ and psychological fields[OR 1.577, 95\% CI (1.247, 1.994), $\mathrm{P}<0.05]$. Combining the duration of sleep at night and the time of falling asleep, those whose sleep time were less than $6.0 \mathrm{~h}$ and the time of sleep $\geq 24: 00$ showed risk factors for both physiological fields[OR 1.433, 95\% CI $(1.089,1.886), \mathrm{P}<0.05]$ and psychological fields [OR 1.359, 95\% CI $(1.029,1.796), \mathrm{P}<0.05]$. Conclusion: Insufficient duration of sleep at night and sleeping passed midnight (24:00) will increases the risk of lower quality of life. For those with insufficient sleep time at night, falling asleep earlier may reduce the negative effect of short sleep time for QOL.

(3136) SNP's association with fatigue and physical activity in head and neck cancer patients: a pilot study for inflammatory mechanisms

Canhua Xiao, Emory University, Atlanta, Georgia, USA; Yixuan Ye, Yale University, New Haven, Connecticut, USA; Andrew Miller, Emory University, Altanta, Georgia, USA; Kristin Higgins, Emory University, Altanta, Georgia, USA; Nabil Saba, Emory University, Altanta, Georgia, USA; Hongyu Zhao, Yale University, New Haven, Connecticut, USA; Rebecca Gary, Emory University, Altanta,

Georgia, USA; Janice Withycombee, Clemson University, Clemson, South Carolina, USA; Evanthia Wommack, Emory University, Atlanta, Georgia, USA; Jennifer Felger, Emory University, Altanta, Georgia, USA; Deborah Bruner, Emory University, Atlanta, Georgia, USA

Aims: The purpose of this pilot study was to examine the associations between inflammation-related SNPs and fatigue, as well as physical activity, for patients with head and neck cancer (HNC) receiving radiotherapy with curative intent. Methods: Patients with HNC without distant metastasis were enrolled; validated patient-reported fatigue and physical activity data were collected prior to and one month post-radiotherapy. Blood samples were collected prior to radiotherapy; SNPs were genotyped using Illumina Omni5 array. Genome-wide association studies (GWAS) summary statistics for C-reactive protein (CRP) from the UK Biobank were used to generate the polygenic risk score (PRS) of inflammation. Linear regression models were used to examine the association between CRP PRS and fatigue as well as physical activity. Logistic regression was used to test whether each inflammation-related SNPs were predictive of dichotomized fatigue and physical activity. Results: Forty-eight patients were enrolled. The majority were male, white, and smokers with a mean age of 58 years; $25 \%$ were obese. Most patients were in the locally advanced cancer stage and received combined chemoradiation. CRP PRS was significantly associated with fatigue at preradiation (beta $=6.02, \mathrm{p}=0.047$ ) after controlling covariates (age, gender, race, BMI, and HPV status). One SNP (rs4251961) in the interleukin-1 receptor antagonist (IL-1RN) gene was predictive of fatigue groups at pre-radiation after adjusting covariates. Patients with $\mathrm{G}$ allele for rs4251961 were more likely to be in the low fatigue group (OR 0.18, p = 0.02). Additionally, patients with $G$ allele for rs1800795 in the IL-6 gene had the attendance of being in the low fatigue group (OR $0.33, \mathrm{p}=0.06$ ) at pre-radiation. IL-1B rs 1143633 was predictive of physical activity at pre-radiation and post-radiation: patients with A allele were more likely to be in the low physical activity group (OR $0.26, p=0.042$; OR $0.28, p=0.057$, for pre and post, respectively). IL4 rs2243248 was predictive of post-radiation physical activity; patients with $\mathrm{C}$ allele were more likely to be in the low physical activity group (OR $0.07, \mathrm{p}=0.05$ ). Conclusion: Findings from this study suggest that CRP PRS is predictive of fatigue, particularly prior to radiotherapy. Inflammation-related SNPs are associated with fatigue and physical activity. Large studies are needed to verify the results.

(3137) Investigation of 3 training methods on the effect of interrater variability in clinical outcome assessment testing

Michael Calcagni, doctor of Physical Therapy, Clinical Assessment Solutions, Narragansett, Rhode Island, USA; Brandon Lum, doctor of Physical Therapy, Clinical Assessment Solutions, Culpeper, Virginia, USA

Aims: The methodological flaws of a study can determine the success or failure of a clinical trial. Adequate clinical evaluator (CE) training is paramount in decreasing testing inconsistencies and variability in clinical outcome assessment (COA) testing. The increased use of COAs as endpoints in clinical trials has created a need for more advanced training practices to ensure testing standardization and $\mathrm{CE}$ rating accuracy to demonstrate a clinically meaningful drug effect. This study evaluated 3 different training methods: asynchronous video training (VIDEO), synchronous remote training (REMOTE), and synchronous in-person training (IN-PERSON), to ascertain which training method yielded the lowest inter-rater variability and the least errors in testing Patient-Reported Outcomes (PRO), Clinician Reported Outcomes (ClinRo), and Performance Outcome Assessments (PerfO). Methods: 29 physical therapy students were randomly divided into 3 training groups, VIDEO $(n=9)$, REMOTE $(n=10)$, and IN-PERSON $(\mathrm{n}=10)$. Each group was trained on a customized clinical assessment tool comprised of elements from validated PRO, ClinRo, and PerfO assessment tests. All groups were video recorded assessing a scripted mock patient and were evaluated by a remote blinded expert reviewer for testing quality and score discrepancy. Results: Across all three tests, the VIDEO group scored substantially lower (more errors) and with higher variability than the REMOTE group and the IN-PERSON groups, which showed similar performance. The mean scores $( \pm \mathrm{SD})$ for the VIDEO group on the PRO, ClinRO, and PerfO were $31 \%( \pm 40 \%), 55 \%( \pm 14 \%)$, and $52 \%$ $( \pm 16 \%)$, respectively. The mean scores for the REMOTE group on the PRO, ClinRO, and PerfO were $80 \%( \pm 21 \%), 90 \%( \pm 9 \%)$, and $89 \%( \pm 9 \%)$, respectively. The mean scores for the IN-PERSON group on the PRO, ClinRO, and PerfO were $88 \%( \pm 13 \%), 91 \%$ $( \pm 4 \%)$, and $95 \%( \pm 4 \%)$, respectively. Conclusion: Results from 
this study illustrate that the CEs who participated in synchronous inperson and synchronous remote training, demonstrated significantly lower inter-rater variability and decreased testing error rates when compared to the asynchronous video training group. Synchronous inperson and synchronous remote training should be highly considered as part of a CE training program for clinical trials to ensure data integrity.

\section{(3138) Accessible patient-reported outcome measure (PROM) systems for adults with disabilities}

Lana Pasek, EdM, MSN, PhD(s), University at Buffalo The State Univeristy of New York, Alden, New York, USA

Aims: Adults with disabilities have a range of physical, sensory, cognitive, and behavioral impairments, and thus, they may have unequal access to Patient-Reported Outcome Measures (PROM) systems. Accommodations for disabilities are available but the extent of their use in PROM systems and research is unknown. Methods: An integrative review was conducted on the accommodations developed or used by researchers to enable the inclusion of adults with disabilities to complete PROMs. The review of peer-reviewed literature through March 2021 was conducted in PubMed and hand searching of articles using the search terms of: patient-reported outcome system, quality of life, computerized adaptive testing, accessibility, disability, and interactive voice response. The integrative literature search strategy used the Prisma-P (preferred reporting items for systematic review and meta-analysis protocols). Results: The review yielded 481 unique articles. Four hundred and fifty-six articles were discarded during the title/abstract and full-text reviews, leaving 25 articles for extraction. Recommendations for accessible PROM systems were typically limited to a single disability, such as cognitive or sensory limitations. For example, an accommodation that was utilized for youths challenged cognitively was the use of enhanced computer systems such as the ATLAS system. The ATLAS system software mediated literacy issues for the youths and was recommended for adults. Other accessibility strategies were identified that could be applied across different limitations. Researchers commonly selected brief scales, short forms, or computerized adaptive testing to reduce burden for adults with disabilities. CAT systems in particular were highlighted for improved measurement precision that may increase power to detect treatment effect in clinical studies. Additional accessibility accommodations for PROM systems included interactive voice response, multimedia to deliver PROMs, and web-based applications. Conclusion: PROM researchers over the last two decades have been actively testing and applying various user interfaces and methodologies to increase accessibility for adults with disabilities, typically with one limitation. Engaging adults with a range of disabilities in the development of PROM systems will improve accessibility. Further development of standards and guidelines is needed for this important effort. More validity work is needed to ensure that PROM systems adequately capture the health experiences of these adults.

\section{(3139) Understanding the relationship between quality of life measures and group therapy outcomes for people with aphasia}

Carlee Wilson, MSc, University of Alberta, Edmonton, Alberta, Canada; Allyson Jones, PhD, University of Alberta, Edmonton, Alberta, Canada; Kara Schick-Makaroff, PhD, University of Alberta, Edmonton, Alberta, Canada; Esther Kim, PhD, University of Alberta, Edmonton, Alberta, Canada

Aims: People with chronic aphasia (PWA) often participate in groupbased therapies; the main outcome measures used to evaluate these therapies focus on language impairment. Patient-centred measurement of health-related quality of life (HRQoL) is necessary as PWA are shown to be at risk for lower HRQoL, decreased social networks, and depression. Group therapy has the potential to improve the HRQoL of PWA. A scoping review directly examining HRQoL outcomes in studies of PWA attending group therapy was completed. This scoping review aimed to identify, select, and appraise research that used HRQoL outcome measures during group therapy with PWA. The following question was used to guide the integration of qualitative and quantitative studies: Does group treatment have an impact on HRQoL for PWA? Methods: A systematic literature search identified studies of group therapy for PWA, with HRQoL as an outcome measure. A total of 22 search terms relating to aphasia, group therapy, and HRQoL were used. Five electronic databases were searched. Studies identified were considered for review if published in a journal before July 2020. Results: The initial search returned 425 citations; thirteen studies met inclusion and exclusion criteria. Interventions focused on improving language or communication of PWA with communication or social participation as the main aspect of group therapy. In the quantitative studies $(\mathrm{n}=10)$, language impairment improved but HRQoL did not, as evidenced by scores on various standard measures. HRQoL measures and feedback from PWA in the qualitative studies $(n=3)$ identified generally positive outcomes of group therapy for PWA. Conclusion: Themes prevalent included that when PWA were supported in their communication, they enjoyed the groups, they felt that their mood improved, their confidence increased, and they felt valued. These benefits of group therapy as told by PWA may help to increase their HRQoL. However, the benefits are not always reflected in quantitative pre/post outcome measures. Further research is required to address how measures can capture HRQoL, and how group therapy impacts HRQoL.

\section{(3140) Comparative study of linguistic validation developer- prescribed processes involved in the development of patient- reported outcomes}

Fiona Miller, Vitaccess, Oxford, UK; Sujyotee Kretz, MSc, Vitaccess, Oxford, UK; Anna Richards, Vitaccess, Oxford, UK; Cathy Spinage, Vitaccess, Oxford, UK

Aims: This comparative study looked at the processes for translating specific patient-reported outcomes (PROs) as stipulated by PRO developers and license holders. The aim was to explore the purpose of each step, the level of validity in the development of an optimal quality translation for patients, and the impact each step has on development and study timelines. Methods: A literature review of PROs used in real-world evidence studies was performed, selecting PROs requiring different types of linguistic validation (LV) processes. The review compared the number of steps and parties involved, and how these variables affect translation quality, validity, and study timelines. Results: $7 \mathrm{LV}$ processes were reviewed: basic methodology, Patient-Reported Outcomes Measurement Information System (PROMIS) measures, EQ-5D, ISPOR guidelines, Hospital Anxiety and Depression Scale (HADS), Health Utilities Index (HUI) and Functional Assessment of Chronic Illness Therapy (FACIT) scale. On average 7 steps are carried out per LV process. The timelines across the 7 processes were between 2 and 10 weeks. The simplest methodology involves 4 people in total (3 linguists and a project manager assuming the quality assurance role); the most complex methodology involves a minimum of 15: 8 linguists, a project manager, the author reviewing the translation at various steps, and 5 subjects for the cognitive debriefing step. The cognitive debriefing step demonstrated its high value in identifying issues in the translation that followed the basic process. The author review step aims to have control over the PRO content but reviews may often lead 
to delays in finalizing translations, thus, affecting the study timeline. Absence of author feedback can lead to the translation not being finalized. Conclusion: Steps that increase the development time are cognitive debriefing, author review, and clinician review; however, they do not compromise translation quality. Cognitive debriefing eliminates risk of any potential miscomprehension, and the end result is a translation that is clear and understood as intended in the source whilst adhering to cultural equivalence. Author and third-party reviews are valuable when completed within the timeline and efficiently. Harmonization of specific PRO LV processes is recommended to ensure that the same translation quality and timelines are achieved across the industry.

\section{(3142) Patient-reported Outcome Measures for Rheumatoid Arthritis Disease Activity: a systematic review}

Tim Pickles, MSc, Centre for Trials Research, Cardiff University, Cardiff, UK; Ernest Choy, Division of Infection and Immunity, Cardiff University, Cardiff, UK; Mike Horton, Psychometric Laboratory for Health Sciences, University of Leeds, Leeds, UK; Karl Bang Christensen, Section of Biostatistics, University of Copenhagen, Copenhagen, Denmark; Rhiannon Phillips, Cardiff School of Sport and Health Sciences, Cardiff Metropolitan University, Cardiff, UK; David Gillespie, Centre for Trials Research, Cardiff University, Cardiff, UK; Rhiannon Macefield, NIHR Biomedical Research Centre, University of Bristol, Bristol, UK; Olalekan Lee Aiyegbusi, Centre for Patient Reported Outcomes Research, University of Birmingham, Birmingham, UK; Claire Beecher, School of Nursing and Midwifery, National University of Ireland, Galway, Galway, Ireland

Aims: Previous research has suggested that Patient-Reported Outcome Measures (PROMs) are the most informative way to assess Rheumatoid Arthritis (RA) Disease Activity (DA) and allow for a more efficient use of NHS resource. PROMs are critical to research and clinical care, as recognised by the U.S. Food and Drug Administration (FDA), who have published guidelines on how to develop and validate PROMs. The objective was to identify all PROMs for RA DA against the internationally recognised COSMIN guidelines. Methods: PubMed and EMBASE searches undertaken by a previous systematic review were expanded to include all papers up to January 2019 (rather than the previous June 2014 date) and those before January 1994. Some papers from the review were excluded as they involved some clinician or laboratory input. All papers selected for final selection were assessed for quality of the measurement property and risk of bias through the COSMIN guidelines by two independent reviewers, to give a recommendation for use. Results: 702 abstracts were retrieved: the 34 previously reviewed had full article review, though 13 were excluded as a tighter lens is applied. Of the remaining 668,128 were selected for abstract review, 58 for full article review and 10 for final selection, thus, 31 in total for final selection. These provided information on 10 different PROMs for RA DA. Following the application of COSMIN guidelines to these 31 articles, none of these PROMs could be recommended for use, as none had sufficient evidence for content validity. 5 PROMs have the potential to be recommended but the other 5 would not be recommended. Conclusion: The lack of content validity is a major drawback for these PROMs, but it is worth noting that all of these were developed long before COSMIN began, and COSMIN have only recently updated their guidelines to increase the relevance of content validity. A 2019 review by the American College of Rheumatology recommended two of the PROMs reviewed here, but these are not based on COSMIN guidelines. More can be done to better assess these PROMs, and the items used within them to fit with COSMIN guidelines.
(3144) Cross-cultural adaptation and face validity of the Sleep Apnea Related Quality of Life Questionnaire

Jairo A Roa, MS, Universidad Santo Tomás, Bucaramanga, Colombia; Sandra Juliana Rueda, MSc, Universidad Santo Tomás, Bucaramanga, Colombia; Angie D Galvis, DDS, Universidad Santo Tomás, Bucaramanga, Colombia; María C Gómez, DDS, Universidad Santo Tomás, Bucaramanga, Colombia; Paula Y. Vargas, DDS, Universidad Santo Tomás, Bucaramanga, Colombia; Adriana E Peñuela, MSc, Universidad Santo Tomás, Bucaramanga, Colombia

Aims: To adapt a version of the Iberian Spanish of the Sleep Apnea Quality of Life Index (SAQLI) questionnaire into Colombian Spanish and evaluate its facial validity with Obstructive Sleep Apnea-Hypopnea Syndrome (OSAHS) patients. Methods: The cross-cultural adaptation was performed according to internationally recommended methodology, using a committee of reviewers and validating the Colombian version with diagnosed OSAHS patients under treatment at an institution in Bucaramanga (Colombia). The final version was submitted to the committee of reviewers consisting of two orthodontists and one otorhinolaryngologist. Fifty-one patients answered the questionnaire and assessed the understanding relevance and syntax of each question; also, they made observations and comments about the 35 items included in the six domains the questionnaire has (daily functioning, social interactions, emotional functioning, symptoms, symptoms related to treatment, and impact). Patients were informed about the purpose of the study and signed their consent. Results: The mean age of participants was $44.4 \pm 10.4$ years, $66.7 \%$ were females, $39.2 \%$ were employees of different types of companies, and $33.3 \%$ were self-employed. It was observed that, in general, the understanding of the questions was good although the participants proposed changing some words within sentences and mentioned that greater clarity is needed when generating some questions. They recommended to simplify and reformulate some questions to facilitate their comprehension and relevance according to Colombian Spanish. Conclusion: Most of the questionnaire's items were understandable and applicable. It was necessary to modify some phrases to Colombian Spanish and correct certain statements according to the observations and suggestions made by participants, so the questionnaire's items could be more easily understood.

(3145) Towards setting-sensitive quality of life assessment in telemedicine-validation of the modular Tele-QoL patientreported outcome measure

Klara Greffin, Dipl.-Psych., University of Greifswald, Greifswald, Germany; Silke Schmidt, Prof. Dr., University Greifswald, Greifswald, Germany; Neeltje van den Berg, Prof. Dr., University Medicine Greifswald, Greifswald, Germany; Wolfgang Hoffmann, Prof. Dr., University Medicine Greifswald, Greifswald, Germany; Oliver Ritter, Prof. Dr., Brandenburg City Hospital, Brandenburg an der Havel, Germany; Michael Oeff, Prof. Dr., Brandenburg City Hospital, Brandenburg an der Havel, Germany; Georg Schomerus, Prof. Dr., University Medicine Leipzig, Leipzig, Germany; Sven Speerforck, Dr., University Medicine Leipzig, Leipzig, Germany; Holger Muehlan, Dr., University Greifswald, Greifswald, Germany

Aims: Aims: A setting-related instrument for assessing quality of life (QoL) in the context of telemedicine (TM) did not exist until now. Within the Tele-QoL project, a content-valid "add-on " instrument was developed and psychometrically tested. In parallel, an index with six items summarizing the content of the respective scales was developed. After the pre- and pilot-testing of the instruments, psychometric performance of the final measures was investigated within a validation study. Methods: Methods: The questionnaires were 
applied along with other standardized instruments for validation purposes (e.g. SECU, MLHFQ, WHOQOL-BREF) within a sample consisting of patients with depression or heart failure, with or without telemedical care $(n=172)$. Data analyses were directed to descriptive and psychometric performance on item, scale, and instrument level. Results: Results: Internal consistency values (Cronbach's Alpha) reach from 0.84 to 0.93 (Tele-QoL multidimensional measure) and 0.91 (Tel-QoL index). The factor structure of the multidimensional Tele-QoL measure was widely confirmed. The Tele-QoL index demonstrates Rasch scalability, all parameters are in accordance with the model assumptions, even though the range of item locations is quite small ( -0.42 to 0.79$)$. Validity of both instruments can be assumed, as indicated by substantial associations between outcome scales and quality of life as well as impact scales and perceived security. As expected, moderate to small associations with health care satisfaction, health literacy, and patient activation could be observed. Conclusion: Conclusion: Both measures show convincing psychometric properties. With two scales per domain, the final multidimensional Tele-QoL comprises six outcome scales and two additional impact scales capturing unintended effects of TM use on QoL. The Tele-QoL measures can be used as a complementary module to existing QoL instruments to capture care-relevant, settingspecific aspects of QoL from the patient's perspective.

\section{(3146) Effectiveness of the timely wholistic care model in residential childcare service: an evaluation study}

Lori Suet Hang Lo, Hong Kong Metropolitan University, Hong Kong, Hong Kong; Bell Pui Ling Fung, University of Manchester, Manchester, UK; Linda Yin King Lee, PhD, MA, MN, BN, Hong Kong Metropolitan University, Hong Kong, Hong Kong; Ka Fai Wong, The Open University of Hong Kong, Hong Kong, Hong Kong; Charlie Chau Lai Wong, Hong Kong Metropolitan University, Hong Kong, Hong Kong; Jessica Chin Kiu Chan, Hong Kong Children's Hospital, Hongkong, Hong Kong; Kitty Kit Lam Tsang, Alice Ho Miu Ling Nethersole Hospital, Hongkong, Hong Kong; Edmond Ngai Man Li, The Hong Kong Polytechnic University, Hongkong, Hong Kong

Aims: Children living in residential homes lack family care, in addition to the monotonous and standardized living environment, they are particularly susceptible to physical and psychosocial problems. Literatures suggested a timely wholistic care model in residential childcare service (consisting of comprehensive health needs assessment, personalised care plan, continuous follow-up and multidisciplinary collaboration) is necessary to address their needs and ultimately reduce undesirable outcomes. This model had been on trial in three children and youth homes in Hong Kong. Children's health problems were identified by their caregivers and timely wholistic care was offered to them until their problems resolved. We aimed to evaluate the effectiveness of the timely wholistic care model in residential childcare service. Methods: This pretest-postest evaluation study recruited children and their primary caregivers from three residential homes between December 2017 and January 2020. The health-related quality-of-life, behavioural problems and adaptive functions of every recruited subject were measured using established questionnaires prior to the commencement of timely wholistic care, every 3 months, and upon the completion of the timely wholistic care when a subject's health problem resolved. Repeated Measures Analysis of Variance was used to compare subjects' performance at different time points. Results: There were 119 pairs of participant (children and their primary caregivers). Children's health-related quality of life and behavioural problems gradually improved, with significant enhancement in physical functioning $[\mathrm{F}(2.00$, $48.00)=311.81, \mathrm{p}=0.02]$ and reduction in cognitive problems $[\mathrm{F}$
$(3.00,81.00)=3.75, \mathrm{p}=0.02]$ after receiving the timely wholistic care. Conclusion: The timely wholistic care model is effective to deliver timely personalized care. We recommend incorporating this model in all residential childcare service. To enhance existing care, we suggest providing healthcare training for caregivers to strengthen their ability in problem identification, launching health education and lifestyle modification programme to address children's physical and psychosocial problems, and introducing nurses to monitor the implementation of timely wholistic care. Despite lacking a control group, this study provides preliminary evidence to support the effectiveness of the timely wholistic care model. Future research adopting a more robust study design is indicated to ensure a more conclusive result.

(3147) Anxiety, depression, and health-related quality of life in adult liver transplant recipients with different survival periods

Yuan He, Master of Science, Université Paris-Saclay, Paris, France; Yanbo Zhu, doctor of Medicine, Beijing University of Chinese Medicine, Beijing, China; Feng Xue, doctor of Medicine, Renji Hospital, Shanghai Jiao Tong University, Shanghai, China; Ooh Chye Tan, Beijing University of Chinese Medicine, Beijing, China

Aims: Liver transplant recipients survive longer with the advancement of surgical techniques and the application of immunosuppressants. This study explored the anxiety, depression, and health-related quality of life (HRQoL) of them with different survival periods. Methods: 290 cases were investigated with the internet-based questionnaire from 17th February 2020 to 14th March 2020. Hospital Anxiety and Depression Scale (HADS) was used to evaluate anxiety and depression. HRQoL evaluation adopted the physical component summary (PCS), mental component summary (MCS) score of the Medical Outcomes Study Short-Form 36 Health Survey (SF-36), and the SF-6D utility value. Results: (1) Samples of 22, 149, 68 and 51 cases from 4 groups of patients, representing 0.25 years, 1 year, 5 years, and 10 years after transplantation were collected. There were no statistically significant differences in gender, marital status, and culture group among them $(\mathrm{P}>0.05)$, and the age difference was statistically significant $(\mathrm{P}<0.05)$. (2) The scores of anxiety and depression were $(5.45 \pm 2.75$ vs. $4.64 \pm 3.19$ vs. $4.78 \pm 3.73$ vs. $3.16 \pm 3.63, \mathrm{~F}=3.54, \mathrm{P}<0.05)$ and $(4.82 \pm 3.84$ vs. $3.72 \pm 3.42$ vs. $3.72 \pm 3.25$ vs. $3.43 \pm 4.30, \mathrm{~F}=0.79, \mathrm{P}>0.05)$ among them. Comparisons showed that the scores of anxiety in the 10-year group were significantly lower than the other 3 groups. (3) The scores of PCS and MCS scores were $(57.86 \pm 17.94$ vs. $74.96 \pm 15.54$ vs. $75.10 \pm 18.08$ vs. $77.38 \pm 19.90, \quad F=7.39$, $\mathrm{P}<0.001)$ and $(56.64 \pm 19.58$ vs. $73.91 \pm 16.85$ vs. $73.84 \pm 15.93$ vs. $78.23 \pm 21.28, \mathrm{~F}=7.88, \mathrm{P}<0.001), \mathrm{SF}-6 \mathrm{D}$ utility value were $(0.64 \pm 0.10$ vs. $0.76 \pm 0.10$ vs. $0.74 \pm 0.13$ vs. $0.79 \pm 0.15$, $\mathrm{F}=7.61, \mathrm{P}<0.001)$. The scores of the PCS, MCS, and the SF-6D in the 0.25 -year group were significantly lower than the other 3 groups. Conclusion: Anxiety level may be lower, and HRQOL may be better for those who survive longer after liver transplant. Special attention should be given to relief anxiety and improve HRQOL in patients with short postoperative time.

(3148) Unpacking the Impact of Chronic Pain as Measured by the Impact Stratification Score

Anthony Rodriguez, PhD, RAND Corporation, Boston, Massachusetts, USA; Maria Orlando Edelen, PhD, Patient Reported Outcomes, Value and Experience (PROVE) Center, Department of Surgery, Brigham and Women's Hospital, Boston, Massachusetts, USA; Patricia Herman, PhD, RAND Corporation, Behavioral 
and Policy Sciences, Santa Monica, California, USA; Ron D. Hays, $\mathrm{PhD}$, Division of General Internal Medicine \& Health Services Research, UCLA Department of Medicine, Los Angeles, California, USA

Aims: The aim is to examine the dimensionality of the Impact Stratification Score (ISS), support for its single total score, and evaluate the psychometric properties of ISS items. Methods: The sample of 1677 chiropractic patients being treated for chronic lower back pain and chronic neck pain, had an average age of $49,71 \%$ female, and $90 \%$ White. Study participants completed the PROMIS29 v2.1 profile survey that contains the 9 ISS items. The ISS was evaluated using item-rest correlations, Cronbach's alpha, factor analysis (i.e., correlated factors and bifactor models), and item response theory (IRT). Reliability indices and item properties were evaluated from bifactor and IRT models, respectively. Results: Itemrest correlations were high $(0.64-0.84)$ with a Cronbach's alpha of 0.93. Eigenvalues suggested the possibility of two factors corresponding to physical function and pain interference/intensity. Bifactor model results indicated that data were essentially unidimensional, primarily reflecting one general construct (i.e., impact) and that after accounting for 'impact' very little reliable variance remained in the two group factors. General impact scores were reliable $($ omegaH $=$ 0.73). IRT models showed that items were strong indicators of impact and provided information across a wide range of the impact continuum and offer the possibility of a shorter 8-item ISS. Finally, it appears that different aspects of pain interference occur prior to losses in physical function. Conclusion: This study presents evidence that the ISS is sufficiently unidimensional, covers a range of chronic pain impact and is a reliable measure. Insights are obtained into the sequence of chronic pain impacts on patients' lives.

\section{(3149) Patient-reported outcomes (PROs) predict the risk of delayed readiness to return to intended oncologic therapy (RIOT) after oncologic hepatic resection: value of implementing PROs in postoperative care}

Xin Wang, MD, MPH, University of Texas, MD Anderson Cancer Center, Houston, Texas, USA; Mona Kamal, University of Texas, MD Anderson Cancer Center, Houston, Texas, USA; Jun Xu, University of Texas, MD Anderson Cancer Center, Houston, Texas, USA; Tsun-Hsuan Chen, University of Texas, MD Anderson Cancer Center, Houston, Texas, USA; Vijaya Gottumukkala, University of Texas, MD Anderson Cancer Center, Houston, Texas, USA; Araceli Garcia-Gonzalez, University of Texas, MD Anderson Cancer Center, Houston, Texas, USA; Charles Cleeland, University of Texas, MD Anderson Cancer Center, Houston, Texas, USA; Thomas Aloia, University of Texas, MD Anderson Cancer Center, Houston, Texas, USA

Aims: To define the value of patient's perspectives that related to important clinical outcome to be used in postoperative care after oncologic hepatic resection. Methods: Perioperative symptoms were assessed using a valid patient-reported outcome (PROs) assessment tool, the MD Anderson Symptom Inventory version for hepatectomy perioperative care (MDASI-PeriOp-Hep). The assessment time points include baseline, postoperative Day (POD) daily during hospitalization and post-discharge Day (PDD) 3, 7, 14, 21, and 28. Regression analysis assessed the association between delay readiness to return to intended oncologic therapy (RIOT) after surgery and perioperative symptoms burden. Results: One hundred twenty-three patients were included in the analysis of this prospective longitudinal study. About $84 \%$ of surgery devolved metastatic disease, colorectal carcinoma $(65 \%)$ was the most common primary cancer type. Most of the patients $(95 \%)$ were under enhanced recovery after surgery (ERAS) program, and $92 \%$ were previously treated. Median hospital stay time was 5 (1-12) days. Thirty-eight percent had delayed RIOT readiness of $>5$ weeks. Patients with prior comorbidities $(P=0.049)$, being not on ERAS program $(\mathrm{P}=0.044)$ or who had laporatomy $(\mathrm{P}=0.001)$ had delayed RIOT. Univariate analysis showed statistical significant associations between delayed RIOT and severity of muscle weakness (OR 1.33), dry mouth (OR 1.23), and abdominal cramping (OR 1.02) at POD3 (all $\mathrm{P}<0.05$ ). After discharged from hospital, severity of abdominal cramping (OR 1.3), and interference with general activity (OR 1.13) at PDD3; drowsiness, fatigue, dry mouth, diarrhea, poor appetite, and interference with walking and work (OR $1.17 \sim 1.80$ ) at PDD 7; abdominal bloating, abdominal cramping, incisional tightness, fatigue, poor appetite, and dry mouth (OR $1.00 \sim 2.08$ ) at PDD 14; poor appetite (OR 2.1), drowsiness (OR 1.4), and incisional tightness (OR 2.3); fatigue (OR 1.41) and incisional tightness (OR 1.69) at PDD 28 were associated with delayed RIOT (all $\mathrm{P}<0.05$ ). Conclusion: This study defined PROs, both postoperative symptom and functioning measure on MDASI-PeriOpHep, may be used to predict patients at higher risk of delayed RIOT after laparotomy for oncologic hepatic resection. The results from the current real-world data support conducting further studies for implementing PROs after oncologic hepatic resection to improve recovery and readiness for further oncology treatments.

(3150) Systematic review and standardized assessment of HealthRelated Quality of Life instruments for ischemic heart disease

Yolanda Pardo, PhD, IMIM-Hospital del Mar Medical Research Institute, CIBERESP, Barcelona, Spain; Olatz Garin, PhD, IMIMHospital del Mar Medical Research Institute, Barcelona, Spain; Cristina Oriol, MD, IMIM- Hospital del Mar Medical Research Institute, Barcelona, Spain; Victor Zamora, IMIM- Hospital del Mar Medical Research Institute, Barcelona, Spain; Itxaso Alayo, IMIMHospital del Mar Medical Research Institute, Barcelona, Spain; Gemma Vilagut, PhD, IMIM- Hospital del Mar Medical Research Institute, Barcelona, Spain; Aida Ribera, PhD, Vall d'Hebron University Hospital and Research Institute, CIBERESP, Barcelona, Spain; Mario Gil, MD, Hospital Universitario Fundación Alcorcón, Madrid, Spain; Laia Redondo, Universitat Autònoma de Barcelona, Barcelona, Spain; Olabode Adisa, Universitat Autònoma de Barcelona, Barcelona, Spain; Marta Biarnés, MD, IMIM- Hospital del Mar Medical Research Institute, Barcelona, Spain; Montserrat Ferrer, PhD, IMIM- Hospital del Mar Medical Research Institute, Barcelona, Spain; submitted on behalf of "EMPRO group"

Aims: Ischemic heart disease (IHD) is ranked first worldwide in burden measured in disability-adjusted life years (Global Burden of Disease Study, 2017). There is a huge number of available instruments for measuring Health-Related Quality of Life (HRQL) in IHD, but how to select the most adequate one for each purpose is a question still to solve. A literature review of HRQL instruments for IHD emphasized that the choice must consider the quality of their psychometric properties and clinical utility (Thompson et al., 2016).The main objective of this study is to perform a standardized assessment of the metric properties of the three most applied instruments for measuring HRQL in IHD patients: MacNew, HeartQoL, and Seattle Angina Questionnaire (SAQ). Methods: We carried out systematic literature reviews in PubMed and Scopus databases to identify manuscripts which contained information regarding either the development process or metric properties. A hand and cited-by search was performed for the name of the instrument. Two researchers independently reviewed the title and abstract, and disagreements were resolved with a third researcher. For standardized assessment we are applying the Evaluating Measures of Patient-Reported Outcomes (EMPRO), a tool designed to assess the quality of instruments by considering all the available evidence. EMPRO considers both the 
methods applied in the studies and the adequacy of the results. EMPRO evaluations are being conducted first independently by two appraisers, who are later required to achieve an agreement. An overall and seven attribute-specific EMPRO scores will be calculated: measurement model, reliability, validity, responsiveness, interpretability, burden, and alternative forms. Results: After a systematic search, 224, 1038 and 97 references were found, respectively, for the MacNew questionnaire, SAQ, and HeartQol. Finally, 59 articles include information about development or psychometric properties for the MacNew questionnaire, 38 for SAQ, and only 17 for HeartQoL, the latter being the most recently developed measure. Conclusion: Our study will allow a panoramic view of metric properties and administration issues of the most applied IHD-specific HRQL instruments, which could help the stakeholders (such as researchers, healthcare professionals, or providers) when selecting the most suitable for each purpose.

\section{(3151) Concept elicitation via structured review of memoirs: The ISROM Method}

\section{Oren Meyers, PhD, IQVIA, New York, New York, USA}

Aims: Qualitative patient interviews are the cornerstone of a series of concept elicitation activities designed to collect data to support the content validity of a clinical outcome assessment (COA). Foreknowledge of likely themes, often coming in the form of a literature review and discussions with medical experts, helps that interviewers craft a discussion guide and shape the interview in a way that balances opportunity for spontaneous patient report with guided inquiry that meets the objective of understanding the patient disease experience. Methods: In this study, the IQVIA Structured Review of Memoirs (ISROM) is introduced, with an example of its application in pancreatic cancer, as a means of efficiently building a knowledgebase of patient experience prior to conducting qualitative interviews. Memoirs, such as biographies in book or blog form, are a source of edited but often unfiltered information about the lived patient experience up to and during the time of the illness. Memoirs go to a depth often not attainable in a typical qualitative interview encounter, and are a medium patients use to describe their experiences in a natural, unstructured setting, with a level of detail and color unconfined by standard scientific research methodology. Results: For this project, five patient memoirs of the experience of pancreatic cancer were read alongside a concept abstraction form which allowed for the structured collection of data on disease- and treatment-related symptoms and the impacts of those symptoms. The resulting data may be used to form general hypotheses about the patient experience which translate into the prompts that underlie the ultimate qualitative interview discussion guide in a later phase of research. Conclusion: The ISROM methodology leverages an often untapped source of rich patientgenerated data. It serves the purpose of educating the research team about the patient's perspective on the disease experience in order to make the time spent with patients in qualitative interviews more productive and efficient, thereby enhancing the quality of the data collected and further amplifying the patient's voice in clinical research.

(3153) Average and Individual Differences between the SF-12v2 and the VR-12 in the Chinese Population

Daniel Fong, PhD, The University of Hong Kong, Hong Kong, Hong Kong; Sha Li, PhD Candidate, The University of Hong Kong, Hong Kong, Hong Kong; Bobo Chan, MAP, The University of Hong Kong, Hong Kong, Hong Kong; Lewis Kazis, PhD, Boston University, Boston, Massachusetts, USA
Aims: The Veterans RAND 12 item Health Survey (VR-12) and the Short-Form 12 version 2 (SF-12v2) are generic quality of life measures, both of which are available for the Chinese population. Their items closely resemble each other with only minor differences in the ordering of response options in four items and item wordings. However, they have not been compared to see if they can be used interchangeably in the Chinese population. Therefore, this study aims to assess the difference between the VR-12 and the SF-12v2 in the Chinese population. Methods: We conducted a household survey of 500 Chinese who were aged at least 18 -year-old in Hong Kong. They self-completed the VR-12 and the SF-12v2. The physical component (PCS) and mental component scores (MCS) of both instruments were scored using the corresponding U.S. population norms. The mean equivalence of VR-12 and SF-12v2 was assessed by a 95\% confidence interval, while the individual agreement was assessed by the Bland-Altman analysis. We adopted 5 units as the minimally important difference (MID) for PCS and MCS. Results: The study participants had a mean age of 38 years (Range: 18 to 80 ) and 168 (34\%) of them were male. Their mean PCS and MCS of the SF-12v2 were 50.3 (standard deviation $[\mathrm{SD}]=6.5)$ and $49.0(\mathrm{SD}=9.0)$, respectively, while those of the VR-12 were $49.6(\mathrm{SD}=6.2)$ and 49.7 $(\mathrm{SD}=8.8)$, respectively. The corresponding paired differences (SF12v2-VR-12) were 0.75 (95\% confidence interval $[\mathrm{CI}]=0.39$ to 1.09 and -0.69 (95\% CI -1.2 to -0.2$)$, respectively. Both CIs fell within the \pm 5 units of MID. However, the limits of agreement in the BlandAltman analysis were $(-7.0,8.5)$ for PCS and $(-11.2,9.9)$ for MCS. Both sets of limits fell outside the \pm 5 units of MID. Conclusion: The VR-12 and the SF-12v2 reached mean equivalence but there can be considerable individual differences. The two instruments can be used interchangeably for assessing group but not individual differences in the quality of life of the Hong Kong Chinese population.

\section{(3154) Re-establish body image in an older lower-limb amputee:} a case report

Linda Yin King Lee, PhD, The Open University of Hong Kong, Kowloon, Hong Kong

Aims: Undesirable change in body appearance or function threatens one's body image. The impact is particularly substantial in persons who have undergone limb amputation. Intense effort is required to reestablish their body image. This case report presents the application of a model for body image care in an older lower-limb amputee. Methods: Peter was a 68-year-old hospitalized patient. He lost his left leg in an accident and received above-knee amputation a week ago. Despite of satisfactory physical recovery, he presented symptoms of disturbance in body image. He was unwilling to communicate with others and showed no interest to his rehabilitation. He presented abusive behaviors. A model for body image care was adopted to guide the delivery of nursing care and facilitate Peter to re-establish his body image. This model specified five dimensions of care. Promotion of body reality aimed to maintain patient's body as it really existed. Nutritious diet was arranged, and wound care was performed to promote wound healing. Improvement of body presentation enhanced the presentation of a desirable body appearance to the outside world. Peter's personal hygiene was attended to and a suitable prosthesis was provided which was helpful to improve his body presentation. Establishment of body ideal facilitated patient to identify his preferred body ideal. Peter was supported to identify his personal attributes and strengths. These qualities enabled him to establish new body ideal. Strengthening patient's coping ability enabled him to cope effectively when adverse situations come. Peter was supported to express his feeling towards the loss, go through the grieving process, and identify positive coping behaviors. Supporting patient to establish social support network facilitated him to re-integrate to the society. Self- 
help group was introduced. Through participation, Peter gained mutual support from those who had encountered a similar situation. Results: Peter presented positive signs indicating acceptance of his body image. He described his body positively. He maintained maximal independence while accepted necessary help. He developed new abilities and engaged in social relationships. Conclusion: Delivery of effective body image care is an intensive process which involves concerted effort of the patient, nurses, and members in the social support network.

\section{(3155) Patient's own perception of health and frailty in HIV}

Mehmet Inceer, PhD, Mcgill University, Montréal, Quebec, Canada; Nancy E Mayo, James McGill professor McGill University, Montreal, Quebec, Canada

Aims: Background: Frailty is a multifactorial clinical syndrome with causes originating from viral infection, treatments, co-morbidities, genetics, lifestyle, and the environment. Frailty is specifically important to people with HIV as the occurrence has been documented about 10 years earlier than in the general population. Systematic identification of frailty is nearly impossible as it would require regular testing of physical function and body composition. Self-rated health $(\mathrm{SRH})$ is a known predictor of future disability and mortality. Could SRH be used to identify people who are at higher risk of having frailty, prioritizing this group for further testing and intervention. Objective: To estimate the extent to which frailty and frailty indicators are statistically predictive of self-reported health (SRH) in people living with HIV. Methods: Methods: Baseline data from the Brain Health Now Study $(\mathrm{n}=856)$ was used. Fried's 5 criteria (exhaustion, low physical activity, slow gait speed, hand weakness, and low BMI) were represented by self-report items. People with ${ }^{3} 3 / 5$ criteria were classified frail. SRH, measured on a 0 to 100 visual analog scale, was regressed on the 5 frailty indicators and on a binary classification frail or not. All models included age and sex. Results: Results: Overall, $14.7 \%$ of the sample were classified as frail and their SRH was, on average, 59.2 (SD: 18.3), lower than those classified as not frail (mean: 78.5; SD: $13.8 ; \beta:-18.7$; SE: 1.4). People classified as frail would be categorized with poor to fair health. In contrast, those not frail would be categorized with good to very good health. The strength of the relationship between frailty and SRH were exhaustion and proxy grip strength $(\beta:-8.1)$; proxy slow gait speed $(\beta:-6.5)$; low physical activity $(\beta$ : -4.9$)$; and low body mass $(\beta$ : -3.0 ; $\mathrm{p}=0.06$ ). Conclusion: Conclusion: While the global concept of "frailty" predicted SRH, no one criteria predominated. However, among those reporting fair or poor health $(n=123), 46.3 \%$ were classified as frail and among those reporting good or better health $(\mathrm{n}=713)$ only $9 \%$ were classified as frail. Thus, using a single item for SHR could prioritize people for in-depth frailty testing.

(3156) A conceptual model describing the impact of family caregiver quality of life on the quality of care provided in dementia

Afeez Hazzan, PhD, SUNY Brockport, Brockport, New York, USA; Joyce Hazzan, MBA, Liberty University, Brockport, New York, USA

Aims: The concept of quality of life is multi-faceted, and there are several definitions for it. These definitions come from several organizations worldwide, including The World Health Organization and the University of Toronto's Quality-of-life Research Unit. Similarly, defining quality or level of care provided in dementia caregiving is cumbersome given the multiplicity of factors (e.g., should we measure the quality of care received from the perspective of the caregiver or care recipient?) that need to be considered. Several organizations have attempted to define quality of care, including the Institute of Medicine. However, there is a paucity of a conceptual framework that specifically explain the link between family caregiver quality of life and the quality or level of care provided in dementia. This study aims to develop a conceptual framework to explain this relationship. Methods: This study relies on the review of the published literature on the meaning of quality of life, level of care, and quality of care provided in dementia. An extensive review of the literature was conducted to identify variables that are pertinent to quality of life, quality of care, and level of care in the context of dementia caregiving. These variables were organized into themes that explain how family caregiver quality of life could explain the quality or level of care provided to older adults with dementia. Results: Important considerations for the impact of family caregiver quality of life on the quality or level of care provided to older adults with dementia include: amount of time spent providing care (i.e. hours per week)/ level of care, caregiver mastery (i.e. mastery of the caregiving process)/quality of care, and task management (i.e. strategies used to manage caregiving tasks)/quality of care. Figure 1 shows the conceptual framework developed. Conclusion: Based on the published literature, a conceptual framework was developed that depicts the assumption that changes in caregiver quality of life could influence both the level and quality of care provided to older adults living with dementia. This conceptual framework highlights the need for the provision of support for family caregivers, particularly those targeted towards improving their quality of life.

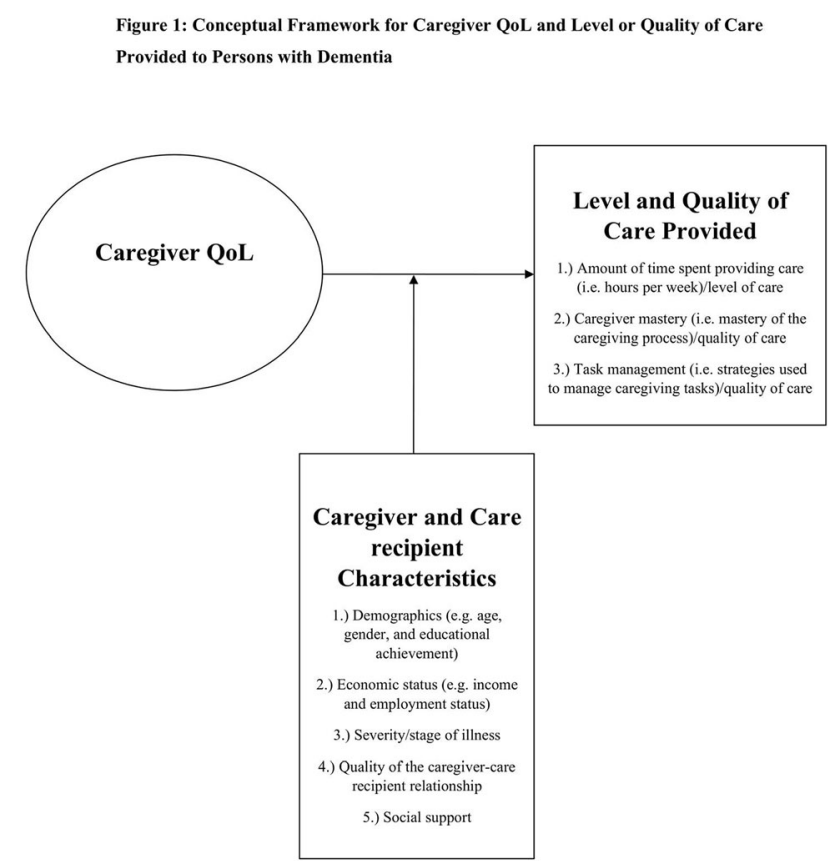

(3157) Interventions to improve Quality of Life in Latin American older adults: a systematic review

Maria Fernanda Casado-Alvarez, Bachelor's student, Nacional University of Mexico, Mexico City, Mexico; Ana L. Gonzalez-Celis, $\mathrm{PhD}$, National University of Mexico, Mexico, Mexico

Aims: To identify and analyze literature concerning health interventions directed to the improvement of the quality of life of the Latin American older adult population. Methods: The systematic review is carried out with a questioning strategy: Population, Intervention, and Outcomes of the publications (PIO). An inquiry was made through the databases: ScieLo, Redalyc, Dialnet, Latindex, Scopus, Pubmed, and PsycInfo. The keywords were Quality of Life (QOL), Health-Related 
Quality of Life (HRQOL), Cognitive Behavioral Intervention, Psychological Intervention, Older Adult, Elderly, and Aging. These words were selected according to the Medical Subject Heading $(\mathrm{MeSH})$ and the Health Sciences Descriptors (DeCS) controlled vocabulary. The inclusion criteria consisted of publications between 2010-2020, such as articles, thesis or electronic books, in English and Spanish languages, with reliable and valid instruments that measure Quality of Life in older adults with not a particular illness. Results: 2626 documents were found, from which only three empirical articles corresponding to the inclusion criteria, were considered. The countries of the original publications were Brazil (2) and Colombia (1) and consisted of quasi-experimental and experimental designs. Conclusion: The study of quality of life, when approached from the health field, is lower in regions such as Latin America, where the population has just started to grow older. The implemented programs were designed by sport and medical professionals and primarily focused on improving the physical aspect, although the multidimensional criteria of quality-of-life construct. Therefore, it is necessary to design interventions that involve psychological variables to provide comprehensive and multidimensional care in the promotion and development of the quality of life of healthy Latin American elders.

\section{(3159) Evaluating the content validity of the Single Assessment Numeric Evaluation (SANE) in shoulder pathologies}

Rochelle Furtado, MSc, Western University, Mississauga, Ontario, Canada; Joy MacDermid, PT PhD, Western University, London, Ontario, Canada

Aims: The Single Assessment Numeric Evaluation (SANE) is a single item outcome measure in where patients rate how normal they feel, relative to a specific problem. Although validated for some orthopedic conditions, it is not yet validated for a population with shoulder pathologies; nor have other studies explored the content validity. This study aims to understand how (1) patients with shoulder conditions interpret and calibrate responses to the SANE and (2) how they define normal. Methods: This study uses cognitive interviewing, a qualitative methodology that focuses on the interpretation of questionnaire items. Patients with shoulder disorders $(n=10)$, clinicians $(n=6)$ and measurement researchers $(\mathrm{n}=10)$ were interviewed using a talk aloud structured interview that evaluated the SANE. All interviews were recorded and transcribed verbatim by one researcher (R.F). Analysis was done through an open coding scheme using a previously established framework for classification of interpretation variances. Results: Overall, the single-item SANE was well received by all participants. Through the interviews, the themes of: Comprehension (20\% of participants), Reference Point (20\% of participants), Relevance (10\% of participants), and Perspective Modifiers (50\% of participants) emerged as potential sources of interpretation variance. Clinicians indicated this tool facilitates discussion when trying to create realistic recovery expectations for patients post-operatively. For patients, the word "normal" was defined relative to their current quality of life, and could further be categorized into subthemes of (1) levels of current pain compared to pre-injury, (2) personal expectations of recovery and (3) pre-injury activity levels. Conclusion: Overall, respondents found the SANE to be cognitively simple, but it was clear that the interpretation of the question and what factors calibrated their responses were highly variable between respondents. The SANE is perceived favorable by patients and clinicians, while providing a low-response burden. However, the construct being measured may vary between patients.

(3160) Quality of Labor Life and risk of mental illness in nursing workers of a health institution in Guadalajara, Mexico
Raquel González-Baltazar, PhD, Universidad de Guadalajara, Guadalajara, Jalisco, Mexico; Silvia G. León-Cortés, PhD, Universidad de Guadalajara, Guadalajara, Jalisco, Mexico; Mónica I. Contreras-Estrada, PhD, Universidad de Guadalajara, Guadalajara, Jalisco, Mexico; Liliana Hidalgo-González, Master, Universidad Guadalajara Lamar, Guadalajara, Jalisco, Mexico; Gustavo HidalgoSantacruz, Master, Universidad de Guadalajara, Guadalajara, Jalisco, Mexico

Aims: The Quality of Labor Life (QLL) and the risk of mental illness in healthcare professionals are determinants for their performance at work and for the relationship with their colleagues and the population they serve. The incidence of mental disorders in the working population is increasing every day. The aim of this study was to know the relationship between the perception of the QLL and the risk of mental illness of the nursing staff of a health institution in Guadalajara, Mexico. Methods: The study was analytical; 197 nursing workers from 98 health units participated. The CVT-GOHISALO questionnaire and the Goldberg General Health questionnaire (GHQ-28) were applied to measure satisfaction with the QLL and the risk of mental illness, respectively, in the latter instrument all people were classified as case or no case; both instruments with high reliability. The relationship between satisfaction in the seven dimensions of the CVTGOHISALO and the cases and non-cases according to the GHQ-28. Results: The percentages of satisfaction with the QLL were high for all dimensions, from 79.5 to $87.9 \%$. Nevertheless, the level of dissatisfaction higher than $12 \%$ in all dimensions of the instrument stands out, reaching up to $20.5 \%$ in the dimension of Job Satisfaction. In the case or non-case classification according to the GHQ-28, 22\% of the personnel were considered cases and $78 \%$ were considered noncases. Satisfaction with the QLL by dimensions and non-Goldberg case were searched, as well as dissatisfaction with the QLL and the Goldberg case. All dimensions have a statistically significant correspondence that ranges from $79.7 \%$ to $82.3 \%$ between satisfaction and no case, with a $\mathrm{p}$ value less than 0.05 . Conclusion: In the participating staff there is a clear link between being satisfied with the QLL and having a lower risk of mental illness. Approximately one fifth of nurses are dissatisfied with the work performed (the Job Satisfaction dimension was rated the lowest).The risk of mental illness in the nursing staff studied is equal to or greater than that of the general population. It is necessary to provide preventive programs among healthcare workers to improve their QLL and mental health.

\section{(3161) Patient-relevant therapy goals and benefits in the treatment of epilepsy}

Toni Maria Klein, M.Sc., University Medical Center HamburgEppendorf, Hamburg, Germany; Christine Blome, PD Dr., University Medical Center Hamburg-Eppendorf, Hamburg, Germany; Vivien Westermayer, Universitätsklinikum Erlangen, Erlangen, Germany; Jeanne Cuny, Dr. med., Universitätsklinikum Erlangen, Erlangen, Germany; Hajo M Hamer, Prof. Dr.med., Universitätsklinikum Erlangen, Erlangen, Germany

Aims: Epilepsy is one of the most common neurological diseases. Besides freedom from (or at least reduction of) seizures, patient's health-related quality of life is one of the most important treatment goals. Patients' specific treatment goals can go beyond quality of life improvement and can also vary considerably between patients. Therefore, the aim of this study was to identify therapy goals of patients with epilepsy and to develop a new version of the Patient Benefit Index (PBI-Epilepsy). The PBI consists of two questionnaires: one assessing the patient's needs at the beginning of the therapy and the other one assessing the achievement of these therapy goals during and after treatment steps. Methods: Patients with epilepsy completed a questionnaire with open questions about their disease burden and 
therapy goals. Afterwards, a physician interviewed them asking for additional details on their responses and noted any additional information. Data werewere analysed using qualitative content analysis. The resulting category system is meant to serve as the basis of an expert discussion (neurologists, psychologists, health scientists, patients) for the development of the PBI-Epilepsy. Results: The 19 patients $(68.4 \%$ female, age range: $18-64)$ named a large number of different burdens and goals, which referred to various dimensions: everyday and leisure activities (e.g. independence, sports), seizures (e.g. seizure reduction, freedom from seizures), profession (e.g. restrictions in choosing/pursuing a career, acceptance by colleagues), body (e.g. reduced performance, seizure-/aura-induced problems), mobility (e.g. ability to drive car or bicycle), psyche (e.g. fears, selfesteem), social relationships (e.g. stigmatisation, concern of relatives), and therapy (e.g., side effects, doctor-patient relationship). Conclusion: From these results, the new PBI-Epilepsy will be developed. This questionnaire is supposed be used in research and clinical practice: its use in research projects provides insights into the patient-defined benefits of different treatment options (beyond the commonly used parameters such as seizure frequency and healthrelated quality of life); the use of the questionnaire in clinical practice supports patient-centred care and shared decision making. Going beyond the mere recording of health-related quality of life, the PBIEpilepsy offers the possibility to consider and discuss individual patient needs from the very beginning.

(3162) Health-related quality of life in patients with obstructive sleep apnea-hypopnea syndrome

Jairo A Roa, MS, Universidad Santo Tomás, Bucaramanga, Colombia; Sandra Juliana Rueda, MSc, Universidad Santo Tomás, Bucaramanga, Colombia; Valentina Camacho, DDS, Universidad Santo Tomás, Bucaramanga, Colombia; Natalia Carreño, DDS, Universidad Santo Tomás, Bucaramanga, Colombia; María C Prada, DDS, Universidad Santo Tomás, Bucaramanga, Colombia; María C Suárez, DDS, Universidad Santo Tomás, Bucaramanga, Colombia; Nathalia Villegas, DDS, Universidad Santo Tomás, Bucaramanga, Colombia; Leslie K Vargas, DDS, Instituto Neumologico del Oriente, Bucaramanga, Colombia

Aims: To determine the health-related quality of life in patients with Obstructive Sleep Apnea-Hypopnea Syndrome (OSAHS) who attended the Instituto Neumológico del Oriente from Bucaramanga (Colombia). Methods: A cross-sectional study was conducted with patients over 18 years of age diagnosed with OSAHS during the first quarter of 2020 who fulfilled the Colombian version of the Sleep Apnea Quality of Life Index (SAQLI). This questionnaire contains 35 items distributed in six domains (daily functioning, social interactions, emotional functioning, symptoms, symptoms related to treatment, and impact) in a 7-point Likert scale that goes from all the time to not at all. Sociodemographic variables (sex, age, socioeconomic condition, marital status, and employment, among others) and variables related to the SAQLI items were analyzed. A descriptive analysis was carried out, parametric and non-parametric tests were applied when appropriate. A p-value $<0.05$ was statistically significant. Patients were informed about the purpose of the study and signed their consent. Results: The questionnaire was applied to 105 people $(53,5 \%$ were men) diagnosed with OSAHS who were not receiving treatment. In general, the highest scores were found in women with a marital status "widow" and occupation "housewife" who belonged to an upper socioeconomic condition. Conversely, the lowest scores were identified in men who belonged to a lower socioeconomic condition and unemployed. Statistically significant differences were observed in the social interaction dimension related to marital status, being widow the option with the higher scores.
Conclusion: Participants diagnosed with OSAHS who presented the lowest scores about health-related quality of life according to SAQLI were men living in a domestic partnership, having a lower socioeconomic condition, and being unemployed.

(3163) Generic health utility measures in exercise and cancer: a scoping review

Joanna F. Parkinson, BKin, Department of Physical Therapy, University of Alberta, Edmonton, Alberta, Canada; Paula Ospina Lopez, BScPT, MSc, PhD Candidate, Department of Physical Therapy, University of Alberta, Edmonton, Alberta, Canada; Margaret L. McNeely, PT, PhD, Department of Physical Therapy, University of Alberta, Edmonton, Alberta, Canada; C. Allyson Jones, PT, PhD, Department of Physical Therapy, University of Alberta, Edmonton, Alberta, Canada

Aims: The number of individuals diagnosed with cancer each year has been rising globally. Advances in early detection and treatments have led to an increase in survival rates for many cancers; however, survivors often experience significant side effects that impact healthrelated quality of life (HRQL). Exercise is effective at mitigating these negative side effects and improving survivors' health status. Utility scores provide an index of HRQL anchored at 0.00 (death) and 1.00 (perfect health). Generic health utility measures such as the EQ5D and Short-Form Six-Dimension, assess non-disease-specific attributes of HRQL and are used to calculate utility scores. The aim of this scoping review is to provide an overview of the generic health utility measures used in exercise intervention studies for cancer survivors. Methods: A systematic search of the literature from January 2000 to February 2021 was conducted using four databases (Medline, EMBASE, CINAHL, Academic Search Complete). The search strategy was developed in consultation with a health sciences librarian. Inclusion criteria consisted of (1) structured exercise interventions delivered by a qualified professional in a class or individual format, (2) adults (18 + years old) with a diagnosis of any type of cancer, (3) an exercise program initiated during or after active treatment, and (4) a generic health utility measure as a primary or secondary outcome. Two reviewers completed title and abstract and full-text screening. One reviewer will extract data, and a second reviewer will check to ensure accuracy. Conflicts will be resolved by consensus. Results: In total, 2166 citations were identified and 157 were included in full text review. Cohen's kappa was 0.9 for title and abstract screening. Over $50 \%$ of the full texts have been reviewed. Most articles were excluded based on not meeting criteria for the intervention or outcomes. Results of the full review will be presented. Conclusion: Although many studies have examined the effects of exercise during and after cancer treatment with cancer-specific measures, generic health measures have not been used to the same extent. The use of generic health measures is critical in evaluating exercise interventions so that comparisons can be made between different cancer types and other patient populations.

\section{(3164) Reliability of the Spanish version of WHOQOL-BREF in a population of Colombian university students}

Martha J. Rodríguez, MSc, Universidad Santo Tomás, Bucaramanga, Colombia; Valentina Navarro, DDS, Universidad Santo Tomás, Bucaramanga, Colombia; Pamela Isaza, DDS, Universidad Santo Tomás, Bucaramanga, Colombia; Juliana Niz, DDS, Universidad Santo Tomás, Bucaramanga, Colombia; Silvia J Rueda, DDS, Universidad Santo Tomás, Bucaramanga, Colombia

Aims: To assess the reliability of the Spanish version of the Abbreviated World Health Organization Quality of Life (WHOQOL-BREF) 
instrument in a population of Colombian university students. Methods: An evaluation of diagnostic technology was conducted with 675 undergraduate university students who filled out the Spanish version of the WHOQOL-BREF questionnaire. Sociodemographic information (sex, age, socioeconomic condition, and marital status, among others), and the four domains (physical health, psychological health, social relationships, and environment) included in the instrument were analyzed. The internal consistency of the item responses was estimated via Cronbach's alpha coefficient, and test-retest was evaluated calculating the Intraclass Correlation Coefficient (ICC) after a subgroup of 75 students who completed the questionnaire on a second occasion, two weeks apart. Values equal to or higher than 0.70 were considered satisfactory. All students signed informed consent. Results: Students' median age was 20 years (range 19-22 years), and $252(37,3 \%)$ were male. There was no evidence of ceiling or floor effect for any of the items. The internal consistency of all four WHOQOL-BREF domains for the entire population was 0.89 . Cronbach's alpha for the physical health domain was 0.69 , for the psychological health 0.80 , social relationships 0.64 , and for the environment domain was 0.79 . Intraclass correlation coefficients were satisfactory for psychological health, social relationships, and environment domains $(0.85,0.70,0.79$, respectively). The physical health domain obtained an ICC of 0.64 , and the ICC for the overall questionnaire was 0.83 . Conclusion: The Spanish version of the WHOQOL-BREF showed satisfactory internal consistency values in three out of four domains; the social relationships domain displayed the lowest values maybe because this domain includes only three items. Test-retest analysis also showed satisfactory values in psychological health, social relationships, and environment domains.

\section{(3166) The burden of migraine on quality of life: the role of interictal and gendered dimensions}

Agathe Le Lay, H. Lundbeck A/S, Valby, Denmark; Martin Bøg, H. Lundbeck A/S, Valby, Denmark; Anders Blædel Lassen, H. Lundbeck A/S, Valby, Denmark; Heather Rae-Espinoza, Clinigma, Copenhagen, Denmark; David Reeves, Clinigma, Copenhagen, Denmark

Aims: Migraine is a common, often severe, and potentially lifelong neurological disease that can lead to negative personal impacts and substantial societal consequences, with many patients experiencing lowered Quality of Life (QoL) as well as associated co-morbidities. This systematic literature review explores patients' burden of disease to support integrating patients' perspective on meaningful important differences into a clinical trial. Methods: Using a selection of biomedical databases, this study follows PRISMA reporting guidelines. 248 English, qualitative studies published in the last decade were screened for data on QoL and burden of disease for adult patients with migraine, and then CASP critical appraisal was conducted resulting in the inclusion of 14 studies. Data was then extracted using a framework-synthesis approach. Results: Although the acute effects of migraine are typically severe, six articles focused specifically on the burden of disease that is experienced intensely in the pain-free interictal period. People living with migraine have difficulty with physical functioning and suffer from a range of emotional challenges, impacting work, social, and family life in both time periods. Patients live in uncertainty and attempt to assert control over their lives with trigger management, but this can lead to isolation from daily activities and interfere with employment continuity and productivity. Seven articles highlighted migraine as especially burdensome for women, noting both greater prevalence and severity of symptoms, along with gendered dismissal to their illness. Applying established sociological theory on gendered lives, this literature review explores QoL phenomena such as social, emotional, and role functioning. Migraine is often countenanced as an invisible disorder, with patients struggling for affirmation in social, employment, and medical interactions. This integration of sociological theory suggests modifications to routine theories of migraine focused on the reduction of pain, to additionally integrate how preventive medication can reduce patient-reported distress to improve QoL. Conclusion: The impact of migraine extends beyond the acute symptomatic phase, with much of the burden of disease occurring in the interictal period. This emphasis on the interictal period points to the necessity for early and effective preventive medication for impairment in functioning and QoL with this under-diagnosed, under-treated, and under-prioritized condition.

(3167) Patients' perception of meaning of life and needed support before and after cancer treatment initiation

Mohana Roy, MD, Stanford Cancer Institute, Stanford, California, USA; Sarah Rosenthal, BA, Biomedical Informatics Research, Stanford University School of Medicine, Stanford, California, USA; Manan Shah, MD, Department of Medicine, Stanford University, Stanford, California, USA; Ali Khaki, MD, Stanford Cancer Institute, Stanford, California, USA; Tina Hernandez-Boussard, PhD,

Biomedical Informatics Research, Stanford University School of Medicine, Stanford, California, USA; Kavitha Ramchandran, MD, Stanford Cancer Institute, Stanford, California, USA

Aims: Many patients with cancer experience significant distress but do not receive additional support when needed. Patients' perception of their overall wellness is a key component of assessing distress. Here, we analyzed patient reported (a) perceived meaning of their life and (b) need for support using responses from a modified PROMISGH questionnaire at Stanford Cancer Center. Methods: We conducted a retrospective analysis of patients who had received at least one cancer medication from June 2015 to December 2020. Baseline questionnaires occurred within one year prior to first treatment, while subsequent questionnaires were between 0 and 6 months after first treatment. We assessed responses to the questions: "'My life lacks meaning'-How true was this before your illness?" and "How true is this now since your illness?". Responses ranged from "not at all" to "very much" on a 5 point scale. We also report on a support question (introduced in 2019, thus, with $\mathrm{N}=129$ ) asking "Overall, how are you doing?". Chi-square testing was used to compare responses. Results: We analyzed 17,645 questionnaires from 5468 patients (Table 1). When asked about lack of meaning during illness, after treatment initiation, there was a significant increase in responses of "quite a bit"/ "very much," compared to patients' recall of prior to illness $(\mathrm{P}<0.001)$. There was a trend for an increase in this sentiment for those with certain disease groups, higher stage of disease, and Medicaid insurance status (Table 2). Patients' rating of meaning of life prior to illness remained similar when asked before vs after treatment initiation $(\mathrm{P}=0.8)$. There was no significant difference in request for support before $(83 \%)$ and during $(87 \%)$ treatment $(\mathrm{P}=0.32)$, with even distribution across categories of care coordination, emotional distress, and physical symptoms. Conclusion: Patient-reported questionnaires capture changes in patients' perceived meaning of life and may serve as a proxy for overall wellness. We did not find a significant increase in need for support from before to after treatment initiation, but limited by small sample size. Further research is needed on how to best assess patients' need for support in the context of perceived lack of meaning during cancer illness. 
Table 1: Baseline Demographics

\begin{tabular}{|l|l|}
\hline & $\mathbf{N}=\mathbf{5 4 6 8}$ patients \\
\hline Median Age at First Questionnaire & 64 \\
\hline Sex & \\
Male & $2189(40 \%)$ \\
Female & $3279(60 \%)$ \\
\hline Race & \\
White, Non-Hispanic & $3027(55.4 \%)$ \\
Black & $119(2.2 \%)$ \\
Asian & $1223(22.4 \%)$ \\
Hispanic, Any Race & $628(11.5 \%)$ \\
Other & $471(8.6 \%)$ \\
\hline Primary Language & \\
English & $4851(88.7 \%)$ \\
Spanish & $185(3.4 \%)$ \\
Asian Languages & $338(6.2 \%)$ \\
Other & $93(1.7 \%)$ \\
\hline Disease Group (at initial contact) & $1438(26.3 \%)$ \\
Breast & $592(10.8 \%)$ \\
Gastrointestinal & $456(8.3 \%)$ \\
Thoracic & $381(7 \%)$ \\
Genitourinary & $412(7.5 \%)$ \\
Hematology and Lymphoma & $233(4.3 \%)$ \\
Gynecologic & $148(2.7 \%)$ \\
Sarcoma & $172(3.1 \%)$ \\
Neurologic/Cutaneous/Endocrine & $1502(27.5 \%)$ \\
Missing Classification & $677(12.4 \%)$ \\
\hline Stage (at initial contact) & $729(13.3 \%)$ \\
I & $560(10.2 \%)$ \\
II & $1043(19 \%)$ \\
III & $2314(42 \%)$ \\
IV & $2175(39.8 \%)$ \\
Missing Data & $287(5.3 \%)$ \\
\hline Insurance Type (at time of first questionnaire) & $2035(37.2 \%)$ \\
Medicare & $499(9.1 \%)$ \\
Medicaid & $472(8.6 \%)$ \\
Private & \\
Other or Self Pay & \\
Missing Data & \\
& \\
\hline
\end{tabular}

Table 2: "My life lacks meaning-Quite a Bit/Very Much"

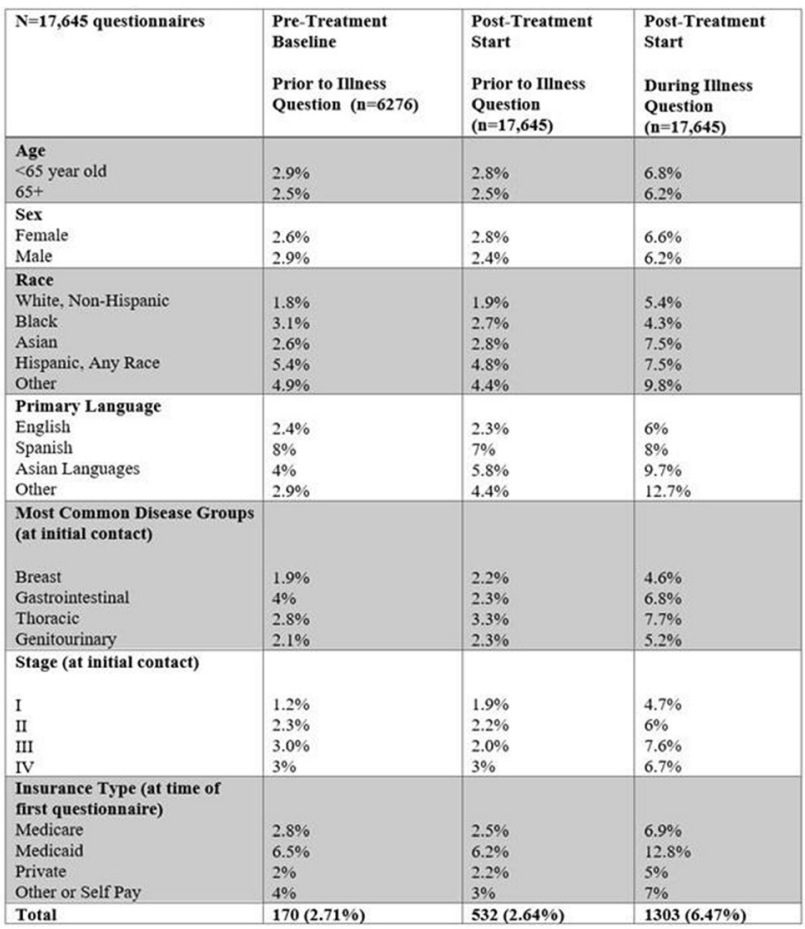

(3168) Chinese patient-reported experience of elderly people living with HIV/AIDS

Ying-Jing Zheng, Department of Social Medicine, School of Public Health, Zhejiang University School of Medicine, Hangzhou, Zhejiang Province, China; Bo-Yan Chen, Department of Social Medicine, School of Public Health, Zhejiang University School of Medicine, Hangzhou, Zhejiang Province, China; Jing-Yi Yun, Department of Social Medicine, School of Public Health, Zhejiang University School of Medicine, Hangzhou, Zhejiang Province, China; Rui Zhou, Department of Social Medicine, School of Public Health, Zhejiang University School of Medicine, Hangzhou, Zhejiang Province, China; Jie Zhou, Department of Social Medicine, School of Public Health, Zhejiang University School of Medicine, Hangzhou, Zhejiang Province, China; Ren-Jun Gu, Department of Social Medicine, School of Public Health, Zhejiang University School of Medicine, Hangzhou, Zhejiang Province, China; Hong-Mei Wang, Department of Social Medicine, School of Public Health, Zhejiang University School of Medicine, Hangzhou, Zhejiang Province, China

Aims: The widespread use of antiretroviral therapy (ART) has expanded the life expectancy of people living with HIV/AIDS (PLWHA) significantly, which created an older patient demographic and revolutionized the landscape of HIV care and patient outcomes. This study sought to explore the perceptions, experiences, and expectations of elderly PLWHA (defined as 50 years and older) which constitute important components of their quality of life, as well as to help clinic professionals to systematically understand the effects of aging, ART, and HIV infection on the health outcomes of PLWHA from patients' perspective. Methods: One-on-one semi-structured interviews were conducted to gather data from elderly PLWHA in three cities with different socioeconomic levels in Zhejiang province, China. Ninety-three participants were recruited including those who were HIV primary infection $(\mathrm{n}=2)$, HIV positive but asymptomatic $(n=48)$, HIV positive and symptomatic but not yet diagnosed with an AIDS infection $(n=12)$ and AIDS $(n=31)$. All participants were currently receiving ART. Each interview was audio taped with informed consent and then transcribed verbatim. A thematic analysis approach was conducted to identify key concepts, categorize themes, and classify domains by NVivo 12.0 software. Results: Four major themes emerged: physical health, mental health, social health, and treatment domain. Fatigue was the most reported symptom by participants. Some participants reported suicidal tendency, depression, and remorse. Most participants worried that HIV infection could cause or exacerbate non-AIDS-related disease, and reported their fear of social stigma. More than $70 \%$ participants reported poor transportation to the hospital and economic pressure from the costs of follow-up examination. Due to drug interactions, underlying diseases or side effects, about a quarter of participant have been recommended to change their treatment plan, while most of them could not afford better but more expensive drugs Conclusion: These thematic findings offer insights into the experiences of elderly PLWHA, with the consideration of the factors including culture, aging, and treatment changes. The findings assured the content validation to develop a culture-dependent patient-reported outcome instrument for use in Chinese elderly PLWHA to measure their well-being, disease burden, and treatment effectiveness.

(3169) Multi-disciplinary team cooperation to build a health education program to improve the health relate quality of life of patients with hepatic encephalopathy

Weijun Ouyang, MA, Shenzhen Traditional Chinese Medicine Hospital Department of Hepatology, Shenzhen, China; Guang-Dong Tong, Dr, Shenzhen Traditional Chinese Hospital, Shenzhen, China; Chun-Shan Wei, Dr, Shenzhen Traditional Chinese Hospital, Shenzhen, China

Aims: The purpose of this single-center prospective observational study was to evaluate the effectiveness of constructing a multidisciplinary team health education program for patients with hepatic encephalopathy to promote their health-related quality of life. Methods: In this research plan, starting from January 2021, the patients recruited from the hepatology ward underwent cognitive 
assessment using West-Haven classification (WHG) and (psychometric hepatic encephalopathy score, PHES), and 100 patients met the diagnosis of hepatic encephalopathy. Carry out prospective follow-up for 1 year. The method of intervention is to construct and implement health education plans for patients with different grades of hepatic encephalopathy through the quality control circle activities of a multidisciplinary team (including doctors, nurses, nutritionists, and social workers), and take the patients before and after the comparison. The main results include the Quality of Life Scale (SF-36), the General Hospital Anxiety and Depression Scale (HADS), and the Social Support Scale (SSRS). The patient's biochemical indicators include liver function (Alt, Ast, Ggt), serum albumin ( Alb), body mass index (BMI), evaluation of cirrhosis of sarcopenia, the composite endpoint of hospitalization related to overt hepatic encephalopathy (OHE), serious complications (such as upper gastrointestinal bleeding), and all-cause mortality during follow-up. Results: This study is ongoing, data is still being collected, and the final results will have to wait until the study is completed. Conclusion: It is currently unavailable.

(3170) Factors associated with low ability to participant in social roles and activities for breast cancer patients: application of latent profile analysis

Changrong Yuan, PhD, Fudan University, Shanghai, China; Qingmei Huang, PhD, Fudan University, Shanghai, China; Fulei $\mathrm{Wu}, \mathrm{PhD}$, Fudan University, Shanghai, China; Tingting Cai, PhD, Fudan University, Shanghai, China; Haozhi Xia, PhD, Fudan University, Shanghai, China

Aims: Cancer and its treatment will affect social functions of patients, and one of the main aims of cancer rehabilitation is to improve participation in social roles and activities. The objective of this study was to explore risk factors which were associated with low ability to participant in social roles and activities for breast cancer patients. Methods: A cross-sectional study was conducted in the sample of breast cancer patients who were undergoing active treatment such as chemotherapy. The Patient-Reported Outcomes Measurement Information System (PROMIS ${ }^{\circledR}$ ) short forms 'Ability to Participate in Social roles and Activities (PROMIS-APS) was used to measure the level of ability to Participate in Social roles and Activities of breast cancer patients. Latent profile analysis was performed to categorize participants into latent sub-groups with distinct social ability profiles. Multinominal logistic regression was conducted to identify final risk factors for patients with low ability profiles. Results: A sample of 155 breast cancer patients was included for analysis. The three-profile model was finally selected as the optimal model after a comprehensive comparison of criterion indices. Profile 1 showed high scores across all of the PROMIS-APS items, profile 2 showed medium scores, and profile 3 showed all low scores across the items, so patients in the above different profiles were labeled as "high ability," "medium ability," and "low ability," respectively. Compared to patients in the high profile, patients in the low ability profile had the worst QOL outcomes, and high anxiety level was found to be the main risk factor ( $\mathrm{OR} 1.237, \mathrm{P}=0.001)$. However, low companionship level was found to be the important factor ( $\mathrm{OR} 0.645, \mathrm{P}=0.003$ ) which will affect the participation in social roles and activities of breast cancer patients with medium profile. Conclusion: In the future intervention, more attention should be paid to breast cancer patients with low ability to participant in social roles and activities. Risk factors identified in this study will help target these vulnerable patients and be given person-centred interventions by healthcare providers.
(3171) Effects of Infant-load positions on trunk muscle activities during front and back Infant-carrying methods.

Chidebele Ojukwu, BMR PT, MSc Women's Health Physiotherapy, $\mathrm{PhD}$ women's health Physiotherapy, Department of Medical Rehabilitation, Faculty of Health Science and Technology, College of Medicine, University of Nigeria, Enugu, Enugu, Nigeria; Ifeoma Blessing Nwosu, BMR PT, MSc in orthopedic and sports Physiotherapy, Department of Medical Rehabilitation, Faculty of Health Science and Technology, College of Health Sciences, Nnamdi Azikiwe University Awka, Nigeria, Enugu, Nigeria; Chidozie Mbada, BSc PT, MSc PT, PhD PT, Obafemi Awolowo University, ile-Ife Osun state, Nigeria, Ile- Ife, Nigeria; Ekundayo Ogunkunle, BSc PT, MSc Exercise Physiology, Sports Physiotherapy Division, Federal Ministry of Youths and Sports Development, Abuja- Nigeria, Abuja, Nigeria; Ayoola Aiyegbusi, BSc PT, MSc PT, $\mathrm{PhD}$ Anatomy, Department of Physiotherapy, College of Medicine, University of Lagos, Lagos, Nigeria

Aims: This study was designed to evaluate trunk muscular responses to Infant-load positions on the trunk during simulated front and back Infant-carrying methods (ICMs). Methods: In this quasi-experimental study, 23 females who performed simulated infant carrying trials in four different trunk positions (upper back, lower back, upper front, and lower front) while walking with a $6 \mathrm{~kg}$ infant dummy. Each trial lasted for five minutes. During each trial, activities of Right (Rt) thoracic erector spinae (ES), lower rectus abdomins (RA), right external oblique (Rt EO) and left external oblique (Lt EO) muscles were simultaneously monitored via surface electromyography. The asymmetry ratio of the normalized EMG values of ES: RA and Rt EO: Lt EO were calculated. Paired T-test was used to compare the average rectified EMG values between the upper and lower back ICMs as well as between the upper and lower front ICMs. Results: Mean age of the participants was $21.00 \pm 2.50$. None of the muscles showed significantly $(\mathrm{p}>0.05)$ different activities between the upper and lower back ICMs. Their asymmetry ratios didn't also differ significantly. Similarly, comparisons the upper and lower front ICMs didn't show significant ( $p>0.05$ ) differences in the muscle activities of all the studied muscles as well as their asymmetry ratios ( $p>0.05)$. Conclusion: Since there were no significant differences, this study concludes that infant load position on the trunk is not a determinant of muscular responses during infant carrying tasks. This could be as a result of short duration of the trials hence we recommend further longitudinal studies to ascertain the long-term effects of trunk-load positions on the musculoskeletal system of nursing mothers.

(3172) Feasibility of monitoring cancer patients with a smart t-shirt: Protocol for the OncoSmartShirt study

Helle Pappot, Rigshospitalet, University Hospital of Copenhagen, Copenhagen, Denmark; Emma Balch Petersen, medical student, Rigshospitalet, Copenhagen, Denmark; Allan Green, MSc, Region Hovedstaden, Copenhagen, Denmark; Cecile Holländer-Mieritz, MD, Rigshospitalet, Copenhagen, Denmark

Aims: Studies have shown that there may be a dissimilar perception on symptoms between cancer patients and health care professionals, which may cause that patients with symptoms notify the clinic irregular or not at all. Wearables may help identifying symptoms earlier. Especially patients with low socioeconomic status and less awareness of own health might benefit from this.A new design of a wearable is a smart t-shirt, which has sensors embedded which generate measurements flows e.g., ECG, thoracic, and abdominal respiration etc. The purpose of this study is to evaluate the feasibility of using a smart t-shirt for remote monitoring of biometric sensor data 
in adolescent and young adult (AYA) and elderly patients during cancer treatment. Methods: Twenty Danish cancer patients $\geq 18$ years in antineoplastic treatment at Department of Oncology Rigshospitalet Denmark will be recruited continuously from all cancer wards at Department of Oncology Rigshospitalet, whether patients are in curative or palliative care. 10 cancer patients under 39 years defined as adolescents and young adults (AYA) and 10 cancer patients over 65 years defined as elderly. Patients with serious cognitive deficits will be excluded. Inclusion in the study will have no interference with the oncological treatment. The OncoSmartShirt study is an explorative study investigating the feasibility of using the ChronolifeTM Smart t-shirt during cancer treatment. This smart T-shirt is designed with multiple sensors and electrodes fully embedded which engender 6 different measurements flow continuously. The intervention being studied is: to wear a smart t-shirt throughout the day (minimum $12 \mathrm{~h}$ pr day) for 3 weeks. Second, qualitative interviews will be carried out, and patients will be asked to fill in a questionnaire concerning their experience with wearing the shirt. Results: Start-up of the trial and inclusion of patients will begin in June 2021. Conclusion: The study will assess the feasibility of using the ChronolifeTM smart T-shirt for home monitoring of vital parameters in cancer patients during their treatment, and bring new insights to how wearables and biometric data can be used as part of symptom recognition in cancer patients during treatment in the quest of increasing patients' quality of life.

(3173) Prevalence of concerns in Indian Head and Neck Cancer (HNC) populations reported on the Patient concerns Inventory (PCI)

Chindhu Shunmuga Sundaram, The University of Sydney, Sydney, Australia; Claudia Rutherford, PhD, The University of Sydney, Sydney, Australia; Phyllis Butow, PhD, The University of Sydney, Sydney, Australia; Puma Sundaresan, The University of Sydney, Sydney, Australia; Mahati Chittem, IIT Hyderabad, Hyderabad, India; Niveditha Akula, IIT Hyderabad, Hyderabad, India; Surendran Veeraiah, Cancer Institute, WIA, Chennai, India; Nagraj Huilgol, Nanavati Hospital, Mumbai, India; Muralidhar Muddusetty, MNJ Institute of Oncology \& Regional Cancer Centre, Hyderabad, India; Haryana Dhillon, PhD, The University of Sydney, Sydney, Australia

Aims: There are approximately 200,000 new cases of head and neck cancer (HNC) annually in India. The anatomy and nature of HNC treatment impacts a person's function, appearance, and socialization. With treatment advances, more HNC patients live longer with some degree of need for monitoring and support. This study aims to explore the primary concerns of Indian HNC populations speaking three languages-Tamil, Telugu, or Hindi. Methods: Three tertiary cancer centres in India participated. Eligibility criteria included HNC diagnosis (excluding thyroid cancer), any cancer stage, in treatment or follow-up, and aged 18 years or older. Consenting participants completed the Patient Concerns Inventory (PCI). We calculated the response rate (percentage of patients who responded 'yes' or 'no') for each concern on the PCI across language groups. Results: Participants included $621 \mathrm{HNC}$ patients $($ Tamil $=205$, Telugu $=216 \&$ Hindi $=$ $200)$. Of the 56 concerns, Telugu (28/56) and Hindi groups (27/56) reported the largest number of concerns. Patients' concerns were highest in the treatment-related domain (100\%), followed by physical and functional well-being $(72 \%)$. Fear of cancer recurrence was reported by $98.5 \%$ Telugu patients, $47.5 \%$ Tamil, and $34 \%$ Hindi. Conclusion: HNC patients in India have a high number of concerns. There are both similarities and differences in concerns across the three language groups. Responses indicate PCI is acceptable and sensitive to the concerns of Tamil, Telugu, and Hindi speaking HNC patients in India. Future research should address the concerns identified to improve the overall health-related quality of life in these patients.

(3175) Log-linear models for psychometric evaluation of sensitivity to change of patient-reported outcomes (PRO)

Giulio Flore, MSc Statistics in Life and Behavioral Sciences, IQVIA, Amsterdam, The Netherlands; Christina Daskalopoulou, PhD in Epidemiology and Medical Statistics, IQVIA, Athens, Greece; Konstantina Skaltsa, PhD in Biometrics, IQVIA, Barcelona, Spain

Aims: Regulatory approval of PRO instruments includes the assessment of their ability to detect change in patient status (Sensitivity to Change-StC). Change in PRO scores is compared to change in other measures of a patient's state on the concept of interest. Analysis of Covariance (ANCOVA) models are often used for this purpose. These models treat PRO scores as continuous variables. However, when PRO scores are ordinal (e.g., COVID-19 symptoms rated 0-3), characterized by skewness (e.g., floor effects), or cluster around few values, alternative methodologies should be employed. The aim of this work is to illustrate the application of log-linear models when assessing StC. Methods: A literature search on the application of loglinear models to $\mathrm{StC}$ analysis for PROs was performed, alongside an examination of their features to assess their suitability for this analysis. Two examples of assessing $\mathrm{StC}$ will be described using: (1) a single-item PRO score rated 0-3, and (2) a scale with high skewness. Results: Our search identified one paper where associations between PRO responses and demographics were examined through log-linear models. No paper described log-linear models assessing StC. Loglinear models are used to assess associations and interactions among categorical variables. The three categorical variables of interest could be: (1) PRO change scores, (2) change in patient status (e.g., PGIC) and (3) baseline PRO score. An interaction term between PRO change scores and change in patient status can be included to test for StC. The significance of the interaction term between PRO change scores and change in patient status, alongside an odds ratio showing improvement in PRO score for those reporting improvement in their status compared to those not reporting improvement can be calculated to support $\mathrm{StC}$ for the PRO score of interest. In cases where PRO change levels are numerous, larger samples may be required. Alternatively, PRO change levels can be grouped. Grouping levels may introduce subjectivity and require careful consideration. Conclusion: Log-linear models constitute a straightforward method to assess STC for categorical PRO scores. They are appropriate when PRO scores are ordinal with few categories, or exhibit skewness.

(3176) The association between work hours, social relationships, and the likelihood of reporting an increase in physical activity among the Canadian workforce

Victory Ugochi Ekwueme, BSc., MSc. (Cand.), Faculty of Health Sciences, University of Lethbridge, Lethbridge, Alberta, Canada; Richard Larouche, Ph.D., Faculty of Health Sciences (Public Health), University of Lethbridge, Lethbridge, Alberta, Canada; Monique Sedgwick, Ph.D. RN, Faculty of Health Sciences (Nursing), University of Lethbridge, Lethbridge, Alberta, Canada; Oluwagbohunmi Adetunji Awosoga, Ph.D. MBA, Faculty of Health Sciences (General), University of Lethbridge, Lethbridge, Alberta, Canada

Aims: Physical activity is vital for the health of the Canadian workforce and the economy. Despite this knowledge, a significant number of Canadians are physically inactive. This study examined associations between total usual hours worked per week, social relationships, and the likelihood of reporting an increase in physical activity in the 
past year among Canadian workers. Methods: A cross-sectional study design was used, and the study data were obtained from the 2018 Canadian Community Health Survey (CCHS). Two independent variables, weekly work hours and a validated social relationships scale, were used. Participants were asked whether they increased their physical activity in the past 12 months. Sociodemographic variables such as age, sex, personal income, occupational group, and education level were treated as confounders in the binary logistic regression model. Results: The majority of the study participants $(73.5 \%)$ responded with 'yes' when asked whether they increased physical activity in the past year. The mean and standard deviation for social relationships are 1.48 (strongly agree/agree) and 0.4 , respectively. While the mean and standard deviation for total usual weekly work hours is 39.17 and 13.9, respectively. The models showed a significant association between weekly work hours and the likelihood of reporting an increase in physical activity $(\mathrm{N}=5719$, OR 0.992 , $\mathrm{P}<0.001,95 \%$ CI 0.992-0.992), and the result remained significant when controlling for confounders (OR 0.994, P $<0.001,95 \%$ CI $0.994-0.995)$. The result suggested that a one unit increase in work hours is associated with lower odds of reporting an increase in physical activity. The models also showed a significant association between social relationships and the likelihood of reporting an increase in physical activity $(\mathrm{N}=6613$, OR $0.806, \mathrm{P}<0.001,95 \%$ CI $0.800-0.812$ ), and the findings remained significant when controlling for confounders (OR 0.837, $\mathrm{P}<0.001,95 \%$ CI $0.830-0.844$ ).

The result suggested that social relationships is associated with lower odds of reporting an increase in physical activity. Conclusion: The results suggested that both work hours and social relationships are associated with lower odds of reporting an increase in physical activity. These findings highlight the need for future research to inform the establishment of recommendation for policy and practices.
Table 1

Regression coefficient showing the association between total weekly work hours and the

likelihood of reporting an increase in physical activity when controlling for confounders.

\begin{tabular}{llllll}
\hline Variable & B & SE & \multicolumn{2}{c}{$\operatorname{Exp(B)}$} & \multicolumn{2}{c}{$95 \%$ CL } \\
Odds Ratio & Lower & Upper \\
\hline $\begin{array}{l}\text { Total usual weekly work } \\
\text { hours }\end{array}$ & -0.006 & 0.000 & 0.994 & 0.994 & 0.995 \\
& & & & & \\
Age & & & & & \\
$18-29$ (Reference) & & & & & \\
$30-39$ & 0.051 & 0.006 & 1.053 & 1.040 & 1.065 \\
$40-49$ & -0.129 & 0.006 & 0.879 & 0.868 & 0.890 \\
$50-59$ & -0.094 & 0.006 & 0.911 & 0.900 & 0.922 \\
$60-69$ & 0.191 & 0.008 & 1.211 & 1.191 & 1.230 \\
$70-79$ & -0.083 & 0.018 & 0.921 & 0.889 & 0.954
\end{tabular}

No income or incom

Less than $\$ 20,000$

$\$ 20,000$ to $\$ 39,999$

$\$ 40,000$ to $\$ 59,999$

$\$ 60,000$ to $\$ 79,999$

$\$ 80,000$ or more (Reference)

0.034

0.029

$\begin{array}{ll}1.191 & 1.230 \\ 0.889 & 0.954\end{array}$

Occupation Group

NOC Code first digit: $0,2,3,4$,

5 (Ref)

(ROC Code first digit: 1

OC Code first digit: 1

NOC Code first digit: 6

NOC Code first digit: 7

NOC Code first digit: 8,9

$0.007 \quad 0.007$

$-0.079$

0.006

0.006

0.967

$0.913 \quad 1.023$

1.007

0.924
0.834

0.834

$\begin{array}{ll}0.993 & 1.022 \\ 0.914 & 0.935\end{array}$

$\begin{array}{ll}0.914 & 0.935 \\ 0.824 & 0.845\end{array}$

$\begin{array}{ll}0.824 & 0.845 \\ 1.194 & 1.228\end{array}$

NOC Code first digit: 8, 9

0.237

0.006

1.268

$\begin{array}{ll}1.253 & 1.282\end{array}$

0.137

0.005

1.146

1.1351 .158

$-0.296$

0.006

0.743

$0.735 \quad 0.752$

Sex

Sex

0.292

0.009

1.339

$1.315 \quad 1.362$

Male (Reference)

$-0.123$

0.004

0.884

$0.877 \quad 0.892$

Education Level

Less than secondary school

graduation

.629

.009

0.533

$0.524 \quad 0.543$

Secondary school graduation,

.354

.005

0.702

$0.695 \quad 0.708$

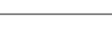


Post-secondary certificate

diploma or university degree

(Reference)

Table 2

Binary logistic regression model showing the association between social relationships and the

and the likelihood of reporting an increase in physical activity when controlling for

confounders.

\begin{tabular}{llllll}
\hline Variable & B & SE & $\begin{array}{l}\text { Exp(B) } \\
\text { Odds Ratio }\end{array}$ & $\begin{array}{l}\text { 95\% CL } \\
\text { Lower }\end{array}$ & Upper \\
\hline Social Relationships & -0.178 & 0.004 & 0.837 & 0.830 & 0.844
\end{tabular}

Age

18-29 (Reference)

30-39

$40-49$

$50-59$

60-69

$70-79$

$\begin{array}{rllll}0.047 & 0.006 & 1.048 & 1.035 & 1.061 \\ -0.143 & 0.006 & 0.866 & 0.856 & 0.877 \\ -0.088 & 0.006 & 0.916 & 0.905 & 0.927 \\ 0.208 & 0.008 & 1.231 & 1.211 & 1.251 \\ 0.022 & 0.018 & 1.022 & 0.987 & 1.058\end{array}$

Sex

Female (Reference)

Personal Income

No income or income loss $\quad 0.034$

Less than $\$ 20,000$

$\$ 20,000$ to $\$ 39,999$

$\$ 40,000$ to $\$ 59,999$

$\$ 60,000$ to $\$ 79,999$

-0.167
0.195

0.882

0.875

0.889

0.029

1.035

$0.977 \quad 1.095$

$0.007 \quad 1.056$

$0.006 \quad 0.952$

0.952
0.847

$1.042 \quad 1.071$

$\begin{array}{ll}0.941 & 0.963 \\ 0.836 & 0.857\end{array}$

$\begin{array}{ll}0.836 & 0.857 \\ 1.198 & 1.232\end{array}$

$\begin{array}{llll}0.006 & 0.847 & 0.836 & 0.857 \\ 0.007 & 1.215 & 1.198 & 1.232\end{array}$

Occupation Group

NOC Code first digit: $0,2,3$,

4,5

(Ref)

NOC Code first digit: 1

NOC Code first digit: 6

NOC Code first digit: 7

NOC Code first digit: 8,9

0.247

0.154

0.006

1.280

$\begin{array}{ll}1.266 \quad 1.295 \\ 1.154 & 1.178\end{array}$

0.00

1.166

$1.154 \quad 1.178$

(Reference)

$\begin{array}{lllll}-0.291 & 0.006 & 0.748 & 0.739 & 0.757 \\ 0.283 & 0.009 & 1.327 & 1.304 & 1.350\end{array}$

Education Level

\begin{tabular}{llllll}
$\begin{array}{l}\text { Education Level } \\
\text { Less than secondary school } \\
\text { graduation }\end{array}$ & -0.635 & 0.009 & 0.530 & 0.521 & 0.540 \\
$\begin{array}{l}\text { Secondary school graduation, } \\
\text { no post-secondary education }\end{array}$ & -0.356 & 0.005 & 0.700 & 0.694 & 0.707 \\
$\begin{array}{l}\text { Post-secondary certificate } \\
\text { diploma or university degree } \\
\text { (Reference) }\end{array}$ & & & & & \\
\hline
\end{tabular}

(3177) Validity of the Japanese HUI3 utility function and the Nippon Health Index in known people

Shinichi Noto, professor Niigata University of Health and Welfare, Niigata, Japan; Ryota Izumi, PhD, Seirei Christopher University, Hamamatsu, Japan; Takeru Shiroiwa, PhD, Center for Outcomes Research and Economic Evaluation for Health $(\mathrm{C} 2 \mathrm{H})$, Wako, Japan

Aims: The Health Utilities Index Mark 3 (HUI3) is a generic multiattribute, preference-based system for assessing health-related quality of life. It is widely used overseas as an outcome measure and for estimating quality-adjusted life years. The HUI3 can calculate a global score and a single score for each of the eight attributes (i.e., vision, hearing, speech, ambulation/mobility, pain, dexterity, selfcare, emotion, and cognition). We developed a scoring function in
2020, and the questionnaire was revised as the Nippon Health Index (NHI). This study validates the newly reformulated NHI. Methods: The survey was conducted at a single hospital near Tokyo, Japan. A total of 200 patients with stroke, cancer, cardiac disease, and orthopedic disease participated in the study. The HUI3 score was validated as both a global score and a single score. Results: The mean age of the subjects was 69.5 years, and $79(39.5 \%)$ were female. The mean EQ5D-5L utility score was $0.775 \pm 0.177$, the mean EQ-VAS was $69.1 \pm 20.9$, and the mean FIM score was $119.9 \pm 11.2$. The mean global score of HUI3 was $0.676 \pm 0.238$. There was a significant difference by disease: $0.668 \pm 0.258$ for stroke, $0.718 \pm 0.239$ for cancer, $0.584 \pm 0.238$ for heart disease, and $0.724 \pm 0.198$ for orthopedic disease. The correlation between the global score of HUI3 and EQ-5D-5L was 0.616, and the correlation with EQ-VAS was 0.353. After adjusting for age and gender, the only single score that showed a significant difference by disease was speech. Conclusion: Findings support the good construct validity of new Japanese multiplicative, multi-attribute utility function, and eight single-attribute utility functions for the HUI3 and the NHI.

(3178) 'As long as I have a restroom somewhere [...], I am fine': a qualitative study on the perspectives of periand postmenopausal women on the impact of the urinary component of the Genitourinary Syndrome of Menopause (GSM)

Michaela Gabes, Otto-von-Guericke University Magdeburg, Magdeburg, Germany; Gesina Kann, University of Regensburg, Regensburg, Germany; Julia von Sommoggy, University of Regensburg, Regensburg, Germany; Petra Stute, Inselspital Bern, Bern, Switzerland; Christian Apfelbacher, Otto-von-GuerickeUniversity, Magdeburg, Germany

Aims: Our aim was to gain insight into the experiences of women suffering from the urinary component of the Genitourinary Syndrome of Menopause (GSM) and to understand the impact of urinary complaints as part of GSM on the lives of affected women. Methods: Postmenopausal women aged from 46 to 85 years reporting vaginal and urinary complaints were recruited to participate in either online or face-to-face focus groups to share their experiences with urinary complaints as part of GSM. Transcripts of sessions were analysed using qualitative content analysis. Results: One online focus group, one face-to-face focus group, and one online-interview were conducted, involving 11 women. Five a priori assumed main themes related to the impact of urogenital symptoms were identified: daily life, emotional well-being, sexual functioning, self-concept and body image, and interpersonal relations and communication. Additionally, two further themes associated with GMS as a clinical condition were inductively found: unmet healthcare needs, including expectations of affected women regarding menopausal symptoms and a lack of adequate health education, and aspects on the personal dealing with the complaints, including personal coping strategies and medical treatment. Conclusion: This study showed that urinary complaints as part of GSM have, similar to vaginal complaints, negative impacts on the daily life, the emotional well-being, the sexual functioning, the selfconcept and body impact as well as interpersonal relations and communication of affected women. We further identified several unmet healthcare needs that should trigger improvements in healthcare.

(3179) Ranking goals by relative importance does not affect Goal Attainment Scaling summary scores

Justin Stanley, Ardea Outcomes, Halifax, Nova Scotia, Canada; Taylor Dunn, MSc, Ardea Outcomes, Halifax, Nova Scotia, Canada; 
Kari Knox, Ardea Outcomes, Halifax, Nova Scotia, Canada; Sanja Stanojevic, Dalhousie University, Halifax, Nova Scotia, Canada; Susan Howlett, Dalhousie University, Halifax, Nova Scotia, Canada; Kenneth Rockwood, Dalhousie University, Halifax, Nova Scotia, Canada

Aims: Goal Attainment Scaling (GAS) is a patient-centered outcome measure used to measure efficacy and capture the patient experience in patient-focused drug development. With GAS, participants can identify and track the treatment-related goals that are most meaningful to them. Defining these goals, setting the GAS scale, and providing a relative rank for each goal has been described as challenging and time consuming by healthcare professionals. Here, we investigated whether goal ranking affected summary GAS scores in two dementia drug trials. Methods: We used data from two clinical trials that used GAS as a primary outcome measure: the Atlantic Canadian Alzheimer Disease Investigation of Expectations (ACADIE, open label) and the Video Imaging Synthesis of Treating Alzheimer disease (VISTA, double blind with open-label extension). Subjects ranked their goals from most to least important. We then set all ranks to 1 and recalculated GAS scores to create an unranked comparison. The mean ranked and unranked GAS scores were then evaluated at 6 months. Responsiveness was defined as the Standardized Response Mean (SRM) at the end of the double-blind phase (4months) in VISTA. Results: Subjects in both trials were older adults $(75.9 \pm 7.8$ and $76.3 \pm 7.6$ years of age), more often women $(73 \%$ and $64 \%$ ), and had mild-moderate Alzheimer disease in ACADIE and VISTA, respectively. There was no difference between ranked and unranked GAS scores in ACADIE (53.7 vs 53.7, $\mathrm{P}=0.99$ ) or VISTA (54.6 vs $54.6, \mathrm{P}=0.99$ ) at 6 months. There was also no difference between subjects who were in the treatment arm (55.6 vs 55.9, $\mathrm{P}=0.88)$ or in the placebo arm $(52.6$ vs $52.9, \mathrm{P}=0.84)$ at the end of the double-blind phase in VISTA for ranked and unranked goals, respectively. Likewise in the VISTA study, the responsiveness was similar at 4 months (ranked $=0.30$, unranked $=0.29$ ). Conclusion: Ranking goals provided no statistical difference in GAS summary scores. Omitting this step in the goal setting process may result in quicker time to completion rates thereby increasing the ease of GAS implementation in trials. There is, however, a trade-off. Knowing which goals are most important (through ranking) may provide key qualitative insights important to characterizing the patient experience.

\section{(3180) The importance of subjective well-being from patients' perspective: The 'Daily Experience Sampling Questionnaire' in psoriasis treatment}

Antonia-Luise Newi, BSc, University Medical Center HamburgEppendorf, Hamburg, Germany; Athanasios Tsianakas, MD, Fachklinik Bad Bentheim, Bad Bentheim, Germany; Sophia von Martial, MD, Fachklinik Bad Bentheim, Bad Bentheim, Germany; Christine Blome, PhD, University Medical Center HamburgEppendorf, Hamburg, Germany

Aims: Patient-reported outcomes (PROs) are essential to determine patient-relevant treatment benefit of medical interventions. The aim of this qualitative study was to investigate the importance of subjective well-being (SWB) as a potential PRO from the patients' perspective in psoriasis treatment. The study focused on the affective component of SWB as assessed with the "Daily Experience Sampling Questionnaire" (DESQ), a validated daily diary assessing positive and negative emotion. Methods: Participants completed the DESQ questionnaire over a period of up to seven days. After this, they took part in semi-structured qualitative telephone interviews (Nov.-Jan. 2021). Patients were asked to reflect on the importance of affective SWB as a treatment goal and its relative importance compared with other outcome measures. They were also asked whether affective
SWB could be an indirect measure of overall treatment benefit in that it may reflect other outcomes important to them. Recruitment took place during inpatient stays at a dermatological rehabilitation clinic. The interview transcripts were analyzed using content analysis. Results: Eleven patients participated in the interviews. The sample was heterogeneous with respect to age (24-63 years) and gender ( 8 male, 3 female); the majority reported moderate to severe psoriasis. Patients described how the disease affected their SWB. Participants uniformly confirmed that DESQ responses reflected benefits of treatment. All but one patient considered affective SWB to be a central aspect of their treatment success-either as the most important goal of their therapy or as an indicator of overall treatment benefit. Regarding other outcomes considered important for their therapy, such as skin clearance and itch, participants described positive associations with SWB. Conclusion: Our findings suggest that SWB is a relevant indicator of treatment benefit from the patients' perspective. Therefore, SWB measures could be used to operationalize patientrelevant benefit of psoriasis treatment, complementing outcome measures currently used. In this context, the DESQ could be a suitable PRO measure for individual and collective assessments of treatment benefit in people with psoriasis.

\section{(3181) Patient-reported experience in head and neck cancer patients treated with radiotherapy: does treatment affect experience?}

Abdulla Al-Rashdan, MD, University of Calgary, Calgary, Alberta, Canada; Demetra Yannitsos, Msc, University of Calgary, Calgary, Alberta, Canada; Petra Grendarova, MD, Msc, University of Calgary, Grande Prairie, Alberta, Canada; Harvey Quon, MD, University of Calgary, Calgary, Alberta, Canada; Robyn Banerjee, MD, University of Calgary, Calgary, Alberta, Canada; Lisa Barbera, MD, MPA, University of Calgary, Calgary, Alberta, Canada

Aims: Head and neck cancer patients have a high prevalence of moderate to severe symptoms at diagnosis and accumulate significant toxicity over the course of their treatment. It is unknown how symptoms and treatment toxicity affect patient experience. The aim of this project was to describe how patient-reported experience changes during a course of radiotherapy. Methods: We conducted a prospective, longitudinal study in the head and neck clinics at a tertiary cancer institution between September and December 2020. Patients with a new cancer diagnosis, who were scheduled to receive radiotherapy with or without chemotherapy and could communicate in English, were approached to participate. The experience was recorded using a modified Your Voice Matters survey (YVM), including seven quantitative questions. The experience data were collected pre-treatment, weekly after radiotherapy review clinic and at one week after treatment completion (for patients post-chemoradiation and those requiring support because of toxicity). Pre-treatment YVM was completed in-person, and the remainder was via phone because of the pandemic. Positive experience was defined as the proportion of positive responses of all valid responses. Fisher's exact test was used to compare experience between visits. Results: 82 patients were approached, 59 agreed to participate of which 32 were treated with radiation and were enrolled. $78 \%$ of patients were older than 60 years, $81 \%$ were males, $59 \%$ received radiotherapy alone, and $41 \%$ received chemoradiotherapy. The YVM response rate was $94 \%$ at pre-treatment and ranged between 78 and $33 \%$ in the following visits. The positive experience in the "overall experience with last visit" was $90 \%$ at pre-treatment and this fluctuated during treatment visits and recorded at $78 \%$ one week after completion $(p=0.93)$. Positive experience increased during treatment compared to pre-treatment for "physical symptom discussion", "emotional worries discussion", "enough time spent", and "quality of care" reaching $100 \%$ after 
completion with no statistically significant differences between visits. The positive experience to the "clinic coordination" and "having needed information" remains unchanged at the end in comparison to pre-treatment level with no significant difference. Conclusion: Head and neck cancer patients had good pre-treatment experience that did not change while receiving radiotherapy.

\section{(3182) eCOA migration for bring-your-own-device technology:} Comparison of user testing versus screenshot review

Anna Richards, MA, Vitaccess, Oxford, UK; Sujyotee Kretz, MSc, Vitaccess, Paris, France; Fiona Miller, BA, Vitaccess, Oxford, UK

Aims: This analysis aimed to evaluate two separate methodologies for quality assurance (QA) during the final stages of linguistic validation of electronic patient-reported outcomes (ePROs) for use in decentralized trials. Following the standard pathway of back translation analysis and cognitive debriefing, the translated clinical outcome assessment (COA) is transformed for electronic use on a device (eCOA). Changes from paper format to electronic can be wide ranging, from updates to instructions to question display format. The traditional QA approach-screenshot review-involves assessing translated screenshots against the source screens; a newer methodology explores testing with a beta version to assess overall functionality, checking all translated content in a test environmentthis is known as user acceptance testing (UAT). Methods: The analysis compared UAT with the screenshot-review methodology to determine which is most effective as a true measure of quality assessment. In screenshot review, the images are static screengrabs of device content, showing one standard version of the screen display for all sizes and types of device (smartphone or tablet). For UAT, a beta version allows for emulation of the eCOA on any device size and type, showing how the content would display and be interacted with on different devices and in different resolutions. For each methodology type, a score was given based on the ability to assess user experience (accessibility, readability), linguistic factors (fluency, cultural equivalence, full localization), and functionality (display, compatibility, no corruptions) across the equivalent of 32 screens of 2 eCOAs. Results: The traditional screenshot review methodology identified linguistic score of 7.5 and resulted in a user experience rating of 2.3, with the main issue deriving from an inability to explore functionality elements further. UAT resulted in a linguistic score of 9 , functionality score of 8.5 and 9 for user experience. Conclusion: The results show that UAT has increased reliability and higher user experience scores. More areas for improvement can be identified through the use of UAT and functionality testing than the standard screenshot QA methodology. The end result from UAT is, therefore, more reliable, with the ability to address issues with functionality, accessibility, and readability, providing an improved overall user experience.

(3183) Utilization of technology while working from home in the US: Quality of Life and associations with age, gender, and employment status

Paul Franco, Ph.D., MBS, Seton Hall University, Nutley, New Jersey, USA; Michelle D'Abundo, Ph.D., MSH, CHES, Seton Hall University, Nutley, New Jersey, USA; Deborah DeLuca, JD, MS, Seton Hall University, Nutley, New Jersey, USA

Aims: Working from home is becoming more common, but little is known about technology usage for quality of life (QOL) and the remote-working experience. QOL is frequently applied to understand subjective health/well-being experiences, but there is little agreement about how to define and measure QOL. The purpose of this study was to understand the contributing factors associated with utilization of technological devices such as smartphones while working from home as a determinant of QOL. Methods: A qualitative study using the Quality of Life Home Workplace Questionnaire (QOLHWQ) was conducted online via SurveyMonkey® between July-September 2020 with 171 US respondents, consisting of demographic items and 11 open-ended questions including the following two, which are the focus of this abstract: (1) What role has technology played in your home workplace experience? and (2) Please select all devices you utilize for working from home (Laptop, Desktop, Smartphone, Tablet, Other). Data were exported into Excel for data analysis including preparing data, open-coding, sorting codes, and organizing themes. Results: The majority of participants utilized a laptop (94.7\%) and a smartphone $(80.1 \%)$ and commented that technology had a positive role $(76 \%)$ in their home workplace experience, citing that it was essential for their employment and allowed them to remain connected and acquire new skills. Some participants $(4.7 \%)$ stated that technology had a negative role, mainly due to WIFI/server connectivity issues and/or their reliance on technology to perform daily tasks leading to burnout. Others cited both positive and negative experiences $(7.6 \%)$ or being unaffected by technology $(11.7 \%)$, having similar at-home experiences as to previous experiences on-site. The associations between age, gender and employment status will be explored. Conclusion: As the trend of more people working from home continues, utilization of technology will become paramount for health promotion practices and overall quality of life in the US. Evaluating barriers to access and utilization of these technologies can help minimize negative experiences and prevent lowered quality of life while working from home. Furthermore, understanding the associations between age, gender and employment status may prove beneficial for employers to strategize initiatives for effective home workplace experiences for employees.

(3184) A prospective study to examine responsiveness and minimally important differences (MID)s for the CLEFT-Q scales following three cleft-specific operations

Anna Miroshnychenko, HBSc, MSc, McMaster University, Hamilton, Ontario, Canada; Charlene Rae, MSc, McMaster University, Hamilton, Ontario, Canada; Karen Wong Riff, MD, $\mathrm{PhD}$, The Hospital for Sick Children, Toronto, Ontario, Canada; Christopher Forrest, MD, MSc, The Hospital for Sick Children, Toronto, Ontario, Canada; Tim Goodacre, BSc MB BS FRCS, Oxford University Hospitals NHS Foundation Trust, Oxford, UK; Marc Swan, DPhil FRCS (Plast), Oxford University Hospitals NHS Foundation Trust, Oxford, UK; Rona SlatOR DPhil FRCS FRCS(Plast), Birmingham Children's Hospital NHS Foundation Trust, Birmingham, UK; Jesse Goldstein, MD, University of Pittsburgh, Pittsburgh, Pennsylvania, USA; Achilleas Thoma, MD MSc, McMaster University, Hamilton, Ontario, Canada; Karen Harman, MD, McMaster University, Hamilton, Ontario, Canada; Anne Klassen, DPhil(Oxon), McMaster University, Hamilton, Ontario, Canada

Aims: CLEFT-Q is a condition-specific patient-reported outcome instrument (PROM) for patients with CLP. The aim of this study was to examine internal responsiveness of the CLEFT-Q scales for 3 cleftspecific surgeries, i.e., rhinoplasty, orthognathic, and lip scar revision surgery. Internal responsiveness refers to the ability of a PROM to detect change over time. Minimally important difference (MID) is the smallest difference in scores that patients perceive as an important deterioration or improvement. Methods: Patients aged 8-29 years were recruited from six cleft centres in Canada, USA, and UK. Participants completed the CLEFT-Q appearance and health-related quality of life (HR-QOL) scales before and six months after surgery. Responsiveness was examined with hypotheses about Cohen's d 
effect sizes (ESs) based on the following interpretation: $0.20-0.49$ small, $0.50-0.79$ moderate, and $\geq 0.80$ large. MIDs were estimated using two distribution-based approaches. Results: Participants had rhinoplasty $(\mathrm{n}=31)$, orthognathic $(\mathrm{n}=21)$ and cleft lip scar revision $(\mathrm{n}=18)$ surgeries. Most participants were males $(56 \%)$ and aged 8-11 years $(41 \%)$. Following rhinoplasty, ESs were larger for the nose $(0.92, \mathrm{p}=0.001)$ and nostrils $(0.94, \mathrm{p}<0.001)$ scales than for the face scale $(0.51, p=0.003)$. MIDs ranged between $6.2-10.4$. For orthognathic surgery, larger ES was observed for the jaws scale (1.80, $\mathrm{p}<0.001)$ compared with the teeth $(1.16, \mathrm{p}<0.001)$, face $(1.15$, $\mathrm{p}=0.001)$ and lips $(0.94, \mathrm{p}<0.001)$ scales. MIDs ranged between 5.9-14.4. In the cleft lip scar revision sample, the largest ES was observed for the nose scale $(0.76, p=0.03)$, followed by lips $(0.58$, $\mathrm{p}=0.009)$ and cleft lip scar $(0.50, \mathrm{p}=0.043)$ scales. MIDs ranged between 6.4-12.3. Conclusion: CLEFT-Q detected change in key outcomes for three cleft-specific surgeries, providing evidence of its responsiveness. MIDs will aid in interpretation of this PROM.

\section{(3185) The schedule of attitudes towards hastened death: a Portuguese experience}

Pedro Ferreira, PhD, CEISUC/FEUC, Coimbra, Portugal; Ana Ramos, MSc, CEISUC, Coimbra, Portugal; Marília Dourado, PhD, CESUC/FMUC, Coimbra, Portugal

Aims: The desire to hastened death is shrouded in great complexity and controversy. It has been the subject of numerous debates among patients, health professionals, families, and recently among government officials, as the decriminalization of euthanasia has recently been discussed. The Schedule of Attitudes Towards Hastened Death (SAHD) is a self-filling instrument that evaluates the desire to hastened death. The main goal of this research was its translation and validation for the Portuguese population. Methods: The methodology used included the translation of the instrument, consensus, backtranslation, evaluation by an expert committee and a cognitive debriefing. We used a sample of 73 patients, both genders, attending ambulatory consultations or inpatient Internal Medicine and Palliative Care Department of the Coimbra Cancer Hospital. The questionnaire implemented included a self-assessment health (SAH) question, the 5Q-5D-5L, the ECOG functional performance scale and the HADS, as well as some sociodemographic questions. Results: $60.3 \%$ of patients were male with an average age of 75 years. EQ-5D-5L index revealed an average score of 0.65 with $20.5 \%$ of patients assuming a bad or very bad SAH. Anxiety was considered normal (HADS-A: 7.3) and depression as light (HADS-D: 9,1). The vast majority of patients surveyed showed low desire to anticipate death. Test-retest reliability was good $(\mathrm{ICC}=0.823)$. By content validly we may prove that SAHD was well accepted and able to assess the desire to anticipate death. SAH, disability, and quality of life scores determine SAHD scores. However, gender, living alone, and age did not present any power to determine SAHD scores. High and moderate correlations were found with depression $(r=0.662)$ and anxiety $(r=0.572)$. Conclusion: SAHD is a reliable and valid measures showing good psychometric scores.

\section{(3186) Establishment of cutpoints to categorize the severity of distress in patients with lung cancer}

Xueyao Su, ChongQing medical university, chongqing, China; Wei $\mathrm{Xu}$, chongqing medical university, Chongqing, China; Hongfan $\mathrm{Yu}$, chongqing medical university, Chongqing, China; Qingsong Yu, chognqing medical university, Chongqing, China; Yang Pu, chognqing medical university, Chongqing, China; Qingling Shi, chognqing medical university, Chongqing, China
Aims: Distress interferes with both mental and physical functions, such as appetite, activity, and sleep. The goals of the current study were to identify optimal cutpoints for differentiating between mild and severe distress interference in patients with lung cancer. Determining appropriate cutpoints for mild, and severe distress is important, because specific interventions may be based on this classification. Methods: Data for this study were obtained from a cohort study of 512 patients with lung surgery, which used MD Anderson Symptom Inventory-Lung Cancer (MDASI-LC) (including an singleitem distress) for symptom management. Patients completed questionnaires daily after surgery to discharge and weekly after discharge to 4 weeks. Optimal cutpoints for categorizing distress interference into 2 levels were derived using multivariate analysis of variance, with mood-related interference (the score of relations with others, enjoyment of life, and mood) as dependent variables and distress score as independent variable. To validate the cutpoints, we assessed whether interference categories were statistically significant in activity related interference. Results: Of 512 patients recruited, 315 had complete data for the MDASI-LC scale were included in analyses. Multivariate analysis suggested that 3 was the threshold to define the severity of distress. we defined $0-3$ as mild or no distress; and Score $\geq 4$ represents moderate to severe distress. Using the threshold to define distress, we demonstrated higher distress scores significant with the higher interference functioning (activity, work, walking) burden ( $\mathrm{p}<0.0001)$. Conclusion: The results of this study sugest that 3 is the most sensitive threshold to classify distress. Using optimal cutpoint for distress may be useful to conduct and interpretation of clinical evaluation, symptom epidemiology, and clinical trials.

\begin{tabular}{ll} 
Table1 Optimal cutpoint analysis using anchors. \\
\hline Cutpoints & $\begin{array}{l}\text { Anchor } \\
\text { Mood } \\
\text { Relation with others } \\
\text { enjoyment of life }\end{array}$ \\
\cline { 2 - 2 } & MANOVN F VALUE \\
\cline { 2 - 2 } & 14.02 \\
1 & 34.27 \\
2 & 34.36 \\
3 & 28.74 \\
4 & 30.90 \\
5 & 21.28 \\
6 & 19.98 \\
7 & 12.46 \\
8 & 12.46 \\
9 & 12.46 \\
10 &
\end{tabular}

\begin{tabular}{|c|c|c|c|c|c|c|c|c|c|}
\hline & WALKING & $z$ & $\mathbf{P}$ & WORK & $z$ & $P$ & $\begin{array}{l}\text { ACTIVIT } \\
\mathrm{Y}\end{array}$ & $z$ & $\mathbf{P}$ \\
\hline Non/mild(0-3) & $2(1-4)$ & 6.77 & $<0.0001$ & $3(1-5)$ & 7.17 & $<0.0001$ & $2(1-4)$ & 8.24 & $<0.0001$ \\
\hline \multicolumn{10}{|l|}{ Moderate to } \\
\hline severe $(4-10)$ & $5(3-6)$ & & & $6(4-8)$ & & & $5(3-6)$ & & \\
\hline
\end{tabular}

(3187) A systematic literature review of patient-reported outcomes (PROs) or health-related quality of life (HRQoL) associated with metastatic colorectal cancer (mCRC)

Mayur Amonkar, Merck \& Co., Inc., Kenilworth, New Jersey, USA; Monica Chase, Merck \& Co., Inc., Kenilworth, New Jersey, USA; Jitender Takyar, Parexel, Mohali, India; Sheetal Sharma, Parexel, Mohali, India

Aims: CRC is the third leading cause of cancer incidence and mortality. A structured review was conducted to understand the humanistic burden of mCRC. Methods: Medline ${ }^{\circledR}$, Embase ${ }^{\circledR}$, Cochrane databases (2014-2020), and 10 cancer or health services research-based conferences (2018-2020) were searched for PRO/ HRQoL information using PRISMA guidelines. The search was limited to first-line (1L) mCRC studies with an additional focus on 
microsatellite instability-high (MSI-H) or mismatch repair-deficient (dMMR) CRC across all lines of therapy. Case studies, case series, and non-English publications were excluded. Results: Of 3615 screened records, 40 (24 randomized/controlled, 5 single arm, and 11 observational) $1 \mathrm{~L} \mathrm{mCRC}$ and 2 non-randomized controlled MSI-H/ dMMR CRC (all-line therapy) studies were identified. All 1L mCRC studies included chemotherapies alone or in combination with targeted therapies (cetuximab, bevacizumab, or panitumumab), while 1 MSI-H/dMMR study included combination immunotherapy and 1 included chemotherapy. The most common instruments in the $1 \mathrm{~L}$ mCRC studies were EORTC QLQ-C30 ( $\mathrm{n}=25$ studies), FACT-G/ FACT-C/FACT-GOG-NTX $(n=7)$ and EQ-5D/-3L/HIS $(n=9)$. The QLQ-C30 domains reported were global health status (GHS/QoL: $\mathrm{n}=25$ studies), functioning (physical: $\mathrm{n}=20$; social: $\mathrm{n}=18$; role/ cognitive/emotional: $\mathrm{n}=16$ ), and symptoms (nausea/vomiting/appetite loss/diarrhea: $\mathrm{n}=15$; pain/fatigue/constipation/dyspnea: $\mathrm{n}=14)$. Studies showed mixed results in terms of improvement $(\mathrm{n}=10)$, deterioration $(\mathrm{n}=6)$, and no change $(\mathrm{n}=9)$ across QLQC30 domains following $1 \mathrm{~L}$ treatment. For EQ-5D-3L, visual analog scale (VAS) scores were mainly reported with only 1 study showing an initial improvement after 1L chemotherapy plus targeted therapy. EORTC QLQ-C30 and EQ-5D were used in 1 MSI-H/dMMR study and MDASI-GI in the other study. Statistically significant and clinically meaningful improvements were reported for QLQ-C30 GHS/ QoL, functioning (role, social), and symptoms (fatigue, pain, appetite loss, constipation, and insomnia) and EQ-5D VAS scores, in the single-arm combination immunotherapy study. The MDASI-GI showed MSI-H CRC patients, refractory to $\geq 1$ line of treatment, had more pain, fatigue, sleep disturbance, and drowsiness compared with microsatellite-stable patients. Conclusion: The EORTC QLQ-C30 was the most used instrument in $1 \mathrm{~L}$ mCRC studies. This structured review revealed a significant humanistic burden associated with mCRC, which varied according to instruments and treatments used. Results highlight the need for newer treatment strategies to help alleviate this burden.

(3188) Facilitators and Barriers of Baduanjin for Breast Cancer Survivors undergoing postoperative adjuvant treatment

Yidan Xie, Master candidate, School of nursing,Shanghai Jiaotong University school of medicine, Shanghai, China; Xiaozhen Xu, Master, Shanghai Ninth People's Hospital, Shanghai Jiao Tong University, School of Medicine, Shanghai, China; Chengrui Zhang, undergraduate, Shanghai Jiao Tong University, School of Nursing, shanghai, China; Yun $\mathrm{Hu}, \mathrm{PhD}$, Associate professor Shanghai Jiao Tong University, School of Nursing, Shanghai, China

Aims: Baduanjin, a Chinese traditional Qigong, is recognized to be an effective exercise to alleviate side effect and increase quality of life for breast cancer survivors (BCSs) during adjuvant treatment. However, exercise adherence is not high among BCSs. The aim was to identify BCSs' perceived facilitators and barriers of a Baduanjin program during adjuvant treatment. Methods: Semi-instructured interviews were conducted with 8 BCSs in a Baduanjin RCT intervention program. The interview asked BCSs' experiences of Baduanjin including facilitators and barriers of exercising, suggestions of future Baduanjin program. Content analysis was performed for data analysis under the guidance of the behavior change wheel. Results: The following five domains emerged from the analysis: (1)Capacity: Facilitators include improvement of treatment related side effects(e.g., sleeping disorder, pain, fatigue) and relief of negative emotions; Barriers include adverse effects from surgery and a adjuvant treatment; feeling low and hopeless; (2)Motivation: Facilitators include belief about beneficial effects of BaDuanJin for health and increasing resistance to chemotherapy; weight management;emphasis on health; and pursuit of healthy lifestyle; (3) opportunity: Facilitators include family environment (e.g., comprehension, support and supervision among family) and create new social links; Barriers include unsupportive family environment and side effects of adjuvant treament; (4) Intervention: Facilitators include using plain and easy-to-understand language, classification guide, and online and offline training pattern; providing professionals knowledge about exercise, diet, and medication; and barriers include the various levels of gym coaches and insufficient exercise; (5) Policy: Facilitators include media publicity(e.g., the promtion on Wuhan mobile cabin hospital and TV); Barriers include medical service(e.g., a little guidance on chemotherapy, rehabilitation, and exercise from their doctor) and social culture, authority, class (e.g., the worry about taking up doctor's working time). Conclusion: Our findings reveal complex factors among individual, intervention, and policy levels corresponding to the facilitation and hindrance of Baduanjin for Chinese BCSs in adjuvant treatment. These factors could be targeted to develop supportive interventions to increase the level and effect of Baduanjin exercise.

(3189) Impact of completing FACE-Q craniofacial module scales on children and young adults with facial differences: an international study

Lucas Gallo, MD, McMaster University, Hamilton, Ontario, Canada; Rakhshan Kamran, BSc(Hon), McMaster University, Hamilton, Ontario, Canada; Charlene Rae, MSc, McMaster University, Hamilton, Ontario, Canada; Karen Wong Riff, MD, PhD, Hospital for Sick Children, Toronto, Ontario, Canada; Anne Klassen, DPhil, McMaster University, Hamilton, Ontario, Canada

Aims: PROMs are increasingly used in clinical care, but little is known about the impact of how patients, particularly children, feel after completing a PROM. The FACE-Q Craniofacial module measures outcomes that matter to patients with craniofacial conditions. Scales contain detailed questions about facial appearance, facial function, health-related quality of life, and adverse effects of treatment. The aim of this study was to investigate the impact of completing the FACE-Q Craniofacial module on patients. Methods: A field-test was conducted for the FACE-Q Craniofacial module that included patients 8 to 29 years of age with a facial difference. Participants were asked 3 questions at the end of the module including: 'Did you like or dislike answering this questionnaire?'; 'Did answering these questions change how you feel about how you look?'; and 'Did answering this questionnaire make you feel unhappy or happy?'. Frequencies were calculated for each question to examine response distribution. Chi-square analysis and one-way ANOVA were used to examine association between both demographic factors and scale scores, and the three impact questions. Results: The sample included 1252 participants, most were female (53.6\%) and were from 9 countries. Most patients responded neutrally to the impact questions: $43.9 \%$ neither disliked or liked questionnaire; $77.6 \%$ felt the same about how they looked after completion; and $73.2 \%$ felt neither unhappy/happy after completion. Negative responses represented a small proportion of patients across all 3 impact questions $(<13 \%)$. Lower scores on Face appearance, Appearance Distress, Psychological, and Social scales were associated with negative responses on all 3 impact questions $(\mathrm{p}<0.001)$. Younger age groups $(8-10,11-13)$ were more likely to respond positively than older age groups (14-17,18-29), who were more likely to respond neutrally to impact questions ( $\mathrm{p}<0.001)$. Conclusion: It is important to understand the impact of completing PROMs with sensitive questions. For the FACE-Q Craniofacial Module, most participants felt neutral about completing the PROM. A small minority of participants reported a negative impact of completing the PROM. It is important to follow-up 
with patients after completing PROMs to address areas of concern in their scale scores; and to ensure that a patient is offered further supports, if necessary.

(3191) Minimal important change in everyday functioning in early Alzheimer's disease: A systematic qualitative investigation

Mark Dubbelman, Alzheimer Center Amsterdam, Department of Neurology, Amsterdam Neuroscience, Vrije Universiteit Amsterdam, Amsterdam UMC, Amsterdam, The Netherlands; Caroline Terwee, Department of Epidemiology and Data Science, Amsterdam UMC, Amsterdam, The Netherlands; Merike Verrijp, Alzheimer Center Amsterdam, Department of Neurology, Amsterdam Neuroscience, Vrije Universiteit Amsterdam, Amsterdam UMC, Amsterdam, The Netherlands; Philip Scheltens, Alzheimer Center Amsterdam, Department of Neurology, Amsterdam Neuroscience, Vrije Universiteit Amsterdam, Amsterdam UMC, Amsterdam, The Netherlands; Sietske Sikkes, Alzheimer Center Amsterdam, Department of Neurology, Amsterdam Neuroscience, Vrije Universiteit Amsterdam, Amsterdam UMC, Amsterdam, The Netherlands

Aims: Everyday functioning is an important outcome for treatment studies of Alzheimer's disease, however, little is currently known about the clinical meaningfulness of changes in functioning. Using a systematic qualitative approach, we aimed to define the minimal important change (MIC) on the Amsterdam Instrumental Activities of Daily Living Questionnaire (A-IADL-Q). Next, we investigated how many memory clinic patients passed the MIC threshold, in an independent cohort. Methods: We invited registrants of the Dutch brain research registry to complete an online survey, based on bookmarking methods described by Cook and colleagues (2017). Vignettes describing difficulties in daily functioning of fictional patients were created using item response theory parameters of the A-IADL-Q. Each respondent viewed seven sets of vignettes, describing the patient's functioning 'one year ago' and 'now'. Respondents indicated whether there was a change in functioning, and, if so, whether they considered the change to have an important impact on daily life. The amount of change varied with each situation, and we investigated both decline and improvement. Individual thresholds were placed at the first vignette set that was rated as showing an important change, and they were averaged over all respondents to obtain the final thresholds. We investigated how many patients who visited a memory clinic for dementia screening passed the MIC threshold on the A-IADL-Q, three, six, nine, and twelve months after the baseline visit. Results: Caregivers ( $\mathrm{N}=1629$, age $62.4 \pm 9.5$ years; $77 \%$ female) completed the MIC survey. Thresholds were established at 2.4 points for decline and +4.7 points for improvement. We applied the MICs to data from 230 patients $(64.3 \pm 7.7$ years; $39 \%$ female). Within one year, 104 patients $(45 \%)$ declined meaningfully, whereas $36(16 \%)$ improved beyond the MIC. Conclusion: Systematic, qualitative appraisal of the clinical meaningfulness of changes is crucial for evaluating possible treatment effects in clinical trials, as well as for monitoring disease progression. In the context of Alzheimer's disease, a larger improvement in functioning was required to be clinically meaningful, as compared to decline. We found that approximately half of unselected memory clinic patients declined meaningfully within one year.
(3192) An updated systematic review on measurement properties of the Patient-Specific Functional Scale

Anupa Pathak, PhD Student, University of Otago, Dunedin, New Zealand; Ross Wilson, PhD, Department of Surgical Sciences, University of Otago, Dunedin, New Zealand; Saurab Sharma, PhD, Neuroscience Research Australia, Sydney, Australia; Yana Pryymachenko, PhD, Department of Surgical Sciences, University of Otago, Dunedin, New Zealand; Daniel Ribeiro, PhD, School of Physiotherapy, University of Otago, Dunedin, New Zealand; Jason Chua, PhD, University of Otago, Dunedin, New Zealand; Haxby Abbott, PhD, Department of Surgical Sciences, University of Otago, Dunedin, New Zealand

Aims: The Patient-Specific Functional Scale (PSFS) is a widely used patient-reported outcome measure in rehabilitation. In 2012, Horn and colleagues conducted a comprehensive review and synthesized findings of 13 studies on measurement properties of the PSFS. Since the publication of the 2012 systematic review, the PSFS has grown in popularity and has additionally been evaluated in new health conditions. Guidelines on how to conduct systematic reviews of PROMs have also since been introduced. In this update, we aimed to systematically review (a) measurement properties of the PSFS (validity, reliability, responsiveness), (b) interpretability, (c) acceptability and feasibility, and (d) current uses of the PSFS using COSMIN guidelines. Methods: We searched 11 databases for published primary articles that either assessed measurement properties of PSFS or used the PSFS. Two independent reviewers screened all records, extracted data, performed risk of bias assessment, and GRADE assessments. We then qualitatively synthesized findings (using median and range) for each measurement property of the PSFS in musculoskeletal and non-musculoskeletal conditions. This study was pre-registered in Open Science Framework. Results: We screened 985 articles, and included 57 articles on measurement properties and 255 articles on the use of PSFS. The PSFS demonstrated sufficient test-retest reliability (28 studies, 1,042 participants) and responsiveness (32 studies, 13,770 participants) in selected musculoskeletal and non-musculoskeletal conditions (very low- to moderate-quality evidence). However, the PSFS had insufficient construct validity as a measure of physical function (21 studies, 2,945 participants: low to moderatequality evidence) since less than $75 \%$ of our hypothesis were met. Median minimum important change (MIC) values ranged from 1 to 3 points. The PSFS was reported to be feasible and acceptable to most participants though some authors reported difficulty with identifying activities. The PSFS was used in 255 studies in participants presenting with 87 unique health conditions, some without prior evidence of validity. Conclusion: The PSFS is an easy to use, reliable, and responsive scale. although the PSFS is commonly grouped with measures of physical function, we found insufficient evidence for this. This suggests that while the PSFS is useful as a measure of clinical improvement, the underlying construct assessed by PSFS remains unclear.

(3193) Health Preference Elicitation Methods in a Rehabilitation Population: A Systematic Review

Prateek Gupta, Masters of Science, Physical Therapy, McGill University, Montreal, Quebec, Canada; Maxime Campbell, Masters of Science, Physical Therapy, School of Physical and Occupational Therapy, Faculty of Medicine, McGill University, Montreal, Quebec, Canada; Melanie Tu, Masters of Science, Occupational Therapy, 
School of Physical and Occupational Therapy, Faculty of Medicine, McGill University, Montreal, Quebec, Canada; Quoc Thai Nguyen, Masters of Science, Physical Therapy, School of Physical and Occupational Therapy, Faculty of Medicine, McGill University, Montreal, Quebec, Canada; Nikki Ow, PhD Rehabilitation Science, Center for Outcomes Research and Evaluation (CORE), Research Institute, McGill University Health Centre; School of Physical and Occupational Therapy, Faculty of Medicine, McGill University, Montreal, Quebec, Canada; Nancy Mayo, Center for Outcomes Research and Evaluation (CORE), Research Institute, McGill University Health Centre; School of Physical and Occupational Therapy, Faculty of Medicine, McGill University, Montreal, Quebec, Canada; Ana Maria Moga, Center for Outcomes Research and Evaluation (CORE), Research Institute, McGill University Health Centre; School of Physical and Occupational Therapy, Faculty of Medicine, McGill University, Montreal, Quebec, Canada

Aims: Health preferences evaluate one's desirability for a health state over another. They are used to identify patients' needs and to allocate healthcare resources. Although different methods exist to elicit such preferences, few studies have investigated their use amongst rehabilitation populations. To close that gap, the literature was systematically reviewed with a twofold aim: 1 . Identify direct healthpreference elicitation techniques used amongst rehabilitation populations 2. estimate the extent to which health elicitation methods affected values for preferences. Methods: A systematic search was conducted on six databases (i.e., MEDLINE, CINAHL, CENTRAL, EMBASE, and PSYCHINFO). Studies were included if the sample was patients receiving physical therapy, occupational therapy, speechlanguage pathology, or chiropractic treatments; study included direct health elicitation methods and were published in English or French until May 2020. The quality of the included articles was appraised using the Appraisal Tool for Cross-Sectional Studies (AXIS) checklist. Results: A total of 61 studies from 20 countries were included. A combination of Standard Gamble (SG) (37.4\%), Time-Trade-Off (TTO) (36.4\%), Visual Analogue Scale (VAS) (25.3\%) and Discrete Choice Experiment (DCE) $(1.0 \%)$ elicitation methods were used to elicit health preferences in rehabilitation population. Within the same conditions, SG scores were reported higher than TTO and VAS. The cardiorespiratory conditions reported mean $\mathrm{SG}=0.698$, $\mathrm{TTO}=$ 0.890 , and VAS $=0.645$; Neurological mean SG 0.757 , TTO $=$ 0.635 and $\mathrm{VAS}=0.573$; and Musculoskeletal mean $\mathrm{SG}=0.754$, $\mathrm{VAS}=0.672, \quad \mathrm{TTO}=0.737$, and $\mathrm{DCE}=0.210$. Most common diagnoses reported amongst all papers included COPD, Spinal Cord Injury, Stroke, and Rheumatoid Arthritis. Conclusion: Within the same rehabilitation conditions, SG yielded the highest scores and VAS the lowest. A meta-analysis is underway to analyze further the utility differences obtained through different elicitation methods. Future research is needed to explore factors that could justify such variability.

\section{(3194) Validation of Computational Sentiment Analysis Methods for Evaluating Free-text Patient Experience Survey Responses}

Robert Fuhrman, University of British Columbia, Vancouver, British Columbia, Canada; Lena Cuthbertson, Providence Healthcare, Vancouver, British Columbia, Canada; Rick Sawatzky, Trinity Western University, Vancouver, British Columbia, Canada; submitted on behalf of "Providence Healthcare"

Aims: Patient experience surveys provide important information for healthcare improvement and accountability purposes. Free-form text responses to open ended survey questions allow patients to disclose issues and concerns, or praise, regarding their healthcare experiences. The efficiency of computational methods can help to address the inherent challenge of analyzing large volumes of unstructured free- text data that would normally require manual hand-coding. The aim of our analysis is to compare computational and manual approaches for evaluating the sentiment of patients' free-form text responses. Methods: We applied an open-source computational sentiment analysis method (VADER -Hutto \& Gilbert, 2014) to a total of 36,423 free-text responses collected from six independent surveys conducted by the British Columbia Office of Patient-Centered Measurement (BCOPCM). Each response was assigned an overall sentiment score on a continuous interval ranging from $[-1,1]$, with -1 indicating highly negative sentiment and 1 being positive. The results of this analysis were compared to hand-labelled sentiment scores, which were assigned on a discrete interval scale on the range $[1=$ best, 4 = worst $]$ for various coded sub-themes present in each response. The hand-coded scores were averaged across themes and rescaled to the range $[-1,1]$ to match the computationally scored scale. Distributions of scores were compared independently for each survey with Wilcoxon signed-rank tests. Results: For all surveys, the distribution of computational and hand-scored sentiment differed significantly ( $p<<0.001$ ). The distributions of computational sentiment scores tend to reflect the general skew of scores obtained by hand (toward positive sentiment scores), while affording greater granularity (See Figure). However, further comparison via empirical cumulative distribution functions suggests a systematic discrepancy where the computational method tends to report more negative sentiment responses than human labelers. Conclusion: Computational sentiment analysis methods can provide a robust means of evaluating the sentiment of patient opinions regarding the quality of care received in clinical settings. Since computational sentiment analysis methods are easily scaled to large volumes of data, they can provide a valuable, and highly efficient, tool for evaluating the sentiment of patients' opinions regarding quality of received care.

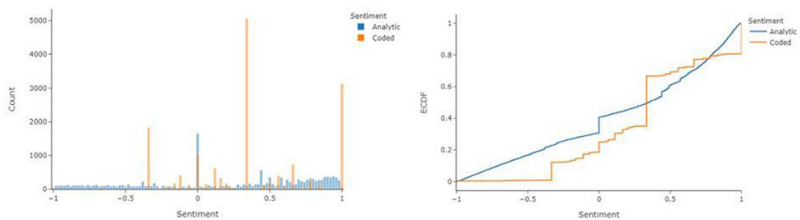

Figure. Distribution of Computational (Analytic) and Hand-coded Sentiment Scores (left) with corresponding Empirical Cumulative Distribution Functions (ECDFs) (right)|

\section{(3195) Assessment of the heterogeneity of DIF using covariates with Rasch family models}

Yseulys Dubuy, UMR INSERM 1246 SPHERE, Nantes, France; Véronique Sébille, UMR INSERM 1246 SPHERE, Nantes, France; Jean-Benoit Hardouin, UMR INSERM 1246 SPHERE, Nantes, France; Myriam Blanchin, UMR INSERM 1246 SPHERE, Nantes, France

Aims: Methods to detect and taking into account differential item functioning (DIF) when analyzing patient-reported outcomes (PROs) data are numerous. However, most of them only enable the detection of DIF considering one covariate. It is a problematic issue when one wants to search for DIF using multiple covariates, as the analysis must be repeated for each covariate. One issue, among others, is that it may lead to the detection of false-positive effects when covariates are correlated). A procedure based on item response theory (named IRTC) has been proposed to incorporate multiple covariate simultaneously, but several limits can be raised, among which: a potential type I error rate above 5\% when no DIF occurs, a questionable and little implemented index to flag items (i.e. the bivariate residuals) and no evaluation of the method in the health research framework (the only simulations available consider many dichotomous items and considerable sample size, which is not representative of PROs data). A simulation study was performed to evaluate and compare the 
performances of two new algorithms inspired by the IRT-C procedure but not relying on bivariate residuals. Methods: Both algorithms were based on Rasch family models. Their performances were evaluated considering different scenarios depending on: the (1) absence or presence of DIF, (2) type and magnitude of DIF, (3) effect of the covariates on DIF or on the latent variable level, and (4) the correlation between the covariates. The performances of the algorithms were assessed regarding the rate of incorrect detection of DIF (when no DIF is simulated), the rates of correct detection of DIF (when DIF is simulated), the DIF assessment, and the bias in the latent variable level estimation. Results: For both algorithms, the rate of incorrect detection of DIF was close to $5 \%$. The DIF effect size and type influenced the rates of correct detection of DIF. Rates of correct detection was higher with increasing DIF effect size. Besides, the algorithm fairly detected and identified uniform differences in the item locations parameters but had more difficulties identifying nonuniform differences. Conclusion: Integrating several covariates during the DIF detection process may allow a better ascertainment of DIF.

\section{(3196) Developing and Implementing an Evidence-based and Patient and Family-informed Program (KidsPRO) to integrate patient-reported outcome measures in pediatrics}

Sumedh Bele, University of Calgary, Calgary, Alberta, Canada; Maria Santana, MPharm, PhD, University of Calgary, Calgary, Alberta, Canada

Aims: Integrating Patient-Reported Outcome Measures (PROMs) in routine clinical care positively impacts healthcare delivery and clinical outcomes. However, in pediatrics, complexities such as, variations in cognitive abilities of patients and involvement of family caregivers in patient care warrants innovative, evidence-based and patient and family informed approaches in developing and implementing eHealth solutions. Thus, we developed an evidence-based and family informed program (KidsPRO), to integrate PROMs into the routine pediatric clinical care. Considering Asthma as the most common chronic condition in pediatric population, we are investigating implementation of KidsPRO in outpatient asthma clinics in Calgary, Canada. Methods: To ensure KidsPRO is a patient and family-informed program, a group of patient partners (pediatric patients aged $8-18$ and their mothers) has been engaged from inception of the project. This multi-phased study used implementation science approaches. The first phase selects an evidence-based (systematic review) and patient-family informed PROM to include in KidsPRO. During the second phase, qualitative interviews were conducted to identify perceived barriers and enablers to the integration of PROMs. Finally, the third phase consists of a survey evaluation of the integration of PROMs using the KidsPRO program. Results: Patient partners were recruited using innovative approaches such as, cartoon videos. They have been engaged in various activities including evidence synthesis, selection of PROM, logo design, codesigning the KidsPRO platform, and development of training modules for KidsPRO. Based on this evidence generated through systematic review and consultation with children and their family caregiver, and stakeholders, PedsQL was chosen as the PROM to be included in KidsPRO system. In second phase, results from qualitative interviews with 17 stakeholders identified the major barrier, lack of integration of KidsPRO with currently used EMR system. Desire to provide patient and family-centered care and ability of PROMs to systematically understand patient's needs were the major enablers. Phase three is currently underway and available results will be presented at the conference. Conclusion: This project adds to growing evidence on the integration of PROMs in pediatric care, including a strong engagement of patient their family caregivers and healthcare system stakeholders from inception using implementation science approaches. Future work includes using a tailored measurement approach.

(3197) Factors influencing health-related quality of life in parents of children born with esophageal atresia in a Swedish-German cohort

Michaela Dellenmark-Blom, RN, PCNS, PhD doctor of Medical Sciences, The Queen Silvia Children's Hospital, Sahlgrenska University Hospital/ Sahlgrenska Academy Gothenburg University, Gothenburg, Sweden; Kate Abrahamsson, MD, Pediatric Surgeon, professor Department of Pediatric Surgery, Queen Silvia Children's Hospital, Sahlgrenska University Hospital \& Institute of Clinical Sciences, Department of Pediatrics, Gothenburg University, The Queen Silvia Children's Hospital, Gothenburg, Sweden; Jens Dingemann, MD, Pediatric Surgeon, Associate professor Center of Pediatric Surgery, Hannover Medical School and Bult Children's Hospital, Hannover, Germany; Stefanie Witt, Pedagogue, Family Scientist, PhD, Post-doc, Department of Medical Psychology, University Medical Center Hamburg-Eppendorf, Hamburg, Germany; Carmen Dingemann, MD, Pediatric Surgeon, Associate professor Center of Pediatric Surgery, Hannover Medical School and Bult Children's Hospital, Hannover, Germany; Linus Jönsson, MD, Pediatric Surgeon, PhD, Department of Pediatric Surgery, Queen Silvia Children's Hospital, Sahlgrenska University Hospital, \& Institute of Clinical Sciences, Department of Pediatrics, Gothenburg University, The Queen Silvia Children's Hospital, Gothenburg, Sweden; Vladimir Gatzinsky, MD, Pediatric Surgeon, PhD, Department of Pediatric Surgery, Queen Silvia Children's Hospital, Sahlgrenska University Hospital \& Institute of Clinical Sciences, Department of Pediatrics, Gothenburg University, The Queen Silvia Children's Hospital, Gothenburg, Sweden; Monika Bullinger, Psychologist, professor Department of Medical Psychology, University Medical Center Hamburg-Eppendorf, Hamburg, Germany; Benno Ure, MD, Pediatric Surgeon, professor Center of Pediatric Surgery, Hannover Medical School and Bult Children's HospitalHannover, Germany; John E Chaplin, Psychologist, Associate professor Institute of Clinical Sciences, Department of Pediatrics, Gothenburg University, The Queen Silvia Children's Hospital, Gothenburg, Sweden; Julia H Quitmann, DipPsych, Associate professor Department of Medical Psychology, University Medical Center Hamburg-Eppendorf, Hamburg, Germany

Aims: Esophageal atresia (EA) is a rare congeital malformation and refers to a discontinuity of the esophagus at birth. Following surgical repair, survival rates of the children exceed $90 \%$, but still EA remains a significant cause of esophageal and respiratory morbidity. Children with EA may suffer from dysphagia, gastrointestinal reflux disease, feeding difficulties, wheeze, chronic cough, and recurrent airway infections. Although it is known that these problems can reduce health-related quality of life (HRQOL) in children with EA, less is understood about the impact on the parents. In order to optimize the child's health and wellbeing, it is crucial also to understand which factors influence their parents' HRQOL. We aimed to identify factors influencing HRQOL in parents of children with EA. Methods: One parent of a child with EA (2-18 years) in 180 families attending three centers in Sweden and Germany answered the PedsQL ${ }^{\mathrm{TM}}$ Family Impact Module (FIM) as the dependent variable, one summary scale which targeted parents' HRQOL (0-100 scale score, with higher scores indicating better HRQOL). The independent variables were proxy-reported generic child HRQOL (PedsQL 4.0), child's current symptoms, school situation and parent/family characteristics together with clinical data from medical records. Regression analyses were used to evaluate factors influencing the Parent HRQOL Summary 
Score. The significant level was $\mathrm{p}<0.05$. Results: Stepwise multivariable regression analysis showed a multifactorial model of the Parent HRQOL Summary Score $(\mathrm{R} 2=0.44)$, with independent factors being the child's overall generic HRQOL,child's schoolabsence $\geq 1 /$ month, a parent with a doctor-diagnosed disease and a parent with no university/college education, $p<0.05$. Logistic regression analysis showed that an increased number of different symptoms in the child the preceding four weeks lowered the Parent HRQOL Summary Score; however, feeding $(\beta \mathrm{O}=83.5, \beta 1=-4.6$, $\mathrm{R} 2=0.25)$ and digestive symptoms $(\beta \mathrm{O}=85.0, \quad \beta 1=-9.4$, $\mathrm{R} 2=0.24)$ explained more in the variation of scores than the child's respiratory symptoms $(\beta \mathrm{O}=81.6, \quad \beta 1=-3.6, \quad \mathrm{R} 2=0.09)$, $\mathrm{p}<0.0001$. Conclusion: There are multifactorial explanations to HRQOL in parents of children with EA. Knowledge of these psychosocial and clinical factors influencing parents' HRQOL is central in clinical contexts such when caring of parents with a newborn child with EA as well as to families whose children attend follow-up care.

(3198) Tulppa-rehabilitation may have a positive effect on the quality of life of coronary artery disease patients: preliminary results

Piia Pietilä, MA Psych, Rehabilitation Foundation, Helsinki, Finland; Ilja Salakka, MA Psych, Rehabilitation Foundation, Helsinki, Finland; Marjaana Lahti-Koski, PhD, Finnish Heart Association, Helsinki, Finland; Anna-Mari Hekkala, PhD, Finnish Heart Association, Helsinki, Finland; Anneli Luoma-Kuikka, MSc, Finnish Heart Association, Helsinki, Finland; Erja Poutiainen, PhD, Rehabilitation Foundation, Helsinki, Finland

Aims: It is important to support coronary artery disease (CAD) patient's quality of life (QoL). Tulppa is a Finnish group-based secondary prevention program for patients with vascular diseases provided at primary health care centers. This study explores the effects of Tulppa on participant's QoL and exhaustion. Methods: Study group (Tulppa participants, $\mathrm{n}=158$ ) was collected from three Finnish health districts. Tulppa rehabilitation includes e.g. health education, peer discussions and physical exercises in 8-10 weekly $3-\mathrm{h}$ group sessions and two follow-up sessions ( 6 and 12 months). Control group $(\mathrm{n}=142)$ included patients living in two health districts that do not provide Tulppa rehabilitation. All participants had diagnosed CAD. QoL was assessed with RAND-36 scale and exhaustion with Maastricht Vital Exhaustion Questionnaire (MQ). Both questionnaires were filled at the baseline and 6 months. Changes in RAND-36 and MQ scores were analysed with repeated measures ANOVA, adjusted by age and education. Baseline differences between the groups were analysed with t-test. Results: At the baseline, the study group reported their QoL lower than the control group (all RAND-36 subscales $\mathrm{p}<0.001-0.05)$. Also, when compared to the Finnish population norms, the QoL of study group was significantly $(\mathrm{p}<0.001)$ lower in 7 subscales of RAND-36 while in control group it was lower only in 4 subscales $(p<0.001-0.04)$. There was a significant time $x$ group interaction in MQ $(\mathrm{p}=0.022)$ and RAND-36 Energy/fatigue subscale $(\mathrm{p}=0.035)$. A positive change in both measures between baseline and 6 months was found in the study group $(\mathrm{p}<0.001$ and $\mathrm{p}=0.028$, respectively), but not in the control group (both measures ns). In other RAND-36 subscales no statistically significant interactions were observed. However, when study and control groups were analysed one by one, the study group assessed their physical functioning $(p=0.022)$ and social functioning $(p=0.022)$ to be better after the intervention (compared to the baseline). In control group there were no within group changes. Conclusion: The results confirm that CAD patients have lower QoL compared to Finnish population. Tulppa rehabilitation may have a positive effect on QoL, especially by increasing energy and reducing exhaustion.

\section{(3201) Scoping review to inform PRO thresholds for use in research and clinical practice: a traumatic brain injury case study}

Christel McMullan, PhD, Centre for Patient Reported Outcomes Research, Institute of Applied Health Research, University of Birmingham; NIHR Surgical Reconstruction and Microbiology Research Centre (SRMRC), University of Birmingham, Birmingham, UK; Birmingham Health Partners Centre for Regulatory Science and Innovation, Birmingham, UK, Birmingham, UK; Sally Bradshaw, Centre for Patient Reported Outcomes Research, Institute of Applied Health Research, University of Birmingham, Birmingham, UK; Ameeta Retzer, Centre for Patient Reported Outcomes Research, Institute of Applied Health Research, University of Birmingham, Birmingham, UK; Anita Slade, Centre for Patient Reported Outcomes Research, Institute of Applied Health Research, University of Birmingham, Birmingham, UK; Antonio Belli, Institute of Inflammation and Ageing, University of Birmingham, Birmingham, UK; Melanie Calvert, Centre for Patient Reported Outcomes Research, Institute of Applied Health Research, University of Birmingham, Birmingham, UK; Grace Turner, Centre for Patient Reported Outcomes Research, Institute of Applied Health Research, University of Birmingham, Birmingham, UK

Aims: Patient-reported outcomes are increasingly used in research and clinical practice to identify early deterioration of symptoms; resulting in a need to identify clinically relevant thresholds to trigger a response.The aim of this review was: (i) to identify how thresholds for three commonly used PRO measures, GAD-7, PHQ-9 and PCL-5, were determined and used in traumatic brain injury (TBI) and (ii) identify potential key principles for threshold selection for other tools and clinical areas. Methods: Using a formal scoping review methodology we systematically searched Medline, Embase, PsycINFO, CINAHL, AHMED, OpenGrey and Google databases for articles where GAD-7, PHQ-9 and PCL-5 were used in a TBI population. In addition, publisher manuals, national level and professional body guidelines including the measures in any population were identified. Data screening and extraction were undertaken by two independent reviewers. The review was registered on ResearchRegistry, including search and screening strategy, explicit inclusion/exclusion criteria, data extraction, and appraisal plan. Results: A total of 1,011 publications were screened and 64 studies included in the review. A total of three publisher manuals and 12 clinical guidelines were identified and also included. Although the publisher manuals for all three PROMs provided clear information on thresholds, GAD-7 and PHQ-9 are not specific to the TBI population. All the guidelines mentioned the tools but did not always provide further information on how to use the thresholds. In the single studies, there was a little variation in the thresholds used. In addition, there was limited information regarding the reasons for using these particular thresholds. The single studies also provided very limited information on reliability, validity, bias, and limitations of the tools. Implications for threshold review in other clinical disciplines will be presented. Conclusion: In order to improve the use of these tools within the field of TBI, authors should be clearer about their motivations behind selecting specific threshold. In addition, more information on applicability to the TBI population as well as more information on the reliability, validity, bias, and limitations of the tools with a TBI population is required. Finally, consideration should be given to applying these thresholds to other tools and clinical areas. 


\section{(3202) Optimizing asthma care through physician and patient} engagement in a remote monitoring platform

Kathy Lasch, Ph.D., Patient Voice Matters Consulting, LLC, Newton, Massachusetts, USA; Jyotsna Mehta, MS, Keva Health, Newton, Massachusetts, USA; Paul Helmuth, MD, Timedoc, Springfield, Massachusetts, USA; Dhrumil Shah, MD, CIO, Compass Health, Springfield, Massachusetts, USA; Robert Michelson, MD, Temple University, Philadelphia, Pennsylvania, USA

Aims: COVID 19 ushered in the implementation of many forms of digital health aimed to improve patient care between visits for several diseases and chronic conditions. Examples include electronic patientreported symptom monitoring during routine cancer care, technologyenabled diabetes self-management education and support. Very few of these pilot projects provide a direct electronic link between providers and patients to engage both. This study assesses the feasibility of a patient-facing mobile app with a provider-facing portal to improve care for patients with Asthma Methods: 100 eligible patients were asked to participate in a 3-month pilot study. Patient consent was obtained. At-home spirometers were provided to document peakflow readings that sync directly with the mobile application using Bluetooth technology. A licensed respiratory therapist provided a 30 min training via video call to demonstrate use of the spirometer. Twenty-five of the patients, recruited through in-app messaging, were asked to participate in a qualitative in-depth interview. An interview guide was developed to guide the conversation and allow patients to provide spontaneous feedback to capture the feasibility, usefulness, and importance of the functions within the application. Objective patient and clinician engagement data were collected through the platform, e.g., number of patients who engage with the app, percentage of patient who fill out a validated asthma questionnaire, number of provider logins, and duration of use by physician/nurse. Results: Patients tended to be engaged when they are feeling well as well as when they are not, using the check-in feature in the app. Patients were very receptive to using the app, device and felt that it was an easy way to track their asthma. The treating physician was able to see the remote patient monitoring report to allow him/her to optimize care for the patient and receives alerts when the patient condition worsens. Conclusion: Interventions that allow both the physician and patient to be engaged in remote care in between visits to the doctor can be helpful for managing chronic illnesses. Digital platforms for remote monitoring should focus on both provider and patient engagement to optimize care through meaningful interpretation of data collected via app and devices.

\section{(3203) Variation in depression among patients undergoing different bariatric procedures: A systematic review and meta- analysis}

Alyssa Budin, Monash University, Melbourne, Australia; Priya Sumithran, Melbourne University, Melbourne, Australia; Wendy Brown, Monash University, Melbourne, Australia

Aims: The rise of bariatric (weight loss) surgery as a mainstream treatment in the battle against climbing worldwide obesity rates has facilitated the recognition of previously underappreciated mental health disturbances associated with both obesity and bariatric surgery. Depression is a well-established comorbidity of obesity that has been subsequently recognised as a significant adverse outcome following bariatric surgery. Understanding the presentation of depressive symptoms following different bariatric procedures, and over the longterm course of patient recovery, is vital to providing meaningful, long-term, patient-centred care. This review aims to identify differences in patient-reported depression outcomes between those undergoing different bariatric surgical procedures for the treatment of obesity. Methods: Medline, Embase, Emcare, PsycINFO, CINAHL, and CENTRAL databases were systematically searched from database inception to March 24th 2021 for articles that evaluated depression before and after a bariatric procedure using a validated, patient-reported outcome measure. An additional manual search was conducted in the reference list of identified articles and reviews. All medically acceptable bariatric procedures were included, with no limits on the age or BMI of included participants. Results: A total of 86 publications describing patient-reported assessment of depression symptoms following bariatric procedures for the treatment of obesity have been included in this comprehensive review. The findings are organised by the depression instruments used and stratified by specific bariatric procedure, as well as by follow-up time points. Statistical analysis using standard mean difference will facilitate the evaluation of patient-reported depression outcomes between various procedures and indicate how this may fluctuate over time. Results of this review and meta-analysis will be presented for the first time at the 2021 ISOQOL Annual Conference. Conclusion: This review represents a first step towards understanding whether the unique physiological effects of different surgical techniques produce observable differences in patient outcomes. This will inform future research, as well as clinical practice, as the adverse psychological effects of various surgical techniques can be considered to ensure appropriate and informed patient care.

(3204) Knowledge, use and attitudes of healthcare professionals towards patient-reported outcome measures (PROMs) at a comprehensive cancer center

Cinzia Brunelli, PhD, Palliative Care, Pain Therapy and Rehabilitation Unit - Fondazione IRCCS Istituto Nazionale dei Tumori, Milan, Italy; Emanuela Zito, Information and Communication technology Unit - Fondazione IRCCS Istituto Nazionale dei Tumori, Milan, Italy; Sara Alfieri, PhD, Clinical Psychology Unit - Fondazione IRCCS Istituto Nazionale dei Tumori, Milan, Italy; Claudia Borreani, Clinical Psychology Unit -

Fondazione IRCCS Istituto Nazionale dei Tumori, Milan, Italy; Augusto Caraceni, Palliative Care, Pain Therapy and Rehabilitation Unit - Fondazione IRCCS Istituto Nazionale dei Tumori, Milan, Italy; Anna Roli, Quality, education and Data Protection Unit, Fondazione IRCCS Istituto Nazionale dei Tumori di Milano, Milan, Italy; Giovanni Apolone, Scientific Directorate - Fondazione IRCCS Istituto Nazionale dei Tumori, Milan, Italy

Aims: Despite evidence of the positive impact of routine assessment of patient-reported outcome measures (PROMs), their systematic collection is not widely implemented in cancer care. This survey was aimed at assessing knowledge, use, and attitude about PROMs among health care professionals (HCP) working in oncology. Methods: A 50 items web-survey was administered to 992 HCPs in an Italian comprehensive cancer centre. The survey investigated which PROMS are known and used, as well as HCPs' opinions about advantages and drawbacks in routine PROM assessment, including electronic assessment (e-PROMs). Likert scales were used to assess knowledge and use, while 0-4 numerical rating scales were used for opinions (higher scores indicate higher agreement). Linear and logistic regression models were used for association analyses. Results: 511 of 992 invited HCPs $(52 \%)$ provided analyzable responses. $68 \%$ were women, $46 \%$ were nurses and $42 \%$ physicians, and $52.5 \%$ had $>20$ years seniority. The average number of PROMs known was six among 17 proposed. All proved to be under-used $(<28 \%)$ except unidimensional and multidimensional pain scales (77\% and $36 \%$ ). Respondents expressed an overall positive attitude towards PROMs, with strengths outweighing weaknesses (mean overall scores 3.6 and 2.9 , respectively, on a $1-5$ scale). $67 \%$ of respondents preferred 
electronic collection over paper and pencil. Profession was associated with knowledge and use (physicians reported knowing more PROMs than other professionals) and with a preference for electronic collection (nurses were less likely to prefer the electronic format than physicians). Senior HCPs were slightly more critical about both PROMs and electronic administration. Conclusion: The results of this survey indicate an acceptable level of knowledge of common PROMs tools but low utilization in practice. However, based on a generally positive attitude of HCPs, routine implementation ePROMs can be promoted as long as adequate resources and training are provided.

\section{(3205) Happiness for Improvement of Premature and Parental Outcome (HIPPO): determining parental stress during and directly after NICU admission (study protocol)}

Marlotte Koopen, Amsterdam UMC, Amsterdam, The Netherlands; Naomi Meesters, Erasmus Medical Center, Rotterdam, The Netherlands; Hedy van Oers, Amsterdam UMC, Amsterdam, The Netherlands; Gerbrich van den Bosch, Erasmus Medical Center, Rotterdam, The Netherlands; Maria Luisa Tataranno, University Medical Center Utrecht, Utrecht, The Netherlands; Wes Onland, Amsterdam UMC, Amsterdam, The Netherlands; Debbie Nuytemans, Amsterdam UMC, Amsterdam, The Netherlands; Willem de Boode, Radboud University Medical Center, Nijmegen, The Netherlands; Peter Dijk, University Medical Center Groningen, Groningen, The Netherlands; Frank Schuerman, Isala Women and Children's Hospital, Zwolle, The Netherlands; Marlou Kouwenberg-Raets, Maastricht University Medical Center, Maastricht, The Netherlands; Christ-jan van Ganzewinkel, Máxima Medical Center, Veldhoven, The Netherlands; Enrico Lopriore, Leiden University Medical Center, Leiden, The Netherlands; Irwin Reiss, Erasmus Medical Center, Rotterdam, The Netherlands; Anton van Kaam, Amsterdam UMC, Amsterdam, The Netherlands; Manon Benders, University Medical Center Utrecht, Utrecht, The Netherlands; Monique van Dijk, Erasmus Medical Center, Rotterdam, The Netherlands; Sinno Simons, Erasmus Medical Center, Rotterdam, The Netherlands; Lotte Haverman, Amsterdam UMC, Amsterdam, The Netherlands

Aims: Premature infants admitted to a Neonatal Intensive Care Unit (NICU) are exposed to painful and stressful medical procedures with long-lasting effects. Former studies mainly focused on the effects of stress on the neonate; however, more extensive research on parental stress is necessary as this influences the parent child relation and outcomes of the child. Therefore, the national multicenter observational cohort 'HIPPO study' is initiated to better understand the role of parental stress during preterm birth, NICU admission, and after discharge. This abstract describes the 'parental stress' study protocol for the HIPPO study. Methods: Parents of preterm infants (aimed $\mathrm{N}=400$ ) born at a gestational age of $<29$ weeks and admitted to a Dutch NICU are eligible. All 10 NICUs will collect data for 12 months; start varied from July 2020 to March 2021. Parent couples are asked to complete Parent Reported Outcome Measures (ParROMs) using the KLIK PROM portal (www.hetklikt.nu) at three time points during and after NICU admission: 8-14 days after birth (T0), 28-34 days after birth (T1) and around expected due date (T2). Parents' answers at T0 are being discussed with them by a NICU psychosocial staff member. Constructs measured are: Post Traumatic Stress Disorder (PCL-5), depression (PROMIS depression CAT), parental distress (Distress Thermometer for Parents) and NICU environmental stressors (PSS:NICU). Medical outcomes of the child and sociodemographic data are collected. Results: The main outcomes are: (1) Levels of PTSD, depression, and parental distress at T0, T1, and T2 and (2) associated variables are identified. We expect data inclusion to be completed in March 2022. Conclusion: This study provides insight into short-term parental consequences of preterm birth and NICU admission and sheds light on which parents should receive additional support during admission. Additional follow-up measurements at 6 (T3) and 12 (T4) months corrected age will be added for long term outcomes and parental outcomes will be related to child outcomes as well.

\section{(3206) Feasibility of a patient-centered method to determine} meaningful change in pain intensity within cancer

Salene Jones, PhD, MA, Fred Hutchinson Cancer Research Center, Seattle, Washington, USA; Joseph Unger, PhD, Fred Hutchinson Cancer Research Center, Seattle, Washington, USA

Aims: Current methods of determining minimally important differences (MID) in patient-reported outcomes do not account for each individual patient's values and preferences. This study tested the feasibility of having cancer patients define for themselves what constituted a meaningful change in pain intensity, a method we have termed Precision PROs. Methods: Adults with cancer and pain in the USA $(n=231)$ were recruited to complete an electronic survey. Participants completed the 3-item PROMIS Pain Intensity measure and were then given their scores with an explanation of what the score meant. Participants then defined their own pain MIDs by indicating the amount of point change that would be needed to be considered a meaningful increase or decrease in pain intensity. Participants also answered three story problems assessing their understanding of the MID concept. A subset of participants $(n=167)$ completed the survey a second time, one to two weeks later. Results: $97 \%(n=223)$ of the sample were able to define an individually meaningful decrease in pain intensity. 98\% $(\mathrm{n}=226)$ of the sample were able to define an individually meaningful increase in pain intensity. On the story problems assessing MID understanding, 72\% $(\mathrm{n}=166)$ answered all three correctly and an additional $26 \%(\mathrm{n}=60)$ answered two of three correctly. The intraclass correlations for the MIDs between the two surveys were $0.72(95 \%$ CI $0.62,0.80)$ for a decrease in pain and 0.58 (95\% CI $0.42,0.69)$ for an increase in pain. Using the individual MID, $32 \%$ of the sample experienced a meaningful change in pain intensity between the two surveys whereas $24 \%$ experienced a meaningful change using a distribution-based MID (X2 = 165.36, $\mathrm{p}<0.001$ ). Conclusion: This study showed that people with cancer may be able to define an individually meaningful difference in pain intensity and that the concept was understandable to most participants. Further studies are needed to compare the individual MID to current methods. Pending further validation, this Precision PRO method could be used in measurement-based care to monitor pain treatment or in clinical trials to define who responds to treatment.

(3207) The development of a mHealth tool for children with longterm illness to enable person-centred communication: a co-design approach

Angelica Wiljén, phd-student, University of Gothenburg, Borås, Sweden; John Chaplin, Associate professor University of Gothenburg, Gothenburg, Sweden; Vanessa Crine, master student, University of Gothenburg, Gothenburg, Sweden; William Jobe, University West, Trollhättan, Sweden; Ensa Johnson, University of Pretoria, Johannesburg, South Africa; Katarina Karlsson, University of Borås, Borås, Sweden; Tomas Lindroth, University of Gothenburg, Gothenburg, Sweden; Annelie Schwarz, Södra Älvsborgs Hospital, Borås, Sweden; Margaretha Stenmarker, Ryhov county hospital, Jönköping, Sweden; Gunilla Thunberg, DART, Gothenburg, Sweden; Joakim Öhlen, professor University of Gothenburg, Gothenburg, Sweden; Stefan Nilsson, Assiciate professor University of Gothenburg, Gothenburg, Sweden 
Aims: Children with long-term illness experience symptoms that negatively affects their daily life, this is underreported in healthcare. Despite existing mHealth tools, few are based on theoretical framework or supported by scientific knowledge. Incorporating universal design ensures that all may benefit from the design and that personcentered communication is facilitated. Our study aimed to develop a person-centred communication support mHealth tool for children (i.e., Pictorial support in person-centred care for children; PicPecc) based on universal design principles for communicating symptoms during long-term illness. Methods: Various stakeholder groups (children with long-term illness; parents; healthcare professionals; developers) were involved in the co-design process. The development process included four phases: (i) interviews with 7 children, 8 parents, and 19 healthcare professionals to determine needs and wishes for support, (ii) workshop with 19 researchers, (iii) workshop with developers, and (iv) interviews with 10 children, 9 parents, and 21 healthcare professionals to evaluate the mock-up (prototype) of the developed mHealth tool. Data were synthesised using interpretive description. Results: Children with long-term illness need to address symptoms such as fatigue, anxiety, pain and nausea. Fatigue and anxiety may be overlooked, and therefore, an easy-to-use tool are needed to facilitate communication. Three common aspects were constructed; gap between perceived and received support, need for an easy tool to assess symptoms and to facilitate communication, as well as mapping the journey to facilitate recall. A need for support when dealing with psychosocial issues were expressed by parents and children. The parents were concerned that anxiety was too alarming to use, the children, however, did not react to the word. Researcher highlighted the importance of easy-to-use mHealth tool for children to communicate symptoms beyond pain. The developers highlighted practical and logistical implications offered by other stakeholders and proposed best options for the development of the tool. Conclusion: PicPecc, demonstrated the capacity to provide support when dealing with multiple symptoms and conditions. PicPecc opens a dialogue between the child and the healthcare professionals, and it addresses symptoms that may otherwise be overlooked. Future research includes usability testing and evaluation in hospitals as well as in a home care setting.

\section{(3208) Cardiff Acne Disability Index (CADI): appraising the relevance of a legacy quality of life measure}

Yasmina Abdelrazik, Medical Student, Division of Infection and Immunity, Cardiff University School of Medicine, Cardiff, UK; Faraz Ali, Medicine, Division of Infection and Immunity, Cardiff University School of Medicine, Cardiff, UK; Sam Salek, Medicine, School of Life and Medical Sciences, University of Hertfordshire, Cardiff, UK; Andrew Finlay, Medicine, Division of Infection and Immunity, Cardiff University School of Medicine, Cardiff, UK

Aims: The Cardiff Acne Disability Index (CADI) is a short five-item questionnaire designed to measure the quality of life (QoL) of teenagers and young adults with acne. It is important for clinicians to fully understand the extent of a patient's QoL impairment to inform treatment decisions. The CADI has been used globally and is one of the five most commonly used acne instruments. 1 This review aims to appraise all published data regarding the clinical and research experience of the CADI, as well as its psychometric properties and validation into a single reference source for researchers and clinicians who wish to assess the QoL impact of acne. Methods: A literature search was conducted using Medline via Ovid, PubMed, EBSCOhost, Web of Science, and Scopus to identify all studies that used the CADI from 1992 until September 2020. The search terms were 'Cardiff Acne Disability Index' or 'CADI' and 'acne'. All full articles in the English language were included. Ninety-six clinical studies were identified and analysed. Results: The CADI has been used in 44 different countries, including four multinational studies. It has undergone full cross-cultural adaptation in nine languages and has 25 validated translations. Twenty-nine therapeutic interventions have used CADI, demonstrating its responsiveness to change and its ability to discriminate between treatments of different effectiveness. Fourteen studies have assessed reliability, fifty-seven studies have demonstrated aspects of its validity and five studies have investigated its dimensionality. There is evidence of high internal consistency, test-retest reliability, responsiveness to change and significant correlation with objective measures. The minimal clinically important difference (MCID) and validated "band descriptors" have not yet been reported. Conclusion: The CADI is a short, practical and effective measure to assess acne related QoL impairment, contributing to holistic medical practice. Question two of the CADI has been rephrased to ensure that it is an appropriate measure for all patients. Further investigation with regard to score banding, and the MCID is needed to assist interpretation of CADI scores for both clinical and research purposes. Finally, validation of CADI delivery by app or over the phone should be carried out.

\section{References \\ 1 Smith $\mathrm{H}$, Layton AM, Thiboutot D et al. Identifying the Impacts of Acne and the Use of Questionnaires to Detect These Impacts: A Systematic Literature Review. American Journal of Clinical Dermatology 2020; https://doi.org/10.1007/s40257- 020-00564-6.}

(3209) Reliability and validity of Comprehensive Score for Financial Toxicity (COST) among cancer patients undergoing radiation therapy in India: a cross-sectional pilot study

Mukhtar Ahmad Dar, PhD (pursuing), National Institute of Pharmaceutical Education and Research (NIPER), Hajipur, Patna, India; Richa Chauhan, MBBS, DNB, Sr. Consultant Department of Radiotherapy, Mahavir Cancer Sansthan and Research Centre (MCSRC), Patna, Patna, India; Sameer Dhingra, PhD, Associate professor Department of Pharmacy Practice, National Institute of Pharmaceutical Education and Research (NIPER), Hajipur, Patna, India

Aims: Besides physical toxicity cancer care imposes significant financial distress referred to as financial toxicity (FT). Researchers have associated FT among cancer patients with clinical outcomes like mortality, negative quality of life and non-adherence. Currently, no reliable tools are available for assessing FT among cancer patients in India. The aim of this pilot study was to test the reliability and validity of the Comprehensive Score for Financial Toxicity (COST) questionnaire among cancer patients undergoing radiation therapy in India. Methods: This cross-sectional pilot study was conducted among Head \& Neck cancer patients who have completed the radiation therapy either as a stand-alone or part of a multimodal treatment regimen. The reliability COST measure was assessed using Cronbach's alpha coefficient. The underlying construct of COST was verified by Exploratory Factor Analysis (EFA). EFA was performed based on Kaiser-Meyer-Olkin (KMO) and Bartlett's test. Results: Based on the inclusion and exclusion criteria, the COST questionnaire was administered to 29 patients using interview method after written informed consent. The COST measure demonstrated excellent reliability with Cronbach's $\alpha$ of 0.92 . A KMO of 0.87 verified the sample adequacy and a p-value of $<0.001$ on Bartlett's sphericity test indicated that the strength of the correlation between 11 COST items was good to perform the EFA. Parallel analysis technique identified one factor on scree plot with eigenvalue of 6.21 explaining $56.5 \%$ of the variance by non-rotated solution. All the factor loadings in the onefactor model were $>0.3$ (range 0.35-0.97). The factor loadings indicated that the underlying construct can be considered as a one- 
factor domain as intended by the original COST development study. However, the Chi-square goodness of fit test revealed the one-factor model did not adequately depict the data. However, the results were consistent with the construct obtained in the original scale development study. Conclusion: This pilot study demonstrated excellent reliability and validity of COST for measuring financial toxicity among head and neck radiation oncology patients. Further studies are warranted in different cancer subsites and treatment modalities across various cancer centres in India. The clinical implications of financial toxicity including impact on Quality of Life (QoL) needs to be evaluated.

(3210) Dimensions of health and wellness individuals with Multiple Sclerosis identify as essential for improving quality of life

Erin Faraclas, PhD, DPT, Massachusetts College of Pharmacy and Health Sciences, Worcester, Massachusetts, USA; Angela Merlo, $\mathrm{PhD}$, Eastern Washington University, Spokane, Washington, USA; Jeffery Lau, PhD, Rocky Mountain University, Provo, Utah, USA; Jeff Lynn, PhD, Slippery Rock University, Slippery Rock, Pennsylvania, USA

Aims: Aims: 1.Identifying and describing the health and wellness needs individuals with relapsing-remitting multiple sclerosis (RRMS) report as essential to their QOL. 2. Investigate why these needs are essential to the individual's overall health and wellness 3 . Identify the perceived barriers to meeting these identified needs. Methods: Methods: Adults with a self-reported diagnosis of RRMS were recruited for this explanatory sequential mixed-method study guided by the phenomenological approach. Study participants $(n=120)$ completed the SF-36v2 survey along with demographic information. One-sample z-tests were completed for all subscales and component means. Semi-structured interviews were then conducted until data saturation was reached $(n=15)$. Interview data were analyzed using a thematic content analysis approach. Results: Results: All values of $\mathrm{z}$ were statistically significant, $p<0.01$. Social function and physical function subscales and the mental health component score had the lowest mean scores compared to the general population. A first stage depression screen revealed that $49 \%$ of the surveyed population were at risk for depression, compared to $18 \%$ in the general population. For years since MS diagnosis, stratification of data revealed $61 \%$ of the ' $0-3$ years since diagnosis' group was at risk for depression. Lifestyle behaviors and social engagement followed as the next most frequent themes emerging from the data. While a decrease in physical function is prevalent in the RRMS population, addressing this was a low priority for optimizing QOL. Conclusion: Conclusions: Overall, the mental health of individuals with RRMS was shown to influence QOL as much as physical health. However, early in the disease course (0-3 years), mental health affected QOL more than physical health. Mental health and lifestyle behaviors were perceived as significant contributors to QOL and the areas reported as having the greatest need. Access to dietary information, exercise guidance, and general education about living with MS were also identified as essential factors to improving QOL. Individuals with RRMS perceive mental, emotional, and social health as essential components to their QOL.

\section{(3211) Comparison of PROPr and EORTC QLU-C10D in breast cancer and fibroadenoma patients}

Christoph Paul Klapproth, M.D., Charité University Medicine, Berlin, Germany; Felix Fischer, PhD, Department of Psychosomatic Medicine, Center for Internal Medicine and Dermatology, Charité Universitätsmedizin Berlin, Germany, Berlin, Germany; Matthias
Rose, PhD, Department of Psychosomatic Medicine, Center for Internal Medicine and Dermatology, Charité -

Universitätsmedizin Berlin, Germany, Berlin, Germany; Anna Maria Hage, Department of Gynecology with Breast Cancer, Charité Universitätsmedizin Berlin, Germany, Berlin, Germany; Karsten Maria Margarete, M.D., Department of Gynecology with Breast Cancer, Charité - Universitätsmedizin Berlin, Germany, Berlin, Germany

Aims: The PROMIS Preference score (PROPr) and the EORTC QLUC10D are new health state utility (HSU) scores for Quality-adjusted life years (QALY) in cost-effectiveness analyses. Both are more comprehensive than existing HSU scores including additional health domains such as fatigue and sleep disturbance. The PROPr was validated in rheumatological patients and the QLU-C10D in patients with myelodysplastic syndrome. Validation for other conditions is still pending. The aim of this study is to validate both scores in a sample of breast cancer and fibroadenoma patients. Methods: We collected PROPr and QLU-C10D from 291 female patients 90 days after either chemotherapy, or breast conserving surgery, or mastectomy in the outpatient clinic of the breast cancer center at Charite University Medicine Berlin between June 2018 and April 2021. We assessed both scores' convergent and known-groups construct validity, agreement, and ceiling and floor effects. Results: The mean PROPr $(0.43,95 \%$ CI $0.41 ; 0.46)$ and the mean QLU-C10D $(0.71$, $95 \%$ CI $0.69 ; 0.74)$ show systematic bias $(0.28,95 \%$ CI $0.27 ; 0.30)$ and moderate agreement (ICC $0.46,95 \%$ CI $0.32 ; 0.57$ ). The Pearson correlation coefficient was 0.83 (95\% CI $0.79 ; 0.86)$. Construct validity for age, treatment, marital status, and education was shown in our sample. The QLU-C10D showed ceiling effects, while the PROPr showed floor effects. Conclusion: Both scores have good psychometric properties in a sample of breast cancer patients. Nevertheless, both scores generally define HSU and in particular "full health" differently. So, they should not be used as equivalent for QALY in cost-effectiveness analyses.

(3212) The theoretical model of healthcare factors influencing Quality of Life in Cancer Survivorship (MoHaQ-CS)

Amanda Drury, $\mathrm{PhD}$, University College Dublin, Dublin, Ireland; Sheila Payne, PhD, Lancaster University, Lancaster, UK; Anne-Marie Brady, PhD, Trinity College Dublin, Dublin, Ireland

Aims: Follow-up care and quality of life (QoL) are important consequences of cancer survivorship. There is limited empirical evidence to support the potential impact of healthcare experiences upon colorectal cancer (CRC) survivors' QOL. Building on the Ashing-Giwa Contextual Model of Health-Related Quality of Life (2005), we propose the theoretical Model of Healthcare factors influencing Quality of Life in Cancer Survivorship (MoHaQ-CS), which depicts the factors directly and indirectly mediating the impact of healthcare experiences upon QoL outcomes among CRC survivors. Methods: The MoHaQ-CS was developed following an integrated literature review (Phase 1), and a sequential explanatory mixed-methods study involving CRC survivors 6-60 months after diagnosis (Phases 2 and 3). In Phase 1, the variables influencing QoL among CRC survivors were mapped to the Contextual Model of Health-Related QoL. Phases 2 (Survey) and 3 (Semi-structured interviews) aimed to identify factors directly and indirectly influencing the impact of healthcare experiences upon CRC survivors' QoL. Results: The MoHaQ-CS incorporates the five quantitative factors which predicted CRC survivors' QoL (Insurance Status, Social Difficulties, Continuity of Care, Use of Cancer Support and Tumour Status). Qualitative findings extend understanding of the statistical factors influencing QoL. The experience of chronic symptoms may create unmet information and supportive care needs, which mediate survivors' perceptions of access 
to, support from and continuity of healthcare, in turn affecting survivors' QoL. CRC survivors sought support beyond healthcare services to Bridge the Gap of Unmet Need, through a Network of Supports. However, Supports were dependent upon the cultural context in which the individual resided and received healthcare, social stigmas about the discussion of chronic effects, difficulties discerning the trustworthiness of online resources, a lack of local cancer support services specific to CRC, or belief that cancer support was not required hindered the accessibility of support beyond the healthcare system. Conclusion: Healthcare experiences may have a significant influence on QoL outcomes among cancer survivors. The MoHaQ-CS specifically identifies multiple healthcare-related factors which may directly and indirectly impact QoL outcomes. This framework may support more in-depth empirical research to enhance understanding of the interactions between healthcare experiences and QoL outcomes.

(3213) Variations in chronic pain intensity and physical function by age and sex for patients with knee osteoarthritis

Ezinne Ekediegwu, B.M.R (Hons.), M.Sc(Hons.), Nnamdi Azikiwe University, Enugu, Nigeria; Adesola Odole, PhD, M.Sc, B.PT, University of Ibadan, Ibadan, Nigeria; Nelson Ekechukwu, PhD, MSc, BMR, University of Nigeria, Nsukka, Enugu, Nigeria; Chigozie Uchenwoke, MSc, BMR, University of Nigeria, Nsukka, Enugu, Nigeria

Aims: Treatment-seeking behaviour, evaluation and management approach, as well as responsiveness to management by individuals with knee osteoarthritis (OA) could be hampered by variations of common confounding (constant) factors of knee OA; age and sex. Studies that integrate age and sex variations in clinical factors such as pain intensity and physical function are limited. The aim of this study was to determine age and sex variations in pain intensity and physical function among individuals with knee OA in Nigeria. Methods: Eighty-nine consecutively sampled patients diagnosed with knee OA from three selected public (secondary and tertiary) hospitals in Enugu, South-East Nigeria, participated in this cross-sectional survey. Instruments used were Visual Analogue Scale (VAS) and Ibadan Knee or Hip Osteoarthritis Measure (IKHOAM). Data were analysed using Analysis of Variance (ANOVA) and Students'-test with Bonferroni correction. The level of significance was set at 0.05. Results: Significant variations of age in pain intensity between age groups of 20-39 and 40-59 years $(\mathrm{md}=-3.68, \mathrm{p}=0.01$ and 60-79 years (md $=-3.23, p=0.04$ ) as well as physical function between age groups of $60-79$ and $20-39$ years $(\mathrm{md} .=20.85, \mathrm{p}=0.02$ ) and $40-59$ years $(\mathrm{md}=10.70, \mathrm{p}=0.03)$ were observed. There was a significant sex variation in physical function, $(\mathrm{F}=9.57, \mathrm{p}<0.05)$ but not in pain intensity $(\mathrm{F}=2.91, \mathrm{p}=0.09)$ on one-way ANOVA, with females reporting higher scores on pain intensity $(5.29 \pm 2.97)$ and lower scores on physical function $(64.06 \pm 17.26)$ than males (3.94 \pm 2.29 and $78.77 \pm 17.08$, respectively). Conclusion: Age and sex should be considered by clinicians in management programs for knee OA so as to address the needs specifically.

\section{(3214) Patient co-design: development of a novel disease-specific PROM.}

Kate Hayes, PhD, BSc, The Wellcome Trust-Wolfson NI Clinical Research Facility - Belfast City Hospital, Belfast, Ireland; Elise Lammertyn, PhD, Cystic Fibrosis Europe, Brussels, Belgium; Hilde De Keyser, PhD, Cystic Fibrosis Europe, Brussels, Belgium; Trudy Havermans, PhD, University Hospital Leuven, Leuven, Belgium; Audrey Chansard, PhD, Epigenetics and Cell Fate Centre, UMR7216 CNRS, Université de Paris, Paris, France., Paris, France; Rosa
Coucke, PhD, Necker Hospital, Paris, France; Jutta Bend, PhD, Cystic Fibrosis Europe \& Mukoviszidose e.V. Bundesverband Cystische Fibrose (CF), Bonn, Germany; Isabelle Sermet, MD, PhD, Necker Hospital, Paris, France; submitted on behalf of "the European Cystic Fibrosis Society-Clinical Trial Network and Cystic Fibrosis Europe Patient Advisory Group"

Aims: The Patient Advisory Group of the European Cystic Fibrosis Society-Clinical Trial Network (ECFS-CTN) and Cystic Fibrosis Europe (CFE), the federation of national CF patient associations in Europe, are conducting a patient-led project which will enhance patient-clinician informed care in CF. The aim is qualitative development and cognitive evaluation of a new patient-led patient-reported outcome measure (PROM) to assess health-related quality of life and symptom report in CF. Utilising research co-design processes such as the 'INVVOLVE' guidelines (invest in co-design; needs assessment; vision roles, responsibilities and rewards; validate participants; organise interaction carefully; lead the engagement; value patient time and input, and evaluate and report) allows a structured approach to the meaningful involvement of all research users. Methods: A previous qualitative study $(n=125)$ of people with $\mathrm{CF}(\mathrm{pwCF})$ and their caregivers identified key themes and 10 structured domains for inclusion in the development of a new CF-specific PROM questionnaire, the Patient-Reported Outcomes in CF (PRO-CF). The results of this study were combined with the expertise of a pan-European expert-patient group who met monthly during 2019-2020 by teleconference to further identify and refine key themes for inclusion in a new CF-specific PROM. A 2nd round review of the tool for content and face validity was conducted $(n=15)$ in 2021 , with additional expert patients reviewing and refining the tool to create the current 10 domain, 68-item questionnaire. Results: Initial review of the tool has confirmed that introductory instructions require clarification, and response scales are relevant and can be clearly interpreted by pwCF. Additional items were also identified for inclusion. The tool is currently being refined, and next steps will address validation and translation including additional psychometric evaluation in patients with $\mathrm{CF}$ including those awaiting transplant. Conclusion: There is a lack of sensitive, standardised patient-reported outcome measures in the current context of new disease modifying therapies in CF. This collaborative project by the ECFS-CTN \& CFE is unique, engaging people with $\mathrm{CF}$ to create a meaningful and sensitive disease-specific PROM, to validate a new questionnaire for use in CF clinical trials and enhance patient-clinician informed care in $\mathrm{CF}$.

(3215) Natural Language Processing in Medicine: a machinebased trend analysis

Safoora Masoumi, MD, Mazandaran University of Medical Sciences, Sari, Iran; Hossein Amirkhani, PhD, Computer and Information Technology Department, University of Qom, Qom, Iran; Najmeh Saedeghian, MPH, Mazandaran University of Medical Sciences, Sari, Iran; Saeid Shahraz, MD PhD, ICON PLC and Tufts Medical Center, San Francisco, California, USA

Aims: Medical Natural Language Processing (NLP) makes our understanding of patient data richer. We used NLP methods to explore how medical NLP has evolved during the last two decades to serve as an instrumental tool to enrich patient data. Methods: Scanning four medical abstract databases, PubMed, Embase, Psychinfo, and CINAHL, we identified 22,294 with a final selection of 12,817 English abstracts published between 2000 to 2020 . We invented a manual classification of medical fields, the context of use, and text sources and created a training dataset by reviewing 485 abstracts. We employed machine learning algorithms to classify the abstracts in the final corpus into those obtained in the trained dataset. This language model, developed by Google, is called Bidirectional Encoder Representations from 
Transformers or BERT for short. The evaluation showed that the trained models' accuracies in classifying abstracts into their text source, the context of use, and the medical field were $78.5 \%, 77.3 \%$, and $87.6 \%$, respectively. We report the growth rate of the publications. Results: Abstracts showed an annual growth rate (AGR) of $17.07 \%$ between 2000 and 2020 (72.01 articles (95\% CI 56.80-78.30) increase per year), with $81.76 \%$ of the abstracts published between 2010 and 2020 . The growth was exponential $(\mathrm{R} 2=0.93)$. Studies on neoplasms constituted $27.66 \%$ of the entire corpus with an annual growth rate of $42.41 \%$, followed by studies on mental conditions with an AGR of $39.28 \%$ and a proportion of $16.27 \%$ of the entire corpus. While electronic health or medical records comprised the highest proportion of text sources (57.12\%), omics databases had the highest growth among all text sources with an AGR of $65.8 \%$. Leaving the miscellaneous uses of NLP in medical fields out of our calculations, the most common NLP application was clinical decision support (25.45\% of all abstracts). Conclusion: Medical NLP, with exponential growth in the last two decades, has been diversely applied to make our understanding of patients and their data at different levels richer. However, this growth was unequal across varying medical fields and applications. Further research is needed to promote the use of NLP in all medical fields and to all patients.

(3216) Experience with the collaborative health platform HUGO EM in patients with multiple sclerosis.

Antonio Torres, Dynamic Science S.L., Madrid, Spain; Esther Álvarez, Evidence Generation Lead, Department of Evidence

Generation, Dynamic Science, Madrid, Spain; Laura Prieto, Evidence Generation Lead, Department of Evidence Generation, Dynamic Science, Madrid, Spain; Santiago Rodríguez, Head of Observational Studies Department, Observational Studies Department, Madrid, Spain

Aims: Patient-generated health data (PHGD) is a primary and reliable data source that uses new technologies to perform research and that has a patient-centered approach. PHGD allows to obtain patientcentric insight not available and, therefore, complementary to traditional healthcare datasets. HUGO EM is a new collaborative technological platform for clinical research for people with multiple sclerosis (MS), presented as a smartphone application (app). The objective of the study is to identify potential factors related to physical activity, fatigue, depressive mood, the evolution of symptoms and disease outcomes in these patients recording in the HUGO EM app. Methods: This is a Real-World Data study with a prospective design conducted in adult patients diagnosed with MS. Patients give their electronic consent for their participation in the study. The data is collected directly (included by the patient in the app; e.g., diagnosis, Expanded Disability Status Scale [EDSS], relapses, history of MS, sociodemographic data, health habits, treatment, symptoms, visits to the neurologist, emergency visits, and fatigue), indirectly (automatically collected by the device; e.g., number of steps, distance, time spent, and consumed energy) and complementary real-time data from external databases (e.g., weather). There is no defined follow-up period in the study; patients can participate until they decide otherwise, revoke their consent, or uninstall the app. Results: Since the launch of the app on 22 April 2021, more than 1. 200 users have downloaded HUGO EM in Spain. In September 2021, available user data will be analyzed, based on the objectives of the study. Conclusion: The results will provide information on the identification of factors related to physical activity, among other aspects impaired in MS, thus, allowing to extract recommendations to help improve the quality of life of these patients.
(3217) Capturing and measuring the patient voice in rare disorders: benefit of pragmatic mixed methods research in NUT Carcinoma

Anna Ciesluk, Modus Outcomes, Cambridge, Massachusetts, USA; Maarten Voorhaar, Boehringer Ingelheim, Ingelheim, Germany; Louise Barrett, Modus Outcomes, Letchworth Garden City, UK; Jessica Baldasaro, Modus Outcomes, Cambridge, Massachusetts, USA; Ingolf Griebsch, Boehringer Ingelheim, Ingelheim, Germany; Patrick Marquis, Modus Outcomes, Cambridge, Massachusetts, USA

Aims: Patient-centered outcome measurement (PCOM) is essential to capture the outcomes important to patients. However, it presents unique challenges in rare diseases, particularly those that are "young" (not diagnosed before the twenty-first century), with limited literature, lack of disease-specific patient-reported outcome (PRO) measures, and difficult sampling and data collection. One example of this is NUT (nuclear protein of the testis) carcinoma (NUTca), a rare and rapidly progressing cancer, with tumors preliminary in the head, neck, and lungs. The published literature on NUTca is scarce. The limited number of case reports focus primarily on the clinical development and presentation of tumors. Currently, there are no publications describing the patient experience of NUTca and no PRO measures for this rare disease. We conducted mixed-methods research, including concept elicitation interviews, cognitive debriefing, and quantitative data analyses, to fill this evidence gap and describe challenges and solutions in the context of NUTca. Methods: Results: First, we interviewed twenty-seven patients $(\mathrm{n}=10)$ and caregivers $(\mathrm{n}=17)$ using a semi-structured format. Caregivers were included, as some patients were too sick or recently deceased. Second, we developed an adaptable conceptual framework allowing the description of locationspecific symptoms-which can be further expanded with new locations-and general manifestations across tumor locations, including pain and systemic effects. Third, we used a bolt-on strategy to develop a bespoke PRO measure based on the EORTC QLQ-C30, supplemented by targeted items from the EORTC item library and new items. Fourth, given the variety of tumor locations and small samples of patients providing response to location-specific symptom items, we used response option endorsement frequencies to illuminate the variability of response for the concepts measured. The small

Table 1. FACE-Q Skin Cancer Scales and Checklist

\begin{tabular}{|c|c|c|c|}
\hline & $\begin{array}{l}\text { Primary repair } \\
(n=55)\end{array}$ & $\begin{array}{l}\text { Second intention } \\
\text { healing ( } n=88 \text { ) }\end{array}$ & p-value \\
\hline Satisfaction with Facial Appearance scale, mean (SD)* & $88.5(16.4)$ & $91.8(15.4)$ & 0.10 \\
\hline Appraisal of the Scar scale, mean (SD)* & $92.1(14.7)$ & $92.8(13.7)$ & 0.92 \\
\hline Appearance-Related Distress scale, mean (SD)* & $20.4(6.6)$ & $19.2(6.2)$ & 0.49 \\
\hline \multicolumn{4}{|l|}{ Adverse Effects, mean (SD) ${ }^{\dagger}$} \\
\hline Pain & $1.0(0.1)$ & $1.0(0.1)$ & 0.74 \\
\hline Discomfort & $1.1(0.3)$ & $1.1(0.4)$ & 0.88 \\
\hline Sensibility & $1.0(0.3)$ & $1.1(0.4)$ & 0.19 \\
\hline Numbness & $1.1(0.3)$ & $1.1(0.3)$ & 0.91 \\
\hline Tingling & $1.1(0.3)$ & $1.1(0.2)$ & 0.70 \\
\hline Tightness & $1.2(0.4)$ & $1.2(0.5)$ & 0.92 \\
\hline Itchiness & $1.1(0.4)$ & $1.1(0.5)$ & 0.91 \\
\hline Swelling & $1.1(0.4)$ & $1.1(0.2)$ & 0.27 \\
\hline Bruising & $1.0(0.1)$ & $1.0(0.2)$ & 0.85 \\
\hline Difficulty facial movements & $1.0(0.4)$ & $1.0(0.1)$ & 0.73 \\
\hline
\end{tabular}


sample sizes precluded any further psychometric analysis, even methods able to handle small samples such as Rasch Measurement Theory (RMT). Conclusion: This study highlights the challenges in implementing patient-centric research to inform and develop PRO measures in rare diseases. Our mixed-methods research used pragmatic solutions to start collecting patient experience data and provides an evidence-base to inform clinical programs in a rapidly progressing rare cancer with high unmet need.

\section{(3218) Kinesiophobia, fall self-efficacy and perceived balance in knee and/or hip osteoarthritis}

Ezinne Ekediegwu, B.M.R (Hons.), M.Sc(Hons.), Nnamdi Azikiwe University, Enugu, Nigeria; Earnest Chigbogu, B.M.R, Nnamdi Azikiwe University, Anambra, Nigeria; Ifeoma Nwosu, B.M.R (Hons.), M.Sc (Hons.), Nnamdi Azikiwe University, Enugu, Nigeria; Nelson Ekechukwu, B.PT, M.Sc, Ph.D, University of Nigeria, Nsukka, Enugu, Nigeria

Aims: Pain was commonly reported among adults with knee and or hip OA. There is a significant correlation between fall self-efficacy and perceived balance in developed countries. There is a dearth in literature on the relationships among kinesiophobia, fall self-efficacy and perceived balance among adults with knee and/or hip OA in a developing country like Nigeria seeing that psychosocial factors could be influenced by ethnicity or race.Aims: To determine the correlation amongst kinesiophobia, fall self-efficacy and perceived balance and also to determine the difference in male and female scores of kinesiophobia, fall self-efficacy and perceived balance among adults with knee and/or hip OA. Methods: This study adopted a cross sectional research design. Seventy individuals with knee and or hip OA were recruited from selected hospitals in southern Nigeria. The Tampa scale for kinesiophobia (TSK), Fall efficacy scale (FES), Timed up and go test (TUG) were used to assess kinesiophobia, fall self-efficacy and perceived balance amongst the participants. Spearman rank order correlation was used to determine the correlation among kinesiophobia, fall self-efficacy, and perceived balance among adults with knee and or hip OA. Mann Whitney U test was used to determine difference between male and female scores of kinesiophobia, fall self-efficacy, and perceived balance among adults with knee and or hip OA. Alpha level was set at 0.05. Results: Pain was commonly reported by all participants. There was significant correlation between fall self-efficacy and perceived balance $(\mathrm{p}<0.01)$. There was no significant correlation between kinesiophobia and fall self-efficacy; and perceived balance. There was no significant difference between the male and female scores of kinesiophobia, fall self-efficacy and perceived balance among adults with knee and/or hip OA. Conclusion: Kinesiophobia increases as one ages, fall selfefficacy and perceived balance suggest that kinesiophobia and fallrisk assessment should be incorporated in physiotherapy assessment of adults with knee and or hip OA.

\section{(3220) User experiences with Patient Activity Treatment Outcome Scale (PATOS) for person-centred practice in patients undergoing surgery for hip- or knee arthritis}

Stine Thestrup Hansen, Postdoc, PhD, MScN, RN, Department of Plasticsurgery and Breast Surgery, Zealand University Hospital/ University of Southern Denmark, Roskilde, Denmark; Rasmus Stig Jensen, Partner, Procordo, Copenhagen, Denmark; Anette Liljensøe, $\mathrm{PhD}, \mathrm{MSc}, \mathrm{RN}$, Procordo/Department of Orthopaedic Surgery, Aarhus University Hospital, Copenhagen, Denmark

Aims: The aim om this study is to investigate patients' and physicians' experiences of person-centred practice after the implementation of PATOS as ePROMs for patients undergoing surgery for knee- or hip arthritis in an orthopaedic outpatient setting. Methods: PATOS is a generic instrument developed as an ePROM to assess the status and preferences of patients with hip- or knee arthritis in four steps:Preoperative the patient identifies issues with daily activities regarding the predefined areas related to their hip- or knee arthritis diagnosis: Self-care work, duties, hobbies, and pastime. The patient prioritizes desirable focus issues of the surgical treatment. Maximum three issues can be selected. The patient rates the prioritized issues with current status plus indication of pain or discomfort on a 10-point likert scale $(1=$ not important at all, $10=$ extraordinary important) Postoperative, three and six months after the surgery, the patient repeats the scores on the selected issues and potential changes in patients' outcomes becomes available. This qualitative study is guided by Braun and Clarke's framework on qualitative research. Qualitative interviews with 20 patients and 10 physicians will be conducted three months after the implementation of PATOS. Interview guides are based on the smart definition of utility including ease of use, time, training and qualifications, format, and interpretation. The approach includes an inductive but transparent thematic step-bystep analysis and theoretical interpretation into thematic findings. Analysis is inspired by McCormack's theory of person-centred care Results: The first results from the study will be presented at the conference. Conclusion: This research investigates whether an innovative approach to integration of ePROMs in a doctor-patient relationship in an outpatient orthopaedic clinic contribute to personcentred practice experienced by patients. Finally, the study will add knowledge on integration of ePROMs in practice experienced by physicians to future PROM-based practice.

\section{(3221) Validation of anger scale on persons} with and without mental illness in Taiwan

Ay Woan Pan, PhD, School of Occupational Therapy, College of Medicine, National Taiwan University, Taipei, Taiwan; Chia-Fang Wu, MS, Department of Psychiatry,Veterans General Hospital, Taipei, Taiwan; Tsyr-Jang Chen, PhD, Department of Mechanical Engineering, Lung Hwa University of Science and Technology, Taoyuan, Taiwan

Aims: The literature has demonstrated that anger is related to aggression and could result in negative consequences. The purpose of this study is to examine the validity and reliability of the anger scale using Rasch analysis when applied to normal persons and persons with mental illness in Taiwan. Methods: Using the anger scale of the Patient-Reported Outcome and Management Information System (PROMIS), translated into traditional Chinese, 720 subjects participated in the study. Among them, 311 subjects were healthy persons without known diseases and 409 subjects were persons with mental illness. The data were analyzed using SPSS 22 and WINSTEPS 3.63.2 Results: The results showed that the anger scale has adequate rating scale structure and is unidimensional, and all items fit the Rasch measurement model except for one item. The resulting scale can discriminate subjects into eight levels of anger degrees. There were significant differences of anger scores among varying levels of psychiatric symptom severity. The correlation of the anger scale with self-reported quality of life (1-100) and the symptom severity score were significant at -0.404 and 0.721 , respectively. Conclusion: The results of the study demonstrated that the traditional Chinese version of the anger scale demonstrate acceptable validity and reliability and can be used as an index to identify subject with emotional problems. 
(3222) Patient satisfaction following primary repair or second intention healing after nasal skin cancer resection; a retrospective cohort study

Frederieke Theelen, BSc, Department of Plastic and Reconstructive Surgery, Catharina Hospital Eindhoven, Eindhoven, The Netherlands; Inge Veldhuizen, MD, Department of Plastic and Reconstructive Surgery, Catharina Hospital Eindhoven, Eindhoven, The Netherlands; Chao Zhou, MD PhD, Department of Plastic and Reconstructive Surgery, Maastricht University Medical Center, Eindhoven, The Netherlands; Erica Lee, MD, Dermatology Division, Memorial Sloan Kettering Cancer Center, New York, New York, USA; Lucy Van Hensbergen, BSc, Department of Plastic and Reconstructive Surgery, Catharina Hospital Eindhoven, Eindhoven, The Netherlands; Coralien Broekhuysen, MD, Department of Plastic and Reconstructive Surgery, Máxima Medisch Centrum Veldhoven, Veldhoven, The Netherlands; René Van Der Hulst, MD PhD, Department of Plastic and Reconstructive Surgery, Maastricht University Medical Center, Maastricht, The Netherlands; Maarten Hoogbergen, MD PhD,

Department of Plastic and Reconstructive Surgery, Catharina Hospital Eindhoven, Eindhoven, The Netherlands

Aims: Nasal reconstruction after wide local non-melanoma skin cancer (NMSC) resection can be challenging. Before pathologic knowledge, a simple reconstruction like primary repair or second intention healing is favored. Yet, little is known about the patient satisfaction following these reconstruction methods. This study aims to evaluate patient satisfaction following nasal skin cancer reconstruction with primary repair or second intention healing using the FACE-Q Skin Cancer. Methods: All patients who underwent wide local excision of nasal NMSC with immediate primary repair or second intention healing between March 2018 and March 2020 at Máxima Medisch Centrum Veldhoven were identified. Patients were contacted $\geq 9$ months after the reconstruction and asked to complete the FACE-Q Skin Cancer Satisfaction with Facial Appearance, Appraisal of the Scar, and Appearance-Related Distress scale, and the Adverse Effects checklist. Results: Of the 183 patients, a total of 140 patients (143 nasal defects) completed the questionnaire. Of these, 55 defects were closed by primary repair $(38.5 \%)$ and 88 by second intention healing $(61.5 \%)$. Thirty-one complications were reported $(16.7 \%)$, of which $87.1 \%(\mathrm{n}=27)$ following second intention healing $(\mathrm{p}=0.004)$. Both groups experienced high satisfaction with facial appearance and scar appraisal, low appearance-related distress, and no to minimal adverse effects (Table 1). Multivariable regression analyses showed that second intention healing had 2.7 higher odds to achieve maximum scar satisfaction compared with primary repair $(p=0.02)$. The occurrence of a complication was independently associated with lower satisfaction with facial appearance $(\mathrm{p}=0.03)$, lower scar appraisal $(p=0.02)$, and lower odds of achieving maximum facial appearance $(\mathrm{p}=0.02)$. Higher age was associated with higher facial and scar satisfaction (both $\mathrm{p}=0.004$ ), higher odds of reporting maximum satisfaction (both $\mathrm{p}=0.02$ ), and less distress $(\mathrm{p}=0.01)$. Previous oncologic facial surgery was associated with lower facial satisfaction $(p=0.02)$. Lower appearance-related distress was seen in patients with a non-aggressive tumor type $(\mathrm{p}=0.03)$. Conclusion: This study shows high satisfaction on facial- and scar appraisal, low appearance-related distress, and no to minimal adverse effects for second intention healing and primary repair after NMSC excision on the nose. Insight into which factors influence these outcomes provides opportunities for clinicians to maximize these outcomes for patients.

Publisher's Note Springer Nature remains neutral with regard to jurisdictional claims in published maps and institutional affiliations. 